Claremont Colleges

Scholarship@Claremont

2012

\title{
Analyzing Best Practices in the Schooling of Secondary-Level Latino Newcomer Immigrant Youth: A Comparison Study of Two Yearlong Specialized Programs
}

Amanda Keri Matas

Claremont Graduate University

\section{Recommended Citation}

Matas, Amanda Keri, "Analyzing Best Practices in the Schooling of Secondary-Level Latino Newcomer Immigrant Youth: A Comparison Study of Two Yearlong Specialized Programs" (2012). CGU Theses \& Dissertations. Paper 68.

http://scholarship.claremont.edu/cgu_etd/68

DOI: $10.5642 /$ cguetd $/ 68$

This Open Access Dissertation is brought to you for free and open access by the CGU Student Scholarship at Scholarship @ Claremont. It has been accepted for inclusion in CGU Theses \& Dissertations by an authorized administrator of Scholarship @ Claremont. For more information, please contact scholarship@cuc.claremont.edu. 


\section{ANALYZING BEST PRACTICES IN THE SCHOOLING OF SECONDARY-LEVEL LATINO NEWCOMER IMMIGRANT YOUTH: A COMPARISON STUDY OF TWO YEARLONG SPECIALIZED PROGRAMS}

\section{BY}

AMANDA KERI MATAS

A dissertation submitted to the Faculty of Claremont Graduate University and San Diego State University in partial fulfillment of the requirements for the degree of Doctor of Philosophy in the Graduate Faculty of Education

Claremont, California

San Diego, California

2012

(C) Copyright Amanda Matas, 2012

All rights reserved. 


\section{APPROVAL OF THE REVIEW COMMITTEE}

This dissertation has been duly read, reviewed, and critiqued by the Committee listed below, which hereby approves the manuscript of Amanda Matas as fulfilling the scope and quality requirements for meriting the degree of Doctor of Philosophy in the Graduate Faculty of Education.

Dr. Karen Cadiero-Kaplan, Co-Chair

San Diego State University

Dr. William Perez, Co-Chair

Claremont Graduate University

Dr. Valerie Ooka Pang, Committee Member

San Diego State University

Dr. Carl Cohn, Committee Member

Claremont Graduate University 


\begin{abstract}
OF THE DISSERTATION
ANALYZING BEST PRACTICES IN THE SCHOOLING OF SECONDARY-LEVEL LATINO NEWCOMER IMMIGRANT YOUTH: A COMPARISON STUDY OF TWO YEARLONG SPECIALIZED PROGRAMS
\end{abstract}

\author{
By \\ Amanda Keri Matas \\ Claremont Graduate University \& San Diego State University
}

2012

The purpose of this research study is to compare two yearlong program models designed specifically to educate secondary-level newcomer immigrant youth within one large, urban school district in Southern California. The two divergent secondary-level programs that are compared in this study, a self-contained newcomer program and a beginning level English as a Second Language program (ESL 1/2), are explored to determine which program more successfully prepares secondary-level Latino immigrant youth to gain the language proficiency, academic skills, and academic self-concept necessary to exit after the requisite year.

The research for this study is informed by scholarly literature that concerns the education of immigrant youth. The literature review is driven by the following four central concepts: an analysis of significant federal and California state language policy, mitigating factors in the education of immigrant youth, existing specialized program models, and guiding theories in the schooling of linguistically and culturally diverse students. 
The data for this study was collected utilizing a mixed-methods multiple case study approach. Three classrooms within each of the two programs were observed over a month-long period as simultaneous stakeholder interviews and focus groups were carried out to illuminate emergent themes and tensions. Additionally, both current and former students from the two programs were surveyed to determine their academic, social, and personal self-concept levels. The qualitative and quantitative data gathered through this study was analyzed and triangulated to determine the effectiveness of each program and answer the guiding research questions.

The results of this study demonstrated mixed findings between the two programs under study. The students gained greater academic skill levels and a higher academic self-concept level as a result of the more supportive environment offered within the newcomer program, yet the ESL 1/2 students made greater gains linguistically, as was evidenced by higher redesignation rates. In addition, after their second year, the students from the newcomer program reported far lower academic self-concept levels than those who had exited the ESL program. Therefore, due to the mixed results, this study incorporated an action plan to assist districts in creating and implementing effective programs for newcomer youth. 


\section{DEDICATION}

I dedicate this dissertation to my loving and supportive family.

The truly heroic level of patience and understanding you have all displayed over the past many years has kept me going through the dissertation process and has led me to the finish line. Thank you Mom, Nicole, Brenda, and Jan for your belief in me, and your ability to listen to endless hours of educational facts and statistics with smiles on your faces.

This dissertation is also dedicated to the two Dr. Matases that came

before me: my father, Dr. Brian Matas, and my grandfather, Dr. Matthew Matas.

Your many accomplishments and continual guidance has inspired me to

follow in your extraordinary footsteps. Thank you for showing me how to work hard for what is important and how to stay true to my passions and my convictions. 


\section{ACKNOWLEDGEMENTS}

First and foremost, I would like to acknowledge the hard work and patience of my committee for guiding me throughout the doctoral process. I would like to specifically thank my co-chair, Dr. Karen-Cadiero Kaplan, for being an exemplary mentor, teacher, coach, and friend. I could not even imagine completing this process without your frequent advice and constant support, and I truly appreciate the many times you kept me moving forward despite the usual bumps in the road. You have been an absolute blessing throughout this process. I would additionally like to thank my co-chair, Dr. William Perez, for inspiring me to work hard and for demonstrating the importance of strong research. I would like to thank Dr. Valerie Ooka Pang and Dr. Carl Cohn for supporting me and encouraging me to become a strong academic. All of your long hours and dedication made this work possible. Finally, I would to thank Dr. Alberto Ochoa for taking the time to meet with me and providing me extensive feedback on my dissertation. You went above and beyond for me and for my research, and I appreciate both your guidance throughout the process, as well as your mentorship as a professor and a strong community advocate.

I would also like to thank my friends and fellow doctoral students, Rachel Stein, Angela Ferreira, Dr. Andrea Yoder Clark, Dr. Gustavo Gonzalez, Dr. Oscar Jimenez-Castellanos, and Dr. Pablo Ramirez. Each of you has played a vital role in my completion of this dissertation. I would like to especially thank Rachel, Angela, and Andrea for being the most amazing "study buddies" and for always showing up to support me along the way. Your friendship is one of the most remarkable outcomes of this degree. 
This process would not have been possible had it not been for my incredible cheerleaders. I need to specifically thank Britt, Christine, April, Summer, Rachel, Tasha, Melissa, Jessica, Kristy, Jen, and Deanna for listening to endless hours of sometimes less than exciting news about my work. You have been the best sports throughout this process and words cannot express how much you all mean to me. You have all played an incredible role in the completion of this research study, as your belief in me has kept me going throughout the many years it has taken to finish this degree, as well as through the many ups and downs I have experienced in completing my Ph.D.

Finally, I would like to thank the individuals who gave their time and their wisdom to this study. The teacher participants who opened their doors to me are truly phenomenal examples of caring and passionate educators who put their students first. Teachers like those in this study are an inspiration to all. I must also thank the administrator and student participants who assisted me with this research. I could have never completed this study without your time and your expertise. 


\section{TABLE OF CONTENTS}

CHAPTER 1: Introduction 1

Statement of the Problem 1

Purpose of the Study 4

Study Objectives $\quad 5$

The Researcher $\quad 6$

$\begin{array}{ll}\text { Conceptual Framework } & 10\end{array}$

$\begin{array}{ll}\text { Study Significance } & 14\end{array}$

$\begin{array}{ll}\text { Definition of Applicable Terms } & 17\end{array}$

CHAPTER 2: Literature Review 20

Theoretical Framework 20

The Sociopolitical and Demographic Contexts of Immigration 24

A Brief History of Language Policy in the United States 27

Early Language Education Policy 28

$14^{\text {th }}$ Amendment of the United States Constitution 30

Bilingual Education Act of $1968 \quad 31$

The Lau v. Nichols Decision of 1974

The Castañeda v. Pickard Decision of 1981

California Proposition 63 of $1986 \quad 36$

California Proposition 187 of $1994 \quad 38$

California Proposition 227 of $1998 \quad 40$

No Child Left Behind Act of 2000

The English-only Movement and Immigrant Youth 46

Mitigating Factors in the Schooling of Immigrant Youth 49

Parallel Versus Non-Parallel Academic Experience $\quad 50$

Age and Language Considerations 52

Guiding Theories in the Education of Immigrant Youth 53

Krashen's Language Acquisition Theory 53

Culturally Relevant Pedagogy $\quad 60$

Multiliteracies Pedagogy and Immigrant Youth 65

Moving Beyond: Transformative Multiliteracies Pedagogy 67

Academic Self-Concept and Academic Achievement 73

Program Models Utilized in the Education of Immigrant Youth 76

$\begin{array}{ll}\text { Two-Way or Maintenance Bilingual Programs } & 77\end{array}$

$\begin{array}{ll}\text { Transitional Bilingual Programs } & 78\end{array}$

English as a Second Language Program and Sheltered 78

English Program Models

The Emergence of Newcomer Programs $\quad 80$ 
$\begin{array}{lr}\text { CHAPTER 3: Methodology } & 88\end{array}$

Research Design $\quad 91$

Selection of Study Participants $\quad 94$

Teacher Participants 98

Student Participants 101

Administrator Participants 105

Qualitative Data Collection Process 108

Quantitative Data Collection Process 111

Qualitative Data Collection Tools 114

Quantitative Data Collection Tool 116

Data Analysis and Triangulation of Findings 118

CHAPTER 4: Results 126

District and State-level Assessment and Placement of 128

English Learners

The Rapid Decline of Access to Bilingual/Biliteracy 133

Education

Overview of the English as a Second Language Program 135

Overview of the New Arrival Center Program 140

Program and Individual Case Level Redesignation Rates 147

Findings of the Student Self-Concept Scale 151

Overall Student Self-Concept Scale Findings $\quad 154$

Academic Self-Concept Subscale Findings 167

Academic Self-Concept Subscale Findings By 172

Sub-Characteristic

Classroom Observation Findings 184

Interview and Focus Group Themes and Tensions 199

English as a Second Language Stakeholders' Attitudes and 203

Expectations

English as a Second Language Program Curricula 208

Language Instruction Within the English as a Second 211

Language Program

Content Instruction Within the English as a Second 216

Language Program

Culturally Responsive Practices Within the English as 220

a Second Language Program

Teachers' Pedagogical Orientations Within the English 
as a Second Language Program

English as a Second Language Students' Academic

Self-Concept

New Arrival Center Stakeholders' Attitudes and Expectations 230

New Arrival Center Program Curricula 235

Language Instruction Within the New Arrival Center Program 239

Content Instruction Within the New Arrival Center Program 242

Culturally Responsive Practices Within the New Arrival 248

Center Program

Teachers' Pedagogical Orientations Within the New Arrival 252

Center Program

New Arrival Center Students’ Academic Self-Concept 256

Comparison and Contrast of the ESL and the NAC Program 260

Findings

CHAPTER 5: Implications $\quad 264$

Analysis and Interpretation of Sub-Question Findings 265

Research Question \#1 266

$\begin{array}{ll}\text { Curricula } & 266\end{array}$

Language Instruction $\quad 268$

$\begin{array}{ll}\text { Content Instruction } & 270\end{array}$

Stakeholders' Attitudes $\quad 273$

Cultural Responsiveness $\quad 277$

Pedagogical Orientations $\quad 280$

$\begin{array}{ll}\text { Research Question \#2 } & 284\end{array}$

$\begin{array}{ll}\text { Research Question \#3 } & 287\end{array}$

Academic Self-Concept $\quad 287$

Language Proficiency Levels 293

Academic Skills $\quad 297$

Analysis and Interpretation of Overall Research Question 299

Discussion of Program Implications 300

Emergent Tensions Regarding the District English Learner Programs 302

An Action Plan to Develop and Improve Specialized English 309

Learner Programs

Other Research Study Recommendations $\quad 321$

Program 321

Curricula $\quad 323$

Language Instruction $\quad 323$

Content Instruction $\quad 324$

Cultural Responsiveness $\quad 325$

Pedagogical Orientations $\quad 326$

Academic Self-Concept $\quad 327$

Research Study Assumptions 328 
Research Study Limitations $\quad 328$

Recommendations for Future Research $\quad 332$

Personal Reflection $\quad 333$

$\begin{array}{ll}\text { REFERENCES } & 335\end{array}$

APPENDICES 343

A. Parental/Guardian Permission/Informed Consent 343

B. Student Participant Informed Consent 349

C. Teacher and Administrator Informed Consent 355

D. Classroom Observation Protocol 358

E. Observation Field Notes Record 362

F. Administrator Interview Questions 363

G. Teacher Interview Questions 364

H. Student Focus Group Questions $\quad 365$

I. SSCS Student Questionnaire 367

J. Disaggregated Academic Self-Concept Subscale Findings 377

K. Chi-Square Analysis of Academic Self-Concept Subscale Findings 387

L. Classroom Observation Indicator Matrix $\quad 390$

M. Completed Classroom Observation Indicator Matrix 392

N. NAC Teacher Interview Analysis 398

O. ESL Teacher Interview Analysis $\quad 430$

P. NAC Administrator Interview Analysis 446

Q. ESL Administrator Interview Analysis 465

R. NAC Focus Group Analysis 481

S. ESL Focus Group Analysis 


\section{LIST OF TABLES}

Table 1. Participating Teacher's Demographics

Table 2. Participating Teachers' Class Compositions

Table 3. Focus Group \#1 Student Demographics - New Arrival Center Year 1

Table 4. Focus Group \#2 Student Demographics - New Arrival Center Year 1

Table 5. Focus Group \#3 Student Demographics - ESL Program Year 1

Table 6. Focus Group \#4 Student Demographics - ESL Program Year 1

Table 7. Focus Group \#5 Student Demographics - New Arrival Center Year 2

Table 8. Focus Group \#6 Student Demographics - ESL Program Year 2

Table 9. Cronbach's Alpha Reliability Analysis for Instrument Subscales

Table 10. Overall Program Percent Redesignation Rates Over Five-Year Period 148

Table 11. Individual Case Study Percent Redesignation Rates Over

Five-Year Period

Table 12. Student Self-Concept Scale Confidence Level Analysis - Total ESL Student Participants and Total NAC Student Participants (Years 1 and 2)

Table 13. Student Self-Concept Scale Item Importance Analysis - Total ESL Student Participants and Total NAC Student Participants (Years 1 and 2)

Table 14. Student Self-Concept Scale Confidence Level Analysis-ESL-Year 1 Student Participants and NAC-Year 1 NAC Student Participants

Table 15. Student Self-Concept Scale Item Importance Analysis-ESL-Year 1 Student Participants and NAC-Year 1 NAC Student Participants

Table 16. Student Self-Concept Scale Confidence Level Analysis-ESL-Year 2 Student Participants and NAC-Year 2 NAC Student Participants

Table 17. Student Self-Concept Scale Item Importance Analysis-ESL-Year 2 Student Participants and NAC-Year 2 NAC Student Participants 
Table 18. Student Self-Concept Scale Confidence Level Analysis-ESL-Year 1 Student Participants and ESL-Year 2 NAC Student Participants

Table 19. Student Self-Concept Scale Item Importance Analysis-ESL-Year 1 Student Participants and ESL-Year 2 NAC Student Participants

Table 20. Student Self-Concept Scale Confidence Level Analysis-NAC-Year 1 165 Student Participants and NAC-Year 2 NAC Student Participants

Table 21. Student Self-Concept Scale Item Importance Analysis-NAC-Year 1 Student Participants and NAC-Year 2 NAC Student Participants

Table 22. Academic Self-Concept Confidence Level Subgroup Analysis 168 Total ESL Student Participants and Total NAC Student Participants (Years 1 and 2)

Table 23. Academic Self-Concept Item Importance Subgroup Analysis 170 Total ESL Student Participants and Total NAC Student Participants (Years 1 and 2)

Table 24. Academic Self-Concept Confidence Level Subscale Characteristic Patterns - Total ESL Student Participants and Total NAC Student Participants (Years 1 and 2)

Table 25. Academic Self-Concept Item Importance Subscale Characteristic Patterns - Total ESL Student Participants and Total NAC Student Participants (Years 1 and 2)

Table 26. Student Self-Concept Scale Confidence Level Analysis ESL-Year 1 Student Participants and NAC-Year 1 Student Participants

Table 27. Student Self-Concept Scale Item Importance Analysis ESL-Year 1 Student Participants and NAC-Year 1 Student Participants

Table 28. Student Self-Concept Scale Confidence Level AnalysisESL-Year 2 Student Participants and NAC-Year 2 Student Participants

Table 29. Student Self-Concept Scale Item Importance Analysis180 ESL-Year 2 Student Participants and NAC-Year 2 Student Participants 
Table 30. Student Self-Concept Scale Confidence Level Analysis ESL-Year 1 Student Participants and ESL-Year 2 Student Participants

Table 31. Student Self-Concept Scale Item Importance AnalysisESL-Year 1 Student Participants and ESL-Year 2 Student Participants

Table 32. Student Self-Concept Scale Confidence Level AnalysisNAC-Year 1 Student Participants and NAC-Year 2 Student Participants

Table 33. Student Self-Concept Scale Item Importance AnalysisNAC-Year 1 Student Participants and NAC-Year 2 Student Participants

Table 34. Number of Effective English Learner Instructional Indicators Recorded During Individual Case Observations

Table 35. Mean Number of Effective English Learner Instructional Indicators Recorded During Program Observations

Table 36. Number of Culturally Relevant Pedagogical Indicators Recorded During Individual Case Observations

Table 37. Mean Number of Culturally Relevant Pedagogical Indicators Recorded During Program Observations

Table 38. Qualitative Findings Overview - English as a Second Language Program

Table 39. Qualitative Findings Overview - New Arrival Center Program 


\section{LIST OF FIGURES}

Figure 1. Conceptual Framework Guiding the Study of Newcomer Immigrant Youth

Figure 2. Theoretical Framework Guiding the Study of Newcomer Immigrant Youth

Figure 3. Nested Pedagogical Orientations

Figure 4. Overview of Methodological Process

90

Figure 5. Two-Tailed Multiple-Case Study Research Design

93

Figure 6. Macro and Micro Levels of Data Collection

Figure 7: District English Learner Flowchart From Identification to Reclassification

Figure 8. Overall Program Percent Redesignation Rates Over Five-Year Period

Figure 9. Individual Case Study Percent Redesignation Rates Over Five-Year Period

Figure 10. Student Self-Concept Scale Item Sub-Characteristic Codes

Figure 11. Effective English Learner Instruction Indicators Observed During First, Second, and Third Case Observations

Figure 12. Number of Effective English Learner Instructional Indicators Recorded During Individual Case Observations

Figure 13. Mean Number of Effective English Learner Instructional Indicators Recorded During Program Observations

Figure 14. Culturally Relevant Pedagogy Indicators Observed During First, Second, and Third Case Observations

Figure 15. Number of Culturally Relevant Pedagogical Indicators Recorded During Individual Case Observations

Figure 16. Mean Number of Culturally Relevant Pedagogical Indicators Recorded During Program Observations 
Figure 17. Pedagogical Orientation Indicators Observed During First, Second, 199 and Third Case Observations 


\section{CHAPTER 1}

Introduction

\section{Statement of the Problem}

The current trend in immigration statistics points to a sharp rise in the number of school-aged children entering secondary level classrooms in the U.S. public school system (Lucas, 1996). According to data gathered from the U.S. Current Population Survey released by the U.S. Census of Population and Housing, "the total foreign-born population passed 34 million in 2004" (Capps, Fix, Murray, Ost, Passel, 2005, p. 5), which was, "more than 3 million people higher than in 2000 and more than triple the figure of 10 million in 1970" (Capps et. al., 2005, p. 5). As of 2010, the foreign-born population increased further to 40 million residents according to the American Community survey (U.S. Census Bureau, 2012). The growth in overall immigration to the United States accounts for the statement that "the share of children of immigrants among school-age population increased rapidly, tripling from six percent in 1970 to 19 percent in 2000" (Fix \& Capps, 2005, p.2). Additionally, a large majority of the newcomer immigrant youth entering the school system have emigrated from countries whose national language is a language other than English. The Urban Institute of Washington D.C. reported that in $2000,80 \%$ of immigrant children attending classes in K-12 schools originated from Latin America and Asian countries, as compared to $36 \%$ in 1970 (Fix \& Passel, 2003; Capps et. al., 2005), over one-third of these same students having emigrated solely from Mexico. While not every student emigrating from these countries is limited English proficient, a system to support those who are new English 
speakers must be in place to best support said students.

As newcomer immigrant youth enroll in our nation's schools, they are commonly placed in grade-level classes and educated solely in English. Consequently, many newcomers are taught in an environment that can be largely incomprehensible linguistically and conceptually, particularly for those who do not have any prior experiences or education in English. A full assimilation approach to mainstreaming newcomer immigrant youth can be particularly difficult for secondary-level students, as these students are additionally contending with rigorous content-area classes rife with both high level and often decontextualized academic English vocabulary and highly abstract concepts (Custodio, 2011). As Jim Cummins (1986) argues, it takes five to seven years for English learners to attain the necessary cognitive-academic language proficiency to be sufficiently fluent in English for success in the context-reduced, cognitively demanding activities of reading, writing, mathematics, science, and other academic subjects. Therefore, students cannot be expected to learn academic concepts in their required grade-level content classes if they cannot understand the highly demanding language of the teacher or the even more complex language present in content-area curricula and textbooks.

The difficulty facing newcomer immigrant youth in understanding others, communicating basic needs, and comprehending new academic vocabulary and concepts can have a negative effect on such students (Lucas, 1996; Zwiers, 2004), and eventually lead to a lower academic self-concept level, or academic view of oneself. Low academic self-concept is a likely contributor to the alarmingly high dropout rates among secondarylevel immigrant youth. In 2003, 39.4 percent of 16- through 24-year-old Latinos born 
outside of the United States were high school dropouts, as compared to the 11.9 percent of 16- to 24-year-old Latinos born in the United States who did not complete high school (Laird, Lew, DeBell, \& Chapman, 2006). As much of the available research related to academic self-concept and academic achievement contends there is a positive correlation between higher academic self-concept and the acquisition of English, as well as overall academic success (Hung Hon \& Yeung, 2005; Kim, 1983; Krashen, 1981; Politzer and Ramirez, 1981), it has become increasingly essential for schools to investigate how to best enable immigrant youth to sustain or develop a strong academic sense of self.

In 1999, the U.S. Census Bureau ascertained that 2.7 million students in K-12 schools were foreign-born, this number accounting for $5 \%$ of the total school-aged population in the United States (Fix \& Passel, 2003; Jamieson, Curry, \& Martinez, 2001). In 2010, the U.S. Census Bureau reported the number of foreign-born youth in the United States to have increased to $7.1 \%$ of the total school-aged population (U.S. Census Bureau, 2012). Knowing that a large percentage of foreign-born students speak a primary language other than English, it has been crucial for schools to develop an alternative manner in which to educate newcomer students (Short \& Boyson, 2004). As a response to the tremendous needs of secondary-level newcomer immigrant youth, many districts have established self-contained newcomer programs, either as part of an existing school site or as a separate school. Newcomer programs concentrate on delivering intensified English acquisition courses, comprehensible content-area courses, and courses to help students adjust to the cultural norms of the United States (Short \& Boyson, 2004). These schools not only offer a supportive environment for students who have newly arrived to the United States, but they also frequently work with parents and families to create an 
educational experience that benefits all family members. The concept of newcomer programs has grown over the past thirty years, and as of the early part of the $21^{\text {st }}$ century, more than 110 newcomer programs have been established in 26 states (Feinberg, 2000). Although often structurally and organizationally different, these programs tend to hold similar goals and values in educating newly arriving immigrant youth.

Newcomer programs are an alternative to the traditional English as a Second Language (ESL) programs in which many secondary-level immigrant youth are placed upon entering the U.S. school system. ESL programs are typically comprised of a class or block of classes where instructors use a variety of strategies to teach beginning English language learners reading, writing, listening, and speaking skills in English. Such classes replace students' mainstream English classes and rather focus on English language acquisition and literacy skills. Historically, ESL classes are not used to teach students content, such as mathematics, history, or science, as students within an ESL program track are typically mainstreamed into their content-area classes. While both programs have strengths and challenges, the question remains as to which program is more effective in educating and supporting the needs of newcomer immigrant youth.

\section{Purpose of the Study}

This research study analyzed the manner in which newcomer programs could potentially support Latino immigrant youth in navigating through secondary school programs, and compared newcomer programs to the more traditional English as a Second Language program placement. This study further investigated how each program model assists students in acquiring language and academic skills, as well as how connections 
between students' home languages and cultures and that of the Unites States are considered within their education. This research additionally examined the connection between academic-self concept and language and academic achievement, comparing students' views of themselves both during and after completing either a newcomer program or a more traditional ESL program.

\section{Study Objectives}

The objective of this research study was to investigate which of two program models best supported secondary-level Latino newcomer immigrant youth as they embarked upon their educational course within the San Diego Unified School District. The San Diego Unified School District was chosen for this study as it was a large urban district in Southern California whose student population contained over 20\% Spanishspeaking English learners (SDUSD, 2011), a number of which were newcomer youth. This study analyzed and evaluated the experiences of Latino newcomer students as they attended or after they had completed their first year of education in either a self-contained newcomer program, or a traditional ESL placement. Based on the scholarly literature and the researcher's extensive classroom experience (more than 12 years teaching in an English as a Second Language classroom) it appeared to the researcher that students are more adequately supported when attending classes in a self-contained newcomer program. Unlike ESL programs, newcomer programs address gaps in students' literacy skills, while accelerating language proficiency and teaching content classes in a highly supported manner (Short \& Boyson, 2004; Custodio, 2011). 
This research study was guided by the following overarching research question and subsequent sub-questions:

How does a specialized newcomer program prepare secondary-level Latino immigrant youth to gain the language proficiency, academic skills, and academic self-concept necessary to be successful within an Englishonly educational environment, as compared to students placed in a traditional ESL program?

1. What are the prevailing pedagogical practices utilized in both a newcomer program and a traditional ESL program?

a. How do the curricula, language instruction, content instruction, teachers' attitudes, level of cultural responsiveness, and pedagogical orientations compare within the programs under study?

2. Is there a relationship between students' academic self-concept and the increase in their language proficiency level within either a specialized newcomer or a traditional ESL program?

3. How are Latino newcomer students' academic self-concept, language proficiency levels and acquisition of academic skills influenced by the program in which they are educated?

\section{The Researcher}

In initiating the process of researching and analyzing data concerning the education of secondary-level immigrant youth, it is essential that I make known the educational and personal route that has brought me to such critical work. During my childhood, I was raised in an affluent, yet politically liberal community in Northern California. This community offered an environment where kids could play safely and attend many after-school activities, yet there was little cultural and linguistic diversity. In addition, the social and economic stratification between this community and that of the neighboring city was quite overt. The schools were, and continue to be, highly supplemented by donations from families of the students, and therefore the education has 
always been specialized to meet individual students' needs. It can be argued that this community remains consciously sheltered by wealth and prosperity, and therefore, though members of the community espouse liberal ideals, little authentic social justice ideology or action is seen among the populace. My political consciousness came about by becoming aware, through outside experiences, of the vast inequities between my upbringing and education and that of the majority living in both this nation and internationally.

As a student in high school, I had the good fortune of spending a summer working in Ecuador with a community service organization. It was both an uplifting experience and one in which I struggled significantly, as my Spanish language skills were at an emergent level and I lived in a non-English speaking town. This two-month experience gave me a new understanding of the value and beauty of language and culture, as well as strong community ties, while also allowing me to experience first-hand the difficulty of not being understood by others around me. It gave me a greater understanding of how students who enter the United States often feel as they struggle to be understood by the educational system and the nation at large. This experience further helped me to appreciate that language and belief systems can either be used to support students in a manner that will give them the space to empower themselves, or conversely, they can be used to destroy students' self-confidence and self-worth by placing value only on the dominant language and ideology in a given populace.

After finishing high school, I earned an undergraduate degree at the University of San Diego, focusing on both education courses and Spanish language courses. This particular course of study led to the attainment of a Bilingual, Crosscultural, Language 
and Academic Development Teaching Credential, a necessity in achieving my ultimate career goal of teaching in a bilingual setting. Upon graduation and based on the school district's placement of me, I taught in a monolingual English self-contained $6^{\text {th }}$ grade class. I also immediately began working on a Master's Degree in the Policy Studies in Language and Cross-Cultural Education Department at San Diego State University. My Master's program opened enlightened me to such critical theorists as Paulo Freire, Donaldo Macedo, Antonia Darder, and bell hooks, to name a few. Through three years of thought-provoking discussion and deep personal contemplation, my sense of advocacy gained momentum and my need to work with immigrant youth became a passion.

Currently, I continue to work with newcomer immigrant youth at the secondary level. I have been fortunate enough to hold a teaching position these past thirteen years with youth who teach me daily about their personal cultures and identities, as well as the struggles many have faced in emigrating from their home countries. My students come from countries all over the world, yet they all face similar struggles as they enter middle school on the first day of classes. Many report a feeling of anxiety and fear, both because of the new language they are charged with learning in an unreasonable amount of time, and because of the new culture within which they must immediately negotiate the world. Such theorists as Baker (2011) and Portes and Rumbaut (2001) assert the notion that language assimilation is thought by many to demonstrate the willingness of immigrants to be part of the host country. Language assimilation is viewed as a symbol of the commitment people must make to the United States to prove their loyalty (Baker, 2011; Portes \& Rumbaut, 2001). Students are made to believe that they must learn English 
quickly and without question to become true Americans, and, with this notion, there is very little space for their own home languages and cultures (Macedo, 1994).

The need to relinquish one's identity, cultural heritage, and language to assimilate to American cultural norms can be a largely traumatic experience for immigrant students. Many immigrant youth enter the United States with the idea that they can and will become American, yet often they find that this is not the case solely by virtue of being in the United States. Laurie Olsen (1997) argues that a surprise for many immigrant students is that coming to America does not necessarily make them American. The common lack of cultural acceptance experienced by immigrant youth can exasperate feelings of isolation as they try to assimilate to the dominant language and culture in the United States. Olsen furthers this point when she writes, "for immigrant students, a lack of English fluency not only precludes them from access to core curriculum, but is a social stigma as well" (1997, p.94). My students face the often-difficult task of adapting to a new cultural, societal, and linguistic identity, while also facing a loss of their home languages and identities. These factors can lead immigrant youth to feel alienated from the educational system in which they are placed, and, furthermore, it denies them the fundamental tools necessary to critically think and reflect within American society (Macedo, 1994).

As an educator, it is my responsibility to ensure my students have a voice in the classroom and it is crucial that I take the time to listen to students as they share about their wants and needs for their education, as well as their personal lives. It is not enough, and can actually be quite detrimental to teach students to listen obediently in the classroom and follow directions without question. Rather, educators and school personnel 
must give youth the space to think and act deliberately upon their needs, both as students and as members of society. Listening to students and giving them true decision-making power while valuing their home languages and cultures will give those in the schooling system a better understanding of how to most effectively educate our youth. The following section will give an overview of the conceptual framework that will direct the proposed research study.

\section{Conceptual Framework}

The conceptual framework utilized to inform and guide this study, as demonstrated in Figure 1, focused on two overarching factors, each of which played a significant role in the creation of the newcomer and the ESL program models, as well as the achievement of the secondary-level immigrant youth. The factors investigated in this study were (1) overall classroom practices and (2) students' personal and academic identity, as the researcher analyzed the manner in which such factors were influenced by having been connected to one of the two programs under study. The two overarching concepts were explored to demonstrate their impact on both the creation and the implementation of the program models within the San Diego Unified School District, as well as the achievement of secondary-level immigrant youth within the district under study. Finally, the collection and analysis of the data took place through a transformative research paradigm, which follows that knowledge and reality are socially constructed and are shaped by political, cultural, ethnic, and economic values, as well as issues of power (Mertens, 2005). This section elaborates further on the aforementioned concepts in the following paragraphs. 
Figure 1: Conceptual Framework Guiding the Study of Newcomer Immigrant Youth

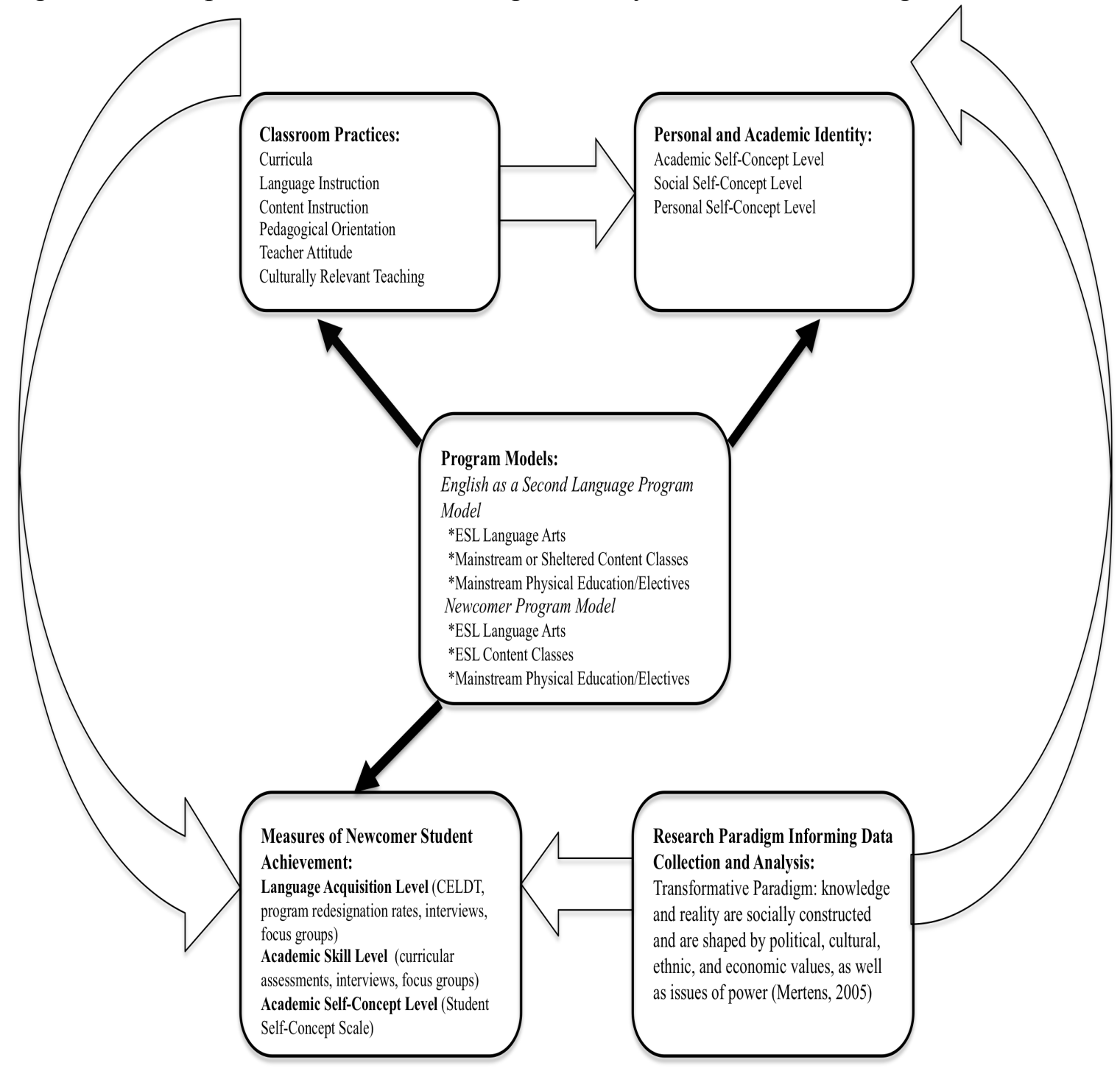

The first concept analyzed the effect of classroom practices and experiences on

the perceptions and achievement of secondary-level newcomer immigrant youth in either a newcomer program or an ESL class. Data was collected that examined the curricula utilized to teach English language acquisition, as well as the curricula used to teach literacy and other content-area skills. Issues of access and choice in the type of curricula available to classroom teachers were further examined to uncover any existing tensions. 
The language and content instruction in these programs was analyzed, looking closely at the pedagogical orientations that were present within each of the two programs (Cummins, 2009). As discussed by Cummins (2009) in his work to close the achievement gap through transformative multiliteracy pedagogy, the instruction in the two programs was viewed through a continuum of pedagogical orientations, ranging from a transmission pedagogical orientation through a social constructivist orientation and, finally, a transformative orientation. The pedagogical orientations will be further defined and expanded upon in the literature review. Lastly, teacher attitude was examined, as well as the use of culturally relevant teaching practices in the classroom setting, as defined by Gay (2010) and Ladson-Billings (1994).

While many factors play a fundamental role in the development and maintenance of students' academic and cultural identities, the second concept looked closely at the personal and academic self-concept levels of secondary-level immigrant youth as they were enrolled in and upon exit from one of the two programs under investigation. The three types of self-concept that were investigated for this study were (1) academic, (2) social, and (3) personal (self-image). Students' academic self-concept, defined as the way in which students view themselves in an academic setting (Hutchinson, Kirby, \& Carson, 2000), was given greater priority in the findings, in that one aim of this research was to demonstrate the influence of the two programs on students' academic sense of self. Students with higher academic self-concepts generally have a stronger sense of belonging in the classroom, as well as the belief that they are able to achieve academically, while students with lower self-concepts often view themselves as not capable to participate effectively in an academic environment (Hutchinson, Kirby, \& Carson, 2000; Hung Hon 
$\&$ Yeung, 2005), therefore making this construct an important focus of this research. The personal and the academic self-concept levels of the immigrant youth under study were viewed in terms of the way such paradigms influence language acquisition and student achievement.

The factors within the conceptual framework were studied to determine their effects on the achievement of secondary-level immigrant youth within the two programs. The measures of student achievement focused on students' English language acquisition levels during and upon exit from the program in which they were enrolled, as well as their academic skill levels and their academic self-concept levels. Changes in students' English language acquisition levels were determined through such oral and written assessments as the California English Language Development Test (CELDT), as well as through the site redesignation rates. Changes in the academic skill levels of the immigrant youth participating in this research study were determined through interview and focus group data. In addition, field observations were used to establish students' academic skill levels during and upon exit from their given program. Lastly, students' academic selfconcept levels, as determined by the self-concept survey entitled the Student SelfConcept Scale (Gresham, Elliot, Evans-Fernandez, 1993), were used to inform the research.

Lastly, the data collection and analysis within this research study was conducted within a transformative research paradigm (Mertens, 2005), as the research was undertaken with the ultimate goal of creating change for a historically oppressed group within the U.S. educational system. This research was performed under the premise that knowledge is socially constructed (Mertens, 2005), yet it further questions the power 
structures present in the educational system and the deficit value systems prevalent in the education of linguistically and culturally diverse children. This research was conducted in an effort to critique the current education of newcomer immigrant youth, as many program models do little to value students' linguistic and cultural backgrounds, and rather serve to ensure students' full assimilation to the dominant language and cultural value system present in the nation's school system (Macedo, 1994). Following an analysis of the programs under study, recommendations were made as to how students' background languages and cultures can and should be valued within a multicultural and multilinguistic society.

\section{Study Significance}

Educators of newcomer immigrant youth need to support students utilizing approaches that promote the acquisition of linguistic and academic skills, as well as an increased sense of belonging and academic self-concept level. The manner in which students view themselves and their academic abilities has a strong impact on their achievement, and, arguably, their continued desire to complete their education. Research has demonstrated that students with higher self-concepts are inclined to outperform those with lower academic self-concepts (Buchholz, n.d.), due, in part, to an increased confidence in their abilities. As the numbers of immigrant youth entering the public school system increases, it is crucial that school districts continue to develop programs to address how to best support these students through both language and skills acquisition, as well as developing students' understanding about how to negotiate this nation's school system. 
This research study explored the perceptions and academic skills of Latino newcomer immigrant youth as they were being educated within or upon exit from one of two specific educational programs provided by the San Diego Unified School District: a self-contained program identified as the New Arrival Center (NAC) or a two-hour English as a Second Language program (ESL). The first program, the New Arrival Center, was a relatively new program as it was in its fourth year of operation. As of the 2011-12 school year, the NAC program had expanded to include self-contained classes at four high schools and two middle school sites. Students within this program received both their English and content-area instruction in a self-contained classroom on a comprehensive school site. The classes were taught by a teacher with training and expertise in working with newcomer immigrant youth. The primary goal of this program, as stated by the district's English Learner department, was to provide a solid foundation in oral and written English for students new to the United States (CAL, 2010). According to the district, "this program strives to engage these students in a rigorous course of study that builds survival and academic English as well as background knowledge across the curriculum" (CAL, 2010). The vision of the district was to ensure that these students learned the concepts and skills necessary for high school graduation and a successful post-high school education. Furthermore, the program worked to make certain that these youth would function successfully within the U.S. cultural norms, while concurrently feeling valued for their own culture.

The youth within the NAC program were compared to students completing a more traditional English as a Second Language course. Historically, immigrant youth at the secondary level (grades 6-12) in the San Diego Unified School District who not placed in 
classes in the New Arrival Center were enrolled in a series of English as a Second Language (ESL) classes to assist them in attaining English language proficiency. These classes took the place of students' grade-level English classes, and these students additionally attended grade-level content classes, such as mathematics, science, and social studies, as well as physical education and elective classes, generally in a mainstream setting with very little language support. The ESL series of classes were utilized for students who tested at the earlier stages of English language acquisition, such as: the beginning, the early intermediate, and the intermediate English language levels. The district encouraged the sites in which the ESL program was located to maintain a small class size of twenty or less students. Students were placed in ESL classes if they had been enrolled in the nation's schools for less than three years and were English language learners (SDUSD, 2012).

In considering the education of beginning English language learners, a group that has historically included newcomer immigrant youth, the ESL classes were developed by the district to provide a balanced approach to English language development, using communication-based, content-based, and literature-based lessons (SDUSD, 2012). The instructional strategies present within this program concentrated on oral and aural activities, focusing on everyday language use, as well as reading and writing activities appropriate for an emergent English acquisition level (SDUSD, 2012). The literacy approaches employed in these classes offered a more modeled and structured pedagogical style; such strategies as shared and modeled reading and writing were utilized in these courses, and these classes also frequently focused on oral language activities to develop students' literacy skills. The key philosophy driving the development of this programs 
was the "Language Experience" approach, which focused on the use of language in the context of everyday situations, while concurrently ensuring the course content was based on the district-adopted English Language Development standards for the beginning proficiency level (SDUSD, 2012). The comparison of the two programs within this study, the NAC and the ESL programs, provided a clearer understanding of how to best support newcomer students as they were completing their first year attending classes in the United States.

\section{Definitions of Applicable Terms}

Academic Self-concept: The manner in which a child views himself or herself in an academic setting.

California English Language Development Test (CELDT): A California state assessment administered to all identified English language learners to assess language proficiency in the areas of reading, writing, speaking and listening. Depending on their scores in each area, students are placed in the beginning, early intermediate, intermediate, early advanced, or advanced band of language proficiency.

California Standards Test (CST): The yearly assessment taken by all students in California's public schools during grades two through eleven. Through a multiple-choice format, the test measures students' progress toward state-adopted content standards in English-language arts, mathematics, science, and history-social science.

English as a Second Language Program (ESL): A class or block of classes where instructors use a variety of strategies to teach beginning English language learners reading, writing, listening, and speaking skills in English. ESL is used synonymously 
with the term English Language Development (ELD). At the secondary level, this class often takes the place of a mainstream English class.

English Learner: Students who are not yet proficient in English. In previous years these students were referred to as Limited English Proficient (LEP) (Ed-Data, 2011).

Fluent English Proficient (FEP): Students whose primary language is other than English and who have met the district criteria for determining proficiency in English (i.e., those students who were identified as FEP on initial identification and students redesignated from Limited English Proficient [LEP] or English learner [EL] to FEP) (CDE, 2011).

Mainstream Content Courses: Traditional grade-level English-only mathematics, science, and social studies courses.

New Arrival Center Program (NAC): The name of the specific secondary-level selfcontained newcomer program researched for this study.

Newcomer: Students who have been studying in the United States for less than one year, and who score at the beginning level on the California English Language Development Test (CELDT).

Newcomer Program: A program designed to develop newcomer immigrant youths' English language skills, help them acculturate to U.S. schools, and make them aware of educational expectations and opportunities (Boyson \& Short, 2003).

Redesignation Rates: The number and/or percentage of former English language learners who have been determined to be Fluent English Proficient according to multiple criteria, standards, and procedures adopted by the specific districts in which the students are enrolled. Students who have been redesignated have been determined to have an 
English language proficiency level comparable to that of average native English speakers (CDE, 2011).

Secondary-level Students: Students attending school at the middle, junior high school, or high school levels, grades 6 or 7 through 12 .

Student Self-Concept Scale (SSCS): A 72-item, multidimensional self-report measure of self-concept. The SSCS provides a norm-referenced measure of children and adolescents in grades $3-12$ in three content domains: Self-Image, Academic, and Social (Gresham, Elliot, \& Evans-Fernandez, 1993).

Sheltered Content Instruction: The use of specialized strategies to make certain content instruction is comprehensible to varying levels of English language learners. 


\section{CHAPTER 2}

Literature Review

The theoretical framework, illustrated in Figure 2, establishes the focus of the literature review for this study, which centers on scholarly literature from the field that concerns the education of immigrant youth. The five central concepts driving this literature review are as follows: (1) the sociopolitical and demographic contexts of immigration in the U.S.; (2) an analysis of federal and California state language policy over the past fifty years; (3) mitigating factors in the education of immigrant youth; (4) guiding theories in education of culturally and linguistically diverse students (5) existing program models for linguistically diverse students. The manner in which sociopolitical contexts, language policy, students' extenuating circumstances, and guiding theories in the education of immigrant youth drive the creation of specialized programs is further addressed and analyzed in this section, as program options are commonly influenced by both the political landscape and student need. 
Figure 2: Theoretical Framework Guiding the Study of Newcomer Immigrant Youth

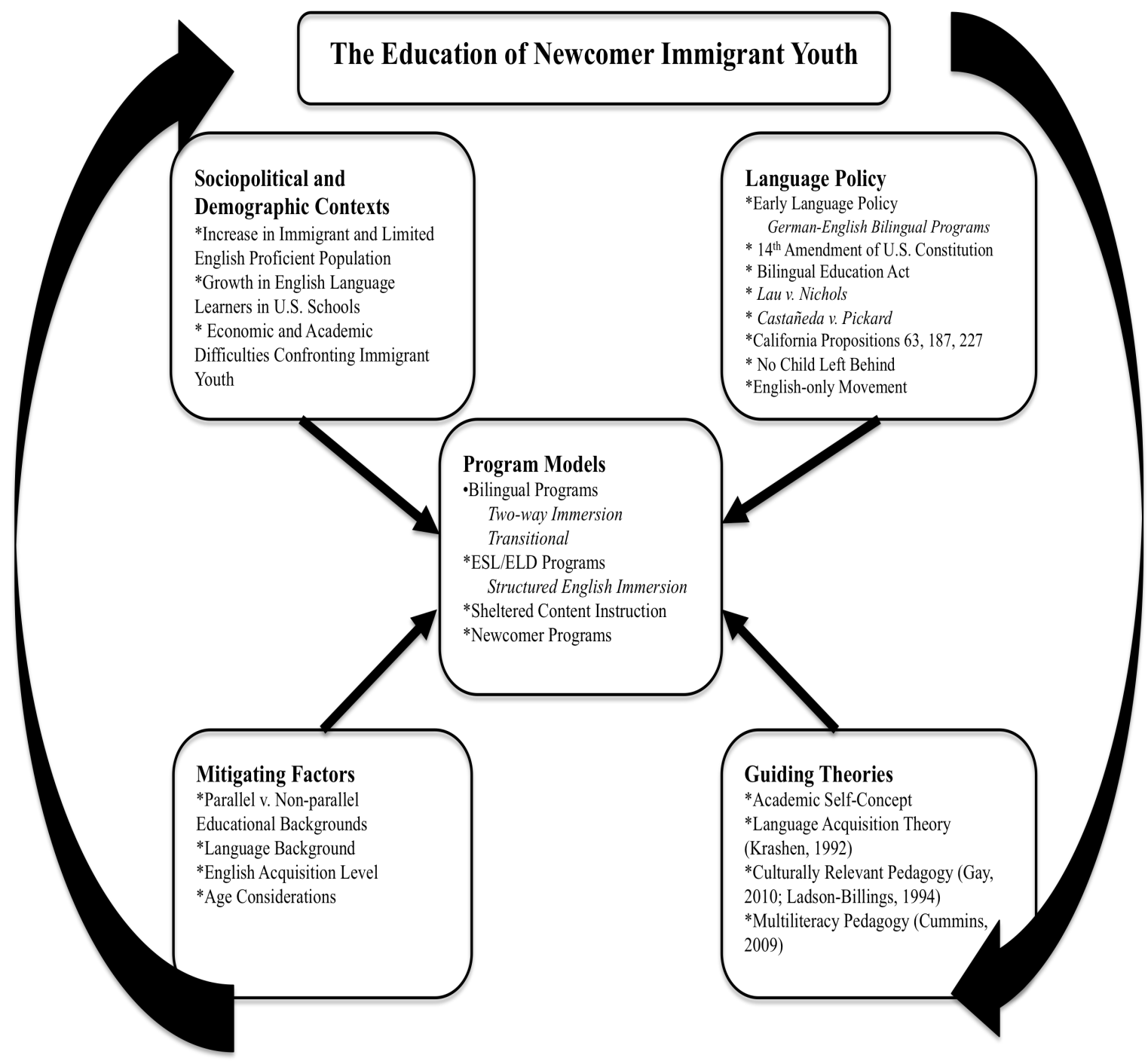

The literature review begins by addressing the dramatic rise in immigration to the

United States in recent years, and how these demographic changes have created the need for specialized programs that address the unique linguistic and academic needs of schoolaged immigrant youth. Significant portions of school-age immigrant youth enter the system with specific academic needs, such as varying past educational experiences and differing academic skills and English language acquisition levels. This section expands 
on the need to consider such factors in the education of secondary-level immigrant youth, in addition to the entering age of these youth and the many primary language backgrounds present in our nation's school-age immigrant population.

The sociopolitical tensions faced by non-English and limited English speaking students, particularly in an era in which the English-only movement is garnering mass support, is brought forth within this section to illustrate the difficulties faced by many newcomer youth. The English-only movement, which has arisen over the past three decades, is analyzed from a historical perspective, closely examining its effects on the education of immigrant youth. Language policy, dating back to the $14^{\text {th }}$ Amendment of the United States Constitution passed in 1869, is reviewed and assessed, both at the federal and the California state level. The relevant pieces of legislation, in particular the Bilingual Education Act of 1968, Lau v. Nichols, Castañeda v. Pickard, California Propositions 63, 187, and 227, and the federal mandate No Child Left Behind, are discussed, giving particular consideration to their influences on the education of immigrant youth in California.

In addition to considering the language policies governing the education of English learners both at the federal and at the state level, school personnel must recognize the varying academic backgrounds and personal circumstances with which the students enter U.S. schools. Immigrant youth arrive at schools in the United States from a variety of socioeconomic, language proficiency, prior schooling, and life experience backgrounds (Short \& Boyson, 2004). Some newcomers arrive at their secondary schools with an adequate English language proficiency level to be effectively mainstreamed into English-only classes upon arrival, while the majority of immigrant youth students enter 
U.S. schools as non-English or limited English proficient (Short \& Boyson, 2004). Some students have experienced parallel schooling experiences in their home countries, while others have experienced interruptions in their formal schooling for a variety of reasons. Due to such interruptions, secondary-level students are at times placed in lower gradelevel classes, creating the reality for such students of being "over-age" in their particular grade level (Custodio, 2011). Finally, teachers and schools often struggle with multiple unfamiliar languages depending on their students' home countries, a situation that can be confusing and overwhelming for site personnel. This chapter will review such mitigating factors in the education of secondary-level immigrant youth.

This literature review examines the education of immigrant youth by analyzing specific academic and linguistic theories in education. Krashen's (1992) theory of language acquisition is outlined within this literature review, as this is a theory of particular importance in the academic field concerning the education of English language learners. Krashen's (1992) theory of language acquisition is discussed in terms of its application to the instruction of secondary-level immigrant youth, describing the connection between the theory and essential classroom practices. Cummins' (2009) work concerning pedagogical orientations within a transformative multiliteracy framework is brought forth to evaluate the manner in which English learners, specifically newcomer youth, are instructed within the varying program options. Both Gay's (2010) and LadsonBilling's (1994) concepts of culturally relevant teaching practices are illustrated in this section, analyzing these practices as they pertain to the instruction of newcomer immigrant youth in the classroom setting. Finally, this literature review will introduce and discuss the notion of academic self-concept, looking closely at both the connection 
between students' academic self-concept levels and their achievement in school and the correlation between academic self-concept and students' English language acquisition.

Lastly, as a result of the political environment in which immigrant youth are being educated, the distinctive linguistic and academic needs of newcomer immigrant youth, and the guiding theories in the field of English learner and immigrant education, various program options have been created to educate such students. This literature review introduces early bilingual education program models and further discusses current traditional and specialized program placements options for newcomer students. More traditional placement such as bilingual, English as a Second Language (ESL) classes, and sheltered content, are compared to an alternative program option, herein referred to as a newcomer program, which has emerged in great numbers over the past three decades (Short \& Boyson, 2004). The manner in which newcomer programs are established is brought forth, looking closely at both the organizational and structural components, as well as the community and parent outreach pieces common to the majority of these programs. The use of these programs to assist students in becoming familiar with the schooling practices in the United States is additionally addressed within this literature review.

\section{The Sociopolitical and Demographic Contexts of Immigration}

Immigrant families comprise a significant segment of the United States population, in fact, as of 2010 immigrants account for 12.9\% of U.S. residents (U.S. Census Bureau, 2012), “the highest percentage in [over] 70 years" (Garcia, 2005, p.10). After a mid-century decline in the number and percentage of foreign-born individuals 
residing in the United States, the sustained rapid growth and high levels of immigration have led to the foreign-born population more than quadrupling in the past 40 years from less than 10 million in the 1970s to 40 million in 2010 (U.S. Census Bureau, 2012). The rapid increase in the number of immigrants relocating to the United States can likely be traced to the tolerant provisions of the 1965 Immigration Act, which abolished prior national-origin quotas, in addition to the formerly sizeable American economy (Portes \&Rumbaut, 2006). The percentage of foreign-born residents of the United States is rapidly climbing towards its former high point, which occurred at the beginning of the twentieth century when immigrants accounted for approximately 14.7 percent of the American population (Portes \& Rumbaut, 2006). The steady increase of foreign-born people entering the United States indicates this segment of the population will continue to grow in the future (Fix \& Passel, 2003).

As the foreign-born population grows in the U.S., the Limited English Proficient (LEP) populace is concurrently increasing at a markedly high rate. Short and Boyson affirm that English language learners are the fastest growing segment of the preK-12 student population, in that "from 1998-1999 to 2008-2009, the English language learner preK-12 population grew 51\%, while total preK-12 enrollment, which includes English language learners grew only 7.2\%” (2012, p. 1). The population of Limited English Proficient persons has increased most rapidly in the six largest immigrant receiving states, California, New York, Texas, Florida, Illinois, and New Jersey, in that as of 2010, $65 \%$ of all foreign-born residents reside in such states (U.S. Census Bureau, 2012). Schools in other states have also seen a dramatic increase in non-English speaking and LEP students in the past decade, and, according to a study recently conducted by the 
Urban institute, "one child in five attending U.S. schools now lives at a home where English is not the primary language" (Custodio, 2011). Despite the fact that the LEP population is growing far more quickly in secondary school than in elementary school (Capps et. al. 2005), the majority of the funding for bilingual resources and language acquisition had been directed to the elementary level (Boyson \& Short, 2003; Capps et. al. 2005), leaving many secondary schools with inadequate funding to support this growing student population.

Finally, though not universal, many immigrant families face economic difficulties upon entry to the United States, in that many have emigrated from poor, developing countries. Camarota reports the poverty rate for immigrants to be 50 percent higher than that of natives, with immigrants and their U.S. - born children (under age 21) making up 22 percent of all persons living in poverty (2001). Fix and Passel stipulate that while one in five total children in the United States are foreign-born or children of immigrants, one in four of the low-income (under $200 \%$ of poverty) children in this country are either first- or second-generation immigrant youth (2003). Additionally, at the secondary schooling level, children of immigrants have seen a consistent increase in the rate of persons living within an economically disadvantaged income level, from 34\% in 1980 to 47\% in 2000 (Capps et. al., 2005). In analyzing such income levels of all LEP students, as of $2000,60 \%$ of secondary-level LEP children lived in economically disadvantaged financial situations, suggesting a high correlation between limited English proficiency and poverty (Capps et. al., 2005).

The poverty faced by many foreign-born persons can be difficult to overcome, as much of the population does not possess the credentialing necessary to achieve upward 
mobilization in the U.S. In his study on immigration and schools, Camarota asserts that the percentage of immigrants without a high school diploma is 30 percent, which is more than three times the rate for natives (2001). Capps et. al. concur with Camarota when they maintain that in $2000,35 \%$ of secondary-level children of immigrants had parents without a high school diploma. In considering parents' education levels, Capps et. al. further contend that in $2000,48 \%$ of the total LEP elementary school student population and $35 \%$ of all LEP secondary-level students had parents who not completed high school, as compared to $11 \%$ and 9\% respectively of English proficient children. Parents' education levels are of particular importance, in that children from families with less formal education tend to achieve at lower levels than children from families that are more highly educated (Garcia, 2005). These statistics further serve as a strong rationale for the creation and implementation of supportive programs designed to specifically target the needs of immigrant youth. The next section will give a succinct overview of the trajectory language policy as it has been passed and implemented both nationally and at the California state level.

\section{A Brief History of Language Policy in the United States}

Language policy in the United States, in particular bilingual educational policy, has seen more permissive periods, from the establishment of the country through the late 1800's and again during the 1950's through the 1970's, and more restrictive periods, from the 1900's to the 1940's, and later from the 1980's until present times (Baker, 2011; Cadiero-Kaplan, 2004; Crawford, 1999). During the permissive periods, the focus has been on quality educational policy, the goal being to educate students to their highest 
potential possible with only a piece of that education centering on the acquisition of English (Brisk, 2005; Cadiero-Kaplan, 2004). In a quality education model, students are taught concepts through their native languages while they acquire English, and, in addition, students' home cultures and value systems are utilized in their instruction. Conversely, during the restrictive periods of language policy, the United States has pushed for a more compensatory educational policy, policy that promotes pedagogical practices that solely focus on the acquisition of English and the path to achieve English proficiency (Brisk, 2005; Cadiero-Kaplan, 2004). In a compensatory model, English is viewed as the fundamental mode through which students are to be educated in their varying academic settings (Brisk, 2005; Cadiero-Kaplan, 2004). These shifts in language policy often follow the changing viewpoints of the political parties in power, as well as the changing perspectives of the people within the nation often due to changes in the economy. These shifts can also be tied to the periods of nativism or, alternately, the periods of cultural acceptance that have encapsulated the country since its inception. The following sections will describe some of the key pieces of language policy that have been passed into law, and their effects on the education of immigrant youth in this nation.

\section{Early Language Education Policy}

In the early days, groups from various European nations established schools that worked to preserve their various heritages and maintain their mother tongues as cultural preservation was one of the reasons, along with the original impetus of religious freedom, that brought the Pilgrims to America (Crawford, 1999). As early as 1694, German immigrants were running schools in their home language, both bilingual and monolingual 
German, which prevailed until the early twentieth century (Crawford, 1999). By the mid 1800 's, both public and private German-English schools were operating in such major cities as Baltimore, Cincinnati, Cleveland, Indianapolis, Milwaukee, and St. Louis (Crawford, 1999). In 1840, in the state of Ohio Germans-Americans even "persuaded the Ohio legislature to pass a law requiring school boards to teach German whenever 'seventy-five freeholders' demanded it in writing” (Tyack, 1974). The resulting schools were some of the first official bilingual schools in the nation, instructing students in the primary grades in both English and German during the literacy portion of their day and in German for their arithmetic, geography, and other subjects; moving to more English instruction as the kids progressed through their years of schooling (Crawford, 1999; Tyack, 1974). In addition, "in 1853 German children residing in districts where there were no special provisions for language instruction were permitted to transfer to German schools" (Tyack, 1974, p. 106), further demonstrating the importance placed on bilingual instruction.

The language policy seen in Ohio was adopted in Louisiana in 1847, though French was the prevailing language rather than German, and in 1848 the Territory of New Mexico authorized Spanish-English bilingual education (Cadiero-Kaplan, 2004; Crawford, 1999). This period of American educational history was one in which the use of multiple languages in school and society was viewed as a means of uniting a nation of diverse language backgrounds and to ensure that all were able to understand the information necessary to validate the political system of the new nation (Cadiero-Kaplan, 2004; Crawford, 1999). This period of permissive language policy lasted until the late nineteenth century, when a resurgence of nativism sparked by such groups as the 
American Protective Association marked the point of decline for bilingual education (Baker, 2011; Cadiero-Kaplan, 2004; Crawford, 1999).

\section{$14^{\text {th }}$ Amendment of the United States Constitution}

On July 7,1868 , the $14^{\text {th }}$ Amendment to the United States Constitution was adopted, creating improved conditions for many within the nation through three largescale clauses: the Citizenship Clause, the Due Process Clause, and the Equal Protection Clause. The Citizenship Clause created a broader definition of citizenship and overruled the Dred Scott v. Sanford decision of 1857, which stated that people of African descent could not be citizens within the United States. The Due Process Clause prohibited the government, either at the state or the federal level, from denying citizens life, liberty, or property without due process of the law. The Equal Protection Clause provided all citizens of the nation equal protection under the law, and has been of great importance within educational policy as it was the basis by which schools were desegregated under the landmark Supreme Court decision of 1954 Brown v. Board of Education of Topeka. As Alberto Ochoa states in his critique of early language policy, "the $14^{\text {th }}$ Amendment (1868) provides four directives: (1) protects the privileges and immunities of all citizens; (2) provides equal protection under the law; (3) gives Congress the power to enforce by legislation; and (4) establishes the principles of equal opportunity" (1995, p. 232).

In considering the implications of the $14^{\text {th }}$ Amendment on language policy, Ochoa asserts "the concept of equal educational opportunity for linguistically diverse persons has evolved from a series of judicial, legislative, and administrative rulings that can be traced back to the U.S Constitution" (1995, p. 231), specifically the $14^{\text {th }}$ Amendment. 
After its passage, the nation's courts struggled to define the parameters of the Equal Protection Clause, which ultimately resulted in the Plessy v. Ferguson Supreme Court decision of 1896. Plessy v. Ferguson "advanced the concept of equal opportunity as meaning 'separate but equal"' (Ochoa, 1995, p. 232), insofar as the Supreme Court interpreted the Equal Protection Clause to mean that equality must be enforced in terms of races, yet they believed it did not mean that distinctions based on color needed to be abolished or they needed to enforce social equality (Ochoa, 1995). It was not until 1954 and the Brown v. Board of Education decision that the courts determined that according to the $14^{\text {th }}$ Amendment, "separate but equal" was in fact unconstitutional according to the Equal Protection Clause, and therefore all students, regardless of ethnicity, must have equal access to a high-quality education. These decisions have played a large role in language policy, as well as policy that affects the education of immigrant youth, as these policies have assured students the opportunity to receive a high-caliber education regardless of ethnicity, and they have laid the groundwork for policies such as the Bilingual Education Act of 1968 to be passed into law.

\section{Bilingual Education Act of 1968}

In 1967, Senator Ralph Yarborough of Texas introduced an initiative that would become the first piece of federal legislation to directly address the needs of Limited English Proficient students. On January 2, 1968, President Lyndon B. Johnson signed the initiative, entitled the Bilingual Education Act, into law making it Title VII of the Elementary and Secondary Education Act of 1965. This action came just a few years after the signing of the historic Civil Rights Act of 1964 and it demonstrated a 
commitment on the part of the federal government to promote students' home languages as a means of educating limited English proficient youth as they were working to acquire English language and literacy skills. In describing the genesis and key components of the law, language policy writer James Crawford states, "the new Title VII of the Elementary and Secondary Education Act (ESEA) authorized resources to support educational programs, to train teachers and aides, to develop and disseminate instructional materials, and to encourage parent involvement" $(1999$, p. 40). In its infancy, the act specifically targeted poor students who were, according to the law, "educationally disadvantaged because of their language" (Crawford, 1999, p. 40), making the focus of the new law compensatory rather than quality in nature (Brisk, 2005; Cadiero-Kaplan, 2004).

This Bilingual Education Act did not require schools and districts to implement bilingual programs, but rather provided funding for school districts enrolling large numbers of language minority students (García \& Kleifgen, 2010). Within two years of its ratification, 21 states enacted bilingual programs, but rather than honoring the original intent of Title VII, most states created transitional bilingual programs, their sole aim being the rapid acquisition of English (Baker, 2011; Cadiero-Kaplan, 2004). The act did not specify the type of bilingual program required or for how long or in what capacity it would be implemented in the school districts, and therefore, due to its ambiguous nature, different states and school districts interpreted the law in the manner they deemed most appropriate (García \& Kleifgen, 2010). For many, this meant the use of students' native languages as a means of English acquisition, rather than as a means of promoting or maintaining students' home dialects. In response to the outcomes of the Bilingual Education Act at the state level, Cadiero-Kaplan asserts, "the legislation that was enacted 
replaced the goal of native language and English development with that of making students proficient in English only (2004, p. 40). Despite issues with implementation, the act was broadened when it was first reauthorized in 1974, as it made limited English proficient students from any socioeconomic background eligible to receive bilingual education services. The shift toward English-only education was made apparent in its second reauthorization in 1978, when the law stipulated "that native languages should only be used to the extent necessary for a child to achieve competence in English" (Cadiero-Kaplan, 2004, p. 45).

\section{The Lau v. Nichols Decision of 1974}

A major U.S. Supreme Court decision, the only of its kind to focus on the rights of language-minority students, was decided in favor of the plaintiffs in 1974 (Baker, 2011; Crawford, 1999). The case, Lau v. Nichols, which originated in 1970 in San Francisco, was filed on behalf of Chinese students who were failing in school because they were unable to understand the language of instruction (Baker, 2011; CadieroKaplan, 2004; Crawford, 1999). After this case was brought forth, the district officials in San Francisco claimed that, "unlike the 1954 Brown case, Lau involved no discrimination because there was no segregation or disparate treatment" (Crawford, 1999, p. 44). They argued that all students were offered the same instruction regardless of their national origin and if the Chinese children had a "language deficiency" it was unfortunate, but not the responsibility of the district (Crawford, 1999). After the case was introduced, both federal and appeals courts sided with school officials until 1974 when the U.S. Supreme Court unanimously overruled the lower courts and sided with the plaintiffs in the case 
Baker, 2011; Crawford, 1999). As Crawford explains, the court declared, "Under Title VI of the Civil Rights Act, the Chinese-speaking children were entitled to special assistance to enable them to participate equally in the school program...sink-or-swim was no longer acceptable" (1999, p. 45). This landmark decision led to the signing of a consent decree by district officials in San Francisco agreeing to provide bilingual education to the city's Chinese, Filipino, and Latino students (Baker, 2011; Cadiero-Kaplan, 2004; Crawford, 1999).

In 1975, the Office for Civil Rights made preliminary visits to 334 school districts with large numbers of language-minority children and found that most districts had failed to comply with their responsibilities under the court's decision (Crawford, 1999). This finding led to the creation of the Lau Remedies, a regulatory framework that "established how school districts should assess and instruct English learners (ELs) and required schools to offer bilingual education to students who were not proficient in English when it could be demonstrated their civil rights had been violated" (Gándara, Losen, August, Uriarte, Gómez, \& Hopkins, 2010, p. 25). The regulations determined that schools, particularly elementary schools, must provide bilingual instruction for students who spoke little to no English, though English as a Second Language was also an integral component of these students' instruction (Crawford, 1999; Gándara et. al., 2010). Though the Lau Remedies lacked the legal status of federal regulations, the mid-1970s were an era in which bilingual education was lauded, and therefore the office of Civil Rights aggressively enforced them and required districts to comply or face losing federal monies (Crawford, 1999; Gándara et. al., 2010). This era, though short lived, proved to be a positive period for advocates of bilingual education, as well as for immigrant youth who 
entered U.S. schools with little to no previous exposure to English. This period allowed for youth to be instructed in a more comprehensible manner and to feel the value of their home dialects, while concurrently learning English and acculturating to their new academic and social environments within the United States.

\section{The Castañeda v. Pickard Decision of 1981}

Despite the progress made through the Lau Remedies promoting bilingual education in many districts across the country during the mid to late 1970s, some districts were still failing to create and implement such programs. In addition, during the late 1970s, the Office of Civil Rights (OCR) began to succumb to more conservative political pressures, and even relented in the case of Fairfax County, Virginia, allowing for an alternative ESL-only approach to educating language minority students rather than the previously required bilingual instruction model (Crawford, 1999). As a result of the OCR's lack of effective action, in 1978 a father named Roy Castañeda filed a case against the Raymondville Independent School District in Texas, arguing the district was segregating his children by tracking them using a grouping system that was racially discriminatory. Mr. Castañeda further claimed this district had failed to establish a bilingual program and, therefore, his children were not being provided the support necessary to overcome the language barrier that prevented them from participating equally in the classroom (Crawford, 1999).

The courts initially found for the defendants in the case of Castaneda v. Pickard, but in 1981 the Fifth Circuit Court of Appeals overturned the original decision, citing the Equal Educational Opportunities Act of 1974. The appeals court decided school districts 
must "take appropriate action to educate language minority students" (García \& Kleifgen, 2010, p. 31). The court outlined the following three criteria for districts to follow in establishing programs that would overcome challenges faced by Limited English proficient students: (1) the program must be based on "sound" educational theory; (2) it must be "implemented effectively" with adequate resources and personnel; (3) after a trial period, the success of the program must be demonstrable (Baker, 2011; Crawford, 1999; Mahoney, MacSwan, Haladyna, \& García, 2010). The Castañeda case was a victory in the education of English learners, in that it assured appropriate resources, qualified teachers, and sound educational theory in implementing programs for such students. However, the case did not mandate a specific program, be it bilingual or ESL, and therefore districts were, and continue to be, free to create such programs at will (García \& Kleifgen, 2010). The lack of a federal mandate and the growing conservatism in the United States throughout the 1980s did little to further the bilingual movement, and, in states such as California, even more decisive measures were passed in the following decades that would virtually eliminate bilingual instruction for all students, including newcomer immigrant youth.

\section{California Proposition 63 of 1986 - The California English Language Amendment}

In November 1986, voters in California approved Proposition 63, a voter initiated state Constitutional Amendment, which declared English to be the official state language of California. The purpose of the initiative, which passed by a 74 percent margin, affirmed English as the official language of the state. The initiative additionally required the state legislature to enforce this provision with appropriate legislation, further 
preserving and enhancing the role of English as the common language of the state (MacKaye, 1990). The initiative further stated that no law may be passed that ignores or diminishes the role of English as the official state language, and it provided for any resident or person doing business within California the ability to sue the state to enforce such provisions (MacKaye, 1990). In passing this legislation, California became only the seventh state to make English the official language and only the second to do so by constitutional amendment. The legislation also paved the way for many other states to pass similar laws, such that "between 1986 and 1988 some forty state legislatures considered similar bills and ten states declared English their official language" (MacKaye, 1990, p. 136).

Though most voters were not aware at the time of the far-reaching and lasting effects of passing such a proposition, it quite effectively furthered the English-only agenda within the field of education and can arguably be viewed as the impetus for later English-only legislation. Crawford asserts that the passage of Proposition 63 dealt a staggering blow against bilingual education in the state, as "at the time [of Proposition 63] California had the nation's most detailed and prescriptive bilingual education law; a year later it had none" (1999, p. 62). Though the law did not specifically mention bilingual education, it was timed by the national group funding and campaigning for the measure (U.S. English) to coincide with the expiration of California's bilingual education statute, a ten-year old law that clarified how Limited English Proficient (LEP) students were to be assessed, instructed, and reclassified, how and when to establish bilingual classrooms, as well as what to do about a shortage of qualified bilingual teachers (Crawford, 1999). Although AB 2813, the bill proposed to extend the bilingual education 
law, breezed through the legislature in the fall of 1986, it was eventually vetoed by thenGovernor George Deukmejian, who cited budget constraints. Contrary to the governor's explanation, though, many felt the veto was in fact due to the English-only political climate of the time (Crawford, 1999).

The passage of Proposition 63 led to an era in which school districts were forced to implement their bilingual education programs without any clear state mandate. In addition, despite the continued efforts to reauthorize the bilingual education law, Governor Deukmejian and his successor Pete Wilson continually vetoed even more modest bilingual education proposals (Crawford, 1999), likely due to the overwhelming influence of the English-only movement in California. As Crawford contended, "bilingual education, conceived as a way to expand opportunities for LEP students and as a superior approach to teaching English, is now attacked as a barrier to students' full participation in American life" (1999, p. 64), a claim that has been continually fostered in the campaign for future English-only legislation within California and across the nation.

\section{California Proposition 187 of 1994 - Save Our State Initiative}

Just eight years after the passage of Proposition 63, an even more highly xenophobic piece of legislation was brought forth to the voters of California. Proposition 187 , or as it was otherwise named the "Save Our State" initiative, was passed by $59 \%$ of California's voters on November 8, 1994, and included such high-profile supporters as then governor of California, Pete Wilson. This proposition specifically targeted undocumented persons in California by denying public services, such as social services, medical services (save for life-threatening emergencies), and public education at the 
elementary, secondary, and post-secondary levels to undocumented immigrants (Garcia, 1995). Proposition 187 charged educators, as well as those providing medical or social services, to verify the legal status of the children attending their institutions, as well as their parents and/or legal guardians, and required school personnel to report any persons without proper documentation to the Immigration and Naturalization Service (INS) and the attorney general of California (Contreras, 2002).

In supporting this piece of legislation, proponents of Proposition 187 flatly ignored the fact that the "educational sections of the initiative were in direct conflict with the Supreme Court's 1982 decision in Plyer v. Doe, which held that the state of Texas could not bar undocumented children from public elementary schools because doing so violates the Equal Protection Clause of the $14^{\text {th }}$ Amendment" (Contreras, 2002, p. 142). In his comment on the legal action, Garcia further argued that one of the goals of Proposition 187 was "to invite the Supreme Court to overturn Plyer, now that the court is politically more conservative than it was in 1982" $\left(1995\right.$, p. 131). On November $9^{\text {th }}$, 1994, such advocacy groups as the Mexican-American Legal Defense/Education Fund (MALDEF) and the American Civil Liberties Union (ACLU) filed federal lawsuits contesting the constitutionality of the proposition. By 1997, a federal court judge issued a permanent injunction against the enforcement of the law, an injunction the state of California has never sought to overturn.

Though it does not specifically address language issues, Proposition 187 has played an important role in the historical trajectory of language and immigration policy in California, and the passage of such a proposition aptly demonstrates the landscape in which immigrant youth have been educated within this state. As Contreras asserts, 
"Proposition 187 attempted to extend the segregation of Hispanic students by denying public education to anyone attending a public elementary, secondary, or postsecondary school who was 'reasonably suspected' of being an illegal alien in the United States" (2002, p. 142). Supporters of the proposition argued this was not an initiative based on race, but rather it was meant to preserve the state's scant resources. However, the use of such terms as "illegal aliens" in reference to undocumented persons demonstrated an attempt on their part to criminalize those without documentation. Analyzing the implications of immigration policy on education reform, Contreras contends, Proposition 187 "disproportionally affected people of color who are stereotyped as illegal aliens" (2002, p. 143) and, additionally, it "reflected the essence of the educational segregation that minorities have historically contested in efforts to gain an equal public education" (Contreras, 2002, p. 143). Though the legislation was eventually deemed unconstitutional, it can be argued that Proposition 187 created an environment in which many students felt unsafe and unwelcome in California's schools. The case can also be made that Proposition 187 paved the way for advocates of English-only education to further their agenda, as was seen in a subsequent piece of legislation, California Proposition 227.

California Proposition 227 of 1998 - English for the Children

Proposition 227, or the English for the Children initiative, was approved by $61 \%$ of California's voters on June 2, 1998 (Baker, 2011; Cadiero-Kaplan, 2004) and had a definitive impact on California's schools by severely limiting students' access to bilingual programs and effectively eliminating bilingual education programs in the state. 
Ron Unz, the chairman of a Palo Alto-based financial services software company, wrote the legislation and both financed and campaigned for its passage (Baker, 2011). Unz maintained that his motive for creating and financing such a proposition was roused after he witnessed a 1996 protest against bilingual education by parents at the Ninth Street Elementary School in Los Angeles (Baker, 2011; Crawford, 1997). He claimed immigrant parents were forced to boycott the school after school officials would not allow their children to be taught in English. Unz asserted that large numbers of children were leaving California's public schools with limited English skills, both oral skills and written skills, and he faulted bilingual education for this claim. Contrary to the assertions made by Unz and his supporters, in their report for the UC Linguistic Minority Research Institute, Gándara countered: Proponents of Proposition 227 contended that bilingual education had failed as a pedagogical strategy and should be abandoned. Evidence for its failure was found in the continuing underachievement of English learners and the low rate that English learners were reclassified as Fluent English Proficient. Yet, the fact was, less than one-third of all English Learners were enrolled in bilingual programs prior to the passage of Proposition 227, so their poor academic achievement could not be attributed to these programs (2000, p.2).

Proposition 227 was written in a manner that would directly impact language minority students in California's K-12 schools. The proposition mandated that students be instructed "overwhelmingly" in English and, therefore, English language learners were to be placed in Structured English Immersion (SEI) classes for a period of approximately one year to gain academic language skills in English. The initiative further stated these 
students were not to remain in SEI classes for a period exceeding two years. According to this proposition, the SEI classes would help support students as they acquired English by utilizing curricula and strategies that assisted students in learning the language. As Gándara explained, "Structured English Immersion classrooms were defined in the law as multi-age classes with students at the same level of English proficiency, in which the focus of instruction was to be the development of English skills" (2000, p.1). These classes were designed with the goal of teaching students English, with only a secondary focus on academic content (Baker, 2011; Brisk, 2005; Cadiero-Kaplan, 2004; MaxwellJolly, 2000).

This proposition included a parent waiver in instances where parents wanted their child to continue in a bilingual program. The parent waiver was considered according to the following three conditions: (1) the child already possessed strong English language skills, as measured by standardized tests of English vocabulary comprehension, reading, and writing, in which the child scored at or above the state average for his or her gradelevel or at or above the 5th grade average; (2) the child was over 10 years of age and it was determined by school personnel that a bilingual approach would best serve this child; (3) the child needed modifications due to a specific learning disability. Each school site must have had a minimum of twenty students with a waiver to create a bilingual class. Additionally, as Palmer and Garcia explained in their article, "the new law specified that children must be placed 'for a period no less than 30 days during the school year in an English language classroom' before a parent waiver would be able to move the child into an 'alternative' (i.e., bilingual) program" (2000, p. 169), thus requiring youth to be immersed in an English-only environment for a period of time regardless of the desires of 
the students and their parents or guardians. Lastly, Proposition 227 included "a provision allowing parents and others to assign personal legal liability to any teacher, school, or district that does not implement the English language program as designated in the initiative" (Maxwell-Jolly, 2000, p.38). The legal responsibility this proposition placed

on school personnel was one that had rarely, if ever, been seen before in California's state educational policy and, arguably, worked to promote the English-only agenda by creating a sense of fear for those implementing bilingual classes. As it has not been successfully challenged or overturned, Proposition 227 continues to be the presiding legal statute under which bilingual programs are implemented within the state of California.

\section{No Child Left Behind Act of 2001}

In January 2002, then President George W. Bush signed into law the federal No Child Left Behind Act of 2001 (NCLB). NCLB was, and continues to be, a highly ambitious reauthorization of the existing Elementary and Secondary Education Act of 1966, a reauthorization that former President Bush and his administration developed with bipartisan Congressional support soon after he took office. Among other mandates, the federal No Child Left Behind Act of 2001 stipulated that all students are to be "proficient" on state assessments by the 2013-14 school year (United States Department of Education, 2002). The goal of proficiency was to be achieved by monitoring districts and their individual school sites utilizing state-developed assessments to make sure they achieved annual targets of Adequate Yearly Progress (AYP) for reading, math, science (Baker, 2011; García \& Kleifgen, 2010; U.S. DOE, 2002). As García \& Kleifgen wrote, "it is not enough for districts or schools to meet their goals in terms of their aggregate 
data; they must also show that all subgroups of students - meaning students of different races, ethnicities, income groups, gender, and so on - are meeting AYP goals" (2010, p. 33). The requirement for districts and individual sites to disaggregate assessment data according to different federally mandated subgroups and, additionally, to meet annual goals for each of the subgroups, has made it increasingly difficult for districts to achieve the federally imposed annual targets for Adequate Yearly Progress (Baker, 2011).

One subgroup that NCLB has required schools and districts to monitor is comprised of students identified as English learners (Baker, 2011). This mandate requires districts to assess their English learners on state-designed assessments without regard to students' given levels of English language acquisition (Baker, 2011). If English learners, or other such subgroups, fail to meet their annual AYP goals at any given site, the school can de designated a "school in need of improvement" and is subject to interventions, or even eventual restructuring or closure (Baker, 2011; García \& Kleifgen, 2010; Wentworth, Pellegrin, Thompson, \& Hakuta, 2010). One way in which some states, such as Arizona and Texas, have dodged the unfair demands placed on them in assessing their English learners under NCLB, is to define how a subgroup must be counted, thereby allowing districts to avoid the creation of an English learner (EL) subgroup in schools with low EL populations (García \& Kleifgen, 2010). By successfully avoiding the creation of an English learner subgroup, these schools have been able to keep the federal government from imposing sanctions on their sites, thus demonstrating some of the many inequities to come from this piece of legislation. As a concession to the various issues raised in assessing emergent English learners, under a 2004 NCLB regulation, states are allowed to exempt newcomer immigrant youth that have attended school in the country 
for less than one year from the states reading/language arts assessment, though the students are still assessed in mathematics and science (Baker, 2011; García \& Kleifgen, 2010).

After it was signed, the federal No Child Left Behind Act of 2001 became "the final stage to date of [the] policy movement away from bilingual education and toward an English-only approach" (García \& Kleifgen, 2010, p. 32). As García \& Kleifgan remark, the word "bilingual" is disappearing in federal language policy, as evidenced by key name changes, such as the change from the former Office of Bilingual Education and Minority Affairs (OBEMLA) in Washington, D.C., to the current Office of English Language Enhancement and Academic Achievement for LEP Students (OELA) (2010). Title VII of the Elementary and Secondary Education Act (the Bilingual Education Act) has been changed to Title III (Language Instruction for Limited English Proficient and Immigrant Students) in the No Child Left Behind legislation, which is "further indicative of the shift away from the support of instruction in students' home languages through bilingual education" (García \& Kleifgen, 2010, p. 35). Furthermore, most English-only states, namely California, Arizona, and Massachusetts, have ignored the fact that the law provides a standard moratorium on testing newcomer youth in English for their first three years, and at times up to five years on a case-by-case basis (Losen, 2010). These same states test all students solely in English, regardless of whether or not the students being tested have the language necessary to comprehend the state standardized assessments (Gándara \& Hopkins, 2010; Losen, 2010). Due to the anti-bilingual political climate in these states, immigrant youth have been forced to endure failure on the state assessments; consequently, these same students have been being tracked into remedial classes as a 
result of the English-only states' unwillingness to test emergent English learners appropriately under federal law (Gándara \& Hopkins, 2010; Losen, 2010). The renaming of policies and offices, along with the one-size-fits-all testing approach apparent in the No Child Left Behind policy initiative (Cadiero-Kaplan, 2004; Gándara \& Hopkins, 2010; Losen, 2010), demonstrates the political move towards a monolingual English-only society in the United States, and a definitive devaluing of the language backgrounds with which immigrant youth enter U.S. schools. The next section will take a closer look at the English-only movement in the United States and its impact on immigrant students.

\section{The English-only Movement and Immigrant Youth}

The current sociopolitical goal in the education of immigrant youth points overwhelmingly to ensuring these children speak English fluently as quickly as possible.

The issue of an English-only curriculum is one that is often found in the national political arena, and can be argued to have "close connections to restrictionist, anti-immigration organizations, which suggests that the English-only movement has a wider, more farreaching, and more negative agenda than simply advocating an official English language policy" (Padilla, Lindholm, Chen, Duran, Hakuta, Lambert, \& Tucker, 1991, p. 120). This movement is one that has far-reaching and exceedingly negative implications on the nation's immigrant youth, yet, as of 1991, 18 U.S. states have enacted laws designating English the official state language (Padilla et al., 1991, p.120).

The movement to solidify parts of the United States as legally monolingual demonstrates an abundantly ethnocentric and Eurocentric mentality on the part of politicians, and, ultimately, the constituencies they serve. It systematically promotes the 
dominant English language and Anglo-American culture as being the most highly revered, without regard to those put in an inferior political and societal position. As Macedo writes in his discussion about the English-only movement and its effects on bilingual education:

The incessant attack on bilingual education, which is based on the claim that bilingual education tongue-ties students in their native language, not only points to a xenophobic culture that blindly negates the multicultural and multilingual nature of U.S. society, but also falsifies the empirical evidence in support of bilingual education, which has been amply documented (1994, p. 125).

Darder further asserts that within the English-only movement "the language that many bicultural students bring to the classroom is systematically silenced and stripped away through values and beliefs that support its inferiority to Standard English" (1991, p. 36). She additionally states that "negating the native language and its potential benefits in the development of the student's voice constitutes a form of psychological violence and functions to perpetuate social control over subordinate language groups through various linguistic forms of cultural invasion" (Darder, 1991, p. 37). The current political movement towards a monolingual English society can be especially disparaging towards immigrant families who are attempting to make the United States their home, and, in particular, to immigrant youth who are required to learn English and adopt the American culture at the expense of their own home languages and cultures (Macedo, 1994).

As previously discussed, Portes and Rumbaut (2001) argue that language assimilation is thought by many to demonstrate the willingness of immigrants to be part of the host country. It is viewed as a symbol of the commitment people must make to the 
United States to prove their loyalty, largely emphasized due to the lack of other forms of commonality amongst the various communities living in this country (Portes \& Rumbaut, 2001). Laurie Olsen concurs with Portes and Rumbaut when she asserts, "the role of the school in Americanizing immigrants and addressing issues of national origin is viewed as a matter of taking non-English-speaking students and making them fluent English speakers" (1997, p.91). The English-only movement has been instrumental in assuring the general public that students are working towards fully assimilating to the dominant culture in the United States, yet the negative impacts of this movement upon immigrant youth is rarely the focus of discussion in the educational and political arenas (Macedo, 1994).

The "English-only" movement in schools propagates the theory that newcomer students must systematically discard their home language and culture in lieu of attaining the English language and American culture to gain acceptance, both academically and socially, in U.S. schools (Valenzuela, 1999). Students are expected to become "American" quickly and without question to attain success in schools and society. Valenzuela refers to the manner in which schools are designed to achieve this goal as "subtractive schooling". Valenzuela looks at the organization of schools as being "subtractive in ways that extend beyond the concept of subtractive cultural assimilation to include the content and organization of the curriculum" (1999, p.27). The curriculum taught in English-only content area classes works to push this deficit agenda that aspires to strip students of their home language and culture while offering little to no support for students to attain academic concepts in a comprehensible manner. Valenzuela further states, "rather than students failing school, schools fail students with a pedagogical logic 
that not only assures the ascendance of a few, but also jeopardizes their access to those among them who are either academically strong or who belong to academically supportive networks" (1999, p.27). In other words, schools are organized to allow a few to succeed and many to fail. The English-only movement only solidifies this reality by denying students support and access to academic content through primary language classes, which would inevitably provide needed comprehensible input to non-native English speakers. The next section will focus on the needs of many secondary-level immigrant youth as they enter schools in the United States.

\section{Mitigating Factors Schooling of Immigrant Youth}

Immigrant youth arrive in the United States with a variety of socioeconomic, English proficiency, prior schooling, and personal experience levels (Short \& Boyson, 2004). Some newcomers enter secondary schools with an adequate English language proficiency level to be effectively mainstreamed into English-only classes upon arrival, while other foreign-born students enter U.S. schools as non-English or limited English proficient. The students, who possess an emergent English language proficiency level, generally require support beyond what is commonly offered in mainstream English classes. This support often comes in the form of primary language or bilingual classes, or English-only classes in which specific strategies are utilized to assist English language learners in comprehending the curricula being taught (Baker 2011; Minaya-Rowe, 2008). The varying considerations that need to be made in the placement of immigrant youth will be expanded upon in this section. 


\section{Parallel Versus Non-Parallel Academic Experience}

Some immigrant youth enter the nation's schools with primary language and literacy skills that equal or exceed their age-appropriate counterparts, due, in part, to their ability to attend school in their home countries, while other youth have significant gaps in their educational backgrounds. Faltis and Coulter refer to these two groups of immigrant youth as having "parallel" or "non-parallel" schooling experiences (2008). About onethird of all adolescent immigrants enter U.S. schools with parallel formal schooling experiences (Faltis \& Coulter, 2008), which is defined as, "immigrant students who have attended formal schooling up to the grade-level at which they enter U.S. schools" (2008, p.51). These students are typically highly literate in their home language, and often proficient in technology (Faltis \& Coulter, 2008). Many students with a parallel formal education have highly educated parents, and have, to varying degrees, been exposed to English prior to their relocation to the U.S., allowing for a generally smooth transition to this country's schools (Faltis \& Coulter, 2008). Olsen recognizes that, despite having a strong prior academic background, students with a parallel formal education may still encounter some difficulties upon entering U.S. schools. She asserted, "even those who arrive as adolescents with strong academic backgrounds face what can be a difficult transition to our forms of schooling, and unexpected academic gaps, particularly in subjects such as social studies or history" (1997, p.153). These academic gaps are often due to differences in the curriculum content and sequence, the teaching pedagogy, and the skills that are emphasized in our nation's schools, which may differ from the skills stressed in students' home countries (Olsen, 1997). Olsen concedes that despite any gaps in academics, "these students (usually from industrialized urban centers of the world and 
from middle-class or professional families) are confident in their abilities as students, and arrive with strong academic skills to apply new content” (1997, p.153), which again reiterates the idea that students with parallel formal educational backgrounds have a far greater ability to achieve success in U.S. schools, due to their prior understanding of schooling, as well as their increased literacy and English language skills.

At the other end of the spectrum, immigrant youth with non-parallel formal schooling, or as they are labeled Students with Interrupted Formal Education (SIFE), "have sporadic formal schooling experiences and are typically underschooled - two or more grades behind where they would be if they had not had interrupted schooling in their home country" (Faltis \& Coulter, 2008, p.52). Jamieson, Curry, and Martinez (2001) spoke to this phenomena with their findings that more than one-third of immigrant adolescents aged 15 to 17 from Latin America were enrolled below grade-level in the United States, arguably due to a disruption in such students' academic course. These students are often from "rural, impoverished or war-devastated regions of the world" (Olsen, 1997, p.153). Non-parallel newcomers are less likely to be familiar with how schools operate, such as: daily routines, school services available to them, and culturallyappropriate classroom behavior, as well as the technology utilized in U.S. classrooms (Faltis \& Coulter, 2008). Addressing the overwhelming academic gaps faced by these students requires "approaches to developing basic literacy in an accelerated fashion, and mechanisms for filling gaps in academic content" (Olsen, 1997, p.153). The undeniable differences in the educational needs of newcomer immigrant youth as they enter U.S. schools demonstrates the necessity for placement of students to be dependant on a variety of factors, including, but not limited to, English language proficiency level and prior 
schooling experiences (Custodio, 2011). These differences further make evident the importance of developing specialized programs to support youth with major prior academic gaps (Custodio, 2011).

\section{Age and Language Considerations}

Additional factors that have been shown to impact the schooling of immigrant youth are such aspects as the advanced age in which many immigrant students are entering U.S. schools, as well as the multitude of language backgrounds that are spoken by many of these same students. The age of newcomer students attending classes at the secondary level can vary greatly, with a general range of 10 to 22 years old (Short, 1998; Short \& Boyson, 2012). These students are often over-age compared to their native grade-level counterparts due to limited formal education and weak academic skills, which inherently places these kids at a higher risk for dropping out of school (Capps, Fix, Murray, Ost, Passel, \& Herwantoro, 2005; Custodio, 2011; Short, 1998). Furthermore, although Spanish is the most prevalent primary language spoken, immigrant youth enter secondary schools speaking up to 150 different languages (Freidlander, 1991). A lack of resources to create effective primary language classes for the considerable number of different primary languages spoken by immigrant students, as well as the need to better support over-age students, are significant factors that demonstrate the need for specialized language programs (Custodio, 2011; Freidlander, 1991). These programs can assist students in effectively gaining the English necessary to comprehend the language and the content being taught in mainstream classes. As Custodio asserted, specialized programs for newcomer youth can "fill in the academic gap for these students through 
courses that develop basic literacy and numeracy skills, and at the same time offer academic subject preparation and orientation to school and society" (2011, p. 7). The next section will focus on several notable theories that pertain to the education of immigrant youth.

\section{Guiding Theories in the Education of Immigrant Youth}

Krashen's Language Acquisition Theory

English language acquisition is a primary focus of both English as a Second Language and newcomer programs, and therefore it is essential to analyze prominent theories of language acquisition within the educational field. As Stephen Krashen has long been considered a preeminent authority in the field, this section will focus on Krashen's five key hypotheses concerning language acquisition. Krashen's five main hypotheses (1992) are as follows: (1) The Acquisition-Learning Hypothesis; (2) The Natural Order Hypothesis; (3) The Monitor Hypothesis; (4) The Input Hypothesis; (5) The Affective Filter Hypothesis Krashen argues that by incorporating his five hypotheses into the language education of English learners, such students will receive the comprehensible input necessary to learn both the language and the literacy skills necessary to achieve success in schools (1992). This section gives an overview of these five hypotheses, as well as their practical classroom applications with regard to the education of immigrant youth, and additionally juxtaposes Krashen's theory of language acquisition with other respected scholars in the field.

Krashen's five main theories of language acquisition, developed and presented in a manner that makes them more easily accessible to a wide variety of practitioners, have 
long been utilized as a basis in developing new theories in this field. Krashen's Acquisition-Learning Hypothesis follows that "language acquisition is a subconscious process; while it is happening we are not aware it is happening", while language learning "is a conscious process; when we are learning, we know we are learning" (Krashen, 1992, p. 1). This language acquisition hypothesis claims acquisition and learning are two independent processes, acquisition being a more innate, subconscious process, and learning being a conscious process guided by the rules of a given language (Krashen, 1992, 2003). He maintains that research does prove that both children and adults can subconsciously acquire new language, though conscious language learning within language classrooms is "of great help to beginners, since classes give them the comprehensible input that the 'outside world' gives them only reluctantly" (Krashen, 1992, p. 42). Therefore, Krashen $(1992,2003)$ contends beginning language learners must be provided courses that provide explicit language instruction and extensive comprehensible input to support them in acquiring the language necessary to better communicate in more decontextualized settings.

Krashen's Natural Order Hypothesis $(1992,2003)$ claims that humans acquire parts of a language in a predictable sequence, some rules of language coming early in the process and other parts coming later as language is acquired. Krashen $(1992,2003)$ believes the order of acquisition is similar between first and subsequent languages, but not identical, as the rules of particular languages vary and, additionally, acquirers might proceed in slightly different manners. He further states "the natural order appears to be immune to deliberate teaching; we cannot change the natural order by explanations, drills, and exercises" (Krashen, 1992, p. 2). 
In an attempt to explain how language acquisition and language learning are used, Krashen developed the Monitor Hypothesis, which differentiates between the roles of acquired and learned language. In his view "language is normally produced using our acquired linguistic competence" (Krashen, 2003, p. 2), and therefore "conscious learning has only one function: as a "Monitor" or editor" (Krashen, 1992, p. 3). Krashen argues, "after we produce some language using the acquired system, we sometimes inspect it and use our learned system to correct errors" (1992, p. 3). He maintains that language learning occurs primarily through acquisition and while some conscious knowledge of language can be helpful, the Monitor can only be used if three conditions are met: the learner has sufficient time to communicate, the learner places a focus on grammatical form or correctness, and the learner has explicit knowledge of the rules that govern the language (Peregoy \& Boyle, 2005). The price to be paid for the use of one's Monitor is that communication can become disrupted and less information can be given as a result of overcorrecting one's own use of the language. In other words "some people overMonitor" and are so concerned with grammar and accuracy that speech is slow and painful to produce as well as to listen to" (Krashen, 2003, p. 3). Krashen (2003) believes the Monitor is best employed when it will not interfere with one's language acquisition or speech, such as in the editing phase of writing, and he further recommends that language teaching focus on communication rather than rote rule learning (Peregoy \& Boyle, 2005).

Krashen asserts the Input, or Comprehension, Hypothesis, answers the crucial question as to how language acquisition actually occurs within a language learner. As previously stated, Krashen (1987) contends that acquisition is central to language development, while language learning is more peripheral, and therefore it is vital that 
learners have the opportunity to acquire language in a natural setting, both within and outside of academic environments. Krashen (2003) argues "we acquire language in only one way: when we understand messages; that is when we obtain "comprehensible input" (2003, p. 4). In other words, when language learners understand what they hear or read, when they understand the message, they acquire language.

Krashen believes language learners acquire language by moving from stage $i$, where $i$ represents current competence or input, to $i+1$, or a level slightly above current competence, by understanding input containing $i+1$ (1987; 2003). In his words "the input hypothesis makes the following claim: a necessary (but not sufficient) condition to move from stage $i$ to stage $i+1$ is that the acquirer understand input that contains $i+1$, where 'understand' means the acquirer is focused on meaning and not the form of the message" (Krashen, 1987, p. 21). He further states "we are able to do this with the help of our previously acquired linguistic competence, as well as our extra-linguistic knowledge, which includes our knowledge of the world and our knowledge of the situation" (Krashen, 2003, p. 4). Krashen believes learners acquire language by using their prior understanding of language, as well as environmental clues, to understand a level of language input that is slightly beyond their current level of proficiency (Peregoy \& Boyle, 2005). In utilizing context and first language knowledge to acquire a new language, beginners rely on such strategies or clues as body movements (Total Physical Response), gestures, pictures, and their prior understanding of language to make meaning of the new language (Krashen, 2003). Krashen (1992) demonstrates the accuracy of the Input Hypothesis by claiming the following four statements to be true: more comprehensible input results in more language acquisition, teaching methods containing 
more comprehensible input have been shown to be more effective than "traditional" methods, the development of second language proficiency can occur without formal instruction, and finally, the complexity of language makes it unlikely that much of language is consciously learned.

Krashen's fifth hypothesis, the Affective Filter Hypothesis, “addresses affective or social-emotional variables related to second language acquisition" (Peregoy \& Boyle, 2005). Krashen argues that affective variables, such as anxiety or low motivation, do not directly hinder language acquisition, but rather prevent input from reaching the part of the brain responsible for language acquisition, an area theorist Noam Chomsky has termed the "language acquisition device" (Krashen, 2003). Krashen further states, "if the acquirer is anxious, has low self-esteem, does not consider himself or herself to be a potential member of the group that speaks the language, he or she may understand the input, but it will not reach the language acquisition device" (2003, p. 6). He believes a block, the Affective Filter, will keep the input from reaching the language acquisition portion of the brain. In addition to a low anxiety environment, Krashen (2003) additionally contends that attitude and motivation is key in language acquisition, in that "those whose attitudes are not optimal for second language acquisition will not only tend to seek less input, but they will have a high or strong Affective Filter" (1987, p. 31). Furthermore, he states, "those with attitudes more conducive to second language acquisition will not only seek and obtain more input, they will also have a lower or weaker filter" (1987, p. 31). In an effort to maintain a low anxiety environment, Krashen urges teachers "not to force production, but rather allow students a 'silent period' during which they can acquire some language knowledge by listening and understanding as 
opposed to learning it through meaningless rote drills" (Peregoy \& Boyle, 2005). In summary, Krashen's five language acquisition theories promote language teaching that focuses on communication, rather than grammatical form, provides comprehensible input at a level slightly above students' current level of language acquisition, allows students a silent period, rather than forcing immediate speech production, and creates a low anxiety environment with which to acquire language (Peregoy \& Boyle, 2005).

While Krashen (2003) makes the assertion that language is primarily acquired and secondarily learned, he supports the notion that beginning language learners must be provided well-taught language classes to receive the comprehensible input necessary to acquire language. Henceforth, newcomer immigrant youth that are beginning language learners must be educated in a classroom that promotes comprehensible input slightly above their current level of comprehension, rather than being mainstreamed into a general education environment that is reluctant to do so. Krashen (2003) lists several teaching strategies that have been proven to provide comprehensible input for beginning language learners, such as the use of pictures and realia (objects from real life) and by using body movements (Total Physical Response) and gestures. Teachers should modify their speech, talking a bit more slowly and using somewhat less complex vocabulary, during instruction and should not place undue expectations on students for a high level of output until the students feel ready, thus ensuring a low anxiety academic environment (Krashen, 2003). Finally, Krashen (2003) believes only the older (high school level and above) beginning level students should be required to study the grammar rules, as these students may want to learn more about the structure of the language and it may help to fill in some of the gaps left by incomplete acquisition. With regard to language classes, 
Krashen states, "the goal is to bring students to the intermediate level" (2003, p. 7), after which time the students will have sufficient language competencies to receive comprehensible input from the mainstream environment at large.

According to Peregoy \& Boyle (2005), Krashen's five theories of language acquisition build upon of the work of the acclaimed linguist Noam Chomsky's innatist theory regarding the manner in which children acquire first languages. Chomsky (2002) argues that children acquire language using an innate, biological language acquisition device (LAD) and they construct grammar using a process of hypothesis testing. Krashen incorporated such concepts into his theories of second language acquisition, as he argues that the majority of language is acquired rather than learned. The innatist theory contradicted such behaviorist theories of language acquisition as B.F. Skinner's hypothesis (1992), which follows that language is developed through stimulus, response, and reinforcement (Peregoy \& Boyle, 2005). In second language acquisition, behaviorists contend that language is best acquired through the memorization of dialogues, as well as pattern drills for practicing verbs and sentence structures (Peregoy \& Boyle, 2005), methods that are counter to the teachings of Krashen. A third theory of language acquisition, the interactionist theory takes into account the necessity of both nature (innatist) and nurture (behaviorist) in the language acquisition process, as it argues that caregivers play a crucial role in the adjustment of acquired language (Peregoy \& Boyle, 2005). Therefore, interactionists contend that while comprehensible input is an important tenet of second language acquisition, non-native speakers are able to exert some control in a conversation with native speakers, asking for repetitions or indicating they don't understand, which forces native speakers to include more comprehensible input into the 
conversation (Peregoy \& Boyle, 2005). This give-and-take process, referred to as the negotiation of meaning, elicits the native speaker to act in the caregiver role, manipulating language through slowed or modified speech, gestures, and pictures, to allow more comprehensible input for the non-native speaker. Ultimately, the interactionist model expands on Krashen's five theories of language acquisition, as it adds a social dimension to the importance of comprehensible input.

\section{Culturally Relevant Pedagogy}

In addition to facing difficulties communicating with others in the educational arena, it is common for newcomer youth to feel isolated due to their differing culture and value systems as they enter the U.S. schools (Olsen, 1997). As was previously stated, Laurie Olsen (1997) argues that it is a surprise for many newcomer students to learn that in coming to America they are often not considered American by the general population, which can cause students to feel quite isolated within their classrooms and schools at large. Immigrants, entering at any age, are expected to acculturate to the dominant, mainstream American culture at a rapid pace, making immigration essentially "a site of cultural stripping away" (Rosaldo, 1993, p. 209). Rosaldo expands on this idea when he argues "from the dominant society's point of view, the process of immigration strips individuals of their former cultures, enabling them to become American citizens transparent just like you and me, people without culture" (1993, p. 209). It can be argued that this process, often called the acculturation or assimilation process, can be harmful to newcomer youth, in that as they enter their new schools, these students are neither valued for the home culture and value systems with which they enter the United States or 
considered culturally American. This perceived cultural void undoubtedly causes many immigrant students to feel disconnected to the U.S. educational system and can be a likely contributor to the disproportionally high dropout rate facing secondary-level Latino immigrant youth (Laird, Lew, DeBell, \& Chapman, 2006).

To ensure newcomer students feel culturally validated as they enter the U.S schooling system, such strategies as those delineated by Gay (2010), Ladson-Billings (1994), and Pang (2005), interchangeably referred to as culturally responsive or culturally relevant teaching strategies, must be incorporated into the education of these youth. The premise of culturally responsive teaching is that it "filters curriculum content and teaching strategies through [students'] cultural frames of reference to make the content more personally meaningful and easier to master" (Gay, 2010). Pang defines culturally relevant teaching as "an approach to instruction that responds to the sociocultural context and seeks to integrate the content of the learner in shaping an effective learning environment" (2005, p. 337). Pang further defines the cultural content of the learner as including "aspects such as experiences, knowledge, events, values, role models, perspectives, and issues that arise from the community" (2005, p. 337), and the cultural context of learners as referring to "behaviors, interactional patterns, historical experiences, and underlying expectations and values of students" (2005, p. 337). According to research conducted by the abovementioned theorists, effective practitioners incorporate aspects of students' home cultures and value systems into their daily teaching practices, thus allowing students to connect with both the content and the instructional practices of said teachers. 
Immigrant youth are charged with both acculturating to the societal norms present in the United States, as well as mastering the dominant language present in this country. Owing to the idea that culture and language are inextricably linked, Gay argues "communication cannot exist without culture, culture cannot be known without communication and teaching and learning are more effective for ethnically diverse students when classroom communication is culturally responsive" (2010, p. 76). Along with teaching the structural and written components of English, culturally responsive teachers consider the sociocultural aspects of the language and encourage connections to be made between newcomer youths' home languages and their newly acquired English language skills. In order to increase student achievement and engagement in courses, Gay suggests while utilizing typical approaches to language instruction teachers of English learners must further:

...identify peer, school, and community informal networks, resources, and relationships that help students advance their oral language proficiency, and use them to facilitate their engagement with the subject matter content and academic language skills taught in schools and students need to have authentic opportunities to practice oral English discourse skills in a wide variety of circumstances (2010, p. 85).

In other words, culturally responsive educators consider the importance of culture while teaching students language acquisition skills, and further consider students' opportunities to authentically practice their oral language schools both within their classes and outside of their school day. 
In addition to considering culture within the context of communication, students should also view themselves and their cultures as a valued part of the educational experience in order to achieve success within the educational system. Gay views the practice of culturally responsive teaching as being essential to the achievement of historically marginalized students. She argues that educators must go beyond blaming students, their families, and their socioeconomic status for the barriers in student achievement, because for many students of color "intragroup variability, differential skills and abilities, stress and anxiety provoked by racial prejudices and stereotypes, and discontinuities between cultures of the school and homes of ethnically diverse students" (2010, p. 17) further play a role in why these students are not doing well in school. Gay believes it is crucial for teachers to understand their own beliefs concerning the education of marginalized students as they enter the classroom, in that "personal beliefs drive instructional behaviors" (2010, p. 216). She asserts, "if teachers have positive beliefs about ethnic and cultural diversity, they will act in accordance with them, and vice versa" (2010, p. 216). It is Gay's (2010) belief that there is a positive correlation between the manner in which teachers view their students culturally and linguistically and the equity of learning experiences and achievement outcomes in their classroom instruction. Gay (2010) contends that a culturally responsive classroom is one that is supportive and facilitative of students' intellectual, personal, social, ethnic, and cultural development, an environment in which students learn cooperatively through inquiry, discourse, and personal involvement. Students should be given choice and play an authentic role in decision-making within the academic environment to create a space of empowerment and to give students a sense of power and authority over their education (Gay, 2010). Finally, 
students must learn by both doing and having the opportunity to critically reflect on their knowledge, beliefs, and practices (Gay, 2010). In providing students a supportive space and authentic voice in their education, while challenging students to critique both their personal beliefs systems, as well as those of the dominant ideology in the United States, students will have a greater opportunity to achieve academic success by being engaged and feeling empowered, both culturally and linguistically, within the academic setting.

The notion of ensuring immigrant youth feel their home culture is significant within the American educational system is arguably a larger sociopolitical action, one that pushes against the current dominant cultural ideology that pervades throughout the system. Ladson-Billings defines the practice of culturally relevant teaching as a "pedagogy of opposition not unlike critical pedagogy but specifically committed to collective, not merely individual, empowerment" (1995, p. 160). In other words, culturally relevant pedagogy is transformative in nature, in that it challenges the status quo of the U.S. educational system by ensuring the system uses students' varying cultures and value systems within the classroom curricula and daily activities, creating a culturally pluralistic classroom, rather than requiring students to merely acculturate to the dominant culture present throughout the U.S. schooling system. To assure students a culturally relevant classroom experience, it is essential the following three criteria be realized: students must experience academic success through the rigorous development of their academic skills, teachers must develop and/or maintain students' cultural competencies by utilizing students' culture as a vehicle for learning, and students must develop a critical consciousness through which they challenge the status quo of the current social order (Ladson-Billings, 1995). Within Ladson-Billing's research $(1994,1995)$, which 
focuses on successful teachers of predominantly African-American students, it is clear that these three criteria can be accomplished in a variety of ways, but ultimately they must each be present to engage students within the daily classroom undertakings to ensure students feel their belief systems and their voices are valued within their education. The use of students' ideologies and cultural backgrounds within the curricula and daily classroom activities provides students the opportunity to connect with their education and to challenge the dominant cultural ideology, which can ultimately lead to greater academic achievement for such historically underserved students.

\section{Multiliteracies Pedagogy and Immigrant Youth}

In addition to ensuring students receive comprehensible input and valuing the cultural backgrounds and the personal and familial ideologies of students within the school environment, educators of immigrant youth must further consider the everchanging concept of literacy within the $21^{\text {st }}$ century. Students are utilizing evermore technologically advanced tools with which to communicate and, due to an increase in immigration, schools within the U.S. are seeing an increase in the cultural and linguistic diversity present in the nation's classrooms (Cummins, 2006). As a response to the aforementioned changes in present-day classrooms, in the mid-1990's a group of Australian, North American, and European academics (who referred to themselves as the New London Group) met to discuss such changes in the use and instruction of literacy in classrooms, coining the term "multiliteracies" to address "the expanding notions of literacy and to inquire into their relevance for education" (Cummins, 2006, p. 4). The New London Group sought to bring to light the importance of utilizing both technology 
and multilingualism to address the ever-changing face of literacy within the classroom and society at large within modern classrooms. According to the New London Group, "if literacy pedagogy is to be effective, it must take account of, and build on, the multilingual competencies that students bring to school and also expand the traditional definitions of literacy beyond the linear text-based reading and writing of western schooling" (Cummins, 2006, p.4). In other words, the instruction present in western classrooms must mirror the technological advances and the linguistic diversity present in today's society, both for native-born students and immigrant youth.

Despite the ever-changing demographics of students and the increased use of technology present in North American society, schools tend to follow a traditionally linear and text-based approach utilizing solely the dominant language in educating youth (Cummins, 2009; Giampapa, 2010; Taylor et al., 2008). Research analyzing the concept of multiliteracies highlights the need for schools to create academic environments that "engage students in a wide range of literacy practices that are creative and cognitively challenging and that bring together text-based and multimedia form of meaning making" (Giampapa, 2010, p. 409), rather than relying on the antiquated practices present in most academic environments. The New London Group proposed a pedagogical framework to make salient the key components of the multiliteracies approach and how these components can be enacted in the classroom, which focused on the following four concepts: situated practice, overt instruction, critical reframing, and transformed practice (Cummins, 2009; Taylor et al., 2008). The core of this framework follows that students should be educated utilizing meaningful and authentic practices (situated practice), and that conceptual learning must be supported by explicit instruction (overt instruction) 
(Cummins, 2009; Taylor et al., 2008). Students should be provided the space necessary to critically reflect on their learning experiences and to connect such experiences to their own lived experiences (critical reframing), and, further, students must be given the opportunity to connect their learning to society and the world at large (transformed practice) (Cummins, 2009; Taylor et al., 2008). Taylor et al. describe classrooms in which a multiliteracies pedagogy is enacted as "innovative learning environments that engage all students in an expanded range of literacy practices, including imaginative and cognitively-demanding text-based and multimedia instruction" (2008, p. 274). Instruction that incorporates the multiliteracies approach allows students the space to connect their personal cultures and languages to their academic experiences, and further to integrate and analyze their learning experiences within a technologically advanced society.

\section{Moving Beyond: Transformative Multiliteracies Pedagogy}

Cummins and his colleagues (2009) noted some issues in the New London Group's pedagogy of multiliteracies, and therefore took it a step further in building their Transformative Multiliteracies Pedagogy framework. The first issue they noted is the fact that the framework combines student-learner perspectives with teacher perspectives. Cummins and his colleagues believe these perspectives should be separated to permit "more specificity with respect to instructional practices" (2009, p. 47). For example, the New London Group framework does not specifically denote the importance of using students' primary languages and students' prior knowledge in instruction, despite their proven relevance for scaffolding dominant-language instruction for culturally and linguistically diverse students in the early stages of language acquisition (Cummins, 
2009). Secondly, Cummins and his contemporaries stated that the categories present in the New London Group framework "do not explicitly link instruction to processes of identity negotiation and societal power relations" (2009, p.47) though the links are implied, and therefore they created a framework that they felt more adequately addressed issues of power and identity within literacy instruction.

Cummins and his colleagues created their transformative framework by collaborating with educators and utilizing observations, stakeholder interviews, and data from case study research, focusing on instructional practices that increased the literacy engagement of culturally and linguistically diverse (CLD) students (Cummins, 2009). They state "the building blocks for Transformative Multiliteracies Pedagogy are: (a) distinctions among transmission, social constructivist, and transformative orientations to pedagogy; (b) an analysis of how societal power relations affect the schooling of CLD students; (c) the construct of multiliteracies $(2009$, p. 42). As the concept of multiliteracies has been discussed at length in the previous section, the notions of pedagogical orientations and societal power relations, as well as their implications with regard to the education of immigrant youth, are further elaborated below.

In analyzing pedagogical orientations, Cummins (2009) introduces the three delineated pedagogical orientations, transmission, social constructivist, and transformative, as being nested within one another, rather than being distinct and isolated from each other. As is seen in the visual representation shown in Figure 3, the innermost circle of the cone represents the pedagogical orientation with the narrowest focus, referred to as Transmission-oriented pedagogy. The goal of Transmission-oriented pedagogy is to "transmit information and skills specified in curriculum (and represented 
in tests) directly to the students", with learning strategy instruction tending to be "narrowly focused on the content of particular lessons rather than integrated into a broader process of collaborative inquiry and knowledge generation" (Cummins, 2009, p. 42). Teachers who solely utilize a Transmission-oriented pedagogy in their practice rely wholly on direct instruction and lecture to convey concepts to students, while students are viewed as passive participants in the academic process. The middle pedagogical space, as seen in Figure 3, is identified as Social Constructivist pedagogy. Social Constructivist pedagogy "acknowledges the relevance of transmission of information and skills, but broadens the focus to include the development of higher-order thinking abilities as teachers and students co-construct knowledge and understanding" (Cummins, 2009, p. 42-43). Teachers with a Social Constructivist pedagogical orientation value students' cultural backgrounds and activate their prior knowledge, while utilizing experiential learning and collaborative inquiry as modes of instructing students. Such educators work to create space for students to take "active control of their learning through the development of metacognitive strategies" (Cummins, 2009, p. 43), though the empowerment is limited as it focuses more on the individual than on larger societal relations, as see in the outermost circle of Figure 3, the Transformative pedagogical orientation. A Transformative pedagogical orientation emphasizes "the relevance not only of transmitting the curriculum and constructing knowledge but also of enabling students to gain insight into how knowledge intersect with power" (Cummins, 2009, p. 43). Transformative pedagogy calls on students to analyze power relations within society through collaborative critical inquiry and to act on inequities through social action. Cummins and his associates nested the abovementioned pedagogical orientations to 
highlight the fact that features of a Transmission-oriented pedagogy are a critical component of the broader two pedagogical orientations, as "explicit instruction and structured guidelines can play an important role in effective teaching/learning" (Cummins, 2009, p. 43).

Figure 3: Nested Pedagogical Orientations

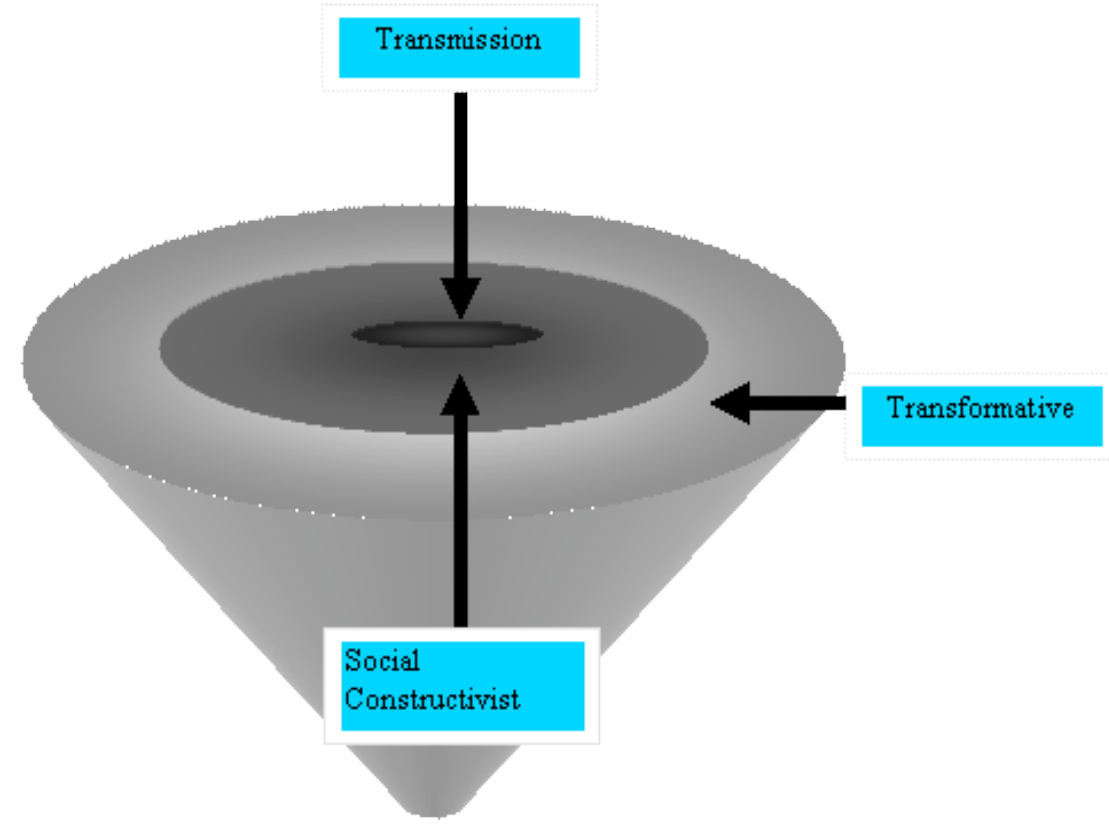

Note. Reprinted from "Multiliteracies pedagogy and the role of identity texts" by Jim Cummins, 2004. In K. Leithwood, P. McAdie, N. Bascia, \& A. Rodigue (Eds.) Teaching for deep understanding: Towards the Ontario curriculum that we need (p. 69). Toronto: Ontario Institute for Studies in Education of the University of Toronto and the Elementary Federation of Teachers of Ontario. Copyright 2004 by V. Kourtis-Kazullis. Reprinted without permission.

A long history of research has been conducted regarding ethnicity and educational achievement (Ladson, Billings, 1994; Portes \& Rumbaut, 2001), which has demonstrated that "groups that experience long-term educational underachievement tend to have experienced material and symbolic violence at the hands of the dominant societal group over generations" (Cummins, 2009, p. 44). Cummins and his contemporaries (2009) 
argue within their Transformative Multiliteracies Pedagogy framework that educators must individually and collectively analyze and challenge inequitable societal power relations within the classroom. Cummins (2009) believes this can be accomplished by ensuring that interactions with minority or subordinated groups of students do not further perpetuate an unjust power system. He illustrates the importance of positive teacherstudent interactions by stating "the ways in which teachers negotiate identities with students can exert a significant impact on the extent to which students will engage academically or withdraw from academic effort" $(2009$, p. 44-45). This concept is particularly salient in the education of immigrant youth as these students often feel pressure from the dominant cultural and linguistic majority to give up their own identity in lieu of that of mainstream society. It is critical for educators to encourage students to question such practices and to maintain and develop their own personal and familial identities to allow them to feel valued within the classroom and to assist them in making connections between their own home cultures and languages and that of the dominant American society. Cummins (2009) recognizes that interactions between students, teachers, and communities are never neutral, as they either reinforce unequal power structures or promote collaborative relations of power, and therefore it is necessary for teachers of immigrant youth to ensure the latter, as a collaborative environment will create the space for students to empower themselves and to challenge unequal power structures within schools and society at large.

In implementing the Transformative Multiliteracies Pedagogy framework (2009) into one's classroom practices, it is understood that teachers often feel powerless to affect change and enact transformative teaching strategies; which is particularly true in the 
current age of high-stakes testing and accountability under the federal No Child Left Behind mandate, a mandate that primarily relies on a Transmission-oriented pedagogical orientation in terms of instruction and assessment. That being acknowledged Cummins argues:

...even under highly constrained conditions, teachers have choices in how they connect the curriculum to the experiences and prior knowledge of CLD students, in the messages about language and culture they convey through their interactions with students, in the levels of cognition they attempt to evoke through instruction, and in how they engage parents with their child's education" (2009, p. 42).

Teachers can choose to enact the principles found in the Transformative Multiliteracies Pedagogy framework (2009), to ensure their students the opportunity to feel valued as individuals from culturally and linguistically diverse backgrounds. Though this framework relies on strategies proven effective in teaching culturally and linguistically diverse students (Cummins, 2009), it is a departure from the current academic environment in which these same students are being educated, an environment that solely relies on Transmission-oriented pedagogical strategies. It is critical that teachers of culturally and linguistically diverse students, namely secondary-level newcomer immigrant youth, afford these students the opportunity to learn in an environment that values their prior educational experiences and linguistic backgrounds, provides space for the co-construction of knowledge between teachers and students, utilizes technologically advanced teaching tools, and engages students in critical dialogue about existing societal power structures, to ultimately ensure all students equitable access to an engaging and rigorous academic experience. 


\section{Academic Self-Concept and Academic Achievement}

A final consideration in the education of immigrant youth is the connection between the students' sense of self and both their academic achievement and the degree to which they acquire language as they progress through their schooling experiences. It has long been argued that students who maintain a strong academic sense of self, or academic self-concept level, are likely to be more successful academically throughout their school careers. As defined by Areepattamannil \& Freeman, a person's academic self-concept is "comprised of a set of attitudes, beliefs, and perceptions held by students about their academic skill sets and performance" (2008, p. 704). Academic self-concept can further be defined as essentially a perception of the self in relation to others within a particular social comparison group (Hutchinson, Kirby, \& Carson, 2000). In other words, the manner in which a student views himself or herself in comparison to others, determines whether the student feels he or she "fits" in an academic setting. Students with higher self-concepts feel they belong and have the ability to achieve in classrooms, whereas students with low self-concepts might view themselves as not capable to participate effectively in the academic realm.

Students with higher academic self-concepts tend to score better and outperform others, due in large part to their belief that they can and should achieve in the classroom setting (Hutchinson, Kirby, \& Carson, 2000; Hung Hon \& Yeung, 2005). In their research analyzing whether or not there exists a causal link from prior academic selfconcept to subsequent achievement, Guay, Marsh \& Boivin (2003) found that for older students in middle and high school academic self-concept is strongly correlated to academic achievement; this finding was additionally tested and was verified in a study of 
primary students in Spain (Peralta Sanchez \& Sanchez Roda, n.d.). Through the use of a highly reputable scale Marsh and his colleagues developed to test students' academic-self concept levels, Marsh (n.d.) also found that older students' academic self-concept levels become more reliable and more stable as they age, likely owing to the fact that students develop a stronger sense of self as they mature. Furthermore, in their extensive work in the field, Guay, Marsh \& Boivin (2003) found that academic self-concept and academic achievement are reciprocally related and mutually reinforcing. In other words, "improved academic self-concepts will lead to better achievement AND improved achievement will lead to better academic self-concepts" (Marsh, n.d., p. 5); a finding that substantiates the importance for teachers to focus on both skills attainment and self-enhancement simultaneously when educating their youth.

Finally, though limited, the available studies have shown a positive correlation between academic self-concept and language acquisition, in that students with high academic self-concept adapt to and achieve within the school environment at greater levels and score at higher levels on language proficiency tests (Kim, 1983; Politzer \& Ramirez, 1981). In his preliminary study analyzing self-concept, language acquisition, and school adaptation in Asian children who have recently immigrated to the United States, Kim found that children with average to high levels of academic self-concept, "showed significantly higher language proficiency progress scores compared to children in the low self-concept group" (1983, p. 74). He further found that children with high academic self-concept demonstrated significantly higher school achievement and adaptation progress scores compared to children with average or low self-concept scores (Kim, 1983). Krashen's theory of the Affective Filter (2003) within language acquisition 
also supports the rationale that high academic self-concept will lead to greater language acquisition, in that anxiety or low self-esteem can cause students to block language acquisition. As was reported in a previous section, Krashen states, "if the acquirer is anxious, has low self-esteem, does not consider himself or herself to be a potential member of the group that speaks the language, he or she may understand the input, but it will not reach the language acquisition device" (2003, p. 6). In other words, English language acquisition requires students to take linguistic risks, and those with low academic self-concept levels are less likely to feel the security necessary to take such chances within the academic environment.

The importance of studying academic self-concept in immigrant youth is without question, particularly in light of the well-documented connection between academic selfconcept and academic achievement. Areepattamannil and Freeman assert "immigrant students with higher levels of achievement during adolescence are more likely to complete high school and to attend and complete college than their peers with lower levels of achievement" (2008, p. 703). In her study of the relationships between student aspirations, student self-concept, and student achievement in Western Australia, Young (1998) found that the classroom environment had a strong, positive effect on student selfconcept, which in turn had a significant effect on student ambition and achievement. It is vital that teachers create classroom environments that provide students the opportunity to connect with their learning processes and to increase their self-concept levels. Immigrant youth are far more likely to acquire language and achieve academic success if they feel they are capable of doing so, which in turn increases the ambition levels of said students and the likelihood that they will be successful within their academic careers in our 
nation's schools. The subsequent section will introduce common program models utilized in the education of immigrant youth

\section{Program Models Utilized in the Education of Immigrant Youth}

Schools in the United States are charged with the daunting task of providing a comprehensible education to a large number of students who do not speak the common societal language. As Valdés stated, "it is not just a question of teaching English; rather it is a question of providing large numbers of students with access to the curriculum at the same time that they are learning English" (2001, p.14). The right for students to attain a comprehensible education is protected under federal legislation, in that "key sources of federal law (Title VI of the Civil Rights Act of 1964, Lau v. Nichols, the Equal Educational Opportunities Act of 1974, Castañeda v. Pickard) prohibit discrimination against students on the basis of language and require that districts take affirmative steps to overcome language barriers" (Valdés, 2001, p.14). To adequately ensure comprehensible input and adhere to the abovementioned laws, districts across the nation offer a range of program options for emergent English learners that encompass both a bilingual and monolingual approach to educating these youth. When considering bilingual and monolingual programs, Minaya-Rowe claimed, "about two-thirds of the existing programs in K-12 schools are organized around five instructional strategies within these two programs... the remaining third weave features of both the bilingual and monolingual models" (2008, p.16). The aforementioned five instructional strategies are: two-way bilingual programs, transitional bilingual programs, sheltered English immersion programs, English as a Second Language (ESL) pull-out or push-in programs, 
or newcomer programs (Minaya-Rowe, 2008). With the emergence of newcomer programs having gained momentum only in recent years, English language learners have been traditionally placed in either a two-way or transitional bilingual program, or in a monolingual sheltered immersion or ESL program (Custodio, 2011; Minaya-Rowe, 2008; Short \& Boyson, 2012).

\section{Two-Way or Maintenance Bilingual Programs}

The general goal of two-way, or maintenance, bilingual program is to promote students' native language as the students acquire English, ultimately resulting in bilingualism and biliteracy (Baker, 2011; García \& Kleifgen, 2010; Minaya-Rowe, 2008; Valdés, 2001). Two-way bilingual programs are also referred to as maintenance programs, given that they are designed for students to maintain and improve their skills in their primary language, while concurrently learning English. These programs also offer content courses in students' home languages to ensure they are able to comprehend the material being taught. The reality of two-way bilingual programs is that they are generally only available to a small group of English learners, particularly in the first three years of schooling (Valdés, 2001), due to a variety of factors, including: a lack of bilingual teachers, the variety of language backgrounds with which students enter schools, a lack of funding sources for specialized programs, and a lack of commitment to the maintenance of students' primary language. Furthermore, in states that have passed anti-bilingual education propositions (such as Proposition 227 in California), even fewer students have access to this type of program due to legal constraints (Gándara, et al., 2010). 


\section{Transitional Bilingual Programs}

The primary goal of transitional bilingual programs, as well as English-dominant programs such as English as a Second Language and English Language Development programs, is the rapid acquisition of the English language. Language education is used as a means of socializing students to the dominant U.S. culture, which is arguably acquired at the cost of students' maintaining and developing students' primary language skills (García \& Kleifgen, 2010; Minaya-Rowe, 2008; Valdés, 2001). Transitional bilingual programs utilize students' home language in teaching content and literacy, yet the intent of this program is to transition students to English-only as rapidly as deemed possible. In transitional bilingual programs the minority language is used to teach concepts, with English becoming the more frequently used language as students progress through the grade-levels (Baker, 2011; García \& Kleifgen, 2010; Valdés, 2001). As Baker explains, "the aim of transitional bilingual education is assimilationist...students are taught briefly through their home language until they are thought to be proficient enough in the majority language to cope in mainstream education" (2011, p. 215). Transitional bilingual programs are generally implemented in grades K-3, after which all students are mainstreamed to English-only classes (Baker, 2011; García \& Kleifgen, 2010; Valdés, 2001).

\section{English as a Second Language Program and Sheltered English Program Models}

English as a Second Language (ESL) and sheltered courses are taught solely in English, utilizing a variety of strategies to assist the students in comprehending the content or language being taught (Baker, 2011; García \& Kleifgen, 2010; McDonnell \& 
Hill, 1993). ESL, also referred to as English Language Development (ELD) classes, are created for "students [to] receive specified periods of instruction aimed at the development of English-language skills, with a primary focus on grammar, vocabulary, and communication rather than content areas" (August \& Hakuta, 1997, p.19). These courses are meant to teach students the domains of reading, writing, listening, and speaking in English, and to prepare them linguistically for mainstream English classes. ESL/ELD courses have historically been taught during a portion of English learners' academic day, either by being "pulled out" of a grade-level academic course by an instructor charged with teaching ESL, or by attending a separate multi-level class in lieu of their grade-level English course. As a result of anti-bilingual education policy in such states as California, Arizona, and Massachusetts, the political term "Structured English Immersion" has been applied to ESL/ELD courses, which have largely replaced a wideranging set of bilingual programs in said states (Gandára et. al., 2010). According to the proponents of this program model, Structured English Immersion courses are meant to expedite students' learning of the language by immersing them in meaningful content and effective interactions in English, and requires that English learners "normally" be placed in such programs for a period not to exceed one academic year (Gandára et. al. 2010). Sheltered instruction focuses on students attaining "subject matter instruction in English, modified so that it is accessible to them at their levels of English proficiency" (August \& Hakuta, 1997, p.19). The overarching goal of sheltered instruction is to ensure English learners are able to understand concepts in grade-level content area classes, such as science, social studies, and mathematics classes. Sheltered courses utilize specific strategies, referred to as specially designed academic instruction in English (SDAIE), to 
assist students in accessing the content in a comprehensible manner, despite the fact that the sole mode of transmission is English, and to help students develop their academic language in English.

Both ESL and sheltered programs are commonly used when educating immigrant youth from a variety of primary language backgrounds, as there may not be sufficient numbers to establish bilingual classes, or if the districts are located in states that have laws limiting the ability for schools to create bilingual classes. The above-mentioned programs, be it bilingual or English-only, offer a variety of language acquisition options, yet these programs do not specifically address the complex acculturation issues immigrant youth face upon entering U.S. schools, a mitigating factor in the establishment of specialized newcomer programs (Short \& Boyson, 2004).

\section{The Emergence of Newcomer Programs}

The need for schools to address the complex issues in educating newcomer immigrant youth has led to the establishment of specialized newcomer programs (Custodio, 2011; Short \& Boyson, 2004). Newcomer programs go beyond language learning as the key focus for immigrant youths' schooling and additionally "emphasize safe educational environments, building bridges to U.S. institutions and society, helping children and families get access to needed services, and involving parents in their children's education" (Chang, 1990, p.17). Furthermore, newcomer programs "are designed for flexibility so they can respond directly to students' needs and to the mobility of the student population" (Chang, 1990, p.17). The goals of newcomer programs include: providing students with a firm academic foundation, developing English 
language proficiency, assisting students in attaining orientation and basic survival skills, developing students' multicultural understanding and promoting inter-cultural communication, encouraging secondary students to continue their education and increasing their access to long-term educational opportunities, and enhancing immigrant students' self-esteem (Friedlander, 1991). These goals are accomplished through a vast array of academic and support services, such as: classes to orient the students to academic and social life in the U.S., a specially designed curriculum, counseling services, parent and family support services, information and referral services, and access to bilingual support personnel, to name a few (Friedlander, 1991). Newcomer programs transcend the language support offered in bilingual and ESL classes to additionally support students and their families on a more holistic level.

\section{Newcomer Program Structure and Design}

Many districts design their newcomer programs on an ad hoc basis to address the immediate issues of newcomer immigrant students (Chang, 1990). There are currently no standard guidelines that govern how these programs are implemented, because most have been developed as a response to local needs and not state or federal educational policies. Therefore, comprehensive research has found there is no unified model for newcomer programs (Chang, 1990; Friedlander, 1991; Short \& Boyson, 2004). Some of the most distinct differences in the structure and development of these programs has been the location of said programs, the length of the daily program, and the duration of program enrollment. While some of these factors are logistic in nature, others are determined by the needs of individual students. 
When developing a newcomer program, site location has often been dependent on the availability of space, the transportation needs of students, as well as the district philosophy about how to best address the needs of newcomer youth. The three basic site models that have been accounted among the nation's newcomer programs are as follows: a class located within a comprehensive school site, a program at a separate school site, or a four-year whole school program (Custodio, 2011; Short \& Boyson, 2004). After conducting an extensive national study of 115 newcomer programs, Short and Boyson reported that "the most common model across the newcomer sites, found in more than $75 \%$ of the programs, is a program located within the larger school setting" $(2004, \mathrm{p} .22)$. This site model often allows students to attend their home schools (depending on their designated attendance area), gives students the opportunity to interact with mainstream students during non-academic periods, electives, and/or physical education classes, and dispels controversial issues related to the separation of one group from another. It can be argued that one of the most important benefits in creating a program within a school is that it allows students with sufficient English language and academic skills from newcomer programs to transfer more smoothly to mainstream classes, as teachers are able to more effectively communicate about such students and students can continue to be supported by the newcomer teacher. Separate site programs, while far less common, are often housed in buildings no longer used by a given district, or space leased or purchased for said purpose of creating a newcomer center. This type of program has often faced criticism due to the segregation of newly arrived immigrant youth from mainstream students, yet it allows districts to consolidate resources by serving all newcomer students at a single site (Custodio, 2011; Short \& Boyson, 2004). The least common site model is 
the whole-school model, which is generally used for schooling high school students with interrupted schooling experiences or students who are over-aged. This program allows students the option of remaining in the school until graduation, or they can be transferred to a comprehensive high school to complete their education (Custodio, 2011; Short \& Boyson, 2004).

Another variation common to the development of newcomer programs is the length of the daily program. While it is most common for these programs to be full day, there is a significant number created to encompass either half of the students' day, or one or two class periods. In their national study Short and Boyson (2004) found that of 115 programs, $56 \%$ were full day, $17 \%$ half day, and just $6 \%$ were less than half day. They also report that $2 \%$ of the programs were solely afterschool and $19 \%$ were a combination full day and half day. The length of daily program is highly dependant on the resources available and the needs of the students being served (Genesee, 1999), as well as the district philosophy on how to best educate immigrant youth.

The final structural variable to be discussed is the length of program enrollment. Most programs are highly dependent on financial resources and the academic and linguistic needs of the students (Custodio, 2011; Genesee, 1999; Short \& Boyson, 2004, 2012). Newcomer programs are specifically designed to assist students in successfully transferring to mainstream classes and thus "the majority of programs, particularly those which students attend for the full day, have set one-year time limits with the understanding that children who are ready to transfer to the next academic program more quickly can do so" (Chang, 1990, p.36). Short and Boyson (2004) found that $43 \%$ of the 115 programs they researched were one-year programs, as compared to $28 \%$ of programs 
where students stayed for more than one year to complete, $23 \%$ that offered either a one year and more than one year option, and 6\% that were created to be implemented over less than a one-year period. The one-year program, the most commonly implemented, serves the multiple purposes of adhering to the legalities making sure students are not fully educated in segregated environments, ensuring that the space is available for more students to be served by such programs, and giving sufficient time for students to become oriented to the schooling and cultural practices of the United States (Chang, 1990). While there is no one structure or organization utilized by districts to facilitate the transfer of students from newcomer programs to mainstream classes, effective articulation involves a sequenced curriculum for English language acquisition as well as a series of courses to help students either further their content knowledge or to attend to gaps in their educational backgrounds (Short \& Boyson, 2004).

\section{Parent and Community Outreach within Newcomer Programs}

Newcomer programs are designed to offer additional services, often beyond academics and English language acquisition. In their abovementioned study, Short and Boyson found that of the 115 programs under study, "sixty-seven percent of the programs offer physical health services, $42 \%$ offer mental health services, and $43 \%$ offer other social services" (2004, p.47), either onsite or through referrals. It is common for newcomer programs to be the point of access for families to learn about services with which they are eligible to participate, and while this sometimes comes about as a result of the formal intake and assessment procedure, or is assigned to a particular staff person, often families are connected to services as a result of the personal efforts of caring school 
personnel who are experienced enough to recognize the problems immigrants face (Chang, 1990). Many programs also offer child care and legal referrals, and participate in community outreach partnerships, such as: local and national government organizations, universities, community and youth organizations, athletic groups, health organizations, libraries, businesses, or social services (Short \& Boyson, 2004).

Additionally, a principal belief in the establishment of newcomer programs is the assumption that increased educational success for immigrant students is more likely to occur when connections between the school and students' families and communities are established and reinforced (Genesee, 1999). Newcomer programs tend to employ nontraditional approaches in establishing contact with families due to the preponderance of immigrant parents who "cannot speak English, are unfamiliar with American institutions, and often lack the time and resources to participate in traditional parent-teacher activities" (Chang, 1990, p.24). The notion that involving parents in the educational process is a critical component triggers many of these programs to seek opportunities to include the whole family in their child's schooling process, through adult ESL classes, family events, GED courses in Spanish, and notices written in students' home languages, among other methods of communication. Through the results of their national study, Short and Boyson $(2004,2012)$ affirm the importance of a strong home-school connection amongst the majority of newcomer programs. Their data shows that seventy percent of these programs conduct parent outreach, and sixty-two percent have a school liaison to work with parents (Short \& Boyson, 2004). Their data further illustrates, "sixtysix of the programs have adult ESL classes available at the program site or another district location, and 36\% make basic adult education available" (Short \& Boyson, 2004, 
p.47). Lastly, in their national study "forty-eight percent of the programs provide orientation for parents to U.S. schools and $43 \%$ provide orientation to the United States" (Short \& Boyson, 2004, p.47). The idea that families must be included in the schooling process is a driving force behind the creation of these specialized programs.

Finally, the vast majority of newcomer programs offer some form of orientation courses or activities as an additional means to assist students in becoming familiar with the U.S. schooling processes, as well as familiarizing them with the social and cultural value system in the United States. These acculturation goals are generally achieved through classroom curricula, and are supplemented with field trips, cultural activities, and special cultural events (Friedlander, 1990; Genessee, 1999), that attempt to familiarize students with American culture, their community and school routines, and the educational expectations of students in the United States (Genessee, 1999). Furthermore, as part of the long-term goal of newcomer programs to encourage secondary students to continue their education and increase their access to long-term educational and career opportunities (Friedlander, 1990), many programs offer some form of career awareness education to assist immigrant youth as they complete school. Over half of the high schools in the national study conducted by Short and Boyson offered career counseling services and forty-two percent of the programs offered career awareness courses (2004). Additionally, "some programs offered vocational education or work internships so that students could develop practical skills and knowledge about job opportunities (Short \& Boyson, 2004, p.36). Vocational training and work internships have been shown to be particularly useful for immigrant students "who were over age and did not have the time to finish high school before reaching the maximum school age" (Short \& Boyson, 2004, 
p.36). Many of these career opportunities utilize students' native language as a resource (Short \& Boyson, 2004), thus valuing the prior knowledge and language with which immigrant students enter U.S. schools. The following section will provide a description of the methodology utilized within this mixed-methods, multiple case study research study. 


\section{CHAPTER 3}

\section{Methodology}

The purpose of this study is to compare the classroom experiences and achievement levels of secondary-level Latino newcomer immigrant youth as they exit two distinctive yearlong program placements. This research focuses on the acquisition of both qualitative data, as it pertains to the experiences of the stake holders within either a newcomer program or an English as a Second Language (ESL) placement, as well as quantitative data, which yields information about students' academic self-concept levels as they exit their program of study. Additional quantitative data attained through the California state educational database Dataquest (CDE, 2011) is used in this study to demonstrate the redesignation rates of English learners within the programs under study. The collection of data for this study, as termed a transformative parallel mixed-methods approach (Mertens, 2005), serves to answer the following central research question and sub-questions:

How does a specialized newcomer program prepare secondary-level Latino immigrant youth to gain the language proficiency, academic skills, and academic self-concept necessary to be successful within an Englishonly educational environment, as compared to students placed in a traditional ESL program?

1. What are the prevailing pedagogical practices utilized in both a newcomer program and a traditional ESL program?

a. How do the curricula, language instruction, content instruction, teachers' attitudes, level of cultural responsiveness, and pedagogical orientations compare within the programs under study?

2. Is there a relationship between Latino students' academic selfconcept and the increase in their language proficiency level within either a specialized newcomer or a traditional ESL program? 
3. How are Latino newcomer students' academic self-concept, language proficiency levels and acquisition of academic skills influenced by the program in which they are educated?

A methodological framework, as illustrated in Figure 4, outlines the methodological process and demonstrates the order in which the steps were undertaken to complete this proposed study. In addition, the methodological framework served as a guide for the researcher during the process of data collection. As is shown in Figure 3, the mixed-methods data for this study was collected utilizing a "two-tailed" multiple case study research design (Yin, 1994), as is further discussed in a subsequent subsection. This section additionally illustrates the process that was employed in selecting participants for this study, as well as the method of data collection. A description of the research tools follows within this chapter, with an explanation about how each of these tools served to effectively gather the qualitative and quantitative data required for this study. The methodology section concludes with a discussion about the data analysis techniques and the manner in which the collected data was methodologically triangulated between multiple sources to substantiate the results and increase the credibility of the findings (Merriam, 2009). 
Figure 4: Overview of Methodological Process

\section{Research Questions}

How does a specialized newcomer program prepare secondary-level Latino immigrant youth to gain the language proficiency, academic skills, and academic self-concept necessary to be successful within an Englishonly educational environment, as compared to students placed in a traditional ESL program?

1. What are the prevailing pedagogical practices utilized in both a newcomer program and a traditional ESL program?

a. How do the curricula, language instruction, content instruction, teachers' attitudes, level of cultural responsiveness, and pedagogical orientations compare within the programs under study?

2. Is there a relationship between the students' academic self-concept and the increase in their language proficiency level within either a specialized newcomer or a traditional ESL program?

3. How are Latino newcomer students' academic self-concept, language proficiency levels and acquisition of academic skills influenced by the program in which they are educated?

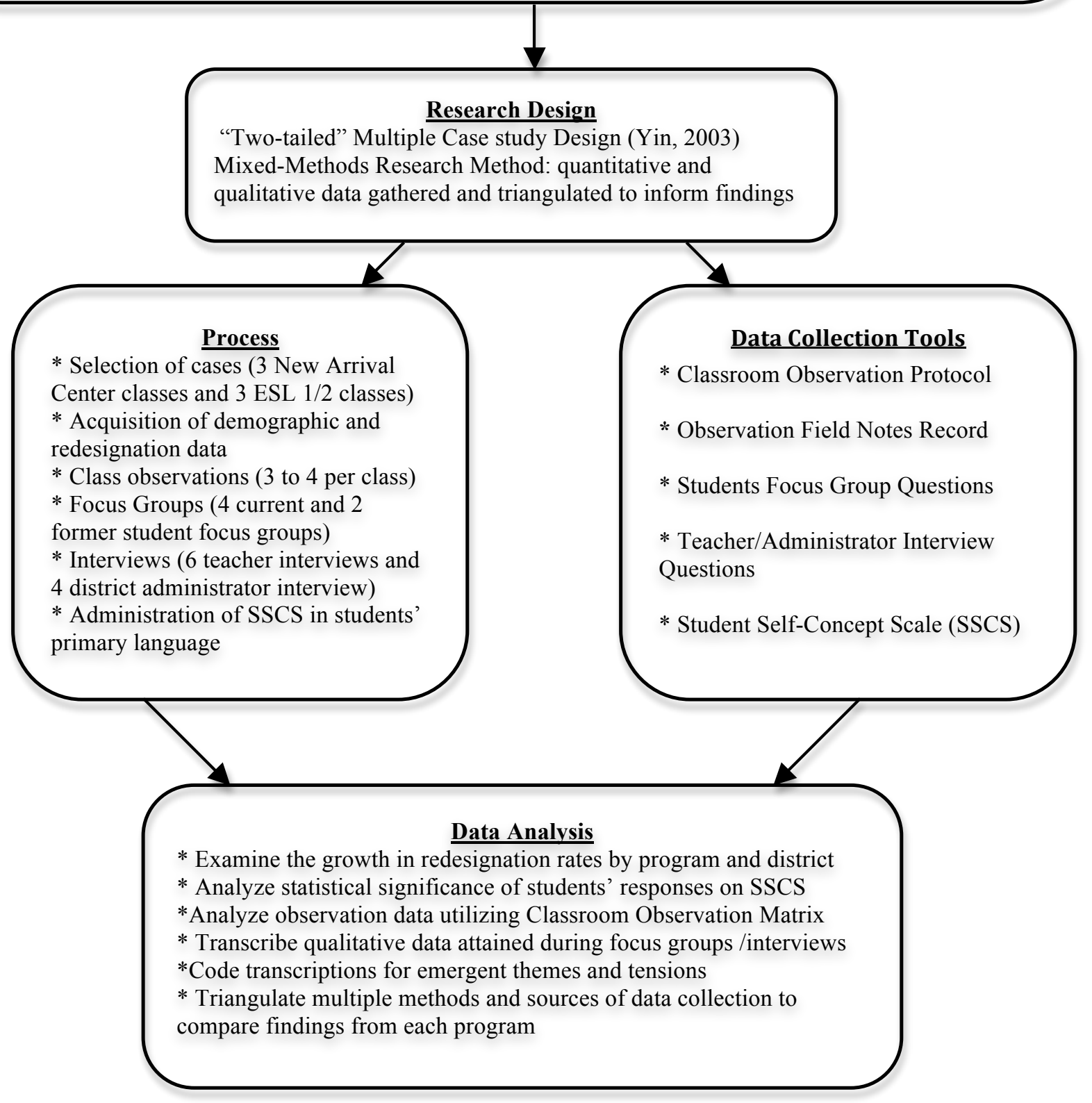




\section{Research Design}

In an effort to better understand the experiences of Latino newcomer immigrant youth as they exit one of two specific programs within the San Diego Unified School District, a case study design was utilized as it most accurately provides an understanding of the natural environment in which the newcomer students under study are being educated. Case studies are effective in understanding complex social phenomena, in that they "allow investigators to retain the holistic and meaningful characteristics of real life events" (Yin, 2003, p.2), this complex social phenomena being the intricate nature of newcomer students' classroom experiences. Yin defines a case study as "an empirical inquiry that investigates the phenomenon within its real-life context, especially when the boundaries between phenomena and context are not clearly evident” (Yin, 2003, p. 13). The case study research design is therefore quite appropriate when considering the language acquisition and self-concept levels of newcomer students within differing program models, as many complex factors must be considered in determining how these programs play a role in said variables. The use of the case study as a research strategy can be viewed as a comprehensive research method in that it takes into account the research design, multiple data collection techniques, and specific data analyses, most commonly being the triangulation of multiple data sources into well-substantiated findings (Yin, 2003). In summary, a case study approach most effectively addresses the many variables associated with researching and attempting to understand the experiences of newcomer immigrant youth as they complete their first year in the U.S. educational system.

This research more specifically employs a multiple-case design, as six total cases, or classrooms, are included in the research process. The advantage of including multiple 
cases in a research study is that these studies are often more robust and compelling in nature, though, on the other hand, they are also more time consuming and thus must not be entered into lightly (Yin, 2003). Multiple-case studies further allow for replication among multiple cases to occur, therefore making the results more reliable (Yin, 2003). This study included three cases, or classrooms, within each of the two programs under study, therefore allowing for literal replications among the similar cases (cases predict similar results) and theoretical replications between the two programs (cases predict contrasting results, but for predictable reasons) (Yin, 2003). The research design is an embedded design in that the experiences and achievement levels of individual students were studied in the context of each classroom case, rather than solely viewing the classroom as one whole case, as seen in a holistic design (Yin, 2003). As seen in Figure 5, the individual experiences of the students within each of the three classes, the micro, have been analyzed in terms of the classrooms as a whole, the meso. The three classrooms in each program were then related to the program itself, as an even greater whole, or the macro, to ensure larger generalizations to be made between the two programs under study. Finally, this case study approach is a "two-tailed" design, defined by Yin (2003) as the selection of cases from two extremes of an important theoretical position, yet this design focuses on two contrasting programs and thus is slightly adapted as neither program represents a true theoretical extreme. 
Figure 5: Two-Tailed Multiple-Case Study Research Design

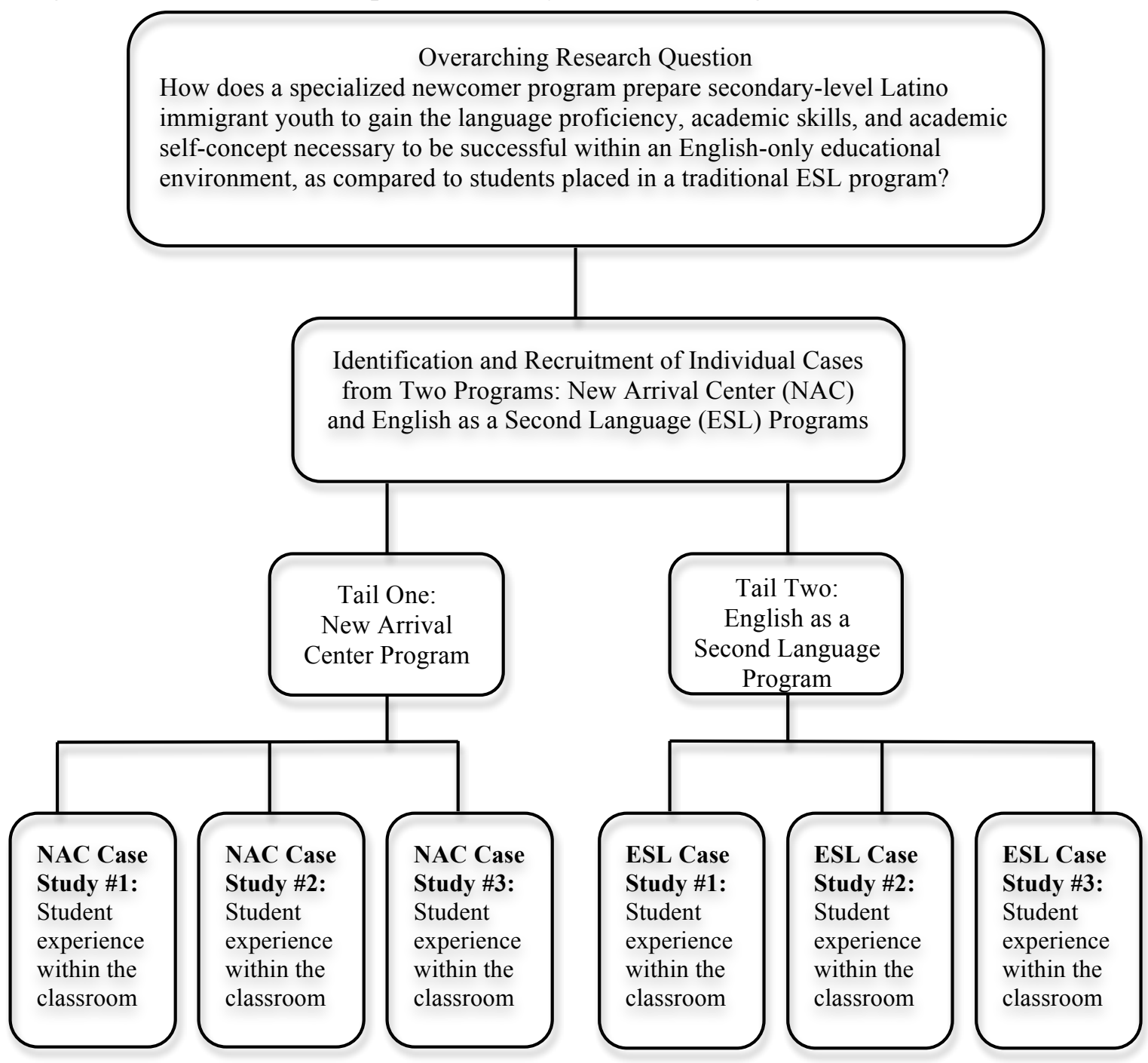

The data for this study was gathered through both qualitative and quantitative research methods, and thus this study is a mixed-methods research study. Descriptive statistics were utilized within the quantitative portion of this study to analyze the demographic information, while database inquiry was used to demonstrate language acquisition through the school-wide redesignation rates. A statistical analysis of students' self-concept levels, as were gathered utilizing the Student Self-Concept Scale (SSCS), was conducted on each of the 72 survey items to address whether or not the self-concept 
data attained from the two groups under study show statistically significant differences for particular survey items. The statistical analysis was then employed to demonstrate whether or not the outcome of participation in a newcomer program effects students' selfconcept levels when compared to the outcome of students educated in a traditional ESL class. The qualitative data for this study was amassed through observations and field notes, as well as student focus groups and teacher and administrator interviews. The results of the focus group and interview data were transcribed and coded for emergent themes and tensions, while the observations were analyzed utilizing a tool of analysis called the Classroom Observation Continuum (Appendix I). The qualitative and quantitative data was collected for this study in a parallel mixed-methods fashion, as both types of data were collected and analyzed simultaneously throughout the study to address the research questions, rather than drawing on one data type to inform the collection of the other data type (Mertens, 2005) as seen in a sequential design.

\section{Selection of Study Participants}

The participants selected for this study were all associated with the San Diego Unified School District, as a student, teacher, or administrator within the district. The San Diego Unified School District is currently ranked as one of the twenty largest urban school districts in the United States (NCES, 2008) with over 134,000 students enrolled during the 2011-12 school year (SDUSD, 2011). In addition, the district is the second largest school district in the state (SDUSD, 2011). Due to its size, this district has a large pool of financial resources, reportedly operating with as much as a $\$ 1.1$ billion dollar budget (SDUSD, 2011). The abovementioned funding sources, coupled with the fact that 
roughly thirty percent of the total school population in the district are English Language Learners of which $77 \%$ are Spanish speakers, has resulted in district personnel having the means and need to develop and implement a variety of specialized English learner programs, namely an ESL program and a self-contained newcomer program. The existence of both self-contained newcomer classes, as well as traditional ESL classes, and the high number of Latino English learners were the primary premises for selecting this school district as an ideal location within which to conduct this research study.

The San Diego Unified School District utilizes a strict policy with which to approve or deny any proposed research studies. Researchers are required to attain sponsorship by a district administrator in the central office, preferably one that oversees the particular area under study. For the purpose of this study, an administrator from the district's English Learner office agreed to sponsor the study, with the guidance of the resource teacher in charge of overseeing the newcomer program, called the New Arrival Center. In accordance with district policy, a research proposal was submitted with a letter of sponsorship from the district sponsor to a panel of district administrators, who then convened to approve or deny the research request. After the research request was approved at the district level, principals and teachers from the individual school sites were contacted as they have the final say as to whether or not they would be willing to be studied for a given research project. Finally, with the consent of the teachers who agreed to be a part of the study, the researcher contacted students and their families within the classes under study, as they must sign the designated consent forms prior to taking part in the research process. 
Prior to selecting the participants for the research study, the researcher obtained approval to conduct human subjects research through the Institutional Review Boards (IRB) at both San Diego State University and Claremont Graduate University. The IRB approved both the scope and methodological practices of the study, as well as the consent forms and tools used within the study. The original approval was to conduct the study solely with high school students, and therefore when middle schools were added a modification to the original protocol was approved as well. After gaining both IRB and district approval to conduct the research project, the cases for the study were chosen according to specific criteria. The three cases illustrating the first tail of the two-tailed study were comprised of full day self-contained newcomer classes, which were chosen from the ten secondary-level newcomer classes present at six middle and high schools throughout the San Diego Unified School District. The second tail of the study was made up of three secondary-level ESL 1/2 classes. ESL 1/2 classes operate in this district as anywhere from an eighty-five minute to a two-hour literacy block, depending on the bell schedule of each school, rather than as a full day self-contained class. The student population of the ESL classes mirrors that of the self-contained newcomer classes, as both contain multi-grade-level students at the beginning level of English language acquisition, as determined by the California English Language Development Test (CELDT). ESL classes, though, solely take the place of grade-level English classes and, therefore, the students in this type of program are mainstreamed into grade-level content and elective classes, while the New Arrival Center classes provide both English language and content instruction to students, only mainstreaming them for Physical Education classes. 
Both the newcomer classes and the ESL 1/2 classes utilized in this study were initially identified by requesting a recommendation from the resource personnel working within the district's central office dedicated to the needs of English learners. The request for a recommendation stipulated that the newcomer and the ESL 1/2 classes were considered by the resource teacher to be exemplary examples of the manner in which either program was designed by the district to function. Henceforth, both the newcomer and the ESL $1 / 2$ classes that were chosen for this study were regarded highly by the district English learner resource personnel and district administrators for their use of successful strategies and instructional practices in the education of immigrant youth. The teachers approached for this study were also considered to be veteran teachers as each of them had been in the classroom for a minimum of ten years, and many had had significant experience teaching English learners. The selection of these classes was additionally dependent on the willingness of the teachers to participate in this study.

The district English learner resource teacher made initial contact with the principals and teachers associated with the recommended classes to introduce both the study and the researcher. She subsequently connected the researcher to the potential teacher participants to ascertain whether or not they would be willing to be involved in the study. The researcher then contacted four potential newcomer teacher participants by email and was approved by three. The newcomer teacher that denied access sent regrets and expressed a feeling of being overwhelmed this year by changes in her teaching schedule and a heavy workload. The researcher contacted ten possible ESL 1/2 teachers, of which five agreed to participate and five declined. The ESL teachers that declined also expressed that they were feeling overwhelmed and they felt unable to accommodate any 
additional pressures. In reviewing the five possible cases, three were chosen according to the number of Latino students in the classes and the experience of the teachers within each class. One of the five teachers willing to participate was excluded as the class contained only one Latino student and the other was left out since the teacher had had less than one years experience teaching beginning English language learners. The students invited to participate in this study either currently attend or had attended classes within one of the two program models under study within the past two years. The students each entered the district's secondary-level schools as newcomer immigrant youth, defined in the introduction as students who have lived in the U.S. and have attended secondary-level schools in the country for one year or less, and Spanish was their primary language. Finally, the four administrators interviewed for the study each oversaw and supported a portion of the newcomer program or the ESL program as an administrator or a resource teacher. The four administrators worked together in the same central office and therefore possessed information about both programs, though they focused more on either the newcomer or the ESL program. The administrators were asked to participate by the researcher and all agreed to do so.

\section{Teacher Participants}

The six teachers that agreed to participate and were selected for the study each were informed that they would be observed three to five times, depending on scheduling availability, and they would be interviewed one time at their convenience. The teachers further agreed to have their Spanish-speaking students surveyed during non-instructional times and to have willing students participate in focus groups with parent consent and 
student assent. Table 1 demonstrates the demographics of the teachers chosen to participate in this study.

\section{Table 1}

Participating Teacher's Demographics

\begin{tabular}{llllll}
\hline & $\begin{array}{l}\text { Case } \\
\text { Study \# }\end{array}$ & Ethnicity & $\begin{array}{l}\text { Total \# } \\
\text { of Years } \\
\text { Teaching }\end{array}$ & $\begin{array}{l}\text { \# of Years } \\
\text { Teaching } \\
\text { in Program }\end{array}$ & $\begin{array}{l}\text { \# of Years } \\
\text { Teaching } \\
\text { Beginner EL's }\end{array}$ \\
\hline Teacher A & NAC \#1 & White & 16 & 2 & 6 \\
Teacher B & NAC \#2 & Latina & 12 & 3 & 12 \\
Teacher C & NAC \#3 & Latina & 16 & 3 & 6 \\
Teacher D & ESL \#1 & White & 17 & 17 & 17 \\
Teacher E & ESL \#2 & Asian & 21 & 18 & 18 \\
Teacher F & ESL \#3 & Latina & 12 & 5 & 8 \\
\hline
\end{tabular}

Note. NAC = New Arrival Center; ESL = English as a Second Language program.

${ }^{a}$ English Learner Support Teacher

The teachers who participated in this study, both in the newcomer program referred to by the district as the New Arrival Center (NAC) and in the ESL program, were each female and, also, had significant classroom experience. Additionally, the teacher participants had been teaching in one of the two programs under investigation for a minimum of two years prior to the research study. As the NAC program was created during the 2008-09 school year, the teachers within this study had only had the opportunity to teach within the program for two to three years, though these same teachers had taught ESL prior and therefore had experience teaching beginning English language learners. The NAC teachers in this study taught their self-contained class for both English language arts and for the majority of the students' content classes, such as mathematics, science, and history. Schools with multiple NAC classes allowed their teachers to team with the other NAC teachers to create leveled math classes to better 
support students at their point of need, and to additionally exchange students for science and history classes to reduce the number of courses about which the teachers would need to prepare. Two of the ESL teachers in this study (Teachers D and E) taught higher levels of ESL classes throughout the day as well as other content areas, while the third ESL teacher (Teacher F) was an English Learner Support Teacher for the remainder of the day. As a support teacher, Teacher F was charged with conducting the CELDT testing for the site, as well as completing the necessary paperwork for redesignating English learners at the site. Additionally, Teacher F supported English learners in their content classes when time permitted, and assisted in site professional development concerning the needs of English learners. Teacher F had worked as an ESL teacher for five years prior to accepting the resource position, which she had held for three years at the time of this study. Though it was not required in her job description, Teacher F had agreed to teach the ESL class at her site after the staff was reduced during the 2011-12 school year, due to overwhelming budget cuts which resulted in the need for an ESL teacher on site.

Table 2

Participating Teachers' Class Compositions

\begin{tabular}{llllll}
\hline & $\begin{array}{l}\text { Grade } \\
\text { Levels }\end{array}$ & $\begin{array}{l}\text { Total \# of } \\
\text { Students }\end{array}$ & $\begin{array}{l}\text { \# of Spanish } \\
\text { Speaking } \\
\text { Students }\end{array}$ & $\begin{array}{l}\text { \# of Different } \\
\text { Languages } \\
\text { in Class }\end{array}$ & $\begin{array}{l}\text { \% Site } \\
\text { Free/Reduced } \\
\text { Price Meals }^{\mathrm{b}}\end{array}$ \\
\hline Teacher A & $9-12$ & 19 & 19 & 1 & \\
Teacher B & $9-12$ & 20 & 2 & 13 & $96.8 \%$ \\
Teacher C & $6-8$ & 11 & 5 & 5 & $93.5 \%$ \\
Teacher D & $9-12$ & 33 & 9 & 5 & $52.7 \%$ \\
Teacher E & $6-8$ & 24 & 2 & 11 & $91.1 \%$ \\
Teacher F $^{\mathrm{a}}$ & $9-12$ & 10 & 8 & 2 & $78.2 \%$ \\
\hline
\end{tabular}

Note. ${ }^{\mathrm{a} C o m b i n a t i o n ~ c l a s s e s ~ c o m p r i s e d ~ o f ~ E S L ~ 1 / 2 ~ a n d ~ E S L ~} 3 / 4$ students, ${ }^{b}$ Free/Reduced Price Meal percentages as reported during the 2010-11 academic year. 
Table 2 illustrates the demographic information of each of the classes, or cases, under study. The two programs being investigated were located in both middle and high school settings, and therefore classes within two high schools and one middle school from each of the two programs were selected to be a part of the study, as these classes best represent the programs in their entirety. An attempt was made to utilize schools with greater percentages of Latino students, though as is demonstrated in Table 2, both the NAC \#2 and the ESL \#2 cases had a small number of Latino students within a vast number of language backgrounds. Despite the small number of Latino students within these two cases, the decision to include them was deliberate as these cases were representative of the realities of the two programs under study, and therefore they added a greater depth of understanding about these two programs as a whole. Finally, due to the need to increase class sizes and fund teacher positions within these programs, two of the ESL cases (ESL \#1 and ESL \#3) were combination classes in which the teachers taught two levels of English learners, ESL 1/2 students and ESL 3/4 students, concurrently within the same class. The results section of this study will further detail the tensions associated with teaching and learning within a combination class.

\section{Students Participants}

The students that participated in the focus groups and the students that were surveyed either attended classes in one of the six cases under study, or they attended classes in other newcomer or ESL classes throughout the district. It was necessary to broaden the sample pool for the focus groups and surveys, as the number of Latino newcomer immigrant youth that fit the criteria of the study was relatively small. The 
surveys were given to 192 total Latino students at 13 different secondary-level schools throughout the district, each of who fit the necessary criteria to participate in the study. The surveys were grouped into four categories: students currently attending classes in the newcomer program, students currently attending classes in the ESL program, students who attended classes in the newcomer program in the past two years, and students who attended classes in the ESL program in the last two years.

The focus groups were made up of between four and nine Latino students each. Two of the student focus groups were comprised of students who currently attended classes in the newcomer program (Focus Groups \#1 and \#2) while two other focus groups were made up of students currently in ESL 1/2 classes (Focus Groups \#3 and \#4). The students currently enrolled in the newcomer (New Arrival Center) or ESL classes were labeled as "Year 1" students in either program. One more focus group each was made up of students who had attended classes in either the newcomer program (Focus Group \#5) or the ESL program (Focus Group \#6) during the past academic year. Students who had attended classes in either program the previous year were labeled "Year 2" students. These students were all currently in ESL 3/4 or ESL 5/6 classes, depending on their level of English language acquisition.

The students who participated in the focus groups each spoke Spanish as a primary language and were selected because of their class placements and their willingness to participate in a focus group during non-instructional time. The focus groups were conducted in English and translated into Spanish by a Spanish translator hired by the researcher to either fully translate or to support students with their primary language when necessary, depending on the needs of the students. All questions were 
translated from English to Spanish during the focus groups, and the students were additionally informed prior to the start of each focus group that they could answer in either Spanish or English. Language choice was an important piece in gathering focus group data, as the researcher wanted to ensure students understood the questions and their peers' answers, both to make certain the data was valid and to create an environment in which students' primary languages were valued and promoted. Tables 3 through 8 illustrate the demographic information of the student focus group participants.

Table 3

Focus Group \#1 Student Demographics- New Arrival Center Year 1

\begin{tabular}{llllll} 
Student & $\begin{array}{l}\text { Grade } \\
\text { Level }\end{array}$ & Age & Gender & $\begin{array}{l}\text { Country of } \\
\text { Origin }\end{array}$ & $\begin{array}{l}\text { Time Attending } \\
\text { School in U.S. }\end{array}$ \\
\hline Student A & 9 & 15 & Male & Mexico & 1 year \\
Student B & 9 & 16 & Male & Mexico & 2 months \\
Student C & 9 & 15 & Male & Mexico & 1 month \\
Student D & 12 & 17 & Female & Mexico & 4 months \\
Student E & 10 & 17 & Female & Mexico & 2 months \\
Student F & 9 & 15 & Female & Mexico & 3 months \\
Student G & 9 & 16 & Male & Mexico & 1 month \\
Student H & 9 & 16 & Female & Mexico & 1 year \\
Student I & 9 & 16 & Male & Mexico & 2 months \\
\hline
\end{tabular}


Table 4

Focus Group \#2 Student Demographics- New Arrival Center Year 1

\begin{tabular}{llllll}
\hline Student & $\begin{array}{l}\text { Grade } \\
\text { Level }\end{array}$ & Age & Gender & $\begin{array}{l}\text { Country of } \\
\text { Origin }\end{array}$ & $\begin{array}{l}\text { Time Attending } \\
\text { School in U.S. }\end{array}$ \\
\hline Student J & 9 & 15 & Female & Mexico & 8 months \\
Student K & 9 & 14 & Male & Mexico & 3 months \\
Student L & 12 & 18 & Male & Mexico & 2 months \\
Student M & 9 & 14 & Male & Mexico & 3 months \\
Student N & 10 & 15 & Female & Mexico & 3 months \\
Student O & 10 & 15 & Female & Mexico & 8 months \\
Student P & 10 & 15 & Male & Mexico & 2 months \\
Student Q & 9 & 15 & Male & Mexico & 6 months \\
\hline
\end{tabular}

Table 5

Focus Group \#3 Student Demographics- ESL Program Year 1

\begin{tabular}{llllll}
\hline Student & $\begin{array}{l}\text { Grade } \\
\text { Level }\end{array}$ & Age & Gender & $\begin{array}{l}\text { Country of } \\
\text { Origin }\end{array}$ & $\begin{array}{l}\text { Time Attending } \\
\text { School in U.S. }\end{array}$ \\
\hline Student R & 11 & 17 & Male & Mexico & 9 months \\
Student S & 9 & 14 & Male & Mexico & 7 months \\
Student T & 10 & 16 & Male & Mexico & 9 months \\
Student U & 9 & 14 & Male & Mexico & 2 years \\
Student V & 11 & 17 & Male & El Salvador & 6 months \\
Student W & 10 & 16 & Male & Mexico & 3 months \\
Student X & 10 & 15 & Female & Mexico & 9 months \\
Student Y & 10 & 15 & Female & Mexico & 2 months \\
\hline
\end{tabular}

Table 6

Focus Group \#4 Student Demographics- ESL Program Year 1

\begin{tabular}{llllll}
\hline Student & $\begin{array}{c}\text { Grade } \\
\text { Level }\end{array}$ & Age & Gender & $\begin{array}{l}\text { Country of } \\
\text { Origin }\end{array}$ & $\begin{array}{l}\text { Time Attending } \\
\text { School in U.S. }\end{array}$ \\
\hline Student Z & 9 & 15 & Male & Mexico & 2 months \\
Student AA & 9 & 15 & Male & Mexico & 3 months \\
Student BB & 9 & 14 & Male & Mexico & 3 months \\
Student CC & 9 & 14 & Male & Mexico & 7 months \\
\hline
\end{tabular}


Table 7

Focus Group \#5 Student Demographics- New Arrival Center Year 2

\begin{tabular}{|c|c|c|c|c|c|}
\hline Student & $\begin{array}{l}\text { Grade } \\
\text { Level }\end{array}$ & Age & Gender & $\begin{array}{l}\text { Country of } \\
\text { Origin }\end{array}$ & $\begin{array}{l}\text { Time Attending } \\
\text { School in U.S. }\end{array}$ \\
\hline Student DD ${ }^{\mathrm{a}}$ & 9 & 14 & Male & Mexico & 1 year, 4 months \\
\hline Student EE & 10 & 15 & Female & Mexico & 1 year, 6 months \\
\hline Student FF & 10 & 15 & Female & Mexico & 1 year, 6 months \\
\hline Student GG & 11 & 16 & Female & Mexico & 1 year, 6 months \\
\hline
\end{tabular}

${ }^{a}$ Attended the New Arrival Center program in middle school

Table 8

Focus Group \#6 Student Demographics- ESL Program Year 2

\begin{tabular}{llllll} 
Student & $\begin{array}{l}\text { Grade } \\
\text { Level }\end{array}$ & Age & Gender & $\begin{array}{l}\text { Country of } \\
\text { Origin }\end{array}$ & $\begin{array}{l}\text { Time Attending } \\
\text { School in U.S. }\end{array}$ \\
\hline Student HH & 7 & 12 & Male & Mexico & 3 years, 5 months \\
Student II & 9 & 15 & Female & Mexico & 2 years, 3 months \\
Student JJ & 9 & 15 & Female & Mexico & 1 year, 2 months \\
Student KK & 7 & 13 & Female & Mexico & 1 year, 6 months \\
Student LL & 7 & 13 & Female & Mexico & 2 years, 3 months \\
Student MM & 7 & 13 & Female & Mexico & 2 years, 3 months \\
Student NN & 11 & 17 & Female & Mexico & 1 year, 2 months \\
\hline
\end{tabular}

Note. ${ }^{\mathrm{a}}$ Gaps in time attending school in the U.S., most recent continuous period of time 1 year, 3 months

\section{Administrator Participants}

In an effort to better understand the creation and the ongoing implementation of

the New Arrival Center and the ESL programs within the district under study, four

administrators based out of the central office that oversees programs and resources for English learners within the district were interviewed about said programs. The administrator who was the district sponsor of the study recommended the administrator participants to the researcher, as these four participants, including the sponsor herself, 
were charged with creating and implementing the two different programs at the school sites across the district. The sponsor (Administrator A), who was the director of the office overseeing the needs of English learners in the district, had played a large role in the creation of the New Arrival Center, and therefore she spoke to this program, in addition to the resource teacher (Administrator B) charged with running the program and supporting the teachers and students within the program. The two ESL administrator participants were resource teachers in charge of the middle (Administrator C) and high school (Administrator D) programs respectively. The sponsor (Administrator A) was the overall head of all English learner programs, and therefore she oversaw both programs and was the supervisor of the other three participants, yet she spoke solely about the New Arrival Center as was previously mentioned. Each of the administrator participants had taught in the district under study prior to applying for their varying positions overseeing programs in the central office. In addition, each administrator participant had had a significant amount of experience working with English learners, both in the classroom and subsequently as a resource teacher working in the central office, and therefore they were awarded their current positions due to their high levels of expertise in this area. According to the district website, the central office for English learners, out of which each of the four administrator participants were employed, is charged with the following district tasks (SDUSD, 2010):

- Designing and supporting the implementation of instructional programs for English learners including SEI, MEC, Bilingual, and Newcomer programs.

- Designing and supporting the implementation of English Language Development instruction and courses in K-12. 
- Providing on-site technical support for EL compliance - Ensuring compliance with both state and federal requirements and procedures.

- Assisting with site English learner program articulation and placement.

- Assisting in coordinating system-wide supports to improve the achievement of ELs.

- Assisting with the design and implementation of the district's World Language program curriculum.

- Designing, delivering, and monitoring professional development for EL and World Language teachers.

- Coordinating procurement of resources and materials for EL and World Languages programs.

Following the recommendation from the district sponsor (Administrator A) concerning viable administrative participants with whom to contact for the study, the additional three administrator participants were emailed to request their involvement in this study. It was explained to each of the four administrator participants that their participation in this study was voluntary and it would involve an approximately one-hour recorded interview, held at a time and a location most convenient to each of them. It was further explained to each participant that they would receive the questions in advance, and that there would be a possible follow-up email or phone call to clarify the participants' responses. Each administrator agreed to take part in the study, though several emails were often sent before a response was received, and therefore the researcher had to be persistent to schedule the interviews. Each administrator participant was interviewed at a time and place they felt was most convenient. Administrators A and 
B were interviewed in their offices at the district central office, while Administrator C was interviewed at a café and Administrator D was interviewed at the researcher's school site, as that was the most convenient time and place chosen by the participant.

\section{Qualitative Data Collection Process}

The qualitative data for this study was collected utilizing several different methods, including conducting focus groups, through individual interviews, and by performing multiple classroom observations. The majority of the data collection took place over a month-long period, during mutually agreed upon times between the participants and the researcher. As a precursor to the class observations, demographic and test score data was accessed utilizing state and district databases, such as the state level databases DataQuest and Ed-Data. These sources of information set the context under which the classroom observations were conducted. Furthermore, the researcher met with each teacher prior to conducting the class observations to explain the study and to address any questions or concerns, and also to get the necessary consent to conduct the study. The initial meetings were held at each teacher participant's site at a time that was convenient for the teacher participant, and lasted no more than one hour.

The observations were carried out with the aim of best attaining a sense of the natural setting and authentic experiences of the students within these programs. Therefore, the observations were performed with a minimal or passive level of participation, as defined by Mertens as an observation in which the researcher is present but does not interact with the participants (2005). The classroom observations occurred three to four times in each of the six classrooms, depending upon the mutually agreed 
upon access and availability of the participants and the researcher. Each of the 23 total observations took place during the language arts portion of the day, as this was the only comparable class within both programs. The classroom observations lasted a minimum of 85 minutes and a maximum of two hours depending on the sites' varying bell schedules. During the classroom observations, the researcher took field notes and filled-out an observation protocol, as are expanded upon in the next section. The researcher attempted to observe one program (New Arrival Center) continuously over a two-week period and the other program (ESL program) over a subsequent two-week period, but due to scheduling conflicts the researcher was compelled to overlap the program observations in an effort to conduct the observations at times that were most convenient for the participants. An attempt was also made to visit each classroom two days in a row each time to view the progressions of the teachers' lessons, but this was not possible for all of the observations, and therefore each classroom was visited over a two-day period at least once.

As the classroom observations were conducted, student focus groups of four to nine Latino students were amassed and undertaken to gain a deeper understanding of the perceptions of students attending each of the two programs under study. Three student focus groups from each of the two programs, six total, were utilized to gather data about the given program. Within the six total student focus groups, two focus groups were made up of students currently attending classes in the New Arrival Center (Year 1), two focus groups were comprised of students currently attending classes within the ESL 1/2 program (Year 1), one focus group was made up of students that had completed the previous year in the New Arrival Center (Year 2), and the final focus group consisted of 
students that had completed the ESL 1/2 class the prior year (Year 2). The students who participated in each of these groups were asked by the researcher to participate with the permission of the students' classroom teachers. With the teachers' permission, the researcher informed the students who qualified for the focus groups about the project and they were told they would be compensated with pizza and soda for their participation. The students were also repeatedly informed that they could opt out before or during the focus group without any negative consequences.

The students who opted to participate in the focus groups received consent from their parents or guardians to take part in the study, which was attained using the Parent/Guardian Informed Consent form (Appendix A). The students further indicated their agreement to participate by signing the Student Participant Informed Consent form (Appendix B). Each of the aforementioned forms was translated into Spanish and presented to the participants in both English and Spanish to assure the participants had a complete understanding of the study and their involvement in it before they signed any of the consent forms. The focus groups were conducted over a one-hour period at the students' sites and were scheduled during non-instructional time at the convenience of the participants. The interview questions were translated into the participants' primary language, as seen in Appendix G, and a Spanish translator was utilized during the focus group portion of the study to interpret the questions and the responses between the researcher and the participants.

One interview per person, ten total, occurred between the researcher and the six teachers and the four chief administrators associated with the two programs under study. The teachers and the administrator participating in the interviews were asked to sign the 
Teacher and Administrator Informed Consent form (Appendix C). The interviews allowed the researcher and the participants to dialogue about the programs under study and it gave the researcher a deeper understanding as to the formation and implementation of both the newcomer and ESL classes. The interviews were conducted over a fiftyminute to ninety-minute time period and took place at the convenience of the participants within the study. The participants chose the time and location for the interview, though they primarily took place at either the school site or central office at which each participant was employed. The teacher and the administrator interview protocols will be described in detail in a subsequent section.

\section{Quantitative Data Collection Process}

The quantitative data for this study was collected utilizing existing database data and by gathering survey data. The database data, attained from such California state educational databases as DataQuest and Ed-Data, gave the researcher both demographic and English learner reclassification data. The demographic data bestowed the information necessary to set the context of the study at the site level and the program level, as well as at the more macro district level, providing information about the locations and the participants about which the study was conducted. The reclassification data, also referred to as redesignation data, demonstrated the rate at which each site within the district reclassified their English learners to Fluent English Proficient (FEP). The district under study used a process in which CELDT scores, California Standards Test scores (CST), and teachers' opinions determined whether or not students had acquired sufficient 
English language skills to function on par with native English speakers, and therefore to be reclassified from English learner to Fluent English Proficient.

The reclassification data was used in this study to demonstrate the rates at which English learners were reclassified within the two programs under study, focusing particularly on students within the three New Arrival Center cases and the three ESL program cases, over a five-year time period. The site level redesignation data was collected by accessing the information on DataQuest, a state educational database, and it was reported out by percentage of students reclassified each year for five years (Table 10). Two New Arrival Center sites, NAC \#1 and NAC\#2, were located on campuses that were comprised of several small schools within one large campus. The newcomer youth at these sites are assigned to different small schools, and therefore the school redesignation rates were computed by averaging the percentages of the total number of small schools on each larger campus. The program level data was gathered by averaging the reclassification rates of the three sites under study within each of the two larger programs (Table 9). The site and program reclassification data was used to demonstrate the rates at which English learners were deemed Fluent English Proficient (FEP) over this same five-year period, which addressed the question as to which program had a greater impact on students' language acquisition levels. The findings will be discussed in detail within the following chapter.

In an attempt to increase the number of students completing the anonymous survey, Latino students within the newcomer and the ESL programs at 13 schools across the district were asked to complete a 72- statement questionnaire (Appendix I), called the Student Self-Concept Scale (Gresham, Elliot, Evans-Fernandez, 1993), to determine their 
academic self-concept levels. The teachers of these classes across the district were identified for the researcher by the district English learner resource teacher, and then were contacted to inquire whether or not the researcher could come to the school and administer the survey during non-instructional time. The researcher contacted 19 classroom teachers, including the six teachers participating in the study, and 13 teachers agreed to have their students surveyed for the study. Four of the teachers who responded indicated they had too few Spanish speakers (one to two total Spanish speakers in each of their classes) to make it worthwhile, or they were uninterested in having their students surveyed. Two teachers did not respond to the researcher's emails, despite several attempts to get in contact, and therefore their students were not surveyed for the study. The survey data collection took place over the same month-long period as the qualitative data collection.

A total of 192 students at the 13 different schools rated the 72 scale items concerning their academic and social self-concept levels, according to how confident they felt about each item and how important the statement was to them. The self-concept scale was translated into the students' primary language to alleviate any confusion and was given over one twenty to thirty minute period at the convenience of the participants and their classroom teachers. The researcher administered the survey and explained the survey directions in Spanish, to assure the students understood the task. The researcher also clarified any questions or confusion as needed in Spanish. The questionnaire was administered according to the instructions provided by the creators of the instrument to ensure reliability and validity, and was coded by the researcher into one of four student groups: (1) ESL - Year 1, (2) ESL - Year 2, (3) New Arrival Center - Year 1, and (4) 
New Arrival Center - Year 2. The findings of this survey will be discussed further in the following chapter.

\section{Qualitative Data Collection Tools}

The qualitative data for this study was gathered utilizing a variety of data collection tools: a classroom observation protocol, an observation field notes record, a student focus group protocol, and a teacher interview protocol. The qualitative data collection began with 23 classroom observations, which were conducted utilizing an observation tool entitled the Classroom Observation Protocol (Appendix D). This data collection tool was adapted from a widely accepted and commonly utilized observation tool, particularly within the district under study, called The Sheltered Instruction Observation Protocol (SIOP) Model created by Echevarria, Vogt, \& Short (2004). The Sheltered Instruction Observation Protocol was designed by its authors to examine the instruction of English language learners with the goal of improving the academic success of said students (Echevarria, Vogt, \& Short, 2004). This model focuses on different components of sheltered instruction, as it is created and implemented, to ensure highquality sheltered instructional practices (Echevarria, Vogt, \& Short, 2004). The Classroom Observation Protocol used in this study contains many of the important components of the SIOP Model, yet some adjustments were made to ensure the protocol addressed the specific research questions about which this research study is being conducted. The adaptations to the SIOP Model were made by utilizing and including the major guiding theories introduced in the literature review, for example Gay's (2010) and Ladson-Billings' (1994) concepts of culturally relevant pedagogy, Krashen's theory 
language acquisition (1992), and Cummin's Transformative Multiliteracies Pedagogy framework (2009). The Classroom Observation Protocol took into account both the sheltered instruction components of the SIOP Model, as well as the guiding theories introduced in Chapter 2, to assure the protocol directly addressed the research questions within this study. The Observation Field Notes Record (Appendix E) was used in tandem with the observation protocol, as this form assisted in collecting and recording anecdotal data throughout the class observations. The Observation Field Notes Record was utilized to record the instruction as it occurred within the classrooms, as well as to record the expectations and actions of the students within the observation period. The researcher typed continuously within the Observation Field Notes Record, recording all that was seen and heard, as well as the time activities and transitions began and ended during the observation. Time permitting, the researcher completed the Classroom Observation Protocol during the observations as well, though immediately after each observation, time was spent completing and reflecting upon the protocol.

The interview and focus group protocols (Appendices $\mathrm{F}$ through $\mathrm{H}$ ) were comprised of questions that had been created utilizing the theories and prior research studies discussed in the literature review, as well as the researcher's extensive experience teaching secondary-level immigrant youth. These protocols were differentiated according to the participating group, be it students, teachers, or the district administrators, as each of these stakeholders offered a different view of the programs under study. The student focus group protocol was translated into the primary language of the participants, as seen in Appendix H, and was provided to the participants in both Spanish and English. Additionally, a translator was employed during the student focus group portion of the 
study to interpret the questions and the responses between the researcher and the participants. The use of the participants' home languages during the focus groups served to ensure the validity and authenticity of the data as it was collected.

\section{Quantitative Data Collection Tool}

The quantitative data measuring students' academic self-concept levels in this study was gathered by administering a 72-item student questionnaire entitled the Student Self-Concept Scale (Appendix I). The Student Self-Concept Scale (SSCS) was created by its authors to measure both the academic and the non-academic self-concept levels of children and adolescents, ranging from grades 3 through 12. For the purpose of this study, the SSCS Level II Questionnaire was administered to the participants as this questionnaire was developed specifically for students in grades 7 through 12 and therefore was more age-appropriate than the SSCS Level I Questionnaire. The SSCS measures students' self-perceptions in three content domains (self-image, academic, and social) and rates these perceptions according to the following three rating dimensions: self-confidence, importance, and outcome confidence (Gresham, Elliot, EvansFernandez, 1993). The self-confidence dimension demonstrates the level of confidence the students have in their ability to perform certain behaviors or possess certain culturally valued attributes (Gresham, Elliot, Evans-Fernandez, 1993). The importance dimension measures the subjective task value that a behavior or personal attribute holds for the student (Gresham, Elliot, Evans-Fernandez, 1993). Finally, the outcome confidence dimension denotes the belief a student has that if he or she performed a particular behavior or task, it will lead to certain anticipated, desired outcomes (Gresham, Elliot, 
Evans-Fernandez, 1993). For the purpose of this study, the self-confidence and outcome confidence dimensions were combined during analysis and reported as student confidence, as the dimensions were determined by the researcher to be sufficiently similar.

Both the internal consistency reliability and the test-retest reliability of Student Self-Concept Scale have been determined by the creators of this instrument, who reported the coefficient alpha reliability and the test-retest reliability coefficients for each of the subscales to be generally high and stable, particularly in older, secondary-level students (Gresham, Elliot, Evans-Fernandez, 1993). The makers of the SSCS reported the coefficient alpha reliability ratings of the three self-confidence subscales (self-image, academic, social) ranged from .78 to .83 in secondary-level students. Additionally, the coefficient alpha reliabilities of the three importance subscales (self-image, academic, social) ranged from .77 to .87 , for these same students (Gresham, Elliot, EvansFernandez, 1993). After administering the SSCS within this particular research study, as seen in Table 9 the rather high coefficient alpha reliabilities reported by the scale creators mirrored the ratings found within the participants under study, in that the three selfconfidence subscale reliabilities (self-image, academic, social) ranged from .80 to .84 and the three importance subscale reliabilities (self-image, academic, social) ranged from .78 to .84 . The creators of the scale further established the construct validity of the SSCS by conducting several studies of the scale, which included investigations of developmental changes and gender differences shown by SSCS ratings, internal consistency of SSCS and Subscale and Composite ratings, correlations between SSCS ratings and scores from other tests, factor analysis of the SSCS item pool, and group separation based on SSCS 
ratings (Gresham, Elliot, Evans-Fernandez, 1993). The authors acknowledge that although continued work is always necessary in developing an effective research tool, they believe the SSCS has been proven to be a reasonable, useful, and efficient approach to the assessment of students' academic and non-academic self-concept levels (Gresham, Elliot, Evans-Fernandez, 1993). The SSCS student questionnaire was translated and administered to students in Spanish, as seen in Appendix I, to ensure accurate responses. The researcher was present at each administration of the SSCS, to clarify any confusion for the students in their primary language.

Table 9

Cronbach's Alpha Reliability Analysis for Instrument Subscales

\begin{tabular}{llll}
\hline $\begin{array}{l}\text { Instrument } \\
\text { Subscale }\end{array}$ & Items & $\begin{array}{l}\text { Valid } \\
\text { Cases }\end{array}$ & $\alpha$ \\
\hline & & & \\
Academic Self-Concept Confidence Level & 22 & 192 & .84 \\
Social Self-Concept Confidence Level & 22 & 192 & .84 \\
Self-Image Confidence Level & 22 & 192 & .80 \\
\hline & & & \\
Academic Self-Concept Item Importance & 18 & 192 & .84 \\
Social Self-Concept Item Importance & 18 & 192 & .84 \\
Self-Image Item Importance & 18 & 192 & .78 \\
\hline
\end{tabular}

\section{Data Analysis and Triangulation of Findings}

The qualitative data and quantitative data collected for this study were analyzed and used to respond to the research questions, to determine which of the two programs under study, the newcomer program or the ESL program, more effectively addressed the linguistic, academic, and self-concept needs of newcomer immigrant youth. The data was collected simultaneously, and therefore the results from the various research tools were 
viewed as independent measures used to contribute to the findings as a whole. The quantitative self-concept data, as attained through the compiled results of the SSCS, was measured both descriptively and to determine its statistical significance, in other words questioning whether or not the results between the two programs would be probable with repeated random sampling under the null hypothesis (Mertens, 2005). The redesignation data, gathered through the state database DataQuest, was utilized to ascertain trends in redesignation data at the site, program, and districts levels, ultimately allowing conclusions to be made with regard to the achievement of English language acquisition of students within the two programs under study. The quantitative results of this research study will be discussed more fully in Chapter 4, Results and the conclusions drawn from the results will be presented in Chapter 5, Implications.

As the quantitative data was being analyzed, concurrently gathered qualitative data, attained through interviews and focus group discussions, were coded and organized according to emergent patterns and tensions. A translator was employed throughout the qualitative date collection process to translate the student focus group data that was gathered in Spanish to ensure its accuracy. The translator was present during the focus groups to orally translate during the discussions, and the same translator was subsequently employed to translate the focus group transcripts to ensure precision and validity of the non-English data. Additionally, the qualitative data was deemed credible through member checks, a process in which qualitative data is verified by the respondents, in this case the subjects who participated in the observations and the interviews, to ensure their agreement with both the content of their individual transcript, as well as the overall constructs and tensions perceived by the researcher (Mertens, 
2005). Each participant was emailed the transcript of his or her interview and the Classroom Observation Indicator Matrix, as is discussed below, and was encouraged to review both for accuracy. Four of the ten participants emailed back and indicated that the transcript was accurate and one respondent clarified an incorrect acronym in the transcript. The significant findings from the interviews and focus groups were organized within the following six tables to aid in the process of analyzing the experiences and insights of the participants within the study: (1) NAC Teacher Interview Analysis (Appendix N), (2) ESL Teacher Interview Analysis (Appendix O), (3) NAC Administrator Interview Analysis (Appendix P), (4) ESL Administrator Interview Analysis (Appendix Q), (5) NAC Student Focus Group Analysis (Appendix R), and (6) ESL Student Focus Group Analysis (Appendix S). The interview and focus group findings are discussed in more detail in Chapter 4, Results.

The classroom observation portion of the qualitative data was categorized and analyzed according to an observation protocol matrix entitled the Classroom Observation Indicator Matrix (Appendix L). The Classroom Observation Indicator Matrix was created utilizing components of the Classroom Observation Protocol (Appendix D) which, as was discussed previously in this chapter, was developed utilizing elements of the SIOP English learner observation protocol developed by Echevarria, Vogt, \& Short (2004), as well as aspects of Gay's (2010) and Ladson-Billings' (1994) concepts of culturally relevant pedagogy, and Cummins' Transformative Multiliteracies Pedagogy framework (2009). The Classroom Observation Indicator Matrix was made up of three major sections and 14 subsections, each derived directly from the Classroom Observation Protocol used during the classroom observations. The three major sections found in the 
matrix are as follows: effective English learner instruction, culturally relevant pedagogical practices, and pedagogical orientations of teachers observed during the study.

The major section of the Classroom Observation Indicator Matrix devoted to effective English learner instruction is comprised of eight subsections, each of which contains between two and 11 total indicators of effectiveness. The subsections and associated indicators of English learner instructional effectiveness are as follows:

- Curriculum - Appropriate for language proficiency, Materials used to supplement language acquisition

- Instruction - Linked to students' experiences/prior knowledge, Connections made between current and prior lessons, Peer interaction used to activate schema

- Key Vocabulary - Academic/content vocabulary introduced in lesson, Vocabulary taught literally, Vocabulary taught contextually, Strategies used to support students' understanding

- Comprehensible Input - Suitable pacing for language proficiency level, Suitable speech for language proficiency level, Clear expectations for students, Checks for understanding, Visuals used during lesson, Realia used during lesson, Modeling used during lesson, Gestures/TPR used during lesson, Primary language support used during lesson, Interactive demonstrations used during lesson, Partner/group activities present during lesson

- Strategies - Think-alouds used during lesson, Word banks present in lesson, Graphic organizer used to support teaching, A variety of question types used 
during lesson (literal, analytical, interpretive), Evidence of a gradual release of responsibility

- Interactions - Frequent interactions between teachers and students, Frequent interactions between students, Students provided sufficient "wait time" when responding

- Practice/Application - Students given opportunities to practice new concept, Activities integrate all language domains (speaking. listening, reading, writing), Oral language opportunities included in lesson, Charts used to support students in applying new concept, Manipulatives used to support students, Visuals used to support students in applying new concept

- Lesson Review/Assessment - Differentiated instruction based on needs of students, Teacher assesses students through oral questions, Teacher assesses students through exit slips, Teacher assesses students through student products, Teacher assess students through tests/quizzes, Teacher provides feedback to students (oral or written)

The second major section of the Classroom Observation Indicator Matrix recounts evidence of culturally responsive pedagogical practices, as were potentially discerned during the classroom observations. The Culturally Relevant Pedagogy section is comprised of three subsections, each of which contains between two and four total indicators of effectiveness. The three subsections and related indicators of culturally relevant pedagogical practices are as follows: 
- Materials - Materials contain culturally relevant content, Materials relevant to students' personal lives/cultures, Materials are thought-provoking, Materials are challenging and complex

- Instruction - Instruction includes flexible grouping, Addresses varying linguistic backgrounds, Instruction includes cooperative learning opportunities, Multiple perspectives on ideas are presented/discussed

- Environment - Multiple cultures, languages, gender represented on walls, Multiple cultures, languages, gender represented in texts The third and final major section of the Classroom Observation Indicator Matrix identifies evidence of teachers' pedagogical orientations, as were noted during the observations. The Pedagogical Orientation section is comprised of three subsections, each of which contains between three and six total indicators of effectiveness. Unlike the other two major sections, the subsections of the Pedagogical Orientation section contain indicators that demonstrate the teachers' pedagogical orientations based on a continuum that ranges from a Transmission-oriented pedagogy, to Social Constructivist-oriented pedagogy, and ending with a Transformative pedagogical orientation. This section is based on Cummins' Transformative Multiliteracies Pedagogy framework (2009), as was discussed in Chapter 2, Literature Review. The three subsections and associated indicators of teachers' pedagogical orientations are as follows:

- Transmission-oriented Pedagogy - Instruction primarily direct instruction/lecture, Instruction focused solely on content of lesson, Students are passive learners

- Social Constructivist-oriented Pedagogy - Teacher activates students' prior knowledge, Cultural backgrounds valued within instruction, Teachers and 
students co-construct understandings through dialogue and discussion, Instruction utilizes collaborative inquiry, Instruction utilizes experiential learning, Higherorder thinking skills promoted

- Transformative Pedagogy - Collaborative critical inquiry present, Instruction promotes analysis of societal power relations, Instruction promotes discussion of ways to act on societal inequalities

The quantitative and qualitative data for this study was collected at both a macro (program) level and a micro (individual case) level. Therefore, the quantitative and qualitative findings for this study, as are discussed in Chapter 4: Results, were demonstrated by presenting the larger findings utilizing the micro level data to substantiate the trends of macro level findings within each of the five sections. The results of the four data gathering methods (the database data, the survey data, the interview and focus group data, and the observation data) were then triangulated through a process in which results from multiple sources are cross-referenced to elicit substantial findings (Merriam, 2009; Mertens, 2005). The data for this study was triangulated to compare the results between the three similar classes within each program, and finally between the two programs as a whole. Figure 6 outlines how data was collected at the program macro level (administrator interviews, student focus groups, redesignation data, and SSCS survey data) and at the case study micro level data (class observations, teacher interviews, and redesignation data) to provide a strong overview of each program from a variety of perspectives and to ensure appropriate contentions were made about each program under study in Chapter 5, Implications, ultimately answering the guiding questions of this research study. 
Figure 6: Macro and Micro Levels of Data Collection

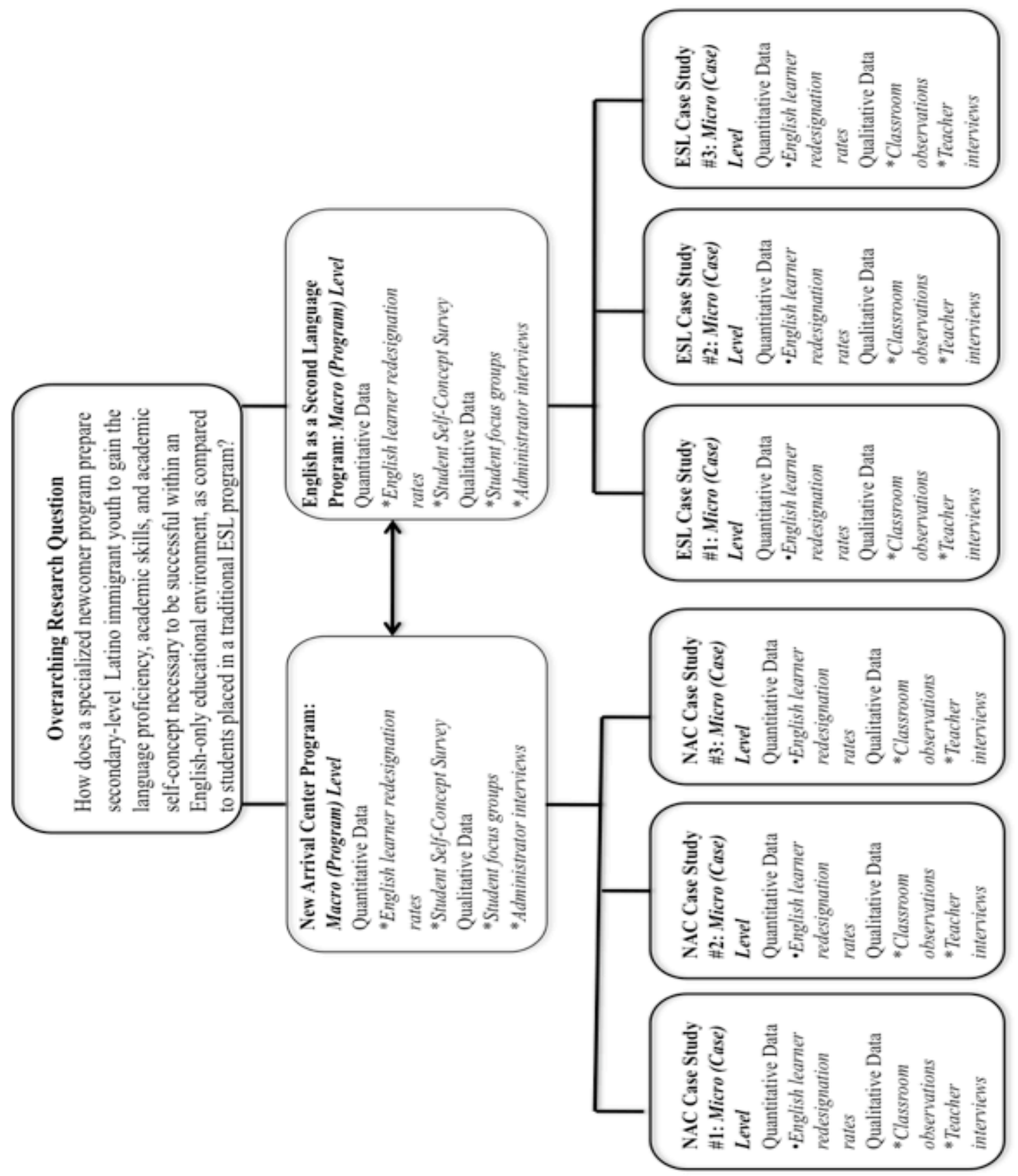




\section{CHAPTER 4}

Results

This study investigated the educational practices and academic outcomes present within two program models that were created and implemented with the ultimate goal of effectively supporting secondary-level Latino newcomer immigrant youth as these students began their academic course within a large urban school district in Southern California, the San Diego unified School District. This study analyzed and compared the experiences of Latino newcomer students, both during and after they completed their first year of education in either a traditional ESL placement or a self-contained newcomer program. This study evaluated the creation and implementation of the two programs, looking closely at the (1) language acquisition, (2) academic skills acquisition, and (3) academic self-concept of the students as they either were attending or after they had completed one of the two programs under study. This research study was guided by the following overarching research question and subsequent sub-questions:

How does a specialized newcomer program prepare secondary-level Latino immigrant youth to gain the language proficiency, academic skills, and academic self-concept necessary to be successful within an Englishonly educational environment, as compared to students placed in a traditional ESL program?

1. What are the prevailing pedagogical practices utilized in both a newcomer program and a traditional ESL program?

a. How do the curricula, language instruction, content instruction, teachers' attitudes, level of cultural responsiveness, and pedagogical orientations compare within the programs under study?

2. Is there a relationship between students' academic self-concept and the increase in their language proficiency level within either a specialized newcomer or a traditional ESL program? 
3. How are Latino newcomer students' academic self-concept, language proficiency levels and acquisition of academic skills influenced by the program in which they are educated?

The results of the research study are first contextualized within the San Diego Unified School District by introducing the overall process by which English learners (El) are assessed and placed in specialized programs designed for the instruction of such students. The findings are subsequently organized into five main sections, each of which addresses the qualitative and quantitative findings that were discerned in both the New Arrival Center (NAC) program and the English as a Second Language (ESL) program. These findings were then used to address the driving research questions, which will be discussed more fully in Chapter 5, Implications. The first section gives detailed background information about each of the two programs under study (New Arrival Center and English as a Second Language). This section provides an overview of each program, describing such components as the program's overarching philosophy, organization, instructional design, and the district's expectations of each program. The second section analyzes the trends in English learner redesignation rates at the micro (case) level and at the macro (program) level, to determine which of the two programs is achieving greater success in moving their students from English learner status to that of Fluent English proficient status. The third section describes the overall results of the Student Self Concept Survey (SSCS), as well as the Academic Self-Concept Subsection results of the survey. The quantitative data was analyzed descriptively to determine the mean responses of each item and comparatively using independent $t$-tests to determine statistical significance for the mean responses of each item. The data was analyzed to 
compare and ascertain statistical significance utilizing five discrete independent variables: Total ESL Student Participants and Total NAC Student Participants, ESL-Year 1 Student Participants and NAC-Year 1 Student Participants, ESL-Year 2 Student Participants and NAC-Year 2 Student Participants, ESL-Year 1 Student Participants and ESL-Year 2 Student Participants, and NAC-Year 1 Student Participants and NAC-Year 2 Student Participants. The fourth section brings forth the findings culled from the classroom observations that were conducted within each of the six cases within the two programs under study. The fifth section discusses the trends found within each of the six focus groups, six teacher interviews, and four administrator interviews that were conducted within the qualitative data collection section of this study. The results disseminated in each of the five sections are analyzed and triangulated within this chapter and in more detail in Chapter 5, Implications, to address each of the guiding research questions within this study.

\section{District and State-level Assessment and Placement of English Learners}

At the state level, the Categorical Program Monitoring division of the California Department of Education has two primary goals in the education of English learners: to ensure English learners gain full proficiency in English as rapidly and effectively as possible and that English learners meet state standards for academic achievement (CDE, 2010). To accomplish these goals, the Categorical Program Monitoring division created seven dimensions through which districts are held accountable when educating English learners (CDE, 2010). The seven program dimensions are as follows (CDE, 2010): 
- Stakeholder Involvement - Parents, staff, students, and community members participate in developing, implementing and evaluating core and categorical programs, through such forums as site-based English Learner Advisory Committee (ELAC).

- Governance and Administration - Policies, plans, and administration of categorical programs meet statutory requirements, such as the administration of a home language survey and the CELDT assessment within a designated time period.

- Funding - Allocation and use of funds meet statutory requirements for allowable expenditures.

- Standards, Assessment, and Accountability - Categorical programs meet state standards, are based on the assessed needs of program participants, and achieve the intended outcomes of the categorical program.

- Staffing and Professional Development - Staff members are recruited, trained, assigned, and assisted to ensure the effectiveness of the program.

- Opportunity and Equal Educational Access - Participants have equitable access to all programs provided by the local educational agency (LEA), as required by law.

- Teaching and Learning - English learners receive a program of instruction in English language development (ELD) in order to develop proficiency in English as rapidly and effectively as possible, while additionally receiving appropriate grade-level academic instruction to meet their districts' content and performance standards in a reasonable amount of time. 
The dimension focused on opportunity and equal educational access requires that districts provide English learners with the specialized programs necessary to meet their individual linguistic and cultural needs (CDE, 2010). It further requires that all students have equitable access to all district programs, as is required by the law. The Categorical Program Monitoring division sees this dimension as requiring all students are to be placed in English-language programs unless a parental waiver has been granted for an alternative bilingual program (CDE, 2010). The opportunity and equal educational access dimension further elaborates that districts are required to place students in program settings that best serve their level of English language fluency, and that school sites are mandated to implement a process of English language acquisition for English learners in which the curriculum and design are created specifically for such students (CDE, 2010). An additional dimension created by the Categorical Program Monitoring division, focuses on the teaching and learning of English learners. This dimension maintains that a primary goal in the teaching of English learners is to assure they receive a program of instruction in English-language development in order to develop proficiency in English as rapidly and as effectively as possible, and to ensure they meet districts' content and performance standards for their respective grade levels in a "reasonable amount of time" (CDE, 2010). The opportunity and equal educational access dimension and the teaching and learning dimension, as created by the state Categorical Program Monitoring division, promoted both the creation and implementation of the New Arrival Center and the English as a Second Language programs within the district under study.

In response to the abovementioned state mandates for English learners, the San Diego Unified School District utilizes a district-wide English learner protocol, as is seen 
in Figure 7, to identify and place English learners in an appropriate program dependent on their level of English language acquisition. Upon enrollment in the district, families of all students are provided a Home Language Survey to complete, detailing the language/s spoken in each child's home. If English is indicated as the only home language, students are placed in grade-level English-only programs. If a language other than English is specified, students' English language levels are assessed utilizing the CELDT assessment to determine their Overall Proficiency Level (OPL) in English, as is discovered through listening, speaking, reading, and writing subtests. The initial CELDT assessment either demonstrates that students are Initially Fluent English Proficient, in which case they are also placed in grade-level English-only programs, or it appropriates students to one of five levels, the Beginning, Early Intermediate, Intermediate, Early Advanced, or Advanced level of English acquisition, depending on their OPL scores. Students at the Beginning and Early Intermediate CELDT levels are placed in Structured English Immersion programs, which include the New Arrival Center and the English as a Second Language programs being analyzed within this research study. Students with an OPL of Intermediate, Early Advanced, or Advanced on the CELDT are placed in Mainstream English Cluster programs, which are often classes that include both English learners and native English speakers.

All English learners are reevaluated on the CELDT annually to measure their English language acquisition progress and to assure proper placement, historically at the start of each academic year. The ultimate goal of the district English learner program is to support students in advancing one OPL per year, and to reclassify students to Fluent English Proficient within a period of no more than five years, dependent on their entering 
English language acquisition level. Upon reclassification, students are termed Fluent English Proficient and are placed in grade-level English-only programs. When available and with approved Parental Exception Waivers, students may also be placed in Biliteracy/Dual Immersion programs, though this placement option primarily exists at the elementary level and is decreasing in availability. The next section will discuss more specifically the decline of primary language placement options within the district.

Figure 7: District English Learner Flowchart From Identification to Reclassification

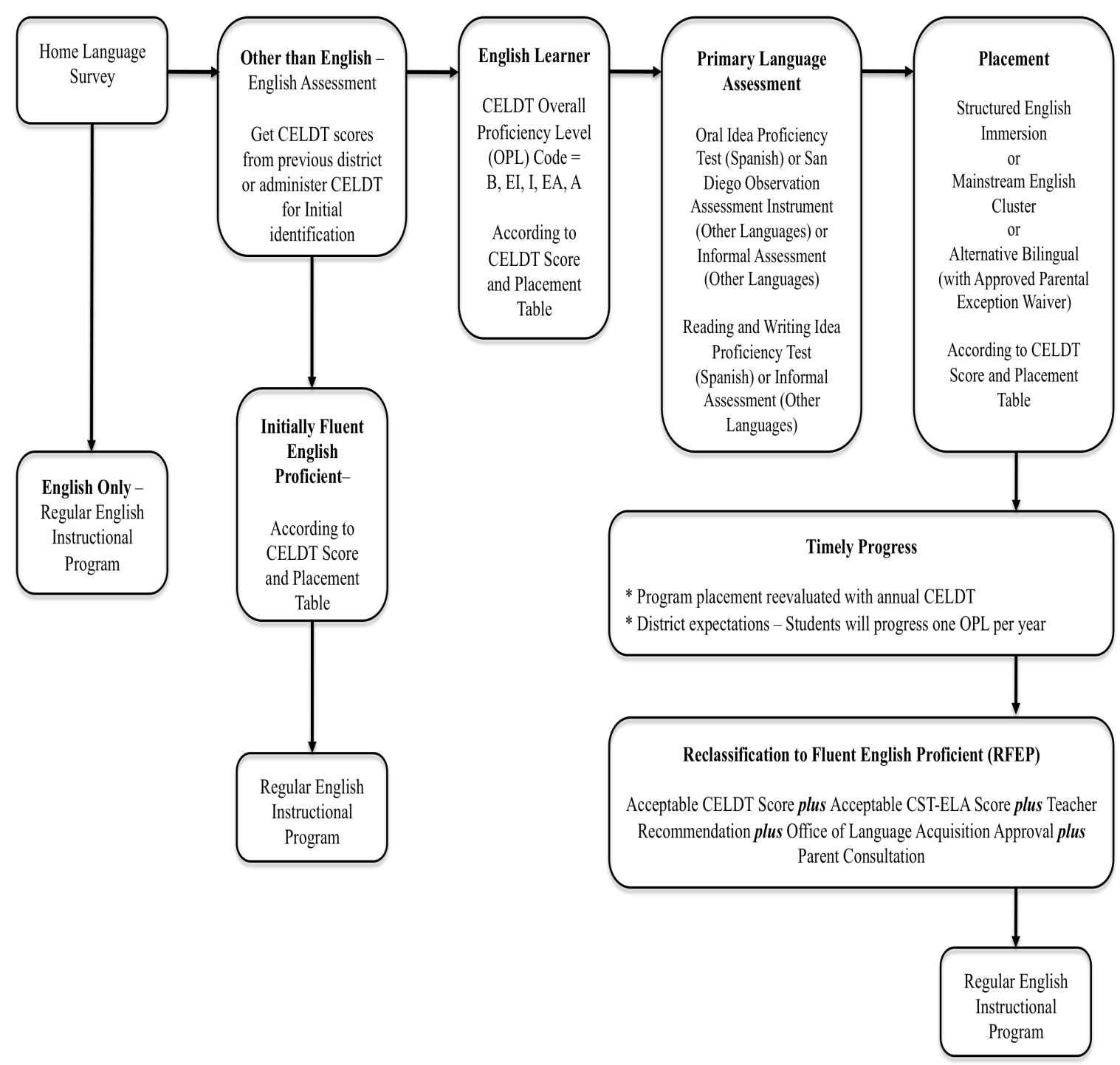


The Rapid Decline of Access to Bilingual/Biliteracy Education

Despite the reality that roughly thirty percent of the students attending classes in the district are English learners and that $77 \%$ of such students are Spanish speakers, the San Diego Unified School district has demonstrated a distinct move towards monolingual, English-dominant instruction since the passage of Proposition 227 in California. Prior to the 1998 passage of Proposition 227, the San Diego Unified School District offered a biliteracy program for Spanish-speaking English learners at a number of its school sites, biliteracy being "the development of academic proficiency in the student's primary language, simultaneous with the development of language and academic proficiencies in a second language" (SDUSD, 2010). In this program literacy and content instruction are provided in both English and Spanish, and while primary language instruction utilized in this program is used to varying degrees at each grade level, it decreases by a percentage each year as the use of English increases. Students are additionally taught English literacy skills, through the use of English language development (ELD) strategies, and they further maintain interaction with students in other programs on campus to promote the use of English in both social and academic contexts. As stated by the district, the goals for students enrolled in the bilingual/biliteracy program are as follows: to demonstrate grade level standards and expectations in all content areas in both languages, to develop proficiency in both languages, and to develop respect for cultural and linguistic diversity.

After the passage of Proposition 227, which requires that all students including newly arriving immigrant youth be instructed "overwhelmingly" in English, the San Diego Unified School District has mirrored other such districts across the state in offering 
a drastically reduced number of bilingual or primary language classes. They have taken the stance that the decision for students to participate in primary language classes rests solely with the parents or guardians, and thus, due to an adherence to state law, the district asserts that the schools are mandated to inform parents of program options for English learners and to assist them in completing the process to enroll their student in the option of their choice (SDUSD, 2012). If parents or guardians choose the biliteracy program option, state law requires each school district to provide them with the waiver request form, to approve or deny the request, and then inform parents of the approval or denial and offer them the chance to appeal if necessary. The parents are required to complete this same waiver each year they opt into the biliteracy program (SDUSD, 2012). This process must be completed in a timely manner, as outlined by the state directive. In each school that has granted 20 or more waivers, an alternative bilingual program must be provided, or the students must be allowed to transfer to a school that provides such services.

Although the San Diego Unified School District possesses a student body made up of almost one-third English learners, of which the large majority are Spanish-speakers, and despite assurances that schools inform parents about the varying program options, in recent years the number of students that have been provided access to bilingual or multilingual instruction has become virtually non-existent. For example, one year prior to the 1998 passage of Proposition 227, 32.96\% of the district's English learners received primary language instruction with ELD support, yet during the 2010-11 school year, only $4.73 \%$ of the English learners were educated using primary language instruction (DataQuest, 2011). In addition, as reported in 2009, only 11 of 181 comprehensive sites 
offer a bilingual/biliteracy program, all of which are located at elementary schools (Alpert, 2009). There were no district bilingual programs at the middle or high school level in 2009 , or at the time of this study. The lack of access to bilingual or multilingual classes for the majority of English learners demonstrates the district's move towards monolingual, English-only education, as is demonstrated in the two programs under analysis within this research study. The following two subsections will provide an overview of the larger English as a Second Language program and the more recently created New Arrival Center program, illustrating the overarching philosophy, organization, instructional design, and district's expectations of each program.

\section{Overview of The English as a Second Language Program}

The English as a Second Language (ESL) program has been more widely available to newcomer immigrant youth in the district, as many middle and high schools in the San Diego Unified School District contain at least one ESL class to service such students. Unlike the New Arrival Center classes discussed below, which only possess Beginning-level ESL 1/2 students, many of the classes are combination classes, in that often both ESL 1/2 and ESL 3/4 students are enrolled in one class to elevate numbers. During the 2011-12 academic year, the district reported that 28 classroom teachers taught 34 sections of ESL at the high school level, either pure ESL 1/2, ESL 3/4, or ESL 5/6 classes or combination classes of two ESL levels. The number had increased by one position from the prior academic year, as there were 27 high school ESL teachers teaching 33 section of ESL during the 2010-2011 school year (District Personnel, email communication, February 12, 2012). Of the 24 typical middle schools in the district, 16 
classroom teachers taught 17 sections of ESL 1/2 or ESL 3/4 during the 2011-12

academic year (District Personnel, email communication, February 22, 2012). As was the case at the high school level, depending on the number of ESL students enrolled at each level, these classes were either purely one level, ESL $1 / 2$ or ESL 3/4, or they were combination classes that included both ESL levels. There were an additional seven ESL support teachers at the middle school sites who taught one-hour ESL support classes, the support classes at these middle school sites being used to supplement the mainstream English classes in which the ESL students were enrolled. Though it is notable that the district funded ESL support teachers at seven sites, the research for this study focused on the traditional two-hour ESL block classes, rather than the one-hour support classes.

The ESL program in the San Diego Unified School District is categorized as part of the Structured or Sheltered English Immersion (SEI) instructional program, which is the state required designation of students at the Beginning, Early Intermediate, and Intermediate levels of English language proficiency according to the CELDT assessment. As it is defined on the California State Department of Education website, "Sheltered English Immersion" is an English language acquisition process for young children in which nearly all classroom instruction is in English, but with curriculum and presentations designed for children who are learning the language (CDE, 2011). In addition, according to the California Department of Education, a typical SEI program includes: (1) English language development (ELD) appropriate to each student's level of English proficiency, (2) content instruction utilizing specially designed academic instruction in English (SDAIE) whenever needed for full access to the core, and (3) it also may include primary language support when appropriate (CDE, 2011). The San 
Diego Unified School District reports that SEI students in the district are purposefully clustered and placed into grade-level classrooms with native and fluent speakers of English. In these classes, referred to as "SEI Clusters", English learners make up roughly one-third of the class population. The district further reports that, in certain cases, an entire class might be made up of students with similar levels of English language proficiency, as long as they qualify for the SEI program. The ESL program researched for this study falls into this latter category, as it solely contains students at the Beginning level of English language acquisition, as determined by the CELDT assessment.

The course of study for students who qualify for ESL 1/2 is designed to ensure students receive a sequential, systematic instructional program that promotes high levels of English proficiency in all English language domains (speaking, listening, reading, and writing). This yearlong course encompasses a two-period block of students' daily schedules, and takes the place of a mainstream English course. At the high school level, the ESL 1/2 course counts for one unit of English language arts credit and one unit of elective credit per semester towards graduation, as it is a two-hour course. Students' eligibility to participate in the ESL $1 / 2$ program is dependent on the following requirements: a Home Language Survey that indicates the student speaks a language other than English at home, a Beginning level score on the CELDT language assessment, and an enrollment date in U.S. schools of one academic year or less (SDUSD, 2011). The general goals and outcomes of the ESL program, as denoted by the district under study, is to provide ESL/ELD curriculum that had been designed to (SDUSD, 2011):

- Provide students with a firm base in English through the development of listening, speaking, reading, and writing skills. 
- Develop students' competence in English to achieve academically in all content areas.

- Develop students' competence in English to communicate in social settings.

- Promote understanding, respect, and appreciation for the traditions and values of the United States.

- Provide exposure to and affirmation of the multicultural nature of the United States.

The primary instructional objective of the ESL $1 / 2$ program is to follow a balanced approach to language development using communication-based, content-based, and literature-based lessons that focus on both oral and aural activities to ensure comprehension of new English concepts and vocabulary. The program asserts that students will learn to understand everyday conversations on a variety of subjects and how to ask for clarification when needed. Students in ESL 1/2 are taught literacy skills appropriate to their emergent level of English proficiency, utilizing a variety of strategies, such as the Language Experience Approach and shared and modeled reading and writing, often accessing students' primary languages when possible to develop their reading and writing skills. Students are additionally given daily opportunities to interact and work collaboratively on activities to encourage communication in English. The content utilized in the ESL 1/2 course is based on the district-adopted English language development standards at the Beginning language proficiency level. Teachers of this course are required to hold a credential issued by the California Commission on Teacher Credentialing authorizing the instruction of English Learners (SDUSD, 2011). 
Students are expected to be enrolled in ESL 1/2 classes for no more than one academic year, though if the need arises, such as a late enrollment date the prior year, schools may opt to place students in this course for a second year. Historically, a sitebased team, which includes such individuals as the student's assigned school counselor, the site English Learner Resource Teacher (ELST), the ESL teacher, and/or the student's parents or guardians, make this decision to ensure the student has acquired sufficient language to advance to the ESL 3/4 level. As students continue to the ESL 3/4 level, they are instructed in a manner that supports them in moving from a literal comprehension of reading passages to a higher level interpretation of reading passages. In ESL 3/4 students are also instructed to develop critical and evaluative reading skills and to apply their reading ability to solve problems critically. ESL 3/4 is a yearlong course that is comprised of a two-period block of students' daily schedules, and it also takes the place of a mainstream English course. At the high school level, this course, much like ESL 1/2, counts for one unit of English language arts credit and one unit of elective credit per semester towards graduation, as it is a two-hour course. The final ESL course, ESL 5/6, is intended to prepare students to achieve in a Mainstream English Cluster (grade-level English courses) by helping them develop appropriate academic language and learning strategies to function successfully within a typical English course. Though a two-hour block, ESL 5/6 is the only ESL course to offer one unit of college preparatory English language arts credit per semester, in addition to one unit of elective credit each semester (SDUSD, 2011).

Although parent involvement in the ESL program varies depending on the site in which the program is located, all schools that contain 21 or more students identified as 
English language learners must have an English Learner Advisory Committee (ELAC), whose sole purpose is to promote and assure academic achievement for English learners. The site ELAC is a voluntary committee comprised of parents of English learners who attend a particular school, as well as parents of students at that site who are not English learners, school personnel, and community members. The site English Learner Resource teacher typically leads the ELAC meetings, which occur on a monthly basis and last approximately one to two hours. The ELAC informs the school principal and school personnel about programs and services for English learners, and serves as the advisory body on the following four key issues: creating and implementing a school plan for English learners, creating and implementing a needs assessment for English learners, administration of the language census, making parents aware of the importance of school attendance and academic development.

\section{Overview of The New Arrival Center Program}

The New Arrival Center (NAC) is a middle and high school level self-contained English learner program that was initially developed and continues to serve newcomer immigrant youth who score at the Beginning level of English language proficiency. The NAC program was originally piloted within four classes at two comprehensive high school sites during the 2008-09 academic school year, and, as of the 2011-12 school year, the program has since grown to include ten classrooms located at four high school sites and two middle school sites (District Personnel, email communication, February 12, 2012). The mission of the New Arrival Center program is to provide a solid foundation in oral and written language for students who are both new to English language and new to 
the United States educational system. This program was developed and continues to engage students in a rigorous course of study that will accelerate their survival and academic English language skills, while developing students' background knowledge in each required curricular area. Students within this program are typically exited after the requisite two semesters with the belief that they will have sufficient language and background knowledge to achieve success in sheltered and mainstream English-only classes across the curriculum. Therefore, the self-contained classes within the NAC program educate the students with the expectation of attaining the following exit goals: Intermediate level of English proficiency, readiness for Algebra I, an understanding of the foundations of democracy, readiness for Earth Science, and the completion of a P.E. and/or a fine/practical arts elective class. The vision of the program follows that the attainment of these goals will ultimately support students in learning the concepts and skills necessary to graduate from high school and to function successfully post-high school, both academically and culturally as members of the United States. Finally, this program makes explicit its desire for students to feel their home culture is valued within this educational program.

Students' eligibility to participate in the NAC program is contingent on the following requirements: a Home Language Survey that indicates the student speaks a language other than English at home, a CELDT score of low to mid-Beginning level, having been enrolled in U.S. schools for less than six months, and parent approval. Additionally, students with little to no literacy in their primary language are given priority to participate in the NAC program. Students who are 17 years or older at the time of enrollment are encouraged to enroll in continuing ESL Continuing Education at the 
local community college district, as the district feels it is unlikely these older age students will have sufficient time to acquire the language necessary to graduate from the high school in which they are enrolled. After such eligibility requirements have been met, the NAC staff enroll students that qualify for the program and reside within the school boundaries in which an NAC program is located or who choose to utilize transportation services provided by the district to attend the closest comprehensive site that houses a NAC program.

According to the district, the content of the New Arrival Center program focuses on providing intensive English Language Development, while assuring students access to foundation skills and language in the core content areas and courses to assist students in orienting to U.S. cultural norms. A typical course of study for students within the NAC program includes the following curricular areas within a primarily self-contained classroom environment:

- ESL Literacy - Focus is on developing reading, writing, speaking, and listening skills to prepare students for successful participation in secondary classes across the content areas. The topics early in the year center on survival skills and topics (school, community, family, health, American culture, personal finance, etc.) and become increasing academic throughout the school year.

- Content-based ESL Science (elective course) - Focus is on vocabulary and basic concepts of earth, life, and physical science.

- Content-based ESL Social Studies (elective course) - Focus is on foundational skills and language for social studies and orientation to U.S. culture and introduction to U.S. and world history. 
- Content-based ESL Mathematics (elective course) - Students who enter with course credit for previous grade-level math courses, or whose entering assessment indicates near grade-level math skills are alternately placed in grade-level math courses for credit, rather than ESL Mathematics.

- Physical Education and /or elective course (i.e.: music, art, band, etc.)

The developers of the NAC program acknowledge that the ESL science, ESL social studies, and ESL mathematics courses utilize K-6 California state standards for each given content area, despite being secondary classes, and therefore receive elective credit at the high school level rather than content area credit. The understanding that newcomer students often need to build a foundation in such content areas justifies the use of below grade-level standards, depending on individual student need. Teachers and students are provided content area resources, such as curricular programs and textbooks that are appropriate for the language and academic skill levels of the students as they progress through the program, which are funded by both the central district office for English learners, and the sites in which the NAC classes are located. The program creators further charge the classroom teachers within the NAC program to integrate career exploration into the course curriculum and encourage real world learning through quarterly field trips, guest speakers, and authentic homework assignments (i.e.: applying for a library card, purchasing groceries, interviewing a parent/community member, etc.). According to the developers of the NAC program, the instruction and design of the program derives from the essential belief that all students have the capacity to develop the high levels of English proficiency that will allow them to successfully engage with grade-level content. Teachers that would like to educate students within the New 
Arrival Center are required to apply through the district office charged with overseeing the English learner programs across the district and must hold a teaching credential issued by the California Commission on Teacher Credentialing authorizing the instruction of English learners. Teachers hired to teach within this program are committed to providing a rigorous educational environment for their students with high academic expectations, differentiating levels of support, and an atmosphere in which cultural and linguistic diversity are not only valued, but are utilized to bridge the curriculum and instruction between students' home cultures and that of the United States. The district's ESL model, which will be discussed further in the English as a Second Language program subsection, is designed around the belief that learning takes place through social interaction, and therefore teachers within the NAC program are encouraged to develop lessons that provide students multiple opportunities to learn through collaborative and active participation, utilizing language within authentic and meaningful activities. Teachers are additionally expected to provide explicit feedback to the students within the NAC program and are required to monitor the students' progress through frequent formative and summative assessments. The developers of the NAC program listed the following elements as being essential to the instructional design of the program:

- High challenge and high support

- Teacher modeling

- Guided practice

- Small group instruction

- Collaborative, productive work 
- Meaningful independent tasks

- Frequent opportunities to use and practice oral and written English

- Explicit instruction in vocabulary and language structure

- Thematic instruction

- Content used as a vehicle to learning language

- Reading, writing, listening, and speaking developed simultaneously and in support of each other

- Print-rich environment where charts, posters, etc. serve as resources for using and practicing language

- Technology used as a tool for learning, research, writing, fluency practice, presentations, etc.

- Realia and real world experiences

- Multimodal instruction utilizing art, music, etc. as vehicles to learn, use, and practice language

- Building upon students' funds of knowledge

- Primary language used to support comprehension

Students are expected to exit the New Arrival Center program after two semesters of instruction within this program, though rare exceptions are made for students that require longer than one full year within this program. The exception for students who need a longer program of study requires approval from the district central offices, for which the NAC staff, the given student, and the student's parents/guardians meet and complete a retention form, in that this is not the typical plan for newcomer students. Conversely, some students may not require a full year in the NAC program, as they are 
ready to progress to a more advanced ESL program for either part of the day or for their full day of instruction. To exit students to a higher level during the school year the NAC staff must complete a Mid-Year Exit Form, provide a student writing sample that demonstrates proficiency at the Early Intermediate or higher level, conduct an oral language assessment that demonstrates Intermediate proficiency or above, provide a specific math assessment that indicates mastery of basic mathematics skills, and receive approval from the district central offices. Typically, students are exited after completing the requisite year in the NAC program and are enrolled in ESL 3/4 or ESL 5/6 courses the following year with sheltered content courses.

Parents of qualifying NAC students are said to be notified of the potential benefits, as well as the limitations, of the New Arrival Center program prior to student placement, in an attempt to allow parents to make an informed decision about whether or not the placement is appropriate for their child. As a part of this program, students will only receive one graduation credit each for English and Physical Education, and will receive elective credit for ESL math, ESL science, and ESL social studies, though students do need elective credits to ultimately graduate from high school within the district. Some students may be required to remain in high school for five years or longer in order to meet graduation requirements, depending on their entering level of English language acquisition, as well as their progress through the pathways to completion of high school credits. Parents of students entering the school aged 17 or higher are informed of alternate paths of education, such as the local community college system. High school NAC teachers are expected to meet with parents twice annually, mid-year and year-end, to explain the pathways to high school graduation, as developed by the 
district offices. According to the developers of the program, parent involvement in the program is encouraged through engaging them in their child's education and providing them with support in accessing community resources. Additionally, the program creators purport that parents are invited to attend field trips and quarterly Parent Nights at their child's NAC class, and also are made aware of available community services such as adult ESL classes.

\section{Program and Individual Case Level Redesignation Rates}

The San Diego Unified School Ditrict utilizes three primary measures in determining if English learners have attained language skills sufficient to be redesignated to Fluent English Proficient (FEP): CELDT scores, CST scores, and teacher judgment. English learners are expected to be reclassified within five years, as it is anticipated that these students will advance one Overall Proficiency Level (OPL) of the total five levels (Beginning, Early Intermediate, Intermediate, Early Advanced, and Advanced) on the CELDT assessment per academic year. Parent/Guardian approval is necessary for general reclassification, as parents must first be given an opportunity to consult with staff regarding English learner programs and to decide if this course will further increase prospects for academic achievement for their child. If the above criteria are met and the district office that oversees the schooling of English learners grants the designation change, district personnel complete the required paperwork to reclassify the individual students from English learner to FEP.

The redesignation rates of the two programs under study greatly exceeded the district total rates over a five-year period, as is seen in Table 10 and Figure 8. The state 
educational database DataQuest (2012) was utilized to attain the redesignation rates of the programs under investigation. The overall NAC and ESL redesignation rates were then calculated by averaging the rates of the three schools housing the New Arrival Center (NAC) classes and by averaging the schools in which the three ESL classes under study were located. These averages were attained over a five-year period to demonstrate the reclassification trends over this time period. The district total redesignation rates were further amassed by accessing DataQuest (2012), and they included the reclassification statistics of all students within the district, both in the ESL or NAC programs and within other English learner placements, at the elementary and the secondary educational levels. Finally, by calculating the differences between the 2006-07 and the 2010-11 academic years, the five-year growth of the two programs and the district total were further reported.

Table 10

Overall Program Percent Redesignation Rates Over Five-Year Period

\begin{tabular}{lllllll}
\hline & $2006-$ & $2007-$ & $2008-$ & $2009-$ & $2010-$ & $\begin{array}{l}\text { Five-Year } \\
\text { Program }\end{array}$ \\
\hline & 2007 & 2008 & 2009 & 2010 & 2011 & Growth $^{\mathrm{a}}$ \\
\hline NAC $^{\mathrm{b}}$ & 3.3 & 5.1 & 8.1 & 11.0 & 13.8 & +10.5 \\
ESL $^{\mathrm{c}}$ & 5.8 & 9.7 & 13.9 & 13.5 & 17.4 & +11.6 \\
District Total & $\mathbf{6 . 4}$ & $\mathbf{6 . 5}$ & $\mathbf{8 . 2}$ & $\mathbf{1 0 . 1}$ & $\mathbf{1 0 . 4}$ & $\mathbf{+ 4 . 0}$ \\
\hline
\end{tabular}

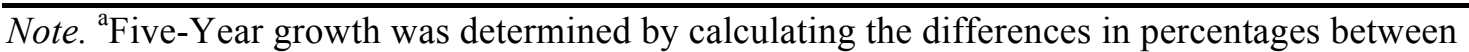
2006-07 and 2010-11, ${ }^{\mathrm{b}}$ Redesignation rate percentages were determined by calculating the averages of the three NAC site cases under study, ${ }^{\mathrm{c}}$ Redesignation rate percentages were determined by calculating the averages of the three ESL site cases under study. 
Figure 8

Overall Program Percent Redesignation Rates Over Five-Year Period

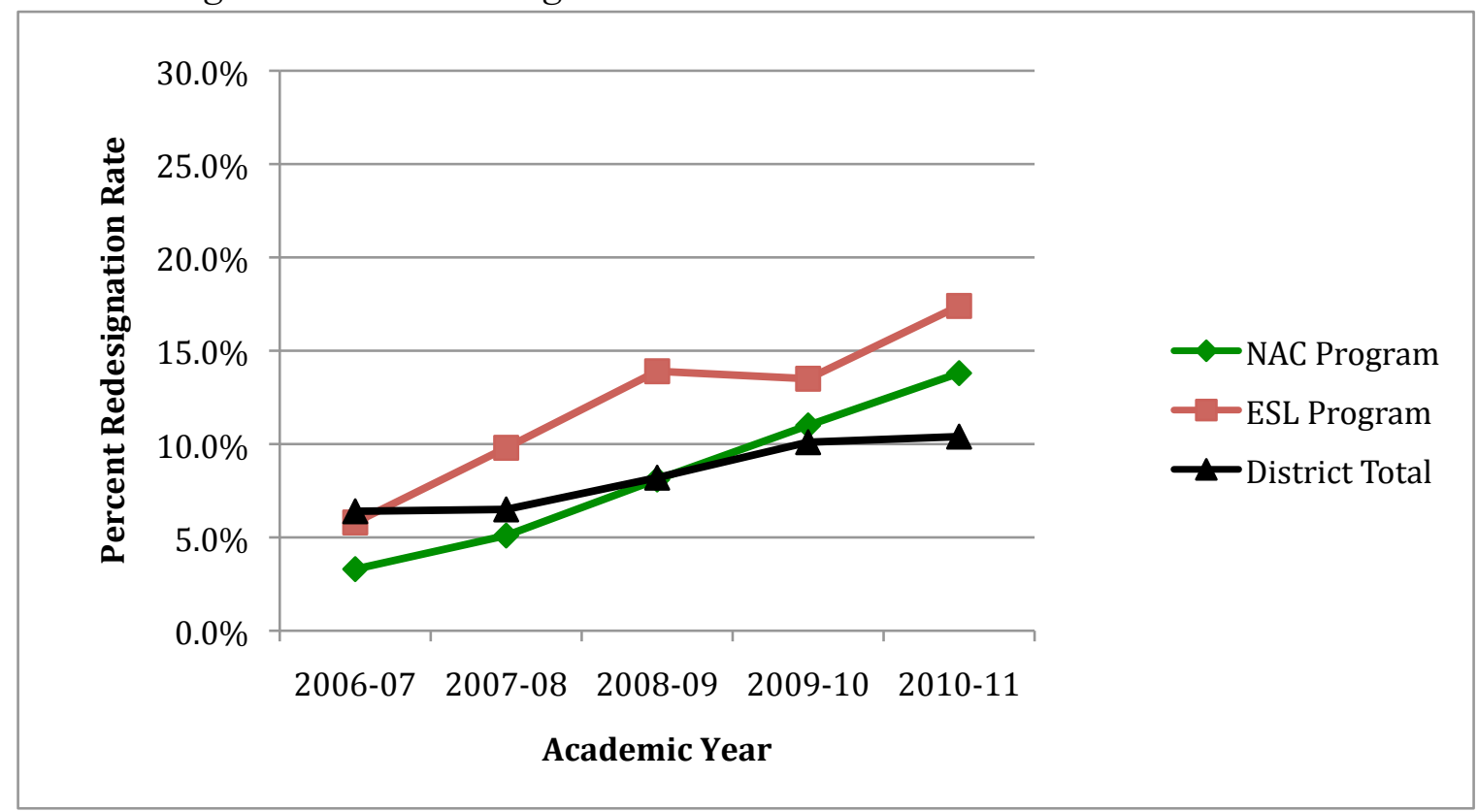

According to the rates over a five-year period, the classes within the NAC program demonstrated a $10.5 \%$ growth in the number of English learners that were redesignated, as compared to a growth of $11.6 \%$ within the ESL classes under study. While the ESL program demonstrated a $1.1 \%$ greater percentage of students redesignated during this time period than the NAC program, both programs were reclassifying their English Learners at a rate that far exceeded that of the district as a whole, which only reported a $4 \%$ increase over these same years. These rates demonstrate successes within both programs under study, in that, when solely viewing redesignation data measures, their students are achieving far greater levels of English language acquisition than the district as a whole.

In reporting the redesignation rates of the individual cases under study (Table 11 and Figure 9), it is apparent that, with the exception of NAC Case \#2, each of the cases 
reclassified their students at a far higher rate than the district total. The growth in reclassification rates for each of these cases (except NAC \#2) over a five-year period were more than double, and in some cases are even triple or quadruple that of the district average. This illuminates the notion that English learners at these sites were more effectively acquiring sufficient language skills to be reclassified. In other words, the gains in language acquisition being made in the schools housing either the ESL or the NAC programs under study exceeded other schools in the district, as is apparent when comparing their redesignation rates to those of the district as a whole.

Table 11

Individual Case Study Percent Redesignation Rates Over Five-Year Period

\begin{tabular}{lllllll}
\hline Case & $2006-$ & $2007-$ & $2008-$ & $2009-$ & $2010-$ & $\begin{array}{l}\text { Five-Year } \\
\text { Growth }\end{array}$ \\
Study \# & 2007 & 2008 & 2009 & 2010 & 2011 & \\
\hline & & & & & & \\
NAC \#1 $^{\text {b }}$ & 5.3 & 5.2 & 6.8 & 7.5 & 17.0 & +11.7 \\
NAC \#2 $^{\mathrm{c}}$ & 1.6 & 3.6 & 4.2 & 6.6 & 5.6 & +4.0 \\
NAC \#3 & 2.9 & 6.4 & 13.3 & 18.9 & 18.9 & +16.0 \\
ESL \#1 & 7.4 & 10.0 & 16.8 & 15.8 & 22.4 & +15.0 \\
ESL \#2 $^{\text {d }}$ & 7.9 & 11.4 & 17.1 & 11.9 & 19.5 & +11.6 \\
ESL \#3 & 2.0 & 7.6 & 7.9 & 12.9 & 10.3 & +8.3 \\
District Total & $\mathbf{6 . 4}$ & $\mathbf{6 . 5}$ & $\mathbf{8 . 2}$ & $\mathbf{1 0 . 1}$ & $\mathbf{1 0 . 4}$ & $+\mathbf{4 . 0}$ \\
\hline
\end{tabular}

Note. ${ }^{\text {a }}$ Five-Year growth was determined by calculating the difference between 2006-07 and 2010-11, ${ }^{\mathrm{b}}$ Overall redesignation rate percentages were determined by averaging the percentages of the six small schools housed on the campus, ${ }^{\mathrm{c}}$ Overall redesignation rate percentages were determined by averaging the percentages of the four small schools housed on the campus, ${ }^{\mathrm{d}}$ Overall redesignation rate percentages were determined by averaging the percentages of the three provisional small schools housed on the campus during the 2006-07 and 2007-08 school years. 
Figure 9

Individual Case Study Percent Redesignation Rates Over Five-Year Period

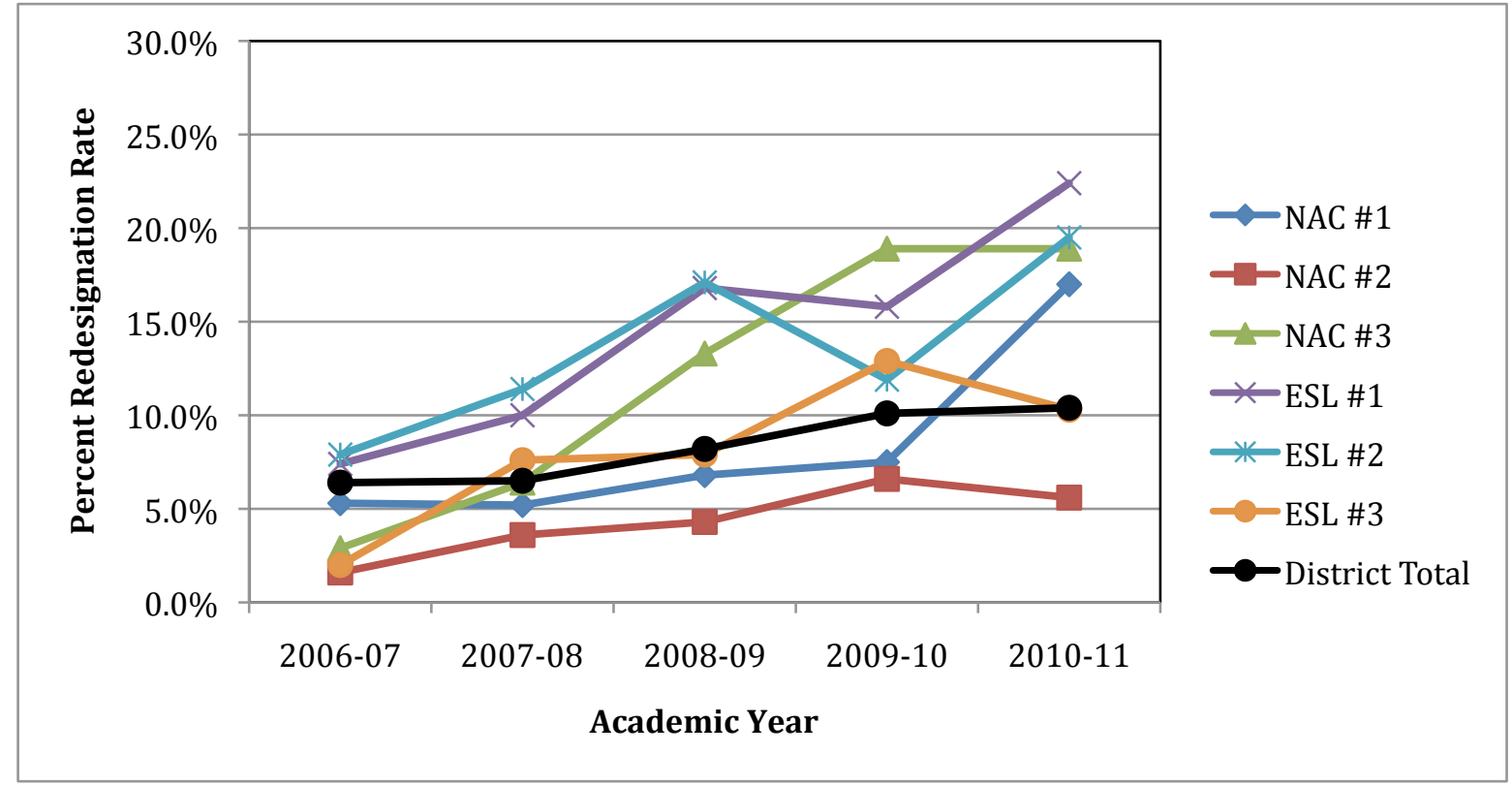

Findings of the Student Self-Concept Scale

The Student Self-Concept Scale (Appendix I) utilized to assess students' academic self-concept levels was administered following specifications indicated by the makers of the scale. The scale was comprised of 72 statements that measured the students' levels in the following three self-concept domains: academic, social, and selfimage. The statements from each of the three domains were scattered throughout the survey. The first 58 statements on the scale asked students to rate how confident they felt about each statement and how important the statement was to them, while the final 14 statements solely asked students to determine how confident they felt about each statement. As was reported in Chapter 3, Methodology, after administering the SSCS within this research study, the relatively high coefficient alpha reliabilities reported by the scale creators were replicated in the ratings found within the particular students 
surveyed for this study. The three self-confidence subscale reliabilities (self-image, academic, social) ranged from .80 to .84 and the three importance subscale reliabilities (self-image, academic, social) ranged from .78 to .84 . The internal consistency reliability of the SSCS was, therefore, determined to be fairly high within this study.

The academic, personal, and social self-concept levels of the students taking part in this study were analyzed by initially grouping the surveys into four groups according to the program in which they attended (ESL or NAC), as well as whether they were currently enrolled in the program (Year 1) or if they had completed the program in the prior year or two (Year 2). The following four codes were used to categorize the group responses: (1) ESL - Year 1; (2) ESL - Year 2; (3) New Arrival Center - Year 1; (4) New Arrival Center - Year 2. Each item response (dependent variable) was then analyzed utilizing SPSS software to determine the mean and the standard deviation, according to the group (independent variable) in which it was coded. An independent $t$-test was additionally employed to ascertain whether or not the items were statistically significantly different, when comparing the average mean responses of each survey item. It was determined that an independent $t$-test was the appropriate statistical analysis for this data, as "the independent $t$-test is used in situations in which there are two experimental conditions and different participants have been used in each condition" (Field, 2005, p. 296). Though there were four groups (experimental conditions) being compared within this portion of the study, conducting several independent $t$-tests allowed for the mean responses of the groups to be compared with specific directionality in mind, year-to-year within each program, and the same year between the two programs (NAC and ESL). Therefore, the following five independent $t$-tests were performed to compare 
the mean responses of the participants in the total survey, as well as each of the three subgroups (academic, social, and self-image) within each specific grouping: Total ESL participants and Total NAC participants (Tables 12 and 13); ESL-Year 1 Student Participants and NAC Year-1 Student Participants (Tables 14 and 15); ESL-Year 2 Student Participants and NAC-Year 2 Student Participants (Tables 16 and 17); ESL-Year 1 Student Participants and ESL-Year 2 Student Participants (Tables 18 and 19); NACYear 1 Student Participants and NAC-Year 2 Student Participants (Tables 20 and 21). As the academic self-concept subgroup of the survey was the subgroup necessary to answer the original guiding research questions for this study, this was the only subgroup of the survey to be analyzed item-be-item in the findings. Tables 22 and 23 demonstrate the mean, standard deviation, and the statistical significance of the 22 items that comprised the academic self-concept confidence level subgroup and the 18 items that made up the academic self-concept item importance subgroup for the total NAC and ESL student populations surveyed. The items that showed statistically significant differences on Tables 22 and 23 were highlighted to illuminate the findings within each table. Appendix $\mathrm{J}$ contains tables demonstrating the mean, standard deviation, and the statistical significance of the 22 items that comprised the academic self-concept confidence level subgroup and the 18 items for each specific grouping: ESL-Year 1 Student Participants and NAC Year-1 Student Participants; ESL-Year 2 Student Participants and NAC-Year 2 Student Participants; ESL-Year 1 Student Participants and ESL-Year 2 Student Participants; NAC-Year 1 Student Participants and NAC-Year 2 Student Participants. In addition, comparisons of the frequencies in the academic self-concept subgroup results were tested on the total NAC and ESL student populations utilizing Pearson's Chi-Square 
statistical analysis. The Chi Square analysis tested the association between the variables, as seen in Appendix K, though the findings were not substantially different than the independent $t$-tests, and therefore were reported solely in the appendix.

The items within the academic self-concept subgroup of the survey were further coded to demonstrate patterns among the findings for the total group comparison, as well as for each of the aforementioned groupings. The researcher coded the items on the scale into the following three codes: (1) sense of self, (2) behavioral, and (3) task-orientation (Figure 8). Tables 24 through 33 illustrate the number of items in which each group within the comparison demonstrated a higher mean score on the academic self-concept subscale, revealing the higher mean response totals for each group according to the three codes. The patterns of responses were analyzed to show which group had greater academic self-concept in the three areas: (1) sense of self, (2) behavior, and (3) task orientation. The patterns additionally drive the discussion as to how each group of students view themselves academically within each of the two programs under analysis.

\section{Overall Student Self-Concept Scale Findings}

The total ESL and total NAC student responses on the Student Self-Concept Questionnaire were compiled by adding together both the current attendees' (Year 1) and the former program participants' (Year 2) scores, so as to initially compare the two programs on the larger program scale. These scores were analyzed both descriptively, in order to compare the difference in total mean responses for each item, as well as to ascertain if there was a statistical significance present for each item. The total confidence level scores, as are demonstrated in Table 12, revealed that as a complete group the ESL 
cluster scored a slightly higher mean difference. Yet, when viewing the scale as a whole, the mean differences were not statistically significant. The confidence level of students on the academic self-concept subscale did not demonstrate any mean difference between the two programs under study. The social self-concept subscale did exhibit a slightly higher score for the ESL cluster, although, again, this different was not statistically significant. The self-image subscale showed that students in the ESL group on average reported more overall personal confidence $(M=1.46, S D=.28)$ than the students in the NAC program $(M=1.35, S D=.35)$. The difference in the mean scores of this item were moderately statistically significant $t(190)=2.32, p<.05$. This demonstrates that as a whole, the only significant difference between students' confidence level reporting occurred when reporting the self-image portion of the survey.

Table 12

Student Self-Concept Scale Confidence Level Analysis - Total ESL Student Participants and Total NAC Student Participants (Years 1 and 2)

\begin{tabular}{|c|c|c|c|c|c|c|c|}
\hline & \multicolumn{3}{|c|}{$\begin{array}{l}\text { ESL } \\
(N=98) \\
\end{array}$} & \multicolumn{3}{|c|}{$\begin{array}{l}\text { NAC } \\
(N=94)\end{array}$} & \multirow{2}{*}{$\begin{array}{l}\text { Mean } \\
\text { Difference }\end{array}$} \\
\hline & $n$ & $\mathrm{M}$ & SD & $n$ & $\mathrm{M}$ & SD & \\
\hline Total SSCS Score & 98 & 1.47 & .24 & 94 & 1.43 & .28 & -.04 \\
\hline Academic Self-Concept Subgroup Score & 98 & 1.52 & .30 & 94 & 1.52 & .32 & .00 \\
\hline Social Self-Concept Subgroup Score & 98 & 1.53 & .29 & 94 & 1.49 & .31 & -.04 \\
\hline Self-Image Subgroup Score & 98 & $1.46^{*}$ & .28 & 94 & $1.35^{*}$ & .35 & -.11 \\
\hline
\end{tabular}

Note. ${ }^{*} \mathrm{p}<.05 . * * \mathrm{p}<.01$.

When analyzing the total level of importance, the students gave the first 58 statements on the SSCS, which asked students to rate both their confidence level and how important the statement was to them, the ESL cluster reported a slightly higher mean 
score than the NAC group (Table 13). Although this difference was not statistically significantly different, it did demonstrate that the ESL group as a whole placed more importance on the self-concept items found in the scale than the NAC group. The academic self-concept subgroup scores showed no difference in mean totals between the two groups, yet the social self-concept and the self-image subgroups, again, showed the ESL group reported a somewhat higher mean score when viewing item importance, than the NAC group. The academic, social, and self-image subgroups did not demonstrate statistically significant differences.

Table 13

Student Self-Concept Scale Item Importance Analysis - Total ESL Student Participants and Total NAC Student Participants (Years 1 and 2)

\begin{tabular}{|c|c|c|c|c|c|c|c|}
\hline & \multicolumn{3}{|c|}{$\begin{array}{l}\text { ESL } \\
(N=98)\end{array}$} & \multicolumn{3}{|c|}{$\begin{array}{l}\text { NAC } \\
(N=94)\end{array}$} & \multirow{2}{*}{$\begin{array}{l}\text { Mean } \\
\text { Difference }\end{array}$} \\
\hline & $n$ & M & SD & $n$ & M & SD & \\
\hline Total SSCS Score & 98 & 1.42 & .30 & 94 & 1.39 & .30 & -.03 \\
\hline Academic Self-Concept Subgroup Score & 98 & 1.52 & .35 & 94 & 1.52 & .32 & .00 \\
\hline Social Self-Concept Subgroup Score & 98 & 1.42 & .35 & 94 & 1.36 & .34 & -.06 \\
\hline Self-Image Subgroup Score & 98 & 1.37 & .35 & 94 & 1.30 & .42 & -.07 \\
\hline
\end{tabular}

To better understand the self-concept levels of students attending classes in either the New Arrival Center program or the English as a Second Language program at the time of study, the mean responses on the SSCS were compared between the NAC-Year 1 student participants and ESL-Year 1 students. As is reported in Table 14, the confidence level analysis demonstrated a slightly higher mean averages for the NAC cluster of 
students, when viewing the total SSCS score. Additionally, both the academic and social self-concept subscales exhibited a somewhat higher mean score for the NAC students than the ESL students, again showing that the NAC-Year 1 students were slightly more confident in such areas than the ESL-Year 1 students. Conversely, in terms of personal self-concept, the ESL-Year 1 group reported a higher mean score than the NAC-Year 1 group on the self-image subset, therefore demonstrating a slightly higher personal selfconcept level. Neither the total survey score nor the academic, social, nor self-image subgroups demonstrated statistically significant differences.

\section{Table 14}

Student Self-Concept Scale Confidence Level Analysis - ESL-Year 1 Student Participants and NAC-Year 1 Student Participants

\begin{tabular}{|c|c|c|c|c|c|c|c|}
\hline & \multicolumn{3}{|c|}{$\begin{array}{l}\text { ESL } \\
(N=49) \\
\end{array}$} & \multicolumn{3}{|c|}{$\begin{array}{l}\text { NAC } \\
(N=51)\end{array}$} & \multirow{2}{*}{$\begin{array}{l}\text { Mean } \\
\text { Difference }\end{array}$} \\
\hline & $n$ & $\mathrm{M}$ & $\mathrm{SD}$ & $n$ & $\mathrm{M}$ & SD & \\
\hline Total SSCS Score & 49 & 1.46 & .28 & 51 & 1.48 & .29 & +.02 \\
\hline Academic Self-Concept Subgroup Score & 49 & 1.48 & .34 & 51 & 1.59 & .33 & +.11 \\
\hline Social Self-Concept Subgroup Score & 49 & 1.53 & .33 & 51 & 1.54 & .31 & +.01 \\
\hline Self-Image Subgroup Score & 49 & 1.45 & .31 & 51 & 1.40 & .38 & -.05 \\
\hline
\end{tabular}

The positive SSCS results exhibited by the NAC-Year 1 student participants were additionally shown in the qualitative focus group findings. During the focus groups, the NAC-Year 1 students discussed feeling there were others enduring similar circumstances, as their classes were comprised solely of newcomer immigrant youth, which made them feel more reassured and supported in their academic environments. They felt more comfortable requesting assistance from other students, and they additionally believed 
their teachers were highly supportive, as they had one primary teacher instructing them throughout their day. The positive environment led these students to have a slightly higher academic self-concept level, as can be seen in Table 14, and as is demonstrated in the following quotes:

The teachers make you feel better, they encourage you to excel [in class]. Initially everyone is quiet then everyone wants to participate (New Arrival Center Student, 2011).

I feel better because there are a lot of people from here that helped me. My friends would help me when I didn't understand and told me how to do it (New Arrival Center Student, 2011).

Table 15 illustrates the total item importance comparison between the ESL-Year 1 students and the NAC-Year 1 students on both the complete SSCS and the three subgroups (academic, social, and self-image). These results demonstrated that the ESL group reported a slightly higher level of item importance than the NAC group in the total survey, as well as on the social self-concept and the self-image subsets. On the contrary, the academic self-concept subgroup revealed the NAC cluster reported a slightly higher item importance mean than the ESL group. Much like the confidence level analysis, neither the total survey score nor the academic, social, nor self-image subgroups demonstrated statistically significant differences. 
Table 15

Student Self-Concept Scale Item Importance Analysis - ESL-Year 1 Student Participants and NAC-Year 1 Student Participants

\begin{tabular}{|c|c|c|c|c|c|c|c|}
\hline & \multicolumn{3}{|c|}{$\begin{array}{l}\text { ESL } \\
(N=49)\end{array}$} & \multicolumn{3}{|c|}{$\begin{array}{l}\text { NAC } \\
(N=51)\end{array}$} & \multirow{2}{*}{$\begin{array}{l}\text { Mean } \\
\text { Difference }\end{array}$} \\
\hline & $n$ & M & SD & $n$ & M & SD & \\
\hline Total SSCS Score & 49 & 1.45 & .33 & 51 & 1.42 & .33 & -.03 \\
\hline Academic Self-Concept Subgroup Score & 49 & 1.52 & .39 & 51 & 1.54 & .36 & +.03 \\
\hline Social Self-Concept Subgroup Score & 49 & 1.45 & .38 & 51 & 1.39 & .36 & -.06 \\
\hline Self-Image Subgroup Score & 49 & 1.39 & .36 & 51 & 1.35 & .44 & -.04 \\
\hline
\end{tabular}

The self-concept levels of Year 2 students, students had completed their classes in either the New Arrival Center program or the English as a Second Language program one to two years prior to the time of the study, were next analyzed to better understand students' self-concept levels after completing one of the two programs under study. As are seen in Table 16, the mean confidence level responses on the SSCS were compared between ESL-Year 2 student participants and NAC-Year 2 students. When viewing the confidence levels as a whole, the ESL-Year 2 cluster $(M=1.48, S D=.20)$ demonstrated a higher self-concept level then the NAC-Year 2 group $(M=1.37, S D=.25)$. The difference in the total mean scores was moderately statistically significant $t(90)=2.36, p$ $<.05$. When examining the academic self-concept subgroup, the ESL-Year $2(M=1.55$, $S D=.25)$ group was again more highly confident than the NAC Year-2 cluster $(M=$ $1.44, S D=.31$ ), yet it was not statistically significant. The social self-concept subgroup additionally demonstrated a higher mean score for the ESL-Year 2 students $(M=1.53$, $S D=.24)$ than the NAC-Year 2 students $(M=1.44, S D=.30)$, which was again was not statistically significantly different. Finally, the self-image subgroup exhibited a higher 
mean score for the ESL-Year $2(M=1.47, S D=.25)$ students than the NAC-Year 2 students $(M=1.30, S D=.31)$. The mean scores of the self-image subset were highly statistically significantly different $t(90)=2.92, p<.01$. The above results demonstrate that the confidence level scores on the total Student Self-Concept Scale, as well as each of the three subgroups, were higher for the ESL-Year 2 students than the NAC-Year 2 students, thus showing the ESL group felt more self-assured than the NAC group.

Table 16

Student Self-Concept Scale Confidence Level Analysis - ESL-Year 2 Student Participants and NAC-Year 2 Student Participants

\begin{tabular}{|c|c|c|c|c|c|c|c|}
\hline & \multicolumn{3}{|c|}{$\begin{array}{l}\text { ESL } \\
(N=49) \\
\end{array}$} & \multicolumn{3}{|c|}{$\begin{array}{l}\text { NAC } \\
(N=43) \\
\end{array}$} & \multirow{2}{*}{$\begin{array}{l}\text { Mean } \\
\text { Difference }\end{array}$} \\
\hline & $n$ & $\mathrm{M}$ & $\mathrm{SD}$ & $n$ & $\mathrm{M}$ & SD & \\
\hline Total SSCS Score & 49 & $1.48^{*}$ & .20 & 43 & $1.37 *$ & .25 & -.11 \\
\hline Academic Self-Concept Subgroup Score & 49 & 1.55 & .25 & 43 & 1.44 & .31 & -.11 \\
\hline Social Self-Concept Subgroup Score & 49 & 1.53 & .24 & 43 & 1.44 & .30 & -.09 \\
\hline Self-Image Subgroup Score & 49 & $1.47 * *$ & .25 & 43 & $1.30 * *$ & .31 & -.17 \\
\hline
\end{tabular}

Note. $* \mathrm{p}<.05 . * * \mathrm{p}<.01$

The statistically significantly higher findings demonstrated by the ESL-Year 2 students on the SSCS were also illustrated during the focus group discussions. During the discussions, the ESL-Year 2 students talked extensively about their increased confidence levels and their greater levels of comfort in classes over the prior academic year. These youth felt more capable in their classes and were able to participate in whole group discussions with greater confidence. The following quote by one ESL-Year 2 focus group 
member speaks to the increased self-concept levels of this group of students, as were also shown through the SSCS findings on Table 16:

[In the beginning] I felt insecure because I felt like I wasn't doing things right. I felt insecure because I sometimes didn't know what people talked about and I felt it was about [me]. I felt less of a person than others... I was insecure and embarrassed because I didn't know the language... Now I feel confident because my teachers tell me my English is better now and my friends do too. (ESL Student, 2011).

Though neither the total survey nor any of the three subgroups exhibited a mean difference that was statistically significantly different when analyzing the item importance reported by the ESL-Year 2 students and the NAC-Year 2 students, the ESL group did report higher mean differences than the NAC group in each of the different areas. The ESL-Year 2 group placed a somewhat greater level of importance on the overall items, as well as in the academic self-concept subset, and relatively higher levels of item importance in the social and self-image portions of the survey. Therefore, despite the fact that none showed statistically significant differences, the ESL group demonstrated greater item importance than the NAC group, as exhibited in Table 17. 
Table 17

Student Self-Concept Scale Item Importance Analysis - ESL-Year 2 Student Participants and NAC-Year 2 Student Participants

\begin{tabular}{|c|c|c|c|c|c|c|c|}
\hline & \multicolumn{3}{|c|}{$\begin{array}{l}\text { ESL } \\
(N=49)\end{array}$} & \multicolumn{3}{|c|}{$\begin{array}{l}\text { NAC } \\
(N=43)\end{array}$} & \multirow{2}{*}{$\begin{array}{l}\text { Mean } \\
\text { Difference }\end{array}$} \\
\hline & $n$ & $\mathrm{M}$ & $\mathrm{SD}$ & $n$ & $\mathrm{M}$ & SD & \\
\hline Total SSCS Score & 49 & 1.40 & .27 & 43 & 1.35 & .27 & -.05 \\
\hline Academic Self-Concept Subgroup Score & 49 & 1.51 & .31 & 43 & 1.49 & .28 & -.02 \\
\hline Social Self-Concept Subgroup Score & 49 & 1.39 & .31 & 43 & 1.32 & .32 & -.07 \\
\hline Self-Image Subgroup Score & 49 & 1.36 & .34 & 43 & 1.24 & .39 & -.12 \\
\hline
\end{tabular}

Table 18 reveals the overall and subgroup mean self-concept scores of the students as they moved from one year to the next within the English as a Second Language (ESL) program. To gain an understanding of the students'self-concept levels during this progression, the average mean responses of ESL-Year 1 students' were compared with the responses of ESL-Year 2 students. The results demonstrated that the ESL-Year 2 group reported a slightly higher confidence level than the ESL-Year 1 group in the total survey, as well as in the self-image subset. The ESL-Year 2 students exhibited a more significant level of confidence than the ESL-Year 1 group when marking responses in the academic self-concept subgroup, but there was no difference between the two groups in the social self-concept subset. These results show that although none are statistically significantly different, the ESL-Year 2 students under investigation did feel more confident, particularly in terms of academics, than the ESL-Year 1 students. 
Table 18

Student Self-Concept Scale Confidence Level Analysis - ESL-Year 1 Student Participants and ESL-Year 2 Student Participants

\begin{tabular}{lcccccccc}
\hline & \multicolumn{7}{l}{$\begin{array}{l}\text { ESL-Year 1 } \\
(N=49)\end{array}$} & \multicolumn{3}{c}{$\begin{array}{l}\text { ESL-Year } 2 \\
(N=49)\end{array}$} & Mean \\
& $n$ & $\mathrm{M}$ & $\mathrm{SD}$ & $n$ & $\mathrm{M}$ & $\mathrm{SD}$ & \begin{tabular}{l} 
Difference \\
\hline
\end{tabular} \\
\hline Total SSCS Score & 49 & 1.46 & .28 & 49 & 1.48 & .20 & +.02 \\
Academic Self-Concept Subgroup Score & 49 & 1.48 & .34 & 49 & 1.55 & .25 & +.11 \\
Social Self-Concept Subgroup Score & 49 & 1.53 & .33 & 49 & 1.53 & .24 & .00 \\
Self-Image Subgroup Score & 49 & 1.45 & .31 & 49 & 1.47 & .25 & +.02 \\
\hline
\end{tabular}

Again the ESL-Year 2 students demonstrated higher academic self-concept mean scores, as demonstrated in Table 18, which can also be seen in the qualitative focus group findings. The ESL-Year 2 group felt the most confident academically of the four subgroups that were queried for the research study, in that they gave the most positive statements in terms of the manner in which they viewed themselves within the academic environment. The following quote again displays the high self-concept level of the ESLYear 2 students within the study:

I feel comfortable [now] because I understand English. I feel better since I know more words (ESL Student, 2011).

Table 19 illustrates the total item importance comparison between the ESL-Year 1 students and the ESL-Year 2 students in both the complete SSCS, as well as in the three subgroups. These results demonstrated that the ESL-Year 1 group reported a slightly higher level of item importance than the ESL-Year 2 group in the total survey, as well as in the academic self-concept, social self-concept, and the self-image subsets. Similar to the confidence level analysis, neither the total survey score nor the academic, social, nor 
self-image subgroups demonstrated statistically significant differences. Although they were not statistically significantly different, these results demonstrated that despite the fact that the ESL-Year 2 students reported a higher level of confidence in the items, the ESL-Year 1 students found the items more important than the ESL-Year 2 students.

Table 19

Student Self-Concept Scale Item Importance Analysis - ESL-Year 1 Student Participants and ESL-Year 2 Student Participants

\begin{tabular}{|c|c|c|c|c|c|c|c|}
\hline & \multicolumn{3}{|c|}{$\begin{array}{l}\text { ESL-Year } 1 \\
(N=49)\end{array}$} & \multicolumn{3}{|c|}{$\begin{array}{l}\text { ESL-Year } 2 \\
(N=49)\end{array}$} & \multirow{2}{*}{$\begin{array}{l}\text { Mean } \\
\text { Difference }\end{array}$} \\
\hline & $n$ & M & SD & $n$ & M & SD & \\
\hline Total SSCS Score & 49 & 1.45 & .33 & 49 & 1.40 & .27 & -.05 \\
\hline Academic Self-Concept Subgroup Score & 49 & 1.52 & .38 & 49 & 1.51 & .31 & -.01 \\
\hline Social Self-Concept Subgroup Score & 49 & 1.45 & .38 & 49 & 1.39 & .31 & -.06 \\
\hline Self-Image Subgroup Score & 49 & 1.39 & .36 & 49 & 1.36 & .34 & -.03 \\
\hline
\end{tabular}

The final confidence level analysis of mean results, as reported on the SSCS, examined the progression of the students' academic self-concept levels as they advanced from one year to the next within the New Arrival Center (NAC) program (Table 20). Hence, the mean item responses of the NAC-Year 1 students were compared with the responses of the NAC-Year 2 participants to uncover differences in average answers between these two groups. When examining the total scores, the NAC-Year 1 cluster ( $M$ $=1.48, S D=.29)$ reported higher overall confidence levels than the NAC-Year 2 students $(M=1.37, S D=.25)$, though the difference in the total mean scores between these two groups was not statistically significant. The academic self-concept subset also demonstrated that the NAC-Year 1 students $(M=1.59, S D=.33)$ felt more confident 
than the NAC-Year 2 students $(M=1.44, S D=.31)$ in terms of their academic abilities. The difference in mean scores between the NAC-Year 1 and NAC-Year 2 clusters of students was moderately statistically significant $t(92)=2.27, p<.05$ on the academic self-concept item subset. Finally, although not statistically significantly different, the social self-concept and self-image subgroups also illustrate that according to the mean scores, the NAC-Year 1 students reported feeling more confident than the NAC-Year 2 students both personally and socially among peers.

Table 20

Student Self-Concept Scale Confidence Level Analysis - NAC-Year 1 Student Participants and NAC-Year 2 Student Participants

\begin{tabular}{|c|c|c|c|c|c|c|c|}
\hline & \multicolumn{3}{|c|}{$\begin{array}{l}\text { NAC-Year } 1 \\
(N=51)\end{array}$} & \multicolumn{3}{|c|}{$\begin{array}{l}\text { NAC-Year } 2 \\
(N=43)\end{array}$} & \multirow{2}{*}{$\begin{array}{l}\text { Mean } \\
\text { Difference }\end{array}$} \\
\hline & $n$ & $\mathrm{M}$ & SD & $n$ & $\mathrm{M}$ & SD & \\
\hline Total SSCS Score & 51 & 1.48 & .29 & 43 & 1.37 & .25 & -.11 \\
\hline Academic Self-Concept Subgroup Score & 51 & $1.59^{*}$ & .33 & 43 & $1.44^{*}$ & .31 & -.15 \\
\hline Social Self-Concept Subgroup Score & 51 & 1.54 & .31 & 43 & 1.44 & .30 & -.10 \\
\hline Self-Image Subgroup Score & 51 & 1.40 & .38 & 43 & 1.30 & .31 & -.10 \\
\hline
\end{tabular}

Note. ${ }^{*} \mathrm{p}<.05 . * * \mathrm{p}<.01$.

The greater self-concept scores exhibited by the NAC-Year 1 student participants, as shown in the SSCS results, were once again apparent in the qualitative focus group findings. During the focus groups, the NAC-Year 1 students spoke of how they felt somewhat comfortable in the academic environment, due to the supportive nature of their classes and the connection they felt with their teachers and their peers. The students were able to foster such positive connections, as they had extended time with the same peers and teachers throughout their academic days. As was previously stated, the encouraging 
nature of their academic environment allowed these students to develop a slightly higher academic self-concept level, as can be seen in Table 20, and as is demonstrated in the following quotes:

I feel better. I feel that I am going to get better because in the beginning I thought that it was going to be very difficult to learn English because of the pronunciation, and I already see that it's not very difficult if you have the desire to move forward (New Arrival Center Student, 2011).

[I felt more confident when] one time my parents and my teacher met and she told them that I more or less understood [concepts] and I help my peers when they don't understand (New Arrival Center Student, 2011).

When comparing the mean importance level responses of both the total combined items on the SSCS as well as the three subgroups (Table 21), the results once again demonstrated that the NAC-Year 1 students found the items more important than the NAC-Year 2 students. Though none of the analyses were statistically significantly different, the mean scores of the NAC-Year 1 cluster were higher in each of the three subgroups (academic, social, and self-image), as well as in the total survey, than the mean responses of the NAC-Year 2 students. Similar to the above reporting of the students' confidence levels on the items, the NAC-Year 1 students found the items to be more important than the NAC-Year 2 students, which indicates that these same students felt more motivated and connected to their respective schools academically, socially, and personally. 
Table 21

Student Self-Concept Scale Item Importance Analysis - NAC-Year 1 Student Participants and NAC-Year 2 Student Participants

\begin{tabular}{|c|c|c|c|c|c|c|c|}
\hline & \multicolumn{3}{|c|}{$\begin{array}{l}\text { NAC-Year } 1 \\
(N=51)\end{array}$} & \multicolumn{3}{|c|}{$\begin{array}{l}\text { NAC-Year } 2 \\
(N=43)\end{array}$} & \multirow{2}{*}{$\begin{array}{l}\text { Mean } \\
\text { Difference }\end{array}$} \\
\hline & $n$ & M & SD & $n$ & $\mathrm{M}$ & $\mathrm{SD}$ & \\
\hline Total SSCS Score & 51 & 1.42 & .33 & 43 & 1.35 & .27 & -.07 \\
\hline Academic Self-Concept Subgroup Score & 51 & 1.54 & .36 & 43 & 1.49 & .28 & -.05 \\
\hline Social Self-Concept Subgroup Score & 51 & 1.39 & .36 & 43 & 1.32 & .32 & -.06 \\
\hline Self-Image Subgroup Score & 51 & 1.35 & .44 & 43 & 1.24 & .39 & -.11 \\
\hline
\end{tabular}

\section{Academic Self-Concept Subscale Findings}

As was stated in the introduction to section three, the academic self-concept subgroup of items on the Student Self-Concept Survey was the subgroup necessary to answer the original guiding research questions for this study and, therefore, this subgroup was analyzed and disseminated item-be-item. In analyzing the total ESL and total NAC student responses on the academic self-concept subsection of the Student Self-Concept Questionnaire, both the current attendees' (Year 1) and the former program participants' (Year 2) scores were compiled to compare the programs as a whole (Tables 22 and 23). The total group comparison on the academic self-concept subscale is highlighted as it gives a more complete picture of the way in which the ESL and the NAC students responded as a whole to each statement within the survey. In addition, the disaggregated findings for each of the year-by-year and program-by-program findings can also be viewed in Appendix J. 
Table 22 demonstrates the results of the confidence levels on each of the 22 items, as were reported by the student participants in each program. The results as analyzed in this manner were mixed, in that the NAC group reported higher mean scores on 13 of the survey statements, while the ESL group reported higher mean scores on nine of the items. Neither group demonstrated overwhelmingly greater mean scores. The analysis showed that for all twenty-two of the items on the academic self-concept subscale of the survey there was no statistical significance in mean responses when comparing the total ESL student scores with the total NAC student scores.

\section{Table 22}

Academic Self-Concept Confidence Level Subgroup Analysis - Total ESL Student Participants and Total NAC Student Participants (Years 1 and 2)

\begin{tabular}{|c|c|c|c|c|c|c|c|}
\hline & \multicolumn{3}{|c|}{$\begin{array}{l}\text { ESL } \\
(N=98)\end{array}$} & \multicolumn{3}{|c|}{$\begin{array}{l}\mathrm{NAC} \\
(N=94)\end{array}$} & \multirow{2}{*}{$\begin{array}{l}\text { Mean } \\
\text { Difference }\end{array}$} \\
\hline & $n$ & M & SD & $n$ & M & $\mathrm{SD}$ & \\
\hline $\begin{array}{l}\text { I can use a nice tone of voice in classroom } \\
\text { discussions with my teacher. }\end{array}$ & 97 & 1.63 & .62 & 92 & 1.72 & .52 & +.09 \\
\hline $\begin{array}{l}\text { I can sit in class without daydreaming } \\
\text { during a lesson. }\end{array}$ & 97 & 1.19 & .77 & 93 & 1.26 & .66 & +.07 \\
\hline I can do my homework on time. & 98 & 1.52 & .61 & 94 & 1.56 & .56 & +.04 \\
\hline $\begin{array}{l}\text { I can read aloud in class without feeling } \\
\text { nervous. }\end{array}$ & 98 & 1.19 & .74 & 92 & 1.32 & .57 & +.13 \\
\hline $\begin{array}{l}\text { I can sit at my desk for } 2 \text { minutes without } \\
\text { moving around or fidgeting. }\end{array}$ & 98 & 1.39 & .80 & 93 & 1.29 & .83 & -.10 \\
\hline I can finish my class work on time. & 97 & 1.46 & .61 & 93 & 1.55 & .54 & +.09 \\
\hline $\begin{array}{l}\text { I can listen to my teacher talk about a } \\
\text { subject for } 20 \text { minutes. }\end{array}$ & 97 & 1.41 & .69 & 92 & 1.50 & .60 & +.09 \\
\hline $\begin{array}{l}\text { I can ask my teacher for help without } \\
\text { feeling ashamed or upset. }\end{array}$ & 95 & 1.60 & .55 & 91 & 1.58 & .62 & -.02 \\
\hline $\begin{array}{l}\text { I can go to the board to do work when my } \\
\text { teacher asks me to. }\end{array}$ & 98 & 1.30 & .69 & 93 & 1.46 & .58 & +.16 \\
\hline
\end{tabular}




\begin{tabular}{|c|c|c|c|c|c|c|c|}
\hline I can laugh when I make silly mistakes. & 98 & 1.41 & .73 & 94 & 1.55 & .65 & +.14 \\
\hline $\begin{array}{l}\text { I can read instructions in a book and } \\
\text { follow them carefully. }\end{array}$ & 97 & 1.56 & .61 & 91 & 1.53 & .62 & -.03 \\
\hline $\begin{array}{l}\text { I can follow my teacher's directions for } \\
\text { doing reading work. }\end{array}$ & 98 & 1.70 & .58 & 92 & 1.71 & .55 & +.01 \\
\hline $\begin{array}{l}\text { I can ignore classmates when they } \\
\text { whisper or talk during class. }\end{array}$ & 97 & 1.28 & .72 & 94 & 1.10 & .67 & -.18 \\
\hline I can do math work without help. & 98 & 1.13 & .74 & 92 & 1.29 & .69 & +.16 \\
\hline $\begin{array}{l}\text { I can speak in class when my teacher } \\
\text { calls on me. }\end{array}$ & 98 & 1.54 & .66 & 92 & 1.64 & .59 & +.10 \\
\hline $\begin{array}{l}\text { I can listen when my teacher } \\
\text { is presenting a lesson. }\end{array}$ & 98 & 1.76 & .50 & 91 & 1.74 & .49 & -.02 \\
\hline $\begin{array}{l}\text { I can remember when class projects } \\
\text { are due. }\end{array}$ & 97 & 1.58 & .56 & 91 & 1.51 & .58 & -.07 \\
\hline I can follow classroom rules. & 98 & 1.69 & .49 & 92 & 1.60 & .56 & -.09 \\
\hline $\begin{array}{l}\text { If I finish my class work on time I } \\
\text { will get good grades in school. }\end{array}$ & 96 & 1.82 & .41 & 92 & 1.85 & .39 & +.03 \\
\hline $\begin{array}{l}\text { If I follow my teacher's directions in } \\
\text { class, I will do my work correctly. }\end{array}$ & 97 & 1.87 & .37 & 92 & 1.90 & .30 & +.03 \\
\hline $\begin{array}{l}\text { If I do my homework on time, my } \\
\text { parents will be proud of me. }\end{array}$ & 96 & 1.85 & .41 & 92 & 1.79 & .46 & -.06 \\
\hline $\begin{array}{l}\text { If I ignore classmates who whisper } \\
\text { in class, I can complete my work. }\end{array}$ & 97 & 1.72 & .54 & 92 & 1.68 & .51 & -.04 \\
\hline
\end{tabular}

The survey additionally asked student to rate the level of importance they gave 18 of the 22 total items on the academic self-concept subset of the SSCS. As is demonstrated in Table 23, the mean differences for these items varied, in that for the ESL group the mean difference was higher for 10 items, while for the NAC group the mean score was higher on seven items. There was no difference in mean scores for one item, demonstrating that both groups gave this item the same average level of importance (SSCS survey item: I can ignore classmates when they whisper or talk during class). Two items had sufficient mean differences to demonstrate statistical significance at the .05 
level $(\mathrm{p}<.05)$. The first of these items demonstrated that on average it was more important to NAC students $(M=1.87, S D=.37)$ to finish class work on time (SSCS survey item: I can finish my class work on time) than it was to the ESL student participants $(M=1.73, S D=.57)$. The difference in the mean scores of this item was moderately statistically significant $t(165)=-2.04, p<.05$. The second item showed that the NAC students $(M=1.74, S D=.53)$ also felt it was more important than the ESL students $(M=1.54, S D=.69)$ to complete math work without help (SSCS survey item: I can do math work without help). The difference of the mean scores on this item was also moderately statistically significant $t(179)=-2.23, p<.05$. Therefore, these results indicated that the students in the New Arrival Center placement placed greater importance in completing class work and being able to achieve independently in math than the ESL students. The findings on the academic self-concept subscales for the total group comparison, as well as the year-by-year and program-by-program group comparisons, will be discussed in more detail in a subsequent analysis of the patterns demonstrated within varying groups' mean item responses.

Table 23

Academic Self-Concept Item Importance Subgroup Analysis - Total ESL Student Participants and Total NAC Student Participants (Years 1 and 2)

\begin{tabular}{|c|c|c|c|c|c|c|c|}
\hline & \multicolumn{3}{|c|}{$\begin{array}{l}\text { ESL } \\
(N=98)\end{array}$} & \multicolumn{3}{|c|}{$\begin{array}{l}\text { NAC } \\
(N=94)\end{array}$} & \multirow{2}{*}{$\begin{array}{l}\text { Mean } \\
\text { Difference }\end{array}$} \\
\hline & $n$ & $\mathrm{M}$ & $\mathrm{SD}$ & $n$ & $\mathrm{M}$ & $\mathrm{SD}$ & \\
\hline $\begin{array}{l}\text { I can use a nice tone of voice in classroom } \\
\text { discussions with my teacher. }\end{array}$ & 96 & 1.58 & .63 & 94 & 1.54 & .62 & -.04 \\
\hline $\begin{array}{l}\text { I can sit in class without daydreaming } \\
\text { during a lesson. }\end{array}$ & 96 & 1.20 & .80 & 92 & 1.12 & .77 & -.08 \\
\hline I can do my homework on time. & 98 & 1.72 & .57 & 93 & 1.75 & .53 & +.03 \\
\hline
\end{tabular}




\begin{tabular}{|c|c|c|c|c|c|c|c|}
\hline $\begin{array}{l}\text { I can read aloud in class without feeling } \\
\text { nervous. }\end{array}$ & 98 & 1.54 & .65 & 94 & 1.46 & .60 & -.08 \\
\hline $\begin{array}{l}\text { I can sit at my desk for } 2 \text { minutes without } \\
\text { moving around or fidgeting. }\end{array}$ & 97 & 1.13 & .80 & 93 & 1.18 & .74 & +.05 \\
\hline I can finish my class work on time. & 97 & $1.73^{*}$ & .57 & 94 & $1.87^{*}$ & .37 & +.14 \\
\hline $\begin{array}{l}\text { I can listen to my teacher talk about a } \\
\text { subject for } 20 \text { minutes. }\end{array}$ & 97 & 1.52 & .69 & 93 & 1.58 & .60 & +.06 \\
\hline $\begin{array}{l}\text { I can ask my teacher for help without } \\
\text { feeling ashamed or upset. }\end{array}$ & 95 & 1.71 & .56 & 92 & 1.63 & .59 & -.08 \\
\hline $\begin{array}{l}\text { I can go to the board to do work when } \\
\text { my teacher asks me to. }\end{array}$ & 98 & 1.57 & .66 & 94 & 1.51 & .65 & -.06 \\
\hline I can laugh when I make silly mistakes. & 98 & 1.04 & .79 & 92 & .90 & .79 & -.14 \\
\hline $\begin{array}{l}\text { I can read instructions in a book and } \\
\text { follow them carefully. }\end{array}$ & 96 & 1.64 & .53 & 93 & 1.63 & .59 & -.01 \\
\hline $\begin{array}{l}\text { I can follow my teacher's directions for } \\
\text { doing reading work. }\end{array}$ & 96 & 1.78 & .51 & 94 & 1.73 & .49 & -.05 \\
\hline $\begin{array}{l}\text { I can ignore classmates when they } \\
\text { whisper or talk during class. }\end{array}$ & 97 & 1.27 & .80 & 92 & 1.27 & .68 & .00 \\
\hline I can do math work without help. & 97 & $1.54^{*}$ & .69 & 91 & $1.74 *$ & .53 & +.20 \\
\hline $\begin{array}{l}\text { I can speak in class when my teacher } \\
\text { calls on me. }\end{array}$ & 98 & 1.54 & .66 & 93 & 1.52 & .65 & -.02 \\
\hline $\begin{array}{l}\text { I can listen when my teacher is } \\
\text { presenting a lesson. }\end{array}$ & 97 & 1.71 & .56 & 92 & 1.75 & .48 & +.04 \\
\hline $\begin{array}{l}\text { I can remember when class } \\
\text { projects are due. }\end{array}$ & 97 & 1.68 & .55 & 93 & 1.78 & .49 & +.10 \\
\hline I can follow classroom rules. & 97 & 1.72 & .47 & 93 & 1.67 & .54 & -.05 \\
\hline
\end{tabular}

Note. ${ }^{*} \mathrm{p}<.05 . * * \mathrm{p}<.01$. 


\section{Academic Self-Concept Subscale Findings By Sub-Characteristic}

In an effort to demonstrate the patterns that emerged from each group comparisons (total, year-by-year, and program-by-program) on the academic self-concept subscale of the Student Self-Concept Scale, the survey items were coded into the following three categories: (1) Sense of Self Items, (2) Behavioral Items, and (3) TaskOriented Items (Figure 10). The Sense of Self Items illustrated the manner in which students viewed themselves as learners in the classroom. The Behavioral Items denoted how the kids felt they were able to behave, both positively and negatively, in the academic environment. Lastly, the Task-Oriented Items demonstrated students' perceived abilities to complete tasks or duties within the school setting. Figure 10 shows the 22 confidence level statements and the 18 item importance statements as they were coded into each of the three categories. As was explained in Chapter 3: Methodology, the confidence level statements demonstrated the level of confidence the students had in their ability to perform certain behaviors, while the item importance statements measured the subjective task value that a behavior or personal attribute held for the student completing the survey (Gresham, Elliot, Evans-Fernandez, 1993). 
Figure 10

Student Self-Concept Scale Item Sub-Characteristic Codes

\begin{tabular}{|c|c|c|}
\hline Category & $\begin{array}{l}\text { SSCS Confidence Level } \\
\text { Statements }\end{array}$ & $\begin{array}{l}\text { SSCS Item Importance } \\
\text { Statements }\end{array}$ \\
\hline Sense of Self Items & $\begin{array}{l}\text { * I can read aloud in class } \\
\text { without feeling nervous. } \\
\text { * I can ask my teacher for help } \\
\text { without feeling ashamed or upset. } \\
\text { * I can go to the board to do work } \\
\text { when my teacher asks me to. } \\
\text { * I can laugh when I make silly } \\
\text { mistakes. } \\
\text { * I can speak in class when my } \\
\text { teacher calls on me. } \\
\text { * If I do my homework on time, } \\
\text { my parents will be proud of me. }\end{array}$ & $\begin{array}{l}\text { * I can read aloud in class } \\
\text { without feeling nervous. } \\
\text { * I can ask my teacher for help } \\
\text { without feeling ashamed or upset. } \\
\text { * I can go to the board to do work } \\
\text { when my teacher asks me to. } \\
\text { * I can laugh when I make silly } \\
\text { mistakes. } \\
\text { * I can speak in class when my } \\
\text { teacher calls on me. }\end{array}$ \\
\hline Behavioral Items & $\begin{array}{l}\text { * I can use a nice tone of voice in } \\
\text { classroom discussions with my } \\
\text { teacher. } \\
\text { * I can sit in class without } \\
\text { daydreaming during a lesson. } \\
\text { * I can sit at my desk for } 2 \\
\text { minutes without moving around } \\
\text { or fidgeting. } \\
\text { * I can listen to my teacher talk } \\
\text { about a subject for } 20 \text { minutes. } \\
\text { * I can ignore classmates when } \\
\text { they whisper or talk during class. } \\
\text { * I can listen when my teacher is } \\
\text { presenting a lesson. } \\
\text { * I can follow classroom rules. } \\
\text { * If I follow my teacher's } \\
\text { directions in class, I will do my } \\
\text { work correctly. } \\
\text { * If I ignore classmates who } \\
\text { whisper in class, I can complete } \\
\text { my work. }\end{array}$ & $\begin{array}{l}\text { * I can use a nice tone of voice in } \\
\text { classroom discussions with my } \\
\text { teacher. } \\
\text { * I can sit in class without } \\
\text { daydreaming during a lesson. } \\
\text { * I can sit at my desk for } 2 \\
\text { minutes without moving around } \\
\text { or fidgeting. } \\
\text { * I can listen to my teacher talk } \\
\text { about a subject for } 20 \text { minutes. } \\
\text { * I can ignore classmates when } \\
\text { they whisper or talk during class. } \\
\text { * I can listen when my teacher is } \\
\text { presenting a lesson. } \\
\text { * I can follow classroom rules. }\end{array}$ \\
\hline Task-Oriented Items & $\begin{array}{l}\text { * I can do my homework on time. } \\
\text { * I can finish my class work on } \\
\text { time. } \\
\text { * I can read instructions in a book } \\
\text { and follow them carefully. } \\
\text { * I can follow my teacher's } \\
\text { directions fordoing reading work. } \\
\text { *I can do math work without } \\
\text { help. } \\
\text { *I can remember when class } \\
\text { projects are due. } \\
\text { * If I finish my class work on } \\
\text { time I will get good grades in } \\
\text { school. }\end{array}$ & $\begin{array}{l}\text { * I can do my homework on time. } \\
\text { * I can finish my class work on } \\
\text { time. } \\
\text { * I can read instructions in a book } \\
\text { and follow them carefully. } \\
\text { * I can follow my teacher's } \\
\text { directions fordoing reading work. } \\
\text { * I can do math work without } \\
\text { help. } \\
\text { * I can remember when class } \\
\text { projects are due. }\end{array}$ \\
\hline
\end{tabular}


Tables 24 to 33 exhibit calculations of the number of higher mean responses given by each of the groups under study within the three categories (sense of self items, behavioral items, and task-oriented items) as calculated on the academic self-concept subscale (Tables 22 and 23, Appendix J). The tables were created to demonstrate the areas in which each group responded with a higher mean score, suggesting a greater level of academic self-concept. The patterns allow one to create assumptions about how each of the groups view themselves and their abilities within the school environment, and it illustrates the comparisons between the groupings (total, year-by-year, and program-by program) in a clear and concise format. The tables were created for both the 22-item confidence level academic self-concept subscale and the 18-item importance academic self-concept subscale.

In tallying the total confidence level mean scores on the academic self-concept subscale (Table 12), the total ESL students and the total NAC students did not demonstrate any overall mean difference between the two programs under study. However, in counting the number of greater mean confidence level scores according to the three categories (Table 24), the total NAC program demonstrate a higher mean score on 13 of the 22 total items, while the ESL group only felt stronger on nine of the 22 statements. Both groups had an equal number of higher scores in the area of sense of self, and, similarly, the total groups were virtually equal when counting the number of behavioral items. The major difference occurred when viewing the task-oriented items, in that the NAC students felt stronger on five of the items, while the ESL group felt more capable on only two of the items. These findings suggest that, though not significantly 
different, the NAC students felt somewhat more able to complete tasks than the ESL group, yet the findings were minimal.

Table 24

Academic Self-Concept Confidence Level Subscale Characteristic Patterns - Total ESL Student Participants and Total NAC Student Participants (Years 1 and 2)

\begin{tabular}{llll}
\hline & $\begin{array}{l}\text { Item Total } \\
(N=22)\end{array}$ & $\begin{array}{l}\text { Total ESL } \\
(N=98)\end{array}$ & $\begin{array}{l}\text { Total NAC } \\
(N=94)\end{array}$ \\
\hline Sense of Self & $n$ & \multicolumn{2}{c}{ Higher Mean Total } \\
$\begin{array}{llll}\text { Behavioral } \\
\text { Task-Oriented }\end{array}$ & 6 & 3 & 3 \\
\cline { 2 - 4 } Total & 9 & 4 & 5 \\
\hline
\end{tabular}

After analyzing the total item importance mean scores on the academic selfconcept subscale (Table 13), the students again did not demonstrate any overall mean difference between the two programs under study. The findings further illustrated, though, that, within the three categories, the total ESL program had a greater mean score on 12 of the 18 total items, while the NAC group had a higher mean score on just five of the 18 statements (Table 25). Both groups had an equal amount of higher mean scores on the behavioral items, yet the significant difference in mean scores was demonstrated on the sense of self statements, in that the total ESL group scored higher than the total NAC group on all 5 of 5 items. Also, the total ESL group had four higher mean scores on the task-oriented statements, as compared to the two higher scores possessed by the total NAC group. The findings imply that the ESL group felt it was more important to have a 
strong academic sense of self and to be able to complete their educational duties than the total NAC group, though both groups viewed the behavioral items evenly.

Table 25

Academic Self-Concept Item Importance Subscale Characteristic Patterns - Total ESL Student Participants and Total NAC Student Participants (Years 1 and 2)

\begin{tabular}{llll}
\hline & $\begin{array}{l}\text { Item Total } \\
(N=18)\end{array}$ & $\begin{array}{l}\text { Total ESL } \\
(N=98)\end{array}$ & $\begin{array}{l}\text { Total NAC } \\
(N=94)\end{array}$ \\
\hline Sense of Self & $n$ & \multicolumn{2}{c}{ Higher Mean Total } \\
Behavioral & 5 & 5 & 0 \\
Task-Oriented & 7 & 3 & 3 \\
\cline { 2 - 4 } Total & 6 & 4 & 2 \\
\hline
\end{tabular}

Note. One behavioral item showed no mean difference.

The total confidence level mean scores on the academic self-concept subscale (Table 14), demonstrated that the NAC-Year $1(M=1.59)$ students had a higher overall mean score than the ESL-Year $1(M=1.48)$ students, yet the difference was not statistically significant. In counting the number of greater mean confidence level scores, the NAC-Year 1 program demonstrate a higher mean score on 17 of the 22 total items, while the ESL-Year 1 group only had greater mean scores on five of the 22 statements. The NAC-Year 1 had greater mean scores on five of the sense of self items (Table 26), compared to one greater mean score for the ESL Year-1 group, and, similarly, the NAC group felt stronger on six behavioral items, while the ESL group solely felt more confident on two items. Lastly, in terms of task-oriented items the NAC-Year 1 students had higher mean scores on six items while the ESL-Year 1 youth only scored higher on 
one task-oriented item. These findings suggest that the NAC-Year 1 group was more confident academically than the ESL-Year 1 group in each of the three categories, as well as the subscale total.

Table 26

Student Self-Concept Scale Confidence Level Analysis - ESL-Year 1 Student Participants and NAC-Year 1 Student Participants

\begin{tabular}{llll}
\hline & $\begin{array}{l}\text { Item Total } \\
(N=22)\end{array}$ & $\begin{array}{l}\text { ESL } \\
(N=49)\end{array}$ & $\begin{array}{l}\text { NAC } \\
(N=51)\end{array}$ \\
\hline Sense of Self & $n$ & \multicolumn{2}{c}{ Higher Mean Total } \\
Behavioral & 6 & 1 & 5 \\
Task-Oriented & 9 & 2 & 6 \\
\cline { 2 - 4 } Total & 7 & 1 & 6 \\
\hline
\end{tabular}

Note. One behavioral item showed no mean difference.

The total item importance mean scores on the academic self-concept subscale (Table 15) illustrated a small overall mean difference between the NAC-Year 1 total mean score $(M=1.54)$ and the ESL-Year 1 total mean score $(M=1.52)$. The findings further demonstrated, that the total ESL-Year 1 program had a greater mean score on 10 of the 18 total items, while the NAC-Year 1 group had a higher mean score on eight of the 18 statements. As seen on Table 27, the ESL-Year 1 youth had high mean scores on sense of self items ( 4 of 5 total) and on behavioral items ( 5 of 7 total), while the NACYear 1 group had more high mean scores when viewing the task-oriented items ( 5 of 6 total). The findings suggest that the ESL-Year 1 group felt it was more important to have a strong academic sense of self and to behave academically, while the NAC-Year 1 group 
found it far more important to be able to complete academic tasks than the ESL-Year 1 students.

Table 27

Student Self-Concept Scale Item Importance Analysis - ESL-Year 1 Student Participants and NAC-Year 1 Student Participants

\begin{tabular}{llll}
\hline & $\begin{array}{l}\text { Item Total } \\
(N=18)\end{array}$ & $\begin{array}{l}\text { ESL } \\
(N=49)\end{array}$ & $\begin{array}{l}\text { NAC } \\
(N=51)\end{array}$ \\
\hline $\begin{array}{llll}\text { Sense of Self } \\
\text { Behavioral }\end{array}$ & $n$ & \multicolumn{2}{c}{ Higher Mean Total } \\
Task-Oriented & 7 & 4 & 1 \\
\cline { 2 - 4 } Total & 6 & 5 & 2 \\
\hline
\end{tabular}

The total confidence level mean findings on the academic self-concept subscale (Table 16) showed that the ESL-Year $2(M=1.55)$ students had a higher overall mean score than the NAC-Year $2(M=1.44)$ students, and the difference was marginally statistically significant. In counting the number of greater mean confidence level scores (Table 28), the ESL-Year 2 program demonstrate a higher mean score on 16 of the 22 total items, while the NAC-Year 2 group only had greater mean scores on five of the 22 statements. The ESL-Year 2 had greater mean scores on three of the sense of self items, compared to two greater mean score for the NAC-Year 2 group, and the ESL-Year 2 group felt stronger on eight behavioral items, while the NAC-Year 2 group solely felt more confident on one item. Finally, in terms of task-oriented items the ESL-Year 2 students had higher mean scores on five items while the NAC-Year 2 youth only scored higher on two task-oriented items. These findings suggest that the ESL-Year 2 group was 
far more confident academically than the NAC-Year 2 group in each of the three categories, as well as the subscale total.

Table 28

Student Self-Concept Scale Confidence Level Analysis - ESL-Year 2 Student Participants and NAC-Year 2 Student Participants

\begin{tabular}{llll}
\hline & $\begin{array}{l}\text { Item Total } \\
(N=22)\end{array}$ & $\begin{array}{l}\text { ESL } \\
(N=49)\end{array}$ & $\begin{array}{l}\text { NAC } \\
(N=43)\end{array}$ \\
\hline Sense of Self & $n$ & \multicolumn{2}{c}{ Higher Mean Total } \\
Behavioral & 6 & 3 & 2 \\
Task-Oriented & 9 & 8 & 1 \\
\cline { 2 - 4 } Total & 7 & 5 & 2 \\
\hline
\end{tabular}

Note. One sense of self item showed no mean difference.

In analyzing the total item importance mean scores on the academic self-concept subscale (Table 17), the students demonstrated a small overall mean difference between the ESL-Year 2 total mean score $(M=1.51)$ and the NAC-Year 2 total mean score $(M$ $=1.49$ ). The sub-characteristic findings further demonstrated (Table 29), that the total ESL-Year 2 program had a greater mean score on 13 of the 18 total items, while the NAC-Year 2 group had a higher mean score on just five of the 18 statements. The ESLYear 2 youth had high mean scores on all five sense of self items and on five of seven total behavioral items, while both groups had the same number of higher mean scores on the task-oriented items. The findings suggest that, like the year one group, the ESL-Year 2 group felt it was more important to have a strong academic sense of self and to behave 
academically, while both groups found it important to be able to complete academic tasks.

Table 29

Student Self-Concept Scale Item Importance Analysis - ESL-Year 2 Student Participants and NAC-Year 2 Student Participants

\begin{tabular}{llll}
\hline & $\begin{array}{l}\text { Item Total } \\
(N=18)\end{array}$ & $\begin{array}{l}\text { ESL } \\
(N=49)\end{array}$ & $\begin{array}{l}\text { NAC } \\
(N=43)\end{array}$ \\
\hline Sense of Self & $n$ & \multicolumn{2}{c}{ Higher Mean Total } \\
Behavioral & 5 & 5 & 0 \\
Task-Oriented & 7 & 5 & 2 \\
\cline { 2 - 4 } Total & 6 & 3 & 3 \\
\hline
\end{tabular}

The total confidence level mean scores on the academic self-concept subscale (Table 18) findings showed that the ESL-Year $1(M=1.48)$ students had a lower overall mean score than the ESL-Year $2(M=1.55)$ students, yet the difference was not statistically different. In counting the number of greater mean confidence level scores (Table 30), the ESL-Year 2 program demonstrate a higher mean score on 16 of the 22 total items, while the ESL-Year 1 group only had greater mean scores on six of the 22 statements. The ESL-Year 2 had the same mean scores on the sense of self items as the ESL-Year 1 youth, however the ESL-Year 2 group felt far stronger than the ESL-Year 1 group on the behavioral items in that the ESL-Year 2 group had higher mean scores on eight items and the ESL-Year 1 group solely felt more confident on one item. Finally, in terms of task-oriented items the ESL-Year 2 students again had higher mean scores on 
five items while the ESL-Year 1 youth only scored higher on two task-oriented items. These findings suggest that the ESL-Year 2 group was also far more confident academically than the ESL-Year 1 in terms of behavioral items and task-oriented items, as well as the subscale total, yet both had similar mean results when discussing their sense of self.

Table 30

Student Self-Concept Scale Confidence Level Analysis - ESL-Year 1 Student Participants and ESL-Year 2 Student Participants

\begin{tabular}{llll}
\hline & $\begin{array}{l}\text { Item Total } \\
(N=22)\end{array}$ & $\begin{array}{l}\text { ESL-Year 1 } \\
(N=49)\end{array}$ & $\begin{array}{l}\text { ESL-Year 2 } \\
(N=49)\end{array}$ \\
\hline & $n$ & \multicolumn{2}{c}{ Higher Mean Total } \\
\hline $\begin{array}{l}\text { Sense of Self } \\
\text { Behavioral }\end{array}$ & 6 & 3 & 3 \\
Task-Oriented & 9 & 1 & 8 \\
\cline { 2 - 4 } Total & 7 & 2 & 5 \\
\hline
\end{tabular}

The total item importance mean scores on the academic self-concept subscale (Table 19) illustrated that the ESL-Year $1(M=1.52)$ students had a higher overall mean score than the ESL-Year $2(M=1.51)$ students. In counting the number of greater mean confidence level scores (Table 31), the ESL-Year 1 program demonstrate a higher mean score on 9 of the 18 total items, while the ESL-Year 2 group had greater mean scores on eight of the 18 statements. The ESL-Year 2 had greater mean scores on the sense of self items ( 2 items) than the ESL-Year 1 group ( 3 items), yet they were virtually the same amount. The ESL-Year 1 group felt stronger on five behavioral items, while the ESL- 
Year 2 group solely felt more confident on two items. Finally, in terms of task-oriented items the ESL-Year 1 students had higher mean scores on two items while the ESL-Year 2 youth scored higher on three task-oriented items. These findings suggest that, despite the slightly different mean scores on the behavioral items, the ESL-Year 1 and the ESLYear 2 groups answered very similarly when considering the importance they placed on the academic self-concept items.

Table 31

Student Self-Concept Scale Item Importance Analysis - ESL-Year 1 Student Participants and ESL-Year 2 Student Participants

\begin{tabular}{llll}
\hline & $\begin{array}{l}\text { Item Total } \\
(N=18)\end{array}$ & $\begin{array}{l}\text { ESL-Year 1 } \\
(N=49)\end{array}$ & $\begin{array}{l}\text { ESL-Year 2 } \\
(N=49)\end{array}$ \\
\hline & $n$ & \multicolumn{2}{c}{ Higher Mean Total } \\
\hline $\begin{array}{l}\text { Sense of Self } \\
\text { Behavioral }\end{array}$ & 5 & 2 & 3 \\
Task-Oriented & 7 & 5 & 2 \\
\cline { 2 - 4 } Total & 6 & 2 & 3 \\
\hline
\end{tabular}

Note. One task-oriented item showed no mean difference

The total confidence level mean scores on the academic self-concept subscale (Table 20) demonstrated that the NAC-Year $1(M=1.59)$ students had a higher overall mean score than the NAC-Year $2(M=1.44)$ students, and the difference was moderately statistically significant. In calculating the number of greater mean confidence level scores (Table 32), the NAC-Year 1 program demonstrate a higher mean score on 15 of the 22 total items, while the NAC-Year 2 group only had greater mean scores on five of the 22 statements (two behavioral items showed no mean difference). The NAC-Year 1 had 
greater mean scores on six of the sense of self items, compared to zero greater mean scores for the NAC-Year 2 group, and the NAC-Year 1 group felt stronger on five behavioral items, while the NAC-Year 2 group solely felt more confident on one item. Lastly, when viewing task-oriented items, the NAC-Year 1 students had higher mean scores on four items while the NAC-Year 2 youth scored higher on three task-oriented items. These findings suggest that the NAC-Year 1 group was significantly more confident academically than the NAC-Year 2 group in each of the three categories, as well as the subscale total.

Table 32

Student Self-Concept Scale Confidence Level Analysis - NAC-Year 1 Student Participants and NAC-Year 2 Student Participants

\begin{tabular}{llll}
\hline & $\begin{array}{l}\text { Item Total } \\
(N=22)\end{array}$ & $\begin{array}{l}\text { NAC-Year 1 } \\
(N=51)\end{array}$ & $\begin{array}{l}\text { NAC-Year 2 } \\
(N=43)\end{array}$ \\
\hline Sense of Self & $n$ & \multicolumn{2}{c}{ Higher Mean Total } \\
Behavioral & 6 & 6 & 0 \\
Task-Oriented & 9 & 5 & 2 \\
\cline { 2 - 4 } Total & 7 & 4 & 3 \\
\hline
\end{tabular}

Note. Two behavioral items showed no mean difference.

The total item importance mean scores on the academic self-concept subscale (Table 21) demonstrated that the NAC-Year 1 total mean score $(M=1.54)$ was higher than the NAC-Year 2 total mean score $(M=1.49)$, though the results were not statistically significant. The findings further demonstrated that the NAC-Year 1 program had an equal amount of higher mean score as the NAC-Year 2 (9 of 18 statement each). As seen on 
Table 33, the NAC-Year 1 youth had high mean scores on sense of self items (3 of 5 total) and on behavioral items (5 of 7 total), while the NAC-Year 2 group had more high mean scores when viewing the task-oriented items ( 5 of 6 total). The findings suggest that the NAC-Year 1 group felt it was more important to have a strong academic sense of self and to behave academically, while the NAC-Year 2 group found it far more important to be able to complete academic tasks than the NAC-Year 1 students, though the groups were fairly similar in the importance they placed on the academic self-concept subset items.

Table 33

Student Self-Concept Scale Item Importance Analysis - NAC-Year 1 Student Participants and NAC-Year 2 Student Participants

\begin{tabular}{llll}
\hline & $\begin{array}{l}\text { Item Total } \\
(N=18)\end{array}$ & $\begin{array}{l}\text { NAC-Year 1 } \\
(N=51)\end{array}$ & $\begin{array}{l}\text { NAC-Year 2 } \\
(N=43)\end{array}$ \\
\hline Sense of Self & $n$ & \multicolumn{2}{c}{ Higher Mean Total } \\
Behavioral & 5 & 3 & 2 \\
Task-Oriented & 7 & 5 & 2 \\
\cline { 2 - 4 } Total & 6 & 1 & 5 \\
\hline
\end{tabular}

\section{Classroom Observation Findings}

As was discussed in detail in Chapter 3, Methodology, an observation protocol matrix entitled the Classroom Observation Indicator Matrix (Appendix L) was used to categorize and analyze the observation data. The researcher developed this tool of analysis to assist in categorizing the observation data into three major sections and 14 
subsections, each of which was acquired directly from the Classroom Observation Protocol used during the classroom observations. The three major sections found in the Classroom Observation Indicator Matrix identified effective English learner instruction, culturally relevant pedagogical practices, and the observed pedagogical orientations of teachers, each of which were discerned during the class observations. Each subsection of the matrix was further partitioned into a series of indicators, in order to determine the strength of each major section according to the observations. Although the majority of the six total cases (three NAC cases and three ESL cases) were observed four times each, three observations from each of the cases were analyzed utilizing the Classroom Observation Indicator Matrix. The determination to solely analyze three cases was made by the researcher as one of the cases (NAC \#1) was only observed three times due to scheduling difficulties and three other cases (ESL \#2, ESL \#3, and NAC \#3) each had one incomplete observation due to unforeseen circumstances that altered the typical class day. The observations were coded numbers one through three according to the date in which the observation took place, and, therefore, the first complete observation was number one, the second complete observation was number two, and the third complete observation was number three. The three completed Classroom Observation Indicator Matrices can be found in Appendix M.

The completed Classroom Observation Indicator Matrices (Appendix M) were used to formulate tables and figures demonstrating the findings within the three major sections of the Classroom Observation Protocol, effective English learner instruction, culturally relevant pedagogical practices, and the observed pedagogical orientations of teachers, each of which were detected during the class observations. The indicators used 
within each of the subsections in order to evaluate evidence of effective English learner instruction and culturally relevant pedagogy were tallied to determine the level of effective English learner instruction and culturally relevant pedagogy that was present during each of the three observations. This data was then disseminated at both the individual case level and at the combined program level. The figure developed to determine the teachers' pedagogical orientations during each of the three observations was developed differently, as the indicators were not equally weighted in the same manner as the prior two major sections. The indicators of teachers' pedagogical orientation placed the teachers on a continuum ranging from transmission-oriented practices to transformative practices, and, therefore, were disseminated in a manner that demonstrated where the teachers' practices were most accurately positioned during each observation. The discussion about the following tables and figures explains in more detail the findings from each of the classroom observations.

The first major section of the Classroom Observation Indicator Matrix focused on determining the levels of effective English learner instruction within each of the two programs (NAC and ESL) under examination, as they were ascertained during the three observations of each individual case. Figure 11 illustrates the indicators of effective English learner instruction apparent during the three observations, the numbers $(1,2$, or 3) denoting the indicators observed during each observation.

As is seen in Figure 11, the indicators of effective English learner instruction illustrate that for each of the cases the teachers used a number of specific strategies in ensuring their students were able to access the curricula and build academic vocabulary in a collaborative and supportive environment. Students in both programs were given 
opportunities to practice and apply their new understandings, and they were frequently assessed orally or in writing to give the instructors ongoing feedback about their students' progress. Overall, the NAC cases demonstrated more consistency in the number of effective English learner instructional indicators present at each observation, yet, with the exception of ESL Case \#1, the differences were somewhat slight between the two programs. It is notable that while five of the six cases possessed teachers that had been highly trained to instruct English learners and held specific credentials from the California State Commission of Teacher Credentialing, the primary instructor in ESL Case \#1 was an assistant to the classroom teacher. ESL Case \#1 was an ESL 1/2 and ESL $3 / 4$ combination class, and, therefore, the credentialed teacher in the class taught the ESL 3/4 group in one part of the classroom, while the assistant taught the ESL 1/2 course in an opposite corner of the room. For this reason, it is understandable that this case demonstrated the lowest average number of indicators of effective instruction for English learners, as the assistant had not received the same training or professional development as the classroom teacher.

In analyzing the overall pattern of indicators of effective English learner instruction, as seen in Figure 11, it became quite apparent that both the NAC and the ESL programs displayed similar strengths and weaknesses throughout the observations. These strengths and weaknesses were determined by either the appearance or the absence of particular indicators of effective English learners instruction, as determined during the classroom observations. Both programs appeared to be exceedingly strong in terms of the following indicators of effective English learner instruction:

- Utilizing appropriate curricula and materials for students' proficiency level 
- Linking instruction to students' prior experiences and lessons

- Directly teaching key vocabulary and providing word banks

- Providing clear expectations for students

- Checking for understanding

- Using visuals and modeling frequently during lessons

- Creating frequent interactions between classroom constituents

- Providing opportunities for students to practice new concepts

- Assessing students through oral questions and student products

- Providing feedback to students

On the contrary, the two programs demonstrated overt weaknesses in several areas according to the Classroom Observation Matrix. The weaknesses were shown in the following areas:

- Using realia during the lessons

- Utilizing primary language support

- Using interactive demonstrations

- Using graphic organizers in the lessons

- Incorporating a variety of questions into the daily activities

- Utilizing manipulatives to support students

- Differentiating instruction based on the need of students

- Assessing utilizing exit slips

Issues related to the above strengths and weaknesses in effective classroom instruction will be further discussed in Chapter 5: Implications. 
Figure 11

\begin{tabular}{|c|c|c|c|c|c|c|}
\hline & $\begin{array}{c}\text { NAC } \\
\# 1\end{array}$ & $\begin{array}{c}\text { NAC } \\
\# 2\end{array}$ & $\begin{array}{c}\text { NAC } \\
\# 3\end{array}$ & $\begin{array}{l}\text { ESL } \\
\# 1^{\text {a }}\end{array}$ & $\begin{array}{c}\text { ESL } \\
\# 2\end{array}$ & $\begin{array}{c}\text { ESL } \\
\# 3\end{array}$ \\
\hline \multicolumn{7}{|l|}{ Curriculum } \\
\hline Appropriate for language proficiency & $1,2,3$ & $1,2,3$ & $1,2,3$ & $1,2,3$ & $1,2,3$ & $1,2,3$ \\
\hline Materials used to supplement language acquisition & $1,2,3$ & $1,2,3$ & $1,2,3$ & $1,2,3$ & $1,2,3$ & $1,2,3$ \\
\hline \multicolumn{7}{|l|}{ Instruction } \\
\hline Linked to student's experiences/prior knowledge & $1,2,3$ & $1,2,3$ & $1,2,3$ & 1,2 & $1,2,3$ & $1,2,3$ \\
\hline Connections made between current and prior lessons & $1,2,3$ & $1,2,3$ & $1,2,3$ & 1,2 & $1,2,3$ & $1,2,3$ \\
\hline Peer interaction used to activate schema & $1,2,3$ & $1,2,3$ & $1,2,3$ & & 3 & $1,2,3$ \\
\hline \multicolumn{7}{|l|}{ Key Vocabulary } \\
\hline Academic/content vocabulary introduced in lesson & 1,3 & $1,2,3$ & $1,2,3$ & $1,2,3$ & 1,2 & 1,2 \\
\hline Vocabulary taught literally & 1,2 & $1,2,3$ & $1,2,3$ & $1,2,3$ & 1,3 & 1 \\
\hline Vocabulary taught contextually & 1,3 & & $1,2,3$ & & 2 & $1,2,3$ \\
\hline Strategies used to support students' understanding & $1,2,3$ & $1,2,3$ & $1,2,3$ & $1,2,3$ & $1,2,3$ & $1,2,3$ \\
\hline \multicolumn{7}{|l|}{ Comprehensible Input } \\
\hline Suitable pacing for language proficiency level & $1,2,3$ & $1,2,3$ & $1,2,3$ & $1,2,3$ & & $1,2,3$ \\
\hline Suitable speech for language proficiency level & $1,2,3$ & $1,2,3$ & $1,2,3$ & $1,2,3$ & & $1,2,3$ \\
\hline Clear expectations for students & $1,2,3$ & $1,2,3$ & $1,2,3$ & 2,3 & $1,2,3$ & $1,2,3$ \\
\hline Checks for understanding & $1,2,3$ & 1,3 & $1,2,3$ & $1,2,3$ & $1,2,3$ & $1,2,3$ \\
\hline Visuals used during lesson & 1,2 & 1,3 & $1,2,3$ & $1,2,3$ & $1,2,3$ & $1,2,3$ \\
\hline Realia used during lesson & & & 1 & & 2 & 1 \\
\hline Modeling used during lesson & $1,2,3$ & $1,2,3$ & $1,2,3$ & 1,3 & $1,2,3$ & $1,2,3$ \\
\hline Gestures/TPR used during lesson & 3 & 1,2 & $1,2,3$ & 1,2 & 1 & 1,2 \\
\hline Primary language support used during lesson & $1,2,3$ & & 1 & 2 & & $1,2,3$ \\
\hline Interactive demonstrations used during lesson & $1,2,3$ & 2,3 & & & & \\
\hline Partner/group activities present during lesson & $1,2,3$ & & $1,2,3$ & & $1,2,3$ & $1,2,3$ \\
\hline \multicolumn{7}{|l|}{ Strategies } \\
\hline Think-alouds used during lesson & $1,2,3$ & $1,2,3$ & $1,2,3$ & 1,3 & 1,3 & $1,2,3$ \\
\hline Word banks are present in lesson & $1,2,3$ & 1,3 & $1,2,3$ & $1,2,3$ & $1,2,3$ & 1 \\
\hline Graphic organizer used to support teaching & & & $1,2,3$ & 1 & 2 & $1,2,3$ \\
\hline A variety of question types used during lesson & & 2 & $1,2,3$ & & & $1,2,3$ \\
\hline Evidence of a gradual release of responsibility & $1,2,3$ & 1 & $1,2,3$ & & $1,2,3$ & $1,2,3$ \\
\hline \multicolumn{7}{|l|}{ Interactions } \\
\hline Frequent interactions between teachers and students & $1,2,3$ & $1,2,3$ & $1,2,3$ & $1,2,3$ & $1,2,3$ & $1,2,3$ \\
\hline Frequent interactions between students & $1,2,3$ & 1,2 & $1,2,3$ & 2 & $1,2,3$ & $1,2,3$ \\
\hline Students provided sufficient "wait time" & $1,2,3$ & $1,2,3$ & $1,2,3$ & $1,2,3$ & 1 & $1,2,3$ \\
\hline \multicolumn{7}{|l|}{ Practice/Application } \\
\hline Students given opportunities to practice new concept & $1,2,3$ & $1,2,3$ & $1,2,3$ & 1,3 & 2,3 & $1,2,3$ \\
\hline Activities integrate all language domains & $1,2,3$ & & $1,2,3$ & & 2 & $1,2,3$ \\
\hline Oral language opportunities included in lesson & $1,2,3$ & $1,2,3$ & $1,2,3$ & & $1,2,3$ & $1,2,3$ \\
\hline Charts used to support students & $1,2,3$ & $1,2,3$ & $1,2,3$ & & 1,3 & \\
\hline \multicolumn{7}{|l|}{ Manipulatives used to support students } \\
\hline Visuals used to support students & $1,2,3$ & 1 & $1,2,3$ & $1,2,3$ & $1,2,3$ & $1,2,3$ \\
\hline \multicolumn{7}{|l|}{ Lesson Review/Assessment } \\
\hline Differentiated instruction based on needs of students & & & & 2 & & $1,2,3$ \\
\hline Teacher assesses students through oral questions & $1,2,3$ & $1,2,3$ & $1,2,3$ & $1,2,3$ & $1,2,3$ & $1,2,3$ \\
\hline Teacher assesses students through exit slips & & & & & & \\
\hline
\end{tabular}




\begin{tabular}{|l|c|c|c|c|c|c|}
\hline Teacher assesses students through student product & $1,2,3$ & $1,2,3$ & $1,2,3$ & 1,3 & $1,2,3$ & $1,2,3$ \\
\hline Teacher assess students through tests/quizzes & $1,2,3$ & 1,3 & 1 & $1,2,3$ & 1 & 1 \\
\hline Teacher provides feedback to students & $1,2,3$ & 2,3 & $1,2,3$ & $1,2,3$ & $1,2,3$ & $1,2,3$ \\
\hline
\end{tabular}

Note. 1 = Observation \#1;2 = Observation \#2;3 = Observation \#3, ${ }^{\mathrm{a}}$ ESL $1 / 2$ students instructed in a small group conducted by teacher's aide.

The number of indicators of effective English learner instruction present for each observation was calculated in order to establish which of the three cases within each of the two overall programs more effectively utilized such strategies with their students, as demonstrated on Table 34 and in Figure 12. The calculations illustrate the program that incorporated a greater amount of such strategies, therefore ensuring a higher level of comprehensible input (Krashen, 1992) present in the instruction of the immigrant youth within their classes. Of the 40 total indicators of effective English learner instruction, each program averaged between 22 and 34 indicators of effective instruction throughout the three observations. Three of the cases (NAC \#1, NAC \#3, and ESL \#3) each averaged over 30 indicators during the three observations, demonstrating the highest level of effective instruction for emergent English speakers within the two programs under study.

Table 34

Number of Effective English Learner Instructional Indicators Recorded During Individual Case Observations

\begin{tabular}{llllll}
\hline Case & & & & & \\
Study \# & Observation \#1 & Observation \#2 & Observation \#3 & Average \# & Total \\
\hline NAC \#1 & 34 & 31 & 32 & 32 & 40 \\
NAC \#2 & 27 & 25 & 26 & 26 & 40 \\
NAC \#3 & 35 & 33 & 33 & 34 & 40 \\
ESL \#1 $^{\text {b }}$ & 23 & 23 & 21 & 22 & 40 \\
ESL \#2 & 25 & 25 & 24 & 25 & 40 \\
ESL \#3 & 35 & 32 & 30 & 32 & 40 \\
\hline
\end{tabular}

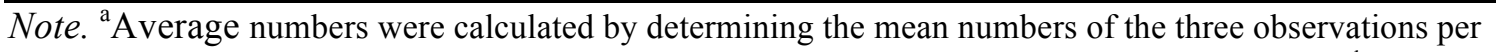
individual case and the calculations were additionally rounded to the nearest whole number, ${ }^{b}$ ESL $1 / 2$ students taught by teacher's aide 
Figure 12

Number of Effective English Learner Instructional Indicators Recorded During Individual Case Observations

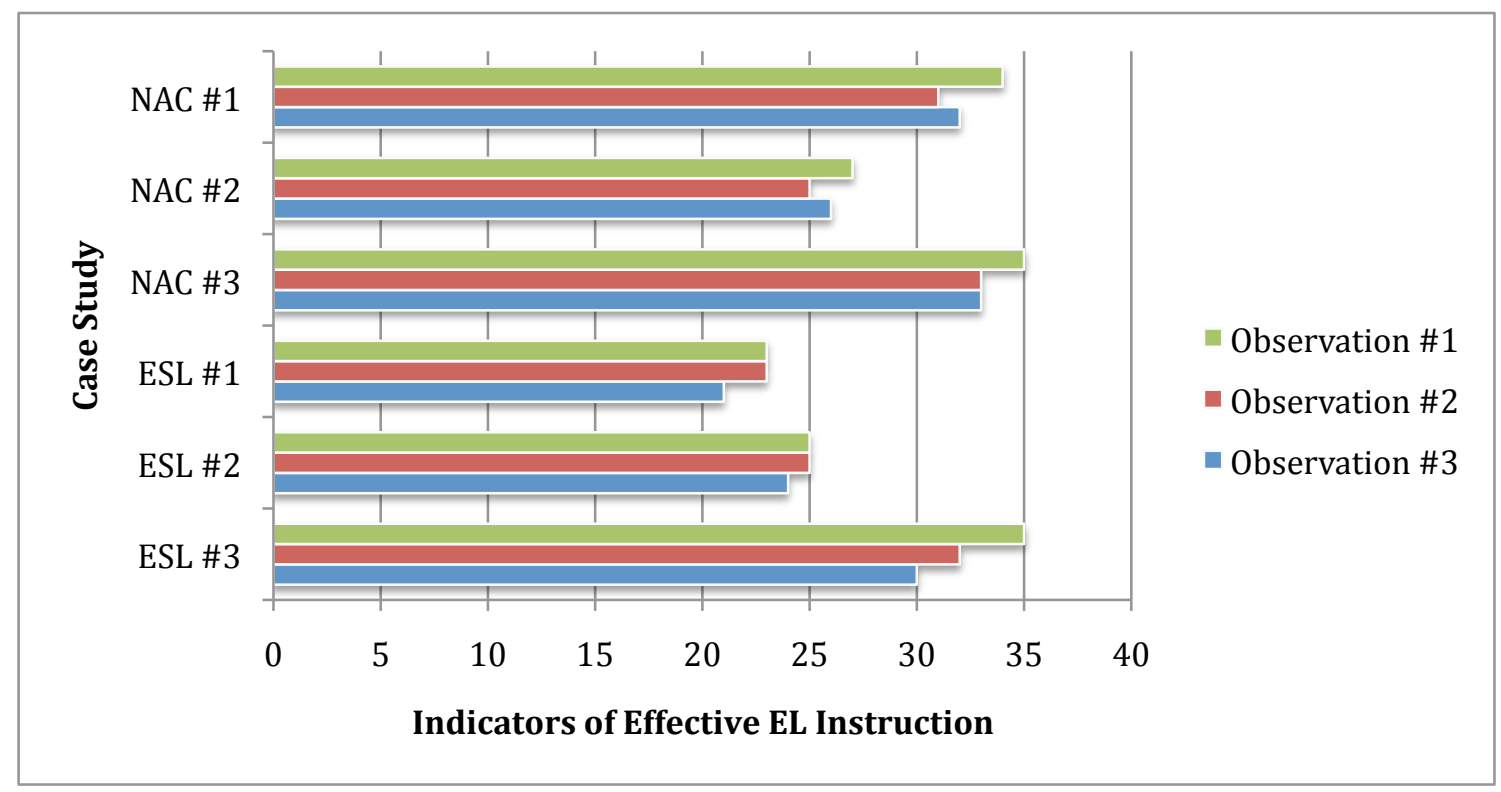

The three total numbers of indicators demarcating effective English learner instruction were averaged for each of the three individual cases to demonstrate the overall mean number of indicators found in each of the two programs under study (NAC and ESL). The totals, as are seen in Table 35 and in Figure 13, allow one to compare the NAC and ESL programs in terms of the one that demonstrates a learning environment more conducive to the needs of English learners, in that more language supports and strategies are provided in said program. Of the 40 total indicators of effective English learner instruction, the NAC program averaged 31 indicators over the three observations, while the ESL program averaged 27 indicators over the same time frame. The averages, as seen in Table 35 and in Figure 13, indicate that the NAC program incorporated more effective English learner strategies into daily instruction and class activities than the ESL program. 
Table 35

Mean Number of Effective English Learner Instructional Indicators Recorded During Program Observations

\begin{tabular}{llllll} 
Program $^{\mathrm{a}}$ & Observation \#1 & Observation \#2 & Observation \#3 & Average $\#^{\mathrm{b}}$ & Total \\
\hline NAC & 32 & 30 & 30 & 31 & 40 \\
ESL & 28 & 27 & 25 & 27 & 40 \\
\hline
\end{tabular}

Note. ${ }^{\mathrm{a}}$ Mean numbers determined by averaging the results within the three NAC cases and the three ESL cases, ${ }^{\mathrm{b}}$ Average numbers were calculated by determining the mean numbers of the three observations per program and the calculations were additionally rounded to the nearest whole number

Figure 13

Mean Number of Effective English Learner Instructional Indicators Recorded During Program Observations

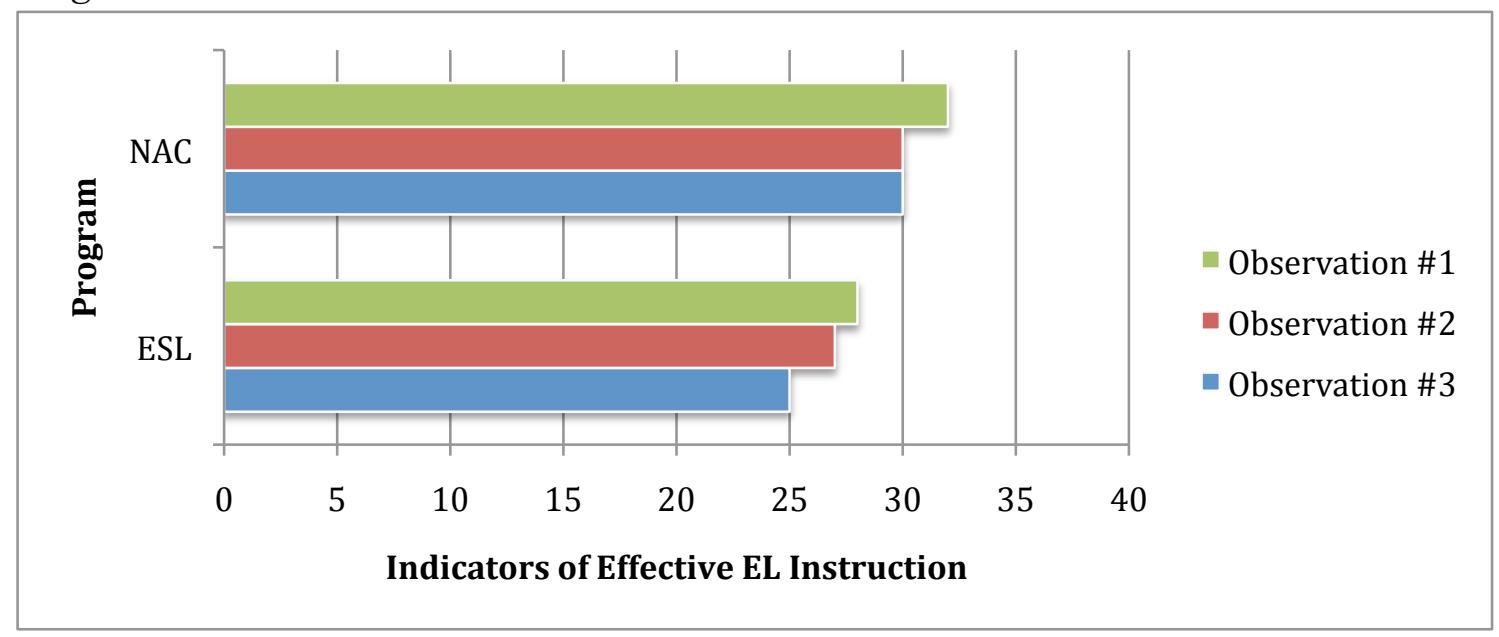

The second major section of the Classroom Observation Indicator Matrix centered on determining the numbers of culturally relevant pedagogical indicators found within each of the two programs under study, which occurred during the three observations of each individual case. Figure 12 illustrates the indicators of culturally relevant pedagogical indicators apparent during the three observations, the numbers $(1,2$, or 3$)$ denoting the indicators observed during each observation. 
As is seen in Figure 14, the culturally relevant pedagogical indicators suggest that for each of the cases the teachers used a number of specific strategies to make certain their students had a culturally congruent experience in their U.S. classroom. Teachers incorporated materials and activities that reflected students' home cultures, as well as those of other societies around the globe, and a few utilized flexible groupings and cooperative learning to provide voice to their students and to allow space for the students to learn from one another. Teachers also challenged their students through thoughprovoking activities and some additionally incorporated multiple perspectives into their daily lessons. The class environments and texts utilized in classes also demonstrated multiple cultures, languages, and genders in most of the cases under observation. Overall, the NAC cases again demonstrated more consistency in the number of culturally relevant pedagogical indicators present at each observation. In addition, ESL Case \#1 again demonstrated the fewest number of indicators, as the support teacher charged with instructing the ESL 1/2 students likely did not have the same training or knowledge of how to best incorporate such strategies into students' daily lessons and class environment as the teachers observed in the other five cases. 
Figure 14

Culturally Relevant Pedagogy Indicators Observed During First, Second, and Third Case Observations

\begin{tabular}{|l|c|c|c|c|c|c|}
\hline & $\begin{array}{c}\text { NAC } \\
\# 1\end{array}$ & $\begin{array}{c}\text { NAC } \\
\# 2\end{array}$ & $\begin{array}{c}\text { NAC } \\
\# 3\end{array}$ & $\begin{array}{c}\text { ESL } \\
\# 1^{\text {a }}\end{array}$ & $\begin{array}{c}\text { ESL } \\
\# 2\end{array}$ & $\begin{array}{c}\text { ESL } \\
\# 3\end{array}$ \\
\hline Materials & & & & & & \\
\hline Materials contain culturally relevant content & $1,2,3$ & $1,2,3$ & $1,2,3$ & & 1 & 2 \\
\hline Materials relevant to students' personal lives/cultures & $1,2,3$ & $1,2,3$ & $1,2,3$ & & 1 & 2 \\
\hline Materials are thought-provoking & 1 & $1,2,3$ & $1,2,3$ & & & 2,3 \\
\hline Materials are challenging and complex & $1,2,3$ & $1,2,3$ & $1,2,3$ & & 2,3 & $1,2,3$ \\
\hline Instruction & & & & & & \\
\hline Instruction includes flexible grouping & & & 2 & 2,3 & 3 & 2,3 \\
\hline Addresses varying linguistic backgrounds & 1 & $1,2,3$ & 1 & 2 & 1 & 1,2 \\
\hline Instruction includes cooperative learning & $1,2,3$ & 1,2 & $1,2,3$ & 2 & $1,2,3$ & $1,2,3$ \\
\hline Multiple perspectives on ideas are discussed & 1 & 1,2 & 2 & & 3 & 2,3 \\
\hline Environment & & & & & & \\
\hline Multiple cultures, languages, gender present on walls & 3 & $1,2,3$ & $1,2,3$ & $1,2,3$ & & \\
\hline Multiple cultures, languages, gender present in texts & $1,2,3$ & & $1,2,3$ & $1,2,3$ & & 2,3 \\
\hline
\end{tabular}

Note. $1=$ Observation \#1;2 = Observation \#2;3 = Observation \#3, ${ }^{\mathrm{a}}$ ESL $1 / 2$ students instructed in a small group conducted by teacher's aide.

As is demonstrated in Table 36 and in Figure 15, the number of indicators observed during each observation was tallied in order to establish which of the three cases within each of the two overall programs most effectively integrated culturally relevant pedagogical practices with their students. The culturally relevant pedagogical indicators were developed utilizing Gay's (2010) and Ladson-Billings' (1994) theoretical and practical concepts of culturally relevant pedagogy, as were discussed in Chapter 2, Literature Review. Of the 10 total indicators of culturally responsive practices, each program averaged between three and eight total indicators of culturally relevant practices throughout the three observations. Two of the NAC cases, NAC \#2 and NAC \#3, each averaged seven and eight average indicators respectively, demonstrating higher levels of culturally relevant practices within their classroom environments during the three class observations. Conversely, two of the ESL cases, ESL \#1 and ESL \#2, each only averaged 
three of ten indicators during the observations, demonstrating a low level of culturally relevant pedagogical occurrences within their academic settings during the three observations.

Table 36

Number of Culturally Relevant Pedagogical Indicators Recorded During Individual Case Observations

\begin{tabular}{lllcll}
\hline Case & & & & & \\
Study \# & Observation \#1 & Observation \#2 & Observation \#3 & ${\text { Average } \#^{\mathrm{b}}}$ & Total \\
\hline NAC \#1 & 8 & 5 & 6 & 6 & 10 \\
NAC \#2 & 8 & 8 & 6 & 7 & 10 \\
NAC \#3 & 8 & 9 & 7 & 8 & 10 \\
ESL \#1 & 2 & 5 & 3 & 3 & 10 \\
ESL \#2 & 4 & 2 & 4 & 3 & 10 \\
ESL \#3 & 4 & 9 & 6 & 6 & 10 \\
\hline
\end{tabular}

Note. ${ }^{\mathrm{a}}$ ESL $1 / 2$ students taught by teacher's aide, ${ }^{\mathrm{b}}$ Average numbers were calculated by determining the mean numbers of the three observations per program and the calculations were additionally rounded to the nearest whole number

Figure 15

Number of Culturally Relevant Pedagogical Indicators Recorded During Individual Case Observations

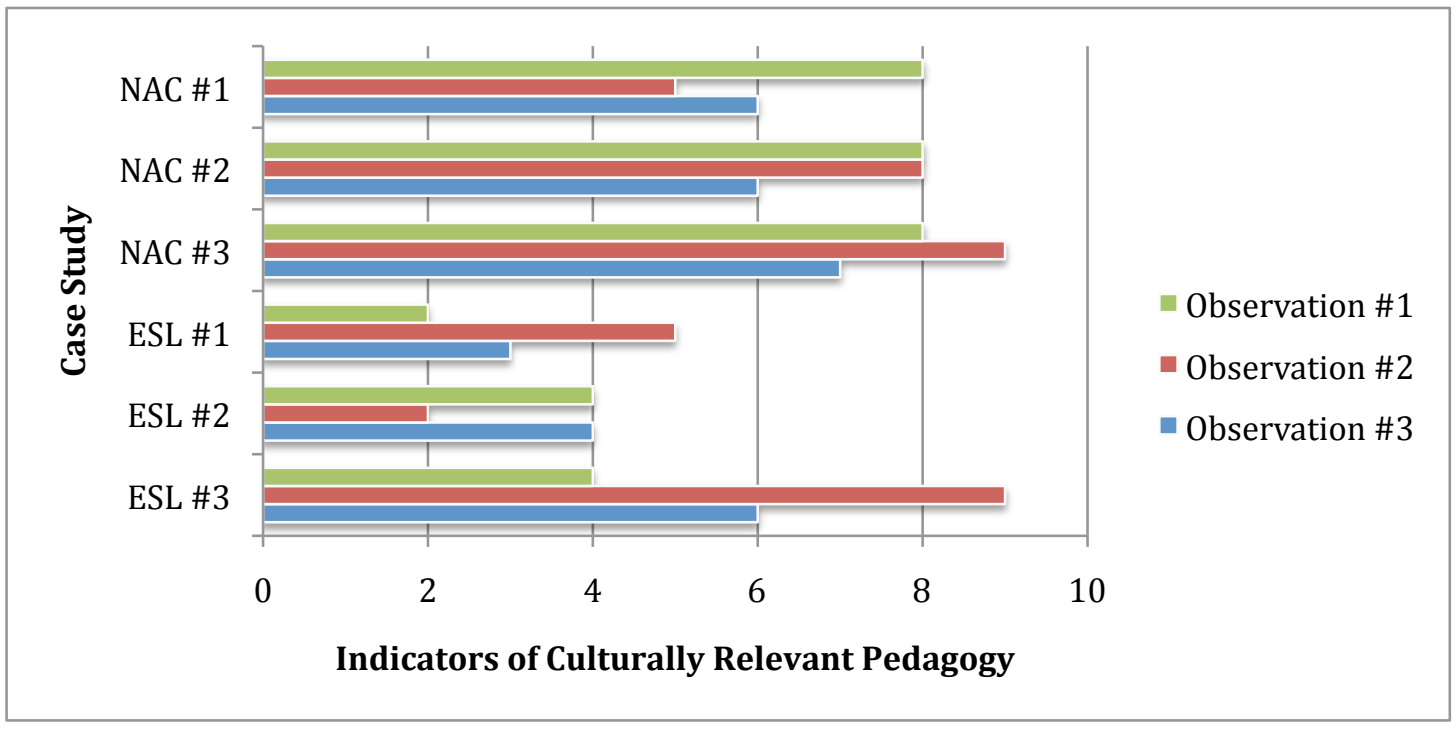


The three total numbers of indicators demonstrating culturally relevant pedagogical practices during the class observations were also averaged for each of the three individual cases with the aim of demonstrating the overall mean number of indicators found in each of the two programs under study (NAC and ESL). The totals, as are seen in Table 37 and in Figure 16, allow one to compare the NAC and ESL programs to determine which program exhibits a learning environment more sensitive to the cultures and languages of the students within the programs, as well as other cultural and linguistic backgrounds not present within the class environment. Of the 10 total indicators of culturally responsive pedagogical practices, the NAC program averaged 7 indicators throughout the three observations, while the ESL program averaged 4 indicators during the same time frame. The mean numbers, as seen in Table 37 and in Figure 16, make clear the notion that as a whole, the NAC program under study incorporated more culturally relevant pedagogical practices into the academic environment then the ESL program.

Table 37

Mean Number of Culturally Relevant Pedagogical Indicators Recorded During Program Observations

\begin{tabular}{llllll} 
Program $^{\mathrm{a}}$ & Observation \#1 & Observation \#2 & Observation \#3 & Average $^{\mathrm{b}}$ & Total \\
\hline NAC & 8 & 7 & 6 & 7 & 10 \\
ESL & 3 & 5 & 4 & 4 & 10 \\
\hline
\end{tabular}

Note. ${ }^{a}$ Mean numbers determined by averaging the results within the three NAC cases and the three ESL cases, ${ }^{b}$ Average numbers were calculated by determining the mean numbers of the three observations per program and the calculations were additionally rounded to the nearest whole number 
Figure 16

Mean Number of Culturally Relevant Pedagogical Indicators Recorded During Program Observations

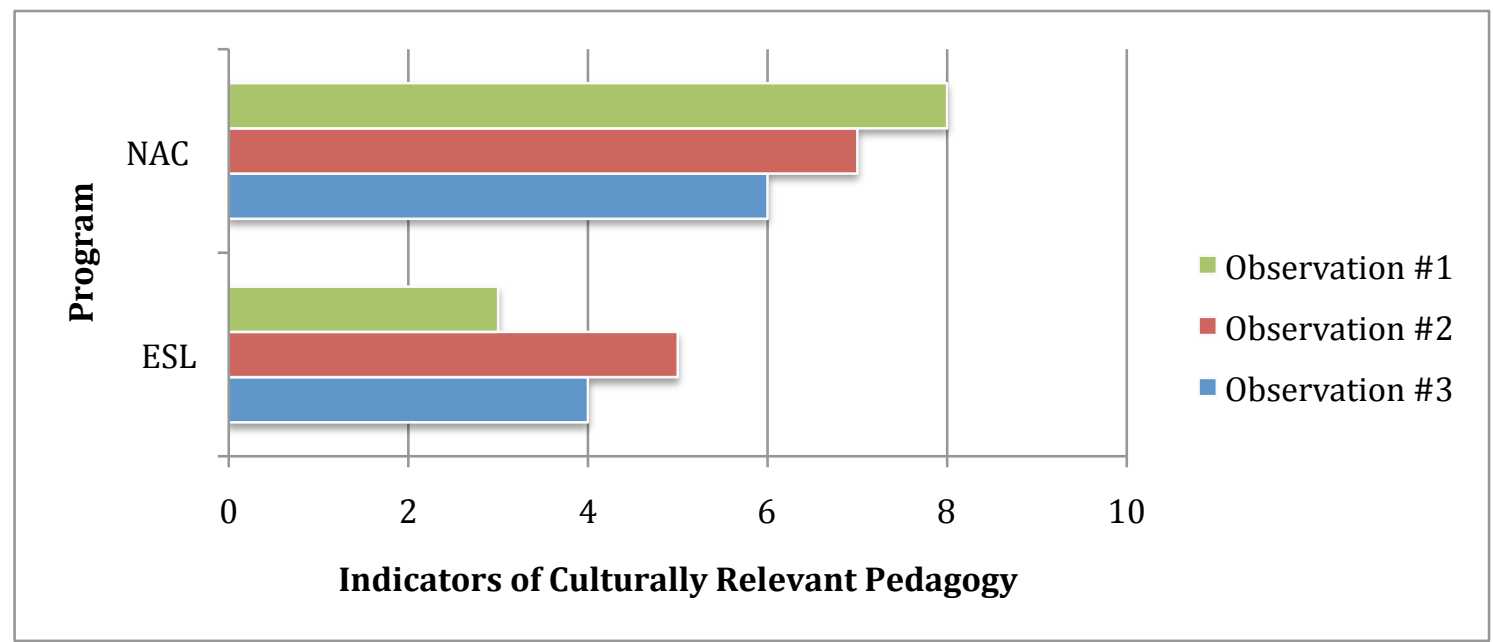

The third major section of the Classroom Observation Indicator Matrix focused on ascertaining the pedagogical orientations of the teachers within the ESL and NAC cases under study. Each of the teachers was observed a minimum of three times, during which indicators from the observation matrix were marked according to the type of pedagogy employed by each teacher under study. The pedagogical orientation indicators were based on Cummins' Transformative Multiliteracies Pedagogy framework, as was discussed in detail in Chapter 2, Literature Review. The continuum, based on Cummins' framework (2009), ranged from a Transmission-oriented pedagogy, to Social Constructivist-oriented pedagogy, and finally a Transformative pedagogical orientation. Cummins' framework nested these pedagogical orientations, as he asserted that a teacher would be unable to educate with a Social Constructivist-oriented or Transformative pedagogy without utilizing some level of Transmission-oriented pedagogical practices (2009). In other words, some direct instruction or lecture is often required in teaching and 
it is necessary to use this type of instruction to move to a more collaborative or transformative classroom environment. Figure 15 demonstrates the placement of each of the teachers on the continuum during the three observations, the numbers $(1,2$, or 3$)$ denote the indicators observed during each observation.

As is seen in Figure 17, the indicators illustrate that for each of the cases, with the exception of ESL Case \#1, the teachers demonstrated an approach to teaching that favored the Social Constructivist-oriented pedagogical orientation. These teachers activated and valued their students' prior knowledge and cultural backgrounds. They also co-constructed understandings about the concepts being taught by engaging students in dialogue and discussion about the topics being studied. While NAC Case \#1 and ESL Case \#2 demonstrated instances in which students were instructed utilizing collaborative inquiry, and the latter additionally promoted higher-order thinking skills during the third observation, only NAC Case \#3 and ESL Case \#3 frequently demonstrated collaborative inquiry and the activation of higher-order thinking skills during the class instruction.

ESL Case \#1 was the only class in which a more Transmission-oriented approach was apparent throughout the three observations. The instruction in this class was primarily focused on direct-instruction and lecture with little collaborative discussion during the lessons and subsequent activities. The students in ESL Case \#1 were essentially passive learners throughout their daily instruction. As was previously stated, ESL Case \#1 was an ESL 1/2 and ESL 3/4 combination class, and, therefore, the credentialed teacher in the class taught the ESL 3/4 group in one part of the classroom, while the assistant taught the ESL 1/2 course in an opposite corner of the room. As the assistant was the teacher that was observed for the study and she had not received the 
same training or professional development as the classroom teacher regarding

collaborative inquiry or the co-construction of knowledge, the pedagogical orientation of this class was understandably a more Transmission-oriented approach. None of the cases had reached the level of a Transformative pedagogical orientation, in that the concepts of societal power relations and inequalities, as well as instances of critical inquiry, were not brought forth during the class observations.

Figure 17

Pedagogical Orientation Indicators Observed During First, Second, and Third Case Observations

\begin{tabular}{|l|c|c|c|c|c|c|}
\hline & $\begin{array}{c}\text { NAC } \\
\# 1\end{array}$ & $\begin{array}{c}\text { NAC } \\
\# 2\end{array}$ & $\begin{array}{c}\text { NAC } \\
\# 3\end{array}$ & $\begin{array}{c}\text { ESL } \\
\# 1^{\mathrm{a}}\end{array}$ & $\begin{array}{c}\text { ESL } \\
\# 2\end{array}$ & $\begin{array}{c}\text { ESL } \\
\# 3\end{array}$ \\
\hline Transmission-oriented Pedagogy & & & & & & \\
\hline Instruction primarily direct instruction/lecture & & $1,2,3$ & & $1.2,3$ & & \\
\hline Instruction focused solely on content of lesson & & 1 & & $1,2,3$ & 1,3 & 1 \\
\hline Students are passive learners & & & & $1,2,3$ & & \\
\hline Social Constructivist-oriented Pedagogy & & & & & & \\
\hline Teacher activates students' prior knowledge & $1,2,3$ & $1,2,3$ & $1,2,3$ & 1 & 1,2 & $1,2,3$ \\
\hline Cultural backgrounds valued within instruction & $1,2,3$ & $1,2,3$ & $1,2,3$ & 2 & 1,2 & 2,3 \\
\hline $\begin{array}{l}\text { Teachers and students co-construct understandings } \\
\text { through dialogue and discussion }\end{array}$ & $1,2,3$ & $1,2,3$ & $1,2,3$ & 2 & $1,2,3$ & $1,2,3$ \\
\hline Instruction utilizes collaborative inquiry & 1,2 & & $1,2,3$ & & 3 & $1,2,3$ \\
\hline Higher-order thinking skills promoted & & & $1,2,3$ & & 3 & $1,2,3$ \\
\hline Transformative Pedagogy & & & & & & \\
\hline Collaborative critical inquiry present & & & & & & \\
\hline $\begin{array}{l}\text { Instruction promotes analysis of societal power } \\
\text { relations }\end{array}$ & & & & & & \\
\hline $\begin{array}{l}\text { Instruction promotes discussion of ways to act on } \\
\text { societal inequalities }\end{array}$ & & & & & & \\
\hline
\end{tabular}

Note. 1 = Observation \#1;2 = Observation \#2;3 = Observation \#3, ${ }^{\mathrm{a}}$ ESL 1/2 students instructed in a small group conducted by teacher's aide.

\section{Interview and Focus Group Themes and Tensions}

In an effort to include stakeholder voice in this research study, six student focus groups (three ESL and three NAC), six teacher interviews (three ESL and three NAC), 
and four administrator interviews (two ESL and two NAC) were conducted as a part of the data collection. This portion of the study was crucial in providing an understanding of "how people interpret their experiences, how they construct their worlds, and what meaning they attribute to their experiences" (Merriam, 2009, p. 5). The people in question, the students, teachers, and administrators within this study, were asked to share their thoughts and opinions in a safe and open environment, with the intent of better understanding the two programs under study from the participants' perspectives, or emic viewpoint, rather than analyzing and evaluating the programs from solely the etic, or outsider's, perspective (Merriam, 2009). As were discussed more fully in Chapter 3, Methodology, the teacher and administrator interview protocols and the student focus group protocol (Appendices $\mathrm{F}$ through $\mathrm{H}$ ) were created utilizing the theories and prior research studies discussed in Chapter 2, Literature Review, as well as the researcher's extensive experience educating secondary-level immigrant youth.

The interviews and focus groups were conducted in the participants' dominant languages to ensure accuracy, and were subsequently transcribed and coded by the researcher to illustrate the significant findings and tensions associated with the two programs under study. The results were organized into the following six tables to assist in the process of analyzing the perceptions and the experiences of the participants within the study: (1) NAC Teacher Interview Analysis (Appendix N), (2) ESL Teacher Interview Analysis (Appendix O), (3) NAC Administrator Interview Analysis (Appendix P), (4) ESL Administrator Interview Analysis (Appendix Q), (5) NAC Student Focus Group Analysis (Appendix R), and (6) ESL Student Focus Group Analysis (Appendix S). The teacher and administrator interview tables were arranged according to nine emergent 
themes: curricula, language instruction, content instruction, attitude/expectations, cultural responsiveness, pedagogical orientations/beliefs, academic self-concept, language proficiency level, and academic skills. The two student focus group tables were categorized according to seven of the above nine themes, with the two exceptions being curricula and academic skills.

In narrating significant findings and tensions within the interview and focus group data, the results were initially separated by program (ESL or NAC) and subsequently arranged according to seven of the abovementioned themes: stakeholders' perceptions and expectations, curricula, language instruction, content instruction, cultural responsive teaching practices, teachers' pedagogical orientations, and students' academic selfconcept. The data that had been categorized within the language proficiency and academic skills portions of the tables were both incorporated into the language instruction and content instruction sections of the narrative, as it was determined that students' skills and proficiency levels directly concerned the manner in which they were instructed within their particular program of study, and therefore it was appropriate to combine such findings. Tables 38 and 39 provide a synthesis of the qualitative findings, illustrating the positive "driving conditions" and the negative "restraining conditions" that emerged during the interviews and focus groups with the stakeholders from each of the two programs under study. The conditions are categorized within their appropriate themes and will be elaborated upon in the discussion section comparing and contrasting the interview and focus group findings. 
Table 38

Qualitative Findings Overview - English as a Second Language Program

\begin{tabular}{|c|c|c|}
\hline Theme & $\begin{array}{l}\text { Driving Conditions } \\
\text { (positive) }\end{array}$ & $\begin{array}{l}\text { Restraining Conditions } \\
\text { (negative) }\end{array}$ \\
\hline $\begin{array}{l}\text { Stakeholders' } \\
\text { Attitudes }\end{array}$ & $\begin{array}{l}\text { A: Support teachers with professional } \\
\text { development, pacing guides } \\
\text { T: Highly qualified to teach Els; } \\
\text { advocate of students; high expectations } \\
\text { S: Important to be fully bilingual; feel } \\
\text { successful in ESL classes }\end{array}$ & $\begin{array}{l}\text { A: Student placement (mixed levels); } \\
\text { budget cuts cause increased workload } \\
\text { T: Focus on high-stakes testing; feel } \\
\text { overwhelmed } \\
\text { S: Failing courses; feel less intelligent, } \\
\text { nervous; pressure to learn English }\end{array}$ \\
\hline Curricula & $\begin{array}{l}\text { A: New curricula is stronger; Teachers } \\
\text { had input in curricula adoption; } \\
\text { creating pacing guide and assessments } \\
\text { T: Teachers had input in curricula pilot } \\
\text { committee; Create own relevant, } \\
\text { interesting supplemental curricula }\end{array}$ & $\begin{array}{l}\text { A: Prior to adoption no consistent } \\
\text { curricula } \\
\text { T: Prior to adoption no consistent } \\
\text { curricula; Vocabulary and dialogue } \\
\text { sections not authentic; Mixed levels no } \\
\text { pacing guides }\end{array}$ \\
\hline $\begin{array}{l}\text { Language } \\
\text { Instruction }\end{array}$ & $\begin{array}{l}\text { A: Pacing guides promote consistency; } \\
\text { Centers support multiple levels; many } \\
\text { oral language opportunities } \\
\text { T: Differentiate instruction to meet } \\
\text { students' needs; encompass all } \\
\text { language domains; El strategies } \\
\text { S: Primary language support; frequent } \\
\text { modeling; music, technology help }\end{array}$ & $\begin{array}{l}\text { A: Impractical for teachers to } \\
\text { simultaneously teach multiple levels } \\
\text { T: Multiple levels and needs in one class } \\
\text { S: Pressure to rapidly acquire English }\end{array}$ \\
\hline $\begin{array}{l}\text { Content } \\
\text { Instruction }\end{array}$ & $\begin{array}{l}\text { A: QTEL strategies and professional } \\
\text { development provided content teachers } \\
\text { T: Often supported students in content } \\
\text { classes; students often placed in } \\
\text { language intensive courses later } \\
\text { S: Primary language support helped; } \\
\text { content taught differently (math) }\end{array}$ & $\begin{array}{l}\text { A: Content taught in mainstream or } \\
\text { sheltered classes } \\
\text { T: No time/ability to "push in" to } \\
\text { content classes } \\
\text { S: Struggled most in language intensive } \\
\text { courses (history and science); content } \\
\text { taught differently (math); lecture format }\end{array}$ \\
\hline $\begin{array}{l}\text { Culturally } \\
\text { Responsive }\end{array}$ & $\begin{array}{l}\text { A: Build on students' prior academic } \\
\text { knowledge } \\
\text { T: Use multicultural texts and discuss } \\
\text { various cultures; support with primary } \\
\text { language } \\
\text { S: Learned about U.S. culture in history }\end{array}$ & $\begin{array}{l}\text { A: Hard to comprehend students' } \\
\text { backgrounds } \\
\text { T: Teachers cannot communicate with } \\
\text { all students in home languages } \\
\text { S: Students not in history classes do not } \\
\text { discuss culture; only taught about } \\
\text { holidays; home cultures not valued; } \\
\text { teachers do not promote bilingualism }\end{array}$ \\
\hline $\begin{array}{l}\text { Pedagogical } \\
\text { Orientations }\end{array}$ & $\begin{array}{l}\text { A: Consistency across school } \\
\text { sites/uniformity } \\
\text { T: Teachers as facilitators and coaches } \\
\text { S: Learned best when connected to } \\
\text { teachers }\end{array}$ & $\begin{array}{l}\text { A: Lack of time to work with ESL } \\
\text { teachers } \\
\text { T: Expected to articulate content } \\
\text { curricula with ELD acquisition } \\
\text { S: Issues with use of dictionaries/ } \\
\text { translators; Teachers' false assumptions }\end{array}$ \\
\hline $\begin{array}{l}\text { Academic Self- } \\
\text { Concept }\end{array}$ & $\begin{array}{l}\text { A: Students became more actively } \\
\text { involved in class; youth more } \\
\text { confident; increased graduation rates } \\
\text { T: More confident; more successful in } \\
\text { ESL classes } \\
\text { S: Felt better/tried harder in classes } \\
\text { they connected to/enjoyed more }\end{array}$ & $\begin{array}{l}\text { A: None } \\
\text { T: ESL 5/6 students embarrassed to be } \\
\text { classified ESL; students more } \\
\text { nervous/higher anxiety in mainstream } \\
\text { classes } \\
\text { S: Uncomfortable in new schools; } \\
\text { frustrated in difficult classes }\end{array}$ \\
\hline
\end{tabular}

Note. $\mathrm{A}=$ Administrators $1 ; \mathrm{T}=$ Teaches $; \mathrm{S}=$ Students 
English as a Second Language Stakeholders' Attitudes and Program Expectations

The program administrators charged with overseeing the English as a Second Language program at the district level held that one of their major ongoing tensions surrounded the issue of student placement. Many sites across the district expressed that they did not have sufficient numbers to create a stand-alone ESL 1/2 course at their site, and therefore they incorporated ESL 3/4, and at times even ESL 5/6, into one larger ESL class. The mix of levels in one course can be problematic as the students have such differing language needs and there are different curricula associated with each ESL level. In addition, other sites opted to mainstream their ESL students into general English classes. When students were to be mainstreamed, the program administrators strongly encouraged the sites to place such students with teachers that were willing and able to work with newcomer students. The administrators asserted that purposeful placement was paramount in assuring effective instruction for all ESL students. In the following quote, one program administrator illuminated the common tensions in student placement:

Every year it seems like we continually have to hit the schools hard and say okay, how are you placing them...? What teachers are you putting them with; who are you mixing them with because we don't think that mixing your Special Education and your Beginning English learners is really the best mix. They don't provide role models for each other...So, it's just -- this is just an ongoing struggle (ESL Administrator, 2011).

Another major tension for the program administrators was that despite having been hired to support ESL teachers and the program as a whole, due to staff reductions and budget cuts they had been asked to take on more central office responsibilities, which ultimately gave them less time to co-plan and co-teach with ESL teachers on site. They also reported that there were fewer resources available to sites and teachers, also due to 
budget decreases, but any resources they did acquire were distributed to the ESL classes.

In addition to resources and materials, the program administrators claimed they were able to support teachers with newcomer youth through extensive professional development opportunities that concentrated on strong English learner teaching strategies. The administrators further supported teachers by helping them to institute necessary small group instruction in their mainstream classes to meet the needs of their ESL students and by creating pacing guides and curricula for them to use with their newcomer youth.

Finally, the administrators stated that support classes were used to assist students with their acquisition of English and their class work at many sites.

[When we have to mainstream newcomer students] that's where I think professional development comes in... And we also get smarter about what we're doing in a support classes. We usually come out and kind of assess the situation and just see what we can do to improve the situation in terms of placement, but if that can't be done [then] how can we support the teacher to work with small groups to... do that. It is very much site by site (ESL Administrator, 2011).

The ESL teachers held high expectations of their students, but many felt they were unable to be as involved or creative as they had been in years past. They felt they had more time, energy, and resources to connect with students when they began teaching, and now the sole focus in school was on the state-mandated high stakes testing. Teachers reported feeling overwhelmed by a lack of time to complete their work and by their many responsibilities. They did report feeling that they were highly qualified to teach ESL students, as they had very strong backgrounds and extensive training in working with newcomer youth, and they were incredibly passionate about teaching such students. In the subsequent quote, one teacher reflected upon the change she felt in her approach to teaching, as high stakes testing had increasingly become the focus at her site: 
Back in the day I would come in here for like Halloween and I have my cake, my big pumpkin and I would be very, very involved and I guess I am not as, I don't know, touchy feely as I used to be and I think that might be because of the high stakes with the exit exam that we have to pass. We don't have time... Yeah, I had the time and the energy I guess to do that and now I think the bit push is to pass the exit test and to get my CST scores up, so I think that's where my mind shift is. (ESL Teacher, 2011).

The ESL teachers felt they were more than solely classroom teachers for their newcomer students; the teachers felt as though they were a point of contact for students since the students often came to them for a variety of needs as they youth were most comfortable with their ESL teachers. The ESL teachers stated it was important for all teachers of newcomers to understand the plight of the students and to attempt to empathize with the difficult situation many ESL students face in attending school in the U.S. and acquiring English. One teacher reported she was an English learner herself and therefore she felt she could better understand the difficult nature her students face in learning and being educated in a new language. The ESL teachers felt that all teachers must know their students inside and outside of the classroom to better connect with them. Finally, the ESL teachers believed it was crucial to hold high expectations for the students and they frequently encouraged students to think about and pursue higher education, in addition to acquiring sufficient language to be mainstreamed the following year.

You have people that really care for English learners and you have people that really feel that you should speak English before you come to America and so... sorry I can't help you...[Students need] a teacher that actually cares and that you can tell that they care about you and they respect that you might be struggling because English is your second language (ESL Teacher, 2011).

During the student focus groups, many ESL students reported having immigrated to the United States because their families wanted them to have greater educational 
opportunities and more job prospects. The students felt it was important to be fully bilingual to have increased opportunities, both academically and personally. Despite the positive intentions with which many moved to the U.S., one student stated that a supposed better life in the U.S. comes with much hardship, as people often work too much and learning the language can be very difficult. In addition, although they were good students in their home countries, many students reported they were failing their courses in the U.S., which made it difficult for them to believe they could attain the opportunities in life for which they moved. Students did not feel as intelligent in this country as they could not communicate in the common language, which ultimately affected their performance in school. In the following quote, one ESL student expressed his discontent with having immigrated to the U.S.:

A better life here means to come and suffer. This isn't life, not knowing the language; everyone works too much, and goes to school. So for me this isn't a better life (ESL Student, 2011).

Students reported having felt nervous when they first came to the U.S., and it was difficult for many of them to be forced to ask other students when they needed assistance. A few newcomers felt no one would speak Spanish when they arrived, but they happily learned people did speak Spanish after they reached the United States. Many students disclosed that they had cried when they first started their new school, as they were extremely nervous about the entire process. Additionally, some students felt anxious when they saw the classroom and the technology available in the U.S., as they weren't sure how to use the computers. During the focus groups, one ESL student reported: 
When I first came I felt] nervous because I didn't know English...I was nervous and didn't know what to say...because you don't know where things are and everything is in English and you have to ask people you don't know (ESL Student, 2011).

Additionally, while conversing about how students felt upon entering U.S. schools during the focus group discussions, one ESL student stated:

I felt bad because I didn't know what I was going to do the day I started... I saw a lot more computers and things hanging from the walls and I felt like I wouldn't know how to use them (ESL Student, 2011).

The newcomers asserted they must become fluent in English to be successful in the U.S. and they felt that the pressure to acquire English was overwhelming, yet, at times the pressure motivated them to work harder. Other students felt discriminated against at school, as they believed their peers viewed them negatively and were unwilling to help them. A few students believed they were teased because they were not born in the U.S. and they maintained that Spanish was not valued in this country. One student reported not being able to concentrate in class because he did not understand what the teachers were saying during the lessons. In the subsequent quote, this student expressed his frustration with his inability to understand his teacher and his difficulty concentrating in class:

Not knowing one word [stops me from learning]...I just can't seem to concentrate. I know I am not learning anything and no matter how much the teacher tries to explain my mind wonders off to other things and by the end of class I didn't learn anything (ESL Student, 2011).

After some time, the students reported feeling better in school, yet they still struggled with the language. They affirmed that their ESL class was their most important class, and, in addition, it was the class in which they felt the most successful because the 
concepts taught in this class were meant for students at their proficiency level. A few students had the opportunity to take Spanish class, in which they felt the most successful, as they are able to be leaders and help others in such a class. Some students supported the newcomer youth by translating and showing them how to do the work, or by allowing them to copy some of the work as well. Lastly, the students stated in the focus groups that a couple of their teachers taught them about the necessary process in applying to institutions of higher education, as well as about the steps they must complete to finish their high school education.

\section{English as a Second Language Program Curricula}

According to the administrators of the English as a Second Language Program (ESL), the curriculum and training utilized within this program have become far stronger over the past three years. Three years prior to the study, the district had funded a new ESL curriculum about which the teachers were increasingly required to be trained, in addition to intense professional development the teachers received concerning how to best teach explicit language development to their students, a training referred to as Focused or Systematic English Language Development. The district had made the decision to fund an ESL textbook adoption, as the official state adoption date was believed to be too far in the future and the need for ESL materials across the district was apparent to all. The district administrators, with the desire to make sure that ESL teachers had input in the adoption process, created a curriculum adoption committee of $15 \mathrm{ESL}$ teachers and several district administrators, who were charged with choosing between four ESL curricula options. Ultimately, the teachers and administrators chose Pearson 
Longman's Keys to Learning and Shining Star curricula, as they felt, of the choices

available, it was the most comprehensive and language intensive curricula.

Prior to the ESL textbook adoption, there was no consistent ESL curriculum, and therefore the district resource teachers were additionally entrusted with creating a pacing guide and assessments to utilize with the new ESL textbook adoption. Along with the textbook adoption, the administrators reported that the locally established Writing Reform Institute for Teaching Excellence (WRITE) curriculum and professional development provided the writing component of the ESL program. As will be discussed in the subsequent results section, the NAC program utilized the same curricula and materials for the English Language Development portion of their program as those adopted by the ESL program. The following quote illustrates the perspective held by the ESL administrators concerning the curricula and training provided by the district central office:

I would say that this year... starting last year, but absolutely this year I would say we have a solid quality curriculum that we are requiring teachers to come in every quarter to get trained on... because we now know it. We have it. We know the books that we're using. We have our assessments down. We have the protocol down for how assessments are going to be given and collected and all those things. And so now we're at a point where we're only offering professional development to brand new teachers... all of our veteran ESL teachers just they have their binders now. It's set and they're good to go (ESL Administrator, 2011).

In accordance with the assertion made by the ESL administrators, one ESL teacher stated that there was no actual ESL curriculum when she began teaching in the program 18 years prior. At that time, ESL teachers were solely provided worksheets that were thematically based, rather than being focused on the much needed language development. According to the ESL teacher, about six years ago the district recognized 
the need for a strong ESL curriculum and started gathering resources to create a more effective program. ESL teachers were later invited to be a part of the ESL pilot committee, as noted in the previous paragraph. Ultimately, the teachers in the pilot committee felt the Keys to Learning curriculum was the most effective for ESL 1/2 students, as it has focused on grammar and syntax practice and contained short dialogues for oral practice. While most teachers were satisfied with this curriculum, others questioned if the vocabulary building and dialogue sections within the text were authentic enough for students to retain over time. Finally, the ESL teachers utilized the WRITE curricula for the writing portion of their daily instruction, about which many reported to be quite content. One ESL teacher spoke of her experience on the pilot committee:

We had several books to choose from, and then we had to go through each of the books and there were criteria that we had to evaluate the books on. Then we met as a team, I think we met four times that year to decide...It was a long process and it was good, but you still wanted more than what was offered. So you chose the best (ESL Teacher, 2011).

In addition to the district-provided curricula, teachers also created their own supplemental curricula with topics that they felt were more interesting and relevant to their students' lives. They additionally reported supplementing the curricula with images they have found online. Teachers used the computer program Rosetta Stone to enhance their teaching, though for many it took up to three years to get the appropriate technology and the necessary headphones to operate the program within the classrooms. One significant tension mentioned by the teachers was that although they were provided a pacing guide by the district, it was created for stand-alone classes and not mixed level classes, as most ESL classes were arranged at the time of this study. Therefore, many teachers felt they were unable to use the pacing guides as they were intended by the 
district and had to create their own manner of assuring the varying levels received the appropriate curricula and instruction throughout the school year.

There is a pacing guide. Now the pacing guide is meant for stand-alone classes. And not so much for mixed classes like mine, and so I try to find at least one common curriculum that I can use for both. So, I have small groups, specialized more for their level and then one whole group application and so WRITE tends to lend itself for that whole group (ESL Teacher, 2011).

\section{Language Instruction Within the English as a Second Language Program}

In speaking about expectations the administrators held for the language instruction component of the ESL program, the program administrators emphasized that curricular pacing guides were distributed to all ESL teachers across the district to ensure consistency within the program. They felt it was important for teachers to maintain similar pacing within the ESL classes, as it was crucial for students to complete the intended units before the end of the school year. Teachers were advised by the program administrators to establish learning centers in classes with multiple ESL levels. The administrators asserted that it was important for the different levels of curricula to be used with the students, yet the students would need to be grouped for such instruction to take place. During whole-class instruction, the administrators maintained that ESL teachers should teach to the middle ESL level in their class, and utilize English learner strategies to scaffold more for lower level ESL students and to give less support to students at a higher acquisition level. Teachers were not expected to simultaneously cover multiple ESL curricular levels, as this would be impractical. One administrator spoke to the manner in which teachers were to instruct in multi-level ESL courses in following quote: 
I mean because our curriculum is divided into $1 / 2,3 / 4$, and $5 / 6$ and while we look at...we are very genre based, there are no major differences in that instruction and so really [it's about] working with those teachers to kind of... teach to the high middle of the class... and then, scaffold more for your lower students and scaffold less for your higher students (ESL Administrator, 2011).

Teachers were expected by the administrators to conduct explicit language instruction utilizing such language development philosophies as Systematic or Focused English Language Development, which utilizes language frames and specific vocabulary instruction to support students in acquiring and practicing both oral and written language in English. Additionally, literacy strategies, such as shared and guided reading, and the creation of multiple authentic reading and language experiences were considered important components of the ESL program. It was expected that newcomer youth have frequent opportunities to speak and present in class, as their oral language skills must be developed in a highly supportive and low-anxiety environment. The ESL administrators asserted that reluctant speakers were to be encouraged and given frequent opportunities to speak in class through the use of specific oral language teaching strategies and activities. They did not believe there should be a silent period, in that they believed reluctant students could, at the very least, repeat others' answers or give one-word answers; such an assertion contradicting the highly respected work of Krashen (1992), which illuminated the existence of a distinct silent period for beginning English learners. Finally, the administrators stated that technology, such as the aforementioned Rosetta Stone and Learning Upgrade computer programs, were also encouraged to be used within daily instruction to assist students in acquiring language skills and thematic vocabulary. In the following quote, one administrator expressed her desire to see all students participating in their ESL classes regardless of their language acquisition levels: 
I don't know if there should be a silent period. I think kids that are reluctant, there is things you can do...you know they don't always have to come up with something original. They can repeat what somebody else has said and they can, you know, say one word. They don't always have to...it's not about coming up with this beautiful sentence. It's saying the one word and it's repeating what somebody else said. It's making them what we call a legitimate peripheral participator...In whatever is happening in the classroom that yes they might not have this fabulous response, but they do have something to contribute to the work as a whole... and that's what we're looking for the kids to constantly give them opportunities to be contributors... no matter what's happening in the classroom, no matter where they are and the stages (ESL Administrator, 2011).

During the teacher interviews, the participants maintained that they created their lessons by first looking at their students' strengths, both oral and written, and then determining the best course of instruction to assist students in acquiring English. Teachers created long-term objectives and taught with the "end in mind" to assure the students were building the necessary language skills to be successful in later mainstream classes. The lessons the teachers created for their classes encompassed all language domains, reading, writing, listening, and speaking, with one major objective to achieve, for example descriptive writing or comparing and contrasting. The content and academic vocabulary taught during the reading portion, was additionally illuminated in students' writing, to demonstrate the connections between the various language domains. Finally, teachers reported frontloading vocabulary and using peer interaction to better support students in their language acquisition.

I do try to frontload as much as I can for students who I know will need more support. I also differentiate by getting in proximity. So, I have the ones that need me the most very, very close by, I also use a lot of peer support and I think you saw that. I know there is one of me and more of them and so I make sure that where the students are seated, a weaker student is seated with a stronger student and that stronger student is encouraged to help the other one. And also for example, at this point, I have an idea of where my students need more enrichment and so I'm willing to move them to another group. I also do some a lot of reteaching (ESL Teacher, 2011). 
The teachers reported frequently modeling the writing process for the students to support them in creating their own writing pieces. The students were permitted to copy the teachers' models as needed, but they were encouraged to challenge themselves and create their own responses when possible. Most teachers did not teach phonics to the students, as their students entered as readers and writers in their home languages, but some sites did report using support or resource teachers to work with the students needing more explicit phonics instruction. Teachers also used such strategies as showing students pictures and videos to assist them in visualizing new terms, using authentic or real-life experiences in class and around the school site, and bringing realia to class to teach students new language. One teacher also asserted she concentrated on slowing her rate of speech in class and she had students use the microphone in her class to help them to understand one another in class. An ESL teacher explained how she connected students' oral language skills to their writing and how she helped students to develop their needed vocabulary in the following quote:

They practice their oral language first with their friends in the classroom and then they write it. I, first of all, I don't care about whether they have a lot of mistakes, that's okay but I encourage them to write...Also, the cafeteria ladies told us that our kids didn't know how to order food. So what I did was I went to the cafeteria to take pictures of the food, and then I sent them to my colleagues so they could [create authentic] lessons... like hamburger... so my camera is with me at all of the time, because everything can be taught through pictures (ESL Teacher, 2011).

In addressing oral language development, the teachers created frequent opportunities for their student to develop and practice their oral language skills. Students used sentence starters to have short conversation in English and sometimes did small oral presentations in front of a small group or the whole class. Teachers asserted that students must have frequent authentic conversations to build their language skills, and therefore 
teachers challenged reluctant speakers to have short conversations, yet in a comfortable and low anxiety manner. Reluctant speakers were often provided with a script or visual cues to assist them, as it was recognized that students must work at their own level and pace. Finally, many teachers used such computer programs as Rosetta Stone in class and Learning Upgrade at school and at home, to practice reading skills and develop language. A few teachers had access to additional computer programs, Teen Biz and Lexia, if their site had chosen to purchase such technology licenses.

During the focus groups, the students stated that teachers helped them to learn English by modeling language. They also felt they learned more by having friends that only spoke English or other bilingual students that could help them by explaining English concept to students in their primary languages. Students practiced speaking English with family members and often had words repeated to them in an effort to learn to pronounce them correctly. Much like with friends and other students, the focus group participants reported that it helped them to acquire language when their family members did not speak Spanish, as they felt it forced them to practice English.

I practice [English] here with my friends because some don't speak Spanish, so I practice with them and sometimes in my English class because I feel more confident there... I [also] speak to my grandparents [in English] because they don't speak much Spanish (ESL Student, 2011).

Many students also asserted that they learned English outside of school, at parties or when shopping, yet it was more informal, oral language. Students further maintained that reading helped them to learn the language, as they would translate unknown words in dictionaries or online and attempt to memorize them in English. Music and TV helped students acquire language at home; students often translated songs and watched TV with 
subtitles to help them learn English. Finally, one student reported that computer programs, such as Rosetta Stone and Learning Upgrade, helped her to learn English. In the following quote, one student illustrated how her teacher encouraged her to practice English:

Books [help me learn English]... my history teacher pressures me a lot and she spoke to my dad and told him to have me read books...she speaks Spanish but pretends she doesn't understand because she wants me to practice. Even though I feel pressured I feel like its something good (ESL Student, 2011).

\section{Content Instruction Within the English as a Second Language Program}

Secondary-level content teachers historically taught the content classes for students enrolled in the English as a Second Language program, as, unlike the New Arrival Center program, this was not a self-contained program. Therefore, the students were often mainstreamed or placed in sheltered content classes, depending on the available classes at each site. As was previously introduced by the NAC administrators, the ESL administrators confirmed that the Quality Teaching for English Learner (QTEL) training was the primary professional development for content teachers. The QTEL training emphasized strategies for teaching English learners in all mainstream content or sheltered courses, and the district maintained that all teachers at the secondary-level sites were to be using such strategies to help students access content and the lessons within their classes. In addition, the English Learner Support Teachers (ELSTs) employed by most sites were charged with placing students with content teachers who are willing and able to teacher English learners effectively. Often the ELSTs also taught a support class at their site to give students extra assistance with content classes, and assisted content teachers through co-planning and co-teaching in such classes. 
The content area support has strictly been through QTEL... that's why we've just started making roads into the content area support, because we've always maintained [for those classes] that nothing is really going to change unless we get everybody to use language strategies... and that during the students' whole day they're learning English not just you know in the English class or the ESL class (ESL Administrator, 2011).

While the ESL teachers were not charged with explicitly teaching their students content from mathematics, science, or history classes, ESL teachers often supported their students during class time by helping them with class work and homework for their content classes. The teachers supported their ESL students in their mainstream classes by giving them time and assistance to complete work. One teacher reported receiving questions from the ELD history teacher beforehand to allow her to frontload her ESL students with the questions and answers before they were covered in the history class. This same ESL teacher stated that she had attempted to push-in to her students' math classes, but it was problematic as she had four total grade levels in her ESL class, and each grade level at her high school was enrolled in a different math class. She also took it upon herself to work with a newer biology teacher and a history teacher to help them in scaffolding the content for their ESL students.

One year, the biology teacher was new. So, I spent my third period with the biology teacher. Last year, I spent it with the history teacher, which was nice, but now I feel like you know because I spend a lot of my time on my testing...now I'll go in there just because of who we are (ESL Teacher, 2011).

Despite the claim of the district administrators that students received content instruction through the use of QTEL strategies, one teacher stated that many mainstream teachers do not truly differentiate instruction for their English learners, so she felt it was crucial that students enter their mainstream classes with enough basic skills and language 
to function and achieve in those classes. She further ascertained that the academic rigor was not too high in content classes, and therefore if kids worked hard they would pass their content courses. Finally, she stated that students at her site were purposefully held out of language intensive classes such as history until they had achieved the ESL 3/4 level of language acquisition, which at times would not occur until mid-year the following year. These students often took less language intensive courses, such as art, during their first year of enrollment in U.S. schools.

They're not in history, none of the ESL $1 / 2$ students are in history and that's purposeful, we don't... we won't put them in until they are ESL 3/4 and maybe even second semester of 3/4...instead [the students are in] art because it's not language intensive, so we picked their classes intentionally we picked them not to have very language intensive courses (ESL Teacher, 2011).

In discussing their content classes, the students stated that they struggled most with language intensive classes such as history, as they did not recognize many content words and it was difficult for them since the text and instruction exclusively occurred in English. The students appreciated when their content teachers allowed them to speak Spanish with one another to help each other with the new concepts, such as in their math class. A student reflected on a time when one of his teachers translated an entire math exam, which helped him and the other Spanish-speaking newcomers in that class greatly. Many of the students were familiar with the math concepts from their schooling in Mexico, which helped them in comprehending and transferring the skills to English. One student stated that mathematics classes were actually more advanced in Mexico, and therefore he felt his class in the U.S. was substantially easier. The students did report though that for many of them math was taught differently in their home countries, and therefore it was difficult, especially when trying to comprehend the word problems in 
math. The students felt they lacked the basic knowledge in how to read instructions, which made completing problems feel virtually impossible.

We all lack basic knowledge in how to read instructions. Without knowing how to do that we can't do a basic problem like two plus two equals four since we don't know what the problem is asking us to solve (ESL Student, 2011).

The students further asserted that content classes, such as their science classes, in which the teachers utilized lecture format as the primary mode of instruction were far more difficult for them to understand. Furthermore, the youth maintained that classes were harder for them when they felt the teachers did not adequately assist them in understanding the directions to assignments, they reported that everyone else would get to work in such classes while they would solely sit nervously, unable to complete their work. Students had a hard time understanding when teachers did not clearly pronounce words or spoke too quickly. Students needed to connect to their teachers and when they felt as though their teachers cared about them, they believed they could be far more successful in the class. The following quote demonstrates an example of how a particular student felt supported by his content teacher:

In math our teacher has been very helpful. He even translated an entire exam once and he is [often] finding new ways to help (ESL Student, 2011).

The second year ESL students stated that language intensive classes, such as history and science, were the hardest for them to understand. They felt the most nervous in classes in which they had to read aloud or participate in front of others. Some specific issues they had in their content classes were that in science class, the use of scientific terms was difficult for them, as many of them didn't know what the terms meant in Spanish or English, and therefore had no background knowledge of the particular term or 
concept. In addition, these students agreed that directions were hard for them to comprehend, especially in such classes as science and math, and it made it far more difficult for students when they did not understand what to do on the daily assignments or on their tests. The following quote illustrates the difficulties that one ESL student faced in her content classes during her first year in U.S. schools:

History class and I think science class [were hard for me] because I really didn't understand. In history we did a lot of writing and I always did really bad on the tests and in math too because I didn't know what I was suppose to do on the tests (ESL Student, 2011).

Culturally Responsive Teaching Practices Within the English as a Second Language Program

While discussing the need for culturally relevant teaching practices within ESL classrooms, the program administrators asserted that newcomer students come from many academic environments, both parallel and non-parallel to the U.S. academic system, which can be difficult for many teachers to comprehend. It is vital that teachers make the effort to understand students' cultural and educational backgrounds to better understand the manner in which students know how to learn. Often students are not educated in a setting analogous to that of the U.S. school system, and therefore these students must be explicitly taught how schools function in the U.S. The teachers should build on students' prior academic knowledge and experiences to assure students understand how to succeed, both academically and personally, in U.S. schools. One administrator, who had herself taught many students with limited formal schooling, expressed the need for teachers to understand students' prior schooling experiences: 
For [some this is the] first time they have been in a traditional school, so when they're running around and they don't know what to do, it's because they've never been in an environment where you sit at a desk or you listen to a teacher... they've never experienced that and that's a huge wake up call for teachers (ESL Administrator, 2011).

ESL teachers reported using multicultural texts and discussing different cultures and customs from around the world with their students, in an effort to assure more culturally relevant teaching practices. They would additionally have students compare cultures from around the world to their own home cultures. Teachers maintained that they made comparisons between various cultures to help students better connect to and understand the texts they were utilizing for instruction, and they encouraged students to share about their home cultures and customs in class. Additionally, if possible the ESL teachers would support students with their primary language, though this type of support historically only occurred with the majority Spanish-speaking population. One teacher reported utilizing her native language, Chinese, whenever possible, and she could also communicate with the youth in Lao, Thai, and Vietnamese, which were prevalent languages in her school community. This same ESL teacher also posted signs around her room in multiple languages. Finally, the teachers relied on parent liaisons and community organizations to assist them in communicating with parents in the languages with they were unable to communicate.

During the focus groups the students recounted that they were taught about U.S. culture and customs within their history classes. Students that were not enrolled in a history class did not feel they were explicitly taught about culture, but they were taught about common U.S. holidays in their ESL classes. Other students learned about the holidays outside of school, through friends, at times from teachers, and by living in the 
U.S. The second year ESL students stated that their teachers had them conduct research about U.S. culture and customs on the computer, and some teachers showed videos and had students write about U.S. culture. Although the newcomer students stated that they learned a bit about the U.S. culture, they felt their home cultures were not valued, in that students are not taught about their personal cultures and do not see their cultural backgrounds within the curricula. Finally, one second year ESL students further maintained that teachers only teach in English and do not speak about the importance of being bilingual to students, as is seen in the following quote:

Teachers don't have much to say [about being bilingual] they just give me the class in English and don't say anything about Spanish (ESL Student, 2011).

Teachers' Pedagogical Orientations Within the English as a Second Language Program

The program administrators viewed their primary role in the program as ensuring consistency across all ESL programs around the district. They believed that program uniformity would allow for the necessary collaboration among ESL teachers at various sites. Additionally, the administrators provided resources and training to the teachers in the program, in that ESL teachers were typically the only person at a given site servicing such students. The program administrators asserted it was their responsibility to co-plan and co-teach with the teachers to model best practices, yet, as was previously discussed, they often lacked the time necessary to work with the many teachers within their jurisdiction. One solution to the issue of time that was mentioned in the interviews was to use the new district technology to assist the ESL teachers in collaborating with one another across the district. 
ESL teachers kind of work in isolation, because what they do isn't like the regular English class.... And it's not like any other class campus, so my hope was that I could bring those teachers together a little bit more so they would work together. And I think you know I'm excited about the new technology because I think we'll have a lot of different venues where we can just converse with one another you know post lessons, get comments from other people, you know do all kinds of things like... (ESL Administrator, 2011).

Teachers in the ESL program viewed themselves as facilitators or coaches within their classrooms, as their responsibility was to teach students linguistic and academic skills and then allow students a chance to practice such skills. They were involved with their students both in their classes, but also by monitoring them as they progressed through their mainstream content classes. Teachers took highly active roles in their individual students' education and often advocated for them, both academically and personally to ensure their needs were being met. Students' needs were viewed on an individual basis to ensure they were best supported at their particular point of need. Finally, when grouping students for cooperative work, the teachers asserted that students were grouped according to their proficiency levels and particular areas of strength and need. Teachers grouped students heterogeneously according to both language and proficiency level, in an attempt to mix languages and to ensure language models within each grouping. In the following quote, one ESL teacher reflects upon her role as an educator in the ESL program:

[I am a] facilitator, I guess I would say. I have to be your second mother more so than just a regular teacher because I am monitoring not just our class but all of the classes and all of the teachers and what they are doing well and what they are not doing well (ESL Teacher, 2011).

As students entered the ESL program, many stated that their teachers attempted to speak Spanish to help them feel more welcome, and also assigned bilingual students to 
translate for them. Students appreciated the gesture, but most still felt uncomfortable in the new environment. At times, the teachers would modify independent work, which the students felt was quite supportive, and they would use gestures to help students understand. It assisted students understanding when teachers repeated concepts several times. The bilingual teachers were additionally very helpful for students, even when they instructed only in English, as the students knew they could speak in their primary language if necessary. Students' understanding was further facilitated when teachers had after school tutoring to allow students extra time to learn about concepts with their teachers in a more one-on-one setting. Overall, students learned best when they felt connected to their teachers.

My geometry teacher sometimes uses words in Spanish that help me understand ...he's always telling us to stay after school and he's the only one I've stayed with for help (ESL Student, 2011).

Students expressed significant tensions during the focus groups, as they often struggled in classes without primary language or English learner support. While it helped students when teachers utilized student tutors, dictionaries, computers, and student translators to assist them in comprehending instruction, they still reported struggling to understand the lessons and activities. They also felt it took too long and wasted time to use a dictionary to look up every word in a paragraph. The students asserted that teachers needed to be careful in using pictures to illustrate a concept, as often times the picture did not actually represent the concept about which they were teaching. Finally, some students felt that assigning a partner to tutor and translate was not always beneficial to them, as other students would get annoyed and have to complete their own work before they were able or willing to help the newcomers. 
Some resources teachers use are tutors, dictionaries, laptops, textbooks, translators and even that sometimes doesn't work. A dictionary wont look up an entire paragraph word by word... it takes time. A tutor won't always help because they first have to do their own work. That's a lot of time wasted (ESL Student, 2011).

Lastly, the a few students reported feeling put aside by the teachers, as they believed the teachers assumed they did not value education, which was completely untrue. The teachers often stressed the importance of learning English, but some students maintained that they did not discuss the process of finishing school or about how to attain a higher education with their youth. One student poignantly expressed the negative perception he held about how the teachers viewed him as a student in the below quote:

I also think that they see us as Latinos who do not value education and will not continue to go to school after high school. They set us aside and they don't explain to us anything other than stressing the important to learn English. We have a lot of pressure (English as a Second Language Student, 2011).

\section{English as a Second Language Students' Academic Self-Concept}

When asked about the academic self-concept levels of students within the ESL program, they program administrators stated teachers had informed them that students who had never participated before did become actively involved in their ESL classes. The youth appeared far more confident as a result of the supportive environment and their acquisition of language skills in their ESL classes. In addition, these same students were achieving great successes in attaining English and were able to communicate more effectively and comprehend the class instruction more fully, thus giving many of them the confidence necessary to take risks in class. The administrators maintained that within the program the challenges students faced in being English learners were addressed and discussed, which gave students a chance to reflect upon their experiences and to build 
quality interactions between students. Students were able to find others in their same position, which was very comforting to them in such an overwhelming setting. The administrators argued that the augmented self-confidence of the newcomer students who had complete the ESL program became apparent when viewing the increased high school exit exam passage rates and the higher high school graduation rates, which they asserted were a direct result of the ESL program. The following quote illustrates the view of the administrators about students' self-concept within the ESL program:

I believe that students are more confident academically and socially as a result of their participation in our program. They are able to find a niche for themselves in a very new and sometimes overwhelming setting. Our students are improving at a rapid rate. Many of our students are making one year's growth in one year's time. Many are exceeding that goal (ESL Administrator, 2011).

The teachers asserted that students in ESL 1/2 classes appeared more confident because they knew they needed the more supportive class and they felt safe, yet students at the ESL 5/6 level were more embarrassed to still be qualified as an ESL student. In addition, students often returned to the teachers for support and guidance, even as they were mainstreamed into higher-level classes. Teachers also reported attempting to espouse the importance of being bilingual to the students and empathizing with the difficult plight many of their students faced. They believed students were far more successful and confident in ESL classes than in mainstream and sheltered content classes, as they were more comfortable in an environment with peers in their same position. The teachers maintained the students were quieter in their content classes, in that most felt uncomfortable participating in front of native-born peers, and many were failing such classes. Although they often had other newcomer students with them in their mainstream classes, which helped alleviate their high anxiety levels, they still appeared far more 
nervous in mainstream classes. Therefore, the teachers were careful about how they placed students in mainstream classes, to attempt to transition the kids as seamlessly as possible.

Well, at first, I think in the morning class they jumped really, I think they feel lucky to be in here because they feel safe and they feel comfortable. In the afternoon, in the 5/6 you are kind of on that bubble, some of them... where we'll be in here and they will want to close the door and we close it because they don't want people to know that they are is an ESL student...Overall, though I guess I would have to say, yes [students appear more confident academically], because they come back and ask me even when they are not in my class any more. They come back for help with English or graduation or whatever. So, I think, yes (ESL Teacher, 2011).

During the focus groups, the students reported feeling uncomfortable when they entered their new schools as they felt they could not fully communicate with others in English. At the start, some worried other students were talking about them when they couldn't understand what was being said around them. Overall, the students felt insecure and embarrassed at school.

[In the beginning] I felt insecure because I felt like I wasn't doing things right. I felt insecure because I sometimes didn't know what people talked about and I felt it was about [me]. I felt less of a person than others...I was insecure and embarrassed because I didn't know the language (ESL Student, 2011).

In their easier classes the students felt they had the space to adjust to life in the U.S. and they often tried harder when they enjoyed the subject and felt connected and safe. ESL class was the course in which the students felt the most comfortable and it was the class where most were willing to participate and take an active role in the instruction. Students tried to make the best of difficult classes, but they often felt quite frustrated. They felt nervous when they have to present in front of others, and a few students even reported they wanted to cry out of frustration during their more difficult classes. Many 
students worried about the poor grades they received in difficult and incomprehensible classes as they didn't know how to remedy their poor grades. Finally, some students reported feeling a bit better after a few months, but they expressed they still had a lot to learn and they articulated feeling a bit hopeless. They did feel calmer and more independent as they began to understand more language, and some maintained that the teachers encouraged them to learn more and helped them feel positive about what they had acquired thus far.

I still feel strange in front of the rest but more confident in what I say...[Teachers] tell us we are improving and they show us our good grades and congratulate us because we are learning (ESL Student, 2011). 
Table 39

Qualitative Findings Overview - New Arrival Center Program

\begin{tabular}{|c|c|c|}
\hline Theme & $\begin{array}{l}\text { Driving Conditions } \\
\text { (positive) }\end{array}$ & $\begin{array}{c}\text { Restraining Conditions } \\
\text { (negative) }\end{array}$ \\
\hline $\begin{array}{l}\text { Stakeholders' } \\
\text { Attitudes }\end{array}$ & $\begin{array}{l}\text { A: Teacher-student connectedness; } \\
\text { schools happy about program } \\
\text { T: Specifically applied for program; } \\
\text { passionate; feel connected to students } \\
\text { S: Felt safer in U.S.; second year } \\
\text { students felt more comfortable }\end{array}$ & $\begin{array}{l}\text { A: Only one year; site tensions, NAC } \\
\text { classes isolated } \\
\text { T: Felt isolated; English acquisition } \\
\text { primary goal; little accountability } \\
\text { S: Nervous about starting school; } \\
\text { Uncomfortable participating in class; } \\
\text { culture different }\end{array}$ \\
\hline Curricula & $\begin{array}{l}\text { A: Curriculum committee chose ESL } \\
\text { adoption } \\
\text { T: District provided ESL } \\
\text { curriculum/pacing guide }\end{array}$ & $\begin{array}{l}\text { A: No prior content curricula } \\
\text { T: Pacing guides too extensive; teachers } \\
\text { have to create content curricula }\end{array}$ \\
\hline $\begin{array}{l}\text { Language } \\
\text { Instruction }\end{array}$ & $\begin{array}{l}\text { A: Many oral language opportunities; } \\
\text { technological support } \\
\text { T: Frequent language opportunities; } \\
\text { encompass all language domains; El } \\
\text { strategies; } \\
\text { S: Constant authentic practice; frequent } \\
\text { modeling; real-world, technology help }\end{array}$ & $\begin{array}{l}\text { A: None } \\
\text { T: Multiple levels and needs in one class } \\
\text { S: None }\end{array}$ \\
\hline $\begin{array}{l}\text { Content } \\
\text { Instruction }\end{array}$ & $\begin{array}{l}\text { A: Students with higher skills } \\
\text { mainstreamed } \\
\text { T: Focus on content language; more } \\
\text { flexibility; co-planning when possible } \\
\text { S: Concepts explained step-by-step; } \\
\text { learned foundation in home country }\end{array}$ & $\begin{array}{l}\text { A: ESL content courses receive elective } \\
\text { credit } \\
\text { T: Not confident/trained to teach content } \\
\text { areas } \\
\text { S: Math higher level in Mexico; History } \\
\text { too language intensive/new concepts }\end{array}$ \\
\hline $\begin{array}{l}\text { Culturally } \\
\text { Responsive }\end{array}$ & $\begin{array}{l}\text { A: Build on students' prior academic } \\
\text { knowledge/home cultures; primary } \\
\text { language support encouraged } \\
\text { T: Use multicultural texts and discuss } \\
\text { various cultures; support with primary } \\
\text { language } \\
\text { S: Learned about culture with } \\
\text { pictures/videos; English-only students } \\
\text { help connect languages }\end{array}$ & $\begin{array}{l}\text { A: Teachers should not instruct in } \\
\text { primary language (only support) } \\
\text { T: Teachers cannot communicate with all } \\
\text { students in home languages; program did } \\
\text { not address emotional needs of students } \\
\text { S: Mainly taught about holidays; home } \\
\text { cultures not valued; teachers do not } \\
\text { promote bilingualism }\end{array}$ \\
\hline $\begin{array}{l}\text { Pedagogical } \\
\text { Orientations }\end{array}$ & $\begin{array}{l}\text { A: Consistency across school } \\
\text { sites/uniformity; Support system } \\
\text { T: Teachers as facilitators /counselors; } \\
\text { student choice valued } \\
\text { S: Learned best when connected to } \\
\text { teachers; teacher more patient }\end{array}$ & $\begin{array}{l}\text { A: Lack of time to work with ESL } \\
\text { teachers } \\
\text { T: English acquisition primary focus of } \\
\text { instruction } \\
\text { S: Less connected to teachers second } \\
\text { year; feel nervous asking teachers for } \\
\text { clarification/rather ask peers }\end{array}$ \\
\hline $\begin{array}{l}\text { Academic Self- } \\
\text { Concept }\end{array}$ & $\begin{array}{l}\text { A: Students more } \\
\text { comfortable/connected to teachers; } \\
\text { youth more confident; increased } \\
\text { graduation rates } \\
\text { T: More confident; more successful in } \\
\text { NAC classes } \\
\text { S: Felt better/tried harder in classes } \\
\text { they connected to/enjoyed more }\end{array}$ & $\begin{array}{l}\text { A: None } \\
\text { T: Students more nervous/higher anxiety } \\
\text { in mainstream classes } \\
\text { S: Silent/gave up in difficult classes; } \\
\text { nervous/anxious in new school }\end{array}$ \\
\hline
\end{tabular}

Note. $\mathrm{A}=$ Administrators $1 ; \mathrm{T}=$ Teaches $; \mathrm{S}=$ Students 
New Arrival Center Stakeholders' Attitudes and Expectations

The primary expectation of the NAC program administrators was to create a setting in which newcomers would have intensive opportunities to learn and acquire English in a meaningful way, to become oriented to what it means to live in the United States, and to prepare students for some of the courses they would be taking for graduation credit upon finishing the specialized program. One administrator stated that the NAC program was originally created in response to the diverse needs of newcomer students at the secondary level. Despite the fact that they are a small population, newcomers' issues are so distinct from other English learners that schools struggled to adequately address the needs of such students. She went on to assert that, for the most part, schools were thrilled to have NAC programs on their campuses, though tensions occurred at times in the implementation of the program.

Our expectations are really to create a setting in which kids could accelerate their acquisition of English, become oriented to what it means to live in the United States and some other things that we take for granted because we lived here for a while our whole lives that we know and are familiar with. So just to help orient students to U.S., to being a student and to give them really intensive opportunities to learn and acquire English in a meaningful way and also then to prepare them for some of the courses they'll be taking for graduation credit the following year, so that was one of the designs as well as at the first year, basically all the courses students take are electives (New Arrival Center Administrator, 2011).

In creating the program, it would have been far easier logistically to implement the program at one separate site, rather than having centers at several comprehensive sites, in that certain tensions, such as negotiating with various comprehensive sites' master schedules, intake procedures, and class numbers, would have been alleviated if the NAC had been at one site. Ultimately, though, comprehensive sites were chosen as the program model due to budget deceases, as well as the underlying program principle that 
the students' school experiences should be as close to possible as those of where they will be transitioning after they have acquired sufficient language. Therefore, the administrators asserted that it was important to make this program as much a part of the comprehensive site as possible.

In implementing the program, the administrators stressed that teachers are considered the primary person to advocate for students and are charged with determining placement for the following year. Although a two-year program was originally envisioned for NAC classes, students are generally only permitted to attend the program for one year. In rare circumstances students have been permitted to repeat it for a second year if it was determined to be absolutely necessary. Students enter the centers with very diverse academic needs, so there needed to be a significant amount of flexibility built into program. The district contended that most students enrolled in the NAC program will be on a five-year track to complete high school. In the following quote, one administrator spoke to role of the teachers as the primary advocates of the students within the NAC program:

... because the teachers are so knowledgeable about their own students and about what they need, the teacher really is the primary person to advocate for the student and do all the placement and make sure everything happens the way it needs to happen for the student (New Arrival Center Administrator, 2011).

New Arrival Center teachers felt very passionate about teaching newcomer youth. One NAC teacher even recounted her struggle learning Spanish and believed she could empathize with the students as they learned English. The teachers all specifically applied for the program, and therefore, they were very devoted to the needs of newcomer youth. They were aware of the great need for a program devoted to newcomers, as many of 
these students had come to the U.S. with differing prior education levels. The teachers reported feeling connected to the students, and they believed there was great importance in learning about the backgrounds of their students, in that it was crucial in the process of educating the kids. The ultimate goal of the teachers within the program was to get them speaking English as quickly as possible, and therefore, the teachers reported constantly reminding students to practice English while in class. The below quote demonstrates one NAC teacher's understanding of the program expectations as she transitioned from her former elementary position to her current NAC position:

I felt that a lot of the things that I had been doing in my current classroom as an [elementary] teacher, I felt like that was going to be the expectation... but I [also] knew that that was going to be the ultimate goal was to get them speaking English as quickly as possible (NAC Teacher, 2011).

Some tensions reported by teachers were that they felt isolated at their sites, particularly if they were the only NAC teachers at their particular site. One teacher mentioned that she felt a certain level of hostility from the general education teachers at her site, likely due to their lack of understanding about the NAC program and their belief that the program was utilizing much needed resources. The interaction among NAC teachers at different sites was more limited than teachers expected. They were hoping for more discussion and collaboration among colleagues. The teachers enjoyed the professional development, which allowed them the chance to speak with other NAC teachers, but they had been far more limited in recent times. One teacher even stated that there had been no meetings or trainings that year for veteran NAC teachers. Teachers felt there was not enough professional development and they worried they will not grow as teachers because of a lack of training. Finally, one teacher reported that she hadn't been 
observed all year, as the program administrators were more concerned with the newer NAC teachers than the veteran NAC teachers, and her site administration did not appear concerned with her program or instruction. She wanted feedback about her teaching, but no leadership from her site or from the program had visited her room. She felt there was a lack of support and accountability within the program at the district level.

I expected there to be more interaction among the teachers in the program, you know, instead of just kind of like here this is what you are teaching, you know ... more dialogue, .... and I was a little, I mean last year I was a little surprised because there wasn't and I just thought that was odd. You are the only one who has observed me [this year]. I want feedback. I have no clue... I am sitting here thinking, you know, if they don't... I mean thank goodness that I'm a diligent, conscientious person. I am doing this because I want do this, but, nobody comes in (New Arrival Center Teacher, 2011).

During the focus groups, several students discussed feeling nervous about starting school in the U.S., in that they believed no one at their school spoke Spanish. After the students arrived they reported feeling more comfortable, particularly after realizing that people did, in fact, speak Spanish at their schools. A few of the students also worried they would be the oldest students in their classes, but they realized they were incorrect after starting school. At the start, many felt uncomfortable participating and reading aloud in class, since they felt they had not acquired sufficient English, and, therefore, the students who had studied English prior to moving to the U.S. felt far more comfortable in their classes.

The first day I got home I wanted to cry because I was scared. Even though I had my cousins in the same school and hung out with them, in my classes I thought it was going to be the same as in Mexico, but it was not the same (New Arrival Center Student, 2011).

NAC students reported that people in the U.S. felt culturally different from them, yet they maintained that the difference was not generally an issue. The problem many had 
was when native-born students spoke English and the newcomers couldn't understand, which made them feel like outsiders in their schools. Most newcomer students felt nervous about not knowing other students when they started, particularly when considering such concerns as with whom they would eat lunch or spend other breaks. Additionally, for many students, it was hard to leave family behind in Mexico and they reported feeling their family was incomplete, as some family members were in their home countries and others were here in the U.S. They also reported missing their friends and their lives in Mexico. Other students, though, maintained they felt safer in the U.S., as they believed there was less crime in the U.S. than in Mexico.

I don't have anything against anyone, everyone is different. There are certain people I don't like and others who don't like me. But it is a bit uncomfortable... I don't want to feel uncomfortable. There are times people are talking and I can't understand so I get bothered (New Arrival Center Student, 2011).

Students who had completed the program the prior year felt more comfortable because they had acquired more language and therefore understood more, but they were still hesitant to speak English since other kids teased them. They reported not completing homework because they did not like doing it, though many did have people to go to if they did not understand a concept. Others also did not do the work because they did not understand what they needed to complete. Overall, these same students did not feel valued in the U.S., given that they felt there was a lot of discrimination against them and they thought students often made fun of them at school. According to the focus group participants, American-born students made blatantly disparaging remarks to them at school, and although some teachers helped in such situations, the students generally had to stand up for one another. Many of these students stated that they wanted to return to 
Mexico because they felt more connected with the people and the culture of Mexico and, in their view, everyone there felt similar to them. They also reported missing their friends and family, and the food to which they were more accustomed in Mexico.

[I didn't feel respected] because last year they were really racist... when you spoke to [the other students] in Spanish they pretended they didn't know because they didn't want to talk to you. One day [some kid] threw a soup on us and said that we wouldn't do anything because we are Mexicans (New Arrival Center Student, 2011).

\section{New Arrival Center Program Curricula}

During the interviews, the administrators reported that after they were given the task of developing the New Arrival Center program (NAC), they opted to utilize the ESL adoption for the English Language Development portion of the NAC instructional day. The Pearson Longman's Keys to Learning and WRITE materials that were being used within the ESL 1/2 program were then purchased for the New Arrival Center classes, in addition to supplemental materials that were bought for the ELD portion of the NAC day. A curricula committee made up of ESL teachers and resource teachers had initially chosen the Pearson Longman's Keys to Learning materials through the regular adoption process several years before, and the WRITE curriculum had been a component of the ESL program for many years prior to the inception of the New Arrival Center. One NAC administrator detailed the process of choosing curriculum for the New Arrival Center in the following quote:

...we use the Keys to Learning for the ESL and that was chosen by regular school adoption committee through the regular adoption process. We used the WRITE institute materials also and that was chosen really by the department. We've used WRITE Institute training materials for a number of years and with a lot of success and so, we've been integrating that in there. The other materials were chosen through talking to publishers and looking at materials and teachers who have had experience teaching ESL 1/2 (New Arrival Center Administrator, 2011). 
The content materials for the New Arrival Center program were bought "from scratch", in that there were no appropriate materials for the social studies, science, and math components of the NAC program at its beginning. The content materials were decided on after eliciting recommendations from ESL teachers who had used such materials, in particular the social studies texts, in their ESL 1/2 classes. The program administrators chose the science materials after having viewed them in various presentations. The administrators stated that the overarching intent of the content instruction and materials were to provide foundation knowledge and content language, rather than grade-level content knowledge, and therefore the essential standards for the NAC content courses encompassed both high school and K-8 standards. The standards were chosen for the program because they were more concrete concepts, and were meant to develop language and teach key concepts to ensure later success in mainstream classes. The administrators claimed each NAC teacher was provide with a year-at-a-glance pacing guide, which was aligned to the standards in each content area. The following quote demonstrates the process that occurred in choosing content materials for the NAC classes:

Well, it was... some was trial and error but it was, you know getting recommendations from teachers who had been...who had used some social studies and science materials, particularly social studies, in their ESL 1/2 classrooms. Science we just...I'd been to some presentations I'd seen some materials that I knew were appropriate for ELD and for ESL. So, we purchased those for the very beginning [levels] and then for higher levels as kids go through the year. We just had to really experiment. We tried to use the elementary Foss kits. And we did use them the first year but we haven't been able to... we can't get on their rotation and we don't really have enough people, enough classrooms to be able to support the program ourselves. So, we've gone to other materials that look at...that address grade level standards. The kind of foundation knowledge needed for the grade level standards and [that] also have a focus on language (New Arrival Center Administrator, 2011). 
Throughout the New Arrival Center teacher interviews, the teachers reported using Pearson Longman's curriculum Keys to Learning, as well as Champion Reader, and the locally created WRITE curricula, all of which were provided by the district for the ELD portion of the New Arrival Center daily instructional schedule. The Keys to Learning curriculum provides specific vocabulary and grammar instruction, as well as short dialogue pieces, and while the teachers preferred this text, many felt it is much too short and wished they could have more. One teacher claimed the WRITE curriculum was too advanced for her students and felt it was not sensitive to her students' needs and their cultural differences. Conversely, a different teacher stated that the WRITE curriculum offered a plethora of units and was highly supportive of the students. She felt it could be used effectively in conjunction with the explicit language instruction found in the Systematic ELD training provided by the district, which focused on providing students with language and sentence frames to support them in developing their language proficiency levels. The teachers further supplemented their ELD curriculum with short stories and songs to give students a more authentic language experience. The following quote illuminates one NAC teacher's perceptions about the WRITE curriculum and how she was able to incorporate it into her class:

I love WRITE...I love having a writing program because the district doesn't have a writing program and [in elementary school] it was very difficult so when I came here and they had actual lessons and suggestions and sentence frames, all that scaffolding done for the writing...I just thought wow.. How perfect can it be! And there are so many different units! So I love the WRITE... I use it along with the systematic ELD because it works in correlation and I think the students really master it because they have so much support (New Arrival Center Teacher, 2011).

The district provided pacing guides and curriculum for the ELD class, though one teacher believed they were too extensive and moved too quickly for her newcomer youth 
to have time to effectively process the material. The students were not being provided sufficient time to practice, as the teacher felt she had to move too quickly through the material. The pacing guide provided for math instruction was helpful, though, as the teacher was not as comfortable teaching math. The teachers reported having to create and supplement a significant portion of the science, social studies, and math curricula. According to the teachers, the district had not supplied the teachers with well-developed pacing guides or specific curricula, though more recently it appeared some curricula and pacing guides were being developed and improved. The content texts provided to the teachers, Access Science and America's Story, were more akin to supplemental materials and were not actual curricula, therefore forcing the teachers to develop a considerable portion of the content instruction and activities utilized with the students. The NAC teachers often shared ideas to assist one another in developing content units, as they were charged with ensuring the students gained the necessary content language and foundational knowledge to be sufficiently prepared to enter sheltered and mainstream content courses the following year. One NAC teacher described her frustrations with the lack of content curricula:

They provide for us a pacing guide for the Keys for Learning, which is our literacy book, but also the Champion Reader. They tie in the lessons, and what is also going to be helpful from the WRITE Institute...but it's all just for literacy and writing...nothing else. We have started to build some curriculum guides for science and social studies, but I feel like that is in the making, like every time that we got together for professional development through [the central office] we were still building that...so there's no pacing for social studies and science (New Arrival Center Teacher, 2011). 


\section{Language Instruction Within the New Arrival Center Program}

The administrators overseeing this study reported that an essential component of the English Language Development (ELD) portion of the day within the New Arrival Center program involves ensuring students have daily opportunities to practice all language domains, reading, writing, listening, and speaking, in English. They stated that the teachers in the NAC program should know that the end goal of their program is to accelerate language, and therefore, they are charged with structuring the day in a way that encourages and supports students in using language throughout the day. Students are provided daily language teachings through explicit language instruction and the WRITE curriculum and activities. According to the program administrators, the computer program called Rosetta Stone is also to be utilized for instruction, giving teachers the opportunity to work with students in small groups. NAC students are encouraged to practice authentic oral and written language frequently within the program, and reluctant speakers are encouraged to participate through partner work and collaborative opportunities. As all district teachers are provided technological supports, reluctant speakers may also use the classroom microphones to ensure others hear them as they speak. Finally, the program administrators asserted that students must be given frequent opportunities to practice language before being asked to speak before others, in an effort to ensure students remain comfortable and confident within their classes. One program administrator stated the following about the language instruction within the NAC program:

[Students are encouraged to practice language both orally and written] which is built into the curriculum and built into their school day. Part of it is instructional pedagogy and practice and part of that would be the tasks that are part of the ESL 
curriculum and then the other subject areas as well (New Arrival Center Administrator, 2011).

In discussing the language instruction that took place in their NAC classes, the teachers reported creating frequent opportunities during class time for students to experience using authentic oral language. Some examples of such opportunities included the use of daily morning messages, a short time each day in which one student would share out a greeting in front of the class, as well as answering phone calls within the classroom and greeting visitors as they entered the room. One teacher reported using similar language strategies in her high school NAC class as those she had used when teaching elementary school, such as conducting read-alouds and using shared reading as a part of literacy instruction, and implementing a dedicated time for independent reading. The teacher did acknowledge, though, that these literacy strategies must be elevated to ensure they are age appropriate for her high school students. Additionally, the teachers utilized choral reading strategies to guarantee a low-anxiety environment in which kids felt comfortable practicing reading out loud in English.

I'm always dealing with second language learners, so a lot of the scaffolding that I was doing for third graders I have to do here. Of course, the instruction is bumped up quite a bit, but because the language of these students is so low a lot of the strategies, a lot of the scaffolding, I'm able to apply them here... so I have a lot of background based on the curriculum that I used to use, and I actually have applied it here...(New Arrival Center Teacher, 2011).

During the interviews, the teachers recounted that they often had students work in partners to more effectively support them in accessing information and to help them understand new concepts. Students were purposefully placed with partners and in cooperative groups, and the teachers attempted to make sure that each grouping had at 
least one student with high English language skills to model language for others. In other words, teachers utilized stronger speakers to help demonstrate language for the newer students. Other common strategies utilized in the classes were as follows: teaching at a slower rate and repeating information to help students access the lesson, modeling responses for students to scaffold information, and using visuals and pictures to support students in acquiring new vocabulary. One teacher asserted that her students came in as readers and writers in their primary languages and therefore the focus of her class was to build vocabulary, rather than teaching phonics in English. As the year progressed, students were expected to advance beyond simply identifying pictures, to analyzing and interpreting texts at their appropriate language proficiency level. Students were also required to begin using their newly acquired skills to identify new and unknown words.

One NAC teacher described the strategies she used during her instruction to support students in understanding the language:

I speak at a slower rate. I use my hands a lot. I repeat things, so I don't just ask a question once and then expect them to answer it. So, I'll ask it, I'll say it again, I kind of wait. I try to give visuals, a model of what or how their response should be, and then I'll also... like with the stronger students I'll call on them first so that they can kind of help [the others] out (New Arrival Center Teacher, 2011).

While conversing about the manner in which they acquired English, New Arrival Center students expressed that for many of them it was common to both practice and learn new English terms while speaking with friends and family members outside of school. Many students reported having English-speaking friends and family members who helped support them in learning English.

... my cousins who live here don't speak Spanish well and they help me understand... when we go to the store they will say what they are talking about and I answer them (New Arrival Center Student, 2011). 
Students recounted using a district-provided computer program, Learning Upgrade, at home to practice language and many asserted that they would also listen to songs and translate the lyrics to learn new terms in a way that they believed was more interesting and enjoyable. One student additionally remarked that he practiced reading English texts after school to try to learn new vocabulary words. Students also felt they were able to use English to negotiate their world outside of school, for example when going to the store or while speaking to agents while crossing the border to Mexico, which assisted them in learning the language.

I learn [English] when I go to Tijuana and I cross back... the agents there ask me and I answer them in English. They ask how old I am, what school I go to, what grade I am in, and they ask where I live and I tell them... questions like that and sometime I get to chat with them (New Arrival Center Student, 2011).

\section{Content Instruction Within the New Arrival Center Program}

During their interviews, the administrators from the New Arrival Center program stated that the content classes within the NAC were considered elective classes, and therefore, in attending these classes, the students enrolled in this program received elective credit toward high school graduation. Students need a number of elective credits to graduate, so the ESL Mathematics, ESL Science, and ESL Social Studies courses that are a part of their self-contained classes within the program counted towards this graduation requirement. The focus of the content cases was primarily centered on developing content language and strengthening students' fundamental skills, such as reading maps in social studies, using rulers and other mathematics tools, and observation and investigation within their science courses. In addition, the ESL social studies part of 
their daily instruction focused on U.S. history to help newcomer students orient to the United States.

...with the content area standards in science and social studies...they are basically focusing on elementary and middle school standards that align to high school. So, because it's an elective class we are not bound by the high school standards. If we were... they would be earning grade level credit, but we realized that it's not realistic... they cannot really learn photosynthesis as well as they need to [in order to] meet the grade level standards. And then as far as math goes, again it's elective credit with a focus on language [which] strengthens their basic math skills, so that they can go out to algebra the following year (New Arrival Center Administrator, 2011).

Students that entered with higher-level mathematics skills or who attained sufficient language during their time in their NAC classes, were often mainstreamed for math, and some were also placed in grade-level science courses. Students that were able to attend such classes were able to start accruing graduation credits in the different content areas, and therefore it was desirable to mainstream students that were ready for such courses. The NAC students were all mainstreamed for PE and, in some cases, a nonlanguage intensive elective such as art. After the students had completed their requisite year in the NAC program, the schools were encouraged by the program administrators to cluster the students into content classes with teachers that would be able to best support students newly acquiring English. Finally, the district administrators stated that most secondary-level content-area teachers in the district had been trained in an approach, referred to as Quality Teaching for English Learners (QTEL), that offered a high level of support for all levels of English Learners, and ideally these teachers were utilizing such strategies in their content instruction.

In preparing to teach within the New Arrival Center, one teacher stated she felt overwhelmed when considering how to teach unfamiliar content areas, for example 
mathematics and science. After she learned that these courses involved teaching content language and foundations, the teacher reported feeling far more comfortable. As a result of attending such a program, she had witnessed student successes in their content areas, for example students were being adequately prepared for grade-level sheltered Algebra the following year. This was significant for her newcomer students, in that many were learning a different kind of math than they were used to, for example in many countries students are not taught using algebraic variables, which made it a difficult concept for them to conceptualize in their U.S. math classes. Additionally, many students had studied world history in their home countries, so the ELD Social Studies class within the NAC program focused on the important events in American history to build students background knowledge for the following year, at which time they would take sheltered or mainstream U.S. History. The subsequent quote demonstrates one NAC teacher's initial insecurity in teaching a content area about which she had little training or experience:

... last year, which was my first year, I was kind of in a panic and feeling overwhelmed because I was teaching math which is not my forte. But now I look at it more like it's a linguistic class versus an academic [class]... Yeah, it's not algebra. It's just kind of getting the language and bringing their basic skills up. So, I am okay with that (New Arrival Center Teacher, 2011).

As was previously stated, the content classes within the NAC program focused on teaching content language and vocabulary rather than content information. Much like the program administrators, the teachers felt it was important to teach kids foundational skills in math and other content areas to ensure they would have the skills necessary to be mainstreamed the following year into their grade-level content classes. Owing to the fact that the NAC program was a self-contained class, the content instruction was often linked to concepts being taught in English, which created a more seamless flow to the day. 
Teachers often utilized primary language support and connections to students' home cultures to support them in understanding the new vocabulary and concepts in a culturally relevant and linguistically accessible manner. In creating their lessons, the teachers did incorporate grade-level content standards, though they were highly scaffolded to make certain students could access the information. The teachers expressed that it was important to, at the very least, expose NAC students to grade-level content.

We do look at the standards, but our students are not going to be able to perform some of their standards, so we adjust the standards so they are able to get exposure. For example, last year I had my students look at a microscope.... we actually looked at cheek cells and onion cells, and I know that is part of the biology standards later on. My purpose was not so much to get into an in depth study of it, it was mostly exposure... do you know how to use a microscope or would you know how to look for a cheek cell or an onion cell if given that... how to use vocabulary words needed to do such a study... that was my purpose, not so much going deep into that study (New Arrival Center Teacher, 2011).

Although the program administrators were interested in maintaining the fidelity of the NAC program, they did recognize that there would be some variability within the program at the site levels. Owing to this reality, the site administrations had some freedom in deciding the manner in which the program would transpire within their schools, and therefore, one teacher reported that her site opted to mainstream all NAC kids for math, despite their entering foundational skills. The students at this school had historically done very well in math, and the principal wanted to maintain their previous placement policy for math. The teacher did attest to this practice as her newcomers were doing well despite being placed in mainstream math, though she did assert that her students participated far less in the mainstream class than in her NAC class.

Another common difference among NAC classes at different sites was that at schools with multiple NAC classes, teachers were able to group their students and 
conduct rotations for social studies and science, ultimately creating fewer classes for which they would need to prepare. The teachers at these sites were conscious that the grouping did not work if they separated language levels, as it left the lower levels with no language models, and therefore they heterogeneously mixed the content groups. One teacher described the practice in the following quote:

We do a rotation because there are two other NAC teachers here so I take care of the science portion of the class and my colleagues take care of the social studies... and we divide our classes into three different groups. We tried to divide them up by levels, but we found it was really difficult to teach anything because we didn't have any students that...for example the beginners... we didn't have any students that would be able to give us any feedback because they don't have enough language...so [there would be] no modeling in there or supports.

Therefore, we decided to mix our students and mix the language abilities and split up the classrooms in three (New Arrival Center Teacher, 2011).

During the student focus groups, the participants maintained that teachers were diligent in explaining difficult math and other content-related concepts step-by-step to help them better understand. They said that it helped them to comprehend the instruction when the teachers wrote information and worked out problems on the board. For some students, math was easiest because they had already learned the foundation of math in their home countries, such as in Mexico. Some also reported that the math they had taken in Mexico was higher level than what they had learned so far in the U.S. The students felt more comfortable in this class, as they recognized the numbers for math, despite the language and vocabulary differences.

Math [is easiest] because what we are doing, I already saw it in Tijuana....and for me it's easy because the numbers are the same in Mexico as they are here. Often you realize they're reviewing the [same concepts] here... the numbers are the same but they are in a different language... in English here and there in Spanish. All you have to know to understand mathematics are the numbers in English (Mew Arrival Center Student, 2011). 
In discussing their content classes, the students said they found science to be easier than history, in that history was purely based in language and reading, whereas science was more hands-on and teachers frequently used manipulatives to help them access the information. Students also expressed that history was difficult as they were not familiar with U.S. history. At one comprehensive NAC site, Physical Education was hardest for the students, as the teacher did not speak Spanish and they didn't understand him when he instructed in English. The students felt the teacher lowered their grades since they did not understand and could not complete the work. This was a negative experience for them, which was unfortunate as it was their only mainstream class.

$\mathrm{PE}$ [is the hardest class] because the teacher that [we] have does not speak Spanish and he says to do the homework but he speaks purely in English and usually we don't understand him, we don't do it, and our grade is lowered (New Arrival Center Student, 2011).

Students that had completed the NAC program the previous year, reported that they struggled most with teachers with whom they were unable to connect academically and personally. The students had a difficult time in mainstream and sheltered content classes, such as science, in which the subject was taught almost exclusively through lecture, and in which they were expected to present frequently. Second year students did report that math was easiest for them, as the teachers wrote the problems on the board and they could better understand the visuals as presented through their instruction.

[The easiest class is] math because the teacher writes everything on the board and in other classes they don't.... it helps to see what they are talking about. I learn more (New Arrival Center Student, 2011). 


\section{Culturally Responsive Teaching Practices Within the New Arrival Center Program}

While addressing the concept of culturally responsive teaching practices, as were defined by theorists Gay (2010) and Ladson-Billings (1994) in Chapter 2, Literature Review, the administrators maintained that teachers were encouraged within the NAC program to build on students' home cultures as a part of every lesson. In other words, the students should continually feel connected to their home cultures and languages within this program. Students were encouraged to read texts in their primary language, and teachers were advised to use students' primary languages to support their lessons when possible, though owing to the fact that most teachers spoke English and Spanish, this support was knowingly limited to Spanish-speaking students. Though the administrators contended that primary language should be used as a support, teachers were discouraged from using students' home languages as a primary piece of instruction, in that administrators wanted students immersed in as much English as possible. They also worried that this practice would exclude students with language backgrounds other than Spanish from being active members of class discussions. To alleviate the concern of students feeling excluded, students were encouraged to help one another with their own home language support, though teachers were asked to enforce short periods of Englishonly time, as short as ten-minute increments of English-only class time. One administrator acknowledged the importance of incorporating students' primary languages into daily practices in the following quote:

If the purpose is using English and applying English then you know [in class] would be the time...but using their language and validating it and respecting it and demonstrating value and support for it, that's something that needs to be a part of every classroom.... at all the New Arrival Centers (New Arrival Center Administrator, 2011). 
The teachers that had acquired sufficient Spanish language skills themselves were often willing and able to use primary language support with their Spanish-speaking students, though one teacher did report that she did not use Spanish in her class as she felt it was inequitable to the students from other primary language backgrounds. This teacher reported 14 language backgrounds were present in her class, and although her primary language was Spanish, she solely used Spanish language support outside of the students' academic day. Another teacher had acquired Spanish as a second language herself, which she believed gave her greater empathy in teaching her students English. She was able to better understand the difficult process facing her students, as well as the anxiety often felt by those learning a new language. All of the teachers did report that students were permitted to translate for one another during class, in an effort to support newer students in accessing the lessons and activities in class, but all students were encouraged to practice speaking English as much as possible. Finally, to assist communication between teachers and families that spoke languages unfamiliar to the teacher, many reported seeking out translators in the students' communities, or using students as language brokers for their families, though this practice was not considered ideal to the teachers.

In the past when I had, like I had Somali student, I couldn't communicate with her or her mother, [so] I contacted the mosque. I try to contact people that I know or students that I know. I had a Vietnamese kid who would come and [speak] Chinese to the Chinese parent, that sort of thing. ... I don't know if that's, I mean, I know that there are translators out there, but, I don't know, it is easier for me to go and ask somebody than to go to the district (New Arrival Center Teacher, 2011).

In discussing culturally relevant teaching practices, one teacher expressed that she had been studying the value honoring cultural differences in her master's program. She felt it was important to honor her students' cultural backgrounds, and therefore she 
approached her students' education from a collectivist point-of-view, as this was more appropriate within her students' home cultures. She further believed the curriculum utilized in the NAC program should focus more on the experiences and feelings of students after they have arrived in the U.S., as it was important to actively confront the issues students have had to face upon entry, rather than suppose that their experiences are positive and without problem in the U.S. She maintained that the NAC program did not address the anger and discomfort many students felt upon arrival to a new country. Lastly, the teacher reported that some of the units provided the NAC teachers were not culturally appropriate; for example, despite its importance to U.S. history, a writing unit concerning the Vietnam War was unknown to many other cultures and offensive to those from Vietnam.

[The materials are] not culturally sensitive and I think more of the focus shouldn't so much be on the history but, what are the issues you have when you move here... that that you have to deal with, you know, [such as] the missing family, having the [new] food and how it upsets your stomach because ...all of those things that are so different. I think a part of it is because either the people that are guiding it haven't had those experiences when they've lived in another country or [they haven't] been with someone who has moved here from another country and [there is], you know, a honeymoon period where you're really excited and then... why are you here and you're hateful and you get the kids with attitudes and they are angry and...there is so much this program could do (New Arrival Center Teacher, 2011).

The teachers felt it was a part of their responsibility to teach students about the culture of the United States and how to become active members of society. With the goal of teaching students about the important customs and traditions within the U.S., teachers reported explicitly instructing on such topics as the food, language, religion, regional geographical features, holidays, and clothing found within the U.S., and then having kids compare such subject matters to those of their home countries. In the following quote, 
one NAC teacher discussed how she connected students' home cultures to that found within the United States:

I'm always trying to [connect to their cultures] whether it's science, history, English.... how do you say this in your language, what is it, do you have this in your country, you know, so there is always that connection... and so [when students] compare and contrast I want them to do that....and also in their descriptive writing there was a lot about themselves and their country...(New Arrival Center Teacher, 2011).

During the focus groups, students recounted that some teachers spoke to them in Spanish, but they felt that the teachers primarily wanted them to speak only in English. The students had a difficult time understanding many of the teachers, in that they often spoke English very quickly and did not pronounce the words carefully. The NAC students did feel valued, though, when English-speaking students asked them how to say certain words in Spanish and when they were curious about the connections between English and Spanish, and they felt it reinforced the importance of being bilingual. Additionally, at times the students were able help the teachers who were not fluent Spanish speakers.

Sometimes we speak Spanish and they can learn from us and sometimes they ask us how to say certain words in Spanish...they ask what is the difference [between English and Spanish words]...they teach us English and we can teach them Spanish and it can be better for your career and all... in Tijuana it's important to learn English and Spanish. Well, even here it's important to learn Spanish... even already knowing English (New Arrival Center Student, 2011).

The focus groups stated that teachers taught students about U.S. holidays using calendars and pictures, in addition to instructing about the manner in which people operated in the United States. The teachers used videos and books with images to delineate concepts related to U.S. culture and important historical events. The students 
reported feeling people in the U.S. were too sensitive, as they were used to joking with others as they had done in Mexico, which was perceived in the U.S. as being offensive. Finally, the people in the U.S. seemed less carefree and accepting to the students in the focus groups, than those with whom they were accustomed in their home countries.

\section{Teachers' Pedagogical Orientations Within the New Arrival Center Program}

The administrators within the NAC program viewed their role as that of a support system for teachers working within the program, as well as helping to support the program in growing and flourishing. Their goal was to ensure that students acquired English and achieved academically within their classes. The administrators wanted to ensure consistency within the program and to collaborate effectively with site administrators to make sure the program maintained its fidelity while being an integral part of the school site in which it was housed. One administrator also expressed her desire to support the teachers in lesson planning, though with 11 NAC classrooms she felt she was unable to do this as much as she would have liked.

I view my role as support, basically I want to know what's going on in all the classrooms and in the program so that we can maintain consistency. So part of my role is to help ensure that consistency of program. Part of my role is to service the coach to teachers to help them with lesson planning to co-teach, it hasn't happened a lot and with 11 classroom it happens less and less... (New Arrival Center Administrator, 2011).

While the program administrators collaborated with principals, they ultimately wanted the principals to feel ownership, as the NAC program was located at their schools and it educated their students. Furthermore, the site principals were charged with evaluating the NAC teachers, as the teachers were a part of the comprehensive site in which the program was located, and thus it was important for principals to understand the 
philosophy of the NAC program and to have a voice in its implementation. The

administrators, at times, worked with the stakeholders at the sites, negotiating the manner in which the program was to be conducted and serving as an intermediary between the principals, the program, and the teachers. The administrators believed that students needed to be fully participating in their schools, and despite the fact that the central office was funding and supporting the program, they wanted to release responsibility to the site while still maintaining the quality and integrity of the program.

We did a kind of collaboration [with schools and principals] We tried...we are very, very collaborative and very open, but ultimately with the classrooms and the assignments, we had some negotiation that occurred. You know it's their school and they're their students. So, and we want that, we want them to feel ownership for their program as well. And so they have a huge say in how it's all operated...they actually evaluate the teachers and we don't, because they are on the campus, they're teachers on the campus (New Arrival Center Administrator, 2011).

When asked what they perceived their roles were in the classroom, one teacher stated that she viewed herself as a facilitator in the classroom and, depending on what students needed, as a counselor and mediator to students. Students often spent their lunchtime and any free time in her classroom, as this was the location in which they felt the most comfortable. Another teacher reported it was her job to teach students how to be model students in the U.S. She believed it was her role to teach them the skills to survive, both linguistically and culturally, outside of the classroom. The teachers also felt their task was to make sure students acquired sufficient English to be mainstreamed into English-only classes the following year.

I feel like I hold a lot of weight in this classroom and sometimes that could be good and that could be bad...I mean these kids are expecting, they are looking at me, they are depending on me to provide them the necessary skills to survive in the outside world of this classroom, so I see myself as more than just a teacher and so that's how I build a lot of my relationships with these students because, 
like I said, they depend on me to give them the right information...the right guidance so they can move on from this class and be successful, not only in school but in life, and here in America. So I'm teaching them not only the necessary things in the classroom, I'm teaching them how to be a model student in the U.S. (New Arrival Center teacher, 2011).

The teachers each asserted that it was vital for their classrooms to be a safe place for students to take academic and linguistic risks. Therefore, students were given time before being asked to speak in front of others to lower the affective filter within and among students, though all were encouraged to participate when they felt ready. If the students felt anxious devising their own answers, they were asked to repeat a word or phrase that a teacher would speak for them, thus helping them to practice giving an answer in class. Student choice was also valued in the classrooms, and the teachers noticed that students often challenged themselves when provided a choice in activities. Teachers reported utilizing visual, audio, and tactile modes of instruction in their classes to make sure all kinds of learners could access the necessary information. Lastly, one teacher reported that routine was important in providing students security as they entered the school and her classroom, in that students knew what to do each day within her class.

I do use the same routine in all of my...basically the same strategies. I normally get new students, usually we have a student helper, which is like a senior helper and what they do is they review things we covered in the beginning... which is basically to know the days of the weeks, the months, the year. To help them get caught up and so that they can get used to the routine that goes on here. I feel like I have a very set routine in all of my classes that when a new student comes like they get to observe that, it's not really like... well let's see what were going to do as soon as you come in (New Arrival Center Teacher, 2011).

During the focus groups, students reported feeling that the teachers were more patient in the United States than in their home countries. They stated the teachers were welcoming, courteous, and were quite supportive of them as they entered U.S. schools. 
Despite acting courteous and welcoming to students, some newcomers also felt the teachers spoke too fast and explained concepts in a way that was new to them. It was difficult for the students to comprehend the instruction if the teachers did not pronounce the words carefully or if they spoke too softly. It facilitated the students' learning and comprehension when the teachers used pictures, gestures, and flash cards during instruction, and when they rewarded the students for their hard work. It also helped them when teachers would repeat English phrases to allow them to hear the phrases more than once, and when teachers made connections between Spanish and English words and phrases. In the following quote, one student shared how his teacher would help his class to access the instruction:

[To help us] they repeat words in English and Spanish and they write our work in both languages to compare. Also when we ask how to say (for example pencil) they ask us to look it up in the dictionary. When we need a phrase said in English, they will repeat it in English whenever we ask... they will teach us in class and then give us homework and if we don't understand they will explain it better and if they see we still don't understand they will continue...they will focus on having us learn, they will explain and explain until we understand (New Arrival Center Student, 2011).

Although the teachers generally spoke only English in class, it made the students feel more comfortable to know the teachers could understand Spanish, as the students knew they could receive clarification of concepts in a comprehensible manner when necessary. Students were encouraged by teachers to speak English in class, which was sometimes frustrating for the kids as they felt some students tried harder than others, yet everybody received the same credit for participation. Several students reported having learned much of the academic content being taught in their classes in their home 
countries, particularly in such classes as mathematics, and therefore they solely needed to learn the English vocabulary to feel able to participate in classes.

[No class is too hard] because you know it in Spanish and then they guide you in telling you [the concepts in English]...(New Arrival Center Student, 2011).

Students who had completed the NAC program the prior year reported having more difficulty in asking teachers questions. They claimed that teachers appeared less patient and often accused students of not paying attention if they didn't understand a concept, which was frustrating to the youth. These students reported feeling more comfortable asking friends for clarification, rather than their teachers, when they had questions about the lessons taught during their classes. The students appeared to feel less connected to their teachers during their second year of instruction, though some students did return to their prior NAC teacher to ask for help when needed. The students reported having felt safer and more comfortable within the NAC classroom environment.

My last year's teacher [NAC teacher]...the teacher that I had in [the NAC program], she helped me... I am not a student with her anymore but she still helps me after school (New Arrival Center Student, 2011).

\section{New Arrival Center Students' Academic Self-Concept}

Finally, in terms of academic self-concept, the administrators maintained the students were very comfortable in the NAC program, likely owing to the fact that the classes were conducted in a manner that felt more family-like, and because students were able to focus on English for the entire day. The newcomer youth knew that the teachers cared about them and the teachers constantly encouraged them to do well academically and linguistically, and thus students often wanted to return to the program as they missed the safe and caring environment. An example of the caring environment created by NAC 
teachers was relayed by a program administrator when she recounted how one teacher noticed the students were feeling discouraged, and so she began having them write in a journal twice a week detailing their feelings in their primary languages. The kids were able to write whatever they felt, and after some time the teacher noticed a shift in how the students viewed themselves both academically and socially; the students were far more positive. In addition to creating a caring environment, the NAC teachers exposed the students to different cultural and college-going experiences through field trips and a specific college-bound program.

The administrators maintained that it was the responsibility of the NAC program to equip the students with the skills necessary to be mainstreamed the following year, and then it would become the duty of the rest of the site to meet newcomer students' needs in the mainstream environment. Many of these students entered as very highly motivated individuals, a characteristic that served them well within the program. It was therefore the program's obligation to support the students effectively to ensure they maintained their high motivation throughout the school year and that they felt comfortable entering the subsequent year's mainstream placement. In the following quote, one administrator highlighted the responsibility of the entire school in assuring that newcomer youth feel supported throughout their schooling experience, thereby creating the space necessary for students to feel confident academically:

Sometimes what happens at schools is the kids want to come back to the New Arrival Center, because they realized once they were out in the real world that they were really cared for and...you know everything was around developing their language, but we really want to not necessarily continue to support them at that level but really equip them...equip them so that they can handle the challenges in the academic world. The teachers want them to have that kind of support and that kind of success too. So... but then it's then the responsibility shifts more towards the rest of the school to make sure that those other pieces are 
in place, so it calls to light those pieces that may not be as well in place (New Arrival Center Administrator, 2011).

The teacher interviews brought to light the notion that they believed their students felt more confident as a result of participation in the NAC program. They asserted that all of the students were in similar places linguistically, which was a comfort to them and helped them to feel safe. One teacher had witnessed students crying at her former school because they had felt alone and overwhelmed in the mainstream environment. Teachers reported witnessing a positive change in students' self-esteem and academic self-concept, particularly by the end of the school year. They attributed this positive change to the routine and safe environment in which the students were being educated, an environment that helped kids to grow over the school year.

I think because I have just one class, I think they're more confident. They know somebody and they feel like a part of something ...like I know that they feel a part of this, and I know that for the most part I don't have kids crying because they feel alone so... much like [at my former school] I remember I had kids that were 15 years old, boys even, that were just overwhelmed and they were just... you could see it. So, I think this program does support them in a way that's good (New Arrival Center Teacher, 2011).

One teacher stated that her students came in very motivated and were always very delighted to be in her class. She helped her students stay joyful and motivated by creating a classroom environment rife with respect, safety, and excitement. The students were never teased in her class and students were generally willing to help once another when they needed assistance, in that she promoted a safe community for all students. She felt her students' self-confidence soared by the end of the school year and her students were generally upset when they had to leave her class. Finally, this teacher had noticed that her 
students were much quieter in mainstream classes, and which attested to the comfort they felt in her NAC class.

I think it's because they've never been laughed at and everybody...there is always somebody that's showing them [what to do] and then they... and they just feel confident...oh my, gosh I mean, look at these kids, this is like the perfect thing for these kids, like their self esteem soars you know, they're so [confident] (New Arrival Center Teacher, 2011).

As one would expect, in classes with interesting and comprehensible lessons and activities, the students reported that they felt academically strong and more capable. They felt like they were successful since they were learning and acquiring language at an accelerated rate. Conversely, in their more difficult classes, students felt belittled and anxious. During the focus groups, the youth reported that they would stay silent in these classes because they felt nervous and embarrassed. A few students agreed that they would rise to the challenge in more difficult classes, but it took a lot of courage for them to participate in such classes, and though they would set the higher standards for themselves, they felt frustrated by their inability to comprehend that which the instructor was teaching. Therefore, some students reported that they gave up in more difficult classes and would instead solely attempt to calm themselves when they felt too nervous. Finally, one student reported feeling frustrated that his work was lower level, as it made him feel like a Kindergartener, despite being a high school student.

[Harder classes] lower my self-esteem. I try a lot but I stay silent. They make me feel a bit incomplete because I try to get better but sometimes I don't do well but at least I tried...(New Arrival Center Student, 2011).

After several months in the NAC program, students reported feeling better as they understood more English. They felt they could complete work on their own and go 
beyond copying the teachers and other students' work. After some time, the newcomer youth also found individuals, such as other students, who were more willing to help support them with their work. The students also maintained in the focus groups that the teachers helped them to feel more confident and to feel safer taking more academic and linguistic risks in class. Finally, the first year NAC students reported feeling hopeful that they would do well the following year, as their English had already improved during the short time they had been in the U.S. Many students did recognize, though, that they still had a long journey ahead of them in acquiring English and in feeling sufficiently capable to be successful with the higher-level mainstream courses. The optimism felt by the majority of the NAC program newcomer youth is epitomized in the following quote:

[Next year] we are going to learn more English, we will learn more concepts, more words, how to write a sentence. We will not be as nervous because we will have a year of experience.... I know I still have a long way to go to be put in normal classes but I know I will learn what I need to be prepared (New Arrival Center Student, 2011).

Comparison and Contrast of the ESL and the NAC Program Findings

The results of the six cases analyzed within the English as a Second Language (ESL) and the New Arrival Center (NAC) programs were presented in the above chapter. The five main sections of the chapter addressed the qualitative and quantitative findings that were discovered though the data collection process. The first section gave detailed background information about each of the two programs under study (New Arrival Center and English as a Second Language), presenting an overview of the programs and describing both programs' overarching philosophy, organization, instructional design, and the expectations of each program. The second section analyzed the trends in English learner redesignation rates at both the site and at the program levels. The third section 
illuminated the student self-concept results, as attained though the Student Self Concept Survey. The fourth section discussed the findings gathered during the classroom observations that were conducted within each of the six cases under study. Finally, the fifth section revealed the trends found within each of the six focus groups, six teacher interviews, and four administrator interviews that were conducted within the qualitative data collection section of this study.

In comparing and contrasting the English as a Second Language (ESL) and the New Arrival Center (NAC) programs, the results illustrated more similarities than differences between the two programs. The overarching goals and stakeholders' expectations of the programs, as seen in the program overview section, demonstrated that despite the added content potion offered in the NAC program, the aims and underpinnings of the programs under investigation were essentially the same. The curricula, language instruction, language assessment, and the instructional practices found within the programs were fundamentally similar, with the only major difference being the addition of the ESL Mathematics, ESL Science, and ESL Social Studies courses in the NAC program. Finally, though they were purported to be different, parent involvement was addressed in the same manner in both programs, as evidenced by the only parental connections being offered through the volunteer site and district English Learner Advisory Committees. The comprehensive sites and the district central offices did little to connect parents to needed community resources.

The redesignation rates fared well for both programs as compared to the district as a whole, yet the ESL program did redesignate students at a slightly higher rate than the NAC program, suggesting more successful language acquisition among students within 
the ESL program. This finding was congruent with the overall results on the academic self-concept subscale of the Student Self-Concept Scale, as those findings additionally implied that the ESL students were more academically confident as they progressed from their first year in the program to their second year in ESL 3/4. Despite the fact that the NAC students began their schooling more academically confident than the ESL 1/2 students, their academic self-concept levels dramatically decreased the second year (in ESL 3/4 and mainstream or sheltered courses), while the ESL 1/2 students saw an increase in their academic self-concept levels their second year (also in ESL 3/4 and mainstream or sheltered courses). The students in the ESL program appeared to be performing and perceiving themselves in a more academically successful manner, despite assurances to the contrary purported by the leadership overseeing the NAC program.

The redesignation findings and the Student Self-Concept results did not support the classroom observation findings, as the indicators of (1) successful English learner strategies, (2) culturally responsive pedagogy, and (3) the pedagogical orientation continuum (found on the Classroom Observation Matrix) each slightly favored the NAC program, rather than the ESL program. The NAC program demonstrated more of the indicators in each of the three areas of the Classroom Observation Matrix than the ESL program, yet the students were not performing at an accelerated rate according to the redesignation rates and the Student Self-Concept Scale findings. This inconsistency suggests that these two programs were, in reality, quite similar, in that there were few progressive strategies present within the NAC program. The traditional strategies found in the ESL program were also utilized in the NAC program, with the exception of the 
self-contained ESL content-area courses, demonstrating the overall similarities present between the two programs.

Finally, the tensions and themes that arose from the interviews and the focus groups were also similar within the ESL and the NAC programs, as the positive driving conditions and the negative restraining conditions brought forth by the stakeholders (administrators, teachers, and students) within the two programs illustrated more similarities than differences. The analogous positive and negative conditions present within the two programs further demonstrate that the implementation of these programs was in actuality more similar than different. The results disseminated in each of the five sections will be analyzed and triangulated within Chapter 5, Implications, to address each of the guiding research questions within this study. 


\section{CHAPTER 5}

Implications

This study sought to explore the schooling practices and academic outcomes that took place within two program models established to service secondary-level newcomer immigrant youth within one large urban school district in Southern California. The focus of this study was to determine which of the two yearlong programs, the English as a Second Language (ESL) program or the New Arrival Center (NAC) program, provided more effective supports for middle and high school level Latino newcomer immigrant youth as they embarked upon their educational journey within the San Diego Unified School District; a large urban district comprised of approximately one-third English learners (SDUSD, 2011). Three classes from each of the two programs were studied to determine which program more successfully supported secondary-level immigrant youth in acquiring the language proficiency and academic skills necessary to transition into mainstream or sheltered courses the following year. The outcome of the (ESL and NAC) programs on students' academic self-concept was an additional factor analyzed within this study, as research has indicated that students' academic self-concept plays a significant role in their future academic achievement. The guiding research question asked:

How does a specialized newcomer program prepare secondary-level Latino immigrant youth to gain the language proficiency, academic skills, and academic self-concept necessary to be successful within an English-only educational environment, as compared to students placed in a traditional ESL program?

This chapter will synthesize the results of the study, as were gathered using qualitative and quantitative data, and were triangulated to answer both the overall 
research question and the subsequent three sub-questions. The findings of the three subquestions are presented at the onset of the chapter to inform the answer to the overarching research question. This chapter offers recommendations and a potential action plan to improve conditions for the stakeholders within such programs, and it further illuminates the assumptions and limitations of this study as it was conducted. Finally, the study concludes by discussing recommendations for future research. The significance of the study has revealed the need for linguistic and academic interventions to persist and to expand with the goal of ensuring a highly inclusive, rigorous, and supportive schooling environment for all students, in particular secondary-level Latino newcomer immigrant youth.

\section{Analysis and Interpretation of Sub-Question Findings}

The goal of this study was to determine which of two program models more effectively supported secondary-level Latino newcomer immigrant youth as they attended or after they had completed their first year of education in either a self-contained newcomer program, or a traditional ESL placement. The conclusions drawn from the research, as were determined after having analyzed the data collected for this study, are presented in the below sub-questions with the ultimate aim of providing a conclusive response to the original research question. Recommendations to address issues of access to quality education and tensions to improve the schooling conditions for newcomer immigrant youth will be presented in a subsequent section. 
The first research sub-question asked:

What are the prevailing pedagogical practices utilized in both a newcomer program and a traditional ESL program?

How do the curricula, language instruction, content instruction, teachers' attitudes, level of cultural responsiveness, and pedagogical orientations compare within the programs under study?

\section{Curricula}

The findings of this study indicated that both the New Arrival Center (NAC) program and the English as a Second Language (ESL) program utilized the same curricula in teaching the students English Language Development during their language arts portion of the day. The NAC program was created during the same time period as the ESL program administrators and the textbook adoption committee were finalizing their choices for the ESL curricula adoption, and therefore, the NAC program administrators made the determination to incorporate the ESL $1 / 2$ curricula into their program. The ESL curricula had been determined by the textbook adoption committee to be the most effective option in teaching language skills to secondary-level newcomer students, and thus both programs used Pearson Longman's Keys to Learning curriculum, in conjunction with the locally created Writing Reform Institute for Teaching Excellence (WRITE) curriculum. The teachers were trained extensively in how to use the two curricula, as well as such specific vocabulary and sentence development teaching methods as Systematic or Focus English Language Development. Finally, according to the district administrators the teachers were provided yearly pacing guides for their English Language Development portion of their day. 
Despite feeling largely satisfied with the language instruction present within the curricula, the teachers in both the NAC and the ESL programs expressed similar tensions regarding the district-provided programs.

- Teachers felt the ELD curriculum was not authentic or culturally responsive enough to connect with the backgrounds or address the needs of newcomer immigrant youth.

- The curriculum did little to address the emotional difficulties facing many youth as they immigrate to the U.S.

- Teachers felt that pacing guides moved too quickly and did little to address the realities of having multiple language levels in one class, as was the case in many ESL classrooms.

- The teachers spent much of their time supplementing the curricula and many felt they were not provided the time or space necessary to develop lessons that would allow for students to more effectively connect to the ELD class.

- The NAC program had inadequate curricula and pacing guides to effectively teach the students their content classes. Though the NAC classes had been given a pacing guide for mathematics, the teachers did not have a strong understanding of how they were expected to cover the social studies and science portions of the NAC program.

- The teachers reported that the content curricula were more akin to supplemental materials, and felt there was a need for more comprehensive content curricula within this program. Teachers reported a need for more time and space to jointly 
develop their content curricula and the daily lessons and activities to be covered during such classes.

- Teachers struggled in classes they had not previously taught, and therefore a sound understanding of the program expectations and how to best teach such courses was an area of need for many NAC teachers.

- The NAC program administrators acknowledged that they had not had sufficient time to develop the content curricula at the inception of the program, and therefore improving the curricular expectations was an ongoing process for the program administrators and the teachers within the program.

\section{Language Instruction}

While the ESL program focused exclusively on developing students' oral and written English language skills, the NAC program was established to ensure students have extensive language opportunities across the curriculum. Administrators expected teachers within both programs to ensure consistency across the district by utilizing the provided pacing guides to make sure they covered the necessary language instruction throughout the school year. The primary goal of both programs was to create daily language experiences in all language domains, listening, speaking, reading, and writing, in a collaborative, active, and authentic manner. Students in both programs were to be given frequent opportunities to practice language in a highly supportive and low-anxiety environment that promoted linguistic risk-taking for all students. Teachers utilized such strategies as partner work, purposeful cooperative grouping, modeling responses, guided and shared reading, frontloading vocabulary, visuals and manipulatives, primary 
language support, and speaking in English at a slower and more deliberate pace to ensure students comprehended the instruction. Finally, in both programs teachers reported utilizing technology to supplement the language instruction and during small group time to give students purposeful individual language activities while teachers met with smaller groups of students.

In comparing the NAC and the ESL programs, it was apparent that the primary linguistic goals of both programs were the fairly identical, yet during the three class observations of each case within the two programs the researcher recorded that:

- The NAC program incorporated more indicators of effective English learner instruction than the ESL program, as was determined utilizing the Classroom Observation Indicator Matrix (Appendices $\mathrm{J}$ and $\mathrm{K}$ ). The indicators of effective English learner instruction were calculated after each observation to decide which of the two programs more effectively incorporated language supports and English acquisition strategies with their students, thereby allowing for a higher level of needed comprehensible input for newcomer immigrant youth in such classes (Krashen, 1992).

- While all of the teachers reported that they felt very passionate about teaching newcomer immigrant youth and were highly qualified to do so, the NAC teachers had specifically applied for and been chosen to teach in the program, while many of the ESL teachers reported having "fallen into" the position.

- Due to the issue of class numbers, the majority of the ESL classes were multilevel classes, and therefore, unlike in the NAC program, the teachers were unable to concentrate solely on the needs of the ESL $1 / 2$ students. In an effort to remedy 
the issue of multiple levels of ESL students, the teachers conducted leveled groups within their classes and often used support staff to assist in instructing such groups. The teachers and program administrators reported they believed this was the only answer to the issue of multiple levels.

- Often support staff did not possess the same training and professional development as the certificated classroom teachers and were often not credentialed teachers.

- Although the job was posted and funded as a resource and assessment position, due to staff reductions English Learner Resource Teachers (ELST) had assumed the ESL classes at schools. The ELSTs were credentialed and highly trained for such a position, yet this role took away from their ability to conduct assessments and train and support the general and special education teachers to address the needs of all English learners at a given school.

- The lack of consistency within the ESL program was apparent when viewing the many ways in which the students were being serviced at the various sites under study, a major tension that appears to be mirrored across the district.

\section{Content Instruction}

Unlike the ESL program, the NAC program was developed to ensure its newcomer students have access to their content instruction in a highly supportive and comprehensible manner, and thus the NAC program was undertaken as a self-contained class. The teachers within the program were charged with teaching ESL Mathematics, ESL History, and ESL Science classes, in addition to the language arts portion of the day, 
one significant benefit being their ability to connect the curricula across the content areas. Salient findings suggest:

- At the high school level, the NAC content classes received elective credit toward graduation, and though students needed elective credits to graduate, these courses did not give students the content credits necessary for graduation.

- The NAC content classes were less constricted than traditional content classes, as the requirements for graduation-level courses were removed, and therefore they were utilized to teach students fundamental skills and content-specific vocabulary.

- Students that entered with high-level skills, generally in mathematics, were often mainstreamed during that portion of their day, as it was recognized that students needed to begin accruing their graduation credits as soon as they could function in a grade-level course.

- Though the NAC teachers felt the curricula and the pacing guides needed to be more fully developed in the content classes in an effort to ensure a strong and consistent program, the teachers also reported that they had more freedom to instruct their students at the students' point of need than they would in traditional content classes.

- NAC teachers were able to use primary language support and culturally relevant material to connect the concepts within such classes to students' home cultures and languages, while additionally scaffolding grade-level standards to ensure students were exposed to mainstream content material.

- Some sites contained more than one NAC class, at which the teachers could opt to group their students for rotations during their content classes to alleviate the many 
classes for which the teachers needed to prepare. In addition, one middle schoollevel NAC site opted to mainstream all of the students for mathematics, and had reported positive results.

Conversely, in the ESL program, all students were mainstreamed for their content classes into either sheltered or mainstream mathematics, history, and science classes. The teachers at all secondary sites were said to have been encouraged to participate in a fourday training called Quality Teaching for English Learners (QTEL), and sites were also training QTEL apprentices to work with the various site staffs in learning how to best educate English learners across the curriculum. In addition:

- ELSTs at each site were charged with ensuring that English learners were placed with teachers who were highly trained and eager to work with English learners to create a positive and supportive experience for such students.

- ELSTs were also encouraged to push-in to classes with high numbers of English learners to support the teachers and students during the content instruction.

- Many ESL teachers also reported working with their students in their content classes when possible and addressing content material during their ESL class to support students as best as they could during their two-hour block.

- ESL teachers viewed themselves as advocates for their ESL students and therefore they often took it upon themselves to ensure students were feeling successful in their content classes, as it was asserted by the stakeholders that many general education teachers did not sufficiently scaffold their instruction for their English learners despite their district-level training. 


\section{Stakeholders' Attitudes}

Although the sub-question specifically sought to compare the teachers' attitudes within the two programs under study, as the data was collected additional significant findings were discovered during both the administrator interviews and the student focus groups, and therefore such findings suggest:

- In creating the NAC program, the primary expectation of the program administrators was to provide newcomer immigrant youth with extensive opportunities to acquire and utilize English in a meaningful way, while simultaneously preparing students for their later mainstream and sheltered content classes and orienting students to U.S. culture and traditions.

- Administrators stated that newcomer youth are a diverse group with distinct issues, such as acculturation needs and, at times, interruptions in their prior formal schooling.

- Administrators decided to group the NAC classes at comprehensive secondarylevel sites with large immigrant populations, a decision that has been wellreceived by the administration at such sites.

- Program administrators charged the NAC teachers with making placement decisions for the students in their classes to determine whether or not they were ready to be exited from the program after the requisite year, at times approving a second year for students with extreme gaps in their prior schooling experiences.

- NAC teachers felt very passionate about their positions, and had sought out and applied for the position after it was decided to create such a program. The teachers felt especially connected to their youth and reported that their goal was to 
get students literate in English as quickly as possible, while concurrently ensuring their students felt their home countries and languages were valued at school.

- Despite the positive viewpoint the NAC teachers had about the program, they reportedly felt isolated by the staff and administration at the sites in which their classes were located.

- NAC teachers hoped there would be more professional development and interaction among the NAC teachers at the various sites, yet the collaboration among veteran NAC teachers was minimal in that the program leaders had not brought the group together as a whole for some time.

- According to program administrators, budget constraints and a desire to not remove teachers from their daily instruction were the primary reasons for the lack of professional development for veteran NAC teachers, yet this tension created further feelings of isolation for such teachers at their individual sites. Much like the NAC program, the ESL program was developed in response to a distinct need for specialized ESL instruction for secondary-level newcomer youth. Unlike the NAC program though, the ESL program has been adjusted to meet the needs at individual sites, either by mixing two or more ESL levels in one class to create sufficient numbers or by utilizing the site ELST rather than a classroom teacher to instruct the class. The findings additionally suggest:

- There is little consistency in how the program appears across the district. The lack of consistency within the program has led to a potential devaluing of the ESL program at comprehensive sites, which in times of staff reductions could lead to a number of ESL classes being eliminated from sites with lower numbers of ESL 
students, or it might force more ELSTs to assume the role of ESL teacher despite their many assessment and professional development duties.

- The issue of budget decreases has additionally created tension for the central resource staff charged with overseeing the ESL program, as they report having fewer resources and markedly less time with which to support the teachers at their individual sites.

- Analogous to the perspectives of the NAC teachers, the ESL teachers held high expectations for their students, though many reported feeling they had less time and fewer resources with which to be as involved or creative in their classes.

- The ESL teachers reported feeling pressured by the test-taking cultures of their sites to focus their teaching on the state-mandated high stakes assessments and the required high school exit exams.

- The ESL teachers appeared to feel less freedom to teach to students at their point of need than the NAC teachers, though many did make a concerted effort to individualize their instruction to the needs of their youth.

- Also, like the NAC teachers, the ESL teachers believed they had a responsibility to serve as their students' advocates and they took it upon themselves to ensure students were properly placed in mainstream and sheltered content courses. The ESL teachers attempted to support students in their content areas when time permitted.

The students in both the NAC and the ESL programs reported they were exceptionally nervous as they began their schooling in the United States. The students felt 
uncomfortable participating and having to speak in front of others, which caused them great anxiety at school. The findings further imply:

- Both NAC and ESL students reported feeling discriminated against in their schooling environment, as many felt their peers viewed them negatively and they believed they were being teased or other students were talking about them when they could not comprehend the conversations.

- The newcomer youth reported feeling like outsiders at their schools, as they were unable to communicate with their peers and school staff.

- The students reported that they had immigrated to the U.S. for greater educational and career opportunities, though they did not feel that the teachers and other students understood their reason for moving to a new country.

- The students stated that it was important to become fully bilingual, as their language skills would help them greatly in the future.

- The newcomer youth that had completed their year in either the NAC program or the ESL program differed in their views of U.S. schools, as the students who had completed the NAC program the prior year expressed a more disconnected view than the students who had completed their year in the ESL program.

- The second year NAC students stated they did not feel valued in U.S. schools and often did not complete their work because they were unable to comprehend it or did not care to finish their work.

- The second year students felt they had to endure disparaging remarks by nativeborn students and they were often forced to support one another, as many adults did not address such issues. 
- The second year ESL students reported feeling more successful in school as time progressed.

- They students felt they were able to access peer assistance and resources to support them in understanding unfamiliar concepts and, despite the fact that they continued to struggle with the language, they were more optimistic than the NAC students about their future in the U.S.

\section{Cultural Responsiveness}

In comparing culturally responsive pedagogical practices between the NAC and the ESL programs, the administrators from both programs recognized the necessity for the programs to address the differing cultural and academic needs with which newcomer youth entered U.S. classrooms. Some students entered the schooling system with parallel educational backgrounds, while others entered with interruptions in their formal schooling. The administrators asserted that this concept could be difficult for teachers to comprehend, as it did not mirror their personal educational backgrounds. It was therefore crucial for teachers to learn about students' cultural and educational backgrounds to ensure their daily lessons and teaching concepts were linked to students' backgrounds and prior understandings and, in addition, the class environment valued their home cultures and languages. Other salient findings suggest:

- Administrators maintained that teachers needed to use primary language support to assist students in their understanding and to validate their home languages whenever possible, and that they ought to access primary language resources for students from less common language backgrounds. 
- The administrators from both programs emphasized that the acquisition and use of English was the ultimate goal of the program, but that limited primary language support should also be a part of daily instruction, either from the teacher or a peer in class.

- The teachers in both programs reported incorporating students' primary languages and cultures into their lessons to ensure access to students' home cultural and linguistic backgrounds and to connect them to those of the United States.

- The teachers maintained they used multicultural texts and made frequent comparisons between students' home countries and the U.S., to assist students in accessing their prior understandings of cultures, traditions, and value systems and to support them in contrasting such personal concepts with those of the United States.

- Unlike the teachers within the ESL program, the NAC teachers made explicit reference to their need to teach students about how to function effectively and be active citizens within the United States. They asserted that it was their responsibility to acculturate newcomer youth to the customs and traditions in the U.S.

- Teachers within both programs accessed school and community resources to acquire translation services in an effort to assist them in better communicating with families, as teachers in both programs desired a strong home-to-school connection with their students' families.

The students reported learning about common U.S. holidays in their classes, as well as some rudimentary cultural understandings about how people function in the 
United States. The students were also taught about major historical events during their history courses, though the ESL students that were not enrolled in history courses stated they were not taught about U.S. culture or history. In addition:

- Some students asserted they did not believe their culture was valued in the U.S., neither in school nor in society as a whole. They felt disconnected to the culture of the United States and, at times, they struggled to understand the cultural norms of said country.

- Students did not see themselves in the texts or lessons utilized in their classrooms, and though some reported their teachers did speak to them about the importance of being bilingual, they did not see their languages valued in the academic environment.

- Many students reported wanting to return to their home countries as they identified culturally with their birth countries and felt they belonged in such countries.

Despite the similarities found in the teacher and administrator interviews and the tensions demonstrated in the student focus groups, the findings linked to the classroom observations revealed that during the three observations of each case:

- On average more culturally relevant pedagogical indicators were apparent in the NAC program than in the ESL program. The NAC program averaged 7 indicators over the three observations, while the ESL program averaged 4 indicators over the same time frame.

- The mean numbers, as seen in Table 34 and on Figure 11 (Chapter 4: Results), illustrated that as a whole, the NAC program under study consistently 
incorporated a greater number of culturally relevant pedagogical practices into the academic setting then the ESL program.

\section{Pedagogical Orientations}

The program administrators in both the NAC and the ESL programs viewed their primary roles in overseeing their particular programs as of ensuring consistency across the district and of being a support for the teachers within the program. They reportedly wanted to assist teachers with their lesson planning and in collaborating with other like teachers. Other significant findings indicate:

- The administrators from both programs reported feeling "pulled thin" in their positions and felt they lacked the time necessary to work with teachers at their individual sites.

- The administrators, particularly in the NAC program, often worked with site leadership to ensure the fidelity of their programs, however they also expressed the need for some program flexibility as each site was to take ultimate ownership over either its ESL or NAC program and therefore needed to have some say in how the program was to be conducted at their particular school.

- Teachers in both programs viewed themselves as facilitators or coaches within their classrooms, as well as mentors and counselors for students depending on their needs.

- The teachers also viewed their primary responsibility in class as ensuring students have multiple opportunities and sufficient time to both acquire and practice 
English, as they all understood their students were to be completely mainstreamed the following year into sheltered or general content courses.

- Teachers spoke of educating students at their point-of-need and advocating for them in other scholastic environments, to ensure students felt supported within their educational setting.

- Teachers within both programs promoted a low-anxiety learning environment for youth, as it was crucial students felt comfortable taking linguistic and academic risks in school.

- Both programs frequently utilized purposefully created cooperative groups and partner work to ensure students had authentic and meaningful modes in which to communicate and engage one another academically.

- Students from both programs reported that teachers made them feel welcome and many attempted to speak Spanish to them as they entered the classroom.

- Students appreciated when teachers spoke more slowly, pronounced words carefully, and repeated concepts in an effort to help students understand what was being taught.

- Students in both programs stated that gestures, visuals, and technology additionally supported them understanding the new concepts about which they were being instructed.

- The students felt more comfortable in the classes in which they had bilingual instructors, as it put them at ease to know they could communicate with said teachers even when the teachers only taught in English. 
- Students believed it was crucial for them to connect to teachers on a more personal level, in that they then felt that the adults were invested in their educations and believed in their worth.

- Students from both programs stated that though teachers often had good intentions, it did not always help them to be partnered with bilingual students, as at times the bilingual students would get frustrated with them and resent having to assist them with their work. Additionally, the bilingual students had their own work to complete, and therefore did not have the time necessary to assist the newcomer youth. It is important for teachers to use caution when creating such partnerships, as though it can be quite helpful to newcomer students, the abovementioned tensions make it difficult for both parties at times.

- Most students within the NAC program reported feeling as though the teachers encouraged them and viewed them positively, while some students in the ESL program felt they had teachers, primarily in their content areas, who thought they did not value education, which was unequivocally not true.

- Students that had finished the NAC program the prior year reported having more difficulty in their classes as they were mainstreamed in their current year. They felt the teachers were less patient and less willing to answer their questions or support them as needed, and they felt less connected to their current teachers than their NAC teacher from the prior school year.

Using the Classroom Observation Indicator Matrix (Appendices K and L) teachers' pedagogical practices were noted on a continuum, which ranged from a Transmission-oriented pedagogy, to Social Constructivist-oriented pedagogy, and finally 
a Transformative pedagogical orientation (Cummins, 2009). While some direct instruction or lecture was required in teaching, as was seen in the Transmission-oriented pedagogical practices, it was preferable to use such teaching practices to move to a collaborative or a transformative classroom environment (Cummins, 2009). Figure 12 (Chapter 4: Results) yielded the following patterns:

- With the exception of one ESL class, the teachers demonstrated an approach to teaching that favored the Social Constructivist-oriented pedagogical orientation. These teachers activated and valued their students' prior knowledge and cultural backgrounds, and they co-constructed understandings about the concepts being taught by engaging students in dialogue and discussion about the subjects being studied.

- ESL Case \#1 was the only class is which a more Transmission-oriented approach was apparent throughout the three observations, in that the instruction in this class was primarily focused on lecture with little collaborative discussion during the lessons. One possible reason for such a pedagogical approach was that this class was an ESL 1/2 and ESL 3/4 combination class, and an assistant was charged with teaching the ESL 1/2 group, while the credentialed ESL teacher concurrently instructed the ESL 3/4 students.

- The findings demonstrated that none of the cases had reached the level of Transformative pedagogy, in that the concepts of societal power relations and inequalities, instances of critical inquiry, and actions to challenge social inequities were not brought forth in teaching or class discussions. 
The second sub-question examined the impact of students' self-concept on their language acquisition, as was determined utilizing the qualitative findings gathered through both the interview and the focus group data collection. The second sub-question asked:

Is there a relationship between students' academic self-concept and the increase in their language proficiency level within either a specialized newcomer or a traditional ESL program?

Overall, according to the interviews and focus groups, the effect of language acquisition and academic self-concept appeared to be somewhat synergistic, in that students felt more confident as they gained language and they gained language when they felt confident and safe to take linguistic risks in an academic environment. The qualitative findings therefore supported the notion that students' self-concept was related to gains in their language proficiency, as the more confident students felt, the more able they were to take risks in an academic environment. Teachers and administrators in both programs reported witnessing an increase in self-confidence as the year progressed, which they attributed to an increase in language skills and a better understanding of the schooling system as a whole. Students' academic self-concept in class was thus providing kids the confidence necessary to be active and take linguistic risks in class.

I've heard reports...people saying you know the students who never raised their hands or volunteered before are now raising their hands and volunteering information and so I'm hearing more and more about that...I think they're more confident (ESL Administrator, 2011).

In the following quote, an additional ESL administrator expressed her belief when considering the connection between language acquisition and self-concept: 
I believe that students are more confident academically and socially as a result of their participation in our program. They are able to find a niche for themselves in a very new and sometimes overwhelming setting. Our students are improving at a rapid rate. Many of our students are making one year's [language] growth in one year's time. Many are exceeding that goal... (ESL Administrator, 2011).

The teachers and administrators in both the NAC and the ESL programs stated that despite that fact that many students entered the programs feeling very nervous, most were also highly motivated and confident in their abilities, which served them well in acquiring language and academic skills. These students, when properly supported in a low-anxiety, highly scaffolded environment felt able to take the risks necessary to acquire and practice English. The NAC program had the additional advantage of educating students in a highly scaffolded environment for virtually their entire school day, thus providing the students a secure environment in which to acquire language for an extended amount of time. In the following quote, one NAC teacher described her students' confidence levels in her NAC class:

I think they are more confident because they know they are around students who are in a similar situation. They are [all] here from a new country... they're here having some English maybe, or no English and they're all learning the English language so I know they feel safe around their peers because they know they have a common ground. So...I think because we are in this situation... that it helps them build their self-esteem (New Arrival Center Teacher, 2011).

An additional NAC teacher shared her view of students' academic self-concept after completing the requisite year in the program. In the following quote she discussed the manner in which language acquisition and an increased level of comprehension affected her students' views of themselves as learners:

I do see [a difference in their academic self-concept and social self-concept] levels especially at the end of the school year... when we've had that year long of 
study... that's when I really get to see all their growth, you know in all the areas (New Arrival Center Teacher, 2011)

The student participants within the NAC and ESL focus groups mirrored the assertion made by the teachers and administrators that there was a positive correlation between language acquisition and levels of self-concept. The students stated they felt unwilling and unable to participate in classes they believed to be more difficult, as well as in classes in which they felt greater levels of anxiety, thereby preventing them from practicing language. The following quote from one NAC student illustrated this contention:

[Harder classes] lower my self-esteem. I try a lot but I stay silent. They make me feel a bit incomplete because I try to get better but sometimes I don't do well...(New Arrival Center Student, 2011).

Conversely, though, in classes that provided the necessary security for newcomer youth to take linguistic risks, such as their NAC or ESL 1/2 courses, the students reported feeling more confident academically and they possessed higher self-esteem:

In my English class is where I speak it the most because I feel more confident there...[The easier classes make you feel] calm and confident and they give you the courage to speak your mind... (ESL Student, 2011).

The above qualitative findings support the notion that there was a positive relationship between student self-concept levels and language acquisition within the two programs under study, as was demonstrated through the interview and focus group findings. In his extensive work on the process of language acquisition, Krashen stated, "if the acquirer is anxious, has low self-esteem, does not consider himself or herself to be a potential member of the group that speaks the language, he or she may understand the 
input, but it will not reach the language acquisition device" $(2003$, p. 6). As was

previously stated, English language acquisition requires students to take linguistic risks, and students possessing low academic self-concept levels or high anxiety levels are far less likely to feel able to take such risks within their classes.

The final sub-question concerning language acquisition and self-concept between the NAC and the ESL programs asked:

How are Latino newcomer students' academic self-concept, language proficiency levels and acquisition of academic skills influenced by the program in which they are educated?

\section{Academic Self-Concept}

The research literature suggested that students with higher academic self-concepts are inclined to score better and outperform others, due in large part to their certainty that they can and should achieve in the academic environment (Hutchinson, Kirby, \& Carson, 2000; Hung Hon \& Yeung, 2005). Prior research studies have indicated that for secondary-level students academic self-concept is strongly correlated to academic achievement, and therefore it is crucial that educators address this important construct in considering how to best support immigrant youth in their new classroom settings (Guay, Marsh \& Boivin, 2003; Peralta Sanchez \& Sanchez Roda, n.d.).

For the purpose of this study, a survey entitled the Student Self-Concept Scale (Appendix I) was administered to the student participants in the study to assess their academic self-concept levels, both during and after having completed one of the two programs under study. The scale was comprised of 72 statements that measured the students' levels in the following three self-concept domains: academic, social, and selfimage. As was more fully elaborated in Chapter 4: Results, five independent $t$-tests were 
performed to compare the mean responses of the student participants in the study, analyzing both the total group responses, as well as the year-to-year within each program and same year between two program responses (Tables 11 through 20).

These findings were reported according to their mean, standard deviation, and to denote the level of statistical significance, if present, between each of the two groups. Finally, the item-by-item academic self-concept subgroup findings were further elaborated upon in Chapter 4:Results, as academic self-concept was the domain necessary to answer the overall driving research question. The findings demonstrate:

- The total ESL group scored slightly higher than the total NAC higher in terms of total confidence level scores, though these findings were not statistically significant.

- The academic self-concept subscale total score was equal for both the ESL and the NAC groups of students.

- The social self-concept subscale was slightly higher for the total ESL group, yet again not statistically significant.

- The self-image sub-score demonstrated that the ESL group exhibited a great deal more personal self-confidence, which was moderately statistically significant. The above findings illustrate that the ESL group, as a whole, was more confident in each area except academic, therefore allowing one to assert that this program was better supporting students' confidence levels in terms of how they viewed themselves personally and socially.

To more specifically ascertain the differences in self-concept levels among the two programs and the year in which the students were enrolled, these same independent 
tests for statistical significance were conducted looking at the two programs during a given year in which the students attended, and the impact of the programs on the students from one year to the next. When analyzing the findings for both the NAC and the ESL students during their first year of attendance in such a program, it became apparent that:

- The NAC group had a slightly higher mean total response than the ESL group, though this response was not statistically significant.

- The academic and social self-concept subscales exhibited a somewhat higher mean score for the NAC students than the ESL students, though, conversely, in terms of personal self-concept, the first year ESL group reported a higher mean score than the first year NAC group on the self-image subset, therefore demonstrating a slightly higher personal self-concept level.

- None of the sub-scale results, not the academic, social, nor self-image, demonstrated statistically significant differences.

The findings illuminate that the NAC students enter schools in the United States feeling a bit more confident than ESL students, academically and socially, yet the ESL immigrant youth appear to feel slightly more confident when considering themselves on a personal level.

The self-concept levels of students who had completed their classes in either the New Arrival Center program or the English as a Second Language program prior to the time of the study were additionally analyzed to better ascertain students' self-concept levels after completing one of the two programs under study. When analyzing the confidence levels as a whole, the findings suggest: 
- The second year ESL cluster demonstrated a higher self-concept level then the second year NAC group, the difference in the total mean scores being moderately statistically significant.

- When examining the academic self-concept and social self-concept subgroup scores, the second year ESL group was again more highly confident than the second year ESL group of students. The differences in both the academic and the social self-concept subgroup mean scores, though, were not statistically significantly different.

- The self-image subgroup also exhibited a higher mean score for the second year ESL cluster than the second year NAC cluster of participants, and these mean differences were highly statistically significantly different.

The above results reveal that the confidence level self-concept scores were statistically significantly higher for second year ESL students than for second year NAC students, a finding that demonstrates that students exiting the NAC program are not being properly supported by their comprehensive sites and are feeling more anxious, academically, socially, and personally, as they are mainstreamed to sheltered and general education classes.

When analyzing the mean self-concept scores of the students as they moved from one year to the next within the NAC program, the findings show:

- The first year NAC participants reported higher overall confidence levels than the second year NAC students. The difference in the total mean scores between these two groups was not statistically significant. 
- The academic self-concept subset further demonstrated that the year-one NAC students felt more confident than the second year NAC, when considering their academic abilities, these scores being moderately statistically significant.

- Though also not statistically significantly different, the social self-concept and self-image subgroups additionally illustrated that the first year NAC students reported feeling more confident than the second year NAC students on both the social and self-image subscales.

These findings illuminate the notion that students feel highly supported and successful while attending classes within the NAC program, but they are more insecure and express uncertainty about themselves academically and personally as they exit to mainstream and sheltered classes the following school year.

The final confidence level analysis of the mean survey results examined the evolution of students' academic self-concept levels as they advanced from one year to the next within the ESL program. To better determine the students' self-concept levels during this progression, the average mean responses of the first year ESL students' were compared with the responses of the second year ESL students. The results demonstrated:

- The second year ESL participants reported a slightly higher confidence level than the first year ESL students on the total survey, as well as on both the academic and the self-image subsets.

- The two groups reported no mean difference on the social self-concept subset. These results show that although none are statistically significantly different, the second year ESL students under study did feel more confident, particularly in terms of academics, than the first year ESL students. 
The findings from the SSCS illustrated that the influence of the two programs under study on the self-concept levels of newcomer immigrant youth were essentially slight, in that the majority of the results did not demonstrate statistical significance. The finding that was most illuminating from the survey was that the New Arrival Center students felt somewhat more confident as a result of participation in such a program, yet the following year their confidence levels decreased dramatically compared to the students who had completed the ESL program. This finding demonstrated that students were building resiliency while attending ESL classes and being mainstreamed for a portion of their day, and therefore they felt more confident the following year in their mainstream classes. Conversely, the NAC students felt confident in the highly supportive environment present in the NAC program, but they expressed great anxiety in being mainstreamed the following year. As is elaborated on in the recommendations section, these results give rise to the need for the NAC program teachers and administrators to better support such students as they exit the program. They must develop a system of gradual release and check in with the NAC students periodically to continue to support said youth even after they have completed their requisite year in the NAC program. The subsequent recommendations made concerning students' academic self-concept took into account the findings from the above analyses of the SSCS, and additionally utilized the item-by-item academic self-concept subsection results and the item importance results described in detail in Chapter 4:Results. 


\section{Language Proficiency Levels}

Due to an inability to attain a longitudinal record of newcomer students' individual CELDT scores within each of the two programs under study, the researcher opted to use site and program redesignation rates to determine language proficiency growth over time. Redesignation statistics demonstrate students' language acquisition, as it is the rate in which English learners are determined to have acquired sufficient language to be considered Fluent English Proficient. The criteria to redesignate English learners to Fluent English Proficient is as follows: an Overall Proficiency Level (OPL) of Advanced or Early Advanced on the CELDT and a score of Advanced or Early Advanced on at least three of the CELDT subsets, Listening, Speaking, Reading, and Writing, a performance level of Advanced, Proficient, or Basic on the English section of the California Standards Test (CST), with a scale score of 333 or higher, and the recommendation of students' English teachers.

The redesignation findings suggest that sites housing both programs under study made marked growth in the number of students redesignated over a five-year period, as compared to the district redesignation totals. Sites in which NAC programs were located demonstrated a $10.5 \%$ growth in the number of English learners that were redesignated, as compared to a growth of $11.6 \%$ within the sites housing the ESL classes under study. Therefore, the findings illustrated that the ESL program redesignated students at a slightly higher rate during this time period than the NAC program (1.1\% higher), and, additionally, that both programs were reclassifying their English Learners at a rate that far exceeded that of the district as a whole, which only reported a $4 \%$ increase over these same years. These findings make the case that although both programs were far 
surpassing the district rates, the ESL classes were actually making slightly greater gains in redesignating English learners to Fluent English Proficient the NAC program during the same time period.

In comparing language acquisition rates qualitatively, the data collected during the teacher and administrator interviews demonstrated that both programs held high expectations for their students in the acquisition of language during the yearlong program in which the students were enrolled. The NAC program administrators did hold that the students within their program should acquire more than one-years growth in language level, an assertion about which many teachers were in agreement, in that the students were given intensive language instructions throughout their school day. The following quote demonstrates the NAC administrators' viewpoints on the matter:

Our expectation was that students would be able to move to an intermediate level of ESL for the next school year. And that they would be able to be successful in mainstream classes that ideally are sheltered. Yeah, so they would have reached that threshold level of intermediate proficiency. We realized of course that not all students were going to make that goal... but that was and still is our goal. And I think we have done better at it every year (New Arrival Center Administrator, 2011).

As was previously stated, the NAC teachers also felt their students should make an accelerated growth in language proficiency over one year's time. These teachers believed that due to the highly supportive environment in which the students were being educated, they ought to be making greater gains than those in more traditional programs.

Well, I try, I mean, I'm shooting for them to go from beginners to intermediate.... so, during the year they would go through that and by the end of the year test...wonderfully, I mean... if you are going to be here for four periods [and] if the goal is a level a year, but I have you for more, I feel like [we should] show something beyond a level, that's just... and, I have kids, not in everything, but I have kids that maybe in speaking or listening, they become intermediate (New Arrival Center Teacher, 2011). 
Conversely, the ESL administrators believed the students should be making the standard one-year's growth over the time in which they participated in the program. They did relate that at times students would progress more than one language level in one year, but the overall expectation was to see one level increase on the CELDT score the following fall.

I mean the official word is that you know we expect kids to make one year of growth...you know per year. So, if a student enrolled as a beginner we would expect that within about five years they would be reclassified...(ESL Administrator, 2011).

The ESL teachers viewed their students individually in terms of proficiency goals. The majority of ESL courses were made up of multiple levels of ESL students, and therefore the teachers felt it was important to examine the students separately before setting proficiency targets. The following quote aptly describes how the teachers viewed their students' language proficiency within their ESL classes:

I guess my real goal has always been to get you out of ESL as best as possible and I tell them that as well. I don't want you to be English learners for life, which is the case of many of our ELD students. So with that being said, I guess I would I don't really give them a goal at the beginning. It depends on where they enter. Although, we are all ESL [students], we are not all at the same proficiency level. Some [students] are needing help with their writing or the reading or speaking depending on what their issue is that's where I push them. So, it's almost like an individualized educational plan (ESL Teacher, 2011).

The students in both programs reported making gains over the year that they were enrolled in either the NAC or ESL program, though they still felt as though they had a great deal to learn. While in each program, both sets of students felt optimistic about their language growth, as they felt they were able to understand basic conversations and concepts and they were able to speak simple sentences in English. The students that had 
completed each program the year prior differed slightly in discussing their language acquisition levels. The NAC students appeared to feel a bit less secure linguistically in their mainstream classes the following year than the students that had completed the ESL program. The following quote demonstrates the struggle facing one student that had finished the NAC program the prior year:

It's still very difficult because when we arrived they told us that for us to understand English we need to pass seven years and I cannot have a real conversation because I am still getting it slow because people speak very fast (NAC Student, 2011).

On the other hand, the second year ESL students had seemingly acquired resiliency the prior year, perhaps due to the sink-or-swim approach they had endured in their content classes upon arrival to their new school. Consequently, the ESL students reported feeling stronger linguistically, as is demonstrated in the below quote:

I can speak now and understand very well...and I feel a lot more confident with my English than last year because last year I didn't really speak that much...I did not really talk in rooms and now I participate more in classrooms (ESL Student, 2011).

In comparing the gains in language acquisition level between the NAC and the ESL programs, the findings demonstrated that the ESL program had slightly more success than the NAC program in terms of both redesignation rates and focus group responses. Despite the NAC teachers and administrators expectations for greater gains within this program, this research demonstrated that the gains were not significantly higher for the program. As is elaborated in the recommendations section, it would behoove both programs to record students' CELDT scores over a specific period of time to determine more conclusive information about the language gains made within each 
program as well as the areas, listening, speaking, or writing, that need improvement within each program.

\section{Academic Skills}

In determining the influence of either the NAC program or the ESL program on students' acquisition of fundamental skills, it was deduced that both programs utilized the ELD portion of their classes to develop students' literacy skills, through the oral and written strategies common to teaching within the language arts domain. Both programs incorporated explicit vocabulary and grammar instruction, as well as read alouds, shared reading, guided reading, and modeled and guided writing, to both support students in acquiring English and to continue developing their literacy skills. Both programs encouraged students to transfer their literacy skills from their primary languages to English by accessing such abilities through language comparisons and primary language support. Students were expected to develop their basic skills further while acquiring the English vocabulary necessary to cultivate their bilingualism or multilingualism.

As was an essential goal of such a program, the NAC program also worked to develop students' fundamental content skills. A major objective of the program was to ensure students were prepared to enter mainstream or sheltered content classes the following year, and therefore the NAC program focused their day on both supporting students in acquiring English and in developing basic content skills. In the following quote, one NAC administrator discussed the goal of the content instruction within the program:

The way we kind of look at it is well why don't we front load those electives and give students an opportunity to have all of those electives being kind of content 
based ELD courses. So there is a math and social studies and a science and depending on the students and their needs they will take, you know, all of them during the course of the year. But the emphasis is really still on language development, building some of the language and the fundamental skills and knowledge around science or they are going to face the following year or two, focused on the standards and the same with social studies...(New Arrival Center Administrator, 2011).

On the contrary, the ESL program focused primarily on building students literacy skills, and although some ESL teachers took it upon themselves to frontload content vocabulary or basic concepts, it was not the expectation of the program to do so. The ESL program was essentially created to assist students in acquiring English, with little emphasis on building students' content skills, in that it was the responsibility of the content teachers to do so in their sheltered and mainstream content courses through differentiated instruction. Owing to the manner in which each program was created and the aim of each program, it can be argued that the NAC program was far stronger in developing students' fundamental academic skills, outside of literacy development, as this program addressed basic concepts in mathematics, social studies and science. As is expanded upon in the recommendations section, the school district in which these two programs are located should create pre-and post-assessments to evaluate newcomers' academic skills as they embark upon their education in the U.S. and after they have finished their requisite year in either program. Such assessments would give stakeholders a better understanding of the strengths entering students possess, as well as areas requiring improvement, and it would assist such programs in focusing their instruction to best support youth in acquiring their necessary academic skills to be successful in mainstream and sheltered classes. 


\section{Analysis and Interpretation of Overall Research Question}

How does a specialized newcomer program prepare secondary-level Latino immigrant youth to gain the language proficiency, academic skills, and academic self-concept necessary to be successful within an English-only educational environment, as compared to students placed in a traditional ESL program?

The overall findings of this study were mixed as was apparent from the abovementioned results, in that neither the NAC program nor the ESL program was more effective than the other in preparing newcomer youth for success in a mainstream English-only academic environment. Both programs were highly supportive of students within the English Language Development portion of the students' academic day, though as is seen in the program description, the newcomer program additionally supported students in their content classes, through content-specific language instruction and the teaching of fundamental content skills to students. Both specialized programs worked to ensure students were acquiring language and the basic skills necessary to access modified or sheltered instruction the following year, at a rate that far exceeded the district as a whole. The argument can therefore be made that such specialized programs are a necessity for immigrant youth in providing them the strong base crucial to continue acquiring language and content skills.

As was discussed in detail in the previous sub-questions, the ESL program was more successful in terms of students' academic self-concept upon completion of the program, in that the second year ESL students scored consistently higher on the SSCS than the second year NAC students. Despite the finding that the first year NAC participants scored slightly higher than the first year ESL participants on the SSCS, the results were far more conclusive regarding the second year students, as those findings 
were both more sizeable and statistically significant. The ESL program also faired slightly better in terms of redesignation rates, leading one to believe that while both programs were effective in teaching students language, the ESL program was more effective in supporting kids to learn language at a faster rate, arguably due to the academic language students in the ESL program were receiving during their sheltered and mainstream content classes. Finally, the New Arrival Center program was undeniably more effective in addressing the academic skills of newcomer youth, as the NAC program went beyond teaching literacy to also instructing in a manner that developed students' fundamental content skills. Overall, both programs were significantly more effective in supporting students to gain language proficiency, acquire academic skills, and to possess increased academic self-concept than if the students had been fully mainstreamed upon entering U.S. schools, and therefore must be championed by all educators and stakeholders as a minimal support necessary to assist newcomer youth in achieving success in such new and unfamiliar schooling environments.

\section{Discussion of Program Implications}

Despite the abovementioned gains made by the two programs, this research study was undertaken with the supposition that the self-contained New Arrival Center program would far exceed the ESL program in terms of language acquisition, the attainment of academic skills, and a higher overall student academic self-concept level, as well as by utilizing students' home cultures and languages in students' daily activities. This research presumed that the NAC program would promote an environment in which more innovative practices would be utilized, as the increased time and money available to such a program would indicate that it would have the ability to yield positive results. It is, 
therefore, quite troubling to realize that both programs are in reality more indicative of a movement towards a monolingual English-only educational environment. While both programs do use some primary language instruction as a means of explaining concepts and directions when logistically possible, neither program goes beyond a cursory use of students' primary languages or their home cultures during classroom instruction. Therefore, both programs ultimately preclude students from being educated in an environment in which their home languages and cultures can be utilized as a tool of empowerment, as they both promote the acquisition of English as being the primary overarching goal of their instruction.

In addition to the movement towards a monolingual educational environment, as was evident in the findings of this study, neither program goes beyond the acquisition of vocabulary and language skills to analyze the role language and culture plays in this country. As Macedo asserted, "the view of the teaching of English as an education sustains a notion of ideology that systematically negates rather than makes meaningful the cultural experiences of the members of the subordinate linguistic groups who are, by and large, the objects of its policies" (1994, p.131). In other words, the notion that the primary goal of each of the two programs under study is solely the acquisition of English demonstrates that they both do little to ensure value is given to the newcomer students' home languages and cultures, as well as the role students' cultures and personal experiences play in adapting to the norms of the United States. These programs also do little to promote critical inquiry or the development of student voice, as was evident in the lack of transformative teaching practices present in both programs within the study. Ultimately, the results of this study illustrate that these programs are in essence quite 
similar, in that the implementation and the goals of the programs are quite aligned with one another, which disproves the notion that the NAC program was the more innovative, supportive approach to educating newcomer immigrant youth. The following section more deeply analyzes the movement towards an English-only educational environment within the district, as the focus of the district promotes monolingual programs such as the ESL and NAC programs and moves away from the bilingual/biliteracy programs that were previously a hallmark of their English learner programs, as well as the tensions that emerged while researching the two programs within the study.

\section{Emergent Tensions Regarding the District English Learner Programs}

Although extensive research has proven the value of bilingual education in the schooling of language minority students (Baker, 2011; Brisk, 2005; Crawford, 1999; Krashen, 1996) and despite the demographics of the San Diego Unified School District (during the 2011-12 academic year virtually one-third of the total student population were English language learners) the district has experienced a drastic decline in the number of primary language programs offered within the district. As a result of restrictive language policies, such as California Proposition 227, districts such as SDUSD have chosen to implement a more subtractive approach to educating English learners (Valenzuela, 1999), focusing on promoting an English-only educational environment, rather than the additive bilingual/biliteracy options utilized in neighboring districts (Gonzalez, 2010). This movement towards an English-only model of language instruction is apparent when analyzing the reduction in student access to primary language classes, as one year prior to the 1998 passage of Proposition 227, 32.96\% of the district's English learners received 
primary language instruction with ELD support, yet during the 2010-11 school year just $4.73 \%$ of the English learners were enrolled in primary language classes (DataQuest, 2011). As was discussed in Chapter 2: Literature Review, Proposition 227 holds "a provision allowing parents and others to assign personal legal liability to any teacher, school, or district that does not implement the English language program as designated in the initiative" (Maxwell-Jolly, 2000, p.38) which could potentially dissuade educators from promoting such courses, yet it also requires that parents and guardians are informed of their option to complete a waiver that would ensure their child be educated in their home language. Therefore, by informing parents and guardians about the benefits of bilingual/biliteracy classes, and with a strong commitment to such a program, the district could legally and without difficulty remedy the decline in primary language courses, yet the movement continues to be focused on increasing English-only courses in lieu of primary language classes.

Not only does the necessary commitment to promoting the option for bilingual/biliteracy classes seem to be lacking at the district level, individual school sites within the San Diego Unified School District are further required by district leadership to adopt the centralized policies created by the office charged with overseeing the education of English learners. This approach is a stark contrast to the more decentralized approach apparent in the neighboring districts with greater student access to bilingual/biliteracy program options (Gonzalez, 2010). In the neighboring districts, schools have been given the option to choose the manner in which they instruct their English learners, thus allowing them the space to create innovative programs and to utilize such proven methods as a strong primary language approach to literacy and language instruction. The 
result of San Diego Unified School District's more centralized approach is a one-size fitsall approach to educating English learners and, additionally, a newcomer program that is more akin to an offshoot of the district's traditional ESL program than the newcomer program model as described by such theorists in the field as Deborah Short and Beverly Boyson (2004).

Some primary tensions in the implementation of San Diego Unified School District's newcomer program are both the lack of innovative strategies and the undervaluing of student's primary languages and cultures within this program, which are likely reasons that the New Arrival Center (NAC) has shown little difference in the findings when compared to the English as a Second Language (ESL) program. Even more troubling was the decline in academic self-concept experienced by NAC students as they transitioned from their highly supportive program to a less supported ESL 3/4 and sheltered or mainstream content course of study. While the intent of the district to create a more accommodating academic setting for their newcomer immigrant youth is to be commended, the NAC program is still a subtractive model as it does nothing to maintain or promote students' home languages and cultures, thereby creating a feeling for newcomer youth of being linguistically and culturally inadequate in the academic setting. This type of program is a vast improvement over a full assimilation, mainstream model, yet it does not go far enough in creating the necessary setting for students to feel connected and successful in their academic environments, as is seen in maintenance and additive bilingual/biliteracy programs (Baker, 2011; Brisk, 2005; Crawford, 1999; Krashen, 1996). 
An additional tension that became apparent while collecting data concerning the NAC and ESL programs was the lack of a systematic and effective manner of assessing the newcomer students within the two programs, both as they enter and as they exit such specialized programs. As was discussed in Chapter 2: Literature Review, the Castañeda v. Pickard decision of 1981 outlines the following three criteria for districts to follow in establishing programs to educate English learners: (1) the program must be based on "sound" educational theory; (2) it must be "implemented effectively" with adequate resources and personnel; (3) after a trial period, the success of the program must be demonstrable (Baker, 2011; Crawford, 1999; Mahoney, MacSwan, Haladyna, \& García, 2010). Despite the fact that according to Mahoney, MacSwan, Haladyna, \& García currently "there has been no challenge brought against an educational agency on the basis of Castañeda's third prong" (2010, p. 51), it is crucial that programs assess the viability of their English-only program models utilizing evidence-based evaluations.

It is clear that neither the New Arrival Center program nor the ESL program within the San Diego Unified School District has fulfilled the third prong of the Castañeda v. Pickard decision, as there appears to be little accessible data being collected to support the programs. Throughout this study, district leadership were unable or perhaps unwilling to supply evidence of short-term or long-term data to support either program, yet the programs had been in existence for a number of years. The researcher perceived this tension immediately, and, additionally, the teachers within the study confirmed this issue during the teacher interviews, as many NAC and ESL teachers had requested data yet none had received a response to the request. It is imperative the district 
analyze the program in a more effective manner to justify the existence of such a program and to assure the best option possible for newcomer youth.

Throughout the classroom observations and teacher interviews, specific tensions were uncovered in both the perceptions teachers held about their teaching practices, and in the actual strategies the instructors incorporated into their daily lessons and activities. Overall, the teachers in both programs expressed feeling comfortable teaching English learners, yet the NAC teachers felt insecure when instructing in particular content areas, as many held single-subject credentials and were not trained in all content areas. To alleviate such a tension, the district must provide specific content area professional development to the teachers hired to instruct within the NAC program, as well as time for NAC teachers to collaborate and observe one another to witness high-level teaching practices in action.

Conversely, there was additional discussion during the teacher interviews about a perceived lack of understanding and insecurity on the part of the mainstream teachers in comprehending how to best support English learners in their content area classes. This lack of confidence makes apparent the need for credentialing programs at the university level to make certain teachers are specifically educated in strategies to support such students in mainstream and sheltered classes, as well as how to effectively differentiate for the varying language levels present within the majority of the state's secondary-level schools. The changing demographics of California's schools, one child in five attending U.S. schools now lives at a home where English is not the primary language (Custodio, 2011), points to the increasing need for all teachers to be considered language instructors. All teachers must embrace and improve upon their practices to ensure English learners 
are provided the comprehensible input necessary to succeed in such classes. Lastly, it is the responsibility of the district to also make sure that ongoing professional development be provided for content-area teachers to assist them in developing their potentials as language instructors and to alleviate any fears or negative perceptions about teaching English learners at all levels within their specific content courses.

During the classroom observations it became apparent that both the NAC and ESL teachers were quite strong in utilizing effective English learner strategies, yet few differentiated instruction or utilized collaborative inquiry or critical questioning in their daily lessons. While all students were at the beginning level according to the CELDT assessment, there is great variability between overall high beginners and overall low beginners, and, additionally, students are often stronger in different language domains (reading, writing, listening, and speaking). It is crucial that teachers assess and teach in a manner that reaches students at their point of need, through small group and individual instruction, rather than solely relying on whole class instruction as a mode for disseminating the daily lessons and activities. To ensure students are given the opportunity to dialogue about and critique social and cultural norms and differences between their home countries and those of the U.S., teachers should elicit critical dialogue through collaborative inquiry and critical questioning. Such strategies must again be a distinct and valued component of the ESL and NAC programs, and therefore the district must provide professional development concerning the development of critical thinking skills within such programs. Students arrive in U.S. schools possessing a plethora of prior experiences and understandings, and therefore it would benefit such programs to utilize students' prior knowledge as well as students prior linguistic 
understandings, to create a classroom environment in which knowledge is truly coconstructed and the voices of all participants are a valued component in the education of newcomer youth.

A final tension discovered during the research study was the lack of stakeholder input into the development and implementation of both the NAC and the ESL programs. The parents of students within the programs were invited to be a part of the English Learner Advisory Committee, yet this was a state-required mandate, and therefore it appeared the district solely convened such a group to remain compliant with the state, rather than utilizing innovative approaches to include parents and community members in the educational process. A major component of a successful newcomer program includes the connection schools make with students and their families. Newcomer programs can potentially serve as a link between families and social services, as well as offer adult ESL courses for parents or guardians who may desire such classes (Boyson and Short, 2004). Neither the NAC nor the ESL programs offered such services, and therefore there was a distinct lack of parent involvement in the secondary level sites that participated in the study.

The above discussed tensions in the education of newcomer youth within the San Diego Unified School District are likely contributors to the actuality that as of the 20112012 academic year, SDUSD entered its third year of federal "program improvement" status under the No Child Left Behind mandate, due in part to its failure to meet academic goals for several student groups, including both English learners and Latino youth (Devine, 2012). The inability of this district to meet the needs of Latino youth and English learners at the federal level illustrates the necessity for SDUSD to reconsider the 
manner in which such youth are currently being educated and to create and enact a plan of action with a variety of stakeholders to better serve such youth. The next section centers on an action plan for the development and improvement of specialized English learner programs, and it will be followed by additional recommendations concerning the education of newcomer students that have been developed in accordance with this research study.

\section{An Action Plan to Develop and Improve Specialized English Learner Programs}

During their extensive three-year national study analyzing effective programs for newcomer students, Short and Boyson worked to identify exemplary programs that have been created and implemented nationally with the express goal of successfully educating secondary-level newcomer immigrant youth (2012). These theorists subsequently created a report entitled Helping Newcomer Students Succeed in Secondary Schools and Beyond in an effort to disseminate their findings on successful practices and polices that they observed during their research project and that concern the education of newcomer youth (2012). This report includes information regarding a national newcomer program database that was created by the authors which contains program overviews and data concerning 63 newcomer programs across the nation, as well as specific findings concerning ten case studies that were conducted utilizing excellent examples of newcomer programs (Short \& Boyson, 2012). Of the ten case studies researched by Short and Boyson, three case studies (located in Dearborn, MI, Omaha, NE, and Union City, NJ) focused on programs located within a school, much like the New Arrival Center (NAC) and English as a Second Language (ESL) programs analyzed for this research 
study. Therefore, in creating an action plan to develop or improve district-level specialized English learner programs, namely the NAC and ESL programs that serve newcomer youth within the San Diego Unified School District, I revisited these similar programs, as they have been deemed successful within their communities around the nation. The following action plan components are based on the findings of my research, as well as successful examples of newcomer centers. They ought to therefore be considered in the creation or the improvement of newcomer programs, as well as ESL programs when applicable:

1. Program Design - Districts should consider the location of their newcomer centers, as well as the duration of student enrollment and the length of the program day (Custodio, 2011; Short \& Boyson, 2012). Much like the ESL Teen Literacy Center in Omaha, NE, the Port of Entry program in Union City, NJ, and the Salina Intermediate Literacy Newcomer Center in Dearborn, MI, the NAC program in the San Diego Unified School District (SDUSD) utilizes a full-day, program-within-a-school option. This option allows students to gain an understanding of a comprehensive site and provides potential opportunities for students to attend mainstream elective or physical education classes, thus giving newcomer youth a more authentic experience in U.S. schools. Unlike the exemplary programs, though, which offer students the option to attend such courses for up to two years, the NAC program is fairly rigid in requiring students to be exited after one year. The SDUSD must consider lengthening the program form one year to up to two years, as this would support students for a longer period of time and could potentially alleviate the NAC-Year 2 students' drastic 
decline in academic self-concept, as was evidenced in the Chapter 4: Results section.

2. Staffing Considerations - Districts must staff newcomer programs with administrators and teachers who have extensive experience working with culturally and linguistically diverse students, are trained in utilizing effective instructional strategies for newcomer students, express a clear focus and high expectations for the program, and promote a positive and caring environment (Custodio, 2011). In states such as California, teachers must also be credentialed and have significant training working with English learners (Custodio, 2011). When available, it is beneficial to employ bilingual instructional aides to assist in language brokering, particularly in instances where the teacher is unable to communicate in students' primary languages (Custodio, 2011). Much like the NAC and the ESL programs, the exemplary programs each employed multiple instructors for their newcomer programs, yet they further employed instructional aides, part-time social workers, and guidance counselors to support the newcomer students more effectively, both socially and academically. Other than in rare instances, the SDUSD does not employ such support personnel specifically for their NAC or ESL programs, placing the majority of the social and academic responsibility on the NAC or ESL classroom teachers. It would be beneficial for the district to consider hiring bilingual support staff to assist teachers in creating a supportive and successful environment for their newcomer youth.

3. Curricula - Newcomer programs should utilize curricula that focuses on helping students to adjust to the U.S. school culture and norms, develops language 
proficiency, promotes numeracy development, and introduces students to other core content areas (Custodio, 2011). Each exemplary newcomer school has chosen curricula that meet the needs of their particular students. The schools employ a highly supported, sheltered approach to teach content areas, as well as specific ESL curricula to develop students' language skills. The language development curricula utilized in both the NAC and ESL programs at the SDUSD were chosen by a curricula committee, and therefore they are well liked by the instructors. The district must employ a similar process in choosing effective content curricula, as many of the teachers reported these materials were more akin to supplementary materials, than an actual comprehensive program. The district should also provide extensive professional development opportunities to train the teachers to use the content and literacy curricula in a manner that promotes success, in that many teachers felt there was little opportunity to attend professional development or collaborate with other similar teachers.

\section{Entrance Criteria/Placement - A successful newcomer program needs a} comprehensive and centralized intake process, which includes testing for academic content in students' home languages as well as English, assessing students' English language proficiency levels, assisting parents with enrollment procedures, and identifying and providing access to needed health services (Chang, 1990). Students should only be placed in such specialized programs when it is educationally appropriate (Chang, 1990). Though Short and Boyson's report (2012) does not specifically list the entry criteria for students within the exemplary newcomer programs, much like the NAC and ESL programs at the 
SDUSD, each program does have specific criteria, primarily focusing on students' language acquisition levels and their time in U.S. schools, as well as instances of interrupted formal schooling. These criteria determine if the programs are educationally appropriate for the students to attend. While the programs do assess students' entering language acquisition skills, neither the NAC nor the ESL programs in the SDUSD systematically assess students' academic skill levels in the content areas (with the exception of a short math assessment) and they do not, as a centralized program, focus on the health needs of students at their point of entry (though sites may opt to assist students and their families in this regard). These entrance assessments must be created and implemented at a programmatic level to ensure students' academic skills and needs are addressed at their point of entry and to better provide evidence of newcomers' growth throughout the program.

5. Development of Literacy Skills - An effective newcomer program should work to develop students' literacy skills by introducing and practicing basic reading skills, explicitly teaching high-frequency words and academic vocabulary, developing fluency in reading and writing, integrating all four language domains, teaching strategies to monitor comprehension, utilizing language supports to ensure comprehension, and promote daily reading and writing both interactively and independently (Custodio, 2011). These specialized programs also must expose students to grade-level content and instruction in a highly scaffolded manner (Custodio, 2011). Each of the three exemplary programs utilizes intensive English instructional strategies, as outlined above, as rapid English acquisition is 
a primary goal of all three programs. The NAC and ESL programs within the SDUSD mirror the exemplary programs' primary goal of rapid English language acquisition, and therefore the above strategies were observed during the classroom observations of language development instruction within the two programs under study.

6. Development of Numeracy Skills - Despite the oft-held belief that mathematics and numbers are a "universal language", in reality depending on the region in which students are originally schooled, the use of numbers and mathematics instruction can actually be quite different. Therefore, it is important for successful newcomer programs to address the numeracy needs of the students being educated in the programs. The components of an effective numeracy program can be divided into the following broad topics: math vocabulary, number sense, and number usage; operations and basic math skills; measurement; data analysis and statistics; geometric shapes and patterns; word problems; and the use of mathematics tools (Custodio, 2011). Of the three exemplary schools, the Port of Entry program at Union City, $\mathrm{NJ}$, is the only program to use primary language instruction to teach mathematics, as well as other content areas. The other two programs, much like SDUSD's New Arrival Center program, teach math in English utilizing a variety of supports. Each program has a dedicated time period in which mathematics are taught, though the Salina Intermediate Literacy Newcomer Center extends and combines this period with the teaching of science content. The NAC program within this study focuses on numeracy language development, while teaching students basic skills. Students that have adequate 
mathematics skills are further transitioned to a mainstream or sheltered math class for this portion of their academic day. It would be advantageous to utilize students' primary languages in their numeracy instruction, whenever possible, and to connect students' home languages to the English vocabulary taught within this content area. The use of students' primary languages would allow newcomers to develop their skills while further developing their home languages and it would additionally give value to the languages and skills with which the students enter the newcomer programs.

7. Development of Content Skills - A major difference between a specialized newcomer program and a traditional ESL class is the development of students' knowledge and proficiency in science, social studies, technology, and other such academic areas (Custodio, 2011; Short \& Boyson, 2012). Most content instruction for English learners occurs in one of three ways: bilingual classes, content-based ESL instruction, or sheltered content instruction, though, "bilingual content classes are especially beneficial to new arrivals who cannot afford to wait to gain proficiency in English before beginning classes in various subject areas" (Custodio, 2011, p. 76). In instances where bilingual classes are unavailable, due to restrictive language policies or students who speak a language for which no bilingual classes are available, content-based ESL instruction or sheltered instruction are the alternatives, their major difference being whether the focus is placed on language and skills development (content-based instruction) or subject matter (sheltered instruction) (Custodio, 2011). The exemplary schools differ in their content area instruction, in that one of the schools, the Port of Entry program 
at Union City, NJ, uses primary language instruction to teach content classes, while the other two programs, much like SDUSD's New Arrival Center program, teach content classes using a content-based instruction or a sheltered approach. The programs using a sheltered approach incorporate "The Sheltered Instruction Observation Protocol (SIOP) Model” created by Echevarria, Vogt, \& Short (2004) into their content teaching, which focuses on using a plethora of specific English learner strategies to assist students in accessing and understanding the content area instruction. Much like the numeracy instruction, it would be beneficial for classes within the NAC program at the SDUSD to incorporate students' primary languages into their content instruction, whenever possible, in that primary language instruction would assure students effectively gain content skills and knowledge, while concurrently affirming the importance of their home languages. In instances where primary language instruction is not possible, such as if a variety of language backgrounds are present in one class, the content-based instruction or a sheltered approach are both viable alternatives, yet instructors in such programs need ongoing training and collaboration time to ensure the English learner instructional strategies utilized in these approaches are incorporated and used effectively during content instruction.

8. Assessment - Successful newcomer programs utilize a variety of assessment measures, both those required by law and those used for placement and ongoing understanding of students strengths and needs. A federally mandated Home Language Survey and state placement assessments (California's state placement assessment being the aforementioned CELDT) are the first assessments most 
newcomers encounter upon entry to U.S. schools (Custodio, 2011). Most districts use such initial assessments for placement into newcomer programs. In addition, there is no one type of classroom assessment that is sufficient for all students and all subject areas, but some effective assessment instruments for newcomer students include: informal assessments, those used by a teacher throughout a lesson to ensure student comprehension; checklists and anecdotal records; oral proficiency assessments through dialogue or recordings; portfolios; performance assessments; and comprehension and review tests (Custodio, 2011). The three exemplary newcomer programs did not maintain there was one particularly beneficial assessment, but rather successful, newcomer programs utilize a variety of assessments to ensure students are placed appropriately and are progressing sufficiently throughout the programs. The SDUSD uses a variety of assessments required by law, such as the Home Language Survey and the CELDT, as well as district-instituted primary language assessments for placement into such specialized programs as the NAC or ESL programs. These assessments are sufficient in analyzing students' literacy skills, yet there are few centralized assessments given by the district to test students' academic and content area skills. Therefore, the SDUSD must develop a systematic method of assessing newcomers academic and content area skills to demonstrate areas of strength and need and to assess growth over the academic year. District-wide content area assessments, testing students' skills in mathematics, science, and social studies, would give teachers and administrators a better understanding of the impact of 
specialized programs, such as the NAC program, on students' acquisition of content area skills.

9. Community/Family Outreach - Effective newcomer programs offer such academic and social services as academic and postsecondary counseling, extracurricular activities, and health and family support services (Custodio, 2011). Such support services provide students with the basic assistance necessary to be physically and emotionally healthy, and they allow newcomers the opportunity to authentically interact with native speakers (Custodio, 2011). Successful newcomer programs additionally partner with community and civic organizations, institutions of higher education, and social service and health providers to ensure their programs, as well as the newcomers attending their programs, are provided the financial, social, and academic services necessary to assure students are supported both within and outside of the classroom. The three exemplary programs studied by Short and Boyson (2012) each provided both family services and community connections, such as social workers and parent/community liaisons at either the site or district levels, adult ESL classes, access to health and immigration services, as well as employment services. These services were provided by either the particular program or the site in which it was housed, or by the community partnerships universal to all three exemplary programs. The NAC and ESL programs within the SDUSD do little to connect parents or community partners to the specialized programs at the macro or district-wide level. Individual teachers within such programs have reached out to different community groups to attain social or health services for their students, but this is not seen in a 
systematic program-wide fashion. Therefore, such specialized programs should develop a system to connect with newcomer students' families and community partnerships in an effort to more effectively promote home/school connections and to provide the information capital and social and health services necessary to support student and their families in successfully negotiating school practices and life in the U.S.

10. Exit Criteria - Finally, effective newcomer programs create a plan or a continuum of services with specific transition times or criteria to determine when and how students will be exited from such specialized programs (Custodio, 2011). Most newcomer programs exit their students in one of two ways, (1) by gradually transitioning or mainstreaming kids into less language intensive courses like physical education or some electives, or (2) by moving students at certain critical points (the end of a semester or the end of a school year) (Custodio, 2011). It is critical that personnel within such specialized programs develop a plan that includes both the former newcomer teachers and the new mainstream or sheltered teachers to ensure as seamless a transition as possible, in that integration into the mainstream is often arduous and a sometimes unpleasant experience for newcomer youth (Chang, 1990; Olsen, 1997). The three exemplary programs each created their own exit plans to ensure a positive and successful transition for their newcomer youth. The Port of Entry program in Union City, NJ exits their students after they have completed and passed all classes required of ninth-grade students and when recommended by their teachers, generally after four semesters in the program. This transition is smooth, as the students are moved to regular bilingual 
classes and are therefore provided necessary primary language support. The Salina Intermediate Literacy Newcomer Center in Dearborn, MI exits their students into traditional ESL classes after the students have achieved a sufficient literacy level in English, as determined by their Developmental Reading Assessment (DRA) scores. A team at this site, including the newcomer and the ESL teachers, work together to ensure a smooth transition, usually after two years attendance in the program. Finally, the ESL Teen Literacy Center Program (Middle School) in Omaha, NE exits their students into ESL after they have reached a third-grade reading level in English. This transition usually occurs after two years within their newcomer program. If students at the site do not attain a third-grade reading level at the end of eighth grade, they have the option to attend the high school ESL Teen Literacy Center at the separate site in which it is located. The NAC program at the SDUSD exits their newcomer youth after one year in the program, unless rare exceptions are made, into a traditional ESL 3/4 program and mainstream or sheltered content classes. It would behoove the school district to reevaluate the stringent one-year timeframe to allow more students to attend such a program for an additional year. It would also be beneficial to create a transition team that includes both the NAC and the higher-level ESL teachers, to develop a plan for each student during their process of transition from one program to the next. As was discussed above in the program design section of the action plan, the opportunity for students to have extended time in the NAC program and a systematic plan for a successful transition could potentially alleviate the NAC-Year 2 students' decline in academic self-concept and would 
likely ensure their success, both academically and emotionally, within their secondary-level schooling environments.

\section{Other Research Study Recommendations}

The findings from this research study have given rise to specific needs within both the New Arrival Center and the English as a Second Language programs that were not addressed in the above action plan or require further explanation. The additional recommendations I bring forth in this section are informed by the data collected for this study, as well as by both the scholarly literature and the guiding theories presented in Chapter 2: Literature Review, and by my many years of classroom experience. The ensuing recommendations may be applied to a specific program, NAC or ESL, or pertain to the education of newcomer immigrant youth as a whole. Many of my additional recommendations were created after having discussed the particular needs and tensions generated and elucidated by the participants within the focus groups and interviews, and the source for each is listed alongside the specific recommendation. The below recommendations are grouped according to area of need and are not an exhaustive list, as with continued dialogue and research in this area, the list will certainly continue to expand:

\section{Program}

- School districts should develop a consistent ESL program model at sites across the district to ensure clarity of expectations and a coherent program model (Custodio, 2011; Short \& Boyson, 2004). 
- School districts must develop a manner of accounting for student growth over time utilizing valid and reliable language proficiency and academic proficiency assessments (Teacher Interviews).

- School districts should develop a system of recording the assessment scores of newcomer students to demonstrate the effectiveness of the programs in which the students are being educated, both longitudinally and in the short-term (Teacher Interviews).

- ESL and newcomer courses and instructors ought to be considered an integral piece of the site plan and must be included in all professional development and site decision-making processes to avoid isolation (Teacher Interviews).

- District centers should be developed at comprehensive sites in areas that have fewer English learners to ensure the numbers necessary to fund ESL or newcomer courses (Administrator Interviews; Teacher Interviews).

- Classes with multiple ESL levels present have to be provided qualified and highly trained support staff to assist in conducting simultaneous ESL groups, mirroring an effective co-teaching model (Teacher Interviews).

- Students must be purposefully placed in mainstream or sheltered content courses with teachers that are both eager and highly trained in teaching English learners, in particular newcomer immigrant youth (Administrator Interviews; Teacher Interviews).

- All teachers should reflect on their beliefs and practices and they must receive extensive training to ensure they are meeting the needs of their English learners (Teacher Interviews; Student Focus Groups). 
- All staff must address students' emotional well-being and recognize the stresses that student incur as they immigrate to a new country (Teacher Interviews; Student Focus Groups).

\section{Curricula}

- Newcomer programs must outline the expectations for both the English Language Development and the content area classes within the program and create consistent pacing guides for all curricular areas (Teacher Interviews).

- Districts should provide ESL courses with up-to-date pacing guides that address the multiple ESL levels within each classroom (Teacher Interviews).

- Districts ought to create a curricula committee with the necessary stakeholders to preview and choose effective content curricula for the ESL Math, ESL Science, and the ESL History classes within newcomer programs (Teacher Interviews).

\section{Language Instruction}

- Students should have frequent opportunities to practice oral and written language in a highly rigorous, yet low-anxiety environment (Krashen, 1992; Administrator Interviews; Teacher Interviews).

- Students must feel safe to take academic and linguistic risks in the academic environment (Krashen, 1992; Teacher Interviews).

- Students should have oral and written language modeled frequently and independent or partner work must be highly scaffolded with the use of sentence 
frames or choral reading as a means of practicing language (Krashen, 1992;

Administrator Interviews; Teacher Interviews).

- New vocabulary must be explicitly taught with the use of visual and contextual aids or physical gestures and movements (Krashen, 1992; Administrator Interviews; Teacher Interviews).

- A school-wide program ought to be created in which students are paired with a willing English-only partner to attain practice in creating authentic conversations with a strong language model (Student Focus Groups).

- ESL and newcomer teachers should utilize popular culture, for example popular music, movie clips, and videos, to support instruction in an effort to help students connect on a personal level as they acquire English (Student Focus Groups).

\section{Content Instruction}

- Teachers should consider the manner in which students were instructed in their home countries to ensure they understand how to access content area instruction (Student Focus Groups).

- Students must be provided concrete, hands-on activities rather than a majority lecture, particularly in highly language intensive content courses (Krashen, 1992; Student Focus Groups).

- Visuals, manipulatives, or technology should be incorporated into instruction, as students reported not having had experiences with many content concepts in their home countries (Student Focus Groups). 
- Content teachers must speak slowly, pronounce words carefully, and repeat words and phrases periodically to ensure students' comprehension (Krashen, 1992; Student Focus Groups).

\section{Cultural Responsiveness}

- Site and district leadership should provide professional development to all on-site staff that focuses on the incorporation of culturally responsive instructional practices into daily instruction (Gay, 2010; Ladson-Billings, 1995).

- Site staff should be given the space to have honest discussions about how to best instruct and value students' home cultures and backgrounds within a multicultural, multilingual environment (Gay, 2010; Ladson-Billings, 1995; Teacher Interviews).

- Schools must incorporate role models, value systems, perspectives, and relevant issues from students' home cultures (Pang, 2005).

- Schools should address the effect of students' anxieties, stresses, differential skills and abilities, and discontinuities between youths' home and school cultures in examining the barriers to student achievement, rather than blaming students, families, and socioeconomic status (Gay, 2010).

- Teachers must reflect on their levels of cultural awareness and personal biases (Gay, 2010; Ladson-Billings, 1995).

- Teachers should contemplate their capacity to integrate multiple linguistic and cultural backgrounds into their instruction (Gay, 2010; Ladson-Billings, 1995). 
- Students should be provided authentic, real-world linguistic and cultural experiences in the academic environment (Gay, 2010; Ladson-Billings, 1995; Teacher Interviews; Administrator Interviews).

- Students should be purposefully placed in cooperative groups to encourage cocreation of knowledge and understanding (Gay, 2010; Ladson-Billings, 1995).

\section{Pedagogical Orientations}

- Teachers should go beyond teaching fundamental skills to developing students' critical consciousnesses and high-order thinking skills (Cummins, 2009).

- Teachers should move toward transformative teaching practices by explicitly teaching students about how knowledge intersects with power in society and about how to act on societal inequities through social action (Cummins, 2009).

- Teachers must incorporate new literacies into classroom practices by addressing multilingualism and technological literacy in daily instruction (Cummins, 2009)

- Students should be given choices in their education and have authentic decisionmaking power to provide students a sense of power over their education (Gay, 2010).

- Students must be provided a schooling environment rife with academic rigor and high expectations (Gay, 2010; Ladson-Billings, 1995; Administrator Interviews).

- Teachers should be cautious in pairing newcomer youth with bilingual students for translation and communication purposes to ensure the pairings create a positive and synergistic experience for both students (Student Focus Groups). 


\section{Academic Self-Concept}

- Newcomer programs should consider how to better support students academically and personally as they are mainstreamed after they complete their requisite year in the program (Administrator Interviews; Teacher Interviews; Student Focus Groups).

- Newcomer programs need to ensure students are being gradually mainstreamed the following year, by ensuring students are placed with highly supportive and caring teachers in mainstream and sheltered classes (Teacher Interviews; Student Focus Groups).

- Newcomer and ESL programs must periodically check in with the students as they progress through their schooling career to ensure they are supported throughout their years at a given site (Teacher Interviews).

- Teachers of newcomer youth must ensure students feel safe and confident to take chances within the class environment by being cautious when compelling students to participate in front of others (Krashen, 1992; Teacher Interviews).

- Newcomer youth should be given modified assignments to ensure they feel successful in class (Krashen, 1992; Teacher Interviews).

- Teachers should make sure students understand the directions for assignments and projects by supporting students using primary language or peer assistance to help translate the instructions (Krashen, 1992; Teacher Interviews; Student Focus Groups).

- Teachers should consider modifying grades according to the work that newcomers are able to complete at their point of language acquisition, rather than comparing 
their output and their grades to those of native-born peers (Teacher Interviews; Student Focus Groups).

\section{Research Study Assumptions}

In conducting this research study, it was assumed the participants were able to make meaning of and were willing to answer and discuss the questions being posed to them by the researcher, as well as the instruments utilized in this research. The instruments and focus groups were translated to ensure accurate responses and the researcher was present to clarify any confusion, yet this study assumed the participants understood the questions or instruments as they were posed in this study. This study further assumed participants who felt unable to take part in the study did exercise their right to remove themselves from the study, rather than answer falsely. Lastly, it was assumed participants in this study were able to give their full attention to the questions as they answered them, and were able to answer in as straightforward a manner as possible.

\section{Research Study Limitations}

The limitations of this study were the sample size, time constraints, and a lack of record concerning individualized CELDT data and content assessments. According to Mertens (2005), the rule of thumb for sample size in survey research is 100 participants per major subgroup and 20 to 50 participants per minor subgroup. Due to the specific requirements necessary for students to qualify for said study, 192 total students completed the survey portion of this study, 98 ESL students and 94 New Arrival Center students. While the two major subgroups, ESL and New Arrival Center, consisted of 
close to 100 participants each, it would have been preferable to have a larger sample size to better represent a larger population. The four minor subgroups (ESL - Year 1, ESL Year 2, New Arrival Center - Year 1, and New Arrival Center - Year 2) were made up of a minimum of 43 and a maximum of 51 participants per subgroup for each of the four subgroups. Though the number of student participants within each minor subgroup did fall within the range of an acceptable sample size for survey research, it would have been preferable to have a larger sample size to allow for better generalization of the results.

Furthermore, according to Mertens the recommended size for focus groups is 7 to 10 participants per focus group and 4 focus groups for each major audience (2005).

While the goal of this study was to conduct four student current student focus groups and two former student focus groups, it was problematic to create groups of 7 to 10 participants, as participation for this study was voluntary and during non-instructional time. Students were provided pizza and soda as compensation, yet as the focus groups were conducted after school, many students who were approached to take part in the study either opted out or were unable to participate owing to other obligations. Due to such constraints, the six focus groups were made up of 4 to 9 students each. Despite this number being a limitation, the researcher found the smaller groups to be equally productive as the larger focus groups, as all of the students appeared to feel more comfortable participating in the dialogue rather than just the more verbal students, as was seen in the larger focus groups. Finally, the sample was taken from one urban school district in California that employs either newcomer classes or traditional ESL placements in educating their newcomer immigrant youth, making this study generalizable only to similar school districts. 
In addition to sample size, time constraints limited this study, in that this study was conducted during one semester of one academic school year. The actual observation, survey, interview, and focus group data was collected over a one-month period, though the demographic and follow up data collection were completed over a several month period. It would have been preferable to conduct a longitudinal study to show the language acquisition, skills acquisition, and academic self-concept levels of immigrant youth as they progressed through their secondary schooling, but due to a lack of time, this study demonstrates the aforementioned variables at one particular point in time. In an effort to alleviate the issue of time, former students who had completed one of the two programs under study during the prior academic year or two were asked to complete the SSCS and participate in the student focus groups. The data gathered from past students makes evident the long-term effects of participation in the two specialized newcomer programs that are being compared for this study.

As the research was being conducted, it became apparent to the researcher that there was a lack of consistent assessment measures across the district that demonstrated individual language proficiency or the academic skill levels of students, in particular newcomer youth. During the data collection process, one major discovery of the research was that there was no effective mode of attaining a longitudinal record of students' CELDT scores within each of the two programs under study. The researcher worked with the CELDT coordinators at the district level and attempted to access the data utilizing the names of classroom teachers who taught within each of the two programs, yet it was impossible to track student level data over time through this means or any alternate means, primarily because information was not disaggregated and available in this 
fashion, and because many students were not still present within the system. Therefore, the researcher was unable to track the language level growth of students within the individual programs over time using the CELDT measure. The researcher opted to utilize site redesignation data to analyze language acquisition over time, though this was limited as well, in that students are not expected to be redesignated to Fluent English proficient for at least five years, though often it takes longer, and the New Arrival Center had only been in existence for four years at the time of this study. The redesignation data was therefore an indicator of the language acquisition growth of the sites at which the six NAC and ESL cases were located at the time of the study, over a period of time that on occasion may not have included the program under study. The redesignation data was useful in demonstrating the trend of language acquisition at such sites, and therefore is reported in the findings section.

Finally, the growth in students' academic skills were determined through the interviews and focus groups, as there were no consistent pre- or post-tests that analyzed students' skills in the content area, with the exception a short district-created math assessment. The results of the entering math assessment informed placement and academic needs of students in mathematics, yet these scores were seemingly not collected or analyzed as a program whole, and the students were not given a consistent postassessment to demonstrate growth over the year. Finally, students' fundamental science or social studies skills were not assessed as student entered their U.S. classes, and therefore there was no baseline score with which to demonstrate growth over the year. Therefore, the students' academic skill data was attained and disseminated utilizing the interview and focus group data collected for this research study. 


\section{Recommendations for Future Research}

The deficiency of research considering the impact of newcomer programs on secondary-level newcomer youth suggests there is a considerable need to continue studying both the short-term and the long-term impacts of such courses on immigrant youth. In an attempt to increase educators' and policy makers' understanding of the importance of more effectively supporting immigrant youth, further case studies must be conducted in districts across the nation that contain such programs to compare the effects of newcomer and ESL programs at a national level. It is important that this type of research be expanded to create more extensive generalizations about the findings, and therefore this research could explore the effects of such programs on varying ethnic and linguistic backgrounds, rather than solely focusing on Latino immigrant youth. Additionally, most databases currently only disaggregate data about newcomer students within the larger subgroup of English language learners. Researchers must separate the newcomer data to study the needs and the success rates of these students within the varying program models. This data should further be gathered over several years to offer a longitudinal view of newcomer needs and successes within differing programs. Owing to the fact that the high school dropout rates of immigrant youth are significantly higher than those of native-born students (Laird, Lew, DeBell, \& Chapman, 2006), continued research is crucial in disseminating information and devising strong programs to support such a critical and precious group of students. 


\section{Personal Reflection}

After having completed this research study, I have become even more aware of the need for researchers and educators to continue developing programs that will support culturally and linguistically diverse students, namely newcomer immigrant youth, in connecting both personally and scholastically to their academic environments. Progress towards a multilingual/multiliterate environment in U.S. schools must take the place of the current English-only educational drive to ensure students a classroom environment that places value on their home languages and cultures and, additionally, gives students the necessary space to continue developing their own prior languages as they acquire English. Accordingly, educators and school personnel must work collaboratively with parents, students, and community members to challenge inequitable and restrictive language policies that ultimately limit site-level programs and curricula choice. Educators and school personnel should also work with parents and guardians to ensure districts provide the necessary information to all families, both native-born and newcomers, about the value of bilingual/biliteracy courses of study, as well as the processes of enrolling their children in such programs through program waivers. It is critical that researchers and school personnel continue to educate the public about the merits of bilingual/biliteracy programs, as these types of programs provide the greatest opportunity for culturally and linguistically diverse students to connect with their academic institutions.

In instances where bilingual/biliteracy programs are not possible, such as if an insufficient number of students speak a particular language or there are a lack of instructors that are certified bilingual in a specific language, specialized programs such as 
newcomer and English as a Second Language programs must utilize innovate strategies to ensure all students comprehend instruction and feel valued for their unique background knowledge and perspectives. This study has demonstrated to me that such strategies can only be developed after teachers and school personnel reflect on their own beliefs and teaching practices to consider whether or not students' home cultures, languages, and perspectives are in fact present in daily instruction. Until all teachers take the time to reflect on their own pedagogical practices and consider how they can better include their students' backgrounds in their daily instruction, teaching practice will undoubtedly remain unchanged.

In addition to my role as a researcher, I am a classroom teacher and a White native-English speaking female, and, therefore, this study has given me the time and the want to reflect on my own instructional practices to make certain I am also considering my students home cultures and languages, as well as their individual strengths and needs, in my daily instruction. This study has allowed me to observe peers in specialized programs that are regularly utilizing creative and exciting teaching styles, though, there were also times in which the observed instruction was of a more traditional lecture format with little student interaction. Observing such instances of divergent teaching practices has permitted me to consider my own mode of teaching, as well as ways I can improve my daily instruction to ensure students' voices and individual perspectives are valued within our classroom community. This study has therefore contributed to my growth as a classroom teacher and a researcher, and it has further confirmed my need to continue researching and working with others to develop supportive programs and practices for both newcomer students and all culturally and linguistically diverse youth. 


\section{References}

Alpert, E. (2009). Proposed schools policy says two languages better than one. Retrieved May 15, 2012 from: http://www.voiceofsandiego.org/education/article 9a4b573db140-5608-8712-162bc3ce6f2e.html

Areepattamannil, S. \& Freeman, J. (2008). Academic achievement, academic selfconcept, and academic motivation of immigrant adolescents in the greater Toronto area secondary schools. Journal of Advanced Academics, (19)4, p. 700 - 743.

August, D. \& Hakuta, K. (1997). Improving Schooling for Language-Minority Children: A Research Agenda. Washington, DC: National Academy Press.

Baker, C. (2011). Foundations of Bilingual Education and Bilingualism $\left(5^{\text {th }}\right.$ ed.) Buffalo, NY: Multilingual Matters.

Bourdieu, P. \& Passeron, J.C. (1977). Reproduction in Education, Society, and Culture. trans. By Richard Nice. London: Sage Publications.

Bourdieu, P. (1986). The forms of capital. In J.G. Richardson (Ed.), Handbook of Theory and Research for the Sociology of Education (pp.241-258). New York: Greenwood Press.

Boyson, B.A, \& Short, D. J. (2003). Secondary school newcomer programs in the United States (Research Report No. 12). University of California, Santa Cruz: Center for Research on Education, Diversity \& Excellence.

Brisk, M.E. (2005). Bilingual Education: From Compensatory to Quality Education. Mahwah, NJ: Lawrence Erlbaum Associates.

Buchholz, B. (n.d.). Ethnic identity, educational beliefs, and perceived academic competence in African American seventh and eighth graders. Retrieved April 1, 2006, from University of North Carolina at Chapel Hill Web site: http://psychology.unc.edu/honors/Buchholz.htm

Cadiero-Kaplan, K. (2004). The Literacy Curriculum and Bilingual Education. New York, NY: Peter Lang Publishing, Inc.

California Department of Education (2010). English learner instrument for 2010-11 categorical program monitoring. Retrieved March 20, 2011, from: http://www.cde.ca.gov/ta/cr/progins1011.asp

California Department of Education (2011). Language census data. Retrieved November 18, 2011, from: http://dq.cde.ca.gov/dataquest/

Camarota, A. (2001). Immigrants and the Schools. Washington, DC: Urban Institute. 
Capps, R., Fix, M., Murray, J., Ost, J., Passel, J, \& Herwantoro, S (2005). The new demography of America's schools: Immigration and the No Child Left Behind Act. Washington, DC: Urban Institute.

Center for Applied Linguistics (2010). Secondary newcomer programs in the U.S. Retrieved on March 24, 2010 from: http://www.cal.org/CALWebDB/Newcomer/

Chang, H. (1990). Newcomer programs: Innovative efforts to meet the educational challenges of immigrant students. San Francisco, CA: California Tomorrow.

Chomsky, N. (2002). Syntactic Structures. Berlin, Germany: Walter de Gruyter GmbH \& Co.

Contreras, A.R. (2002). The impact of immigration policy on education reform: Implications for the new millennium. Education and Urban Society, 34(2), $134-155$.

Crawford, J. (1997). The campaign against proposition 227: A post mortem. Bilingual Research Journal, 21(1), 1 - 29.

Crawford, J. (1999). Bilingual Education: History, Politics, Theory, and Practice. Los Angeles, CA: Bilingual Educational Services, Inc.

Cummins, J. (1986) Empowering minority students: A framework for intervention. Harvard Educational Review, 56(1), 18 - 24.

Cummins, J. (2004) Multiliteracies pedagogy and the role of identity texts. In K. Leithwood, P. McAdie, N. Bascia, \& A. Rodigue (Eds.) Teaching for deep understanding: Towards the Ontario curriculum that we need (pp. 68 - 74). Toronto: Ontario Institute for Studies in Education of the University of Toronto and the Elementary Federation of Teachers of Ontario.

Cummins, J. (2006). Mulitliteracies and equity: How do Canadian schools measure up? Education Canada, 42(2), $4-7$.

Cummins, J. (2009). Transformative multiliteracies pedagogy: School-based strategies for closing the achievement gap. Multiple Voices for Ethnically Diverse Exceptional Learners, 11(2), $38-56$.

Custodio, B. (2011). How to Design and Implement a Newcomer Program. Boston , MA: Pearson Education, Inc.

Darder, A. (1991). Culture and Power in the Classroom: A Critical Foundation for Bicultural Education. Westport, CT: Bergin \& Garvey. 
Devine, R. (2012) District misses deadline for federal money. Retrieved May 25, 2012 from: http://www.nbcsandiego.com/news/local/District-Misses-Deadline-for-FederalMoney-144718275.html

Echeverria, J., Vogt, M., Short, D. (2004). Making Content Comprehensible for English Learners: The SIOP Model. Boston, MA: Pearson Education, Inc.

Education Data Partnership (2011). Fiscal, demographic and performance data on California's K-12 schools. Retrieved November 18, 2011, from: http://www.ed-data.k12.ca.us/welcome.asp

Faltis, C. \& Coulter, C. (2008) Teaching English Learners and Immigrant Students in Secondary Schools. New Jersey: Prentice Hall.

Feinberg, R. C. (2000). Newcomer schools: Salvation or segregated oblivion for immigrant students? Theory Into Practice, 39(4), 220 - 227.

Field, A. (2005). Discovering Statistics Using SPSS. London, U.K.: Sage Publications.

Fix, M. \& Capps, R. (2005). Immigrant children, urban schools, and the No Child Left Behind Act. Retrieved April 1, 2006, from http://www.migrationinformation.org/ Feature/display.cfm?id=347

Fix, M. \& Passel J. (2003). U.S. Immigration - Trends and Implications for Schools. Paper session presented at the annual National Association of Bilingual Education meeting, New Orleans, LA.

Friedlander, M. (1991). The newcomer program: Helping immigrant students succeed in U.S. schools. . (Report No. 8). National Clearinghouse for Bilingual Education (ERIC Document Reproduction Service No. ED339290)

Gándara, P. (2000). In the aftermath of the storm: English learners in the post-227 era. Bilingual Research Journal, 24(1/2), 1 - 13.

Gándara, P. \& Hopkins, M. (2010). The changing linguistic landscape of the United States. In P. Gándara \& M. Hopkins (Eds.), Forbidden Language: English Learners and Restrictive Language Policies (pp. 7 -19). New York, NY: Teachers College Press.

Gándara, P., Losen, D., August, D., Uriarte, M., Gómez, M.C., \& Hopkins, M. (2010). Forbidden language: A brief history of U.S. language policy. In P. Gándara \& M. Hopkins (Eds.), Forbidden Language: English Learners and Restrictive Language Policies (pp. 20 - 33). New York, NY: Teachers College Press.

Garcia, E.E. (2005). Teaching and Learning in Two Languages. New York, New York: Teachers College Press. 
Garcia, O. \& Kleifgen, J.A. (2010). Educating Emergent Bilinguals. New York, New York: Teachers College Press.

Garcia, R. J. (1995). Critical race theory and proposition 187: The racial politics of immigration law. Chicano-Latino Law Review, 17(118), 118 - 154.

Gay, G. (2010). Culturally Responsive Teaching: Theory, Research, and Practice. New York, New York: Teacher's College Press.

Genesee, F. (1999). Program alternatives for linguistically diverse students. (Educational Practice Report No. 1). University of California, Santa Cruz: Center for Research on Education, Diversity \& Excellence.

Giampapa, F. (2010). Multiliteracies, pedagogy and identities: Teacher and student voices from a Toronto elementary school. Canadian Journal of Education, 33(2), 407 $-431$.

Gonzalez, G. (2010). Language, Policy, and Access for English Learners: Pedagogy, Outcomes, and Accountability (Doctoral Dissertation). Available from ProQuest Dissertations \& Thesis database (3421460).

Gresham, F., Elliot, S., \& Evans-Fernandez, S. (1993). Student Self-Concept Scale. Circle Pines, MN: American Guidance Service, Inc.

Guay, F., Marsh, H.W., \& Boivin, M. (2003). Academic self-concept and academic achievement: Developmental perspectives on their causal ordering. Journal of Educational Psychology, (95)1, 124 - 136.

Hung Hon, R. Y. \& Yeung, A.S. (2005). Low achievers' parent-child relations and liking of school. Retrieved April 1, 2006 from University of Western Sydney, Hong Kong Institute of Education SELF Research Centre Web site: http://72.14.203.104/search ?q=cache:m5-qL-05bcQJ:www.aare.edu.au/05pap/yeu05353.pdf+academic +selfconcepts + with + immigrant + students $\& h l=e n \& g l=u s \& c t=c \operatorname{lnk} \& c d=6 \& i e=U T F-8$

Hutchinson, N., Kirby, J., \& Carson, L. (2000). Phonological processing, family support, and academic self-concept as predictors of early reading [Electronic version]. Canadian Journal of Education, 25(2), n/a.

Jamieson, A., Curry, A., \& Martinez, G. (2001). School enrollment in the United StatesSocial and economic characteristics of students. Current Population Reports. Washington, DC: U.S. Government Printing Office

Kim, S. (1983). Self-concept, English language acquisition, and school adaption in recently immigrated Asian children. Journal of Children in Contemporary Society, 15(3), $71-79$. 
Krashen, S. (1981). Second Language Acquisition and Second Language Learning. Oxford: Pergamon Press.

Krashen, S. (1987). Principles and Practice in Second Language Acquisition. Englewood Cliffs, NJ: Prentice Hall Inc.

Krashen, S. (1992). Fundamentals of Language Education. Chicago, IL: SRA McGrawHill.

Krashen, S. (1996). Under Attack: The Case Against Bilingual Education. Culver City, CA: Language Education Associates.

Krashen, S. (2003). Explorations in Language Acquisition and Use. Portsmouth, NH: Heinemann.

Ladson - Billings, G. (1994). The Dreamkeepers: Successful Teachers of African American Children. San Francisco, CA.: Jossey -Bass Publishers.

Ladson - Billings, G. (1995). But that's just good teaching. Theory into Practice, 34(3), $159-165$.

Laird, J.,Lew, S., DeBell, M., \& Chapman, C. (2006). Dropout Rates in the United States: 2002 and 2003(NCES 2006-062). U.S. Department of Education. Washington DC: National Center for Educational Statistics.

Losen, D. (2010). Challenging limitations: The growing potential for overturning restrictive language policies and ensuring equal educational opportunity. In P. Gándara \& M. Hopkins (Eds.), Forbidden Language: English Learners and Restrictive Language Policies (pp. 195-215). New York, NY: Teachers College Press.

Lucas, T. (1996). Promoting secondary school transition for immigrant adolescents. Retrieved March 29, 2006, from http://firstsearchhoclc.org.libproxy.sdsu.edu/ WedZ/FSFETCH?fetchtypy=fullrecord:sessionid=fsapp2-34409-elfspvq5ly 5 wco:entitypagenum=17:0:recno $=5:$ resultset $=4:$ format $=\mathrm{FI}:$ next $=\mathrm{html} / \mathrm{record} \cdot \mathrm{html}$

Macedo, D. (1994). Literacies of Power. Boulder, CO: Westview Press, Inc.

MacKaye, S. (1990). California proposition 63: Language attitudes reflected in the public debate. The ANNALS of the American Academy of Political and Social Science, $508(1), 135-146$.

Mahoney, K., MacSwan, J., Haladyna, T., \& García, D. (2010). Castañeda's third prong: Evaluating the achievement of Arizona's English learners under restrictive language policy. In P. Gándara \& M. Hopkins (Eds.), Forbidden Language: English Learners and Restrictive Language Policies (pp. 50 - 64). New York, NY: Teachers College Press. 
Marsh, H. (n.d.). Causal ordering of academic self-concept and achievement. SelfConcept Research: Driving International Agendas, p. 1 - 10.

Maxwell-Jolly, J. (2000). Factors influencing implementation of mandated policy change: Proposition 227 in seven northern California school districts. Bilingual Research Journal, 24(1/2), 36 - 56.

Merriam, S. (2009). Qualitative Research: A Guide to Design and Implementation. San Francisco, CA: Jossey-Bass.

Mertens, D. (2005). Research and Evaluation in Education and Psychology: Integrating Diversity with Quantitative, Qualitative, and Mixed Methods. Thousand oaks, CA: Sage Publications.

McDonnell, L. \& Hill, P. (1993). Newcomers in American schools: Meeting the educational needs of immigrant youth. Santa Monica, CA: RAND.

Minaya-Rowe, L. (2008). Options for English language learners: a spectrum of instructional formats exist, from two-way bilingual to short-term newcomer programs. School Administrator, 65(10), 16-22.

National Center for Education Statistics (2008). Characteristics of the 100 largest public elementary and secondary school districts in the United States. Retrieved July 24, 2008, from: http://nces.ed.gov/

Ochoa, A. M. (1995). Language policy and social implications for addressing the bicultural immigrant experience in the United States. In A. Darder (Ed.), Culture and Difference (pp. 227-253). Westport, CT: Bergin \& Garvey.

Olsen, L. (1997). Made In America. New York: The New Press.

Padilla, A., Lindholm, K., Chen, A., Duran, R., Hakuta, K., Lambert, W., \& Tucker, G. R. (1991). The English-only movement: myths, reality, and implications for psychology [Electronic version]. American Psychologist, 46(2), 120-130.

Palmer, D. \& Garcia, E. (2000). Voices from the field: Bilingual educators speak candidly about proposition 227. Bilingual Research Journal, 24(1/2), 169 - 178.

Peralta Sánchez F.J. \& Sánchez Roda, D. (n.d.). Relationships between self-concept and academic achievement in primary students. Electronic Journal of Research in Educational Psychology and Psychopedagogy, 1(1), 95-120.

Peregoy, S. \& Boyle, O. (2005). Reading Writing and Learning in ESL. Boston, MA: Pearson Education, Inc. 
Pollitzer, R. \& Ramirez, A. (1981). Linguistic and communicative competence of students in a Spanish/English bilingual high school journal. NABE Journal, 5(3), 81101.

Portes, A. \& Rumbaut, R.G. (2001). Legacies: The Untold Story of the Immigrant Second Generation. Berkeley, CA: University of California Press.

Portes, A. \& Rumbaut, R.G. (2006). Immigrant America. Berkeley, CA: University of California Press.

Rosaldo, R. (1993). Culture \& Truth: The Remaking of Social Analysis. Boston, MA: Beacon Press.

San Diego Unified School District (2011). About San Diego Unified School District. Retrieved November 2, 2011, from: http://www.sandi.net/page/21

San Diego Unified School District (2012). English learner programs. Retrieved February 12, 2012, from: http://www.sandi.net/page/1361

Short, D. (1998). Secondary newcomer programs: Helping recent immigrants prepare for school success. ERIC Clearinghouse on Languages and Linguistics. (ERIC Document Reproduction Service No. ED419385).

Short, D. \& Boyson, B. (2000). Newcomer programs for linguistically diverse students. NASSP Bulletin, 84(619), 34-42.

Short, D. (2002). Newcomer programs: An educational alternative for secondary immigrant students. Education and Urban Society, 34(2), 173 - 198.

Short, D, \& Boyson, B. (2004). Creating Access: Language and Academic Programs for Secondary School Newcomers. Washington DC: Center for Applied Linguistics.

Short, D. \& Boyson, B. (2012). Helping newcomer students succeed in secondary school and beyond. Washington, DC: Center for Applied Linguistics.

Skinner, B.F. (1992). Verbal Behavior. Acton, MA: Copley Publishing Group.

Stake, R. (2010). Qualitative Research: Studying How Things Work. New York, NY: The Guilford Press.

Suarez-Orozco, C., Suarez-Orozco, M., \& Todorova, I. (2008). Learning a New Land: Immigrant Students in American Society. Cambridge, MA: The Belknap Press of Harvard University Press.

Suarez-Orozco, C. \& Suarez-Orozco, M. (2001). Children of Immigration. Cambridge, MA: Harvard University Press. 
Taylor, L. K., Bernhard, J. K., Garg, S., \& Cummins, J. (2008). Affirming plural belongings: Building on students' family-based cultural and linguistic capital through multiliteracies pedagogy. Journal of Early Childhood Literacy, 8(3), 269 - 294.

Tyack, D. (1974). The One Best System. Cambridge, MA: Harvard University Press.

United States Census Bureau. (2012) The Foreign-Born population in the United States: 2010. Retrieved May 21, 2012, from: http://www.census.gov/population/foreign/

United States Department of Education. (2002). No Child Left Behind Act of 2001, Public Law 107-110. Retrieved May 21, 2012, from: http://www2.ed.gov/policy/elsec/leg/esea02/index.html

Valdés, G. (2001). Learning and Not Learning English: Latino Students in American Schools. New York: Teachers College Press.

Valenzuela, A. (1999). Subtractive Schooling: US - Mexican Youth and the Politics of Caring. New York: The State University of New York Press.

Wentworth, L., Pellegrin, N., Thonpson, K., \& Hakuta, K. (2010). Proposition 227 in California: A long-term appraisal of its impact on English learner student achievement. In P. Gándara \& M. Hopkins (Eds.), Forbidden Language: English Learners and Restrictive Language Policies (pp. 37 - 49). New York, NY: Teachers College Press.

Young, D. (1998). Ambition, self-concept, and achievement: A structural equation model for comparing rural and urban students. Journal of Research in Rural Education, $12(1), 34-44$.

Yin, R. (2003). Case Study Research: Design and Methods. London: Sage Publications.

Zwiers, J. (2004) The third language of academic English. Educational Leadership, 62(4), 60-63. 
Appendix A

\section{Parental/Guardian Permission/Informed Consent:}

\section{Analyzing Best Practices in the Schooling of Secondary-Level Newcomer Immigrant Youth: A Comparison Study of Students' Academic Skills and Perceptions of Self Upon Exit From Specialized English Learner Programs}

You are being asked to allow your child to participate in a research study. Before you give your permission for your child to participate, it is important that you read the following information and ask as many questions as necessary to be sure you understand your child's role as a participant in this research study.

\section{Investigator}

Amanda Matas has taught newcomer immigrant students in a Beginning English Language Development class and in the content area of social studies for several years and is a doctoral student in the San Diego State University /Claremont Graduate University Joint Doctoral Program. Dr. Karen Cadiero-Kaplan, a professor at San Diego State University (SDSU) and Dr. William Perez, a professor at Claremont Graduate University (CGU), are supervising this research.

\section{Purpose of the study}

The purpose of this study is to better understand how students who are new to the United States achieve, both academically and personally, after their first year in U.S. schools. This study will ask students to communicate about how they see themselves as learners in school and how they feel in their individual classes, being that their classes are taught only in English. This study will further explore the experiences of newcomer youth as they complete their first year within one of two different programs, a self-contained newcomer program and an ESL program, examining the curricula, instruction, teachers' perceptions, and students' academic self-confidence as they complete the year within one of the two programs. Finally, this study will examine parents', teachers', and program administrators' perceptions of the two programs and their effects on student achievement and academic success.

\section{Description of the study}

If you agree to allow your student to participate in the study, he or she will be asked to:

- Complete a seventy-two question survey that uses a scale to indicate how true or how false each statement is as it describes your child as a student in the United States, and how important each statement is to your child. These statements ask the students to describe how they feel in school, and how they view themselves as learners. Your child will be asked to complete this survey one time and it will take about thirty minutes. 
- Participate in a focus group that asks the students about how they currently feel in their classes in the United States, as well as how they felt upon entering schools in the United States, both academically and personally. The focus group will occur before or after school, and will last no longer than one hour. The students will receive a copy of the questions ahead of time.

\section{Risks or Discomforts}

Your child may feel uneasy writing or talking about situations that may have made $\mathrm{him} / \mathrm{her}$ uncomfortable. Your child will be informed that he/she does not have to write or talk about or answer anything that makes him/her uncomfortable.

\section{Benefits of the study}

The benefits of this project include helping the researcher to determine which program most effectively supports newcomer immigrant youth, both academically and personally. This information will be used to write a dissertation about the topic and, in addition, the results will be disseminated to the district under study, as well as the academic field at large. This information will only be utilized in a way that maintains the participants' anonymity.

\section{Confidentiality}

Research records will be kept confidential. Your child's name will not be directly connected with responses or used in writing the article about this research. To protect your child's privacy, an identification number will be assigned to the data collected. Any data collected will be kept in a locked file cabinet to which only the investigator will have access. The data will be used solely to write a $\mathrm{PhD}$ dissertation and for possible use in a journal article or conference presentation at a later date. Your child's privacy will be maintained in all publications or presentations resulting from this study, as no actual names of people or schools will be used within this research report. Any audio recordings used to gather focus group data will be used solely to transcribe the data, and will be kept in a locked cabinet until the transcriptions are complete, at which time the recordings will be destroyed. Your student's identity will not be disclosed without consent as required by law.

\section{Voluntary Participation}

Participation in this study is voluntary. Your decision of whether or not to allow your student to participate will not affect your relations with either the researcher or the faculty at your child's school and will have no impact on your child's grades. If you decide to allow your student to participate, you are free to withdraw your consent and to discontinue his/her participation at any time without penalty or loss of benefits to which you are otherwise entitled. 


\title{
Questions about the Study
}

If you have questions about the research, you may contact the Principal Investigator or either professor supervising the study.

\author{
Amanda Matas, M.A., Joint Doctoral Student SDSU/CGU \\ Phone: 619-261-1353_Email: mandymatas@hotmail.com \\ Dr. Karen Cadiero-Kaplan, San Diego State University \\ Phone: 619-594-5155_Email: kcadiero@mail.sdsu.edu \\ Dr. William Perez, Claremont Graduate University \\ Phone: 323-610-2074Ｅmail: William.perez@cgu.edu
}

An institutional review board (IRB) is a committee that has been formally designated to approve, monitor, and review research involving humans, with the aim to protect the rights and welfare of the research subjects. If you questions regarding your child's rights as a human subject and participant, you may contact an Institutional Review Board (IRB) representative in the Division of Research Administration at SDSU (phone: 619-5946622; email: irb@mail.sdsu.edu) or an IRB representative at CGU (phone: 909-6079406; email: IRB@cgu.edu). These boards are responsible for ensuring the protection of research participants.

Your signature below indicates that you have read the information in this document and have had a chance to ask any questions you have about the study. Your signature also indicates that you agree to allow your child to be in the study and have been told that you can change your mind and withdraw your consent to participate at any time.

Name of Student Participant (please print)

Signature of Parent or Guardian

Date 


\section{Consentimiento Informativo Para Padres o Tutores:}

\section{Analizando las mejores prácticas en la escolaridad de los jóvenes inmigrantes de nivel secundario: Un estudio comparativo de las habilidades académicas de los estudiantes y percepciones de sí mismo después terminando de programas especializados de Aprendices de Inglés}

Se le pide que permita que su hijo/a para participar en un estudio de investigación. Antes de dar su permiso para que su hijo/a participe, es importante que lea la siguiente información y haga tantas preguntas como sea necesario para asegurarse de que entienda el papel de su hijo/a como participante en este estudio de investigación.

\section{Investigador}

Amanda Matas ha enseñado a estudiantes inmigrantes recién llegados a los Estados Unidos en clases para el desarrollo del idioma de Inglés y en el área de contenido de estudios sociales durante varios años. Ella tambien es estudiante de doctorado en la Universidad Estatal de San Diego (SDSU) / Claremont Graduate University Común programa de doctorado. Ambos La Dra. Karen Cadiero-Kaplan, profesora en SDSU, y el Dr. William Pérez, profesor de la Claremont Graduate University (CGU), está supervisando la investigación.

\section{Propósito del estudio}

El propósito de este estudio es comprender mejor cómo los estudiantes que son nuevos en los Estados Unidos logran tanto, académicamente como personalmente, después de su primer año en la escuela. En este estudio se le pide a los estudiantes que refleccionen cómo ellos mismos se ven como estudiantes en la escuela y cómo se sienten en sus clases individuales (siendo que sus clases se enseñan únicamente en Inglés). Este estudio seguirá estudiando las experiencias de los jóvenes recién llegados al terminar su primer año en una de dos programas diferentes, un programa recién llegado autónomo o un programa de ESL. Voy a examinar los planes de estudio, la enseñanza, la percepción de los maestros, y su confianza academica en sí mismo al terminar el año escolar en uno de los dos programas. Por último, este estudio examinará los sentimientos de sus padres, profesores, administradores acerca de los dos programas y sus efectos sobre el rendimiento de los estudiantes y el éxito académico.

\section{Descripción del estudio}

Si está de acuerdo para permitir que su estudiante pueda participar en el estudio, él o ella se le pedirá: 
Completar una encuesta de setenta y dos preguntas que utilizan una escala para indicar qué tan cierto o falso cada declaración es, como su hijo/a se describen como estudiantes en los Estados Unidos, y la importancia de cada declaración para su hijo/a. Estas declaraciones piden a los alumnos que describan cómo se sienten en la escuela, y cómo se ven a sí mismos como estudiantes. Su hijo/a tendrá que completar esta encuesta una sola vez y tendrá cerca de treinta minutos para hacerlo.

Participaran en un grupo de enfoque que les preguntara a los estudiantes cómo se siente actualmente en sus clases en los Estados Unidos, así cómo se sintieron al entrar a la escuela en los Estados Unidos, tanto académicamente como personalmente. El grupo de enfoque se producirá antes o después de la escuela, y tendrá una duración no más de una hora. Los estudiantes recibirán una copia de las preguntas antes de tiempo.

\section{Los riesgos o molestias}

Su niño/a puede sentirse incómodo escribiendo o hablando sobre situaciones que pueden haberle pasado. A su hijo/a se le informará de que no tiene que escribir, hablar, o contestar cualquier pregunta que lo/a haga sentir incomodo/a.

\section{$\underline{\text { Beneficios del estudio }}$}

Los beneficios de este proyecto es para ayudar a los investigadores a determinar cual es el programa más eficaz de apoyo para la juventud inmigrante de estudiantes recién llegados, tanto académicamente como personalmente. Esta información se utilizará para escribir una tesis sobre el tema y, además, los resultados se difundirán en la zona de estudio, así como el ámbito académico en general. Esta información sólo se utilizará en forma anonima.

\section{Confidencialidad}

Expedientes de esta investigación se mantendrá confidencial. El nombre de su hijo/a no estará directamente relacionada con las respuestas o utilizados al escribirse un artículo sobre esta investigación. Para proteger la privacidad de su hijo/a, un número de identificación serán asignados. Los datos recopilados se guardarán en un archivador cerrado al cual sólo el investigador tendrá acceso. Los datos serán utilizados exclusivamente para escribir una tesis de doctorado, para el posible uso en un artículo de revista, o en una conferencia de presentación en un futuro. La privacidad de su hijo/a se mantendrá en todas las publicaciones o presentaciones como resultado de este estudio, ya que los datos personales no se utilizarán en este informe de investigación. Las grabaciones de audio utilizadas para recopilar datos de los grupos de enfoque se utilizará exclusivamente para transcribir los datos y se mantendrá en un armario cerrado con llave hasta que las transcripciones sean terminadas. Al final las grabaciones serán destruidas. La identidad de su estudiante no será revelada sin autorización exigida por la ley. 


\title{
Participación voluntaria
}

La participación de este estudio es voluntaria. La decisión para permitir que su estudiante participe no afectará sus relaciones con el investigador o el maestro en la escuela de su hijo. Tampoco tendrá ningún impacto en las calificaciones de su hijo. Si usted decide permitir que su estudiante pueda participar, usted es libre de retirar su consentimiento y de suspender su participación en cualquier momento sin sanción o pérdida de beneficios a los que usted tiene derecho.

\section{Preguntas Sobre el Estudio}

Si usted tiene preguntas acerca de la investigación, puede comunicarse con el investigador principal o el profesor supervisor del estudio.

\author{
Amanda Matas, M.A., Estudiante de Doctorado Conjunto SDSU/CGU \\ teléfono: 619-261-1353_correo electrónico: mandymatas@hotmail.com \\ Dr. Karen Cadiero-Kaplan, Universidad Estatal de San Diego (SDSU) \\ teléfono: 619-594-5155_correo electrónico: kcadiero@mail.sdsu.edu \\ Dr. William Perez, Universidad Claremont Graduate \\ teléfono: 323-610-2074_correo electrónico: William.perez@cgu.edu
}

Un instituto de revision llamado Institutional Review Board (IRB) es un comité que ha sido designado oficialmente para aprobar, supervisar, y revisar las investigaciónes que incluyen a seres humanos, con el objetivo de proteger los derechos y el bienestar de los sujetos de la investigación. Si usted tiene pregunta con respecto a los derechos de su hijo como el participante, puede comunicarse con un representante de la división de administración de esta investigación en SDSU (teléfono: 619-594-6622, correo electrónico: irb@mail.sdsu. edu) o un representante de la IRB en la CGU (teléfono: 909607-9406, correo electrónico: IRB@cgu.edu). Estas instituciones son responsables de garantizar la protección de los participantes en la investigación.

Su firma indica que usted ha leído la información contenida en este documento y han tenido la oportunidad de hacer cualquier pregunta que tenga sobre el estudio. Su firma también indica que usted acepta que su hijo/a participe en el estudio y le han dicho que puede cambiar de opinión y retirar su consentimiento en cualquier momento.

Nombre del alumno participante (en letra de imprenta)

Firma del Padre o Tutor

Fecha 
Appendix B

\section{Student Participant Informed Consent:}

\section{Analyzing Best Practices in the Schooling of Secondary-Level Newcomer Immigrant Youth: A Comparison Study of Students' Academic Skills and Perceptions of Self Upon Exit From Specialized English Learner Programs}

You are being asked to participate in a research study. Before you give your permission to participate, it is important that you read the following information and ask as many questions as necessary to be sure you understand your role as a participant in this research study.

\section{Investigator}

My name is Amanda Matas and I have taught middle school English, ESL, and social studies for twelve years. I am also a graduate student in the San Diego State University /Claremont Graduate University Joint Doctoral Program. The professors supervising my research are Dr. Karen Cadiero-Kaplan, a professor at San Diego State University (SDSU) and Dr. William Perez, a professor at Claremont Graduate University (CGU).

\section{Purpose of the study}

I am conducting a study is to better understand how students who are new to the United States achieve, both academically and personally, after their first year in U.S. schools. This study will ask you to communicate about how you see yourself as a student in school and how you feel in your different classes. This study will ask you about your experiences as you complete your first year within one of two different programs, a selfcontained newcomer program or an ESL program. I will examine the curricula, instruction, teachers' perceptions, and your academic self-confidence as you complete the school year within one of the two programs. Finally, this study will examine your parents', teachers', and program administrators' feelings about the two programs and their effects on student achievement and academic success.

\section{Description of the study}

If you agree to participate in the study, you will be asked to:

- Complete a seventy-two question survey that uses a scale to tell how true or how false each statement is as it describes you as a student in the United States, and how important each statement is to you. These statements ask you to describe how you feel in school, and how you view yourself as a learner. You will be asked to complete this survey one time and it will take about thirty minutes.

- Participate in a focus group that asks you about how you currently feel in your classes in the United States, as well as how you felt upon entering schools in the 
United States, both academically and personally. The focus group will occur before or after school, at a time that is convenient to you, and will last no longer than one hour. You will receive a copy of the questions ahead of time.

\section{Risks or Discomforts}

You may feel uneasy talking about situations that have made you uncomfortable. You do not have to talk about any situations or answer any questions that make you feel uncomfortable and you can leave the study at any time.

\section{Benefits of the study}

The benefits of this project include helping me to determine which program most effectively supports newcomer immigrant youth, both academically and personally. This information will be used to write a dissertation about the topic and the results will be submitted to the district administrators. This information will only be used in a way that maintains your anonymity, in other words no names will be used in this study.

\section{Confidentiality}

Research records will be kept confidential. Your name will not be directly connected with your responses or used in writing the article about this research. To protect your privacy, an identification number will be assigned to the data collected. Any data collected will be kept in a locked file cabinet to which only I have access. The data will be used solely to write a $\mathrm{PhD}$ dissertation and for possible use in a journal article or conference presentation at a later date. Your privacy will be maintained in all publications or presentations resulting from this study, as no actual names of people or schools will be used within this research report. Any audio recordings used to gather focus group data will be used solely to transcribe the data, and will be kept in a locked cabinet until the transcriptions are complete, at which time the recordings will be destroyed. Your identity will not be disclosed without consent as required by law.

\section{Voluntary Participation}

Participation in this study is voluntary. Your decision of whether or not to participate will not affect your relationship with teachers at your school or with me, and it will have no impact on your grades. If you decide to participate, you are free to stop participating at any time without any penalties.

\section{Questions about the Study}

If you have questions about the research, you may contact the Principal Investigator or either professor supervising the study.

Amanda Matas, M.A., Joint Doctoral Student SDSU/CGU

Phone: 619-261-1353_Email:mandymatas@hotmail.com 
Dr. Karen Cadiero-Kaplan, San Diego State University

Phone: 619-594-5155_Email: kcadiero@mail.sdsu.edu

Dr. William Perez, Claremont Graduate University

Phone: 323-610-2074Ｅmail:William.perez@cgu.edu

An institutional review board (IRB) is a committee that has been formally designated to approve, monitor, and review research involving humans, with the aim to protect the rights and welfare of the research subjects. If you questions regarding your rights as a human subject and participant, you may contact an Institutional Review Board (IRB) representative in the Division of Research Administration at SDSU (phone: 619-5946622; email: irb@mail.sdsu.edu) or an IRB representative at CGU (phone: 909-6079406; email: IRB@,cgu.edu). These boards are responsible for ensuring the protection of research participants.

Your signature below indicates that you have read the information in this document and have had a chance to ask any questions you have about the study. Your signature also indicates that you agree to be in the study and have been told that you can change your mind and withdraw your consent to participate at any time.

Name of Participant (please print)

Your signature

Date 


\section{Consentimiento Para los Estudiante Participantes:}

\section{Analizando las mejores prácticas en la escolaridad de los jóvenes inmigrantes de nivel secundario: Un estudio comparativo de las habilidades académicas de los estudiantes y percepciones de sí mismo después terminando de programas especializados de Aprendices de Inglés}

Se le pide participar en un estudio de investigación. Antes de dar su permiso para participar, es importante que lea la siguiente información y haga tantas preguntas como sea necesario para asegurarse de que entienda su papel como participante en este estudio de investigación.

\section{Investigador}

Mi nombre es Amanda Matas y he sido maestra por doce años al nivel de secundaria. He enseñado las materias de Inglés, ESL, y estudios sociales. Yo también soy un estudiante de posgrado en la Universidad Estatal de San Diego (SDSU) y Universidad Claremont Graduate Común Programa de Doctorado. La supervisión de mi investigación son la Dra. Karen Cadiero-Kaplan, profesora en SDSU, y el Dr. William Pérez, profesor de la Claremont Graduate University (CGU).

\section{Propósito del estudio}

Estoy realizando un estudio para entender mejor cómo los estudiantes que son nuevos en los Estados Unidos logran, tanto académicamente como personalmente, después de su primer año en la escuela. En este estudio se le pide refleccionar acerca de cómo se ve usted como estudiante en la escuela y cómo se siente en sus diferentes clases. Este estudio le preguntará acerca de sus experiencias al terminar su primer año en una de dos programas diferentes, un programa recién llegado autónomo o un programa de ESL. Voy a examinar los planes de estudio, la enseñanza, la percepción de los maestros, y su confianza academica en sí mismo al terminar el año escolar en uno de los dos programas. Por último, este estudio examinará los sentimientos de sus padres, profesores, administradores acerca de los dos programas y sus efectos sobre el rendimiento de los estudiantes y el éxito académico.

\section{Descripción del estudio}

Si usted acepta participar en el estudio, se le pedirá:

- Completar una encuesta de setenta y dos preguntas que utilizan una escala para indicar qué tan cierto o falso cada declaración es, como se describe como un estudiante en los Estados Unidos, y la importancia de cada declaración para usted. Estas declaraciones le pedirá que describa cómo se siente en la escuela, y cómo usted se ve como un aprendiz. Se le pedirá completar esta encuesta una sola vez y tendrá cerca de treinta minutos para hacerlo. 
- Participar en un grupo de enfoque que le preguntara acerca de cómo se siente actualmente en sus clases en los Estados Unidos, así cómo se sintió al entrar a las escuelas en los Estados Unidos, tanto académicamente como personalmente. El grupo de enfoque se producirá antes o después de la escuela, en el momento en que sea conveniente para usted, y tendrá una duración no más de una hora. Usted recibirá una copia de las preguntas antes de tiempo.

\section{Los riesgos o molestias}

Usted puede sentirse incómodo/a hablando de algunas situaciones. Usted no tiene que hablar sobre ninjuna situación o tampoco responder a cualquier pregunta que lo haga sentir incómodo/a y puede dejar el estudio en cualquier momento.

\section{Beneficios del estudio}

Los beneficios de este proyecto incluyen ayudarme a determinar el programa más eficaz de apoyar a la juventud inmigrante recién llegados, tanto académicamente como personalmente. Esta información se utilizará para escribir una tesis sobre el tema y los resultados serán presentados a los administradores del distrito. Esta información sólo será utilizada de una manera que lo/a mantentra anonimo/a, es decir su nombre no será utilizado en este estudio.

\section{Confidencialidad}

Expedientes de la investigación se mantendrá confidencial. Su nombre no estará directamente relacionada con sus respuestas o utilizados al escribirse un artículo sobre esta investigación. Para proteger su privacidad, un número de identificación serán asignados a los datos recogidos. Los datos recopilados se guardarán en un archivador cerrado al que sólo yo tendre acceso. Los datos serán utilizados exclusivamente para escribir una tesis de doctorado, para su posible uso en un artículo de revista, o en una conferencia de presentación en un futuro. Su privacidad se mantendrá en todas las publicaciones o presentaciones como resultado de este estudio, ya que los datos personales no se utilizarán en este informe de investigación. Las grabaciones de audio utilizadas para recopilar datos de los grupos de enfoque se utilizará exclusivamente para transcribir los datos y se mantendrá en un armario cerrado con llave hasta que las transcripciones sean terminadas. Al final las grabaciones serán destruidas. Su identidad no será revelada sin autorización exigida por la ley.

\section{Participación voluntaria}

La participación en este estudio es voluntaria. Su decisión para participar no afectará su relación con los maestros en su escuela o conmigo, y no tendrá ningún impacto en sus calificaciones. Si usted decide participar, usted es libre de dejar de participar en cualquier momento sin ningún tipo de consequencia. 


\title{
Preguntas Sobre el Estudio
}

Si usted tiene preguntas acerca de la investigación, puede comunicarse con el investigador principal o el profesor supervisor del estudio.

\author{
Amanda Matas, M.A., Estudiante de Doctorado Conjunto SDSU/CGU \\ teléfono: 619-261-1353correo electrónico: mandymatas@hotmail.com \\ Dr. Karen Cadiero-Kaplan, Universidad Estatal de San Diego (SDSU) \\ teléfono: 619-594-5155 correo electrónico: kcadiero@mail.sdsu.edu \\ Dr. William Perez, Universidad Claremont Graduate \\ teléfono: 323-610-2074＜noBreak>correo electrónico: William.perez@cgu.edu
}

\begin{abstract}
Un instituto de revision llamado Institutional Review Board (IRB) es un comité que ha sido designado oficialmente para aprobar, supervisar, y revisar las investigaciónes que inlcuyen a seres humanos, con el objetivo de proteger los derechos y el bienestar de los sujetos de la investigación. Si usted tiene pregunta con respecto a los derechos de su hijo como el participante, puede comunicarse con un representante de la división de administración de esta investigación en SDSU (teléfono: 619-594-6622, correo electrónico: irb@mail.sdsu. edu) o un representante de la IRB en la CGU (teléfono: 909607-9406, correo electrónico: IRB@cgu.edu). Estas instituciones son responsables de garantizar la protección de los participantes en la investigación.
\end{abstract}

Su firma indica que usted ha leído la información contenida en este documento y han tenido la oportunidad de hacer cualquier pregunta que tenga sobre el estudio. Su firma también indica que usted se compromete a participar en el estudio y le han dicho que puede cambiar de opinión y retirar su consentimiento en cualquier momento.

Nombre (en letra de imprenta)

Firma

Fecha 
Appendix C

\section{Teacher and Administrator Informed Consent:}

\section{Analyzing Best Practices in the Schooling of Secondary-Level Newcomer Immigrant Youth: A Comparison Study of Students' Academic Skills and Perceptions of Self Upon Exit From Specialized English Learner Programs}

You are being asked to participate in a research study. Before you agree to participate, it is important that you read the following information and ask as many questions as necessary to be sure you understand your role as a participant in this research study.

\section{Investigator}

Amanda Matas has taught newcomer immigrant students in a Beginning English Language Development class and in the content area of social studies for several years and is a doctoral student in the San Diego State University /Claremont Graduate University Joint Doctoral Program. Dr. Karen Cadiero-Kaplan, a professor at San Diego State University (SDSU) and Dr. William Perez, a professor at Claremont Graduate University (CGU), are supervising this research.

\section{Purpose of the study}

The purpose of this study is to better understand how students who are new to the United States achieve, both academically and personally, after their first year in U.S. schools. This study will ask students to communicate about how they see themselves as learners in school and how they feel in their individual classes, being that their classes are taught only in English. This study will further explore the experiences of newcomer youth as they complete their first year within one of two different programs, a self-contained newcomer program and an ESL program, examining the curricula, instruction, teachers' perceptions, and students' academic self-confidence as they complete the year within one of the two programs. Finally, this study will examine parents', teachers', and program administrators' perceptions of the two programs and their effects on student achievement and academic success.

\section{Description of the study}

If you agree to participate in the study, you will be asked to:

- Allow the researcher to observe your class for 3 to 5 half-day sessions (approx. 3 hours each). The researcher will take a passive role during the observations and will not interrupt the daily lessons and activities. The researcher will share all field notes taken during the observations. 
- Participate in one interview with the researcher at your convenience describing your experiences as a teacher or administrator in a newcomer or an ESL program. While there will be interview questions guiding the discussion, it will be conducted as a dialogue between the researcher and the interviewee. The interview will occur before or after school, at your convenience, and will last no longer than one hour. You will receive the questions before the actual interview.

\section{Benefits of the study}

The benefits of this project include helping the researcher to determine which program most effectively supports newcomer immigrant youth, both academically and personally. This information will be used to write a dissertation about the topic and, in addition, the results will be disseminated to the district under study, as well as the academic field at large. This information will only be utilized in a way that maintains the participants' anonymity.

\section{Confidentiality}

Research records will be kept confidential. Your name will not be directly connected with responses or used in writing the article about this research. To protect your privacy, an identification number will be assigned to the data collected. Any data collected will be kept in a locked file cabinet to which only the investigator will have access. The data will be used solely to write a PhD dissertation and for possible use in a journal article or conference presentation at a later date. Your privacy will be maintained in all publications or presentations resulting from this study, as no actual names of people or schools will be used within this research report. Any audio recordings used to gather interview data will be used solely to transcribe the data, and will be kept in a locked cabinet until the transcriptions are complete, at which time the recordings will be destroyed. Your identity will not be disclosed without consent as required by law.

\section{Voluntary Participation}

Participation in this study is voluntary. You are free to discontinue your participation at any time.

\section{Questions about the Study}

If you have questions about the research, you may contact the Principal Investigator or either professor supervising the study.

Amanda Matas, M.A., Joint Doctoral Student SDSU/CGU

Phone: 619-261-1353_ Email: mandymatas@hotmail.com

Dr. Karen Cadiero-Kaplan, San Diego State University

Phone: 619-594-5155_Email:kcadiero@mail.sdsu.edu 
Dr. William Perez, Claremont Graduate University

Phone: 323-610-2074_Ｅmail: William.perez@,cgu.edu

An institutional review board (IRB) is a committee that has been formally designated to approve, monitor, and review research involving humans, with the aim to protect the rights and welfare of the research subjects. If you questions regarding your child's rights as a human subject and participant, you may contact an Institutional Review Board (IRB) representative in the Division of Research Administration at SDSU (phone: 619-5946622; email: irb@mail.sdsu.edu) or an IRB representative at CGU (phone: 909-6079406; email: IRB@cgu.edu). These boards are responsible for ensuring the protection of research participants.

Your signature below indicates that you have read the information in this document and have had a chance to ask any questions you have about the study. Your signature also indicates that you agree to be in the study and have been told that you can change your mind and withdraw your consent to participate at any time.

Name of Participant (please print)

Signature of Participant

Date 


\section{Appendix D}

\section{Classroom Observation Protocol}

Date:

School:

Grade-level:

Length of observation: Start: Finish:

\begin{tabular}{|c|c|}
\hline Lesson Components & Observation Notes \\
\hline $\begin{array}{l}\text { Content Objective: } \\
\text { - Clearly defined for students? } \\
\text { - Demonstrated throughout lesson? } \\
\text { - Appropriate for age and proficiency level } \\
\quad \text { of students? }\end{array}$ & \\
\hline $\begin{array}{l}\text { English Language Objective: } \\
\text { - Clearly defined for students? } \\
\text { - Demonstrated throughout lesson? } \\
\quad \text { Appropriate for proficiency level of } \\
\text { students? }\end{array}$ & \\
\hline $\begin{array}{l}\text { Curriculum: } \\
\text { - Materials teacher-created or from a } \\
\text { designated program? } \\
\text { Materials appropriate for language } \\
\text { proficiency level of students? } \\
\text { Materials contain culturally relevant } \\
\text { content? } \\
\text { - Supplementary materials used to augment } \\
\text { the lesson? }\end{array}$ & \\
\hline $\begin{array}{l}\text { Instruction: } \\
\text { Building Background } \\
\text { - Concepts linked to students' background } \\
\text { experiences/prior knowledge? } \\
\text { - Concepts address students varying cultural } \\
\text { and linguistic backgrounds? } \\
\text { - Connections made between current lesson } \\
\text { objectives and past learning? } \\
\text { - Peer interaction used to activate schema? }\end{array}$ & \\
\hline $\begin{array}{l}\text { Key Vocabulary } \\
\text { - Academic or content vocabulary introduced } \\
\text { in lesson? } \\
\text { - Vocabulary taught literally or contextually? } \\
\text { Strategies used to support students in } \\
\text { understanding key vocabulary? }\end{array}$ & \\
\hline
\end{tabular}

${ }^{*}$ Adapted from J. Echevarria, M. Vogt, \& D. Short (2004), The Sheltered Observation Protocol (SIOP) 


\begin{tabular}{|c|c|}
\hline $\begin{array}{l}\text { - Suitable pacing for language proficiency } \\
\text { level? } \\
\text { - Suitable speech (speed and enunciation) for } \\
\text { language proficiency level? } \\
\text { - Clear expectations for students? } \\
\text { Checks for understanding throughout } \\
\text { lesson? } \\
\text { A variety of techniques used during } \\
\text { instruction? } \\
\text { Visuals? } \\
\text { Realia? } \\
\text { Modeling? } \\
\text { Gestures/TPR? } \\
\text { Primary language support? } \\
\text { Interactive demonstrations? } \\
\text { Partner activities? } \\
\text { Group activities? }\end{array}$ & \\
\hline $\begin{array}{l}\text { Strategies } \\
\text { - Use of scaffolding techniques to assist and } \\
\text { support students? } \\
\text { Think-alouds? } \\
\text { Word banks? } \\
\text { Schema building? } \\
\text { Graphic organizers? } \\
\text { - Use of a variety of question types } \\
\text { throughout lesson (literal, analytical, } \\
\text { interpretive)? } \\
\text { Evidence of a gradual release of } \\
\text { responsibility? }\end{array}$ & \\
\hline $\begin{array}{ll}\text { iteractions } \\
\text { - Students given frequent opportunities to } \\
\text { interact with teacher? } \\
\text { - Students given frequent opportunities to } \\
\text { interact with other students? } \\
\text { - } \quad \text { Grouping configurations support lesson? } \\
\text { Students provided sufficient "wait time" to } \\
\text { respond to questions? } \\
\quad \text { Concepts clarified in students' primary } \\
\text { languages when possible? }\end{array}$ & \\
\hline $\begin{array}{l}\text { ractice/Application } \\
\text { - Students given opportunities to practice } \\
\text { new content or language concept? } \\
\text { - Activities integrate all language domains } \\
\text { (reading, writing, speaking listening)? } \\
\text { - Extensive oral language opportunities } \\
\text { included in lesson? } \\
\text { - Supports provided students to help in } \\
\text { applying new knowledge? } \\
\text { Charts? } \\
\text { Manipulatives? } \\
\text { Visuals? }\end{array}$ & \\
\hline
\end{tabular}




\begin{tabular}{|c|c|}
\hline $\begin{array}{ll}\text { Lesson Delivery } \\
\text { - Content objects apparent in lesson } \\
\text { delivery? } \\
\text { - Language objectives apparent in lesson } \\
\text { delivery? } \\
\text { - Lesson responsive to needs of students? } \\
\text { Appropriate pacing for students? }\end{array}$ & \\
\hline $\begin{array}{l}\text { Review/Assessment: } \\
\text { - Desired outcome achieved through lesson? } \\
\text { Instruction differentiated based on the } \\
\text { linguistic and academic needs of students? } \\
\text { Teacher checks for understanding and } \\
\text { gathers information about the students? } \\
\text { Oral questions? } \\
\text { Exit slips? } \\
\text { Student product (written work)? } \\
\text { Tests/Quizzes? } \\
\text { Teacher provides feedback to students (oral } \\
\text { or written)? }\end{array}$ & \\
\hline $\begin{array}{l}\text { Culturally Relevant Teaching: } \\
\text { - Materials contain culturally relevant } \\
\text { content? } \\
\text { Relevant to students' lives and } \\
\text { languages/cultures? } \\
\text { Interesting? } \\
\text { Thought-provoking? } \\
\text { Challenging and complex? } \\
\text { - Instruction differentiated based on needs of } \\
\text { students? } \\
\text { Flexible grouping? } \\
\text { Cooperative learning? } \\
\text { Multiple perspectives on ideas are } \\
\text { presented and discussed? } \\
\text { Environment incorporates multiple } \\
\text { cultures, languages, and gender? } \\
\text { Represented on walls? } \\
\text { Represented in texts? }\end{array}$ & \\
\hline $\begin{array}{l}\text { Pedagogical Orientation: } \\
\text { Transmission-oriented Pedagogy } \\
\text { - Instruction primarily teacher directed? } \\
\quad \text { Direct instruction or lecture? } \\
\text { - Instruction focused solely on content of } \\
\text { lesson (no collaborative inquiry)? } \\
\text { - Students are passive learners? }\end{array}$ & \\
\hline
\end{tabular}




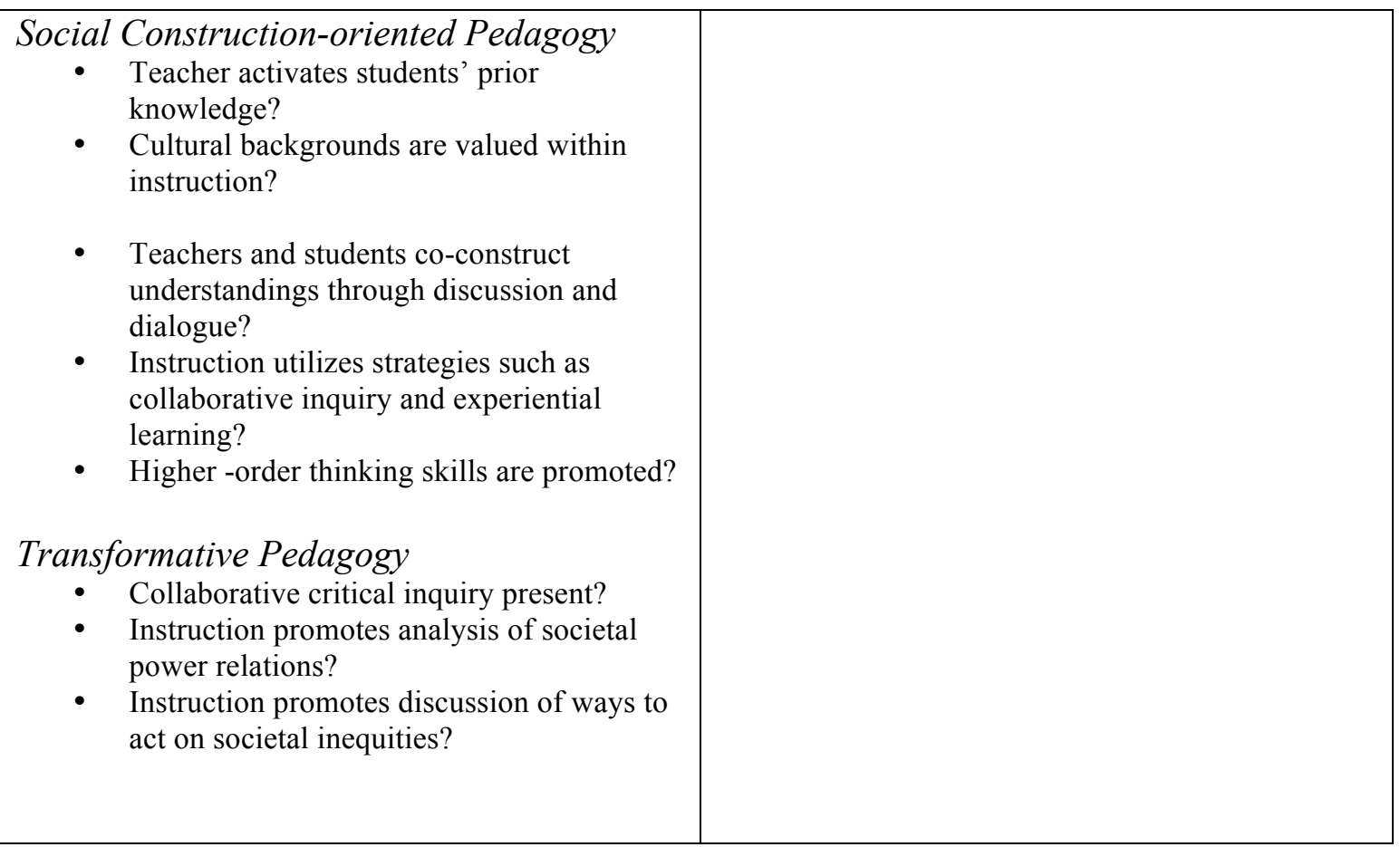


Appendix E

\section{Observation Field Notes Record*}

Date: School: \# Students

Grade-levels: Length of observation: Start:

Finish:

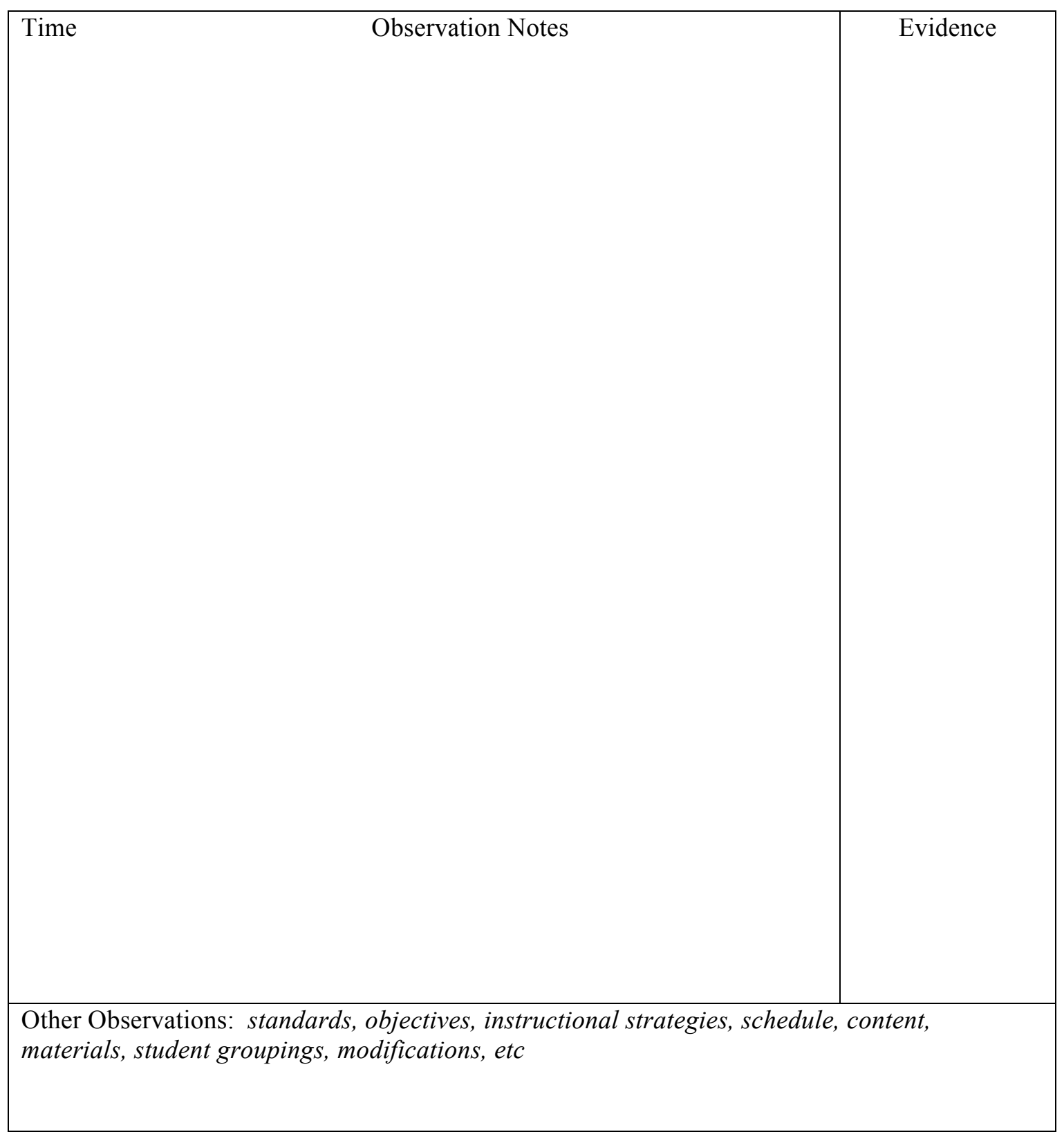

* Adapted from G. Gonzalez (2010), Language Policy and Access for English Learners: Pedagogy, Outcomes, and Accountability 


\section{Appendix F}

\section{Administrator Interview Questions}

Research Theme Interview Questions

Demographic Information

Theme 1: Initial Program Access and Information

Theme 2: Pedagogical Practices

Theme 3: Language Acquisition

Theme 4: Academic Skill Attainment

Theme 5: Academic Self-concept

Theme 6: Stakeholder Support
What is your position in the district? How long have you been an employee of the district? What other positions have you held in the district? How long and in what capacity have you been involved in education? Within what type of teaching or administrative credentials are you employed?

Describe the history of the program about which you are overseeing. When and why was it originally established? Have you encountered hesitance in implementing your program at school sites? What are some successes you have witnessed as a result of this program? How has your program changed over time? What was your role in the creation or continuation of the program? What motivated you to work within this program? What are your current perceptions about your position? How did your prior experience in the classroom prepare you for your current position?

How does the program assess students' language levels? At the beginning, middle, end of school year? How do you use data to drive planning and language instruction in your program? How are students encouraged to practice language, both orally and written? How are reluctant speakers (silent period) encouraged?

How does the program assess students' academic skills levels (literacy, mathematics, etc)? At the beginning, middle, end of school year? How does this program assist students in developing academic content skills? How does the program use data to drive academic content planning and instruction? How are content skills addressed in your program? How is instruction differentiated within this program?

How does this program assist students in developing academic content skills? What is done to accelerate literacy skills in newcomer students? How are other content skills addressed in your program? How is instruction differentiated within this program?

How does this program impact students' academic selfconcept (sense of self)? Do students appear more or less confident academically and socially as a result of participation in this program?

What type of professional development do you provide or receive in your position? What support services are available on campus to assist students and their families? What strategies are used to inform parents and students about the process of completing their education within the U.S.? How are community resources accessed and utilized within your program? 


\section{Appendix G}

\section{Teacher Interview Questions}

\begin{tabular}{ll}
\hline Research Theme & Interview questions
\end{tabular}

Demographic Information

Theme 1: Initial Program Access and Information

Theme 2: Pedagogical Practices

Theme 3: Language Acquisition

Theme 4: Academic Skill Attainment

Theme 5: Academic Self-concept

Theme 6: Stakeholder Support
What is your position in the district? How long have you been an employee of the district? What other positions have you held in the district? How long and in what capacity have you been involved in education? Within what type of teaching or administrative credentials are you employed?

How did you become involved with your program? How were you informed of this position? What were your expectations? What are your current perceptions about your position? How did your prior experience prepare you for your current position?

What are the linguistic and academic expectations you set for yourself and the students in your class? What would you like to accomplish during this school year? What curricula are utilized in your program and how was it chosen? Describe how your program utilizes students' primary languages and home cultures to connect to those of the United States. How do you see your role as a teacher in the classroom? How do you group your students for cooperative work? How do you differentiate instruction for different levels?

How do you assess you students' language levels? At the beginning, middle, end of school year? How does this instruction assist students in acquiring language skills? How do you use data to drive planning and language instruction? What strategies are used to model language for newcomer students? How do you specifically encourage reluctant speakers (silent period)?

How do you assess you students' academic skills levels (literacy, mathematics, etc)? How do you use data to drive academic content planning and instruction? What is done to accelerate literacy skills in newcomer students? How are other content skills addressed in your program? How is instruction differentiated within this program?

How does this program impact students' academic selfconcept (sense of self)? Do students appear more or less confident academically and socially as a result of participation in this program?

What type of professional development do you receive in your position? How are you able to transcend language barriers and communicate with your students' parents and guardians?

What strategies are used to inform parents and students about the process of completing their education within the U.S.? How are community resources accessed and utilized within your program? 


\section{Appendix $\mathrm{H}$}

\section{Student Focus Group Questions}

\begin{tabular}{ll}
\hline Research Theme & Interview questions
\end{tabular}

Demographic Information

Theme 1: Initial Program Access and Information

Theme 2: Pedagogical Practices

Theme 3: Language Acquisition

Theme 4: Academic Skill Attainment

Theme 5: Academic Self-concept

Theme 6: Stakeholder Support
What is your country of origin? Who do you live with in the United States? When did you and your family immigrate to the United States? Why did you and your family emigrate from your home country? How many years of school did you attend in your home country?

Were you a good student in school or did you struggle?

How did you feel on your first day at school in the United States? What did you think about your classes?

How did you teachers welcome you to class? How did you feel about the other students in your classes? Did the students help you when you first arrived at this school? If yes, how?

When you started at your school, did you understand what was being taught in your classes? What helped you or kept you from understanding what was being taught? What did teachers do to help you understand the lessons? How have teachers helped you to understand about U.S. customs and culture? How have teachers helped you to understand U.S. schools?

Did you study English prior to moving to the United States? How much did you understand when you first entered school? How much do you understand now at the finish of the school year? What has helped you in school to learn more English? What has helped you outside of school?

Which classes are the easiest to understand? Why? Which classes are the hardest to understand? Why? Are you able to complete independent work, such as homework? How do you study for your classes? What have teachers done to help you understand hard concepts?

How do the easier classes make you feel about yourself? How do the harder classes make you feel? How did you feel about yourself as a learner when you first started school in the U.S.? How do you feel about yourself today? What have the teachers done to help you feel more confident about yourself?

Are there adults other than your teachers who support you at school? If so, what do they do? What does your family do to help you with school? How do other students help you in class or after school? 


\section{Preguntas Para el Estudiante Grupo de Enfoque}

Tema de investigación

Preguntas de Entrevista

Información Demográfica

Tema 1: Acceso al programa inicial y de la información

Tema 2: Prácticas Pedagógicas

Tema 3: Adquisición del Idioma

Tema 4: El logro de habilidades académicas

Tema 5: Auto-concepto Académico

Tema 6: Apoyo a las partes interesadas
¿Cuál es tu país de origen? ¿Con quién vives en los Estados Unidos? ¿Cuándo emigraron a los Estados Unidos tu y tu familia? ¿Por qué emigraron de tu país de origen? ¿Cuántos años de escuela asististes en tu país de origen?

¿Eras un buen estudiante en la escuela o tuvistes conflictos?

¿Cómo te sentistes el primer día de escuela en los Estados Unidos? ¿Qué piensas de tus clases?

¿Cómo fue la bienvenida de tus maestros en tu clases?

¿Cómo te sientes con los demas estudiantes en tus clases?

$¿$ Otros estudiantes te aydaron cuando llegastes por primera vez a tu escuela? En caso que si, ¿cómo?

Cuando comensastes ir a la escuela, ¿entendistes lo que enseñaban en tus clases? ¿Qué te ayudó o que te detuvo de entender lo que se enseñaba? ¿Qué hacieron los maestros para ayudarte a entender las lecciones? ¿Cómo te han ayudado a los maestros a entender las costumbres y cultura de los EE.UU? ¿Cómo te han ayudado los maestros a entender las escuelas de los EE.UU.?

¿Estudiastes Inglés antes de venir a los Estados Unidos? ¿Cuánto entendias la primera vez que entrastes a la escuela? ¿Cuánto entiendes ahora al final del año escolar? ¿Qué te ha ayudado en la escuela para aprender más Inglés? ¿Qué te ha ayudado fuera de la escuela?

¿Cuales son las clases más fáciles de entender? ¿Por qué? ¿Cuales son las clases más difíciles de entender? ¿Por qué? ¿Eres capaz de completar trabajo independiente, como tu tarea? ¿Cómo estudiar para tus clases? ¿Qué han hecho tus maestros para ayudarte a comprender conceptos difíciles?

¿Cómo te hacen sientes las clases más fáciles? ¿ Cómo te hacen sientes las clases más dicifiles? ¿Cómo te sientes tu mismo como un estudiante cuando comensastes la escuela en los EE.UU.? ¿Cómo te sientes acerca de ti hoy? ¿Qué han hecho los maestros para ayudarte a sentirse más seguro de ti mismo?

$¿$ Hay adultos que no sean tus maestros que te apoyan en la escuela? Si es así, ¿qué hacen? ¿Qué hace su familia para ayudarte con la escuela? ¿Cómo te ayudan los otros estudiantes durante clase o después de escuela? 


\section{Appendix I}

\section{SSCS Student Questionnaire Level 2}

This form lists things that students your age may do. Please read each statement and think about yourself. Then pick your answers. There are no right or wrong answers. All information supplied will be kept strictly confidential.

\section{HOW TO PICK ANSWERS}

Decide how confident (how sure you are that you are able to do what the item says.

- Circle 0 if you are Not at All confident that you can do it.

- Circle 1 if you are Not Sure that you can do it.

- Circle 2 if you are Confident that you can do it.

Decide how important doing it is to you.

- Circle 0 if it is Not Important and wouldn't make a difference in how you feel about yourself.

- Circle 1 if it is Important to you and would make a difference in how you feel about yourself.

- Circle 2 if it is Critical or very important for you and would make a difference in how you feel about yourself.

\section{Look at the examples:}

\begin{tabular}{|c|c|c|c|c|c|c|}
\hline & \multicolumn{3}{|c|}{ HOW CONFIDENT? } & \multicolumn{3}{|c|}{ HOW IMPORTANT? } \\
\hline & $\begin{array}{l}\text { Not at } \\
\text { All }\end{array}$ & $\begin{array}{l}\text { Not } \\
\text { Sure }\end{array}$ & Confident & $\begin{array}{l}\text { Not Im- } \\
\text { portant }\end{array}$ & $\begin{array}{l}\text { Impor- } \\
\text { tant }\end{array}$ & Critical \\
\hline A. I can sing as well as my friends. & $\underline{\mathbf{0}}$ & 1 & 2 & 0 & 1 & $\underline{2}$ \\
\hline $\begin{array}{l}\text { B. I can give a good report in front of the } \\
\text { class. }\end{array}$ & 0 & 1 & $\underline{2}$ & $\underline{\mathbf{0}}$ & 1 & 2 \\
\hline
\end{tabular}

The student is not at all confident that he can sing as well as his friends, and singing well is critical to him - it makes a big difference in how he feels about himself.

The student is confident he can give a good report in front of the class, but giving reports is not important to him - it does not make a difference in how he feels about himself.

For items 1 to 57, you need to mark how confident you are that you can do what the item says, and how important it is to you.

For items 58 to 72, you need to mark only how confident your are.

Begin answering the questions with Item 1 when told to do so.

Do not skip any items. 


\begin{tabular}{|c|c|c|c|c|c|c|}
\hline & \multicolumn{3}{|c|}{ HOW CONFIDENT? } & \multicolumn{3}{|c|}{ HOW IMPORTANT? } \\
\hline & $\begin{array}{l}\text { Not at } \\
\text { All }\end{array}$ & $\begin{array}{l}\text { Not } \\
\text { Sure }\end{array}$ & Confident & $\begin{array}{l}\text { Not Im- } \\
\text { portant }\end{array}$ & $\begin{array}{l}\text { Impor- } \\
\text { tant }\end{array}$ & Critical \\
\hline $\begin{array}{l}\text { 1. I can stand up for my friends when } \\
\text { others treat them unfairly. }\end{array}$ & 0 & 1 & 2 & 0 & 1 & 2 \\
\hline $\begin{array}{l}\text { 2. I can tell adults when they have done } \\
\text { something nice for me. }\end{array}$ & 0 & 1 & 2 & 0 & 1 & 2 \\
\hline 3. I am easy to like. & 0 & 1 & 2 & 0 & 1 & 2 \\
\hline 4. I am proud of who I am. & 0 & 1 & 2 & 0 & 1 & 2 \\
\hline 5. I never get my feelings hurt. & 0 & 1 & 2 & 0 & 1 & 2 \\
\hline $\begin{array}{l}\text { 6. I can use a nice tone of voice in } \\
\text { classroom discussions with my teacher. }\end{array}$ & 0 & 1 & 2 & 0 & 1 & 2 \\
\hline $\begin{array}{l}\text { 7. I can jump as high and as far as my } \\
\text { classmates. }\end{array}$ & 0 & 1 & 2 & 0 & 1 & 2 \\
\hline $\begin{array}{l}\text { 8. I agree with everything other people } \\
\text { say. }\end{array}$ & 0 & 1 & 2 & 0 & 1 & 2 \\
\hline 9. I can look as nice as other kids my age. & 0 & 1 & 2 & 0 & 1 & 2 \\
\hline 10. I am fun to be with. & 0 & 1 & 2 & 0 & 1 & 2 \\
\hline 11.I am liked by everyone I know. & 0 & 1 & 2 & 0 & 1 & 2 \\
\hline 12. I am a happy person. & 0 & 1 & 2 & 0 & 1 & 2 \\
\hline $\begin{array}{l}\text { 13. I can sit in class without daydreaming } \\
\text { during a lesson. }\end{array}$ & 0 & 1 & 2 & 0 & 1 & 2 \\
\hline $\begin{array}{l}\text { 14. I can introduce myself to new people } \\
\text { without being told by others. }\end{array}$ & 0 & 1 & 2 & 0 & 1 & 2 \\
\hline 15. I can do my homework on time. & 0 & 1 & 2 & 0 & 1 & 2 \\
\hline $\begin{array}{l}\text { 16. I can dance as well as other kids my } \\
\text { age. }\end{array}$ & 0 & 1 & 2 & 0 & 1 & 2 \\
\hline $\begin{array}{l}\text { 17. I can read aloud in class without } \\
\text { feeling nervous. }\end{array}$ & 0 & 1 & 2 & 0 & 1 & 2 \\
\hline $\begin{array}{l}\text { 18. I can sit at my desk for } 2 \text { minutes } \\
\text { without moving around or fidgeting. }\end{array}$ & 0 & 1 & 2 & 0 & 1 & 2 \\
\hline
\end{tabular}




\begin{tabular}{|c|c|c|c|c|c|c|}
\hline 19. I like everyone I know. & 0 & 1 & 2 & 0 & 1 & 2 \\
\hline $\begin{array}{l}\text { 20. I can politely refuse to do things that } \\
\text { are wrong even when other kids try to talk } \\
\text { me into doing them. }\end{array}$ & 0 & 1 & 2 & 0 & 1 & 2 \\
\hline 21. I can finish my class work on time. & 0 & 1 & 2 & 0 & 1 & 2 \\
\hline 22. I always tell the truth. & 0 & 1 & 2 & 0 & 1 & 2 \\
\hline $\begin{array}{l}\text { 23. I can listen to my teacher talk about a } \\
\text { subject for } 20 \text { minutes. }\end{array}$ & 0 & 1 & 2 & 0 & 1 & 2 \\
\hline 24. I can make friends easily. & 0 & 1 & 2 & 0 & 1 & 2 \\
\hline $\begin{array}{l}\text { 25. I can ask my teacher for help without } \\
\text { feeling ashamed or upset. }\end{array}$ & 0 & 1 & 2 & 0 & 1 & 2 \\
\hline $\begin{array}{l}\text { 26. I can go to the board to do work when } \\
\text { my teacher asks me to. }\end{array}$ & 0 & 1 & 2 & 0 & 1 & 2 \\
\hline $\begin{array}{l}27 . \text { I can control my temper in arguments } \\
\text { with other kids. }\end{array}$ & 0 & 1 & 2 & 0 & 1 & 2 \\
\hline $\begin{array}{l}\text { 28. I can laugh when I make silly } \\
\text { mistakes. }\end{array}$ & 0 & 1 & 2 & 0 & 1 & 2 \\
\hline $\begin{array}{l}\text { 29. I am as coordinated as other kids my } \\
\text { age. }\end{array}$ & 0 & 1 & 2 & 0 & 1 & 2 \\
\hline $\begin{array}{l}\text { 30. I can ask classmates to play a game } \\
\text { with me. }\end{array}$ & 0 & 1 & 2 & 0 & 1 & 2 \\
\hline $\begin{array}{l}\text { 31. I can read instructions in a book and } \\
\text { follow them carefully. }\end{array}$ & 0 & 1 & 2 & 0 & 1 & 2 \\
\hline $\begin{array}{l}\text { 32. I can follow my teacher's directions } \\
\text { for doing reading work. }\end{array}$ & 0 & 1 & 2 & 0 & 1 & 2 \\
\hline $\begin{array}{l}\text { 33. I can end arguments with my parents } \\
\text { calmly. }\end{array}$ & 0 & 1 & 2 & 0 & 1 & 2 \\
\hline $\begin{array}{l}\text { 34. I can tell my friends that I have } \\
\text { something well without bragging. }\end{array}$ & 0 & 1 & 2 & 0 & 1 & 2 \\
\hline $\begin{array}{l}\text { 35. I can say nice things to classmates } \\
\text { when they have done something well. }\end{array}$ & 0 & 1 & 2 & 0 & 1 & 2 \\
\hline \multirow{2}{*}{$\begin{array}{l}\text { 36. I can ignore classmates when they } \\
\text { whisper or talk during class. }\end{array}$} & 0 & 1 & 2 & 0 & 1 & 2 \\
\hline & 0 & 1 & 2 & 0 & 1 & 2 \\
\hline
\end{tabular}




\begin{tabular}{|c|c|c|c|c|c|c|}
\hline $\begin{array}{l}\text { 37. I can tell kids my age I like them } \\
\text { without feeling embarrassed. }\end{array}$ & 0 & 1 & 2 & 0 & 1 & 2 \\
\hline $\begin{array}{l}\text { 38. I can ask other kids if I may join the } \\
\text { game they are playing. }\end{array}$ & 0 & 1 & 2 & 0 & 1 & 2 \\
\hline $\begin{array}{l}\text { 39. I can show others that I feel good } \\
\text { about myself. }\end{array}$ & 0 & 1 & 2 & 0 & 1 & 2 \\
\hline 40. I can do math work without help. & 0 & 1 & 2 & 0 & 1 & 2 \\
\hline 41. I am as strong as other kids my age. & 0 & 1 & 2 & 0 & 1 & 2 \\
\hline 42. I can run as fast as other kids my age. & 0 & 1 & 2 & 0 & 1 & 2 \\
\hline $\begin{array}{l}\text { 43. I can take turns in games or other } \\
\text { activities. }\end{array}$ & 0 & 1 & 2 & & & \\
\hline $\begin{array}{l}\text { 44. I can speak in class when my teacher } \\
\text { calls on me. }\end{array}$ & 0 & 1 & 2 & 0 & 1 & 2 \\
\hline $\begin{array}{l}\text { 45. I can listen when my teacher is } \\
\text { presenting a lesson. }\end{array}$ & 0 & 1 & 2 & 0 & 1 & 2 \\
\hline $\begin{array}{l}\text { 46. I can tell my teacher when he or she } \\
\text { has accused me of doing something I } \\
\text { didn't do. }\end{array}$ & 0 & 1 & 2 & 0 & 1 & 2 \\
\hline $\begin{array}{l}\text { 47. I can remember when class projects } \\
\text { are due. }\end{array}$ & 0 & 1 & 2 & 0 & 1 & 2 \\
\hline $\begin{array}{l}\text { 48. I can talk things over calmly with kids } \\
\text { my age when we disagree. }\end{array}$ & 0 & 1 & 2 & 0 & 1 & 2 \\
\hline $\begin{array}{l}\text { 49. I can play sports as well as other kids } \\
\text { my age. }\end{array}$ & 0 & 1 & 2 & 0 & 1 & 2 \\
\hline $\begin{array}{l}\text { 50. I can tell classmates how I feel when } \\
\text { they upset me or hurt my feelings. }\end{array}$ & 0 & 1 & 2 & 0 & 1 & 2 \\
\hline 51. I never get mad. & 0 & 1 & 2 & 0 & 1 & 2 \\
\hline 52. I am a nice person. & 0 & 1 & 2 & 0 & 1 & 2 \\
\hline 53. I can do anything I want. & 0 & 1 & 2 & 0 & 1 & 2 \\
\hline $\begin{array}{l}\text { 54. I can share my possessions with } \\
\text { others. }\end{array}$ & 0 & 1 & 2 & 0 & 1 & 2 \\
\hline 55. I can follow classroom rules. & 0 & 1 & 2 & 0 & 1 & 2 \\
\hline 56. I like to be with others. & 0 & 1 & 2 & 0 & 1 & 2 \\
\hline
\end{tabular}




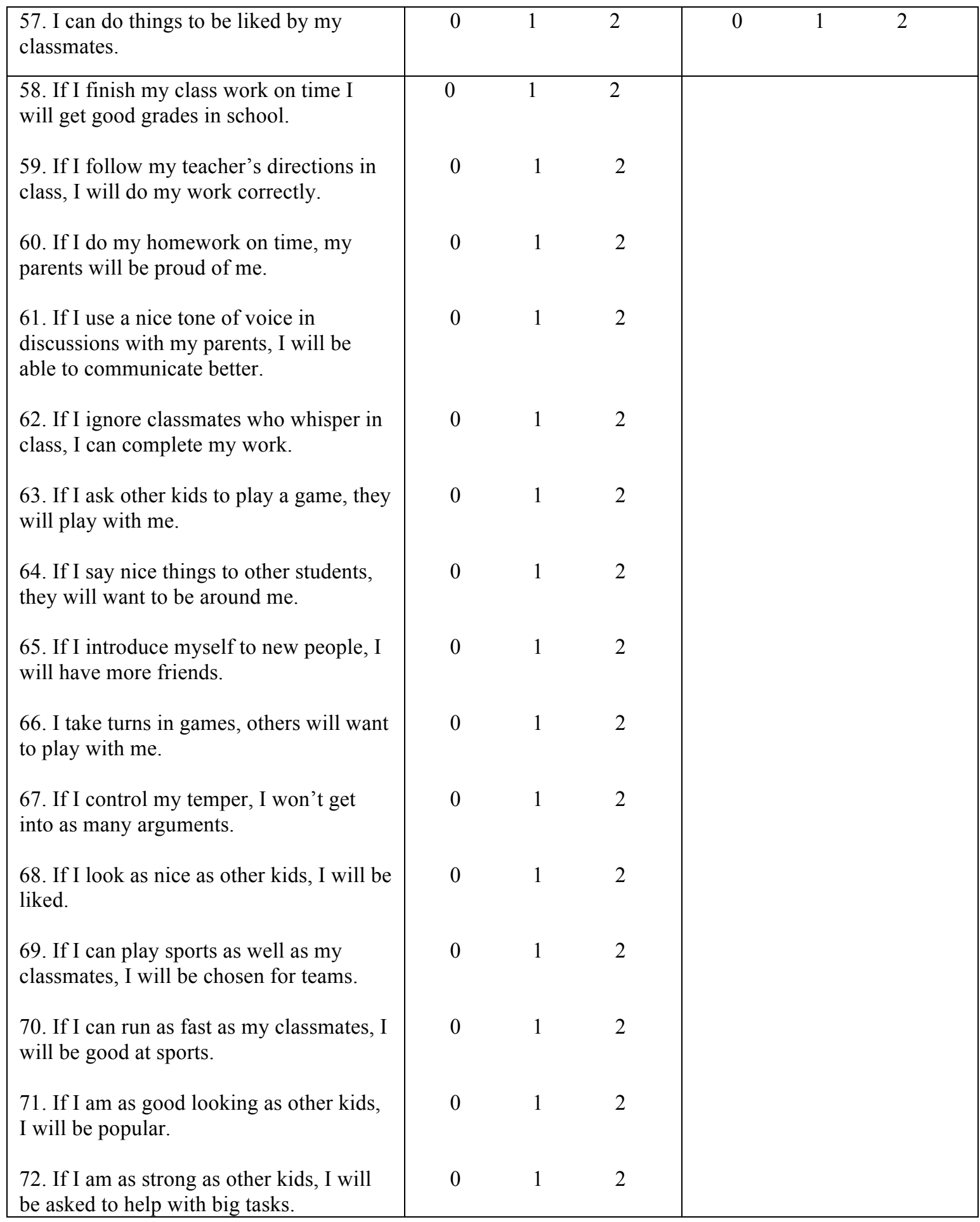




\section{SSCS Estudiante Cuestionario \\ Nivel 2}

Esta forma de listas de cosas que los estudiantes de su edad puede hacer. Por favor, lea cada frase y pensar en ti mismo. A continuación, elegir sus respuestas. No hay respuestas correctas o incorrectas. Toda la información proporcionada se mantendrá estrictamente confidencial.

CÓMO ESCOGER RESPUESTAS

Decidir el grado de confianza (como seguro está de que usted es capaz de hacer lo que dice el artículo).

- Círculo 0 si NO estas seguro de que puedas hacerlo.

- Círculo 1 si no está muy seguro de que puedas hacerlo.

- Círculo 2 si ESTAS seguro de que puedas hacerlo.

Decida la importancia de hacerlo es a usted.

- Círculo 0 si NO ES IMPORTANTE y NO haría una diferencia en cómo te sientes sobre ti mismo.

- Círculo 1 si es IMPORTANTE para ti y haría una diferencia en cómo te sientes sobre ti mismo.

- Círculo 2, si es CRÍTICO o MUY IMPORTANTE para ti y haría una diferencia en cómo te sientes sobre ti mismo.

\section{Ve los ejemplos:}

¿QUÉ TAN SEGURO?

Nada No está Seguro muy seguro
¿QUÉ TAN IMPORTANTE?

No está Un poco Muy importante importante importante

\begin{tabular}{|l|lll|llr|}
\hline A: Puedo cantar tan bien como mis amigos. & $\underline{0}$ & 1 & 2 & 0 & 1 & $\underline{\mathbf{2}}$ \\
$\begin{array}{l}\text { B. Puedo dar un buen reporte en el frente } \\
\text { de la clase. }\end{array}$ & 0 & 1 & $\underline{\mathbf{2}}$ & $\underline{\mathbf{0}}$ & 1 & 2 \\
\hline
\end{tabular}

El estudiante no es en absoluto seguro de que puede cantar, así como sus amigos, y cantar bien es muy importante para él - que hace una gran diferencia en cómo se siente acerca de sí mismo.

El estudiante confía en que puede dar un buen informe al frente de la clase, pero los informes de dar no es importante para él - no hacer una diferencia en cómo se siente acerca de sí mismo.

Para los artículos 1 a 57, necesitas marcar el nivel de confianza que tu eres y el nivel de importancia que es para ti.

Para los artículos 58 a 72, solo tienes que marcar el nivel de confianza que tu eres.

Comienza a responder las preguntas con la primer pregunta cuando lo indique.

No te saltes ningúna pregunta. 


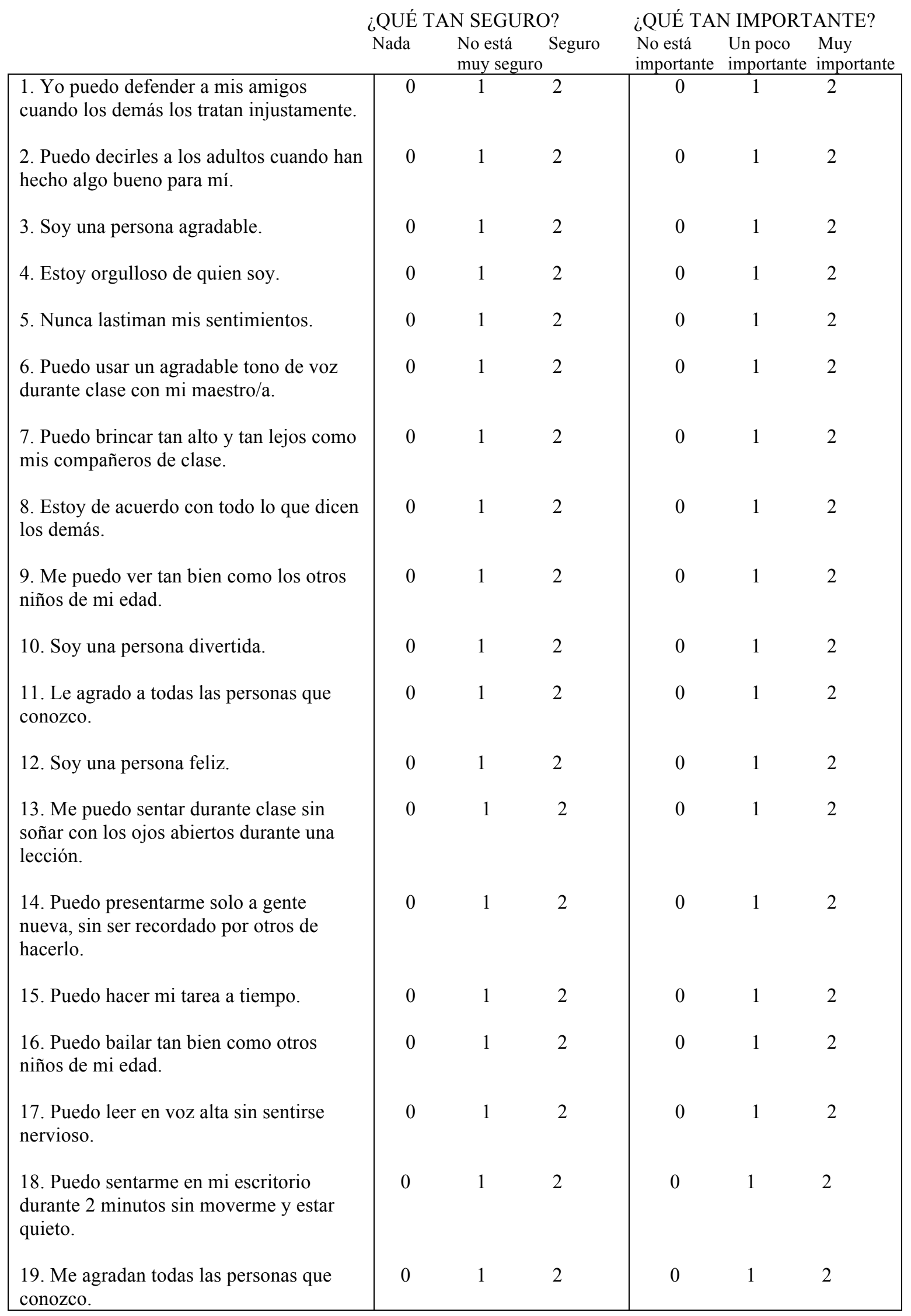


20. Cortésmente puedo negar a hacer las cosas que están mal, incluyendo cuando otros niños quieren que las haga.

21. Puedo terminar mi trabajo de clase a tiempo.

22. Yo siempre digo la verdad.

23. Puedo escuchar a mi maestro/a hablar sobre un mismo tema por 20 minutos.

24. Me resulta fácil hacer amigos.

25. Puedo pedir ayuda a mi maestro sin sentirme avergonzado/a o molesto/a.

26. Puedo hacer mi trabajo en el pizarron a cuando mi maestro/a me lo pide.

27. Puedo controlar mi temperamento en discusiones con otros niños.

28. Me río cuando cometo errores tontos.

29. Estoy igual coordinado/a como otros niños de mi edad.

30. Le puedo pedir a mis compañeros que juegen un juego conmigo.

31. Soy capaz de leer las instrucciones en un libro y seguirlas con mucha atención.

32. Puedo seguir instrucciones de mi maestro para hacer el trabajo de lectura.

33. Puedo terminar discusiones con mis padres con calma.

34. Puedo decirles a mis amigos que tengo algo sin presumir.

35. Puedo decirles cosas agradables a mis compañeros cuando han hecho algo bien.

36. Puedo ignorar a mis compañeros de clase cuando susurran o hablan durante la clase.

37. Puedo decirle a los niños de mi edad que me agradan sin sentirme avergonzado/a.

38. Puedo preguntarle a otros niños si me permiten entrar en el juego que están

\begin{tabular}{|c|c|c|c|c|c|}
\hline 0 & 1 & 2 & 0 & 1 & 2 \\
\hline 0 & 1 & 2 & 0 & 1 & 2 \\
\hline 0 & 1 & 2 & 0 & 1 & 2 \\
\hline 0 & 1 & 2 & 0 & 1 & 2 \\
\hline 0 & 1 & 2 & 0 & 1 & 2 \\
\hline 0 & 1 & 2 & 0 & 1 & 2 \\
\hline 0 & 1 & 2 & 0 & 1 & 2 \\
\hline 0 & 1 & 2 & 0 & 1 & 2 \\
\hline 0 & 1 & 2 & 0 & 1 & 2 \\
\hline 0 & 1 & 2 & 0 & 1 & 2 \\
\hline 0 & 1 & 2 & 0 & 1 & 2 \\
\hline 0 & 1 & 2 & 0 & 1 & 2 \\
\hline 0 & 1 & 2 & 0 & 1 & 2 \\
\hline 0 & 1 & 2 & 0 & 1 & 2 \\
\hline 0 & 1 & 2 & 0 & 1 & 2 \\
\hline 0 & 1 & 2 & 0 & 1 & 2 \\
\hline 0 & 1 & 2 & 0 & 1 & 2 \\
\hline 0 & 1 & 2 & 0 & 1 & 2 \\
\hline 0 & 1 & 2 & 0 & 1 & 2 \\
\hline
\end{tabular}




\begin{tabular}{|c|c|c|c|c|c|c|}
\hline jugando. & & & & & & \\
\hline $\begin{array}{l}\text { 39. Puedo mostrarle a los demás que me } \\
\text { siento bien conmigo mismo. }\end{array}$ & 0 & 1 & 2 & 0 & 1 & 2 \\
\hline $\begin{array}{l}\text { 40. Puedo hacer el trabajo de matematicas } \\
\text { sin ayuda. }\end{array}$ & 0 & 1 & 2 & 0 & 1 & 2 \\
\hline $\begin{array}{l}\text { 41. Yo soy tan fuerte como otros niños de } \\
\text { mi edad. }\end{array}$ & 0 & 1 & 2 & 0 & 1 & 2 \\
\hline $\begin{array}{l}\text { 42. Puedo correr tan rápido como otros } \\
\text { niños de mi edad. }\end{array}$ & 0 & 1 & 2 & 0 & 1 & 2 \\
\hline $\begin{array}{l}\text { 43. Puedo tomar turnos en los juegos o } \\
\text { otras actividades. }\end{array}$ & 0 & 1 & 2 & 0 & 1 & 2 \\
\hline $\begin{array}{l}\text { 44. Puedo hablar en clase cuando mi } \\
\text { maestro me lo pide. }\end{array}$ & 0 & 1 & 2 & 0 & 1 & 2 \\
\hline $\begin{array}{l}\text { 45. Puedo escuchar cuando mi maestro } \\
\text { presenta una lección. }\end{array}$ & 0 & 1 & 2 & 0 & 1 & 2 \\
\hline $\begin{array}{l}\text { 46. Puedo decirle a mi maestro cuando él } \\
\text { o ella me ha acusado de hacer algo que no } \\
\text { hice. }\end{array}$ & 0 & 1 & 2 & 0 & 1 & 2 \\
\hline $\begin{array}{l}\text { 47. Recuerdo cuando los proyectos de } \\
\text { clase se deben de entregar. }\end{array}$ & 0 & 1 & 2 & 0 & 1 & 2 \\
\hline $\begin{array}{l}\text { 48. Puedo hablar tranquilamente con } \\
\text { niños de mi edad cuando estemos en } \\
\text { desacuerdo. }\end{array}$ & 0 & 1 & 2 & 0 & 1 & 2 \\
\hline $\begin{array}{l}\text { 49. Puedo practicar deportes igual que } \\
\text { otros niños de mi edad. }\end{array}$ & 0 & 1 & 2 & 0 & 1 & 2 \\
\hline $\begin{array}{l}\text { 50. Puedo decirle a mis compañeros de } \\
\text { clase lo que siento cuando me molestan o } \\
\text { lastiman mis sentimientos. }\end{array}$ & 0 & 1 & 2 & 0 & 1 & 2 \\
\hline 51. Nunca me enojo. & 0 & 1 & 2 & 0 & 1 & 2 \\
\hline 52. Soy una buena persona. & 0 & 1 & 2 & 0 & 1 & 2 \\
\hline 53. Puedo hacer lo que yo quiera. & 0 & 1 & 2 & 0 & 1 & 2 \\
\hline $\begin{array}{l}\text { 54. Puedo compartir mis bienes con los } \\
\text { demás. }\end{array}$ & 0 & 1 & 2 & 0 & 1 & 2 \\
\hline 55. Puedo seguir las reglas del salón. & 0 & 1 & 2 & 0 & 1 & 2 \\
\hline 56. Me gusta estar con los demás. & 0 & 1 & 2 & 0 & 1 & 2 \\
\hline
\end{tabular}




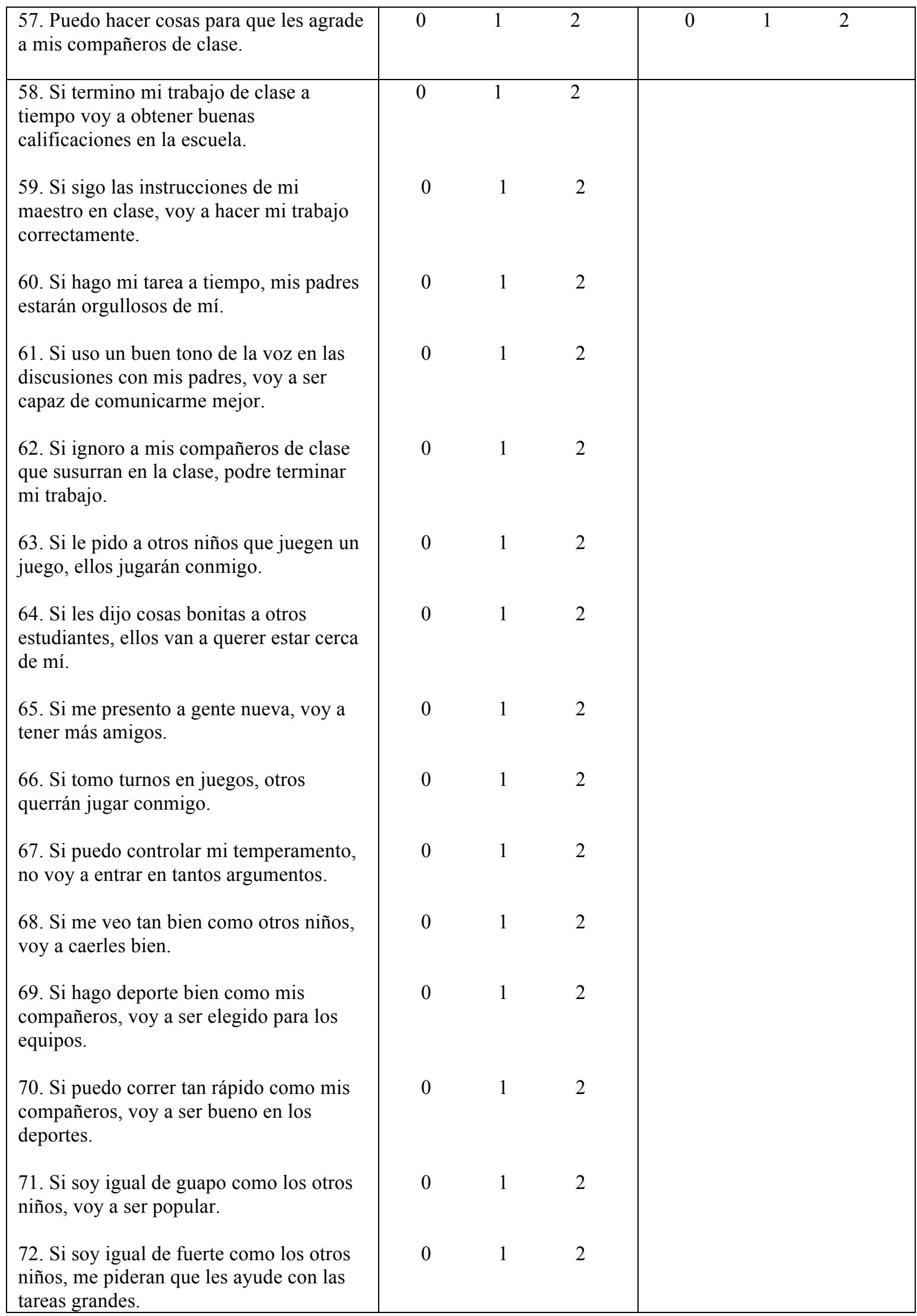




\section{Appendix J}

\section{Disaggregated Academic Self-Concept Subscale Findings}

\section{Academic Self-Concept Confidence Level Subgroup Analysis-ESL-Year 1 Student}

Participants and NAC-Year 1 Student Participants

\begin{tabular}{|c|c|c|c|c|c|c|c|}
\hline & $\begin{array}{l}\mathrm{ES} \\
(N= \\
\end{array}$ & & & $\begin{array}{l}\mathrm{NA} \\
(N=\end{array}$ & & & \\
\hline & $n$ & M & SD & $n$ & M & $\mathrm{SD}$ & Difference \\
\hline I can use a nice tone of voice in classroom & 48 & $1.56^{*}$ & .68 & 50 & $1.82 *$ & .44 & +.26 \\
\hline $\begin{array}{l}\text { I can sit in class without daydreaming } \\
\text { during a lesson. }\end{array}$ & 48 & 1.17 & .78 & 51 & 1.25 & .66 & +.08 \\
\hline I can do my homework on time. & 49 & 1.53 & .65 & 51 & 1.67 & .52 & +.14 \\
\hline $\begin{array}{l}\text { I can read aloud in class without feeling } \\
\text { nervous. }\end{array}$ & 49 & 1.20 & .79 & 50 & 1.40 & .57 & +.20 \\
\hline $\begin{array}{l}\text { I can sit at my desk for } 2 \text { minutes without } \\
\text { moving around or fidgeting. }\end{array}$ & 49 & 1.29 & .87 & 51 & 1.29 & .80 & .00 \\
\hline I can finish my class work on time. & 49 & 1.47 & .68 & 51 & 1.65 & .52 & +.18 \\
\hline $\begin{array}{l}\text { I can listen to my teacher talk about a } \\
\text { subject for } 20 \text { minutes. }\end{array}$ & 49 & 1.39 & 67 & 50 & 1.58 & .61 & +.19 \\
\hline $\begin{array}{l}\text { I can ask my teacher for help without } \\
\text { feeling ashamed or upset. }\end{array}$ & 48 & 1.63 & .57 & 49 & 1.71 & .50 & +.08 \\
\hline $\begin{array}{l}\text { I can go to the board to do work when my } \\
\text { teacher asks me to. }\end{array}$ & 49 & $1.27 *$ & .70 & 51 & $1.57 *$ & .56 & +.30 \\
\hline I can laugh when I make silly mistakes. & 49 & $1.45^{*}$ & .74 & 51 & $1.69 *$ & .55 & +.24 \\
\hline $\begin{array}{l}\text { I can read instructions in a book and } \\
\text { follow them carefully. }\end{array}$ & 48 & 1.50 & .65 & 49 & 1.57 & .61 & +.07 \\
\hline $\begin{array}{l}\text { I can follow my teacher's directions for } \\
\text { doing reading work. }\end{array}$ & 49 & 1.61 & .67 & 51 & 1.69 & .58 & +.08 \\
\hline $\begin{array}{l}\text { I can ignore classmates when they } \\
\text { whisper or talk during class. }\end{array}$ & 49 & 1.22 & .77 & 51 & 1.18 & .59 & -.04 \\
\hline I can do math work without help. & 49 & 1.10 & .80 & 51 & 1.35 & .66 & +.25 \\
\hline $\begin{array}{l}\text { I can speak in class when my teacher } \\
\text { calls on me. }\end{array}$ & 49 & $1.49 *$ & .68 & 51 & $1.75^{*}$ & .52 & +.26 \\
\hline $\begin{array}{l}\text { I can listen when my teacher is } \\
\text { presenting a lesson. }\end{array}$ & 49 & 1.69 & .59 & 50 & 1.72 & .54 & +.03 \\
\hline
\end{tabular}




\begin{tabular}{|c|c|c|c|c|c|c|c|}
\hline $\begin{array}{l}\text { I can remember when class } \\
\text { projects are due. }\end{array}$ & 49 & 1.53 & .58 & 51 & 1.47 & .64 & -.06 \\
\hline I can follow classroom rules. & 49 & 1.71 & .50 & 51 & 1.61 & .57 & -.10 \\
\hline $\begin{array}{l}\text { If I finish my class work on time } \\
\text { I will get good grades in school. }\end{array}$ & 47 & 1.79 & .46 & 51 & 1.82 & .39 & +.03 \\
\hline $\begin{array}{l}\text { If I follow my teacher's directions in } \\
\text { class, I will do my work correctly. }\end{array}$ & 48 & 1.77 & .47 & 51 & 1.90 & .30 & +.13 \\
\hline $\begin{array}{l}\text { If I do my homework on time, my } \\
\text { parents will be proud of me. }\end{array}$ & 47 & 1.83 & .43 & 51 & 1.82 & .43 & -.01 \\
\hline $\begin{array}{l}\text { If I ignore classmates who whisper in } \\
\text { class, I can complete my work. }\end{array}$ & 48 & 1.71 & .54 & 51 & 1.73 & .50 & +.02 \\
\hline
\end{tabular}

Note. ${ }^{*} \mathrm{p}<.05 . * * \mathrm{p}<.01$.

Academic Self-Concept Item Importance Subgroup Analysis-ESL-Year 1 Student Participants and NAC-Year 1 Student Participants

\begin{tabular}{|c|c|c|c|c|c|c|c|}
\hline & \multicolumn{3}{|c|}{$\begin{array}{l}\text { ESL } \\
(N=49)\end{array}$} & \multicolumn{3}{|c|}{$\begin{array}{l}\text { NAC } \\
(N=51)\end{array}$} & \multirow{2}{*}{$\begin{array}{l}\text { Mean } \\
\text { Difference }\end{array}$} \\
\hline & $n$ & M & SD & $n$ & M & SD & \\
\hline $\begin{array}{l}\text { I can use a nice tone of voice in classroom } \\
\text { discussions with my teacher. }\end{array}$ & 49 & 1.69 & .60 & 51 & 1.67 & .60 & -.02 \\
\hline $\begin{array}{l}\text { I can sit in class without daydreaming } \\
\text { during a lesson. }\end{array}$ & 48 & 1.23 & .83 & 50 & 1.16 & .79 & -.07 \\
\hline I can do my homework on time. & 49 & 1.67 & .63 & 50 & 1.76 & .52 & +.09 \\
\hline $\begin{array}{l}\text { I can read aloud in class without feeling } \\
\text { nervous. }\end{array}$ & 49 & 1.55 & .68 & 51 & 1.41 & .64 & -.14 \\
\hline $\begin{array}{l}\text { I can sit at my desk for } 2 \text { minutes without } \\
\text { moving around or fidgeting. }\end{array}$ & 49 & 1.08 & .86 & 50 & 1.16 & .74 & +.08 \\
\hline I can finish my class work on time. & 49 & 1.73 & .61 & 51 & 1.84 & .42 & +.11 \\
\hline $\begin{array}{l}\text { I can listen to my teacher talk about a } \\
\text { subject for } 20 \text { minutes. }\end{array}$ & 49 & 1.47 & .71 & 50 & 1.66 & .48 & +.19 \\
\hline $\begin{array}{l}\text { I can ask my teacher for help without } \\
\text { feeling ashamed or upset. }\end{array}$ & 48 & 1.65 & .67 & 49 & 1.61 & .64 & -.04 \\
\hline $\begin{array}{l}\text { I can go to the board to do work when my } \\
\text { teacher asks me to. }\end{array}$ & 49 & 1.53 & .74 & 51 & 1.59 & .64 & +.06 \\
\hline I can laugh when I make silly mistakes. & 49 & 1.12 & .83 & 50 & .98 & .74 & -.14 \\
\hline
\end{tabular}




\begin{tabular}{|c|c|c|c|c|c|c|c|}
\hline $\begin{array}{l}\text { I can read instructions in a book and } \\
\text { follow them carefully. }\end{array}$ & 47 & 1.49 & .59 & 50 & 1.60 & .61 & +.11 \\
\hline $\begin{array}{l}\text { I can follow my teacher's directions for } \\
\text { doing reading work. }\end{array}$ & 47 & 1.74 & .53 & 51 & 1.71 & .54 & -.03 \\
\hline $\begin{array}{l}\text { I can ignore classmates when they } \\
\text { whisper or talk during class. }\end{array}$ & 49 & 1.41 & .76 & 50 & 1.34 & .63 & -.07 \\
\hline I can do math work without help. & 49 & 1.55 & .68 & 51 & 1.71 & .54 & +.16 \\
\hline $\begin{array}{l}\text { I can speak in class when my } \\
\text { teacher calls on me. }\end{array}$ & 49 & 1.49 & .71 & 51 & 1.63 & .60 & +.14 \\
\hline $\begin{array}{l}\text { I can listen when my teacher is } \\
\text { presenting a lesson. }\end{array}$ & 49 & 1.76 & .56 & 50 & 1.74 & .53 & -.02 \\
\hline $\begin{array}{l}\text { I can remember when class } \\
\text { projects are due. }\end{array}$ & 49 & 1.69 & .55 & 51 & 1.73 & .57 & +.04 \\
\hline I can follow classroom rules. & 49 & 1.73 & .45 & 51 & 1.71 & .54 & -.02 \\
\hline
\end{tabular}

Note. ${ }^{*} \mathrm{p}<.05 . * * \mathrm{p}<.01$

Academic Self-Concept Confidence Level Subgroup Analysis-ESL-Year 2 Student Participants and NAC-Year 2 Student Participants

\begin{tabular}{|c|c|c|c|c|c|c|c|}
\hline & \multicolumn{3}{|c|}{$\begin{array}{l}\text { ESL } \\
(N=49)\end{array}$} & \multicolumn{3}{|c|}{$\begin{array}{l}\text { NAC } \\
(N=43) \\
\end{array}$} & \multirow{2}{*}{$\begin{array}{l}\text { Mean } \\
\text { Difference }\end{array}$} \\
\hline & $n$ & M & SD & $n$ & M & SD & \\
\hline $\begin{array}{l}\text { I can use a nice tone of voice in classroom } \\
\text { discussions with my teacher. }\end{array}$ & 49 & 1.69 & .55 & 42 & 1.60 & .59 & -.09 \\
\hline $\begin{array}{l}\text { I can sit in class without daydreaming } \\
\text { during a lesson. }\end{array}$ & 49 & 1.20 & .76 & 42 & 1.26 & .67 & +.06 \\
\hline I can do my homework on time. & 49 & 1.51 & .58 & 43 & 1.44 & .59 & -.07 \\
\hline $\begin{array}{l}\text { I can read aloud in class without feeling } \\
\text { nervous. }\end{array}$ & 49 & 1.18 & .70 & 42 & 1.21 & .57 & +.03 \\
\hline $\begin{array}{l}\text { I can sit at my desk for } 2 \text { minutes without } \\
\text { moving around or fidgeting. }\end{array}$ & 49 & 1.49 & .71 & 42 & 1.29 & .86 & -.20 \\
\hline I can finish my class work on time. & 48 & 1.46 & .54 & 42 & 1.43 & .55 & -.03 \\
\hline $\begin{array}{l}\text { I can listen to my teacher talk about a } \\
\text { subject for } 20 \text { minutes. }\end{array}$ & 48 & 1.44 & .71 & 42 & 1.40 & .59 & -.04 \\
\hline $\begin{array}{l}\text { I can ask my teacher for help without } \\
\text { feeling ashamed or upset. }\end{array}$ & 47 & 1.57 & .54 & 42 & 1.43 & .70 & -.14 \\
\hline
\end{tabular}


I can go to the board to do work when my teacher asks me to.

I can laugh when I make silly mistakes.

I can read instructions in a book and follow them carefully.

I can follow my teacher's directions for doing reading work.

I can ignore classmates when they whisper or talk during class.

I can do math work without help.

I can speak in class when my teacher calls on me.

I can listen when my teacher is presenting a lesson.

I can remember when class projects are due.

I can follow classroom rules.

If I finish my class work on time I will get good grades in school.

If I follow my teacher's directions in class, I will do my work correctly.

If I do my homework on time, my parents will be proud of me.

If I ignore classmates who whisper in class, I can complete my work.

$\begin{array}{lllllll}49 & 1.33 & .69 & 42 & 1.33 & .57 & .00\end{array}$

$\begin{array}{lllllll}49 & 1.37 & .73 & 43 & 1.40 & .73 & +.03\end{array}$

$\begin{array}{lllllll}49 & 1.61 & .57 & 42 & 1.48 & .63 & -.13\end{array}$

$\begin{array}{lllllll}49 & 1.80 & .46 & 41 & 1.73 & .50 & -.07\end{array}$

$\begin{array}{lllllll}48 & 1.33 * & .66 & 43 & 1.00 * & .76 & -.33\end{array}$

$\begin{array}{lllllll}49 & 1.16 & .69 & 41 & 1.22 & .73 & +.06 \\ 49 & 1.59 & .64 & 41 & 1.51 & .64 & -.08 \\ 49 & 1.82 & .39 & 41 & 1.76 & .44 & -.06 \\ 48 & 1.63 & .53 & 40 & 1.55 & .50 & -.08 \\ 49 & 1.67 & .47 & 41 & 1.59 & .55 & -.08 \\ 49 & 1.86 & .35 & 41 & 1.88 & .40 & +.02 \\ 49 & 1.96 & .20 & 41 & 1.90 & .30 & -.06 \\ 49 & 1.88 & .39 & 41 & 1.76 & .49 & -.12 \\ 49 & 1.73 & .53 & 41 & 1.63 & .54 & -.10\end{array}$

Note. $* \mathrm{p}<.05 . * * \mathrm{p}<.01$.

Academic Self-Concept Item Importance Subgroup Analysis-ESL-Year 2 Student Participants and NAC-Year 2 Student Participants

\begin{tabular}{|c|c|c|c|c|c|c|c|}
\hline & \multicolumn{3}{|c|}{$\begin{array}{l}\text { ESL } \\
(N=49)\end{array}$} & \multicolumn{3}{|c|}{$\begin{array}{l}\mathrm{NAC} \\
(N=43)\end{array}$} & \multirow{2}{*}{$\begin{array}{l}\text { Mean } \\
\text { Difference }\end{array}$} \\
\hline & $n$ & $\mathrm{M}$ & SD & $n$ & M & $\mathrm{SD}$ & \\
\hline $\begin{array}{l}\text { I can use a nice tone of voice in classroom } \\
\text { discussions with my teacher. }\end{array}$ & 48 & 1.48 & .65 & 43 & 1.40 & .62 & -.08 \\
\hline $\begin{array}{l}\text { I can sit in class without daydreaming } \\
\text { during a lesson. }\end{array}$ & 48 & 1.17 & .78 & 42 & 1.07 & .75 & -.10 \\
\hline
\end{tabular}


I can do my homework on time.

49

1.78

I can read aloud in class without feeling

49

1.53

.51

43

$1.74 \quad .54$

$-.04$

nervous.

I can sit at my desk for 2 minutes without

49

moving around or fidgeting.

I can finish my class work on time.

48

$1.73 \quad .54$

43

$1.51 \quad .55$

$-.02$

I can listen to my teacher talk about a

48

$1.56 \quad .68$

43

1.91

.21

.74

$-.02$

subject for 20 minutes.

I can ask my teacher for help without

47

$$
1.77
$$

.43

43

$1.65 \quad .53$

$-.12$

I can go to the board to do work when my

49

1.6

teacher asks me to.

I can laugh when I make silly mistakes.

49

49

I can read instructions in a book and

follow them carefully.

I can follow my teacher's directions for

49

doing reading work.

I can ignore classmates when they

whisper or talk during class.

I can do math work without help.

I can speak in class when my

teacher calls on me.

I can listen when my teacher is

presenting a lesson.

I can remember when class

projects are due.

48

48

$1.13 \quad .82$

42

1.19

$1.42 \quad .66$

$-.19$

I can follow classroom rules.

\section{8}

49

$$
1.52
$$

$$
.71
$$

$1.59 \quad .61$

40

1.78

$1.78 \quad .53+.16$

48

$1.67 \quad .56$

42

$1.38 \quad .70$

$-.21$

48

1.6

48

$\begin{array}{llllll}1.71 & .50 & 42 & 1.62 & .54 & -.09\end{array}$

Note. ${ }^{*} \mathrm{p}<.05 .{ }^{* *} \mathrm{p}<.01$. 


\begin{tabular}{|c|c|c|c|c|c|c|c|}
\hline & \multicolumn{3}{|c|}{$\begin{array}{l}\text { ESL-Year } 1 \\
(N=49)\end{array}$} & \multicolumn{3}{|c|}{$\begin{array}{l}\text { ESL-Year } 2 \\
(N=49)\end{array}$} & \multirow{2}{*}{$\begin{array}{l}\text { Mean } \\
\text { Difference }\end{array}$} \\
\hline & $n$ & M & $\mathrm{SD}$ & $n$ & M & $\mathrm{SD}$ & \\
\hline $\begin{array}{l}\text { I can use a nice tone of voice in classroom } \\
\text { discussions with my teacher. }\end{array}$ & 48 & 1.56 & .68 & 49 & 1.69 & .55 & +.13 \\
\hline $\begin{array}{l}\text { I can sit in class without daydreaming } \\
\text { during a lesson. }\end{array}$ & 48 & 1.17 & .78 & 49 & 1.20 & .76 & +.03 \\
\hline I can do my homework on time. & 49 & 1.53 & .65 & 49 & 1.51 & .58 & -.02 \\
\hline $\begin{array}{l}\text { I can read aloud in class without feeling } \\
\text { nervous. }\end{array}$ & 49 & 1.20 & .79 & 49 & 1.18 & .70 & -.02 \\
\hline $\begin{array}{l}\text { I can sit at my desk for } 2 \text { minutes without } \\
\text { moving around or fidgeting. }\end{array}$ & 49 & 1.29 & .87 & 49 & 1.49 & .71 & +.20 \\
\hline I can finish my class work on time. & 49 & 1.47 & .68 & 48 & 1.46 & .55 & -.01 \\
\hline $\begin{array}{l}\text { I can listen to my teacher talk about a } \\
\text { subject for } 20 \text { minutes. }\end{array}$ & 49 & 1.39 & .67 & 48 & 1.44 & .71 & +.05 \\
\hline $\begin{array}{l}\text { I can ask my teacher for help without } \\
\text { feeling ashamed or upset. }\end{array}$ & 48 & 1.63 & .57 & 47 & 1.57 & .54 & -.06 \\
\hline $\begin{array}{l}\text { I can go to the board to do work when my } \\
\text { teacher asks me to. }\end{array}$ & 49 & 1.27 & .70 & 49 & 1.33 & .69 & +.06 \\
\hline I can laugh when I make silly mistakes. & 49 & 1.45 & .74 & 49 & 1.37 & .73 & -.08 \\
\hline $\begin{array}{l}\text { I can read instructions in a book and } \\
\text { follow them carefully. }\end{array}$ & 48 & 1.50 & .65 & 49 & 1.61 & .57 & +.11 \\
\hline $\begin{array}{l}\text { I can follow my teacher's directions for } \\
\text { doing reading work. }\end{array}$ & 49 & 1.61 & .67 & 49 & 1.80 & .46 & +.19 \\
\hline $\begin{array}{l}\text { I can ignore classmates when they } \\
\text { whisper or talk during class. }\end{array}$ & 49 & 1.22 & .77 & 48 & 1.33 & .66 & +.11 \\
\hline I can do math work without help. & 49 & 1.10 & .80 & 49 & 1.16 & .69 & +.06 \\
\hline $\begin{array}{l}\text { I can speak in class when my } \\
\text { teacher calls on me. }\end{array}$ & 49 & 1.49 & .68 & 49 & 1.59 & .64 & +.10 \\
\hline $\begin{array}{l}\text { I can listen when my teacher is } \\
\text { presenting a lesson. }\end{array}$ & 49 & 1.69 & .58 & 49 & 1.82 & .53 & +.13 \\
\hline $\begin{array}{l}\text { I can remember when class } \\
\text { projects are due. }\end{array}$ & 49 & 1.53 & .58 & 48 & 1.63 & .53 & +.10 \\
\hline I can follow classroom rules. & 49 & 1.71 & .50 & 49 & 1.67 & .47 & -.04 \\
\hline
\end{tabular}


If I finish my class work on time I

47

$1.79 \quad .46$

49

$\begin{array}{lll}1.86 & .35+.07\end{array}$

will get good grades in school.

If I follow my teacher's directions in 48

$1.77 * \quad .47$

49

$1.96 * .20$

$+.19$

class, I will do my work correctly.

If I do my homework on time, my parents will be proud of me.

47

If I ignore classmates who whisper in class, I can complete my work.

Note. ${ }^{*} \mathrm{p}<.05 . * * \mathrm{p}<.01$.

Academic Self-Concept Item Importance Subgroup Analysis-ESL-Year 1 Student Participants and ESL-Year 2 Student Participants

\begin{tabular}{|c|c|c|c|c|c|c|c|}
\hline & \multicolumn{3}{|c|}{$\begin{array}{l}\text { ESL-Year } 1 \\
(N=49)\end{array}$} & \multicolumn{3}{|c|}{$\begin{array}{l}\text { ESL-Year } 2 \\
(N=49)\end{array}$} & \multirow{2}{*}{$\begin{array}{l}\text { Mean } \\
\text { Difference }\end{array}$} \\
\hline & $n$ & M & SD & $n$ & M & $\mathrm{SD}$ & \\
\hline $\begin{array}{l}\text { I can use a nice tone of voice in classroom } \\
\text { discussions with my teacher. }\end{array}$ & 48 & 1.69 & .59 & 48 & 1.48 & .65 & -.21 \\
\hline $\begin{array}{l}\text { I can sit in class without daydreaming } \\
\text { during a lesson. }\end{array}$ & 48 & 1.23 & .83 & 48 & 1.17 & .78 & -.06 \\
\hline I can do my homework on time. & 49 & 1.67 & .63 & 49 & 1.78 & .51 & +.11 \\
\hline $\begin{array}{l}\text { I can read aloud in class without feeling } \\
\text { nervous. }\end{array}$ & 49 & 1.55 & .68 & 49 & 1.53 & .62 & -.02 \\
\hline $\begin{array}{l}\text { I can sit at my desk for } 2 \text { minutes without } \\
\text { moving around or fidgeting. }\end{array}$ & 49 & 1.08 & .86 & 48 & 1.19 & .73 & +.11 \\
\hline I can finish my class work on time. & 49 & 1.73 & .61 & 48 & 1.73 & .54 & .00 \\
\hline $\begin{array}{l}\text { I can listen to my teacher talk about a } \\
\text { subject for } 20 \text { minutes. }\end{array}$ & 49 & 1.47 & .71 & 48 & 1.56 & .68 & +.09 \\
\hline $\begin{array}{l}\text { I can ask my teacher for help without } \\
\text { feeling ashamed or upset. }\end{array}$ & 48 & 1.65 & .67 & 47 & 1.77 & .43 & +.12 \\
\hline $\begin{array}{l}\text { I can go to the board to do work when my } \\
\text { teacher asks me to. }\end{array}$ & 49 & 1.53 & .74 & 49 & 1.61 & .57 & +.08 \\
\hline I can laugh when I make silly mistakes. & 49 & 1.12 & .83 & 49 & .96 & .74 & -.16 \\
\hline $\begin{array}{l}\text { I can read instructions in a book and } \\
\text { follow them carefully. }\end{array}$ & 47 & $1.49 * *$ & .59 & 49 & $1.78 * *$ & .42 & +.29 \\
\hline $\begin{array}{l}\text { I can follow my teacher's directions for } \\
\text { doing reading work. }\end{array}$ & 47 & 1.74 & .53 & 49 & 1.82 & .49 & +.08 \\
\hline
\end{tabular}


I can ignore classmates when they whisper or talk during class.

I can do math work without help.

I can speak in class when my teacher calls on me.

I can listen when my teacher is presenting a lesson.

I can remember when class projects are due.

I can follow classroom rules.
49

$$
1.41 * \quad .77
$$

48

$1.13 * \quad .66$

$-.28$

49

$1.55 \quad .68$

48

$1.52 \quad .71$

$-.03$

49

1.49

.71

49

$\begin{array}{lll}1.59 & .61+.10\end{array}$

49

1.76

.56

48

$1.67 \quad .56$

$-.09$

49

1.69

.55

48

1.67

.56

$-.02$

49

1.73

48

$1.71 \quad .50$

$-.02$

Academic Self-Concept Confidence Level Subgroup Analysis-NAC-Year 1 Student Participants and NAC-Year 2 Student Participants

\begin{tabular}{|c|c|c|c|c|c|c|c|}
\hline & \multicolumn{3}{|c|}{$\begin{array}{l}\text { NAC-Year } 1 \\
(N=51)\end{array}$} & \multicolumn{3}{|c|}{$\begin{array}{l}\text { NAC-Year } 2 \\
(N=43)\end{array}$} & \multirow{2}{*}{$\begin{array}{l}\text { Mean } \\
\text { Difference }\end{array}$} \\
\hline & $n$ & M & SD & $n$ & M & SD & \\
\hline $\begin{array}{l}\text { I can use a nice tone of voice in classroom } \\
\text { discussions with my teacher. }\end{array}$ & 50 & $1.82 *$ & .44 & 42 & $1.60 *$ & .59 & -.22 \\
\hline $\begin{array}{l}\text { I can sit in class without daydreaming } \\
\text { during a lesson. }\end{array}$ & 51 & 1.25 & .66 & 42 & 1.26 & .67 & +.01 \\
\hline I can do my homework on time. & 51 & 1.67 & .52 & 43 & 1.44 & .59 & -.23 \\
\hline $\begin{array}{l}\text { I can read aloud in class without feeling } \\
\text { nervous. }\end{array}$ & 50 & 1.40 & .57 & 42 & 1.21 & .57 & -.19 \\
\hline $\begin{array}{l}\text { I can sit at my desk for } 2 \text { minutes without } \\
\text { moving around or fidgeting. }\end{array}$ & 51 & 1.29 & .81 & 42 & 1.29 & .86 & .00 \\
\hline I can finish my class work on time. & 51 & 1.65 & .52 & 42 & 1.43 & .55 & -.22 \\
\hline $\begin{array}{l}\text { I can listen to my teacher talk about a } \\
\text { subject for } 20 \text { minutes. }\end{array}$ & 50 & 1.58 & .61 & 42 & 1.40 & .59 & -.18 \\
\hline $\begin{array}{l}\text { I can ask my teacher for help without } \\
\text { feeling ashamed or upset. }\end{array}$ & 49 & $1.71 *$ & .50 & 42 & $1.43 *$ & .70 & -.28 \\
\hline $\begin{array}{l}\text { I can go to the board to do work when my } \\
\text { teacher asks me to. }\end{array}$ & 51 & 1.57 & .58 & 42 & 1.33 & .57 & -.24 \\
\hline I can laugh when I make silly mistakes. & 51 & $1.69 *$ & .55 & 43 & $1.40 *$ & .73 & -.29 \\
\hline
\end{tabular}


I can read instructions in a book and

follow them carefully.

I can follow my teacher's directions for

doing reading work.

I can ignore classmates when they

whisper or talk during class.

I can do math work without help.

I can speak in class when my teacher calls on me.

I can listen when my teacher is

presenting a lesson.

I can remember when class

projects are due.

I can follow classroom rules.

If I finish my class work on time I

will get good grades in school.

If I follow my teacher's directions in class, I will do my work correctly.

If I do my homework on time, my parents will be proud of me.

If I ignore classmates who whisper in class, I can complete my work.

Note. ${ }^{*} \mathrm{p}<.05 . * * \mathrm{p}<.01$.

\begin{tabular}{|c|c|c|c|c|c|c|}
\hline 49 & 1.57 & .61 & 42 & 1.48 & .63 & -.09 \\
\hline 51 & 1.69 & .58 & 41 & 1.73 & .50 & +.04 \\
\hline 51 & 1.18 & .59 & 43 & 1.00 & .76 & -.18 \\
\hline 51 & 1.35 & .66 & 41 & 1.22 & .73 & -.13 \\
\hline 51 & 1.75 & .52 & 41 & 1.51 & .64 & -.24 \\
\hline 50 & 1.72 & .54 & 41 & 1.76 & .44 & +.04 \\
\hline 51 & 1.47 & .64 & 40 & 1.55 & .50 & +.08 \\
\hline 51 & 1.61 & .57 & 41 & 1.59 & .55 & -.02 \\
\hline 51 & 1.82 & .39 & 41 & 1.88 & .40 & +.06 \\
\hline 51 & 1.90 & .30 & 41 & 1.90 & .30 & .00 \\
\hline 51 & 1.82 & .43 & 41 & 1.76 & .49 & -.06 \\
\hline 51 & 1.73 & .49 & 41 & 1.63 & .54 & -.10 \\
\hline
\end{tabular}

Academic Self-Concept Item Importance Subgroup Analysis -NAC-Year 1 Student Participants and NAC-Year 2 Student Participants

\begin{tabular}{|c|c|c|c|c|c|c|c|}
\hline & \multicolumn{3}{|c|}{$\begin{array}{l}\text { NAC-Year } 1 \\
(N=51)\end{array}$} & \multicolumn{3}{|c|}{$\begin{array}{l}\text { NAC-Year } 2 \\
(N=43)\end{array}$} & \multirow{2}{*}{$\begin{array}{l}\text { Mean } \\
\text { Difference }\end{array}$} \\
\hline & $n$ & $\mathrm{M}$ & $\mathrm{SD}$ & $n$ & $\mathrm{M}$ & $\mathrm{SD}$ & \\
\hline $\begin{array}{l}\text { I can use a nice tone of voice in classroom } \\
\text { discussions with my teacher. }\end{array}$ & 51 & $1.67^{*}$ & .59 & 43 & $1.40^{*}$ & .62 & -.27 \\
\hline $\begin{array}{l}\text { I can sit in class without daydreaming } \\
\text { during a lesson. }\end{array}$ & 50 & 1.16 & .79 & 42 & 1.07 & .75 & -.09 \\
\hline I can do my homework on time. & 50 & 1.76 & .52 & 43 & 1.74 & .54 & -.02 \\
\hline
\end{tabular}




\begin{tabular}{|c|c|c|c|c|c|c|c|}
\hline $\begin{array}{l}\text { I can read aloud in class without feeling } \\
\text { nervous. }\end{array}$ & 51 & 1.41 & .64 & 43 & 1.51 & .55 & +.10 \\
\hline $\begin{array}{l}\text { I can sit at my desk for } 2 \text { minutes } \\
\text { without moving around or fidgeting. }\end{array}$ & 50 & 1.16 & .74 & 43 & 1.21 & .74 & +.05 \\
\hline I can finish my class work on time. & 51 & 1.84 & .42 & 43 & 1.91 & .29 & +.07 \\
\hline $\begin{array}{l}\text { I can listen to my teacher talk about a } \\
\text { subject for } 20 \text { minutes. }\end{array}$ & 50 & 1.66 & .48 & 43 & 1.49 & .70 & -.17 \\
\hline $\begin{array}{l}\text { I can ask my teacher for help without } \\
\text { feeling ashamed or upset. }\end{array}$ & 49 & 1.61 & .64 & 43 & 1.65 & .53 & +.04 \\
\hline $\begin{array}{l}\text { I can go to the board to do work when } \\
\text { my teacher asks me to. }\end{array}$ & 51 & 1.59 & .64 & 43 & 1.42 & .66 & -.17 \\
\hline I can laugh when I make silly mistakes. & 50 & .98 & .74 & 42 & .81 & .83 & -.17 \\
\hline $\begin{array}{l}\text { I can read instructions in a book and } \\
\text { follow them carefully. }\end{array}$ & 50 & 1.60 & .61 & 43 & 1.67 & .57 & +.07 \\
\hline $\begin{array}{l}\text { I can follow my teacher's directions for } \\
\text { doing reading work. }\end{array}$ & 51 & 1.71 & .54 & 43 & 1.77 & .43 & +.06 \\
\hline $\begin{array}{l}\text { I can ignore classmates when they } \\
\text { whisper or talk during class. }\end{array}$ & 50 & 1.34 & .63 & 42 & 1.19 & .74 & -.15 \\
\hline I can do math work without help. & 51 & 1.71 & .54 & 40 & 1.78 & .53 & +.07 \\
\hline $\begin{array}{l}\text { I can speak in class when my teacher } \\
\text { calls on me. }\end{array}$ & 51 & 1.63 & .60 & 42 & 1.38 & .70 & -.25 \\
\hline $\begin{array}{l}\text { I can listen when my teacher is } \\
\text { presenting a lesson. }\end{array}$ & 51 & 1.74 & .53 & 42 & 1.76 & .43 & +.02 \\
\hline $\begin{array}{l}\text { I can remember when class projects } \\
\text { are due. }\end{array}$ & 51 & 1.73 & .57 & 42 & 1.86 & .35 & +.13 \\
\hline I can follow classroom rules. & 51 & 1.71 & .54 & 42 & 1.62 & .54 & -.09 \\
\hline
\end{tabular}

Note. ${ }^{*} \mathrm{p}<.05 .{ }^{* *} \mathrm{p}<.01$. 


\section{Appendix K}

\section{Chi-Square Analysis of Academic Self-Concept Subscale Findings}

\section{Academic Self-Concept Confidence Level Subgroup Analysis - Total ESL Student}

Participants and Total NAC Student Participants (Years 1 and 2)

\begin{tabular}{|c|c|c|c|c|c|c|}
\hline & & $\begin{array}{l}\text { ESL } \\
\text { Freque } \\
(N=98\end{array}$ & ies & & $\begin{array}{l}\text { NAC } \\
\text { Frequer } \\
(N=94)\end{array}$ & \\
\hline & $\begin{array}{l}\text { Not } \\
\text { Secure }\end{array}$ & $\begin{array}{l}\text { Some- } \\
\text { what } \\
\text { Secure }\end{array}$ & $\begin{array}{l}\text { Very } \\
\text { Secure }\end{array}$ & $\begin{array}{l}\text { Not } \\
\text { Secure }\end{array}$ & $\begin{array}{l}\text { Some- } \\
\text { what } \\
\text { Secure }\end{array}$ & $\begin{array}{l}\text { Very } \\
\text { Secure }\end{array}$ \\
\hline $\begin{array}{l}\text { I can use a nice tone of voice in classroom } \\
\text { discussions with my teacher. }\end{array}$ & 7 & 22 & 68 & 3 & 20 & 69 \\
\hline $\begin{array}{l}\text { I can sit in class without daydreaming } \\
\text { during a lesson. }\end{array}$ & 21 & 37 & 39 & 11 & 47 & 35 \\
\hline I can do my homework on time. & 6 & 35 & 57 & 3 & 35 & 56 \\
\hline $\begin{array}{l}\text { I can read aloud in class without feeling } \\
\text { nervous. ** }\end{array}$ & 19 & 41 & 38 & 5 & 53 & 34 \\
\hline $\begin{array}{l}\text { I can sit at my desk for } 2 \text { minutes without } \\
\text { moving around or fidgeting. }\end{array}$ & 19 & 22 & 57 & 22 & 22 & 49 \\
\hline I can finish my class work on time. & 6 & 40 & 51 & 2 & 38 & 53 \\
\hline $\begin{array}{l}\text { I can listen to my teacher talk about a } \\
\text { subject for } 20 \text { minutes. }\end{array}$ & 11 & 35 & 51 & 5 & 36 & 51 \\
\hline $\begin{array}{l}\text { I can ask my teacher for help without } \\
\text { feeling ashamed or upset. }\end{array}$ & 3 & 32 & 60 & 6 & 26 & 59 \\
\hline $\begin{array}{l}\text { I can go to the board to do work when my } \\
\text { teacher asks me to. }\end{array}$ & 13 & 43 & 42 & 4 & 42 & 47 \\
\hline I can laugh when I make silly mistakes. & 14 & 30 & 54 & 8 & 26 & 60 \\
\hline $\begin{array}{l}\text { I can read instructions in a book and } \\
\text { follow them carefully. }\end{array}$ & 6 & 31 & 60 & 6 & 31 & 54 \\
\hline $\begin{array}{l}\text { I can follow my teacher's directions for } \\
\text { doing reading work. }\end{array}$ & 6 & 17 & 75 & 4 & 19 & 69 \\
\hline $\begin{array}{l}\text { I can ignore classmates when they } \\
\text { whisper or talk during class. }\end{array}$ & 15 & 40 & 42 & 17 & 51 & 26 \\
\hline I can do math work without help. & 21 & 43 & 34 & 12 & 41 & 39 \\
\hline $\begin{array}{l}\text { I can speak in class when my teacher } \\
\text { calls on me. }\end{array}$ & 9 & 27 & 62 & 5 & 23 & 64 \\
\hline
\end{tabular}




\begin{tabular}{|c|c|c|c|c|c|c|}
\hline $\begin{array}{l}\text { I can listen when my teacher } \\
\text { is presenting a lesson. }\end{array}$ & 3 & 18 & 77 & 2 & 20 & 69 \\
\hline $\begin{array}{l}\text { I can remember when class projects } \\
\text { are due. }\end{array}$ & 3 & 35 & 59 & 4 & 37 & 50 \\
\hline I can follow classroom rules. & 1 & 28 & 69 & 3 & 31 & 58 \\
\hline $\begin{array}{l}\text { If I finish my class work on time I } \\
\text { will get good grades in school. }\end{array}$ & 1 & 15 & 80 & 1 & 12 & 79 \\
\hline $\begin{array}{l}\text { If I follow my teacher's directions in } \\
\text { class, I will do my work correctly. }\end{array}$ & 1 & 11 & 85 & 0 & 9 & 83 \\
\hline $\begin{array}{l}\text { If I do my homework on time, my } \\
\text { parents will be proud of me. }\end{array}$ & 2 & 10 & 84 & 2 & 15 & 75 \\
\hline $\begin{array}{l}\text { If I ignore classmates who whisper } \\
\text { in class, I can complete my work. }\end{array}$ & 4 & 19 & 74 & 2 & 25 & 65 \\
\hline
\end{tabular}

Note. ${ }^{*} \mathrm{p}<.05 .{ }^{* *} \mathrm{p}<.01$.

Academic Self-Concept Item Importance Subgroup Analysis - Total ESL Student Participants and Total NAC Student Participants (Years 1 and 2)

\begin{tabular}{|c|c|c|c|c|c|c|}
\hline & & \multicolumn{2}{|c|}{$\begin{array}{l}\text { ESL } \\
\text { Frequencies } \\
(N=98)\end{array}$} & & \multicolumn{2}{|c|}{$\begin{array}{l}\text { NAC } \\
\text { Frequencies } \\
(N=94)\end{array}$} \\
\hline & $\begin{array}{l}\text { Not } \\
\text { Secure }\end{array}$ & $\begin{array}{l}\text { Some- } \\
\text { what } \\
\text { Secure }\end{array}$ & $\begin{array}{l}\text { Very } \\
\text { Secure }\end{array}$ & $\begin{array}{l}\text { Not } \\
\text { Secure }\end{array}$ & $\begin{array}{l}\text { Some- } \\
\text { what } \\
\text { Secure }\end{array}$ & $\begin{array}{l}\text { Very } \\
\text { Secure }\end{array}$ \\
\hline $\begin{array}{l}\text { I can use a nice tone of voice in classroom } \\
\text { discussions with my teacher. }\end{array}$ & 7 & 26 & 63 & 6 & 31 & 57 \\
\hline $\begin{array}{l}\text { I can sit in class without daydreaming } \\
\text { during a lesson. }\end{array}$ & 23 & 31 & 42 & 22 & 37 & 33 \\
\hline I can do my homework on time. & 6 & 15 & 77 & 4 & 15 & 74 \\
\hline $\begin{array}{l}\text { I can read aloud in class without feeling } \\
\text { nervous. }\end{array}$ & 8 & 29 & 61 & 5 & 41 & 48 \\
\hline $\begin{array}{l}\text { I can sit at my desk for } 2 \text { minutes without } \\
\text { moving around or fidgeting. }\end{array}$ & 25 & 34 & 38 & 18 & 40 & 35 \\
\hline I can finish my class work on time. & 6 & 14 & 77 & 1 & 10 & 83 \\
\hline $\begin{array}{l}\text { I can listen to my teacher talk about a } \\
\text { subject for } 20 \text { minutes. }\end{array}$ & 11 & 25 & 61 & 5 & 29 & 59 \\
\hline $\begin{array}{l}\text { I can ask my teacher for help without } \\
\text { feeling ashamed or upset. }\end{array}$ & 5 & 18 & 72 & 5 & 24 & 63 \\
\hline
\end{tabular}




\begin{tabular}{|c|c|c|c|c|c|c|}
\hline $\begin{array}{l}\text { I can go to the board to do work when } \\
\text { my teacher asks me to. }\end{array}$ & 9 & 24 & 65 & 8 & 30 & 56 \\
\hline I can laugh when I make silly mistakes. & 28 & 38 & 32 & 33 & 35 & 24 \\
\hline $\begin{array}{l}\text { I can read instructions in a book and } \\
\text { follow them carefully. }\end{array}$ & 2 & 31 & 63 & 5 & 24 & 64 \\
\hline $\begin{array}{l}\text { I can follow my teacher's directions for } \\
\text { doing reading work. }\end{array}$ & 4 & 13 & 79 & 2 & 21 & 71 \\
\hline $\begin{array}{l}\text { I can ignore classmates when they } \\
\text { whisper or talk during class.* }\end{array}$ & 21 & 29 & 47 & 12 & 43 & 37 \\
\hline I can do math work without help. & 11 & 23 & 63 & 4 & 16 & 71 \\
\hline $\begin{array}{l}\text { I can speak in class when my teacher } \\
\text { calls on me. }\end{array}$ & 9 & 27 & 62 & 8 & 29 & 56 \\
\hline $\begin{array}{l}\text { I can listen when my teacher is } \\
\text { presenting a lesson. }\end{array}$ & 5 & 18 & 74 & 2 & 19 & 71 \\
\hline $\begin{array}{l}\text { I can remember when class } \\
\text { projects are due. }\end{array}$ & 4 & 23 & 70 & 3 & 14 & 76 \\
\hline I can follow classroom rules. & 1 & 25 & 71 & 3 & 25 & 65 \\
\hline
\end{tabular}

Note. ${ }^{*} \mathrm{p}<.05 .{ }^{* *} \mathrm{p}<.01$. 


\section{Appendix L}

\section{Classroom Observation Indicator Matrix}

\begin{tabular}{|c|c|c|c|c|c|c|}
\hline & $\begin{array}{c}\text { NAC } \\
\# 1\end{array}$ & $\begin{array}{c}\text { NAC } \\
\# 2\end{array}$ & $\begin{array}{c}\text { NAC } \\
\# 3\end{array}$ & $\begin{array}{c}\text { ESL } \\
\# 1\end{array}$ & $\begin{array}{c}\text { ESL } \\
\# 2\end{array}$ & $\begin{array}{c}\text { ESL } \\
\# 3\end{array}$ \\
\hline \multicolumn{7}{|l|}{ Effective EL Instruction } \\
\hline \multicolumn{7}{|l|}{ Curriculum } \\
\hline \multicolumn{7}{|l|}{ Appropriate for language proficiency } \\
\hline \multicolumn{7}{|l|}{ Materials used to supplement language acquisition } \\
\hline \multicolumn{7}{|l|}{ Instruction } \\
\hline \multicolumn{7}{|l|}{ Linked to student's experiences/prior knowledge } \\
\hline \multicolumn{7}{|l|}{ Connections made between current and prior lessons } \\
\hline \multicolumn{7}{|l|}{ Peer interaction used to activate schema } \\
\hline \multicolumn{7}{|l|}{ Key Vocabulary } \\
\hline \multicolumn{7}{|l|}{ Academic/content vocabulary introduced in lesson } \\
\hline \multicolumn{7}{|l|}{ Vocabulary taught literally } \\
\hline \multicolumn{7}{|l|}{ Vocabulary taught contextually } \\
\hline \multicolumn{7}{|l|}{ Strategies used to support students' understanding } \\
\hline \multicolumn{7}{|l|}{ Comprehensible Input } \\
\hline \multicolumn{7}{|l|}{ Suitable pacing for language proficiency level } \\
\hline \multicolumn{7}{|l|}{ Suitable speech for language proficiency level } \\
\hline \multicolumn{7}{|l|}{ Clear expectations for students } \\
\hline \multicolumn{7}{|l|}{ Checks for understanding } \\
\hline \multicolumn{7}{|l|}{ Visuals used during lesson } \\
\hline \multicolumn{7}{|l|}{ Realia used during lesson } \\
\hline \multicolumn{7}{|l|}{ Modeling used during lesson } \\
\hline \multicolumn{7}{|l|}{ Gestures/TPR used during lesson } \\
\hline \multicolumn{7}{|l|}{ Primary language support used during lesson } \\
\hline \multicolumn{7}{|l|}{ Interactive demonstrations used during lesson } \\
\hline \multicolumn{7}{|l|}{ Partner/group activities present during lesson } \\
\hline \multicolumn{7}{|l|}{ Strategies } \\
\hline Think-alouds used during lesson & & & & & & \\
\hline Word banks are present in lesson & & & & & & \\
\hline Graphic organizer used to support teaching & & & & & & \\
\hline $\begin{array}{l}\text { A variety of question types used during lesson } \\
\text { (literal, analytical, interpretive) }\end{array}$ & & & & & & \\
\hline Evidence of a gradual release of responsibility & & & & & & \\
\hline Interactions & & & & & & \\
\hline Frequent interactions between teachers and students & & & & & & \\
\hline Frequent interactions between students & & & & & & \\
\hline Students provided sufficient "wait time" when responding & & & & & & \\
\hline Practice/Application & & & & & & \\
\hline Students given opportunities to practice new concept & & & & & & \\
\hline $\begin{array}{l}\text { Activities integrate all language domains (speaking. } \\
\text { listening, reading, writing) }\end{array}$ & & & & & & \\
\hline
\end{tabular}




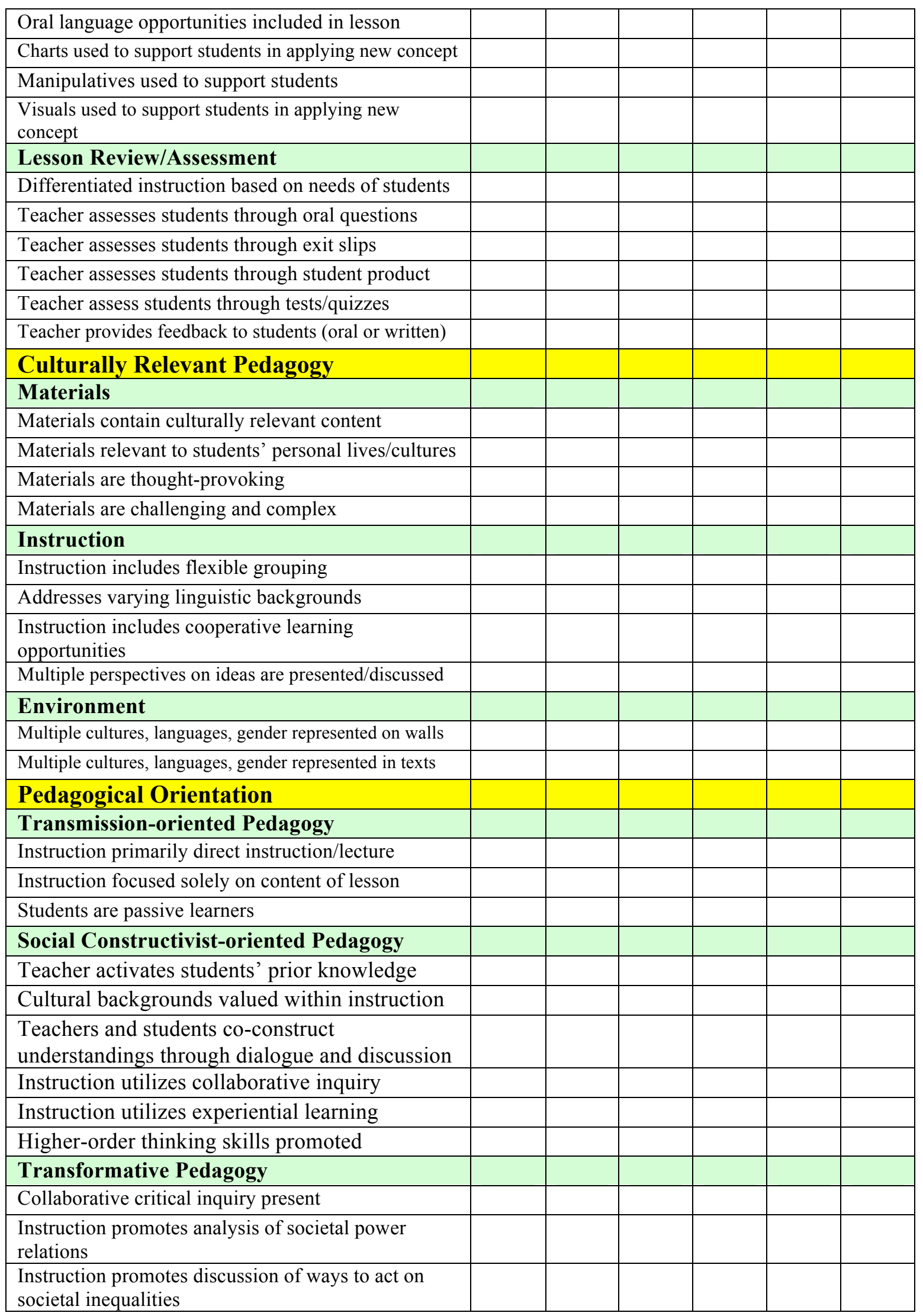


Appendix M

\section{Completed Classroom Observation Indicator Matrix}

Observation \#1

\begin{tabular}{|c|c|c|c|c|c|c|}
\hline & $\begin{array}{c}\text { NAC } \\
\# 1\end{array}$ & $\begin{array}{c}\text { NAC } \\
\# 2\end{array}$ & $\begin{array}{c}\text { NAC } \\
\# 3\end{array}$ & $\begin{array}{l}\text { ESL } \\
\# 1^{\text {a }}\end{array}$ & $\begin{array}{c}\text { ESL } \\
\# 2\end{array}$ & $\begin{array}{c}\text { ESL } \\
\# 3\end{array}$ \\
\hline \multicolumn{7}{|l|}{ Effective EL Instruction } \\
\hline \multicolumn{7}{|l|}{ Curriculum } \\
\hline Appropriate for language proficiency & $\mathrm{x}$ & $\mathrm{x}$ & $\mathrm{x}$ & $\mathrm{x}$ & $\mathrm{x}$ & $\mathrm{x}$ \\
\hline Materials used to supplement language acquisition & $\mathrm{x}$ & $\mathrm{x}$ & $\mathrm{x}$ & $\mathrm{x}$ & $\mathrm{x}$ & $\mathrm{x}$ \\
\hline \multicolumn{7}{|l|}{ Instruction } \\
\hline Linked to student's experiences/prior knowledge & $\mathrm{x}$ & $\mathrm{x}$ & $\mathrm{x}$ & $\mathrm{x}$ & $\mathrm{x}$ & $\mathrm{x}$ \\
\hline Connections made between current and prior lessons & $\mathrm{x}$ & $\mathrm{x}$ & $\mathrm{x}$ & $\mathrm{x}$ & $\mathrm{x}$ & $\mathrm{x}$ \\
\hline Peer interaction used to activate schema & $\mathrm{x}$ & $\mathrm{x}$ & $\mathrm{x}$ & & & $\mathrm{x}$ \\
\hline \multicolumn{7}{|l|}{ Key Vocabulary } \\
\hline Academic/content vocabulary introduced in lesson & $\mathrm{x}$ & $\mathrm{x}$ & $\mathrm{x}$ & $\mathrm{x}$ & $\mathrm{x}$ & $\mathrm{X}$ \\
\hline Vocabulary taught/reviewed literally & $\mathrm{x}$ & $\mathrm{x}$ & $\mathrm{x}$ & $\mathrm{x}$ & $\mathrm{x}$ & $\mathrm{x}$ \\
\hline Vocabulary taught/reviewed contextually & $\mathrm{x}$ & & $\mathrm{x}$ & & & $\mathrm{x}$ \\
\hline Strategies used to support students' understanding & $\mathrm{x}$ & $\mathrm{x}$ & $\mathrm{x}$ & $\mathrm{X}$ & $\mathrm{X}$ & $\mathrm{x}$ \\
\hline \multicolumn{7}{|l|}{ Comprehensible Input } \\
\hline Suitable pacing for language proficiency level & $\mathrm{x}$ & $\mathrm{x}$ & $\mathrm{x}$ & $\mathrm{x}$ & & $\mathrm{x}$ \\
\hline Suitable speech for language proficiency level & $\mathrm{x}$ & $\mathrm{x}$ & $\mathrm{x}$ & $\mathrm{x}$ & & $\mathrm{x}$ \\
\hline Clear expectations for students & $\mathrm{x}$ & $\mathrm{x}$ & $\mathrm{x}$ & & $\mathrm{x}$ & $\mathrm{x}$ \\
\hline Checks for understanding & $\mathrm{x}$ & $\mathrm{x}$ & $\mathrm{x}$ & $\mathrm{x}$ & $\mathrm{x}$ & $\mathrm{x}$ \\
\hline Visuals used during lesson & $\mathrm{x}$ & $\mathrm{x}$ & $\mathrm{x}$ & $\mathrm{x}$ & $\mathrm{x}$ & $\mathrm{x}$ \\
\hline Realia used during lesson & & & $\mathrm{x}$ & & & $\mathrm{x}$ \\
\hline Modeling used during lesson & $\mathrm{x}$ & $\mathrm{x}$ & $\mathrm{x}$ & $\mathrm{x}$ & $\mathrm{x}$ & $\mathrm{x}$ \\
\hline Gestures/TPR used during lesson & & $\mathrm{x}$ & $\mathrm{x}$ & $\mathrm{x}$ & $\mathrm{x}$ & $\mathrm{x}$ \\
\hline Primary language support used during lesson & $\mathrm{x}$ & & $\mathrm{x}$ & & & $\mathrm{x}$ \\
\hline Interactive demonstrations used during lesson & $\mathrm{x}$ & & & & & \\
\hline Partner/group activities present during lesson & $\mathrm{x}$ & & $\mathrm{x}$ & & $\mathrm{x}$ & $\mathrm{x}$ \\
\hline \multicolumn{7}{|l|}{ Strategies } \\
\hline Think-alouds used during lesson & $\mathrm{x}$ & $\mathrm{x}$ & $\mathrm{x}$ & $\mathrm{x}$ & $\mathrm{x}$ & $\mathrm{x}$ \\
\hline Word banks are present in lesson & $\mathrm{x}$ & $\mathrm{x}$ & $\mathrm{x}$ & $\mathrm{x}$ & $\mathrm{x}$ & $\mathrm{x}$ \\
\hline Graphic organizer used to support teaching & & & $\mathrm{x}$ & $\mathrm{x}$ & & $\mathrm{x}$ \\
\hline $\begin{array}{l}\text { A variety of question types used during lesson } \\
\text { (literal, analytical, interpretive) }\end{array}$ & $\mathrm{x}$ & & $\mathrm{x}$ & & & $\mathrm{x}$ \\
\hline Evidence of a gradual release of responsibility & $\mathrm{x}$ & $\mathrm{x}$ & $\mathrm{x}$ & & $\mathrm{x}$ & $\mathrm{x}$ \\
\hline \multicolumn{7}{|l|}{ Interactions } \\
\hline Frequent interactions between teachers and students & $\mathrm{x}$ & $\mathrm{x}$ & $\mathrm{x}$ & $\mathrm{x}$ & $\mathrm{x}$ & $\mathrm{x}$ \\
\hline Frequent interactions between students & $\mathrm{x}$ & $\mathrm{x}$ & $\mathrm{x}$ & & $\mathrm{x}$ & $\mathrm{x}$ \\
\hline Students provided sufficient "wait time" when responding & $\mathrm{x}$ & $\mathrm{x}$ & $\mathrm{x}$ & $\mathrm{x}$ & $\mathrm{x}$ & $\mathrm{x}$ \\
\hline \multicolumn{7}{|l|}{ Practice/Application } \\
\hline Students given opportunities to practice new concept & $\mathrm{x}$ & $\mathrm{X}$ & $\mathrm{x}$ & $\mathrm{x}$ & & $\mathrm{x}$ \\
\hline Activities integrate all language domains & $\mathrm{X}$ & & $\mathrm{x}$ & & & $\mathrm{x}$ \\
\hline Oral language opportunities included in lesson & $\mathrm{x}$ & $\mathrm{X}$ & $\mathrm{X}$ & & $\mathrm{X}$ & $\mathrm{X}$ \\
\hline
\end{tabular}




\begin{tabular}{|c|c|c|c|c|c|c|}
\hline $\begin{array}{l}\text { Charts used to support students in applying new } \\
\text { concept }\end{array}$ & $\mathrm{X}$ & $\mathrm{X}$ & $\mathrm{X}$ & & $\mathrm{X}$ & \\
\hline \multicolumn{7}{|l|}{ Manipulatives used to support students } \\
\hline Visuals used to support students in applying new concept & $\mathrm{X}$ & $\mathrm{X}$ & $\mathrm{X}$ & $\mathrm{X}$ & $\mathrm{X}$ & $\mathrm{X}$ \\
\hline \multicolumn{7}{|l|}{ Lesson Review/Assessment } \\
\hline Differentiated instruction based on needs of students & & & & & & $\mathrm{X}$ \\
\hline Teacher assesses students through oral questions & $\mathrm{X}$ & $\mathrm{X}$ & $\mathrm{X}$ & $\mathrm{X}$ & $\mathrm{X}$ & $\mathrm{X}$ \\
\hline \multicolumn{7}{|l|}{ Teacher assesses students through exit slips } \\
\hline Teacher assesses students through student product & $\mathrm{X}$ & $\mathrm{X}$ & $\mathrm{X}$ & $\mathrm{X}$ & $\mathrm{X}$ & $\mathrm{X}$ \\
\hline Teacher assess students through tests/quizzes & $\mathrm{X}$ & & & & & \\
\hline $\begin{array}{l}\text { Teacher provides feedback to students (oral or } \\
\text { written) }\end{array}$ & $\mathrm{X}$ & & $\mathrm{X}$ & $\mathrm{X}$ & $\mathrm{X}$ & $\mathrm{X}$ \\
\hline \multicolumn{7}{|l|}{ Culturally Relevant Pedagogy } \\
\hline \multicolumn{7}{|l|}{ Materials } \\
\hline Materials contain culturally relevant content & $\mathrm{x}$ & $\mathrm{X}$ & $\mathrm{X}$ & & $\mathrm{x}$ & \\
\hline Materials relevant to students' personal lives/cultures & $\mathrm{X}$ & $\mathrm{X}$ & $\mathrm{X}$ & & $\mathrm{X}$ & \\
\hline Materials are thought-provoking & $\mathrm{x}$ & $\mathrm{X}$ & $\mathrm{X}$ & & & \\
\hline Materials are challenging and complex & $\mathrm{X}$ & $\mathrm{X}$ & $\mathrm{X}$ & & & $\mathrm{X}$ \\
\hline \multicolumn{7}{|l|}{ Instruction } \\
\hline Instruction includes flexible grouping & & & & & & $\mathrm{X}$ \\
\hline Addresses varying cultural/linguistic backgrounds & $\mathrm{X}$ & $\mathrm{X}$ & $\mathrm{X}$ & & $\mathrm{X}$ & $\mathrm{X}$ \\
\hline Instruction includes cooperative learning opportunities & $\mathrm{X}$ & $\mathrm{X}$ & $\mathrm{X}$ & & $\mathrm{X}$ & $\mathrm{X}$ \\
\hline Multiple perspectives on ideas are presented/discussed & $\mathrm{X}$ & $\mathrm{X}$ & & & & \\
\hline \multicolumn{7}{|l|}{ Environment } \\
\hline Multiple cultures, languages, gender represented on walls & & $\mathrm{X}$ & $\mathrm{X}$ & $\mathrm{X}$ & & \\
\hline Multiple cultures, languages, gender represented in texts & $\mathrm{X}$ & & $\mathrm{X}$ & $\mathrm{X}$ & & \\
\hline \multicolumn{7}{|l|}{ Pedagogical Orientation } \\
\hline \multicolumn{7}{|l|}{ Transmission-oriented Pedagogy } \\
\hline Instruction primarily direct instruction/lecture & & $\mathrm{X}$ & & $\mathrm{x}$ & & \\
\hline Instruction focused solely on content of lesson & & $\mathrm{X}$ & & $\mathrm{X}$ & $\mathrm{X}$ & $\mathrm{X}$ \\
\hline Students are passive learners & & & & $\mathrm{X}$ & & \\
\hline \multicolumn{7}{|l|}{ Social Constructivist-oriented Pedagogy } \\
\hline Teacher activates students' prior knowledge & $\mathrm{X}$ & $\mathrm{X}$ & $\mathrm{X}$ & $\mathrm{X}$ & $\mathrm{X}$ & $\mathrm{X}$ \\
\hline Cultural backgrounds valued within instruction & $\mathrm{X}$ & $\mathrm{X}$ & $\mathrm{X}$ & & $\mathrm{X}$ & \\
\hline $\begin{array}{l}\text { Teachers and students co-construct understandings } \\
\text { through dialogue and discussion }\end{array}$ & $\mathrm{X}$ & $\mathrm{X}$ & $\mathrm{X}$ & & $\mathrm{X}$ & $\mathrm{X}$ \\
\hline Instruction utilizes collaborative inquiry & $\mathrm{X}$ & & $\mathrm{X}$ & & & $\mathrm{X}$ \\
\hline Higher-order thinking skills promoted & $\mathrm{X}$ & & $\mathrm{X}$ & & & $\mathrm{X}$ \\
\hline \multicolumn{7}{|l|}{ Transformative Pedagogy } \\
\hline \multicolumn{7}{|l|}{ Collaborative critical inquiry present } \\
\hline \multicolumn{7}{|l|}{$\begin{array}{l}\text { Instruction promotes analysis of societal power } \\
\text { relations }\end{array}$} \\
\hline $\begin{array}{l}\text { Instruction promotes discussion of ways to act on } \\
\text { societal inequalities }\end{array}$ & & & & & & \\
\hline
\end{tabular}

Note. ${ }^{\text {a}}$ ESL $1 / 2$ students taught by teacher's aide 


\section{Completed Classroom Observation Indicator Matrix Observation \#2}

\begin{tabular}{|c|c|c|c|c|c|c|}
\hline & $\begin{array}{c}\text { NAC } \\
\# 1\end{array}$ & $\begin{array}{c}\text { NAC } \\
\# 2\end{array}$ & $\begin{array}{c}\text { NAC } \\
\# 3\end{array}$ & $\begin{array}{c}\text { ESL } \\
\# 1^{\text {a }} \\
\end{array}$ & $\begin{array}{c}\text { ESL } \\
\# 2\end{array}$ & $\begin{array}{c}\text { ESL } \\
\# 3\end{array}$ \\
\hline \multicolumn{7}{|l|}{ Effective EL Instruction } \\
\hline \multicolumn{7}{|l|}{ Curriculum } \\
\hline Appropriate for language proficiency & $\mathrm{x}$ & $\mathrm{x}$ & $\mathrm{x}$ & $\mathrm{x}$ & $\mathrm{x}$ & $\mathrm{x}$ \\
\hline Materials used to supplement language acquisition & $\mathrm{x}$ & $\mathrm{x}$ & $\mathrm{x}$ & $\mathrm{x}$ & $\mathrm{x}$ & $\mathrm{x}$ \\
\hline \multicolumn{7}{|l|}{ Instruction } \\
\hline Linked to student's experiences/prior knowledge & $\mathrm{x}$ & $\mathrm{x}$ & $\mathrm{x}$ & $\mathrm{x}$ & $\mathrm{x}$ & $\mathrm{x}$ \\
\hline Connections made between current and prior lessons & $\mathrm{x}$ & $\mathrm{x}$ & $\mathrm{x}$ & $\mathrm{x}$ & $\mathrm{x}$ & $\mathrm{x}$ \\
\hline Peer interaction used to activate schema & $\mathrm{x}$ & $\mathrm{x}$ & $\mathrm{x}$ & & & $\mathrm{x}$ \\
\hline \multicolumn{7}{|l|}{ Key Vocabulary } \\
\hline Academic/content vocabulary introduced in lesson & & $\mathrm{x}$ & $\mathrm{x}$ & $\mathrm{x}$ & $\mathrm{x}$ & $\mathrm{x}$ \\
\hline Vocabulary taught/reviewed literally & $\mathrm{x}$ & $\mathrm{x}$ & $\mathrm{x}$ & $\mathrm{x}$ & & \\
\hline Vocabulary taught/reviewed contextually & & & $\mathrm{x}$ & & $\mathrm{x}$ & $\mathrm{x}$ \\
\hline Strategies used to support students' understanding & $\mathrm{x}$ & $\mathrm{x}$ & $\mathrm{x}$ & $\mathrm{x}$ & $\mathrm{x}$ & $\mathrm{X}$ \\
\hline \multicolumn{7}{|l|}{ Comprehensible Input } \\
\hline Suitable pacing for language proficiency level & $\mathrm{x}$ & $\mathrm{x}$ & $\mathrm{x}$ & $\mathrm{x}$ & & $\mathrm{x}$ \\
\hline Suitable speech for language proficiency level & $\mathrm{x}$ & $\mathrm{x}$ & $\mathrm{x}$ & $\mathrm{x}$ & & $\mathrm{x}$ \\
\hline Clear expectations for students & $\mathrm{x}$ & $\mathrm{x}$ & $\mathrm{x}$ & $\mathrm{x}$ & $\mathrm{x}$ & $\mathrm{x}$ \\
\hline Checks for understanding & $\mathrm{x}$ & & $\mathrm{x}$ & $\mathrm{x}$ & $\mathrm{x}$ & $\mathrm{x}$ \\
\hline Visuals used during lesson & $\mathrm{x}$ & & $\mathrm{x}$ & $\mathrm{x}$ & $\mathrm{x}$ & $\mathrm{x}$ \\
\hline Realia used during lesson & & & & & $\mathrm{x}$ & \\
\hline Modeling used during lesson & $\mathrm{x}$ & $\mathrm{x}$ & $\mathrm{x}$ & & $\mathrm{x}$ & $\mathrm{x}$ \\
\hline Gestures/TPR used during lesson & & $\mathrm{x}$ & $\mathrm{x}$ & $\mathrm{x}$ & & $\mathrm{x}$ \\
\hline Primary language support used during lesson & $\mathrm{x}$ & & & $\mathrm{X}$ & & $\mathrm{x}$ \\
\hline Interactive demonstrations used during lesson & $\mathrm{x}$ & $\mathrm{x}$ & & & & \\
\hline Partner/group activities present during lesson & $\mathrm{x}$ & & $\mathrm{x}$ & & $\mathrm{x}$ & $\mathrm{x}$ \\
\hline \multicolumn{7}{|l|}{ Strategies } \\
\hline Think-alouds used during lesson & $\mathrm{x}$ & $\mathrm{x}$ & $\mathrm{x}$ & & & $\mathrm{x}$ \\
\hline Word banks are present in lesson & $\mathrm{x}$ & & $\mathrm{x}$ & $\mathrm{x}$ & $\mathrm{x}$ & \\
\hline Graphic organizer used to support teaching & & & $\mathrm{x}$ & & $\mathrm{x}$ & $\mathrm{x}$ \\
\hline $\begin{array}{l}\text { A variety of question types used during lesson } \\
\text { (literal, analytical, interpretive) }\end{array}$ & & $\mathrm{x}$ & $\mathrm{x}$ & & & $\mathrm{x}$ \\
\hline Evidence of a gradual release of responsibility & $\mathrm{x}$ & & $\mathrm{x}$ & & $\mathrm{x}$ & $\mathrm{x}$ \\
\hline \multicolumn{7}{|l|}{ Interactions } \\
\hline Frequent interactions between teachers and students & $\mathrm{x}$ & $\mathrm{x}$ & $\mathrm{x}$ & $\mathrm{x}$ & $\mathrm{X}$ & 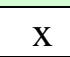 \\
\hline Frequent interactions between students & $\mathrm{x}$ & $\mathrm{x}$ & $\mathrm{x}$ & $\mathrm{x}$ & $\mathrm{x}$ & $\mathrm{X}$ \\
\hline Students provided sufficient "wait time" when responding & $\mathrm{x}$ & $\mathrm{x}$ & $\mathrm{x}$ & $\mathrm{x}$ & & $\mathrm{x}$ \\
\hline \multicolumn{7}{|l|}{ Practice/Application } \\
\hline Students given opportunities to practice new concept & $\mathrm{x}$ & $\mathrm{x}$ & $\mathrm{x}$ & & $\mathrm{x}$ & $\mathrm{X}$ \\
\hline Activities integrate all language domains & $\mathrm{x}$ & & $\mathrm{x}$ & & $\mathrm{x}$ & $\mathrm{x}$ \\
\hline Oral language opportunities included in lesson & $\mathrm{x}$ & $\mathrm{x}$ & $\mathrm{x}$ & & $\mathrm{x}$ & $\mathrm{x}$ \\
\hline Charts used to support students in applying new concept & $\mathrm{x}$ & $\mathrm{x}$ & $\mathrm{x}$ & & & \\
\hline
\end{tabular}




\begin{tabular}{|c|c|c|c|c|c|c|}
\hline \multicolumn{7}{|l|}{ Manipulatives used to support students } \\
\hline Visuals used to support students in applying new concept & $\mathrm{x}$ & & $\mathrm{x}$ & $\mathrm{x}$ & $\mathrm{x}$ & $\mathrm{x}$ \\
\hline \multicolumn{7}{|l|}{ Lesson Review/Assessment } \\
\hline Differentiated instruction based on needs of students & & & & $\mathrm{x}$ & & $\mathrm{x}$ \\
\hline Teacher assesses students through oral questions & $\mathrm{x}$ & $\mathrm{x}$ & $\mathrm{x}$ & $\mathrm{X}$ & $\mathrm{X}$ & $\mathrm{x}$ \\
\hline \multicolumn{7}{|l|}{ Teacher assesses students through exit slips } \\
\hline Teacher assesses students through student product & $\mathrm{x}$ & $\mathrm{x}$ & $\mathrm{x}$ & & $\mathrm{X}$ & $\mathrm{x}$ \\
\hline Teacher assess students through tests/quizzes & $\mathrm{x}$ & & & $\mathrm{X}$ & & \\
\hline Teacher provides feedback to students (oral or written) & $\mathrm{x}$ & $\mathrm{x}$ & $\mathrm{x}$ & $\mathrm{x}$ & $\mathrm{x}$ & $\mathrm{x}$ \\
\hline \multicolumn{7}{|l|}{ Culturally Relevant Pedagogy } \\
\hline \multicolumn{7}{|l|}{ Materials } \\
\hline Materials contain culturally relevant content & $\mathrm{x}$ & $\mathrm{x}$ & $\mathrm{x}$ & & & $\mathrm{x}$ \\
\hline Materials relevant to students' personal lives/cultures & $\mathrm{x}$ & $\mathrm{x}$ & $\mathrm{x}$ & & & $\mathrm{x}$ \\
\hline Materials are thought-provoking & & $\mathrm{x}$ & $\mathrm{x}$ & & & $\mathrm{x}$ \\
\hline Materials are challenging and complex & $\mathrm{x}$ & $\mathrm{x}$ & $\mathrm{x}$ & & $\mathrm{X}$ & $\mathrm{x}$ \\
\hline \multicolumn{7}{|l|}{ Instruction } \\
\hline Instruction includes flexible grouping & & & $\mathrm{x}$ & $\mathrm{x}$ & & $\mathrm{x}$ \\
\hline Addresses varying cultural/linguistic backgrounds & & $\mathrm{x}$ & & $\mathrm{x}$ & & $\mathrm{x}$ \\
\hline Instruction includes cooperative learning opportunities & $\mathrm{x}$ & $\mathrm{x}$ & $\mathrm{x}$ & $\mathrm{x}$ & $\mathrm{X}$ & $\mathrm{x}$ \\
\hline Multiple perspectives on ideas are presented/discussed & & $\mathrm{x}$ & $\mathrm{x}$ & & & $\mathrm{x}$ \\
\hline \multicolumn{7}{|l|}{ Environment } \\
\hline Multiple cultures, languages, gender represented on walls & & $\mathrm{x}$ & $\mathrm{x}$ & $\mathrm{x}$ & & \\
\hline Multiple cultures, languages, gender represented in texts & $\mathrm{x}$ & & $\mathrm{x}$ & $\mathrm{X}$ & & $\mathrm{x}$ \\
\hline \multicolumn{7}{|l|}{ Pedagogical Orientation } \\
\hline \multicolumn{7}{|l|}{ Transmission-oriented Pedagogy } \\
\hline Instruction primarily direct instruction/lecture & & $\mathrm{x}$ & & $\mathrm{x}$ & & \\
\hline Instruction focused solely on content of lesson & & & & $\mathrm{x}$ & & \\
\hline Students are passive learners & & & & $\mathrm{x}$ & & \\
\hline \multicolumn{7}{|l|}{ Social Constructivist-oriented Pedagogy } \\
\hline Teacher activates students' prior knowledge & $\mathrm{x}$ & $\mathrm{x}$ & $\mathrm{x}$ & & $\mathrm{x}$ & $\mathrm{x}$ \\
\hline Cultural backgrounds valued within instruction & $\mathrm{x}$ & $\mathrm{x}$ & $\mathrm{x}$ & $\mathrm{x}$ & $\mathrm{x}$ & $\mathrm{x}$ \\
\hline $\begin{array}{l}\text { Teachers and students co-construct understandings } \\
\text { through dialogue and discussion }\end{array}$ & $\mathrm{x}$ & $\mathrm{x}$ & $\mathrm{x}$ & $\mathrm{x}$ & $\mathrm{x}$ & $\mathrm{x}$ \\
\hline Instruction utilizes collaborative inquiry & $\mathrm{x}$ & & $\mathrm{x}$ & & & $\mathrm{x}$ \\
\hline Higher-order thinking skills promoted & & & $\mathrm{x}$ & & & $\mathrm{x}$ \\
\hline \multicolumn{7}{|l|}{ Transformative Pedagogy } \\
\hline \multicolumn{7}{|l|}{ Collaborative critical inquiry present } \\
\hline \multicolumn{7}{|l|}{$\begin{array}{l}\text { Instruction promotes analysis of societal power } \\
\text { relations }\end{array}$} \\
\hline $\begin{array}{l}\text { Instruction promotes discussion of ways to act on } \\
\text { societal inequalities }\end{array}$ & & & & & & \\
\hline
\end{tabular}

Note. ${ }^{a}$ ESL $1 / 2$ students taught by teacher's aide 


\section{Completed Classroom Observation Indicator Matrix Observation \#3}

\begin{tabular}{|c|c|c|c|c|c|c|}
\hline & $\begin{array}{c}\text { NAC } \\
\# 1\end{array}$ & $\begin{array}{c}\text { NAC } \\
\# 2\end{array}$ & $\begin{array}{c}\text { NAC } \\
\# 3\end{array}$ & $\begin{array}{l}\text { ESL } \\
\# 1^{\text {a }}\end{array}$ & $\begin{array}{c}\text { ESL } \\
\# 2\end{array}$ & $\begin{array}{c}\text { ESL } \\
\# 3\end{array}$ \\
\hline \multicolumn{7}{|l|}{ Effective EL Instruction } \\
\hline \multicolumn{7}{|l|}{ Curriculum } \\
\hline Appropriate for language proficiency & $\mathrm{x}$ & $\mathrm{x}$ & $\mathrm{x}$ & $\mathrm{x}$ & $\mathrm{x}$ & $\mathrm{x}$ \\
\hline Materials used to supplement language acquisition & $\mathrm{x}$ & $\mathrm{x}$ & $\mathrm{x}$ & $\mathrm{x}$ & $\mathrm{x}$ & $\mathrm{x}$ \\
\hline \multicolumn{7}{|l|}{ Instruction } \\
\hline Linked to student's experiences/prior knowledge & $\mathrm{x}$ & $\mathrm{x}$ & $\mathrm{x}$ & & $\mathrm{x}$ & $\mathrm{x}$ \\
\hline Connections made between current and prior lessons & $\mathrm{x}$ & $\mathrm{x}$ & $\mathrm{x}$ & & $\mathrm{x}$ & $\mathrm{x}$ \\
\hline Peer interaction used to activate schema & $\mathrm{x}$ & $\mathrm{x}$ & $\mathrm{x}$ & & $\mathrm{x}$ & $\mathrm{x}$ \\
\hline \multicolumn{7}{|l|}{ Key Vocabulary } \\
\hline Academic/content vocabulary introduced in lesson & $\mathrm{x}$ & $\mathrm{x}$ & $\mathrm{x}$ & $\mathrm{X}$ & & \\
\hline Vocabulary taught/reviewed literally & & $\mathrm{x}$ & $\mathrm{x}$ & $\mathrm{x}$ & $\mathrm{x}$ & \\
\hline Vocabulary taught/reviewed contextually & $\mathrm{x}$ & & $\mathrm{x}$ & & & $\mathrm{x}$ \\
\hline Strategies used to support students' understanding & $\mathrm{x}$ & $\mathrm{x}$ & $\mathrm{x}$ & $\mathrm{X}$ & $\mathrm{x}$ & $\mathrm{X}$ \\
\hline \multicolumn{7}{|l|}{ Comprehensible Input } \\
\hline Suitable pacing for language proficiency level & $\mathrm{x}$ & $\mathrm{x}$ & $\mathrm{x}$ & $\mathrm{x}$ & & $\mathrm{x}$ \\
\hline Suitable speech for language proficiency level & $\mathrm{x}$ & $\mathrm{x}$ & $\mathrm{x}$ & $\mathrm{x}$ & & $\mathrm{x}$ \\
\hline Clear expectations for students & $\mathrm{x}$ & $\mathrm{x}$ & $\mathrm{x}$ & $\mathrm{x}$ & $\mathrm{x}$ & $\mathrm{x}$ \\
\hline Checks for understanding & $\mathrm{x}$ & $\mathrm{x}$ & $\mathrm{x}$ & $\mathrm{x}$ & $\mathrm{x}$ & $\mathrm{x}$ \\
\hline Visuals used during lesson & & $\mathrm{x}$ & $\mathrm{x}$ & $\mathrm{x}$ & $\mathrm{x}$ & $\mathrm{x}$ \\
\hline \multicolumn{7}{|l|}{ Realia used during lesson } \\
\hline Modeling used during lesson & $\mathrm{x}$ & $\mathrm{x}$ & $\mathrm{x}$ & $\mathrm{x}$ & $\mathrm{x}$ & $\mathrm{x}$ \\
\hline Gestures/TPR used during lesson & $\mathrm{x}$ & & $\mathrm{x}$ & & & \\
\hline Primary language support used during lesson & $\mathrm{x}$ & & & & & $\mathrm{x}$ \\
\hline Interactive demonstrations used during lesson & $\mathrm{x}$ & $\mathrm{x}$ & & & & \\
\hline Partner/group activities present during lesson & $\mathrm{x}$ & & $\mathrm{x}$ & & $\mathrm{x}$ & $\mathrm{x}$ \\
\hline \multicolumn{7}{|l|}{ Strategies } \\
\hline Think-alouds used during lesson & $\mathrm{x}$ & $\mathrm{x}$ & $\mathrm{x}$ & $\mathrm{x}$ & $\mathrm{x}$ & $\mathrm{x}$ \\
\hline Word banks are present in lesson & $\mathrm{x}$ & $\mathrm{x}$ & $\mathrm{x}$ & $\mathrm{x}$ & $\mathrm{x}$ & \\
\hline Graphic organizer used to support teaching & & & $\mathrm{x}$ & & & $\mathrm{x}$ \\
\hline $\begin{array}{l}\text { A variety of question types used during lesson } \\
\text { (literal, analytical, interpretive) }\end{array}$ & & & $\mathrm{x}$ & & & $\mathrm{x}$ \\
\hline Evidence of a gradual release of responsibility & $\mathrm{x}$ & & $\mathrm{x}$ & & $\mathrm{x}$ & $\mathrm{x}$ \\
\hline \multicolumn{7}{|l|}{ Interactions } \\
\hline Frequent interactions between teachers and students & $\mathrm{x}$ & $\mathrm{x}$ & $\mathrm{x}$ & $\mathrm{x}$ & $\mathrm{x}$ & $\mathrm{x}$ \\
\hline Frequent interactions between students & $\mathrm{x}$ & & $\mathrm{x}$ & & $\mathrm{x}$ & $\mathrm{x}$ \\
\hline Students provided sufficient "wait time" when responding & $\mathrm{x}$ & $\mathrm{x}$ & $\mathrm{x}$ & $\mathrm{x}$ & & $\mathrm{x}$ \\
\hline \multicolumn{7}{|l|}{ Practice/Application } \\
\hline Students given opportunities to practice new concept & $\mathrm{x}$ & $\mathrm{x}$ & $\mathrm{x}$ & $\mathrm{x}$ & $\mathrm{x}$ & $\mathrm{x}$ \\
\hline Activities integrate all language domains & $\mathrm{x}$ & & $\mathrm{x}$ & & & $\mathrm{x}$ \\
\hline Oral language opportunities included in lesson & $\mathrm{x}$ & $\mathrm{x}$ & $\mathrm{x}$ & & $\mathrm{x}$ & $\mathrm{x}$ \\
\hline Charts used to support students in applying new concept & $\mathrm{x}$ & $\mathrm{x}$ & $\mathrm{x}$ & & $\mathrm{x}$ & \\
\hline
\end{tabular}




\begin{tabular}{|c|c|c|c|c|c|c|}
\hline \multicolumn{7}{|l|}{ Manipulatives used to support students } \\
\hline Visuals used to support students in applying new concept & $\mathrm{X}$ & & $\mathrm{X}$ & $\mathrm{X}$ & $\mathrm{X}$ & $\mathrm{X}$ \\
\hline \multicolumn{7}{|l|}{ Lesson Review/Assessment } \\
\hline Differentiated instruction based on needs of students & & & & & & $\mathrm{X}$ \\
\hline Teacher assesses students through oral questions & $\mathrm{X}$ & $\mathrm{X}$ & $\mathrm{X}$ & $\mathrm{X}$ & $\mathrm{X}$ & $\mathrm{X}$ \\
\hline \multicolumn{7}{|l|}{ Teacher assesses students through exit slips } \\
\hline Teacher assesses students through student product & $\mathrm{X}$ & $\mathrm{X}$ & $\mathrm{X}$ & $\mathrm{X}$ & $\mathrm{X}$ & $\mathrm{X}$ \\
\hline Teacher assess students through tests/quizzes & $\mathrm{X}$ & $\mathrm{X}$ & & $\mathrm{X}$ & & \\
\hline Teacher provides feedback to students (oral or written) & $\mathrm{X}$ & $\mathrm{X}$ & $\mathrm{X}$ & $\mathrm{X}$ & $\mathrm{X}$ & $\mathrm{X}$ \\
\hline \multicolumn{7}{|l|}{ Culturally Relevant Pedagogy } \\
\hline \multicolumn{7}{|l|}{ Materials } \\
\hline Materials contain culturally relevant content & $\mathrm{X}$ & $\mathrm{X}$ & $\mathrm{X}$ & & & \\
\hline Materials relevant to students' personal lives/cultures & $\mathrm{X}$ & $\mathrm{X}$ & $\mathrm{X}$ & & & \\
\hline Materials are thought-provoking & & $\mathrm{X}$ & $\mathrm{X}$ & & & $\mathrm{X}$ \\
\hline Materials are challenging and complex & $\mathrm{X}$ & $\mathrm{X}$ & $\mathrm{X}$ & & $\mathrm{X}$ & $\mathrm{X}$ \\
\hline \multicolumn{7}{|l|}{ Instruction } \\
\hline Instruction includes flexible grouping & & & & $\mathrm{X}$ & $\mathrm{X}$ & $\mathrm{X}$ \\
\hline Addresses varying cultural/linguistic backgrounds & & $\mathrm{X}$ & & & & \\
\hline Instruction includes cooperative learning opportunities & $\mathrm{X}$ & & $\mathrm{X}$ & & $\mathrm{X}$ & $\mathrm{X}$ \\
\hline Multiple perspectives on ideas are presented/discussed & & & & & $\mathrm{X}$ & $\mathrm{X}$ \\
\hline \multicolumn{7}{|l|}{ Environment } \\
\hline $\begin{array}{l}\text { Multiple cultures, languages, gender represented on } \\
\text { walls }\end{array}$ & $\mathrm{X}$ & $\mathrm{X}$ & $\mathrm{X}$ & $\mathrm{X}$ & & \\
\hline Multiple cultures, languages, gender represented in texts ${ }^{b}$ & $\mathrm{X}$ & $\mathrm{n} / \mathrm{a}$ & $\mathrm{X}$ & $\mathrm{X}$ & $\mathrm{n} / \mathrm{a}$ & $\mathrm{X}$ \\
\hline \multicolumn{7}{|l|}{ Pedagogical Orientation } \\
\hline \multicolumn{7}{|l|}{ Transmission-oriented Pedagogy } \\
\hline Instruction primarily direct instruction/lecture & & $\mathrm{X}$ & & $\mathrm{X}$ & & \\
\hline Instruction focused solely on content of lesson & & & & $\mathrm{X}$ & $\mathrm{X}$ & \\
\hline Students are passive learners & & & & $\mathrm{X}$ & & \\
\hline \multicolumn{7}{|l|}{ Social Constructivist-oriented Pedagogy } \\
\hline Teacher activates students' prior knowledge & $\mathrm{X}$ & $\mathrm{X}$ & $\mathrm{X}$ & & & $\mathrm{X}$ \\
\hline Cultural backgrounds valued within instruction & $\mathrm{X}$ & $\mathrm{X}$ & $\mathrm{X}$ & & & $\mathrm{X}$ \\
\hline $\begin{array}{l}\text { Teachers and students co-construct understandings } \\
\text { through dialogue and discussion }\end{array}$ & $\mathrm{X}$ & $\mathrm{X}$ & $\mathrm{X}$ & & $\mathrm{X}$ & $\mathrm{X}$ \\
\hline Instruction utilizes collaborative inquiry & & & $\mathrm{X}$ & & $\mathrm{X}$ & $\mathrm{X}$ \\
\hline Higher-order thinking skills promoted & & & $\mathrm{X}$ & & $\mathrm{X}$ & $\mathrm{X}$ \\
\hline \multicolumn{7}{|l|}{ Transformative Pedagogy } \\
\hline \multicolumn{7}{|l|}{ Collaborative critical inquiry present } \\
\hline \multicolumn{7}{|l|}{$\begin{array}{l}\text { Instruction promotes analysis of societal power } \\
\text { relations }\end{array}$} \\
\hline $\begin{array}{l}\text { Instruction promotes discussion of ways to act on } \\
\text { societal inequalities }\end{array}$ & & & & & & \\
\hline
\end{tabular}

Note. ${ }^{a}$ ESL $1 / 2$ students taught by teacher's aide, ${ }^{b}$ texts not used during lesson (not applicable) 


\section{Appendix N}

\section{NAC Teacher Interview Analysis}

\begin{tabular}{|c|c|c|c|c|}
\hline & $\begin{array}{l}\text { Teacher } \\
\text { NAC \#1 }\end{array}$ & $\begin{array}{l}\text { Teacher } \\
\text { NAC \#2 }\end{array}$ & $\begin{array}{l}\text { Teacher } \\
\text { NAC \#3 }\end{array}$ & Significant Theme \\
\hline Curricula & $\begin{array}{l}\text { * I do not know } \\
\text { how it was } \\
\text { chosen. It's the } \\
\text { Keys to Learning } \\
\text { which I think is } \\
\text { Longman and } \\
\text { Pearson and then } \\
\text { it's the WRITE } \\
\text { Institute } \\
\text { curriculum which } \\
\text { the district has } \\
\text { been using for } \\
\text { many years... last } \\
\text { year I found we } \\
\text { started reading it } \\
\text { to early they just } \\
\text { weren't grabbing } \\
\text { much of it. So, I } \\
\text { did use it more } \\
\text { into the second } \\
\text { semester... you } \\
\text { know what. It's } \\
\text { boring. } \\
\text { * The Keys is, } \\
\text { well that's, you } \\
\text { know, specifically } \\
\text { grammar and } \\
\text { vocabulary in } \\
\text { teaching } \\
\text { language. I wish } \\
\text { that we had more } \\
\text { like leveled } \\
\text { readers that were } \\
\text { not meant for } \\
\text { kindergarten, } \\
\text { because we've } \\
\text { got, you know, } \\
\text { some of that, but, } \\
\text { we don't have, it's } \\
\text { either way too } \\
\text { easy or way too } \\
\text { you know, the } \\
\text { descriptive }\end{array}$ & $\begin{array}{l}\text { * I know that they, } \\
\text { from what I had } \\
\text { heard from people } \\
\text { that were in the } \\
\text { program already, } \\
\text { that they didn't } \\
\text { really have } \\
\text { specific } \\
\text { curriculum for the } \\
\text { math and for the } \\
\text { science. I knew } \\
\text { that the language } \\
\text { portion and the } \\
\text { writing were very } \\
\text { specific, but that } \\
\text { other component } \\
\text { like I said, the } \\
\text { math, social } \\
\text { studies and } \\
\text { science, there're } \\
\text { not really a } \\
\text { specific } \\
\text { curriculum. Now } \\
\text { we've begun to } \\
\text { develop some } \\
\text { curriculum, pacing } \\
\text { maps cause the } \\
\text { need is there } \\
\text { obviously, and our } \\
\text { whole purpose is } \\
\text { to teach them the } \\
\text { language. } \\
\text { * OLA has a very } \\
\text { specific writing } \\
\text { curriculum that we } \\
\text { need to follow and } \\
\text { a lot of the literacy } \\
\text { and more } \\
\text { specifically the } \\
\text { dextbook that we has a lot of } \\
\text { students, it has } \\
\text { some grammar, it } \\
\text { has some word } \\
\text { study... so just } \\
\end{array}$ & 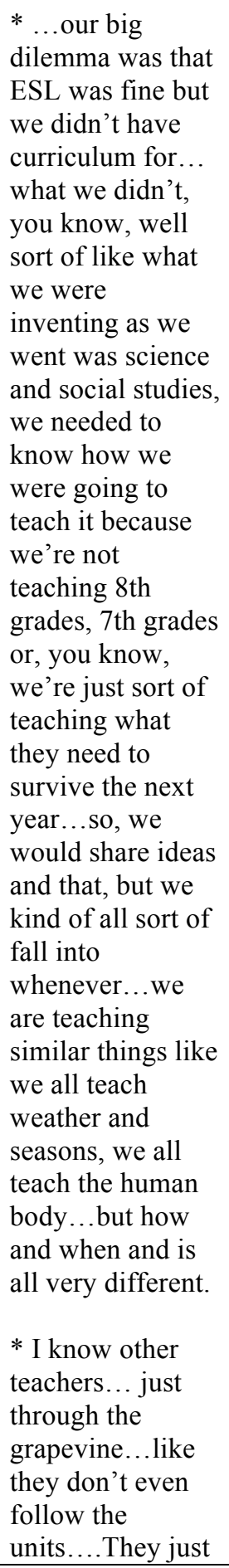 & $\begin{array}{l}\text { The teachers use } \\
\text { Keys to Learning, } \\
\text { Champion Reader, } \\
\text { and WRITE } \\
\text { curricula provided } \\
\text { by the district for } \\
\text { ESL portion of } \\
\text { class. } \\
\text { The Keys provides } \\
\text { specific vocabulary } \\
\text { and grammar } \\
\text { instruction, as well } \\
\text { as short dialogue } \\
\text { pieces. The text is } \\
\text { preferred, but is too } \\
\text { short. Teachers } \\
\text { supplement with } \\
\text { short stories and } \\
\text { songs. } \\
\text { The WRITE } \\
\text { Teachers need more } \\
\text { language- } \\
\text { appropriate leveled } \\
\text { high for the students } \\
\text { and is not sensitive } \\
\text { to the students, } \\
\text { needs and their } \\
\text { different cultures. } \\
\text { WRITE does offer a } \\
\text { plethora of units } \\
\text { and is highly } \\
\text { supportive. It can be } \\
\text { used in conjunction } \\
\text { with the explicit } \\
\text { language } \\
\text { instruction found in } \\
\text { the Systematic ELD } \\
\text { training. Bits and } \\
\text { prices of each } \\
\text { WRITE to tean be } \\
\text { anit as } \\
\text { anted }\end{array}$ \\
\hline
\end{tabular}




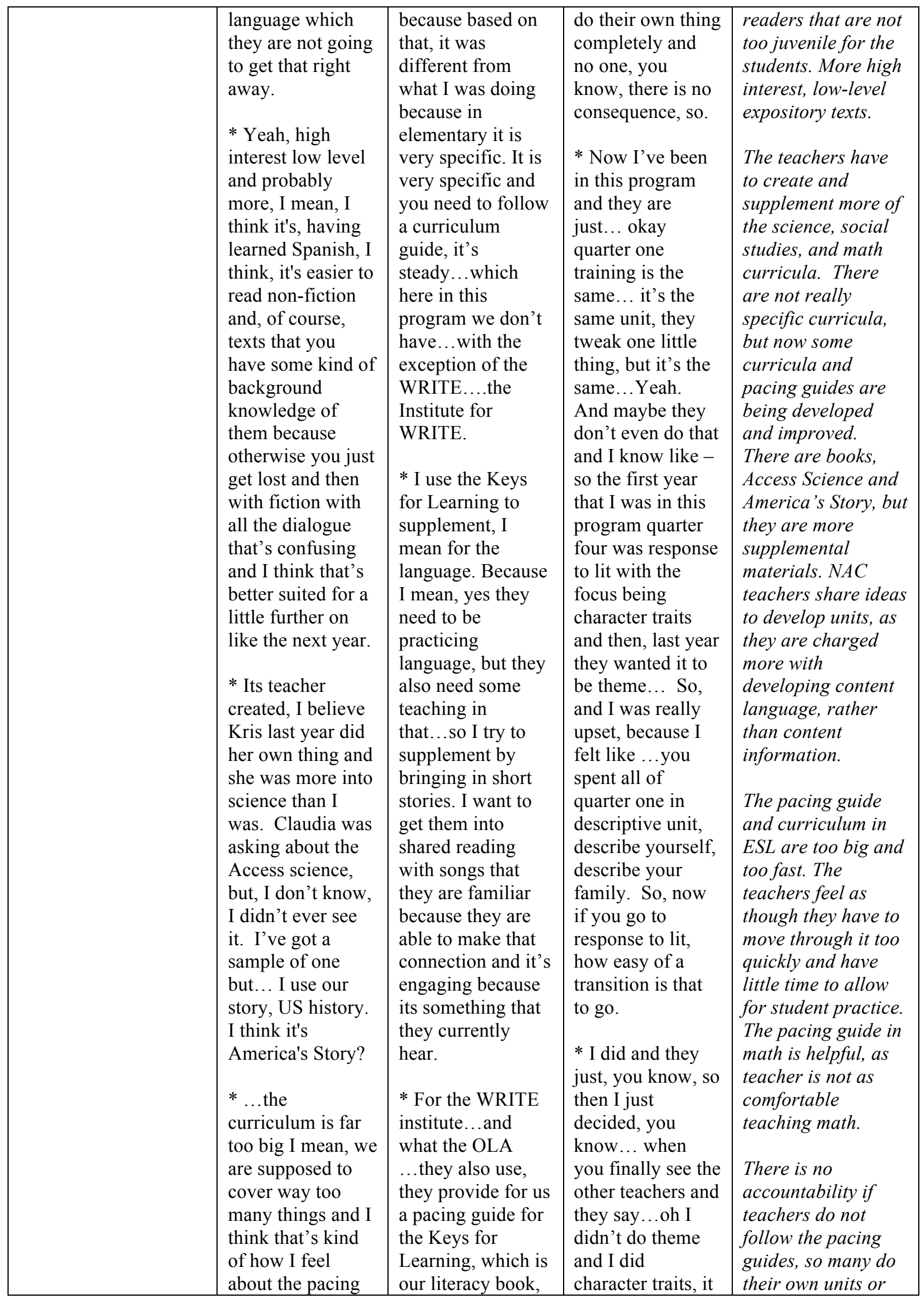




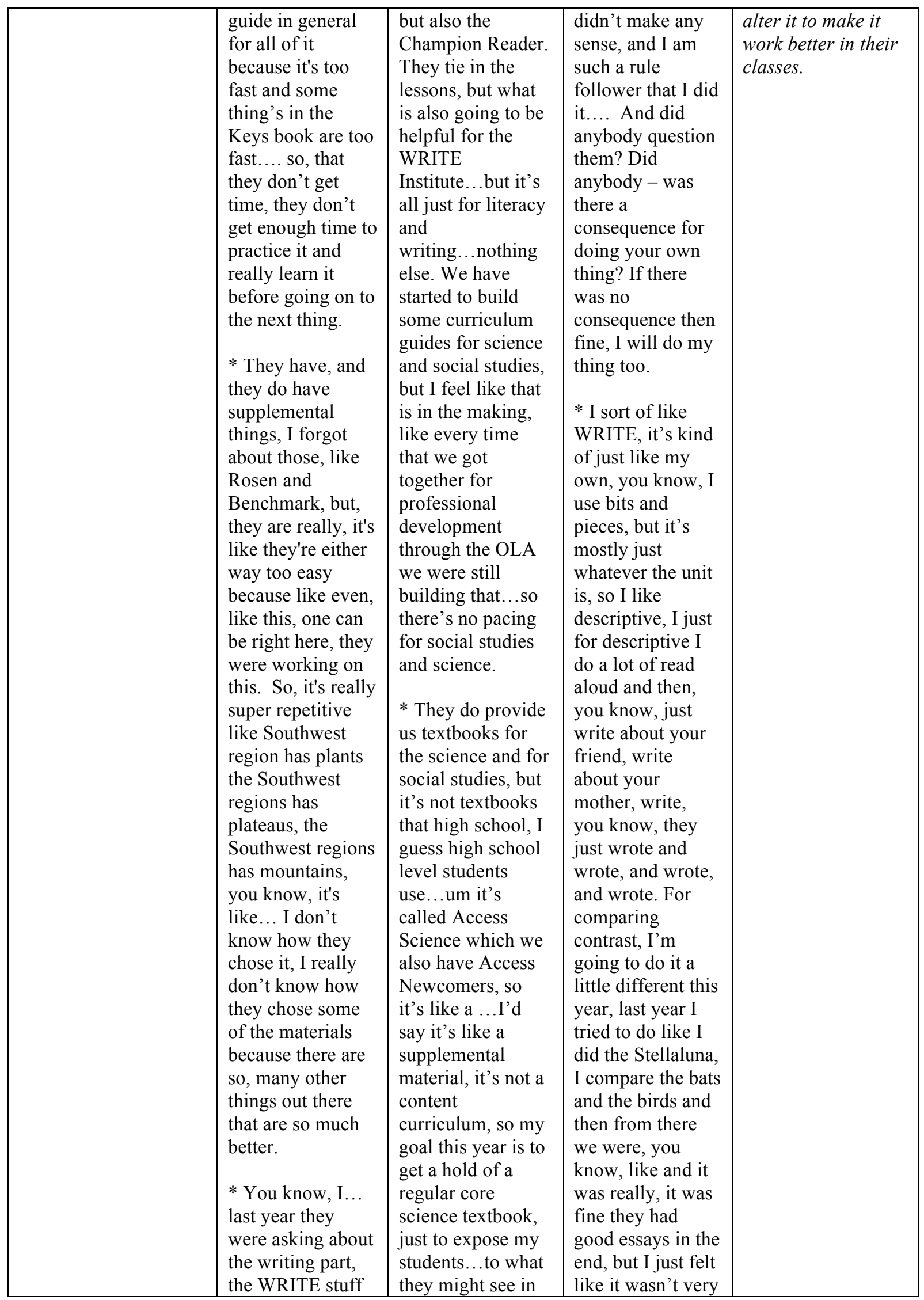




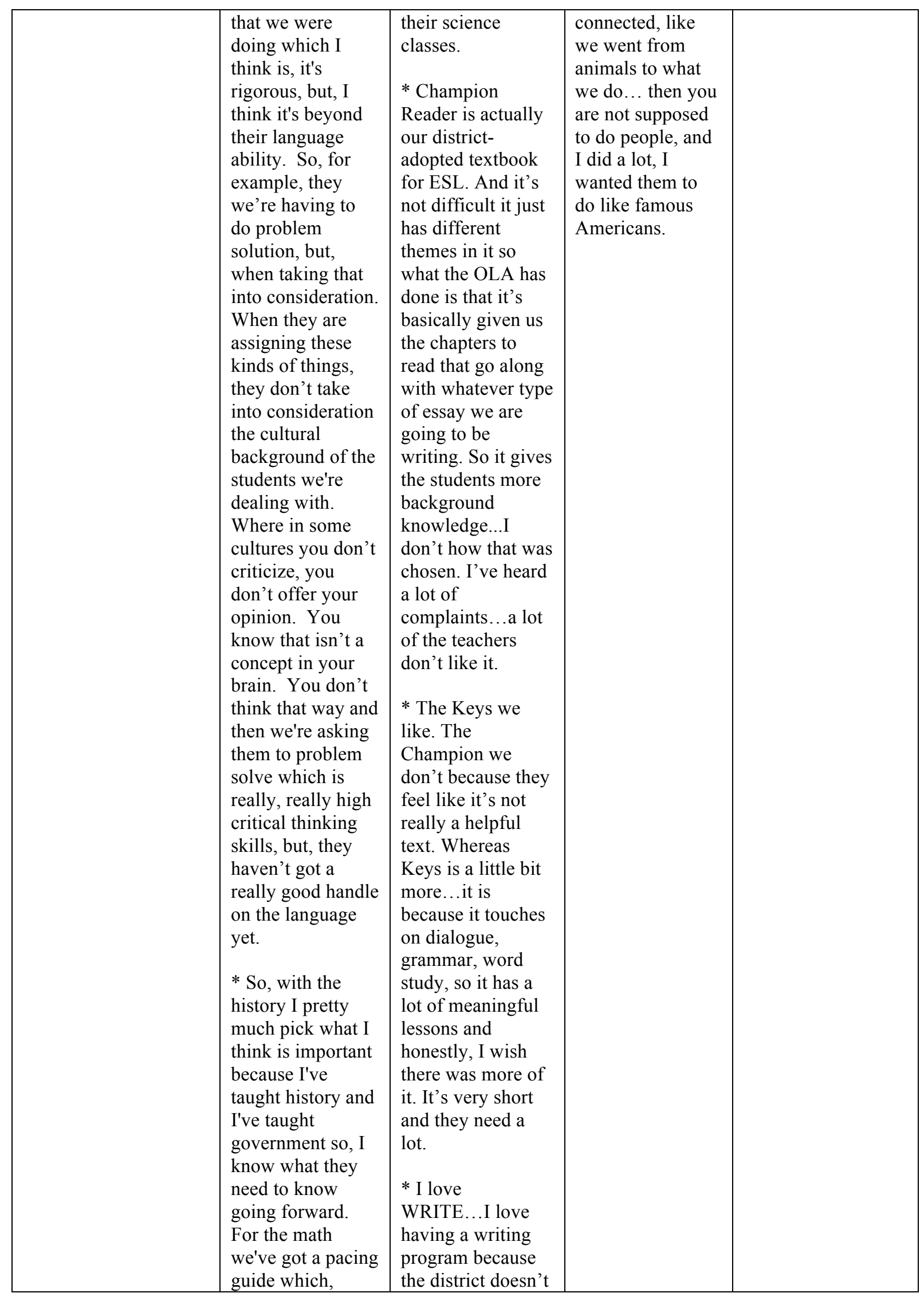




\begin{tabular}{|c|c|c|c|c|}
\hline & $\begin{array}{l}\text { because math is } \\
\text { not my forte I } \\
\text { follow it, but, then } \\
\text { some things even } \\
\text { I look at and go } \\
\text { up okay that's not } \\
\text { going to come and } \\
\text { I kind of mix it } \\
\text { around and then I } \\
\text { kind give them } \\
\text { quizzes everyday } \\
\text { just to see who } \\
\text { has gotten it and } \\
\text { there are a few } \\
\text { that really } \\
\text { struggle and they } \\
\text { don't get it and } \\
\text { they'll actually } \\
\text { come in to, I've } \\
\text { said come on in } \\
\text { during lunch if } \\
\text { you don't get it. } \\
\text { We'll spend } 10,15 \\
\text { minutes that's all } \\
\text { we'll spend so you } \\
\text { just can try to get } \\
\text { it. }\end{array}$ & $\begin{array}{l}\text { have a writing } \\
\text { program and third } \\
\text { grade it was very } \\
\text { difficult so when I } \\
\text { came here and } \\
\text { they had actual } \\
\text { lessons and } \\
\text { suggestions and } \\
\text { sentence frames, } \\
\text { all that scaffolding } \\
\text { done for the } \\
\text { writing...I just } \\
\text { thought } \\
\text { wow...how perfect } \\
\text { can it be... and } \\
\text { there are so many } \\
\text { different units! So } \\
\text { I love the } \\
\text { WRITE... I use it } \\
\text { along with the } \\
\text { systematic ELD } \\
\text { because it works } \\
\text { in correlation and I } \\
\text { think the students } \\
\text { really master it } \\
\text { because they have } \\
\text { so much support. } \\
\text { * Access is the } \\
\text { other curricula, it } \\
\text { is not district- } \\
\text { adopted } \\
\text { curricula...it is a } \\
\text { choice of our } \\
\text { program. } \\
\text { * I don't reinvent } \\
\text { anything, the I } \\
\text { only thing I do is } \\
\text { sometimes I make } \\
\text { up my own math } \\
\text { quiz using some of } \\
\text { the materials } \\
\text { provided in the } \\
\text { curriculum, just to } \\
\text { test where they're } \\
\text { at during the } \\
\text { chapter. }\end{array}$ & & \\
\hline $\begin{array}{l}\text { Language } \\
\text { Instruction }\end{array}$ & $\begin{array}{l}* \text { I guess, like I } \\
\text { see my students } \\
\text { needing more } \\
\text { authentic hands } \\
\text { on oral practice } \\
\text { and I think }\end{array}$ & $\begin{array}{l}\text { * I'm always } \\
\text { dealing with } \\
\text { second language } \\
\text { learners, so a lot of } \\
\text { the scaffolding } \\
\text { that I was doing }\end{array}$ & $\begin{array}{l}\text { * Cooperative } \\
\text { work, I usually } \\
\text { will always have } \\
\text { one really strong } \\
\text { person in the } \\
\text { group mixed with }\end{array}$ & $\begin{array}{l}\text { Students need } \\
\text { authentic, oral } \\
\text { language practice. } \\
\text { It's important for } \\
\text { teachers to create } \\
\text { opportunities. }\end{array}$ \\
\hline
\end{tabular}




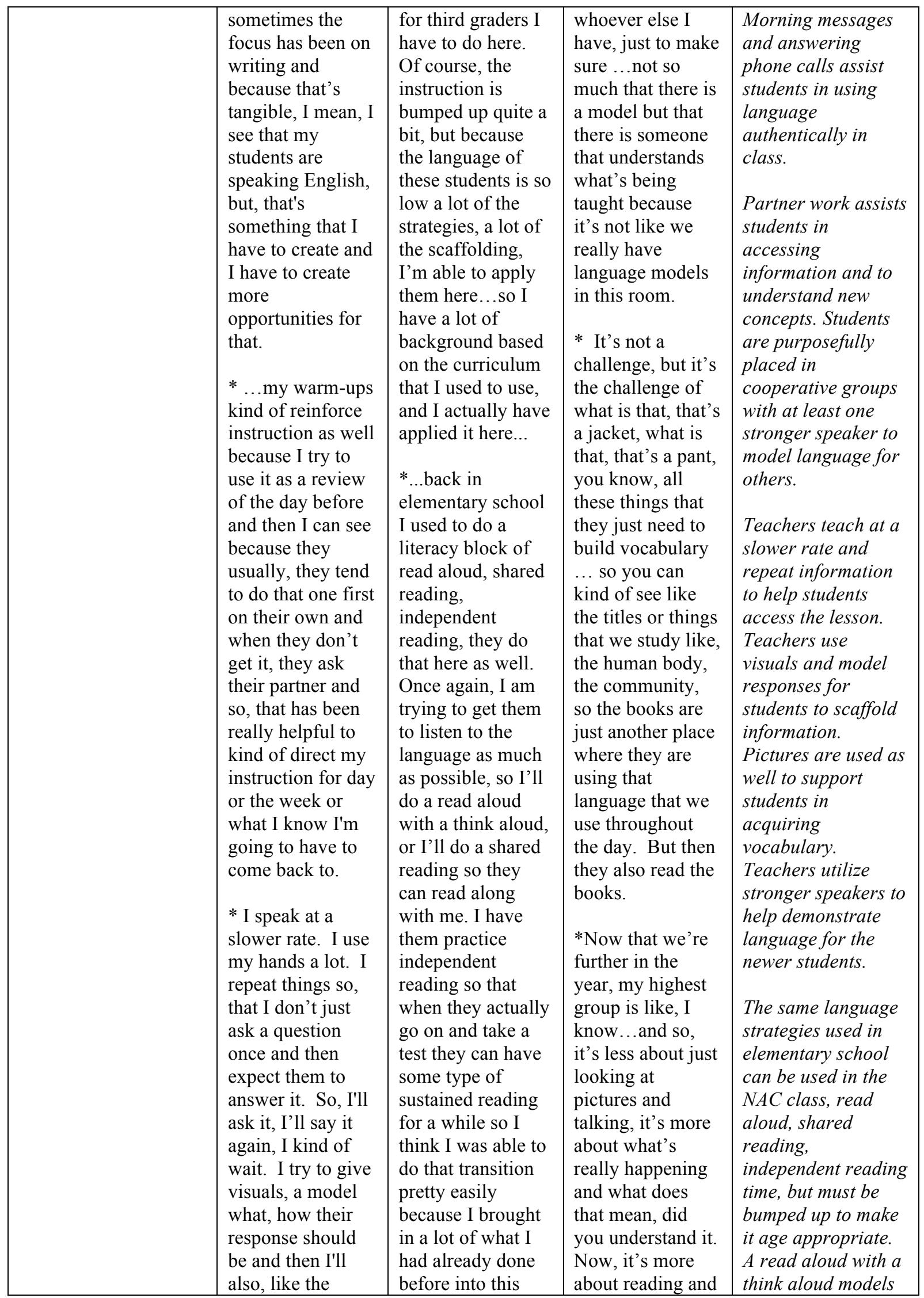




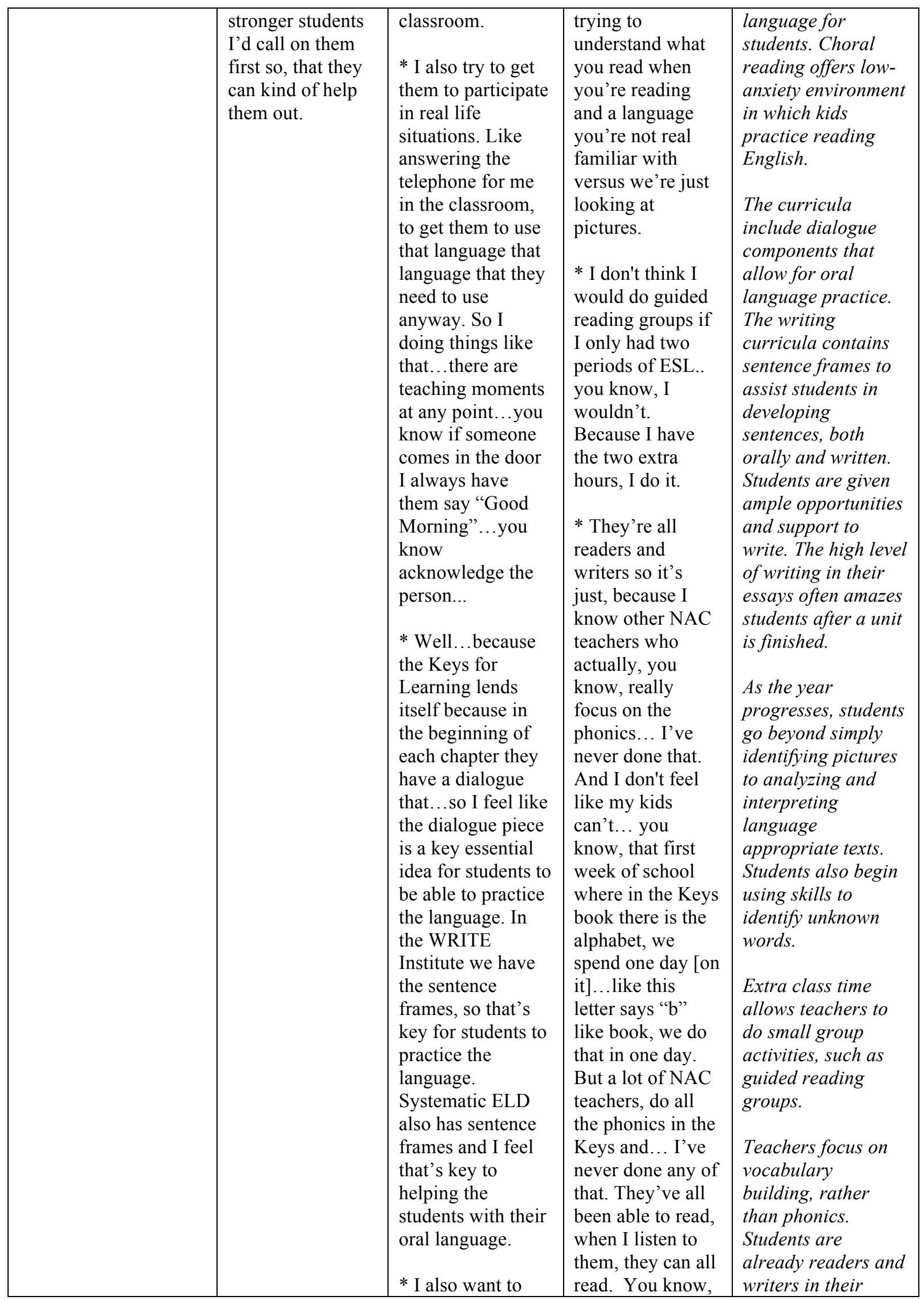




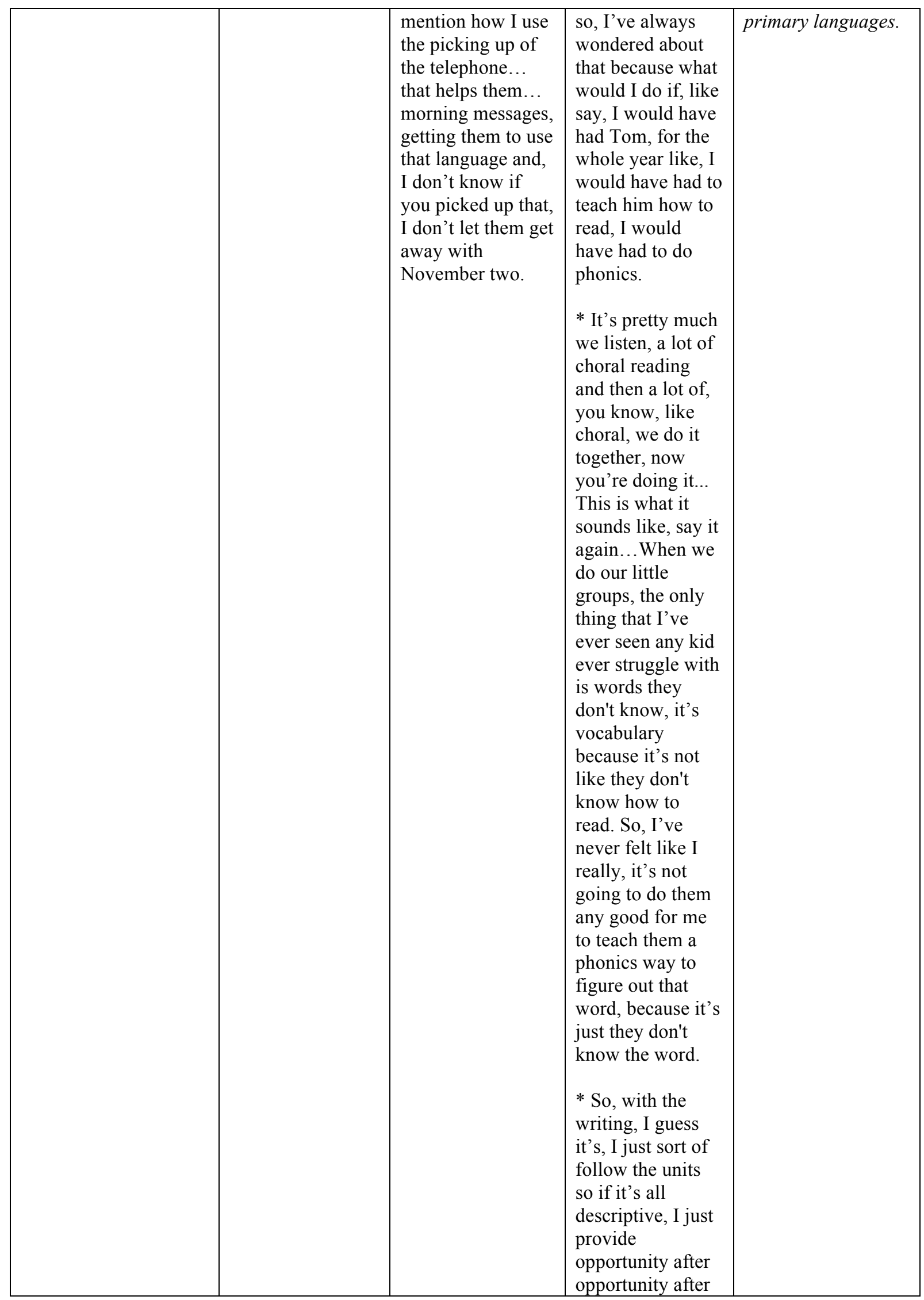




\begin{tabular}{|c|c|c|c|c|}
\hline & & & $\begin{array}{l}\text { opportunity to do } \\
\text { whatever it is, and } \\
\text { then I use } \\
\text { Systematic ELD } \\
\text { to support that, } \\
\text { and then every } \\
\text { time they finish a } \\
\text { unit, they write a } \\
\text { paragraph. So, } \\
\text { like when it was } \\
\text { the descriptive } \\
\text { unit, everything } \\
\text { that we did, every } \\
\text { week they're } \\
\text { writing about } \\
\text { themselves, a } \\
\text { friend their } \\
\text { family, over and } \\
\text { over so by the } \\
\text { time we got to the } \\
\text { end of that unit, } \\
\text { they all had just } \\
\text { beautiful essays } \\
\text { and, they didn't } \\
\text { even have to } \\
\text { think, they didn't } \\
\text { even realize how } \\
\text { easy that was. }\end{array}$ & \\
\hline Content Instruction & $\begin{array}{l}* . . \text { last year } \\
\text { which was my } \\
\text { first year I was } \\
\text { kind of in a panic } \\
\text { and feeling } \\
\text { overwhelmed } \\
\text { because I was } \\
\text { teaching math } \\
\text { which is not my } \\
\text { forte. But, now } \\
\text { that I look at more } \\
\text { like it's a } \\
\text { linguistic class } \\
\text { versus an } \\
\text { academic... Yeah, } \\
\text { it's not algebra. } \\
\text { It's just kind of } \\
\text { getting the } \\
\text { language and } \\
\text { bringing their } \\
\text { basic skills up. } \\
\text { So, I am okay } \\
\text { with that. } \\
\text { * Last year the } \\
\text { other teacher took }\end{array}$ & $\begin{array}{l}\text { * I feel like our } \\
\text { ultimate goal is to } \\
\text { get them using the } \\
\text { English language, } \\
\text { but also to prepare } \\
\text { them writing in } \\
\text { English and get } \\
\text { them ready to be } \\
\text { able to survive in } \\
\text { their regular core } \\
\text { classrooms. That's } \\
\text { why for math, my } \\
\text { focus is a lot of } \\
\text { language, and } \\
\text { language they will } \\
\text { be able to } \\
\text { understand. In the } \\
\text { next class, which } \\
\text { will be for some } \\
\text { students geometry } \\
\text { and others } \\
\text { algebra...because } \\
\text { since we are small } \\
\text { schools they have } \\
\text { different pathways } \\
\text { as far as where }\end{array}$ & $\begin{array}{l}\text { * Science, we just } \\
\text { kind of thought } \\
\text { about okay, like } \\
\text { we started with } \\
\text { the human body } \\
\text { like body parts, } \\
\text { just vocabulary } \\
\text { that they } \\
\text { need....So, it was } \\
\text { like body parts, } \\
\text { what else did we } \\
\text { do in science, my } \\
\text { mind is going } \\
\text { blank, went from } \\
\text { body parts to, } \\
\text { food... Nutrition } \\
\text { and things are } \\
\text { healthy, } \\
\text { unhealthy, I } \\
\text { know, there was } \\
\text { something before } \\
\text { weather, or } \\
\text { maybe just went } \\
\text { body parts food, } \\
\text { weather, } \\
\text { seasons....And }\end{array}$ & $\begin{array}{l}\text { One teacher felt } \\
\text { overwhelmed about } \\
\text { teaching new } \\
\text { content areas, for } \\
\text { example math. Once } \\
\text { it was understood it } \\
\text { was more important } \\
\text { to teach language } \\
\text { and content terms, } \\
\text { teacher felt more } \\
\text { comfortable. } \\
\text { Successes occurring } \\
\text { in content areas, } \\
\text { students are being } \\
\text { adequately } \\
\text { prepared for grade- } \\
\text { level sheltered math } \\
\text { the following year } \\
\text { (algebra). Students } \\
\text { are learning a } \\
\text { different kind of } \\
\text { math they are used } \\
\text { to, for example in } \\
\text { Mexico they do not } \\
\text { teach using }\end{array}$ \\
\hline
\end{tabular}




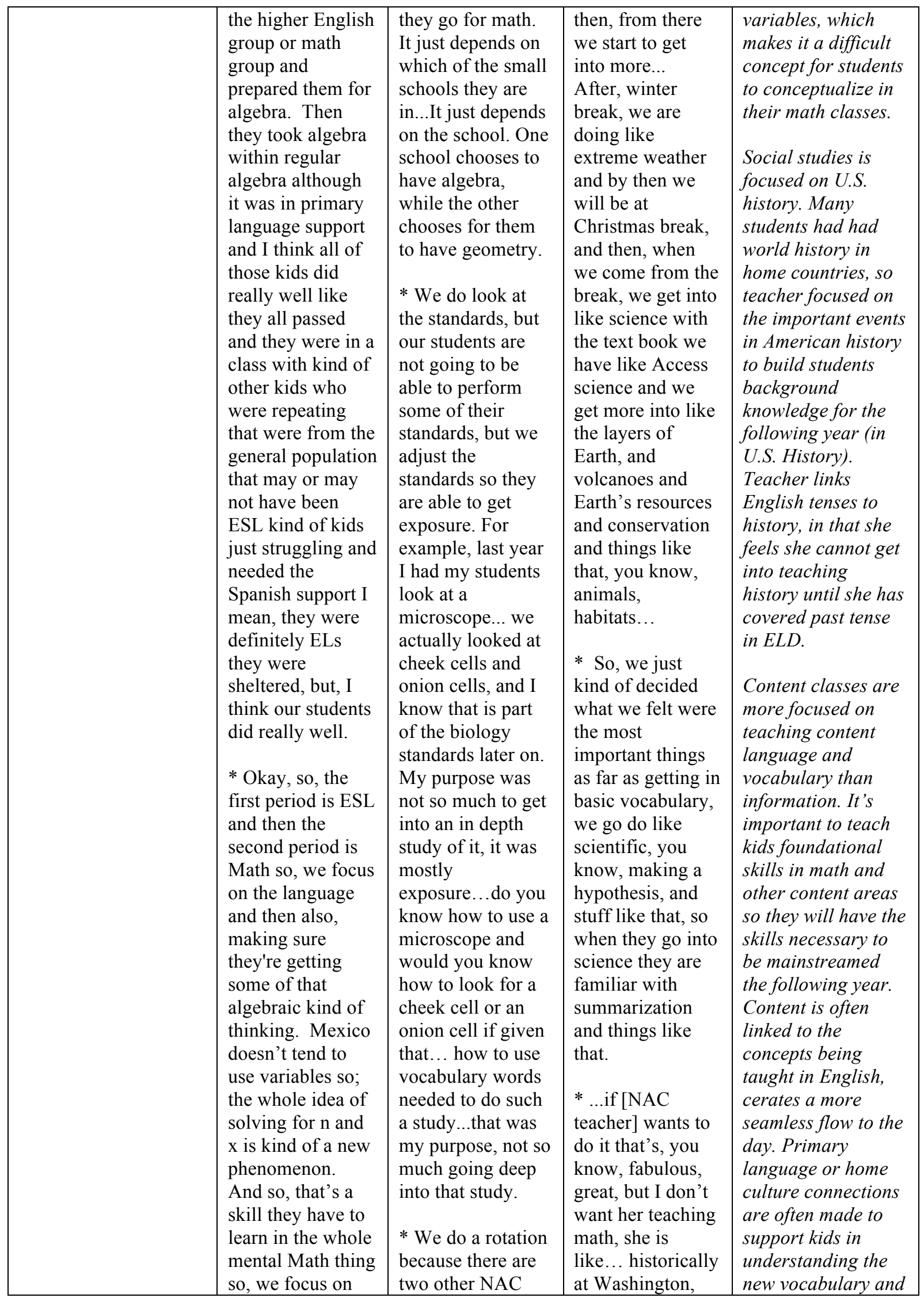




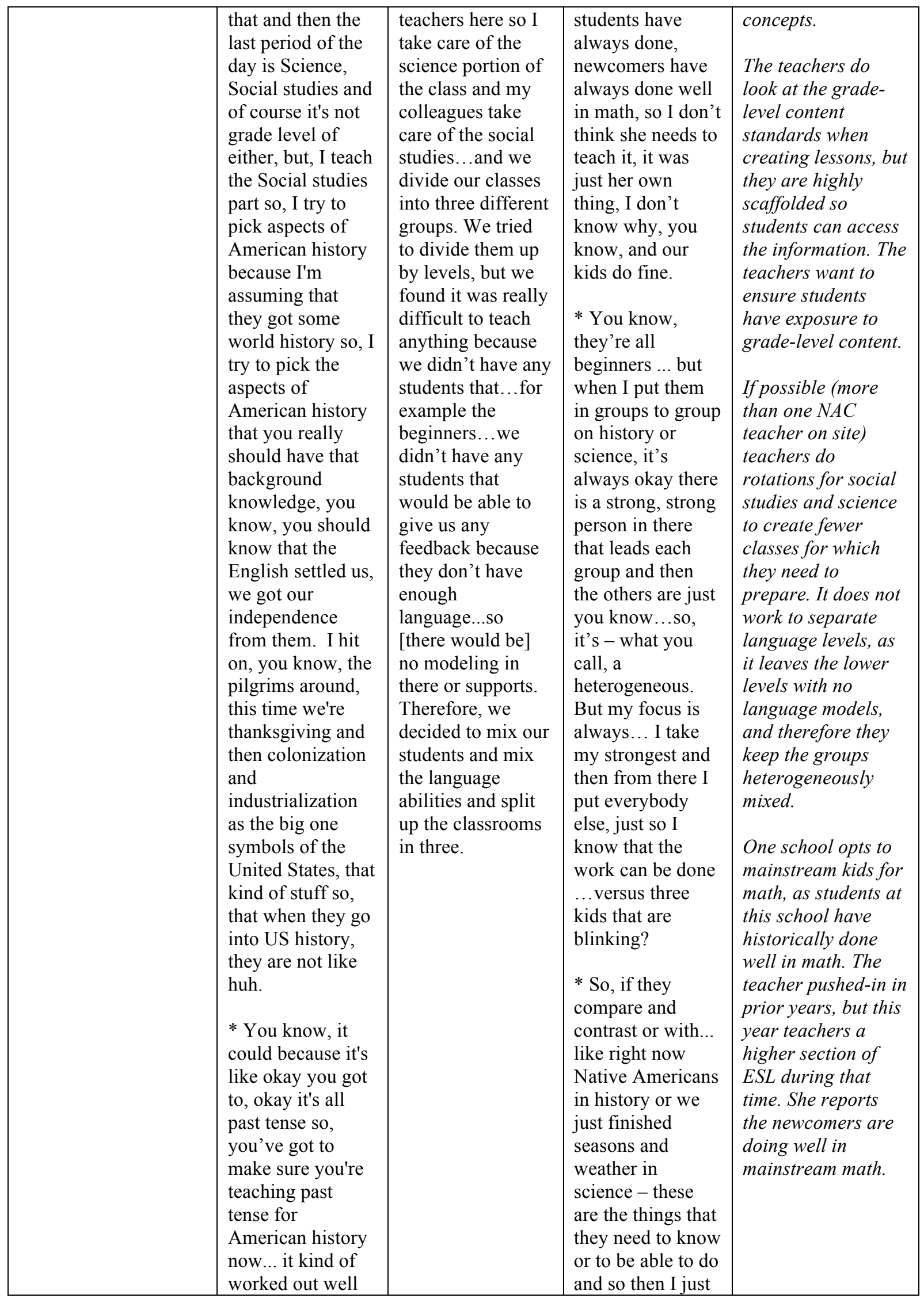




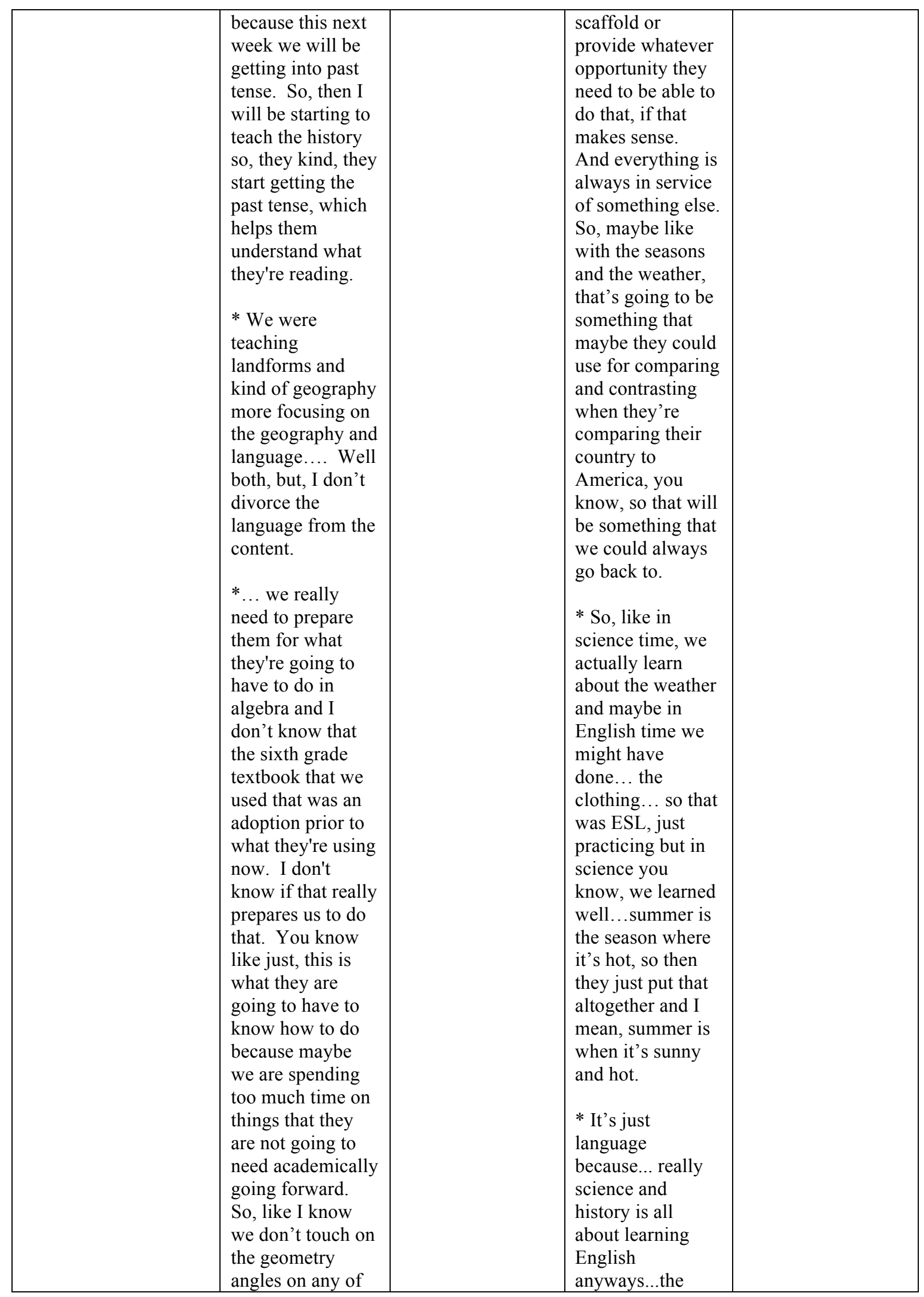




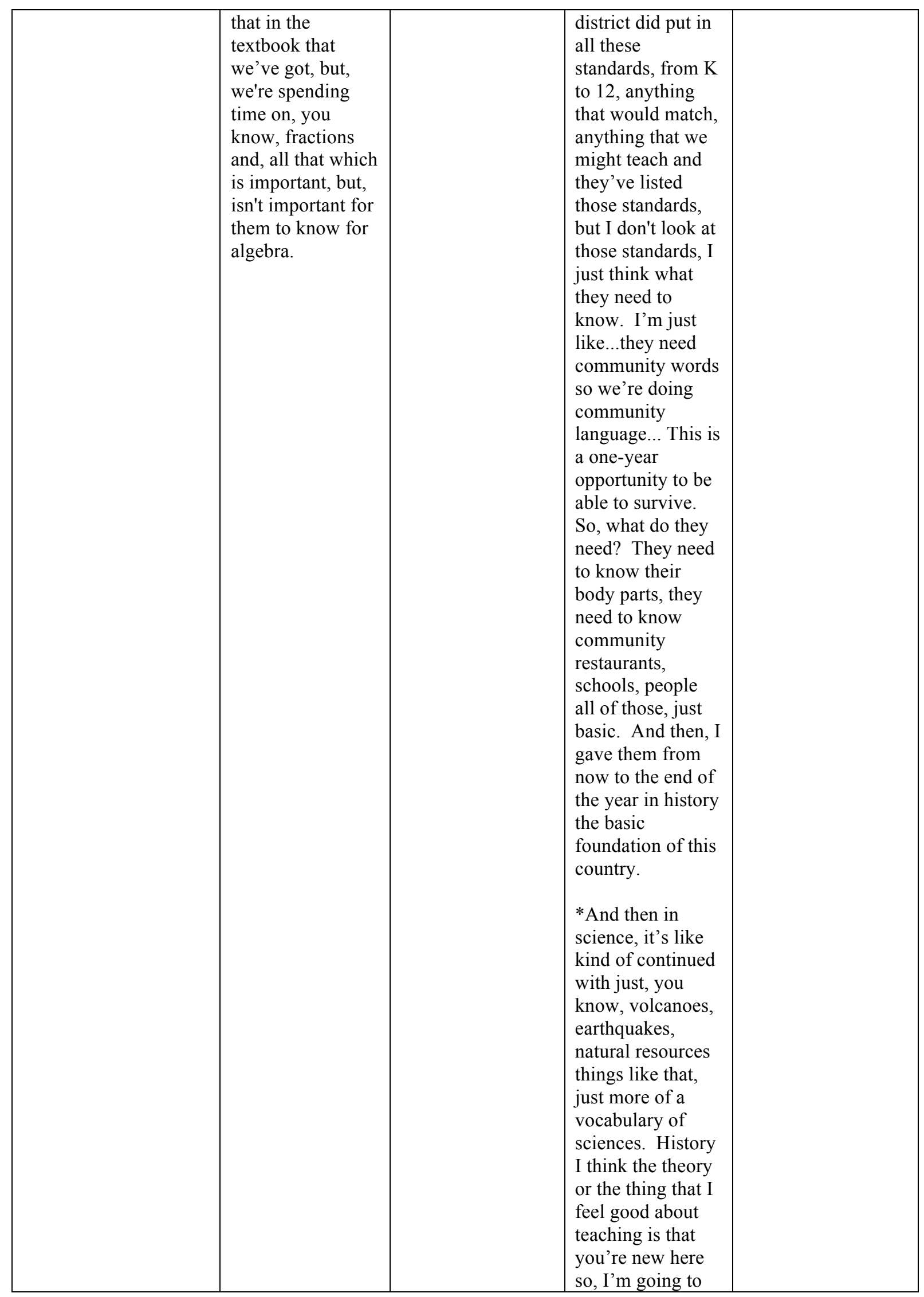




\begin{tabular}{|c|c|c|c|c|}
\hline & & & $\begin{array}{l}\text { tell you what the } \\
\text { history of this } \\
\text { country, just the } \\
\text { basics. And then, } \\
\text { in science it's } \\
\text { more about } \\
\text { continuing to } \\
\text { build language. } \\
\text { * ... lot of times } \\
\text { like, when we're } \\
\text { doing vocabulary } \\
\text { type words, or in } \\
\text { history or science } \\
\text { we'll make, like } \\
\text { cards and it will } \\
\text { divided up and } \\
\text { it's like English } \\
\text { word, your } \\
\text { language, picture, } \\
\text { you know, so they } \\
\text { always, and } \\
\text { always try to say } \\
\text { them, and we are } \\
\text { always making } \\
\text { that connection. } \\
\text { Do you have that? } \\
\text { Is it the same? Is } \\
\text { it different? } \\
\text { Things like that. }\end{array}$ & \\
\hline Teacher's Attitudes & $\begin{array}{l}\text { *I like the } \\
\text { population and the } \\
\text { kids are really } \\
\text { nice and I know } \\
\text { how much, I } \\
\text { know the struggle } \\
\text { I had trying to } \\
\text { learn Spanish and } \\
\text { so, I just felt like } \\
\text { people weren't } \\
\text { getting that, they } \\
\text { didn't have the } \\
\text { empathy and they } \\
\text { were approaching } \\
\text { it like, you're just } \\
\text { teaching a } \\
\text { kindergartener } \\
\text { how to read and } \\
\text { write where really } \\
\text { you're teaching, } \\
\text { it's a whole } \\
\text { different thing. } \\
\text { * When I was }\end{array}$ & $\begin{array}{l}\text { * What drew me to } \\
\text { this position was } \\
\text { that the group I } \\
\text { was going to be } \\
\text { working with was } \\
\text { specifically aimed } \\
\text { for English } \\
\text { language learners, } \\
\text { and though I have } \\
\text { always worked } \\
\text { with English } \\
\text { language learners } \\
\text { there's always } \\
\text { been a mix } \\
\text { with...I've worked } \\
\text { with students that } \\
\text { are Anglo- } \\
\text { American and } \\
\text { African-American, } \\
\text { but this was } \\
\text { specifically geared } \\
\text { towards a specific } \\
\text { group and it } \\
\text { was...just students }\end{array}$ & $\begin{array}{l}\text { * Yeah, I knew } \\
\text { that because I } \\
\text { knew that they } \\
\text { were starting this } \\
\text { new program, like } \\
\text { I would hear } \\
\text { about it at my } \\
\text { trainings and I } \\
\text { thought like, oh } \\
\text { that's such a cool } \\
\text { idea. And then, } \\
\text { so there was one } \\
\text { year that I didn't } \\
\text { do it and then the } \\
\text { second year was } \\
\text { when I was like, } \\
\text { okay, you know, } \\
\text { that sounds like a } \\
\text { really amazing } \\
\text { program. And I } \\
\text { want to go back } \\
\text { to the classroom, } \\
\text { maybe that's } \\
\text { where I need to }\end{array}$ & $\begin{array}{l}\text { Teachers feel very } \\
\text { passionate about } \\
\text { teaching newcomer } \\
\text { youth. One teacher } \\
\text { recounted her } \\
\text { struggle learning } \\
\text { Spanish and could } \\
\text { empathize with the } \\
\text { students as they } \\
\text { learn English. } \\
\text { Teachers } \\
\text { specifically applied } \\
\text { for program, and } \\
\text { were very devoted } \\
\text { to the needs of } \\
\text { newcomer youth. } \\
\text { They thought the } \\
\text { program sounded } \\
\text { interesting, and one } \\
\text { teacher, who was } \\
\text { out of the } \\
\text { classroom, felt it } \\
\text { would be a good } \\
\text { opportunity to get }\end{array}$ \\
\hline
\end{tabular}




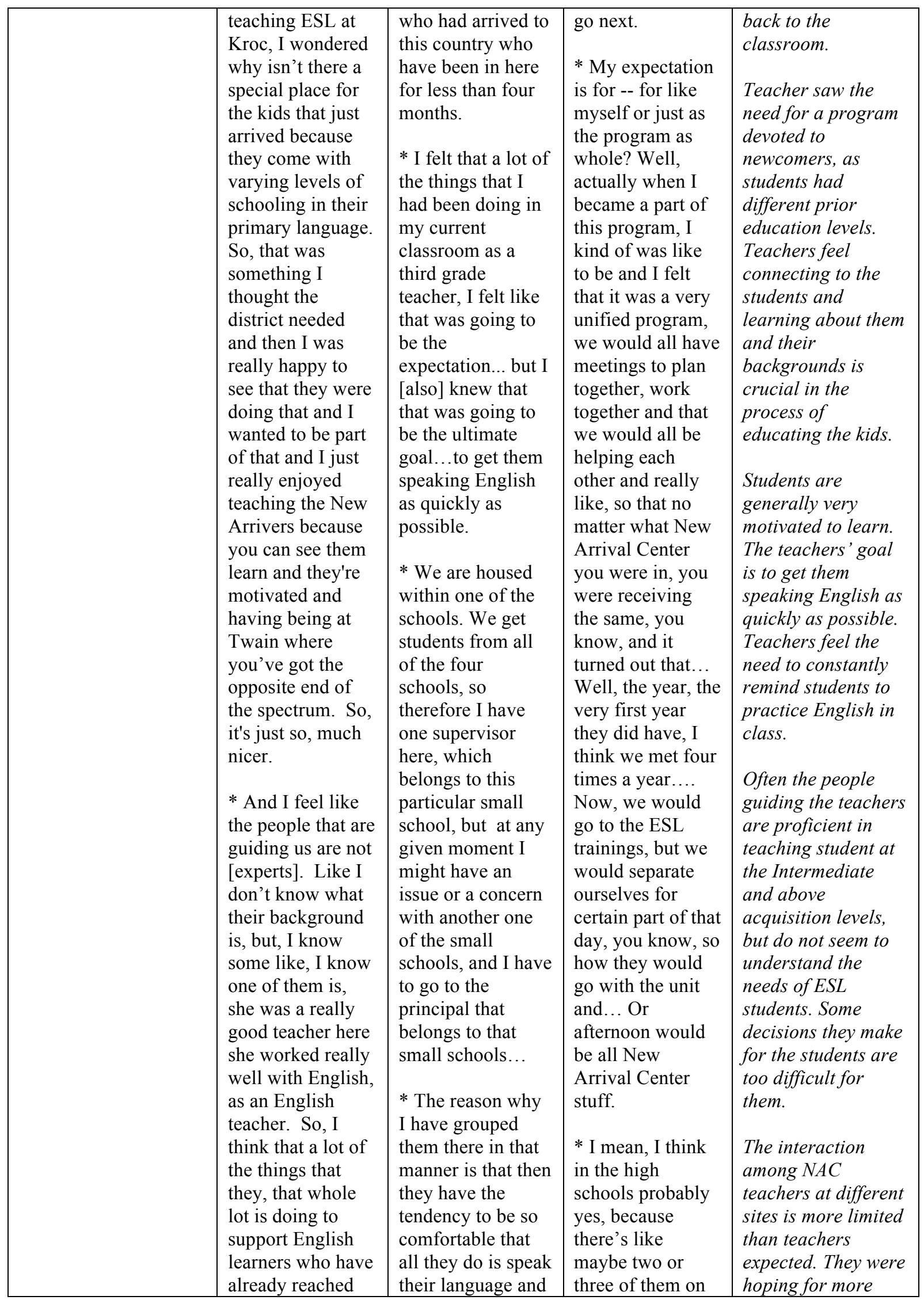




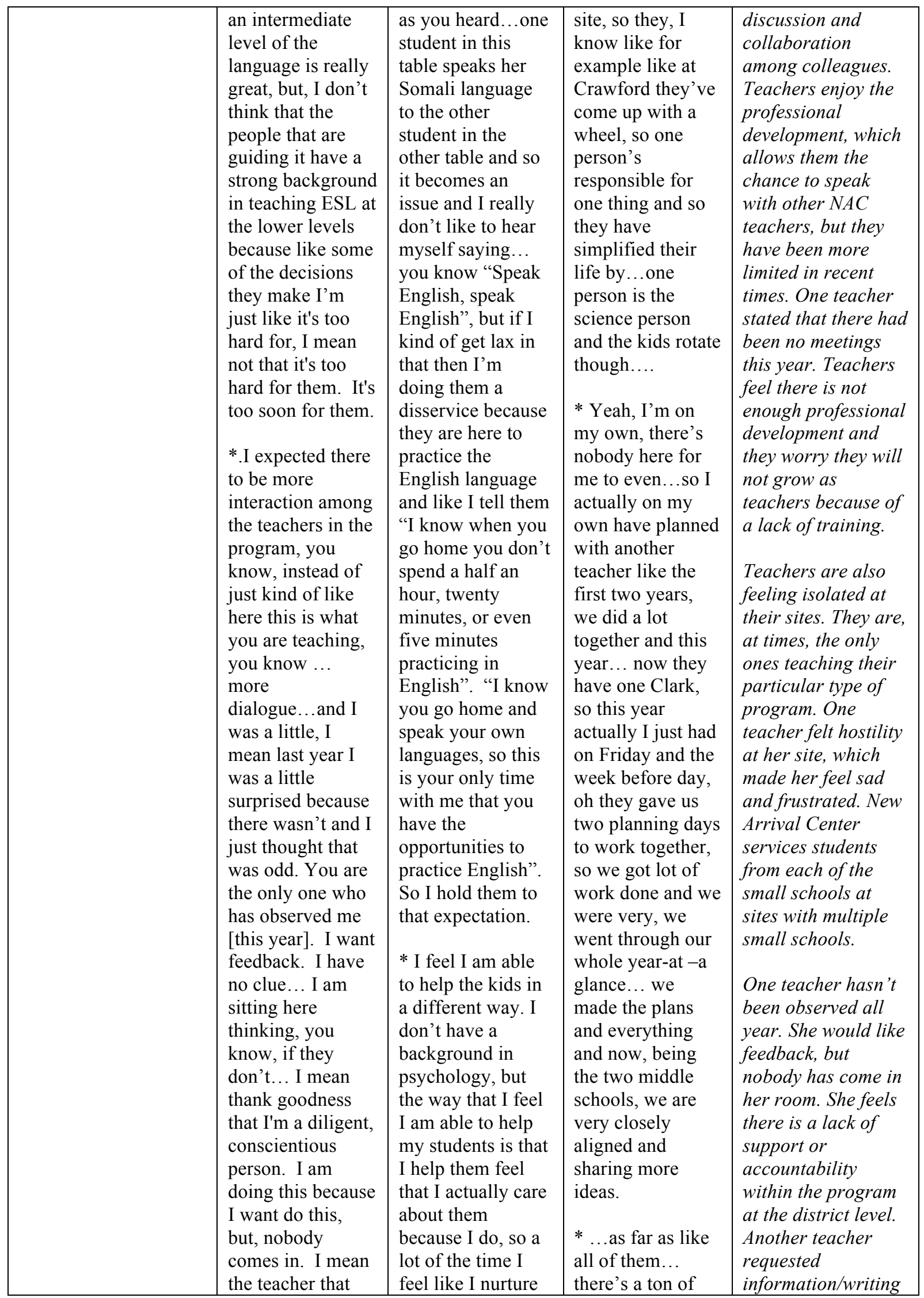




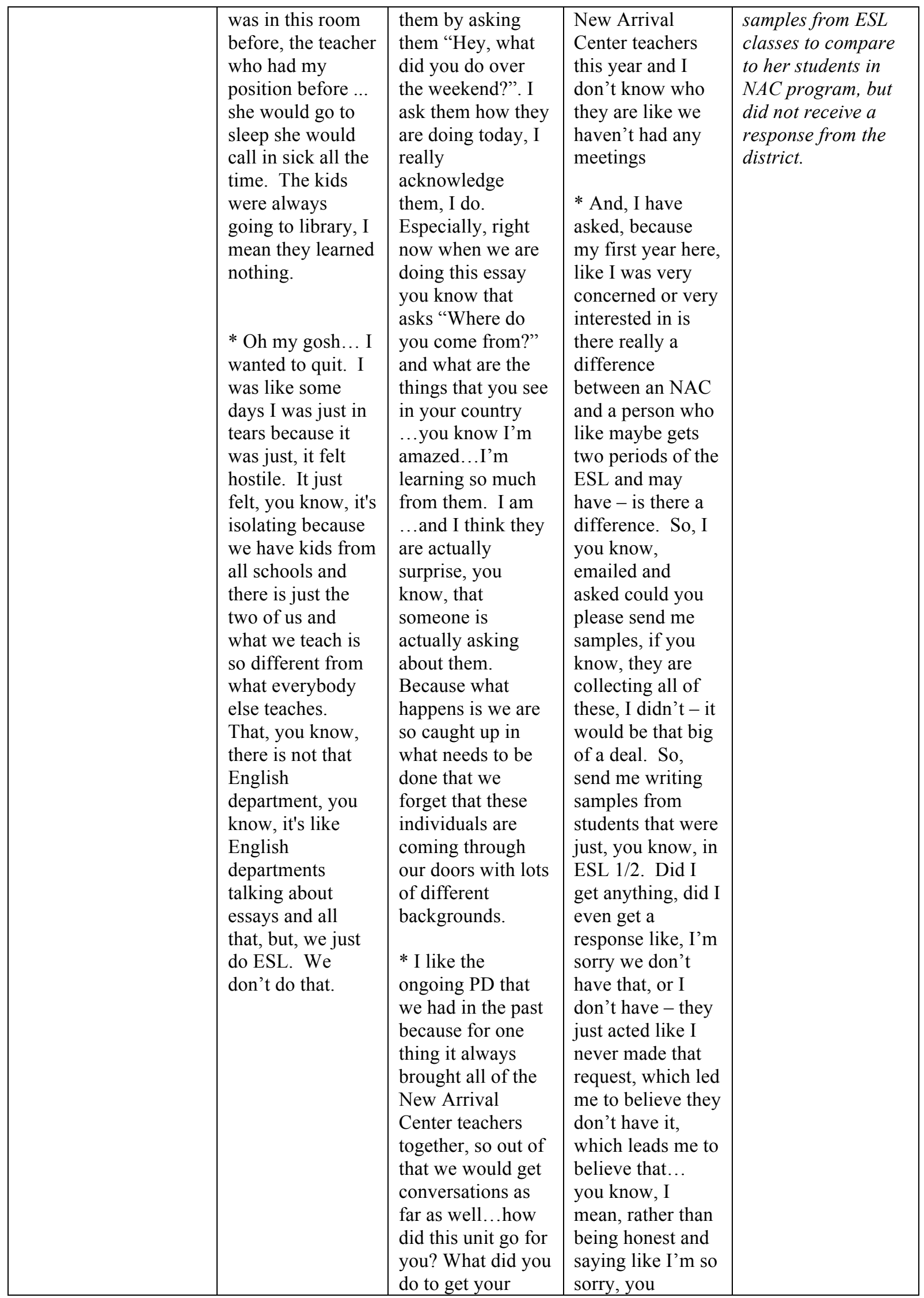




\begin{tabular}{|c|c|c|c|}
\hline & 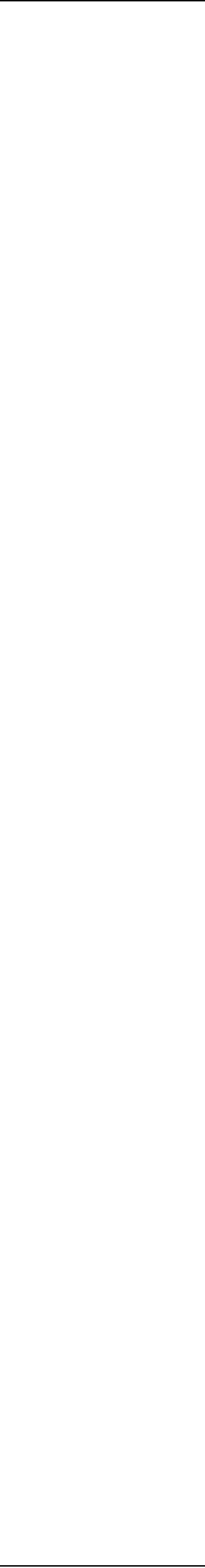 & \begin{tabular}{|l|} 
students to \\
produce what they \\
produced?...So it's \\
always fun to hear \\
what other \\
teachers are doing \\
instead of just \\
being in one place \\
and thinking well I \\
guess they're \\
doing well. It's \\
always interesting \\
to see what other \\
teachers are doing \\
at different sites \\
because everyone \\
has a different \\
teaching style and \\
everyone brings in \\
their own unique \\
style of integrating \\
the curriculum \\
with something \\
else.
\end{tabular} & $\begin{array}{l}\text { know...teachers } \\
\text { never sent that to } \\
\text { me or I didn't } \\
\text { follow through... } \\
\text { I know, we ask } \\
\text { for it every year, } \\
\text { but... just feel } \\
\text { like, I should } \\
\text { have been told } \\
\text { something of that } \\
\text { nature. } \\
\text { * So, I just had } \\
\text { have a lot of } \\
\text { training. So, I } \\
\text { feel very } \\
\text { confident like in } \\
\text { what I'm doing, } \\
\text { you know, what I } \\
\text { mean.... But, I } \\
\text { also, I'm afraid, } \\
\text { like I'm going to } \\
\text { stagnate like } \\
\text { because now that } \\
\text { I'm in the...like } \\
\text { when you are a } \\
\text { resource teacher, } \\
\text { you are invited to } \\
\text { go to everything, } \\
\text { you get every } \\
\text { training because } \\
\text { the idea is that } \\
\text { you are supposed } \\
\text { to go back and } \\
\text { you know, train } \\
\text { or coach staff. I } \\
\text { feel like, now I } \\
\text { feel like, I don't } \\
\text { know what's } \\
\text { going on any } \\
\text { more and I'm } \\
\text { afraid like I'm } \\
\text { going to get, I } \\
\text { don't know what } \\
\text { you even call it, } \\
\text { like everything, } \\
\text { you know, } \\
\text { progress and } \\
\text { things continue, } \\
\text { people keep } \\
\text { learning and } \\
\text { growing and I feel } \\
\text { like I just don't } \\
\text { want to be stuck, } \\
\text { like I love being } \\
\text { here. But, at the }\end{array}$ \\
\hline
\end{tabular}




\begin{tabular}{|c|c|c|c|c|}
\hline & & & $\begin{array}{l}\text { same time when } \\
\text { you are not a } \\
\text { resource teacher, } \\
\text { you don't go to } \\
\text { trainings. } \\
\text { * You know, it's } \\
\text { there [the } \\
\text { accountability] - I } \\
\text { mean, maybe my } \\
\text { principal and } \\
\text { maybe every now } \\
\text { and then we'll } \\
\text { just check and see } \\
\text { how the NAC } \\
\text { kids are doing but } \\
\text { nobody is worried } \\
\text { about them } \\
\text { because they're } \\
\text { all - first of all } \\
\text { they have all been } \\
\text { very successful } \\
\text { within my classes } \\
\text { and I've never } \\
\text { complained or } \\
\text { worried or so they } \\
\text { kind of leave me } \\
\text { alone. }\end{array}$ & \\
\hline $\begin{array}{c}\text { Cultural } \\
\text { Responsiveness }\end{array}$ & $\begin{array}{l}\text { * So, I can speak } \\
\text { Spanish and I } \\
\text { know the Spanish } \\
\text { culture, but, I } \\
\text { shouldn't say } \\
\text { Spanish culture. } \\
\text { My ex-husband } \\
\text { was from Peru. } \\
\text { So, I know, and I } \\
\text { have got many } \\
\text { Latino friends, So, } \\
\text { I know Latin } \\
\text { culture. So, I do } \\
\text { use that when I'm } \\
\text { talking to the kids. } \\
\text { And because I } \\
\text { have been } \\
\text { studying in my } \\
\text { master's the } \\
\text { cultural } \\
\text { differences, I try } \\
\text { to pull that in so, } \\
\text { instead of holding } \\
\text { the carrot to the } \\
\text { student that, oh, } \\
\text { you're going to }\end{array}$ & $\begin{array}{l}\text { * A lot of these } \\
\text { students come } \\
\text { from countries } \\
\text { where they're not } \\
\text { allowed to even } \\
\text { look at the person } \\
\text { in the face. So } \\
\text { throughout the } \\
\text { year I am doing a } \\
\text { lot of "Well in this } \\
\text { country it's ok to } \\
\text { look at the other } \\
\text { person in the face } \\
\text { and talk to } \\
\text { them"...it's ok to } \\
\text { say "Excuse me, I } \\
\text { think you've made } \\
\text { a mistake". I'm } \\
\text { doing that } \\
\text { constantly because } \\
\text { they come from } \\
\text { countries where } \\
\text { they've been told } \\
\text { no, it's not ok for } \\
\text { you to talk back to } \\
\text { your teachers, it's }\end{array}$ & $\begin{array}{l}\text { * You know, so } \\
\text { like but I'm still } \\
\text { not sure how I'm } \\
\text { going to do it. } \\
\text { But, it will } \\
\text { basically be like, } \\
\text { so in the end they } \\
\text { can compare their } \\
\text { country and } \\
\text { United States and } \\
\text { they can compare } \\
\text { religions, they can } \\
\text { compare holidays, } \\
\text { whatever... So, } \\
\text { for the next eight } \\
\text { weeks I will just } \\
\text { be reading and } \\
\text { learning a lot } \\
\text { about, you know, } \\
\text { United States, } \\
\text { Vietnam, you } \\
\text { know, all of the } \\
\text { countries and } \\
\text { focus on maybe } \\
\text { four areas like } \\
\text { food, holiday, }\end{array}$ & $\begin{array}{l}\text { Teachers spoke } \\
\text { Spanish and were } \\
\text { able to use primary } \\
\text { language support } \\
\text { with their Spanish- } \\
\text { speaking students. } \\
\text { One teacher did } \\
\text { report that she did } \\
\text { not use Spanish in } \\
\text { her class, as it was } \\
\text { inequitable to the } \\
\text { students from other } \\
\text { language } \\
\text { backgrounds. One } \\
\text { teacher learned } \\
\text { Spanish as a second } \\
\text { language, which she } \\
\text { believed gave her } \\
\text { greater empathy in } \\
\text { teaching her } \\
\text { students English. } \\
\text { After she had } \\
\text { children, she } \\
\text { focused more on } \\
\text { learning the } \\
\text { language. }\end{array}$ \\
\hline
\end{tabular}




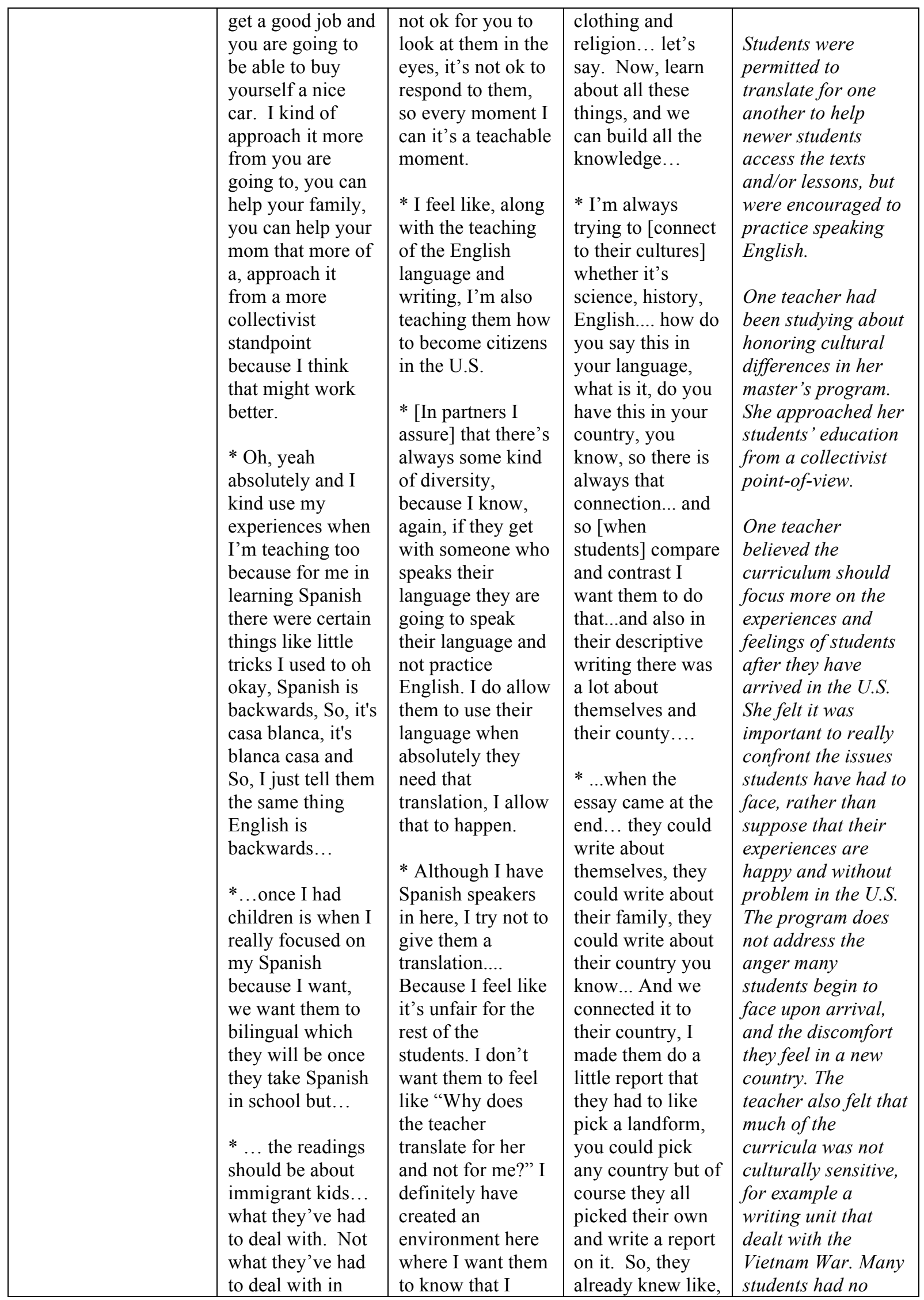




\begin{tabular}{|c|c|c|c|c|}
\hline & $\begin{array}{l}\text { their country, but } \\
\text { what they've had } \\
\text { to deal with } \\
\text { having arrived } \\
\text { here. Most of it } \\
\text { tends to be, I } \\
\text { don't know. It's } \\
\text { like everybody's } \\
\text { happy, you know, } \\
\text { we're here, we've } \\
\text { got a great life, } \\
\text { you know, and } \\
\text { just even the } \\
\text { assumption I } \\
\text { mean, we're so, } \\
\text { ethnocentric. } \\
\text { *The assumption } \\
\text { that like the } \\
\text { Vietnam War was } \\
\text { important to the } \\
\text { whole world. It } \\
\text { wasn't. It was } \\
\text { important to us } \\
\text { and to Vietnam } \\
\text { and Vietnamese } \\
\text { they don't really } \\
\text { like to talk about } \\
\text { it. So, you know, } \\
\text { the WRITE } \\
\text { institute we have } \\
\text { this, I remember } \\
\text { The Wall....I had } \\
\text { one Vietnamese } \\
\text { student and part of } \\
\text { it was to go back } \\
\text { and talk to your } \\
\text { family. She is } \\
\text { like I am not } \\
\text { talking to my } \\
\text { family about this. } \\
\text { We are not, we } \\
\text { don't talk about } \\
\text { this. The } \\
\text { Mexican kids, } \\
\text { they don't even } \\
\text { know there was a } \\
\text { Vietnam War. } \\
\text { *It's not culturally } \\
\text { sensitive and I } \\
\text { think more of the } \\
\text { focus shouldn't } \\
\text { so, much be on } \\
\text { the history, but, } \\
\text { what are the } \\
\end{array}$ & $\begin{array}{l}\text { respect where they } \\
\text { come from and } \\
\text { that I understand } \\
\text { some of the } \\
\text { conditions they } \\
\text { left their country } \\
\text { in and that I'm } \\
\text { here to help them } \\
\text { and not make them } \\
\text { feel less then } \\
\text { anyone else. } \\
\text { * I depend on } \\
\text { students to help } \\
\text { me if I need to talk } \\
\text { to parents. I } \\
\text { depend a lot on the } \\
\text { students to help } \\
\text { me to translate and } \\
\text { I know that's not } \\
\text { the best way, } \\
\text { because they are } \\
\text { learning English } \\
\text { and I know the } \\
\text { message doesn't } \\
\text { get completely } \\
\text { across, but that's } \\
\text { the situation that } \\
\text { we are dealing } \\
\text { with and that's } \\
\text { how it is in the } \\
\text { district as well. I } \\
\text { can translate the } \\
\text { Spanish because I } \\
\text { have a Spanish } \\
\text { background, and I } \\
\text { can depend on a } \\
\text { Somali translator } \\
\text { because we have a } \\
\text { Somali resource } \\
\text { person on site who } \\
\text { speaks Somali, I } \\
\text { know I can depend } \\
\text { on a Vietnamese } \\
\text { translator because } \\
\text { we also have a } \\
\text { person who speaks } \\
\text { Vietnamese on our } \\
\text { campus who is an } \\
\text { adult, but all the } \\
\text { other languages I } \\
\text { mean....I have } \\
\text { other students } \\
\text { translate for } \\
\text { students' parents, } \\
\text { especially now }\end{array}$ & $\begin{array}{l}\text { such and such } \\
\text { river in my } \\
\text { country is } \\
\text { beautiful, you } \\
\text { know, and they } \\
\text { were able to } \\
\text { incorporate that } \\
\text { into their writing } \\
\text {...so in the end, } \\
\text { the language is so } \\
\text { natural it's just } \\
\text { going to flow. }\end{array}$ & $\begin{array}{l}\text { prior knowledge of } \\
\text { this conflict, and } \\
\text { Vietnamese students } \\
\text { did not want to } \\
\text { discuss the war (a } \\
\text { painful experience). } \\
\text { Teachers also } \\
\text { reported having } \\
\text { students compare } \\
\text { their home } \\
\text { countries to the } \\
\text { U.S., focusing on } \\
\text { food, language, } \\
\text { religion, regional } \\
\text { features, holidays, } \\
\text { and clothing. } \\
\\
\text { Teachers felt part of } \\
\text { their job was to also } \\
\text { teach students about } \\
\text { the culture in the } \\
\text { United States, how } \\
\text { to become citizens } \\
\text { in the U.S. } \\
\text { When speaking to } \\
\text { parents in } \\
\text { unfamiliar } \\
\text { languages, teachers } \\
\text { have found } \\
\text { translators in the } \\
\text { community, or used } \\
\text { students as } \\
\text { language brokers } \\
\text { for their families. }\end{array}$ \\
\hline
\end{tabular}




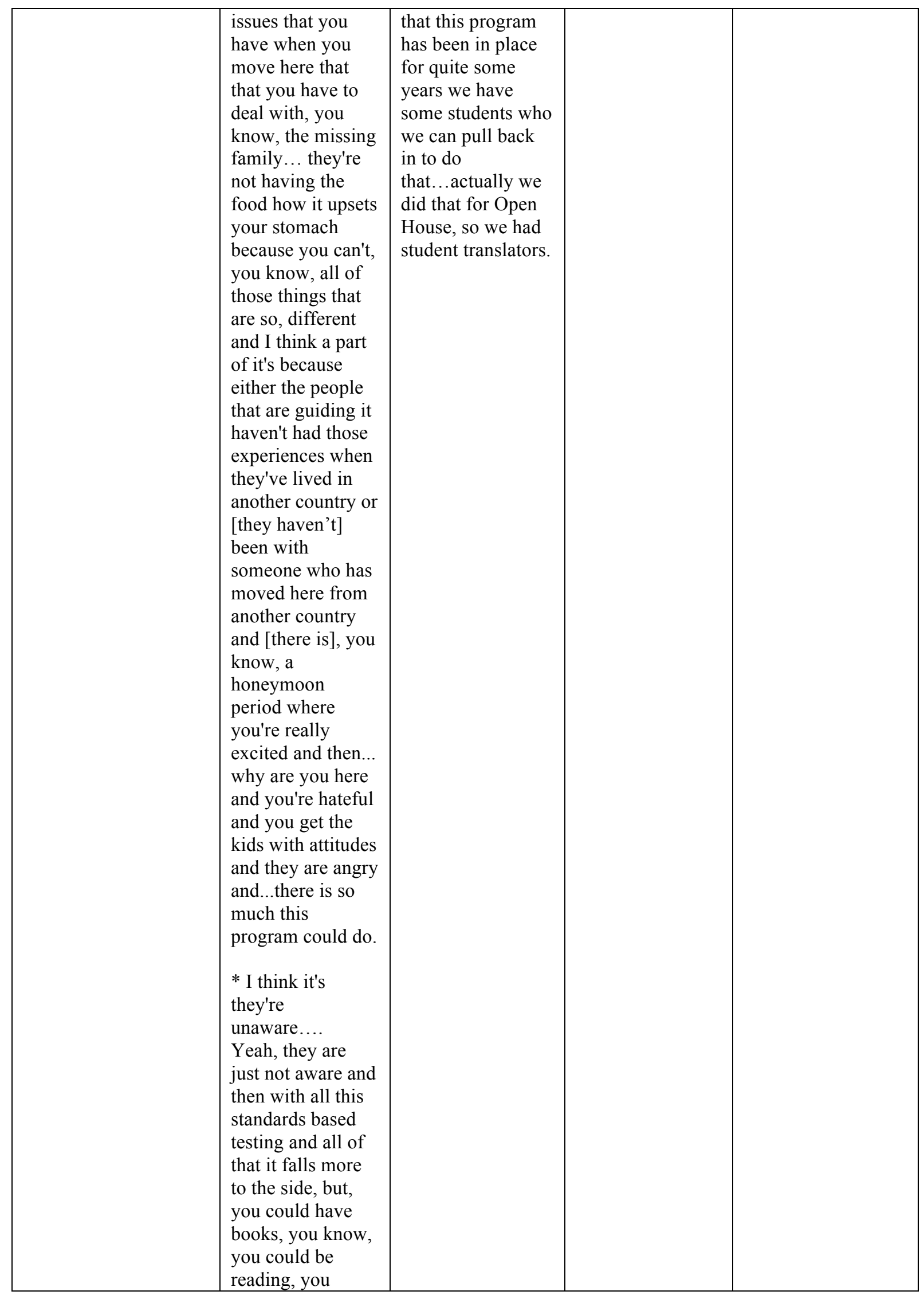




\begin{tabular}{|c|c|c|c|c|}
\hline & $\begin{array}{l}\text { know, acquiring } \\
\text { language, but, at } \\
\text { the same time } \\
\text { connecting with } \\
\text { something } \\
\text { because of that } \\
\text { piece [The Wall] } \\
\text { like I think the } \\
\text { High Point book } \\
\text { had a little bit } \\
\text { more of that. } \\
\text { * In the past when } \\
\text { I had, like I had } \\
\text { Somali student. I } \\
\text { couldn't } \\
\text { communicate with } \\
\text { her or her mother, } \\
\text { I contacted the } \\
\text { mosque.... } \\
\text { I try to contact } \\
\text { people that I knew } \\
\text { or students that I } \\
\text { knew. I had a } \\
\text { Vietnamese kid } \\
\text { who would come } \\
\text { and talk Chinese } \\
\text { to the Chinese } \\
\text { parent that sort of } \\
\text { thing. ... I don't } \\
\text { know if that's, I } \\
\text { mean, I know that } \\
\text { there are } \\
\text { translators out } \\
\text { there, but, I don't } \\
\text { know, it is easier } \\
\text { for me to go and } \\
\text { ask somebody } \\
\text { than to go to the } \\
\text { district. }\end{array}$ & & & \\
\hline $\begin{array}{c}\text { Pedagogical } \\
\text { Orientations/Beliefs }\end{array}$ & $\begin{array}{l}\text { * Well, I'm a } \\
\text { facilitator, but, I } \\
\text { am also, I don't } \\
\text { want to say a } \\
\text { counselor, but, in } \\
\text { a sense I am. I'm } \\
\text { a mediator. I'm a } \\
\text { facilitator. I'm an } \\
\text { instructor. It just } \\
\text { depends what had } \\
\text { is needed at the } \\
\text { time, because with } \\
\text { this group of kids } \\
\text { they just, there are }\end{array}$ & $\begin{array}{l}\text { * I feel like I hold } \\
\text { a lot of weight in } \\
\text { this classroom and } \\
\text { sometimes that } \\
\text { could be good and } \\
\text { that could be } \\
\text { bad...I mean these } \\
\text { kids are expecting, } \\
\text { they are looking at } \\
\text { me, they are } \\
\text { depending on me } \\
\text { to provide them } \\
\text { the necessary } \\
\text { skills to survive in }\end{array}$ & $\begin{array}{l}* \text { [I see my role as } \\
\text { a teacher as] } \\
\text { just making sure } \\
\text { they learn as } \\
\text { much English as } \\
\text { they can learn } \\
\text { * I try to keep } \\
\text { them motivated, I } \\
\text { try to keep them } \\
\text { excited, I try to } \\
\text { you know, keep } \\
\text { them happy, just } \\
\text { like this is a very }\end{array}$ & $\begin{array}{l}\text { One teacher viewed } \\
\text { herself as a } \\
\text { facilitator in the } \\
\text { classroom and as a } \\
\text { counselor and } \\
\text { mediator to } \\
\text { students, depending } \\
\text { on what students } \\
\text { needed. Students } \\
\text { spent free } \\
\text { time/lunch time with } \\
\text { her, since they were } \\
\text { so comfortable. } \\
\text { Another teacher }\end{array}$ \\
\hline
\end{tabular}




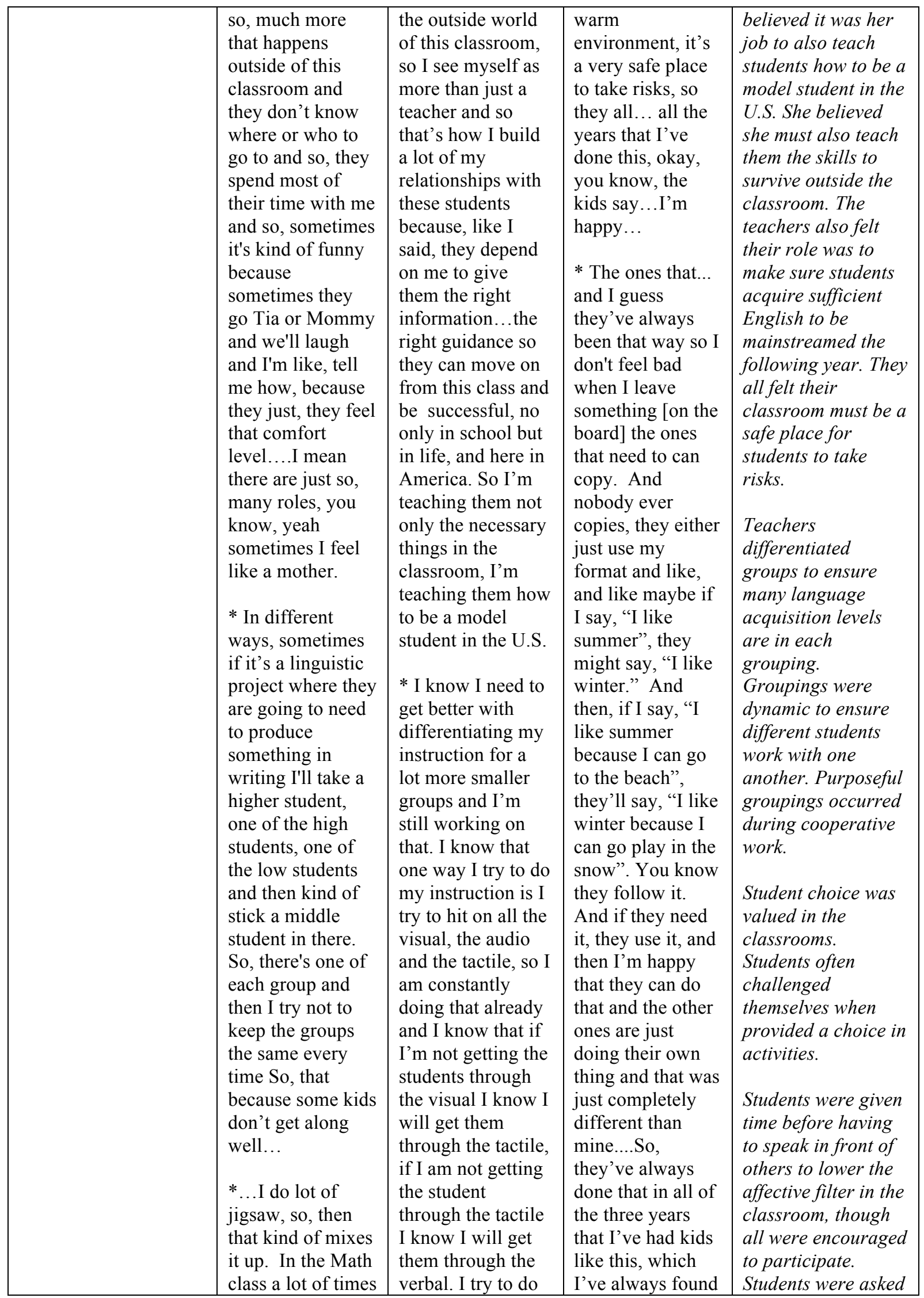




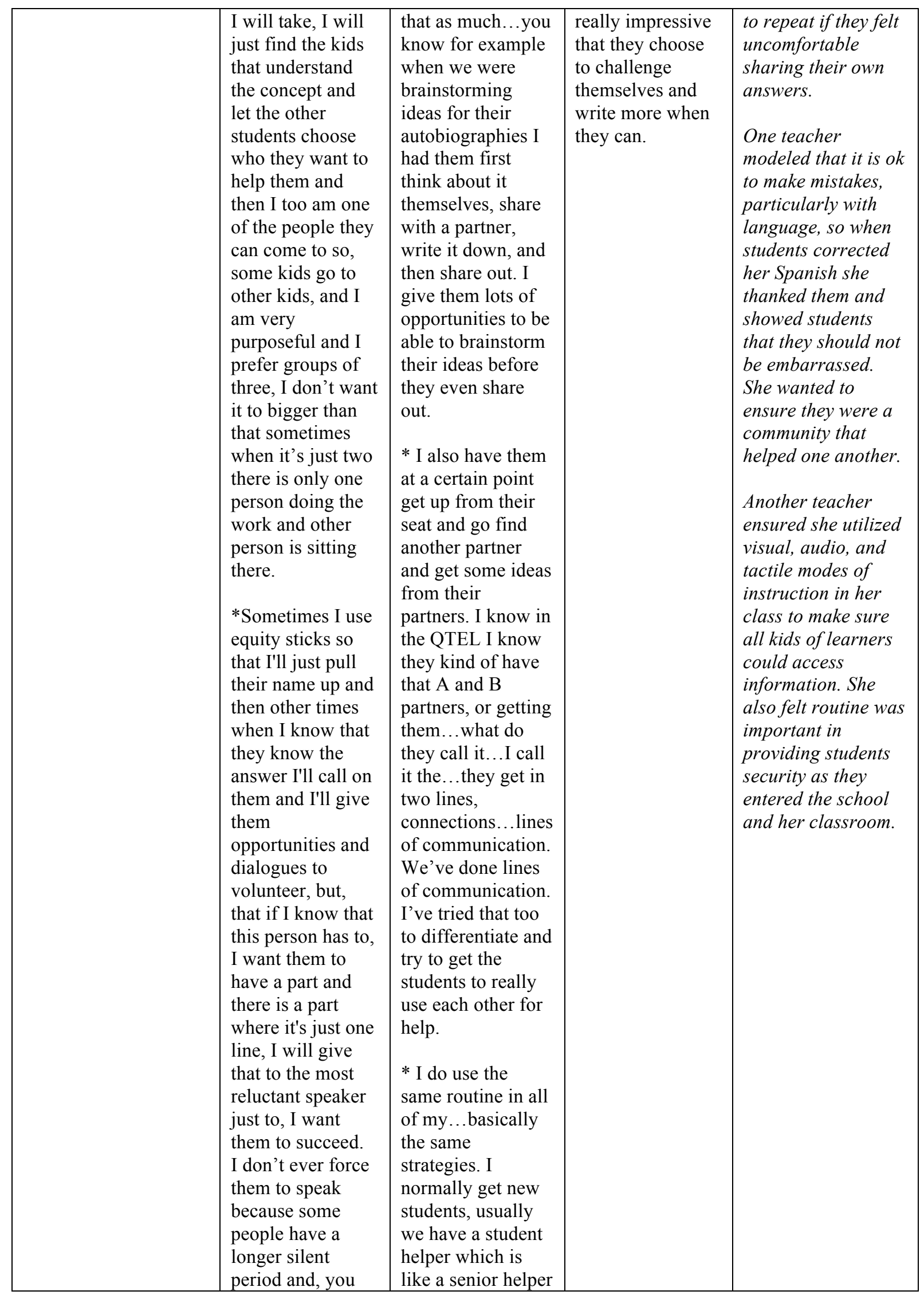




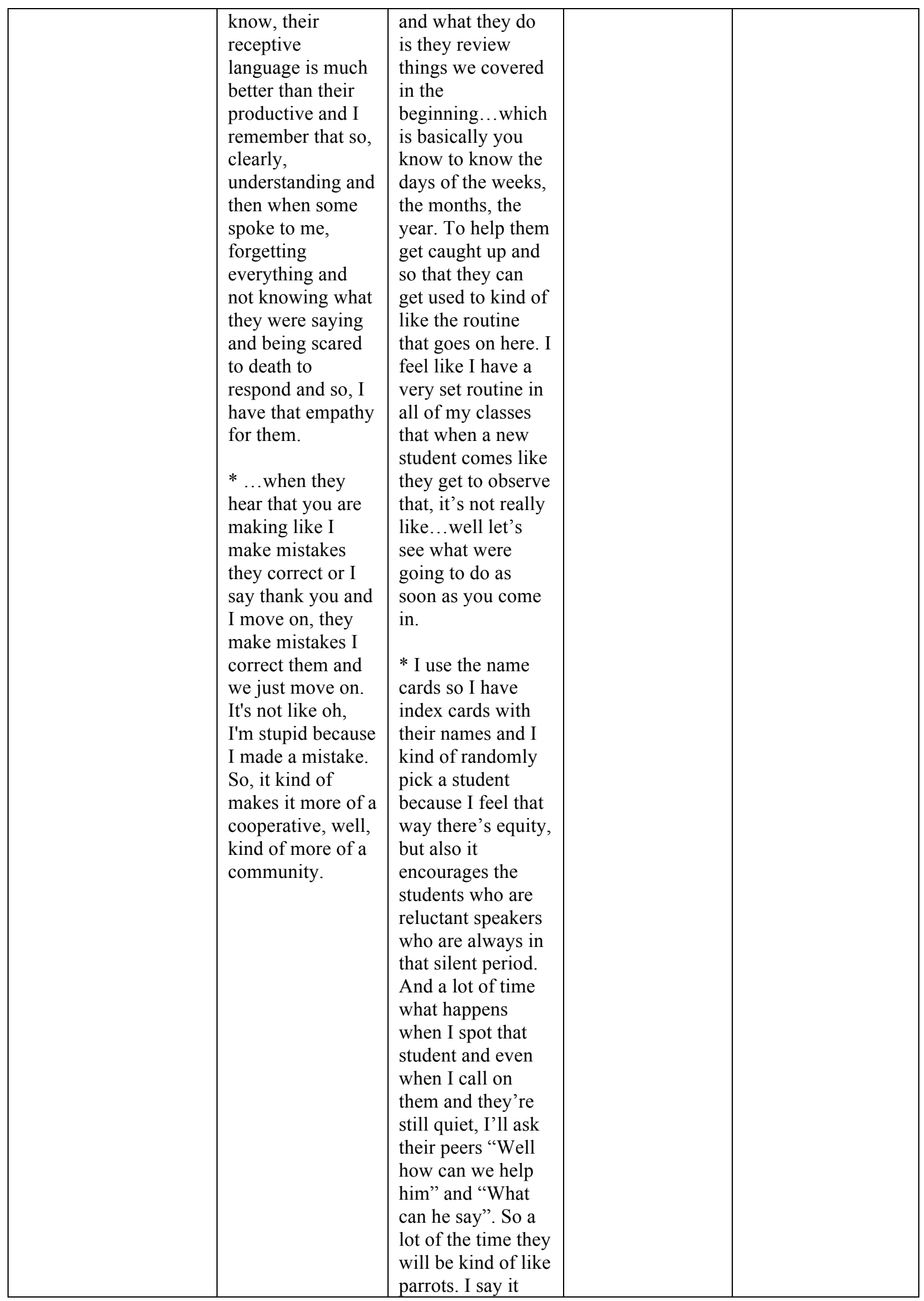




\begin{tabular}{|c|c|c|c|c|}
\hline & & $\begin{array}{l}\text { and then have } \\
\text { them repeat it. }\end{array}$ & & \\
\hline $\begin{array}{l}\text { Academic Self- } \\
\text { Concept }\end{array}$ & $\begin{array}{l}\text { * I think because I } \\
\text { have just one } \\
\text { class, I think } \\
\text { they're more } \\
\text { confident. They } \\
\text { know somebody } \\
\text { and they feel like } \\
\text { a part of } \\
\text { something ...like } \\
\text { I know that they } \\
\text { feel a part of this, } \\
\text { and I know that } \\
\text { for the most part I } \\
\text { don't have kids } \\
\text { crying because } \\
\text { they feel alone } \\
\text { so... much like [at } \\
\text { my former school] } \\
\text { I remember I had } \\
\text { kids that were } 15 \\
\text { years old, boys } \\
\text { even, that were } \\
\text { just overwhelmed } \\
\text { and they were } \\
\text { just... you could } \\
\text { see it. So, I think } \\
\text { this program does } \\
\text { support them, in a } \\
\text { way that's good. }\end{array}$ & $\begin{array}{l}\text { * I think they are } \\
\text { more confident. } \\
\text { Because they } \\
\text { know they are } \\
\text { around student } \\
\text { who are in a } \\
\text { similar situation. } \\
\text { They are here } \\
\text { from a new } \\
\text { country they're } \\
\text { here having some } \\
\text { English maybe or } \\
\text { no English and } \\
\text { they're all learning } \\
\text { the English } \\
\text { language so I } \\
\text { know they feel } \\
\text { safe around their } \\
\text { peers because they } \\
\text { know they have a } \\
\text { common ground. } \\
\text { So...I think } \\
\text { because we are in } \\
\text { this situation... } \\
\text { that it helps them } \\
\text { build their self- } \\
\text { esteem. } \\
\text { * I do see [a } \\
\text { difference in their } \\
\text { academic self- } \\
\text { concept or social } \\
\text { self-concept] } \\
\text { especially at the } \\
\text { end of the school } \\
\text { year...when we've } \\
\text { had that year long } \\
\text { of study, they've } \\
\text { basically have } \\
\text { picked up on what } \\
\text { they're learning as } \\
\text { far as... I know } \\
\text { Keys is for my } \\
\text { grammar and word } \\
\text { study, I know } \\
\text { WRITE is for my } \\
\text { writing and I know } \\
\text { math... that's } \\
\text { when I really get } \\
\text { to see all their } \\
\text { growth, you know } \\
\text { in all the areas. }\end{array}$ & $\begin{array}{l}\text { *... the } \\
\text { [students] always } \\
\text { seem to be happy, } \\
\text { they always seem } \\
\text { to enjoy coming, } \\
\text { you know, so I } \\
\text { just feel like, they } \\
\text { are motivated in } \\
\text { here, I don't feel } \\
\text { like, I have to, I } \\
\text { think, the } \\
\text { motivation is their } \\
\text { own, it's not } \\
\text { anything I do that, } \\
\text { but everything } \\
\text { else like the } \\
\text { excitement, the } \\
\text { respect, the } \\
\text { safety. } \\
\text { * You } \\
\text { know...Arlen is } \\
\text { quiet but his } \\
\text { CELDT was } \\
\text { straight } \\
\text { intermediate, it's } \\
\text { like he's ready If } \\
\text { feel like if I keep } \\
\text { him I'm holding } \\
\text { him back and he's } \\
\text { been so upset } \\
\text { about it for the } \\
\text { last two days. I } \\
\text { told them on } \\
\text { Wednesday and } \\
\text { everyday, he } \\
\text { says... 'I want to } \\
\text { stay, I want to } \\
\text { stay". And I'm } \\
\text { like ...I'm sorry, } \\
\text { I'll be doing a } \\
\text { disservice if I } \\
\text { keep you, you'll } \\
\text { be fine and ... you } \\
\text { know, he knows } \\
\text { everybody he was } \\
\text { with them before, } \\
\text { I think it's just } \\
\text { safe and } \\
\text { comfortable } \\
\text { here... And that's } \\
\text { what I say, come }\end{array}$ & $\begin{array}{l}\text { Teachers reported } \\
\text { students felt more } \\
\text { confident. They felt } \\
\text { like all of the } \\
\text { students were in the } \\
\text { same position, } \\
\text { which was a } \\
\text { comfort to them and } \\
\text { helped them to feel } \\
\text { safe. One teacher } \\
\text { had witnessed } \\
\text { students crying at } \\
\text { her former school } \\
\text { because they felt } \\
\text { alone and } \\
\text { overwhelmed. } \\
\text { Teachers did } \\
\text { witness a change in } \\
\text { students' self- } \\
\text { esteem and } \\
\text { academic self- } \\
\text { concept, } \\
\text { particularly by the } \\
\text { end of the school } \\
\text { year. The routine } \\
\text { and safe } \\
\text { environment helped } \\
\text { kids to grow over } \\
\text { the school year. } \\
\text { One teacher } \\
\text { believed that her } \\
\text { students came in } \\
\text { very motivated and } \\
\text { were always very } \\
\text { happy to be in her } \\
\text { class. She helped } \\
\text { her students stay } \\
\text { happy and } \\
\text { motivated through } \\
\text { respect, safety, and } \\
\text { excitement in her } \\
\text { class. The students } \\
\text { were never laughed } \\
\text { at in her class and } \\
\text { other students were } \\
\text { generally willing to } \\
\text { help students when } \\
\text { they need } \\
\text { assistance, as she } \\
\text { promoted a safe }\end{array}$ \\
\hline
\end{tabular}




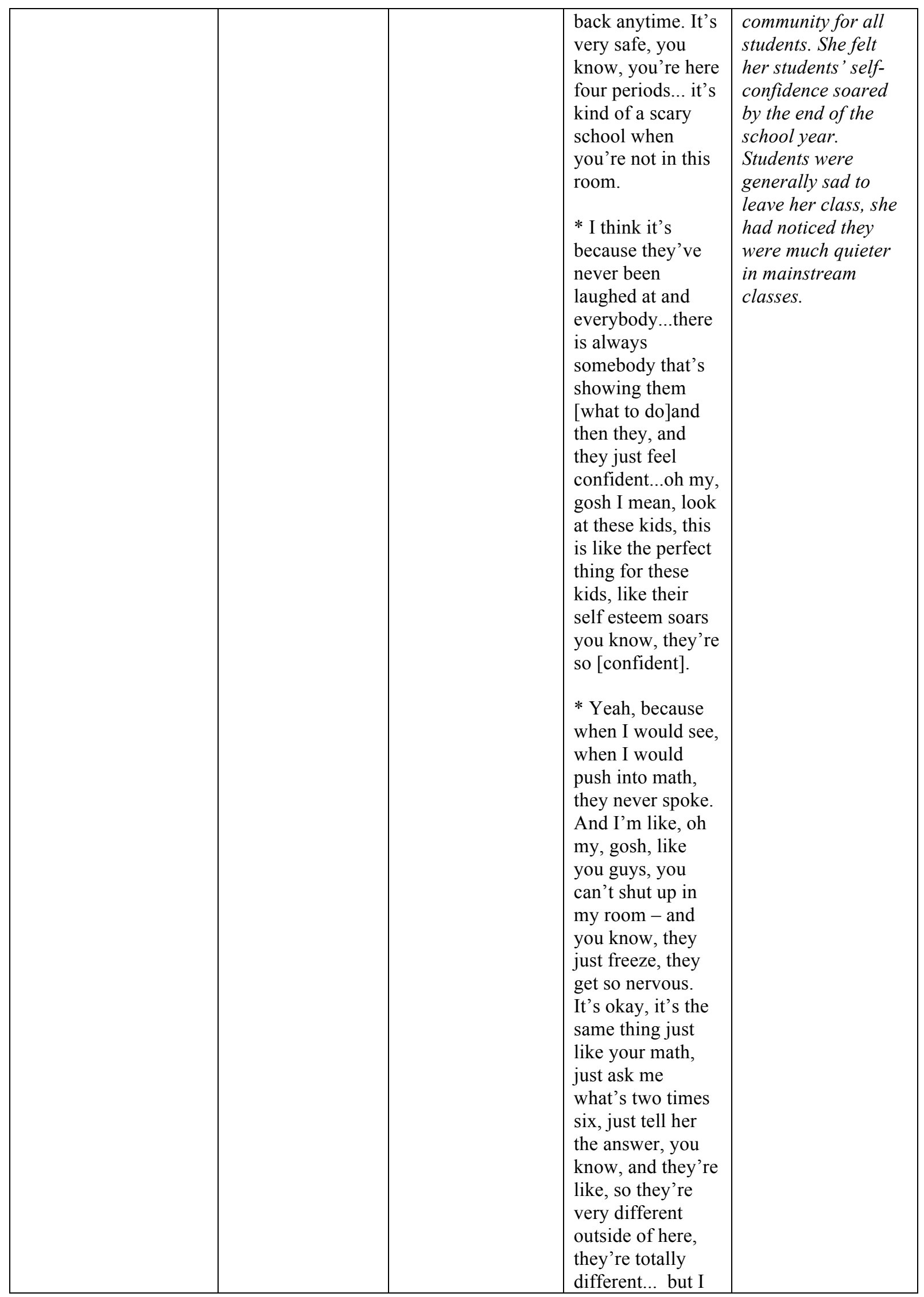




\begin{tabular}{|c|c|c|c|c|}
\hline & & & $\begin{array}{l}\text { think in time, this } \\
\text { will allow them... } \\
\text { if they didn't have } \\
\text { this, I think they } \\
\text { would be quiet a } \\
\text { lot longer. }\end{array}$ & \\
\hline $\begin{array}{c}\text { Language } \\
\text { Proficiency } \\
\text { Level }\end{array}$ & $\begin{array}{l}\text { * I see them being } \\
\text { able to first } \\
\text { communicate their } \\
\text { needs and wants } \\
\text { with, you know, } \\
\text { an ample } \\
\text { vocabulary. I see } \\
\text { them being able to } \\
\text { read more. I don't } \\
\text { want to say grade } \\
\text { level text, but, to } \\
\text { read text and have } \\
\text { an understanding. } \\
\text { To be able to } \\
\text { write at the very } \\
\text { least in the present } \\
\text { with, you know } \\
\text { basic vocabulary } \\
\text { because they're } \\
\text { not going to get } \\
\text { all of the } \\
\text { academic...but, } \\
\text { that they can go } \\
\text { and they can } \\
\text { function. } \\
\text { * So, when they } \\
\text { come in, they take } \\
\text { the Express which } \\
\text { kind of gives us } \\
\text { an idea of how } \\
\text { much do they } \\
\text { really hear, you } \\
\text { know, how much } \\
\text { can they } \\
\text { understand and } \\
\text { how much can } \\
\text { they say and then } \\
\text { if they're too high } \\
\text { for us, they go out } \\
\text { to the next year } \\
\text { ESL class. We } \\
\text { also, give on } \\
\text { demand writing } \\
\text { that goes with the } \\
\text { Keys box and then } \\
\text { I give, you know, } \\
\text { this unit tests and }\end{array}$ & $\begin{array}{l}\text { * Well...that } \\
\text { depends on the } \\
\text { students. If the } \\
\text { student comes in } \\
\text { with very little to } \\
\text { no English } \\
\text { language ability, I } \\
\text { expect at least that } \\
\text { by half of the } \\
\text { school year, they } \\
\text { are responding } \\
\text { with one or two } \\
\text { phrases and } \\
\text { speaking some } \\
\text { English. If the } \\
\text { student comes in } \\
\text { with some } \\
\text { English, I expect } \\
\text { them by the } \\
\text { middle of the } \\
\text { school year to be } \\
\text { able to hold } \\
\text { somewhat of a } \\
\text { conversation and it } \\
\text { all depends on the } \\
\text { student. } \\
\text { * So I have } \\
\text { different linguistic } \\
\text { expectations for } \\
\text { different students. } \\
\text { So just like in } \\
\text { regular classrooms } \\
\text { in elementary, and } \\
\text { I know here in } \\
\text { high school they } \\
\text { have them, you } \\
\text { have mixed } \\
\text { language abilities, } \\
\text { so you have } \\
\text { different } \\
\text { expectations...that } \\
\text { 's why it's really } \\
\text { hard to grade them } \\
\text { because how do } \\
\text { you grade a } \\
\text { student who has } \\
\text { zero English but is } \\
\end{array}$ & $\begin{array}{l}\text { * Well, I try, I } \\
\text { mean, I'm } \\
\text { shooting for them } \\
\text { to go from } \\
\text { beginners to } \\
\text { intermediate.... } \\
\text { So, during the } \\
\text { year like they } \\
\text { would go through } \\
\text { that and by the } \\
\text { end of the year } \\
\text { test, that's my } \\
\text { goal.... You } \\
\text { know, wonderful, } \\
\text { I mean, like you } \\
\text { know, new } \\
\text { arrivals and if you } \\
\text { are going to be } \\
\text { here for four } \\
\text { periods.... You } \\
\text { know, if the goal } \\
\text { is a level a year, } \\
\text { but I have you for } \\
\text { more, I feel like } \\
\text { show something } \\
\text { beyond a level, } \\
\text { that's just...And, } \\
\text { I have kids not in } \\
\text { everything, but I } \\
\text { have kids that } \\
\text { maybe in } \\
\text { speaking or } \\
\text { listening, they } \\
\text { become } \\
\text { intermediate. } \\
\text { * Every year I } \\
\text { decide, if students } \\
\text { repeat NAC or if } \\
\text { they go on to the } \\
\text { next level and I } \\
\text { was on the fence } \\
\text { with them, like oh } \\
\text { gosh, what do? I } \\
\text { made the decision } \\
\text { to keep them. } \\
\text { And I think it was } \\
\text { probably the right }\end{array}$ & $\begin{array}{l}\text { The teachers give } \\
\text { the students a } \\
\text { picture-based } \\
\text { assessment called } \\
\text { the Express as they } \\
\text { enter the class to } \\
\text { assess their } \\
\text { language } \\
\text { proficiency. If they } \\
\text { are too high they } \\
\text { can be placed in a } \\
\text { higher ESL class. } \\
\text { Teachers also use } \\
\text { On-Demand writing } \\
\text { to assess the } \\
\text { students' } \\
\text { proficiency levels, } \\
\text { as well as chapter } \\
\text { tests and exit slips. } \\
\text { By the end of the } \\
\text { school year the } \\
\text { teachers expect that } \\
\text { the students will be } \\
\text { able to } \\
\text { communicate their } \\
\text { wants and needs, } \\
\text { have short } \\
\text { conversations, and } \\
\text { be able to read } \\
\text { more fluently in } \\
\text { English. The } \\
\text { students will not } \\
\text { necessarily be } \\
\text { reading grade-level } \\
\text { texts, but will have a } \\
\text { greater } \\
\text { understanding of } \\
\text { what they do read. } \\
\text { They should be able } \\
\text { to write in at least } \\
\text { the present tense } \\
\text { with basic } \\
\text { vocabulary. The } \\
\text { expectations depend } \\
\text { on the level of } \\
\text { English with which } \\
\text { the students enter }\end{array}$ \\
\hline
\end{tabular}




\begin{tabular}{|c|c|c|c|c|}
\hline & $\begin{array}{l}\text { things like that, } \\
\text { but, then I kind of } \\
\text { just give little } \\
\text { things throughout } \\
\text { the week, the } \\
\text { month, you know, } \\
\text { exit slips So, that I } \\
\text { can see where } \\
\text { they're lost, what } \\
\text { is going to need } \\
\text { more } \\
\text { reinforcement... } \\
\text { * I know that last } \\
\text { year the principal } \\
\text { here was trying to } \\
\text { put together a } \\
\text { monitoring thing } \\
\text { where you could } \\
\text { see, so, we had to, } \\
\text { you know, figure } \\
\text { out, what do they } \\
\text { have to figure out, } \\
\text { what their } \\
\text { attendance was, } \\
\text { what their Express } \\
\text { was, you know, } \\
\text { kind of look at } \\
\text { where they were } \\
\text { in their writing to } \\
\text { see if they were } \\
\text { going up or } \\
\text { they're flat lining, } \\
\text { or they were } \\
\text { making any } \\
\text { progress. }\end{array}$ & $\begin{array}{l}\text { making a lot of } \\
\text { effort and that is } \\
\text { something we } \\
\text { don't have } \\
\text { set...yet...but I } \\
\text { think there's an } \\
\text { urgency to do it. }\end{array}$ & $\begin{array}{l}\text { thing to do, I } \\
\text { think they needed } \\
\text { that beginning } \\
\text { that had missed, } \\
\text { all that basic } \\
\text { foundational stuff } \\
\text { and like Arlen } \\
\text { and Ricky are } \\
\text { totally ready to } \\
\text { go, but Lee... I'll } \\
\text { keep probably the } \\
\text { whole year, she is } \\
\text { still real quiet. } \\
\text { She is good on } \\
\text { paper but she } \\
\text { doesn't have the } \\
\text { oral. } \\
\text { * I know they're } \\
\text { going to be with } \\
\text { me next year, if } \\
\text { they're starting in } \\
\text { May unless they } \\
\text { come with a lot of } \\
\text { English. If } \\
\text { they're brand, } \\
\text { brand new in } \\
\text { May, I know I'm } \\
\text { going to have you } \\
\text { next year. }\end{array}$ & $\begin{array}{l}\text { the school. One } \\
\text { teacher would like } \\
\text { them to jump from } \\
\text { beginner to } \\
\text { intermediate on the } \\
\text { CELDT assessment. } \\
\text { Depending on the } \\
\text { site, some teachers } \\
\text { submit information } \\
\text { to their } \\
\text { administration } \\
\text { regarding the } \\
\text { students' language } \\
\text { progress. Other } \\
\text { sites do not require } \\
\text { any form of } \\
\text { accountability. } \\
\text { NAC teachers } \\
\text { determine if } \\
\text { students are ready } \\
\text { to be mainstreamed } \\
\text { the following year. } \\
\text { At times, it is } \\
\text { necessary to keep } \\
\text { students longer if } \\
\text { they haven't } \\
\text { developed sufficient } \\
\text { language skills. If } \\
\text { students arrive late } \\
\text { in the school year } \\
\text { (generally Spring), } \\
\text { they will repeat the } \\
\text { following year. }\end{array}$ \\
\hline Academic Skills & $\begin{array}{l}\text { * I knew what I } \\
\text { was going to be } \\
\text { confronted with } \\
\text { like it's not just } \\
\text { learning the } \\
\text { language, there is, } \\
\text { you know, the } \\
\text { fear of being in an } \\
\text { American school } \\
\text { or there are } \\
\text { deficits in their } \\
\text { home country } \\
\text { education. } \\
\text { * Well, we do } \\
\text { [level] because } \\
\text { I've got a lower } \\
\text { group of kids... }\end{array}$ & $\begin{array}{l}\text { * You have } \\
\text { students that come } \\
\text { with high } \\
\text { academic } \\
\text { background } \\
\text { knowledge but } \\
\text { then they don't } \\
\text { have the English } \\
\text { language, and then } \\
\text { you have students } \\
\text { who have nothing, } \\
\text { not the language } \\
\text { nor the } \\
\text { academics... so } \\
\text { what I try to do } \\
\text { I'm doing more so } \\
\text { in math because } \\
\text { it's somehow a lot }\end{array}$ & $\begin{array}{l}\text { * Well, so far in } \\
\text { all my three } \\
\text { years, I've been } \\
\text { fortunate that } \\
\text { everybody has } \\
\text { come as readers } \\
\text { and writers. You } \\
\text { know, whether - } \\
\text { you know, I don't } \\
\text { think I've really } \\
\text { had anybody } \\
\text { that's actually } \\
\text { grade level. But } \\
\text { they all read and } \\
\text { write. So, you } \\
\text { can have, except } \\
\text { for that boy last } \\
\text { year, he was the }\end{array}$ & $\begin{array}{l}\text { Teachers report that } \\
\text { students come in } \\
\text { with varying } \\
\text { academic } \\
\text { backgrounds and } \\
\text { prior education } \\
\text { levels. Some just } \\
\text { need to learn } \\
\text { English vocabulary } \\
\text { and grammar, and } \\
\text { therefore can } \\
\text { transfer their skills, } \\
\text { while others have } \\
\text { interrupted formal } \\
\text { schooling and need } \\
\text { to acquire literacy } \\
\text { and mathematics } \\
\text { skills as well. The }\end{array}$ \\
\hline
\end{tabular}




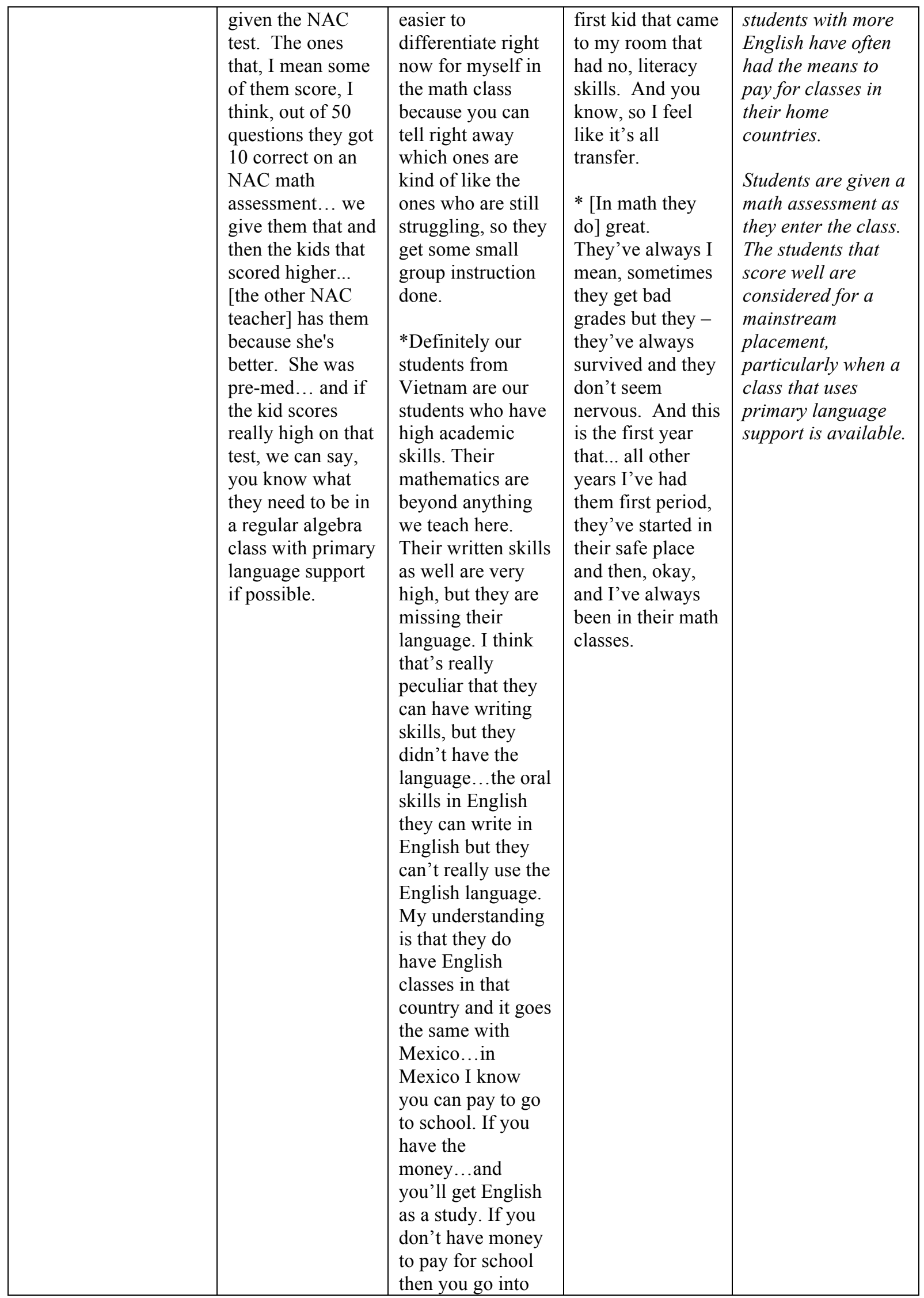




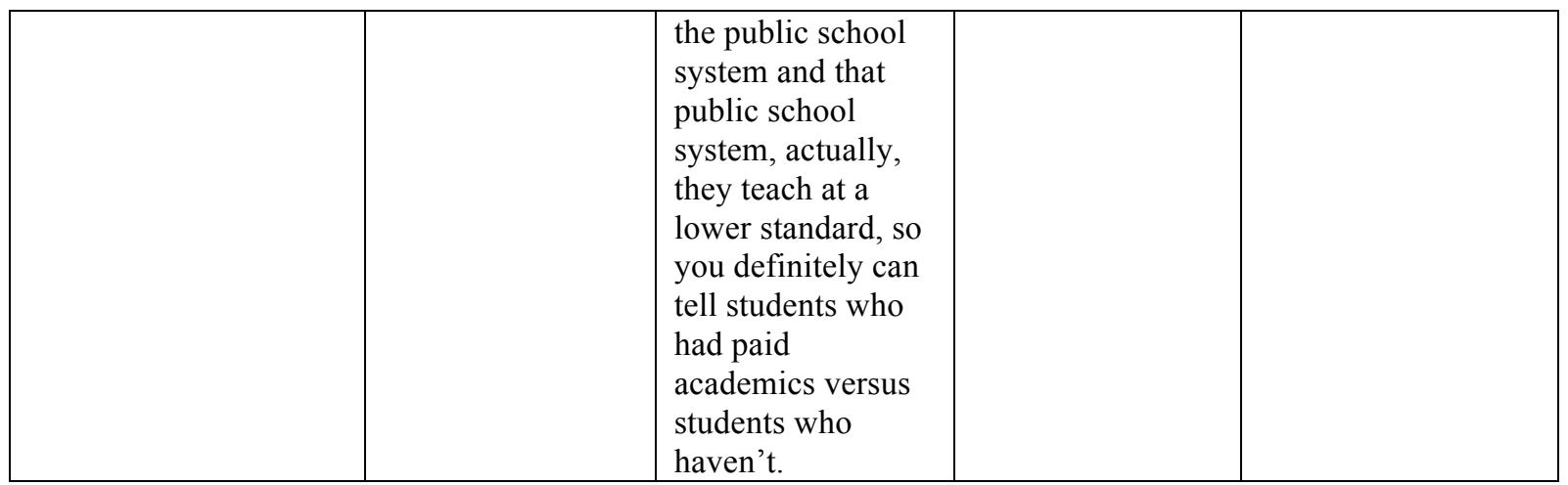




\section{Appendix O}

\section{ESL Teacher Interview Analysis}

\begin{tabular}{|c|c|c|c|c|}
\hline & $\begin{array}{l}\text { Teacher } \\
\text { ESL \#1 }\end{array}$ & $\begin{array}{l}\text { Teacher } \\
\text { ESL \#2 }\end{array}$ & $\begin{array}{l}\text { Teacher } \\
\text { ESL \#3 }\end{array}$ & Significant Themes \\
\hline Curricula & $\begin{array}{l}\text { * When I first } \\
\text { started there } \\
\text { wasn't any real } \\
\text { curriculum and so } \\
\text { that was a little bit } \\
\text { of a challenge. } \\
\text { They had } \\
\text { something called } \\
\text { ELEPS, which } \\
\text { teachers had } \\
\text { created. It didn't } \\
\text { have anything to } \\
\text { do with language } \\
\text { development } \\
\text { really. It was } \\
\text { thematic and it } \\
\text { was all on } \\
\text { worksheets. At } \\
\text { that point the } \\
\text { worksheets were } \\
\text { provided for you } \\
\text { though in a box. } \\
\text { Here are your } \\
\text { worksheets for this } \\
\text { week and for next } \\
\text { week. } \\
\text { * After } 18 \\
\text { years..like maybe } \\
\text { about six or seven } \\
\text { years ago, it } \\
\text { started to change } \\
\text { and people } \\
\text { books, so I did that } \\
\text { bealized that we } \\
\text { need to books for } \\
\text { English learners } \\
\text { much like the } \\
\text { books that they } \\
\text { have for Spanish } \\
\text { classes here. } \\
\text { we going to } \\
\end{array}$ & $\begin{array}{l}\text { * Well, the } \\
\text { curriculum is chosen } \\
\text { by the district...All } \\
\text { at our office and I } \\
\text { think they want us } \\
\text { to use kids to } \\
\text { learning and also the } \\
\text { WRITE unit and } \\
\text { some other stuff, so } \\
\text { far I've - we aligned } \\
\text { to WRITE unit a lot. } \\
\text { * KEYs, I love only } \\
\text { the first part...And } \\
\text { the second part is I } \\
\text { don't think it's - I } \\
\text { don't think that that } \\
\text { is very effective in } \\
\text { terms of the lot of } \\
\text { dialogues and this } \\
\text { and that you } \\
\text { know... WRITE } \\
\text { program and also I } \\
\text { create something for } \\
\text { my students...My } \\
\text { experience and also } \\
\text { I use that the } \\
\text { WRITE program as } \\
\text { a base, so a little } \\
\text { unit - those are the } \\
\text { fixed, an } \\
\text { autobiography } \\
\text { incident, descriptive } \\
\text { writing... Summary } \\
\text { writing, compare } \\
\text { and contrast, those } \\
\text { are things that we } \\
\text { need to do, to have } \\
\text { the kids, especially } \\
7^{\text {th }} \text { graders, writing. }\end{array}$ & $\begin{array}{l}\text { * I use [the district } \\
\text { curriculum], I use it } \\
\text { at times when for } \\
\text { example in a pinch } \\
\text { when I don't have } \\
\text { time to plan anything } \\
\text { or when I need a } \\
\text { certain type of } \\
\text { structure but, when I } \\
\text { can't... I see the } \\
\text { value in creating an } \\
\text { organic curriculum } \\
\text { with things that are } \\
\text { relevant to the } \\
\text { students. } \\
\text { * So, I use mostly } \\
\text { the district-adopted } \\
\text { text for ESL } 1 / 2 \text { its } \\
\text { Keys to Learning, } \\
\text { and Champion's red } \\
\text { for ESL three, four } \\
\text { its champion's blue. } \\
\text { Some of the Quest } \\
\text { and then I do } \\
\text { supplement with a } \\
\text { lot of my own } \\
\text { material, so } \\
\text { sometimes I'll use } \\
\text { Breaking News } \\
\text { English, sometimes } \\
\text { I'll just use images } \\
\text { from Google and } \\
\text { then also some } \\
\text { holidays and some } \\
\text { culture too } \\
\text { depending on the } \\
\text { season and so... } \\
\text { *Well in the, in a } \\
\text { more ideal world } \\
\text { they are, there would } \\
\text { be much more } \\
\text { it's just kind of } \\
\text { going off of what's } \\
\text { in the curriculum and }\end{array}$ & $\begin{array}{l}\text { One ESL teacher } \\
\text { stated that there was } \\
\text { no actual ESL } \\
\text { curriculum when she } \\
\text { began teaching. } \\
\text { There was a } \\
\text { program that utilized } \\
\text { worksheets and was } \\
\text { thematically based } \\
\text { rather than focusing } \\
\text { on language } \\
\text { development. } \\
\text { About six or seven } \\
\text { years ago the district } \\
\text { recognized the need } \\
\text { for an ESL } \\
\text { curriculum. ESL } \\
\text { teachers were invited } \\
\text { to be a part of the } \\
\text { ESL pilot committee, } \\
\text { which met four times } \\
\text { during that year, and } \\
\text { the committee ended } \\
\text { up choosing Key's to } \\
\text { Learning for the ESL } \\
\text { l/2 students, as it } \\
\text { was unanimously } \\
\text { thought to be the } \\
\text { best choice. } \\
\text { Teachers like Keys, } \\
\text { as it has good } \\
\text { grammar and syntax } \\
\text { practice and } \\
\text { dialogues, but they } \\
\text { questions if the } \\
\text { information is } \\
\text { authentic enough for } \\
\text { students to retain. } \\
\text { The teachers have } \\
\text { also had WRITE } \\
\text { curricula for the past } \\
\text { ten years or so and } \\
\text { program. }\end{array}$ \\
\hline
\end{tabular}




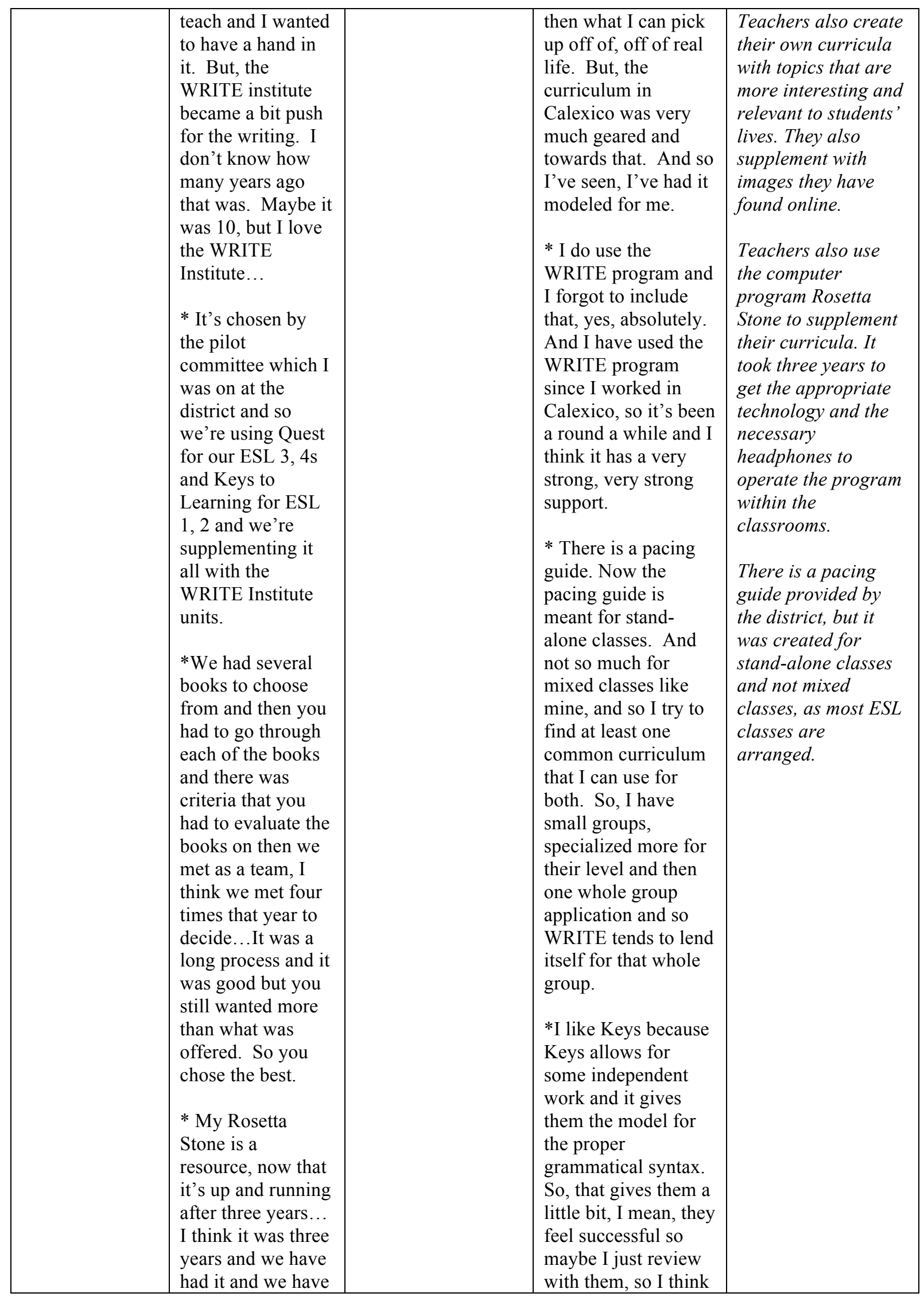




\begin{tabular}{|c|c|c|c|c|}
\hline & $\begin{array}{l}\text { not been able to } \\
\text { use it...For } \\
\text { multiple reasons. } \\
\text { First one, were } \\
\text { there computers } \\
\text { that were back } \\
\text { there we are not } \\
\text { able to load } \\
\text { Rosetta Stone } \\
\text { because they } \\
\text { didn't have enough } \\
\text { memory. Then, I } \\
\text { asked for new } \\
\text { computers. I was } \\
\text { told, I could not } \\
\text { have new } \\
\text { computers because } \\
\text { I was going to be } \\
\text { getting this lovely } \\
\text { system here but } \\
\text { that wasn't here } \\
\text { yet because the } \\
\text { district took six, } \\
\text { seven, eight } \\
\text { months }\end{array}$ & & $\begin{array}{l}\text { it's great. Now, I } \\
\text { don't know that } \\
\text { they're retaining } \\
\text { with Keys. I don't } \\
\text { know if the practice } \\
\text { is authentic enough } \\
\text { for them to retain it. }\end{array}$ & \\
\hline $\begin{array}{l}\text { Language } \\
\text { Instruction }\end{array}$ & $\begin{array}{l}\text { * [Language } \\
\text { instruction] } \\
\text { depends on what } \\
\text { your strength is. } \\
\text { Some people can } \\
\text { speak and some } \\
\text { can't write and do } \\
\text { other things. } \\
\text { * Well, it depends } \\
\text { like if we were } \\
\text { doing comparison } \\
\text { and contrast. So } \\
\text { whatever our long- } \\
\text { term objective is, } \\
\text { like that's kind of } \\
\text { born. But, when } \\
\text { we come back } \\
\text { from break, we're } \\
\text { doing comparison } \\
\text { and contrasting. } \\
\text { So, we will start } \\
\text { by learning } \\
\text { comparison and } \\
\text { contrast words and } \\
\text { then, we'll apply } \\
\text { language and then } \\
\text { we'll speak using } \\
\text { the language and }\end{array}$ & $\begin{array}{l}\text { * Okay for the } \\
\text { phonics right now, } \\
\text { we just hired a } \\
\text { teacher to do the } \\
\text { very, very beginning } \\
\text { levels. She is the } \\
\text { one that helps me } \\
\text { with that...It's } \\
\text { helpful because } \\
\text { somebody like } \\
\text { filters, some of } \\
\text { those - the ability } \\
\text { for us and then } \\
\text { before they come to } \\
\text { me. So, that means } \\
\text { that they can move } \\
\text { faster. } \\
\text { *... we depend on } \\
\text { computers, we use } \\
\text { Learning Upgrade... } \\
\text { And TeenBiz and } \\
\text { also Lexia, it's a } \\
\text { program for them to } \\
\text { work on. So they } \\
\text { build their levels } \\
\text { with in that program } \\
\text { right there... and } \\
\text { then that's how - }\end{array}$ & $\begin{array}{l}\text { * Yeah the text is, so } \\
\text { for example I know } \\
\text { that, so I do try to } \\
\text { frontload as much as } \\
\text { I can for students } \\
\text { who I know will } \\
\text { need more support. I } \\
\text { also differentiate like } \\
\text { getting in proximity. } \\
\text { So, I have, the ones } \\
\text { that need me the } \\
\text { most very, very close } \\
\text { by, I also use a lot of } \\
\text { peer support and I } \\
\text { think you saw that. } \\
\text { Where I know there } \\
\text { is one of me and } \\
\text { more of them and so } \\
\text { I make sure that the } \\
\text { students were seated, } \\
\text { a weaker student is } \\
\text { seated with a } \\
\text { stronger student and } \\
\text { that stronger student } \\
\text { is encouraged to help } \\
\text { the other one. And } \\
\text { also for example, at } \\
\text { this point, I have an } \\
\text { idea of where my }\end{array}$ & $\begin{array}{l}\text { Teachers instruct } \\
\text { students by first } \\
\text { looking at their } \\
\text { strengths, oral or } \\
\text { written, and then } \\
\text { determining the best } \\
\text { course of instruction. } \\
\text { Teachers create } \\
\text { long-term objectives } \\
\text { and teach with the } \\
\text { "end in mind" to } \\
\text { assure the students } \\
\text { are building } \\
\text { necessary language } \\
\text { skills. The lessons all } \\
\text { fit together with one } \\
\text { major objective, } \\
\text { such as compare and } \\
\text { contrast, descriptive } \\
\text { writing, etc. the } \\
\text { vocabulary taught } \\
\text { during reading also } \\
\text { assists students with } \\
\text { their writing. } \\
\text { Teachers frontload } \\
\text { vocabulary and use } \\
\text { peer interaction in } \\
\text { supporting students, } \\
\text { language }\end{array}$ \\
\hline
\end{tabular}




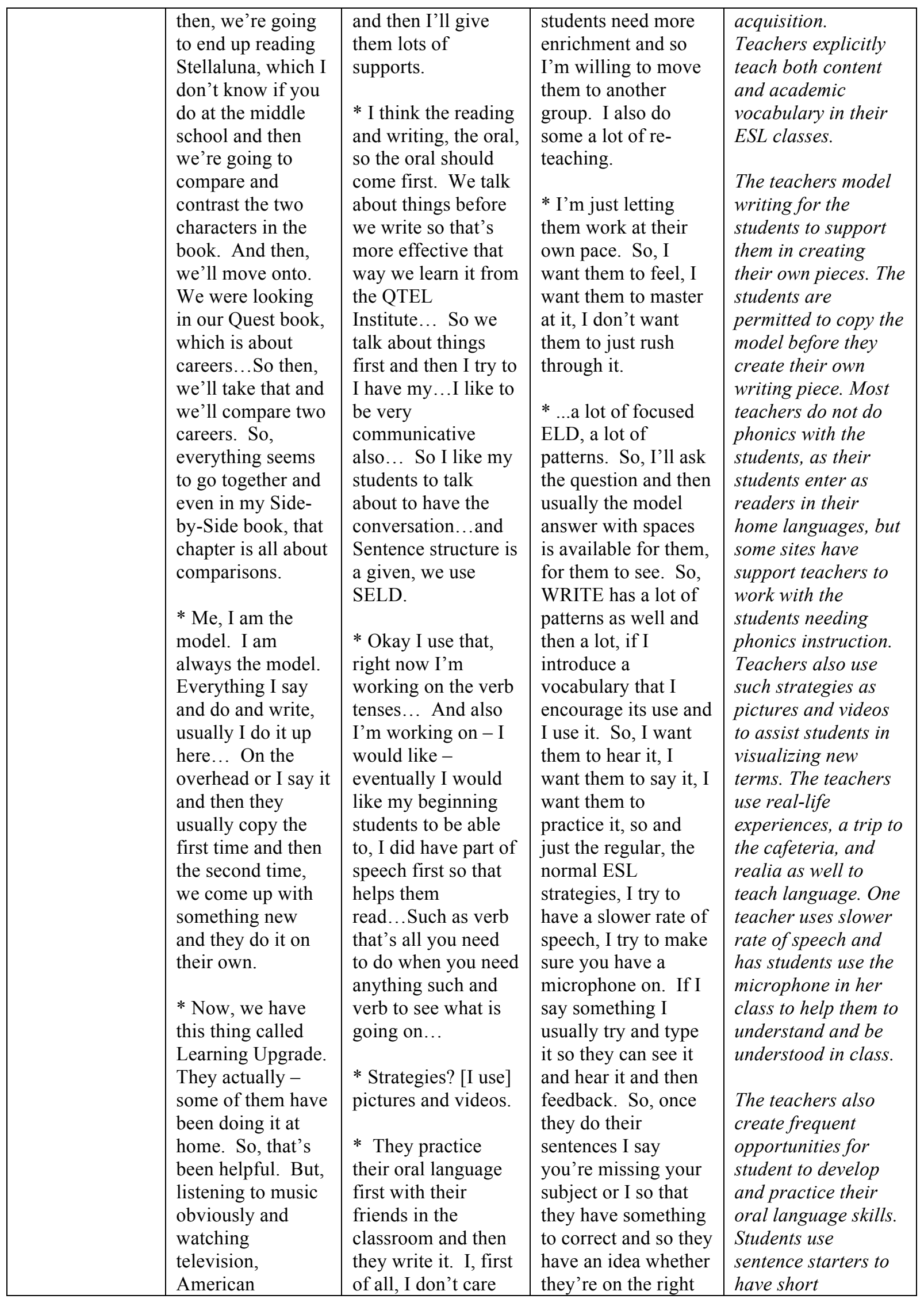




\begin{tabular}{|c|c|c|c|c|}
\hline & $\begin{array}{l}\text { television, are } \\
\text { things that I ask } \\
\text { them to do at } \\
\text { home... and } \\
\text { writing, I guess I } \\
\text { haven't really } \\
\text { asked them to do } \\
\text { on their own. I } \\
\text { mean they could } \\
\text { write journals, but, } \\
\text { most boys are not } \\
\text { going to write } \\
\text { journals. } \\
\text { *[With reluctant } \\
\text { speakers]...I am } \\
\text { just right up in } \\
\text { your face. Hi, how } \\
\text { are you? You } \\
\text { have anything to } \\
\text { say today. So I } \\
\text { guess you want to } \\
\text { spot it without } \\
\text { taking that ... you } \\
\text { know... some } \\
\text { people would say } \\
\text { the affective filter, } \\
\text { you don't want to } \\
\text { damage that and I } \\
\text { don't think I } \\
\text { would do that right } \\
\text { off the gate but I } \\
\text { do after a while } \\
\text { and they } \\
\text { understand. No } \\
\text { one has cried } \\
\text { about it, I don't } \\
\text { think. }\end{array}$ & $\begin{array}{l}\text { about whether they } \\
\text { have a lot of } \\
\text { mistakes, that's } \\
\text { okay but I } \\
\text { encourage them to } \\
\text { write...Also, the } \\
\text { cafeteria ladies told } \\
\text { us that our kids } \\
\text { didn't know how to } \\
\text { order food. So what } \\
\text { I did was I went to } \\
\text { the cafeteria to take } \\
\text { pictures of the food, } \\
\text { and then I sent them } \\
\text { to my colleagues so } \\
\text { they could [create } \\
\text { authentic] lessons... } \\
\text { like hamburger... so } \\
\text { my camera is with } \\
\text { me at all of the time, } \\
\text { because everything } \\
\text { can be taught } \\
\text { through pictures. }\end{array}$ & $\begin{array}{l}\text { track or not. } \\
\text { Well reading skills, I } \\
\text { think, I do separate } \\
\text { them. And so } \\
\text { reading mostly } \\
\text { happens with the } \\
\text { Champion, there is } \\
\text { great text in there } \\
\text { with great } \\
\text { vocabulary. And so } \\
\text { we do, and we go } \\
\text { through the regular } \\
\text { reading strategies } \\
\text { looking at the } \\
\text { pictures, making } \\
\text { productions, looking } \\
\text { at words in context, } \\
\text { trying to get clues for } \\
\text { definitions and so we } \\
\text { do practice the } \\
\text { regular reading } \\
\text { strategies. Writing, I } \\
\text { think, is much more } \\
\text { targeted through } \\
\text { writing, so every } \\
\text { creating period there } \\
\text { is an essay that is } \\
\text { required and that that } \\
\text { also provides a } \\
\text { springboard for } \\
\text { speaking } \\
\text { opportunities and so } \\
\text { and a lot of group, } \\
\text { work and a lot of } \\
\text { presentations. } \\
\text { * Now when I saw } \\
\text { this one, right, this is } \\
\text { a trigger for me, so } \\
\text { to develop academic } \\
\text { vocabulary because } \\
\text { you know this is the } \\
\text { brick and mortar } \\
\text { words and so as we } \\
\text { were doing our four } \\
\text { snorms], or as we're } \\
\text { developing ours, so } \\
\text { teachers didn't feel }\end{array}$ & $\begin{array}{l}\text { conversation in } \\
\text { English and } \\
\text { sometimes do short } \\
\text { oral presentations. } \\
\text { Teachers encourage } \\
\text { authentic } \\
\text { conversations. } \\
\text { The district } \\
\text { encourages students } \\
\text { to use a computer } \\
\text { program called } \\
\text { Learning Upgrade at } \\
\text { school and at home, } \\
\text { to practice reading } \\
\text { skills and develop } \\
\text { language. Some } \\
\text { teachers have access } \\
\text { to other computer } \\
\text { programs, Teen Biz } \\
\text { and Lexia, if their } \\
\text { site has purchased } \\
\text { such technology } \\
\text { licenses. } \\
\text { The teachers } \\
\text { challenge reluctant } \\
\text { speakers, yet in a } \\
\text { comfortable and low } \\
\text { anxiety manner. } \\
\text { Students are } \\
\text { permitted to work at } \\
\text { their own pace. } \\
\text { Reluctant speakers } \\
\text { may also be } \\
\text { provided with a } \\
\text { script or visual cues } \\
\text { to assist them. }\end{array}$ \\
\hline
\end{tabular}




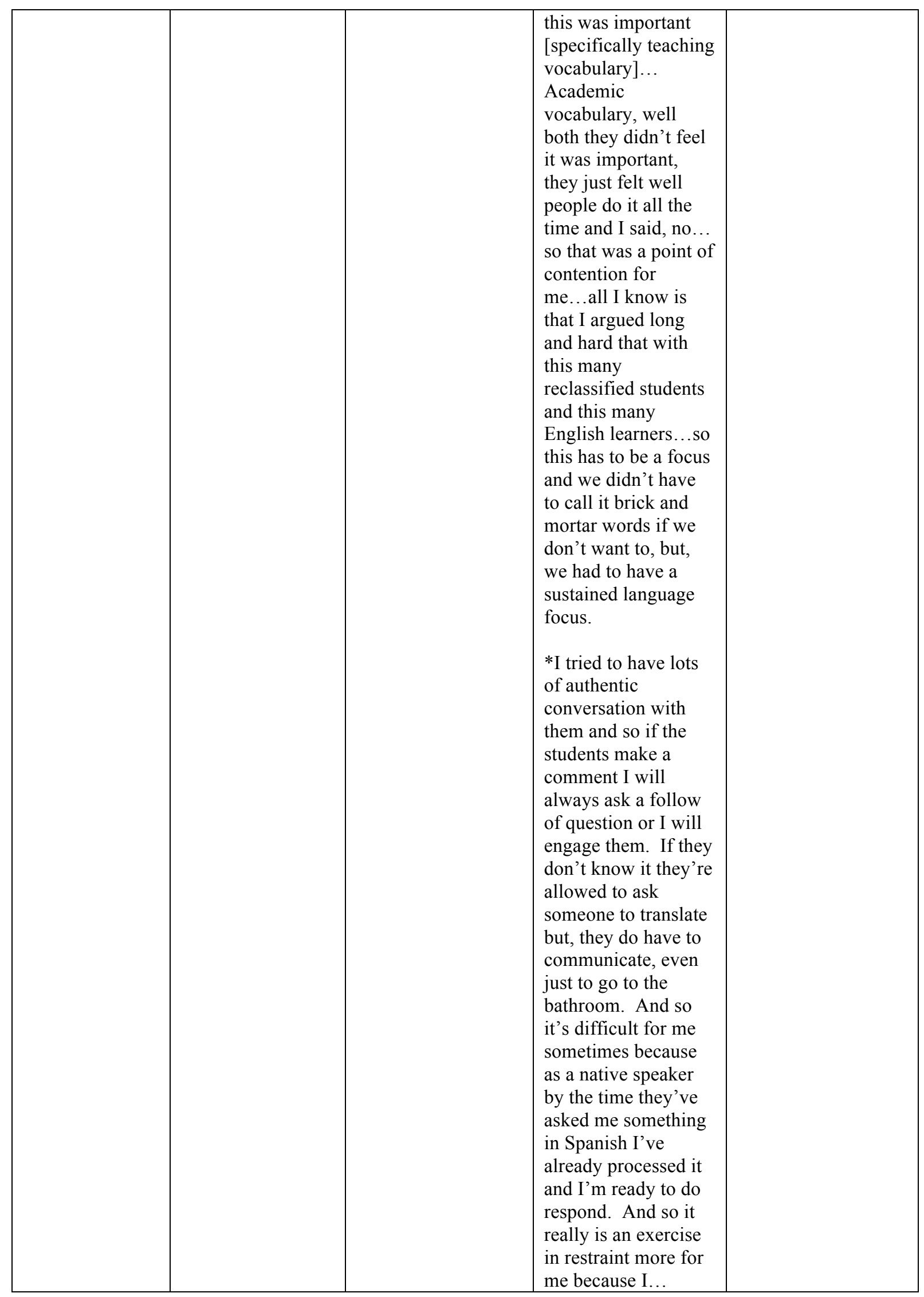




\begin{tabular}{|c|c|c|c|c|}
\hline & & & $\begin{array}{l}\text { * Well they need } \\
\text { support they're } \\
\text { reluctant than they } \\
\text { could have a visual } \\
\text { or they can have a } \\
\text { cue or they can have } \\
\text { a script or to just we } \\
\text { focus on the content. } \\
\text { And so I think } \\
\text { reluctant speakers } \\
\text { can be reluctant for a } \\
\text { little bit. I think } \\
\text { there is a time, I } \\
\text { mean } 30 \text { days I think } \\
\text { is a lot for basic } \\
\text { yes... no... I need to } \\
\text { go the bathroom and } \\
\text { so I want to make } \\
\text { sure that they have } \\
\text { the words that they } \\
\text { need and even if it's } \\
\text { like [one ESL } \\
\text { student] starting to } \\
\text { very slowly produce } \\
\text { but, I'm worried so } \\
\text { that silent period for } \\
\text { me is also like a } \\
\text { monitoring piece of, } \\
\text { if it's too long then } \\
\text { that to me is } \\
\text { signaling there is } \\
\text { other things going } \\
\text { on. }\end{array}$ & \\
\hline $\begin{array}{c}\text { Content } \\
\text { Instruction }\end{array}$ & $\begin{array}{l}\text { *Yeah, I've had... } \\
\text { because just like } \\
\text { we have them for } \\
\text { two hours. So the } \\
\text { second hour I } \\
\text { might - they might } \\
\text { be like having } \\
\text { their math } \\
\text { homework over } \\
\text { here and other one } \\
\text { is copying. So, } \\
\text { I've tried to train } \\
\text { them that they } \\
\text { need to ask me for } \\
\text { time. They need } \\
\text { to ask me for the } \\
\text { questions and they } \\
\text { are starting to get } \\
\text { that now...now } \\
\text { that's nine weeks }\end{array}$ & $\begin{array}{l}\text { * So my principal } \\
\text { decided to have me } \\
\text { just focus on the } \\
\text { things it and also my } \\
\text { ability to do the } \\
\text { math thing, because } \\
\text { I use the language a } \\
\text { lot heavily and the } \\
\text { language support } \\
\text { and so that they can } \\
\text { move the math so } \\
\text { fast. } \\
\text { * Okay these are } \\
\text { things I created for } \\
\text { my students by } \\
\text { using language... } \\
\text { Plus, minus, } \\
\text { multiply and } \\
\text { divide...[I use }\end{array}$ & $\begin{array}{l}\text { * The district made a } \\
\text { strong commitment } \\
\text { to work with West } \\
\text { Ed and QTEL and I } \\
\text { mean we're } \\
\text { expecting some kind } \\
\text { of a yield and as part } \\
\text { of that agreement } \\
\text { they did train the } \\
\text { principles, now some } \\
\text { get it more than } \\
\text { others and again I } \\
\text { think it's such a } \\
\text { complex issue, it's } \\
\text { really, really hard to } \\
\text { understand and so } \\
\text { you know it kind of } \\
\text { sort of I mean I don't } \\
\text { know my principle } \\
\text { has an idea... }\end{array}$ & $\begin{array}{l}\text { Teachers give } \\
\text { students time in ESL } \\
\text { class to work on } \\
\text { homework for } \\
\text { content classes. They } \\
\text { support students with } \\
\text { their mainstream } \\
\text { classes by giving } \\
\text { them time and } \\
\text { assistance. } \\
\text { Students are } \\
\text { permitted to help one } \\
\text { another, and } \\
\text { students use online } \\
\text { resources to } \\
\text { supplement their } \\
\text { content classes. One } \\
\text { teacher receives } \\
\text { questions from the }\end{array}$ \\
\hline
\end{tabular}




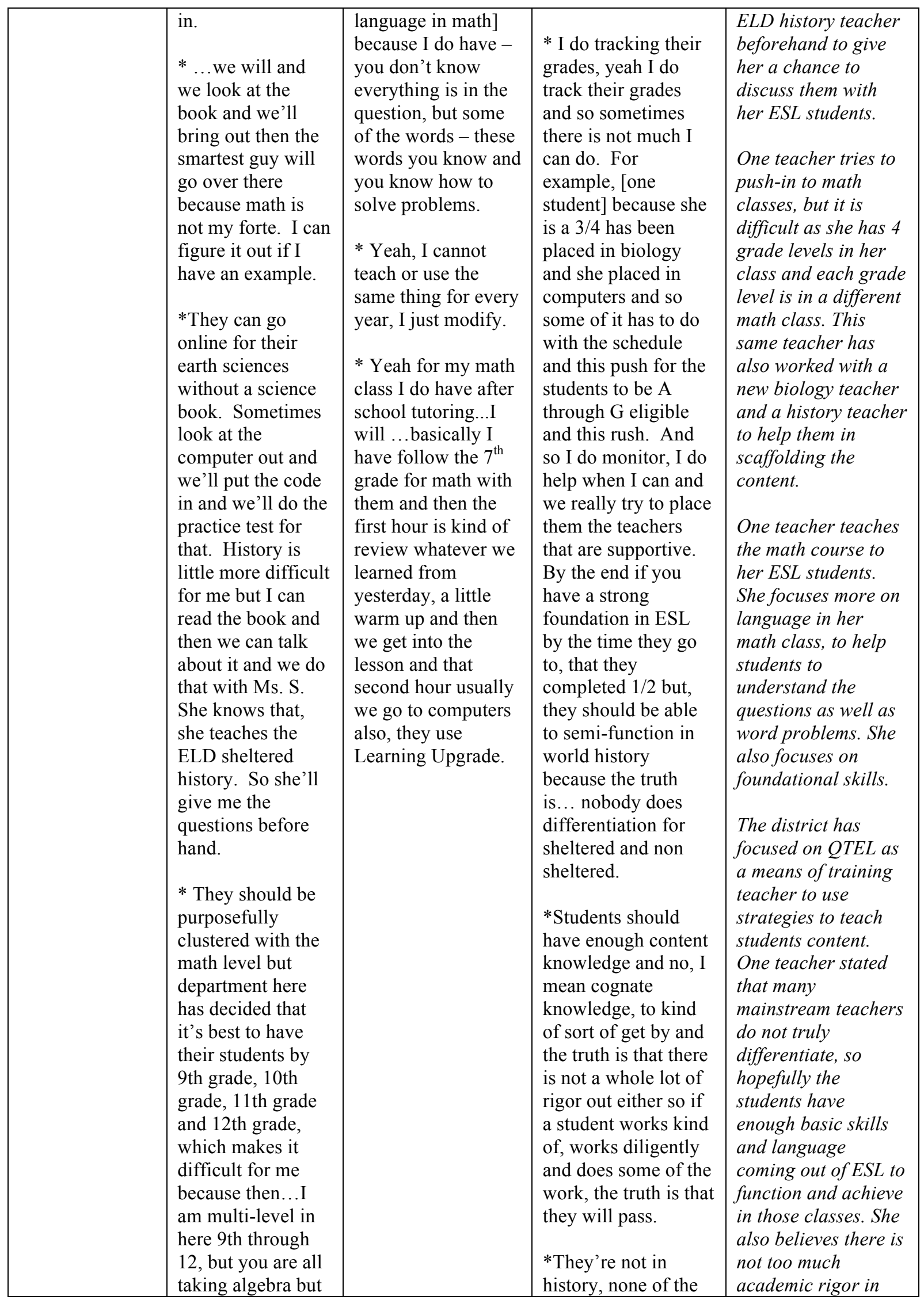




\begin{tabular}{|c|c|c|c|c|}
\hline & $\begin{array}{l}\text { you are not in the } \\
\text { same class. So, I } \\
\text { come in and } \\
\text { support you but } \\
\text { you are in four } \\
\text { different periods. } \\
\text { * One year, the } \\
\text { biology teacher } \\
\text { was new. So, I } \\
\text { spent my third } \\
\text { period with the } \\
\text { biology teacher. } \\
\text { Last year, I spent it } \\
\text { with the history } \\
\text { teacher, which is } \\
\text { nice but now I feel } \\
\text { like you know } \\
\text { because I spend a } \\
\text { lot of my time on } \\
\text { my testing. Now, } \\
\text { I'll go in there just } \\
\text { because of who we } \\
\text { are. }\end{array}$ & & $\begin{array}{l}\text { ESL } 1 / 2 \text { students are } \\
\text { in history and that's } \\
\text { purposeful, we } \\
\text { don't...we won't put } \\
\text { them in until they are } \\
\text { ESL } 3 / 4 \text { and maybe } \\
\text { even second } \\
\text { semester of } \\
\text { 3/4...instead [the } \\
\text { students are in] art } \\
\text { because it's not } \\
\text { language intensive, } \\
\text { so we picked their } \\
\text { classes intentionally } \\
\text { we picked them not } \\
\text { to have very } \\
\text { language intensive } \\
\text { courses, and so art, } \\
\text { physics for some } \\
\text { depending on the } \\
\text { teacher interview and } \\
\text { the student...Physics } \\
\text { and Spanish. So, } \\
\text { they have Spanish, } \\
\text { PE, and then two } \\
\text { periods of English. }\end{array}$ & $\begin{array}{l}\text { content classes, so if } \\
\text { kids work hard, they } \\
\text { will pass. Students } \\
\text { are purposefully not } \\
\text { placed in language } \\
\text { intensive classes } \\
\text { such as history until } \\
\text { they are at the ESL } \\
\text { 3/4 level, and } \\
\text { sometimes even } \\
\text { second semester. }\end{array}$ \\
\hline $\begin{array}{c}\text { Teacher's } \\
\text { Attitudes/ } \\
\text { Expectations }\end{array}$ & $\begin{array}{l}\text { * Back in the day I } \\
\text { would come in } \\
\text { here for like } \\
\text { Halloween and I } \\
\text { have my cake, my } \\
\text { big pumpkin and I } \\
\text { would very, very } \\
\text { involved and I } \\
\text { guess I am not as I } \\
\text { don't know, } \\
\text { touchy feely as I } \\
\text { used to be and I } \\
\text { think that might be } \\
\text { because of the } \\
\text { high stakes with } \\
\text { the Exit exam that } \\
\text { we have to pass it. } \\
\text { We don't have } \\
\text { time... } \\
\text { * Yeah, I had the } \\
\text { time and the } \\
\text { energy I guess to } \\
\text { do that and now I } \\
\text { think the bit push } \\
\text { is to pass the Exit } \\
\text { test and to get my } \\
\text { CST scores up. So }\end{array}$ & $\begin{array}{l}\text { * I believe [I wanted } \\
\text { to teach ESL] } \\
\text { because I'm a } \\
\text { second language } \\
\text { learner } \\
\text { myself... Yeah, so I } \\
\text { can relate to } \\
\text { whatever I } \\
\text { do...Yeah I just } \\
\text { wanted to share my } \\
\text { experience in my } \\
\text { teaching too. } \\
\text { * So that's when I } \\
\text { started - I had a } \\
\text { chance to sit with } \\
\text { them and one-on- } \\
\text { one - I work with } \\
\text { them one-on-one } \\
\text { and then I just loved } \\
\text { to the way they } \\
\text { learn thing and they } \\
\text { are very eager. } \\
\text { * High, all of my } \\
\text { students would go to } \\
\text { college.... That is } \\
\text { my ultimate }\end{array}$ & $\begin{array}{l}\text { * I offered to take } \\
\text { the ESL class, since I } \\
\text { was already there } \\
\text { and since I have the } \\
\text { content knowledge } \\
\text { and... } \\
\text { * ...it's a highly } \\
\text { specialized and we } \\
\text { don't have a person } \\
\text { with the... } \\
\text { methodology and } \\
\text { pedagogy, for ESL... } \\
\text { we don't. So, I } \\
\text { didn't want it, I } \\
\text { didn't want to go, I } \\
\text { wanted to train } \\
\text { someone and it just } \\
\text { kind of happened } \\
\text { that I ended up } \\
\text { taking over. } \\
\text { * As an ESL teacher } \\
\text { when I first started I } \\
\text { started in Calexico } \\
\text { and I didn't want to } \\
\text { teach ESL, it was } \\
\text { the, I wanted, this is }\end{array}$ & $\begin{array}{l}\text { Teachers felt they } \\
\text { were able to be more } \\
\text { involved in years } \\
\text { past. They had more } \\
\text { time, energy, and } \\
\text { resources to connect } \\
\text { with students prior } \\
\text { to push for high } \\
\text { stakes testing. } \\
\text { Teachers feel } \\
\text { overwhelmed by lack } \\
\text { of time and their } \\
\text { many } \\
\text { responsibilities. They } \\
\text { also feel they are } \\
\text { highly qualified to } \\
\text { teach ESL, as they } \\
\text { have very strong } \\
\text { backgrounds and are } \\
\text { passionate about } \\
\text { teaching such } \\
\text { students. } \\
\text { One teacher also } \\
\text { worked as an ELST. } \\
\text { Due to budget cuts } \\
\text { and staff reductions, } \\
\text { there was no one to }\end{array}$ \\
\hline
\end{tabular}




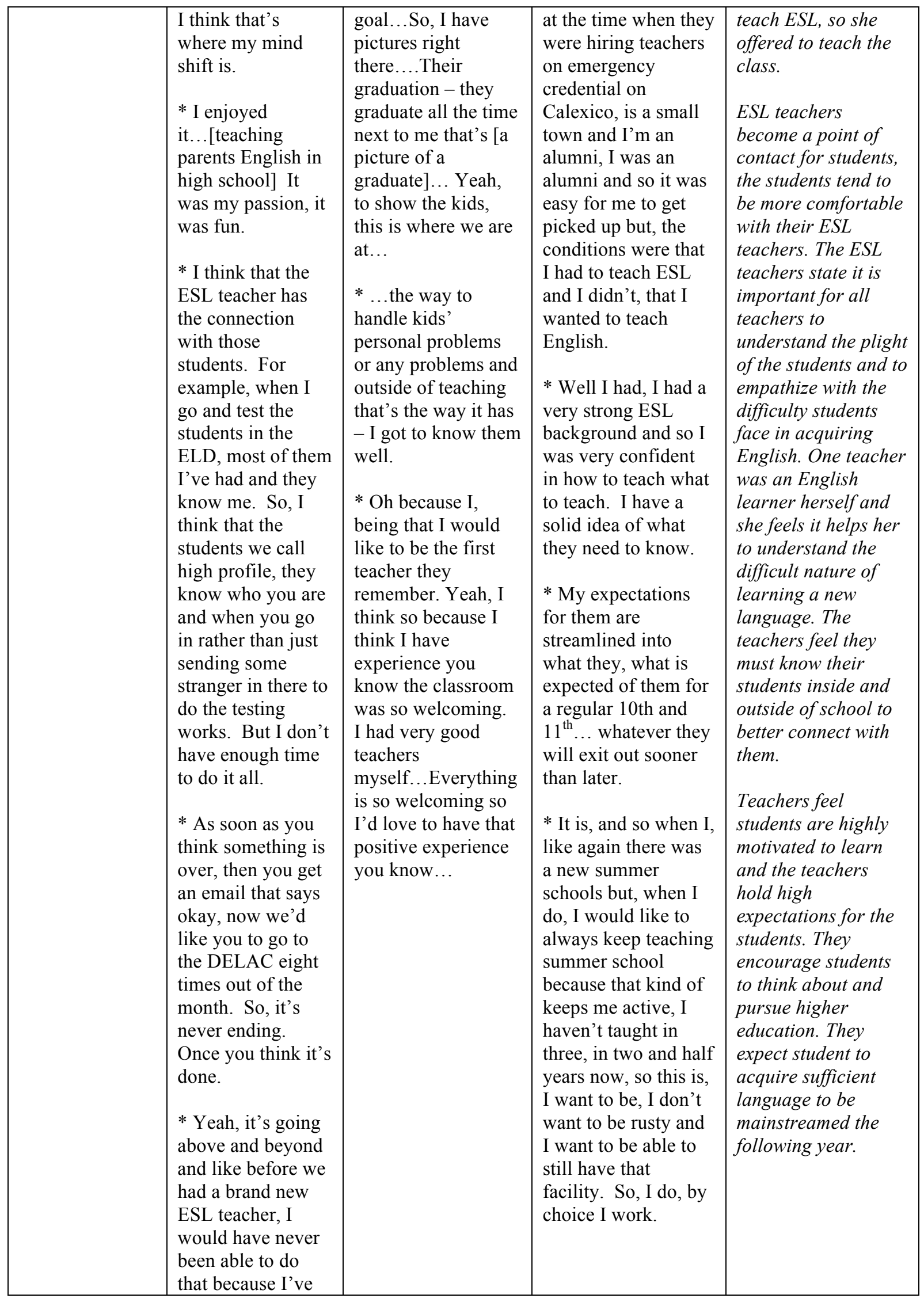




\begin{tabular}{|c|c|c|c|c|}
\hline & $\begin{array}{l}\text { been doing it for } \\
18 \text { years, I can do } \\
\text { this without having } \\
\text { to spend hours and } \\
\text { hours on the } \\
\text { planning. } \\
\text { *You have people } \\
\text { that really care for } \\
\text { English learners } \\
\text { and you have } \\
\text { people that really } \\
\text { feel that you } \\
\text { should speak } \\
\text { English before you } \\
\text { come to America } \\
\text { and so, sorry I } \\
\text { can't help you. } \\
\text { *[Students need] a } \\
\text { teacher that } \\
\text { actually cares and } \\
\text { you can tell that } \\
\text { they care about } \\
\text { you and they } \\
\text { respect that you } \\
\text { might be } \\
\text { struggling because } \\
\text { English is your } \\
\text { second language. }\end{array}$ & & & \\
\hline $\begin{array}{c}\text { Culturally } \\
\text { Responsive } \\
\text { Teaching }\end{array}$ & $\begin{array}{l}\text { * Many kids are } \\
\text { Vietnamese and } \\
\text { that's not my forte. } \\
\text { But we have } \\
\text { students that, for } \\
\text { example, I do have } \\
\text { students that come } \\
\text { back and they TA, } \\
\text { like I have the one } \\
\text { in the morning } \\
\text { who ended with } \\
\text { me with ESL 5/ } 6 \\
\text { and now she is in } \\
\text { the regular } \\
\text { program and she } \\
\text { comes back to } \\
\text { tutor her own } \\
\text { ethnicity. She uses } \\
\text { her Spanish with } \\
\text { her students. So, } \\
\text { it's kind of like a } \\
\text { payback, which I } \\
\text { love. I love it } \\
\text { when they come }\end{array}$ & $\begin{array}{l}\text { * Children, yes I } \\
\text { [give primary } \\
\text { language support] in } \\
\text { Chinese and also I } \\
\text { speak Lao, Thai a } \\
\text { little bit and } \\
\text { Vietnamese a little } \\
\text { bit. } \\
\text { * My family again, } \\
\text { they were refugees } \\
\text { they came here } \\
\text { before me, so I just } \\
\text { joined them. And } \\
\text { most of my kids all } \\
\text { here are refugees...I } \\
\text { told them about my } \\
\text { story, I told them } \\
\text { that I left home } \\
\text { when I was } 15,14- \\
15 \text { years old. } \\
\text { * Okay, for example } \\
\text { when we talk about }\end{array}$ & $\begin{array}{l}\text { * I try to have them } \\
\text { compare and contrast } \\
\text { with what situations } \\
\text { are like in their home } \\
\text { country. And then } \\
\text { here, there is; a lot of } \\
\text { cognates Spanish, } \\
\text { now Vietnamese } \\
\text { there isn't that many, } \\
\text { but, they do } \\
\text { have...Lee } \\
\text { especially has a lot } \\
\text { of language and } \\
\text { more so then Anh. } \\
\text { And so I'm able to } \\
\text { use him to give a } \\
\text { different perspective, } \\
\text { so the kids will ask } \\
\text { him is there } \\
\text { Christmas in } \\
\text { Vietnam or what was } \\
\text { your school like and } \\
\text { so he offers a lot of } \\
\text { information on what }\end{array}$ & $\begin{array}{l}\text { One teacher } \\
\text { encourages students } \\
\text { to come back and } \\
\text { tutor newer students } \\
\text { after they have } \\
\text { completed the } \\
\text { program. She feels it } \\
\text { is a good "pay } \\
\text { back" for old } \\
\text { students to help } \\
\text { newer students. } \\
\text { Teachers use } \\
\text { multicultural texts } \\
\text { and discuss different } \\
\text { cultures and customs } \\
\text { from around the } \\
\text { world, and compare } \\
\text { them to students, } \\
\text { home cultures. } \\
\text { Teachers make } \\
\text { comparisons } \\
\text { between cultures to } \\
\text { help students better }\end{array}$ \\
\hline
\end{tabular}




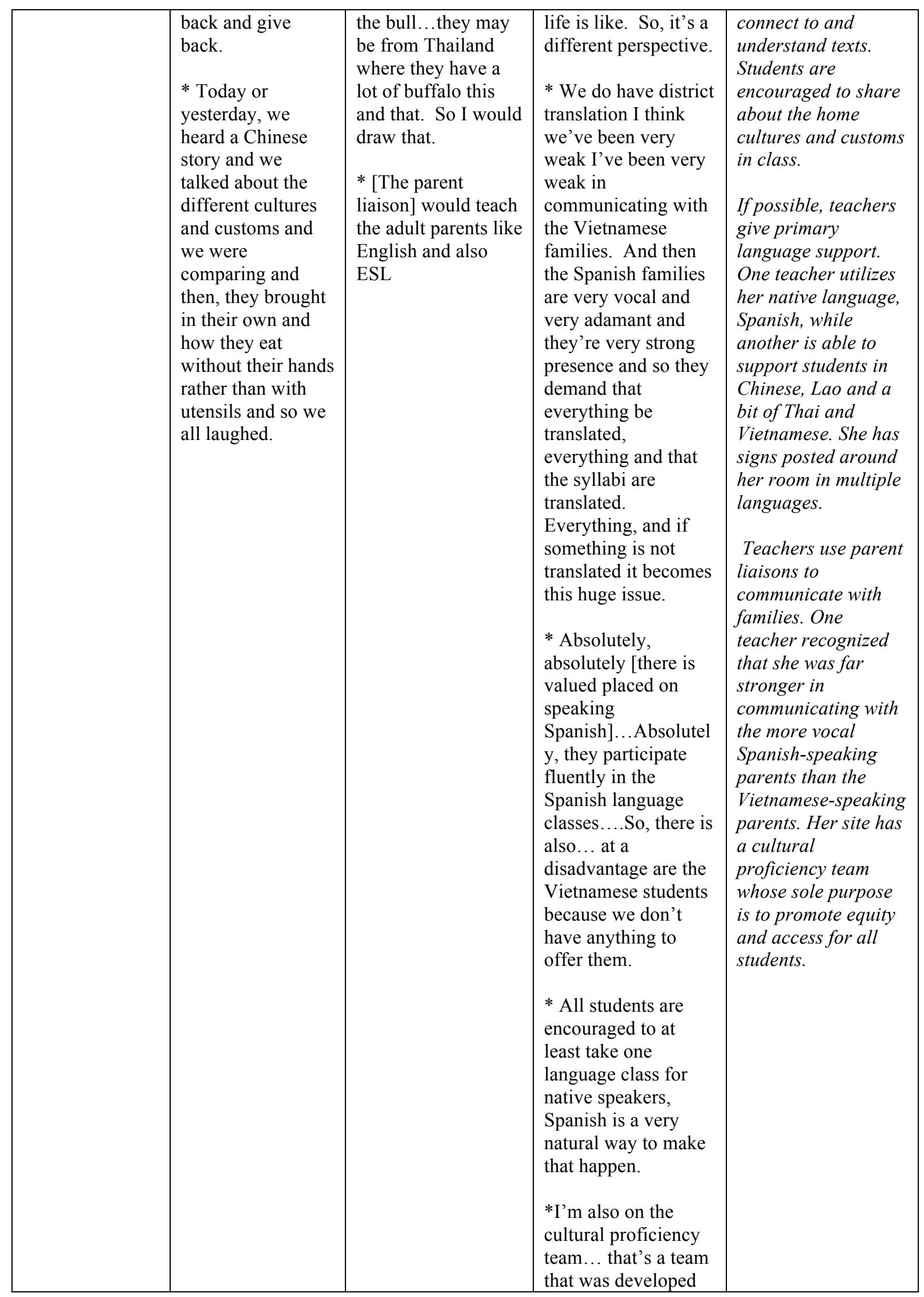




\begin{tabular}{|c|c|c|c|c|}
\hline & & & $\begin{array}{l}\text { here to help promote } \\
\text { equity and access. }\end{array}$ & \\
\hline $\begin{array}{c}\text { Pedagogical } \\
\text { Orientations/ } \\
\text { Beliefs }\end{array}$ & $\begin{array}{l}\text { * Facilitator, I } \\
\text { guess I would say. } \\
\text { I have to be your } \\
\text { second mother } \\
\text { more so than just a } \\
\text { regular teacher } \\
\text { because I am } \\
\text { monitoring not just } \\
\text { our class but all of } \\
\text { the classes and all } \\
\text { of the teachers and } \\
\text { what they are } \\
\text { doing well and } \\
\text { what they are not } \\
\text { doing well. } \\
\text { * I am in your } \\
\text { business but it's to } \\
\text { help you because } \\
\text { at home I don't } \\
\text { think that they are } \\
\text { always } \\
\text { knowledgeable of } \\
\text { what they need to } \\
\text { have done like we } \\
\text { don't have the } \\
\text { parents online } \\
\text { looking at the } \\
\text { grades and the } \\
\text { attendance and } \\
\text { then we have right } \\
\text { phone numbers } \\
\text { and I think that's } \\
\text { where I just kind } \\
\text { of take over that } \\
\text { role that they are } \\
\text { missing. } \\
\text { * [Cooperative } \\
\text { grouping] depends } \\
\text { on what the } \\
\text { assignment is. If } \\
\text { I'll do different } \\
\text { levels of } \\
\text { proficiency or } \\
\text { different } \\
\text { ethnicities and } \\
\text { that's usually how } \\
\text { I always started } \\
\text { because if I put all } \\
\text { my Vietnamese }\end{array}$ & $\begin{array}{l}\text { * Yeah as a coach... } \\
\text { Coaching them, I } \\
\text { teach the skills just } \\
\text { like as a sports } \\
\text { player and then I } \\
\text { teach them the skills } \\
\text { and they learn the } \\
\text { skills and they can } \\
\text { perform well... } \\
\text { How do I group the } \\
\text { kids, I will look at } \\
\text { their access like } \\
\text { writing piece...And } \\
\text { also ability wise and } \\
\text { I group them, I } \\
\text { would draw, pull } \\
\text { them, you have not } \\
\text { seen it yet you will } \\
\text { see that...In groups } \\
\text { and I work with } \\
\text { them to their } \\
\text { specific needs. } \\
\text { * Yeah and my goal } \\
\text { is I'm not like - I } \\
\text { kind of even though } \\
\text { we don't have to do } \\
\text { this... we just have } \\
\text { to focus on the kid } \\
\text { who is very highly } \\
\text { motivated and give } \\
\text { them the extra pat } \\
\text { on their shoulders to } \\
\text { push them, give } \\
\text { them special } \\
\text { attention. } \\
\text { * Yeah my trick [for } \\
\text { good behavior] is } \\
\text { you know... I don't } \\
\text { know... what I think } \\
\text { I have developed a } \\
\text { presence that you } \\
\text { know. }\end{array}$ & $\begin{array}{l}\text { * I didn't want to } \\
\text { take over the class } \\
\text { and micromanage, } \\
\text { what I wanted to do } \\
\text { was build capacity. } \\
\text { And we never got to } \\
\text { that point. } \\
\text { * What I have done, } \\
\text { again because I don't } \\
\text { want to take over the } \\
\text { situation, what I've } \\
\text { done is I have } \\
\text { prepared people. So, } \\
\text { if I find someone } \\
\text { that's capable or } \\
\text { someone who is a } \\
\text { solid English } \\
\text { teacher, well I do set } \\
\text { up the program and } \\
\text { make sure that } \\
\text { they're supported } \\
\text { with materials, with } \\
\text { training and with, } \\
\text { whatever supplies, } \\
\text { whatever they need. } \\
\text { * Well it's to prepare } \\
\text { them, to prepare } \\
\text { them to exit to the } \\
\text { regular classes and } \\
\text { also my role as } \\
\text { ELST kind of } \\
\text { expands that teacher } \\
\text { role and so it goes } \\
\text { beyond what } \\
\text { happens with the } \\
\text { curriculum and so if } \\
\text { the children need an } \\
\text { advocate or if they } \\
\text { need someone to } \\
\text { change their classes } \\
\text { or if they need } \\
\text { someone to talk to } \\
\text { them about behavior } \\
\text { and to contact the } \\
\text { parents, to make sure } \\
\text { that they're eating } \\
\text { lunch and so my role } \\
\text { as an ELST allows } \\
\text { me to go beyond the }\end{array}$ & $\begin{array}{l}\text { Teachers view } \\
\text { themselves as } \\
\text { facilitators or } \\
\text { coaches, they teach } \\
\text { students skills and } \\
\text { then students have a } \\
\text { chance to practice } \\
\text { the new skills. They } \\
\text { are involved with } \\
\text { their students both in } \\
\text { their classes, but } \\
\text { also by monitoring } \\
\text { them in mainstream } \\
\text { classes. Teachers } \\
\text { take a highly active } \\
\text { role in the individual } \\
\text { students' education } \\
\text { and advocate for } \\
\text { them, both } \\
\text { academically and } \\
\text { ensuring that their } \\
\text { needs are being met. } \\
\text { Teachers group } \\
\text { students } \\
\text { heterogeneously } \\
\text { according to both } \\
\text { language and } \\
\text { proficiency level, } \\
\text { trying to mix } \\
\text { languages and } \\
\text { providing language } \\
\text { models. } \\
\text { Students' needs are } \\
\text { viewed individually } \\
\text { to best support them } \\
\text { at their point of } \\
\text { need. Students are } \\
\text { grouped according } \\
\text { to proficiency levels } \\
\text { and particular areas } \\
\text { of need. }\end{array}$ \\
\hline
\end{tabular}




\begin{tabular}{|c|c|c|c|c|}
\hline & $\begin{array}{l}\text { together, they all } \\
\text { start speaking } \\
\text { Vietnamese rather } \\
\text { than the target } \\
\text { language. So, both } \\
\text { ways... It's } \\
\text { flexible with my } \\
\text { terms. } \\
\text { * That's been like } \\
\text { when they go back } \\
\text { to that, there you } \\
\text { have an } \\
\text { individualized } \\
\text { plan, I might give } \\
\text { the lesson for all } \\
\text { but I know that she } \\
\text { can only write a } \\
\text { paragraph or he } \\
\text { can actually infer } \\
\text { and can do more. } \\
\text { * ...everything } \\
\text { else that I do } \\
\text { throughout the } \\
\text { year, to me are } \\
\text { things that will be } \\
\text { on the CAHSEE. } \\
\text { So I don't think I } \\
\text { need the direct } \\
\text { instruction on that. } \\
\text { * ...it's just } \\
\text { building that } \\
\text { community before } \\
\text { you pull out [the } \\
\text { language] }\end{array}$ & & $\begin{array}{l}\text { expectations of just, } \\
\text { of not just, but, of } \\
\text { being the teacher } \\
\text { there, the provider of } \\
\text { the academic } \\
\text { content. }\end{array}$ & \\
\hline $\begin{array}{c}\text { Academic } \\
\text { Self-Concept }\end{array}$ & $\begin{array}{l}\text { * We are really } \\
\text { comfortable in } \\
\text { here but the } \\
\text { teachers that walk } \\
\text { in here say that } \\
\text { they have them in } \\
\text { their regular } \\
\text { classes and they } \\
\text { never say a word, } \\
\text { but they won't } \\
\text { shut up in here } \\
\text { because they are } \\
\text { comfortable. } \\
\text { * Let's say Ann, } \\
\text { because Ann is } \\
\text { silent. She sits }\end{array}$ & $\begin{array}{l}\text { * Well, I think that } \\
\text { they have problems } \\
\text { especially when } \\
\text { they get to see } \\
\text { themselves moving } \\
\text { into a regular class } \\
\text { thing. } \\
\text { * They are confident } \\
\text { when they are still } \\
\text { here...I think so, } \\
\text { yeah I think so.... } \\
\text { * I do [think } \\
\text { students are nervous } \\
\text { when they move to a } \\
\text { higher class] but, I }\end{array}$ & $\begin{array}{l}\text { *I also want to have } \\
\text { a really supportive } \\
\text { environment so that } \\
\text { kids are comfortable } \\
\text { to speak and that's } \\
\text { always a struggle } \\
\text { with ESL because } \\
\text { they're, they make } \\
\text { fun of each other of } \\
\text { if they make a } \\
\text { mistake, they're not } \\
\text { as patient. } \\
\text { * They do feel, they } \\
\text { do feel, they } \\
\text { understand that } \\
\text { they're language }\end{array}$ & $\begin{array}{l}\text { Students are far } \\
\text { more talkative in } \\
\text { their ESL classes } \\
\text { because they are } \\
\text { more comfortable. } \\
\text { They sometimes } \\
\text { tease each other } \\
\text { when they make } \\
\text { mistakes, teacher } \\
\text { tries to create } \\
\text { supportive non- } \\
\text { threatening } \\
\text { environment. } \\
\text { Students are far } \\
\text { more successful and } \\
\text { confident in ESL }\end{array}$ \\
\hline
\end{tabular}




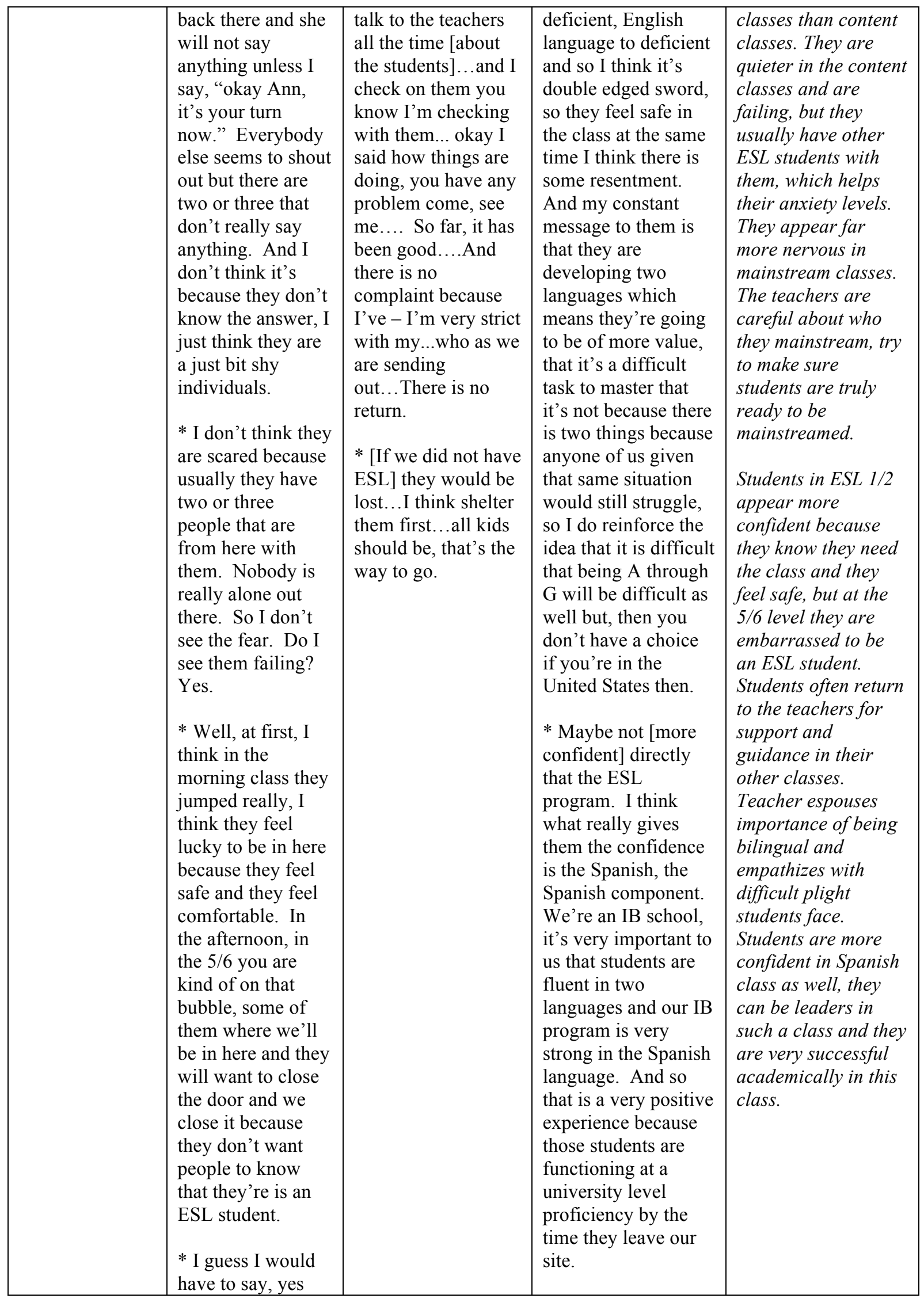




\begin{tabular}{|l|l|l|l|l|}
\hline & $\begin{array}{l}\text { [students appear } \\
\text { more confident } \\
\text { academically], } \\
\text { because they come } \\
\text { back and ask me } \\
\text { even when they } \\
\text { are not in my class } \\
\text { any more. They } \\
\text { come back for help } \\
\text { with English or } \\
\text { graduation or } \\
\text { whatever. So, I } \\
\text { think, yes. }\end{array}$ & & & \\
\hline
\end{tabular}


Appendix $\mathrm{P}$

NAC Administrator Interview Analysis

\begin{tabular}{|c|c|c|c|}
\hline & $\begin{array}{c}\text { Administrator \#1 } \\
\text { ESL Program }\end{array}$ & $\begin{array}{c}\text { Administrator \#2 } \\
\text { ESL Program }\end{array}$ & Significant Themes \\
\hline Curricula & $\begin{array}{l}\text { * Well, we had to buy } \\
\text { materials from scratch... } \\
\text { there was nothing.... } \\
\text { other than the ESL } \\
\text { adoption, so we } \\
\text { purchased, we had to } \\
\text { buy things for social } \\
\text { studies, for science, for } \\
\text { math and then additional } \\
\text { things for English as } \\
\text { well. So, I don't know } \\
\text { what our budget was at } \\
\text { first year. And then the } \\
\text { second year we made } \\
\text { some changes in the } \\
\text { materials that we were } \\
\text { using so, we did by } \\
\text { some additional things } \\
\text { and now we've pretty } \\
\text { much settled into a } \\
\text { routine of what we are } \\
\text { able to purchase every } \\
\text { year. And we have } \\
\text { supported these classes } \\
\text { with a lot of classroom } \\
\text { supplies and we're not } \\
\text { able to do that as much } \\
\text { as we did the first three } \\
\text { years. } \\
\text { * ...we use the Keys to } \\
\text { Learning for the ESL } \\
\text { and that was chosen by } \\
\text { regular school adoption } \\
\text { committee through the } \\
\text { regular adoption } \\
\text { process. We used the } \\
\text { WRITE institute } \\
\text { materials also and that } \\
\text { was chosen really by the } \\
\text { department. We've } \\
\text { used WRITE institute } \\
\text { training materials for a } \\
\text { number of years and } \\
\text { with a lot of success and } \\
\text { so, we've been } \\
\text { integrating that in there. } \\
\text { The other materials were }\end{array}$ & $\begin{array}{l}\text { * What we, we looked at } \\
\text { just the range of what } \\
\text { was out there in both } \\
\text { considered core } \\
\text { curriculum and } \\
\text { supplemental resources. } \\
\text { Knowing that, well first } \\
\text { of all the ELD, I should } \\
\text { go back to ELD is our, } \\
\text { basically our year-one } \\
\text { ELD, that had been } \\
\text { redesigned and } \\
\text { redeveloped anyway and } \\
\text { then we just would kind } \\
\text { of add on that so there is } \\
\text { some enrichment that's } \\
\text { brought on that. But } \\
\text { it's, the ESL } 1 / 2 \text { level, } \\
\text { all those resources and } \\
\text { materials that just } \\
\text { moved into the New } \\
\text { Arrival Center. } \\
\text { * In terms of the other } \\
\text { content areas math, } \\
\text { social studies, science } \\
\text { with that, again what we } \\
\text { are trying to accomplish } \\
\text { within those. An } \\
\text { orientation to what you } \\
\text { get in science, an } \\
\text { orientation to what you } \\
\text { get in social studies, not } \\
\text { the exact same } \\
\text { curriculum that they are } \\
\text { going to be taking the } \\
\text { following year. But } \\
\text { resources and materials } \\
\text { which help develop the } \\
\text { language and key } \\
\text { concepts so that they } \\
\text { would have a better } \\
\text { opportunity of being } \\
\text { successful... Their } \\
\text { second year and in their } \\
\text { regular science, math, } \\
\text { social studies classes. } \\
\text { So we looked at all of }\end{array}$ & $\begin{array}{l}\text { Materials were bought } \\
\text { from scratch for social } \\
\text { studies science and math } \\
\text { and supplemental } \\
\text { English materials. The } \\
\text { ESL adoption and } \\
\text { supplemental materials } \\
\text { were utilized for the } \\
\text { English portion. } \\
\text { The Keys to Learning } \\
\text { and WRITE materials } \\
\text { used within the ESL } 1 / 2 \\
\text { program were also used } \\
\text { in the New Arrival } \\
\text { center. A curriculum } \\
\text { committee made up of } \\
\text { ESL teachers and } \\
\text { resource teachers } \\
\text { initially chose the } \\
\text { materials through a } \\
\text { regular adoption } \\
\text { process. } \\
\text { The content materials } \\
\text { were decided on after } \\
\text { getting } \\
\text { recommendations from } \\
\text { teachers who had used } \\
\text { such materials, in } \\
\text { particular social studies, } \\
\text { in ESL } 1 / 2 \text { classes. The } \\
\text { program administrator } \\
\text { chose Science materials } \\
\text { after having seen them } \\
\text { in presentations. } \\
\text { Materials are meant to } \\
\text { provide foundation } \\
\text { knowledge and content } \\
\text { language. } \\
\text { The essential standards } \\
\text { for the NAC content } \\
\text { courses encompass both } \\
\text { high school and K-8 } \\
\text { standards. The } \\
\text { standards chosen for the } \\
\text { program are more }\end{array}$ \\
\hline
\end{tabular}




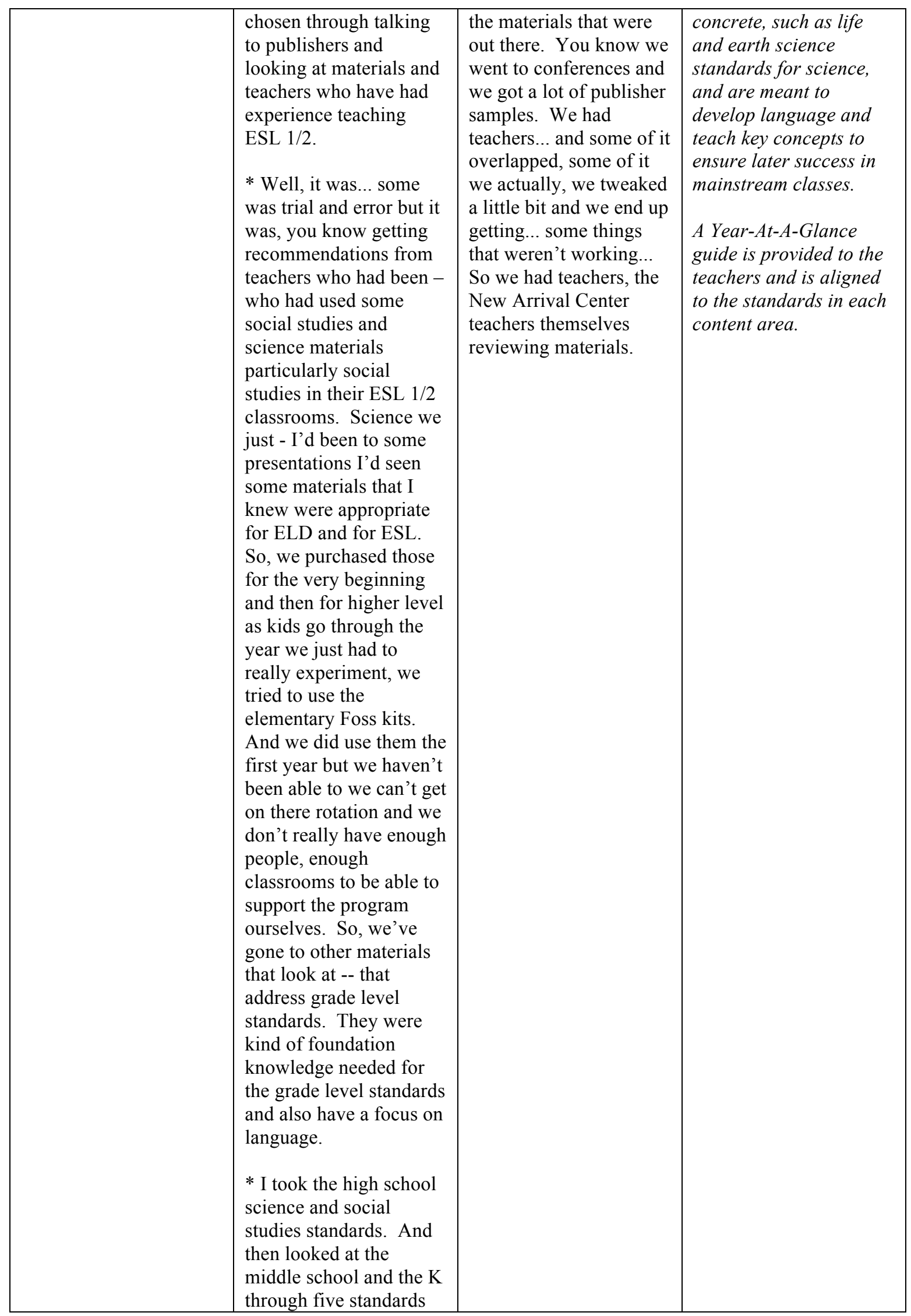




\begin{tabular}{|c|c|c|c|}
\hline & $\begin{array}{l}\text { that aligned with some } \\
\text { of those - we had } \\
\text { selected some essential } \\
\text { standards that we felt } \\
\text { kids would need } \\
\text { primarily in life and } \\
\text { earth science because } \\
\text { it's a very concrete. } \\
\text { Life and earth would be } \\
\text { the first science from } \\
\text { many of the kids, } \\
\text { physics might ...at some } \\
\text { schools would be the } \\
\text { first science they would } \\
\text { mainstream into but that } \\
\text { requires a lot of math as } \\
\text { well. So, we really } \\
\text { focused on life and earth } \\
\text { partly because it just } \\
\text { aligns really well with } \\
\text { the things that you need } \\
\text { to teach beginning ESL } \\
\text { kids, in the first place it } \\
\text { aligns with real life. It's } \\
\text { very concrete, so they } \\
\text { can use it that language } \\
\text { right away... where as } \\
\text { physics that kind of } \\
\text { language and those ideas } \\
\text { maybe not so much. So, } \\
\text { once we looked at those } \\
\text { standards that aligned } \\
\text { with the high school } \\
\text { standards... } \\
\text { * We have a curriculum } \\
\text { guide for the year, sort } \\
\text { of the year at a glance } \\
\text { and then all the } \\
\text { standards are listed for } \\
\text { that. So, we haven't } \\
\text { gone to more detailed } \\
\text { than a year at a glance, } \\
\text { partly because we've } \\
\text { been working on just } \\
\text { continuing to set up the } \\
\text { program. }\end{array}$ & & \\
\hline $\begin{array}{l}\text { Language } \\
\text { Instruction }\end{array}$ & $\begin{array}{l}\text { * ...we use a method } \\
\text { called growing } \\
\text { sentences that you may } \\
\text { be familiar with.... the } \\
\text { WRITE institute also } \\
\text { focuses on language } \\
\text { everything that kids say } \\
\text { we want them writing so }\end{array}$ & $\begin{array}{l}\text { * Express is something } \\
\text { useful and provides } \\
\text { information and that } \\
\text { could, we could use that } \\
\text { a couple of times during } \\
\text { the year and then some } \\
\text { of the, again the ELDPI } \\
\text { and the other }\end{array}$ & $\begin{array}{l}\text { Daily reading, writing, } \\
\text { listening, and speaking } \\
\text { is expected in the ESL } \\
\text { portion of the day, } \\
\text { through explicit } \\
\text { language instruction } \\
\text { and the WRITE } \\
\text { curriculum and }\end{array}$ \\
\hline
\end{tabular}




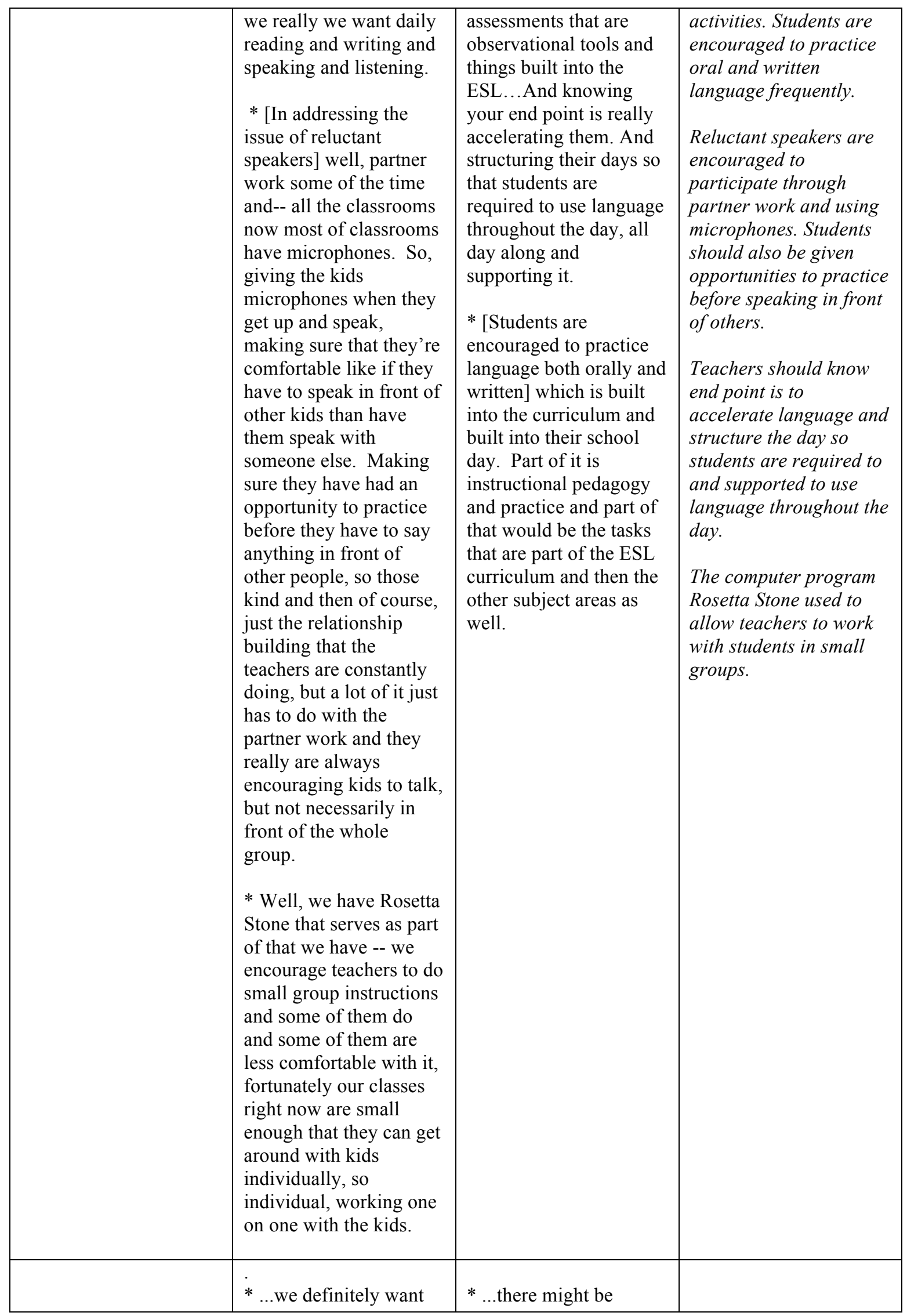




\begin{tabular}{|c|c|c|c|}
\hline Content Instruction & $\begin{array}{l}\text { kids to start earning } \\
\text { graduation credits as } \\
\text { soon as they can. So, } \\
\text { we send them out to } \\
\text { algebra when they're } \\
\text { ready or even geometry. } \\
\text { If some of them come in } \\
\text { having had algebra or } \\
\text { geometry so, they go } \\
\text { directly to a mainstream } \\
\text { class and then we } \\
\text { support them as best we } \\
\text { can through after school } \\
\text { or during their other } \\
\text { class periods to make } \\
\text { sure that they are } \\
\text { understanding what's } \\
\text { going on even though } \\
\text { they have the math } \\
\text { skills. Or a couple of } \\
\text { kids we've been able to } \\
\text { send them out to science } \\
\text { and there have been } \\
\text { some kids that we've } \\
\text { been able to have them } \\
\text { go out to mainstream in } \\
\text { the middle of the school } \\
\text { year or the semester. } \\
\text { * ...with the content area } \\
\text { standards in science and } \\
\text { social studies...they are } \\
\text { basically focusing on } \\
\text { elementary and middle } \\
\text { school standards that } \\
\text { align to high school. So, } \\
\text { because it's an elective } \\
\text { class we are not bound } \\
\text { by the high school } \\
\text { standards. If we were... } \\
\text { they would be earning } \\
\text { grade level credit, but } \\
\text { we realized that its not } \\
\text { realistic... they cannot } \\
\text { really learn } \\
\text { photosynthesis as well } \\
\text { as they need to [in order } \\
\text { to] meet the grade level } \\
\text { standards. And then as } \\
\text { far as math goes, again } \\
\text { it's elective credit with a } \\
\text { focus on language } \\
\text { [which] strengthens } \\
\text { so that basic math skills, } \\
\text { to algebra the following }\end{array}$ & $\begin{array}{l}\text { sheltered courses or } \\
\text { clusters [for their } \\
\text { following year of } \\
\text { school], so what } \\
\text { we... We at least ask } \\
\text { them to do is to cluster } \\
\text { those students and place } \\
\text { them in the content area } \\
\text { classes with teachers } \\
\text { who would be you know } \\
\text { best equipped to deal } \\
\text { with students a year into } \\
\text { school here... That's } \\
\text { what we're, that's what } \\
\text { we definitely } \\
\text { recommend schools to. } \\
\text { * The way we kind of } \\
\text { look at it is well why } \\
\text { don't we front load } \\
\text { those electives and give } \\
\text { students an opportunity } \\
\text { to have all of those } \\
\text { electives being kind of } \\
\text { content based ELD } \\
\text { courses. So there is a } \\
\text { math and social studies } \\
\text { and a science and } \\
\text { depending on the } \\
\text { students and their needs } \\
\text { they will take, you } \\
\text { know, all of them during } \\
\text { the course of the year. } \\
\text { But the emphasis is } \\
\text { really still on language } \\
\text { development, building } \\
\text { some of the language } \\
\text { and the fundamental } \\
\text { skills and knowledge } \\
\text { around science or they } \\
\text { are going to face the } \\
\text { following year or two, } \\
\text { focused on the standards } \\
\text { and the same with social } \\
\text { studies... mainly U.S. } \\
\text { history again because } \\
\text { we want that orientation } \\
\text { to the United States and } \\
\text { all of that and them } \\
\text { math and math is } \\
\text { trickier because some of } \\
\text { our kids come and bring } \\
\text { some math skills...and } \\
\text { we want to recognize } \\
\text { that. So some students } \\
\text { will go out, we call it }\end{array}$ & $\begin{array}{l}\text { If students are ready, } \\
\text { they may be } \\
\text { mainstreamed for math } \\
\text { or science. They can } \\
\text { start accruing } \\
\text { graduation credits if } \\
\text { they are mainstreamed } \\
\text { into regular classes. } \\
\text { The NAC content classes } \\
\text { are elective classes, so } \\
\text { the focus is primarily on } \\
\text { content language } \\
\text { acquisition and } \\
\text { strengthening } \\
\text { fundamental skills. The } \\
\text { elementary and middle } \\
\text { school content } \\
\text { standards that align to } \\
\text { high school content } \\
\text { standards are used } \\
\text { within NAC content } \\
\text { classes. } \\
\text { Content skills such as } \\
\text { reading maps, using } \\
\text { rulers, and observation } \\
\text { and investigation are } \\
\text { used in content classes. } \\
\text { Schools are encouraged } \\
\text { to cluster the students } \\
\text { the following year into } \\
\text { content classes that } \\
\text { would be best equipped } \\
\text { to deal with students a } \\
\text { year into school here. } \\
\text { Social studies focuses on } \\
\text { U.S. history to help } \\
\text { newcomer students } \\
\text { orient to the United } \\
\text { States. } \\
\text { QTEL is a training } \\
\text { approach and strategies } \\
\text { mainsed to assist teachers } \\
\text { in supporting English } \\
\text { learners more effectively } \\
\text { in content classes. This } \\
\text { approach is being } \\
\text { widely disseminated } \\
\text { around the district. } \\
\end{array}$ \\
\hline
\end{tabular}




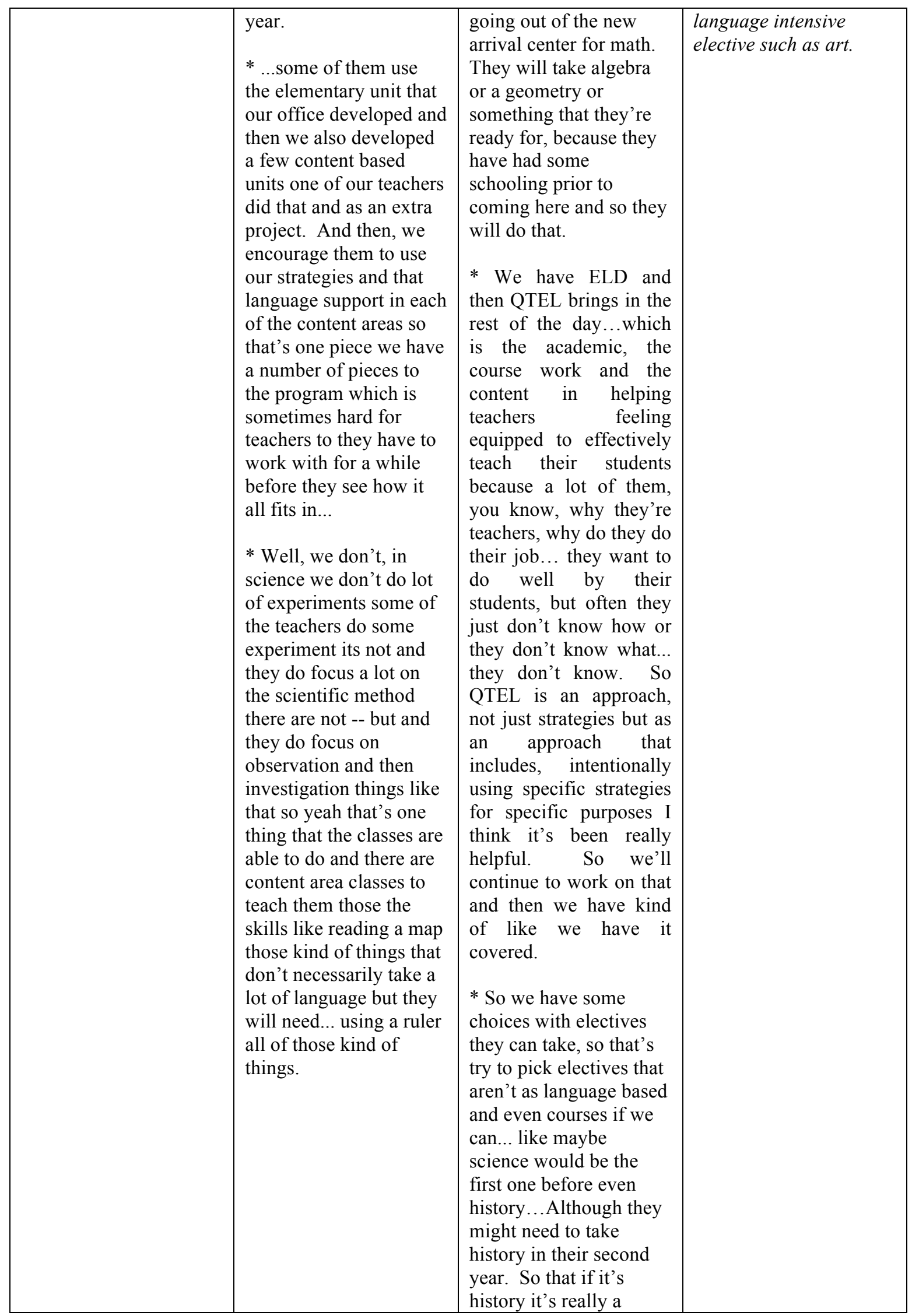




\begin{tabular}{|c|c|c|c|}
\hline & & $\begin{array}{l}\text { language development } \\
\text { approach as well. } \\
\text { * And there are also } \\
\text { opportunities in some } \\
\text { schools for them to go } \\
\text { out for another } \\
\text { elective...like maybe } \\
\text { art...they all go out for } \\
\text { PE, so they all take PE } \\
\text { as part of the general ed } \\
\text { mainstream program. } \\
\text { But sometimes they'll } \\
\text { go out for another } \\
\text { elective as well. } \\
\text { * No, they haven't [gone } \\
\text { out] for science and they } \\
\text { haven't for history } \\
\text { because history is so } \\
\text { language based...And } \\
\text { that would be a tough } \\
\text { one...plus most of them } \\
\text { have not had U.S. } \\
\text { history... }\end{array}$ & \\
\hline $\begin{array}{c}\text { Administrator } \\
\text { Attitude/ } \\
\text { Expectations }\end{array}$ & $\begin{array}{l}* . . . \text { and because the } \\
\text { teachers are so } \\
\text { knowledgeable about } \\
\text { their own students and } \\
\text { about what they need, } \\
\text { the teacher really is the } \\
\text { primary person to } \\
\text { advocate for the student } \\
\text { and do all the placement } \\
\text { and make sure } \\
\text { everything happens the } \\
\text { way it needs to happen } \\
\text { for the student they call } \\
\text { in the ELST when they } \\
\text { need to. } \\
\text { * The schools are } \\
\text { thrilled to have the } \\
\text { program there... the } \\
\text { administrators love it. } \\
\text { The other teachers seem } \\
\text { very happy to have it } \\
\text { and the tension just } \\
\text { comes in just the actual } \\
\text { implementation and the } \\
\text { logistical kinds of } \\
\text { things... not in terms of } \\
\text { philosophy at all or }\end{array}$ & $\begin{array}{l}\text { * I do oversee them all, } \\
\text { but specifically the New } \\
\text { Arrival Centers. It's a } \\
\text { program that started just } \\
\text { a few years ago, really } \\
\text { in response to the kids } \\
\text { who were brand new to } \\
\text { English... brand new to } \\
\text { the country and the } \\
\text { secondary level and we } \\
\text { don't have a huge } \\
\text { number of them, even a } \\
\text { huge percentage of them } \\
\text { in San Diego but their } \\
\text { issues are so distinct and } \\
\text { so different that it throw } \\
\text { schools off. You know } \\
\text { so they don't have to do } \\
\text { or they over generalize } \\
\text { that all the English } \\
\text { learners are like the new } \\
\text { arrivals because they are } \\
\text { so present and their } \\
\text { needs are so distinct. So } \\
\text { at that time we talked } \\
\text { about how we could best } \\
\text { support them, what kind } \\
\text { of New Arrival Center }\end{array}$ & $\begin{array}{l}\text { The NAC program was } \\
\text { started in response to } \\
\text { the diverse needs of } \\
\text { newcomer students at } \\
\text { the secondary level. } \\
\text { They are a small } \\
\text { population, but their } \\
\text { issues are so distinct } \\
\text { and different than other } \\
\text { English learners that } \\
\text { schools struggle to } \\
\text { adequately address the } \\
\text { needs of such students. } \\
\text { Schools are thrilled to } \\
\text { have NAC programs on } \\
\text { their campuses, though } \\
\text { tensions can occur in } \\
\text { the implementation of } \\
\text { the program. } \\
\text { Teachers are considered } \\
\text { the primary person to } \\
\text { advocate for students } \\
\text { and are charged with } \\
\text { determining placement } \\
\text { for the following year. } \\
\text { Students generally only }\end{array}$ \\
\hline
\end{tabular}




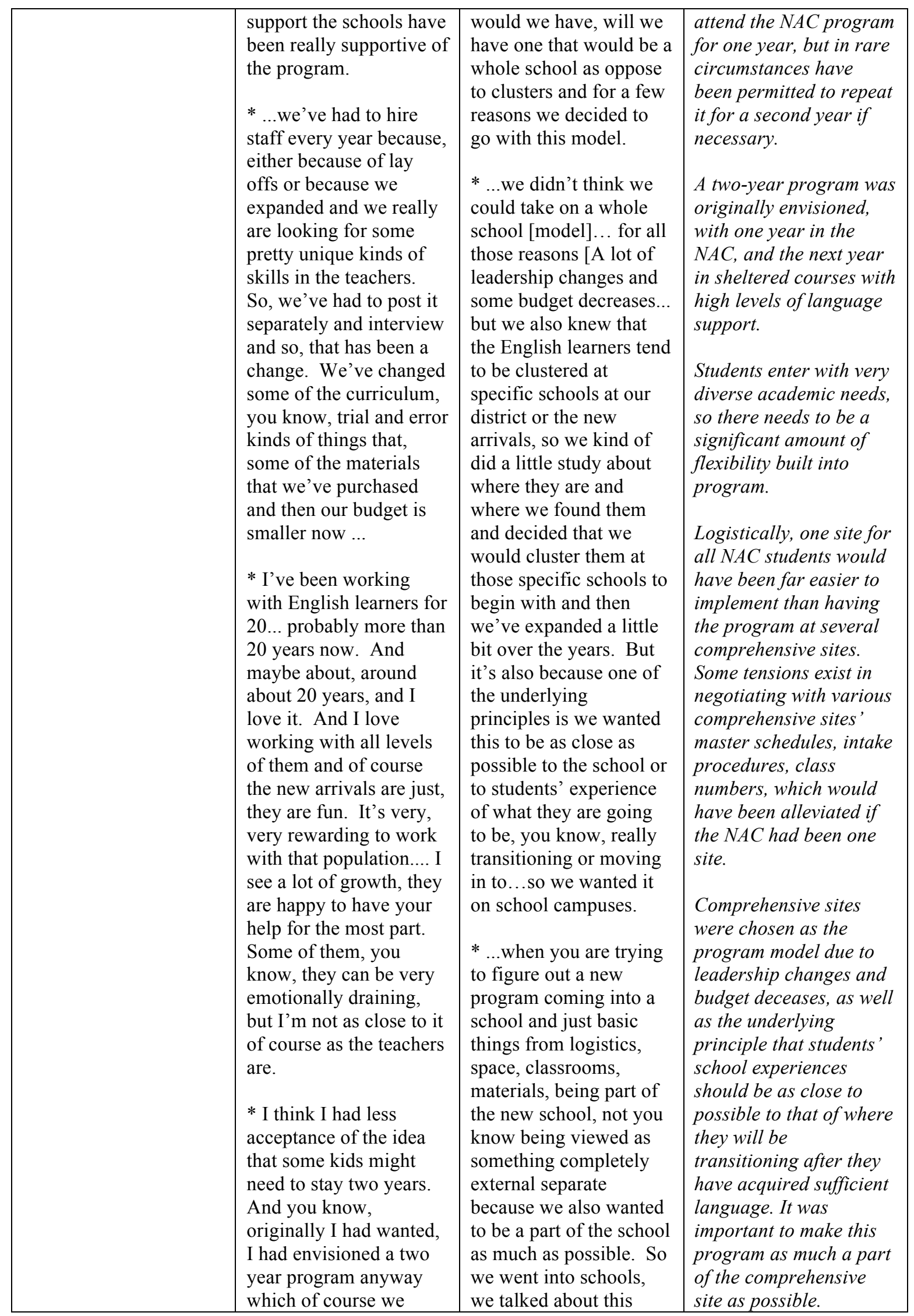




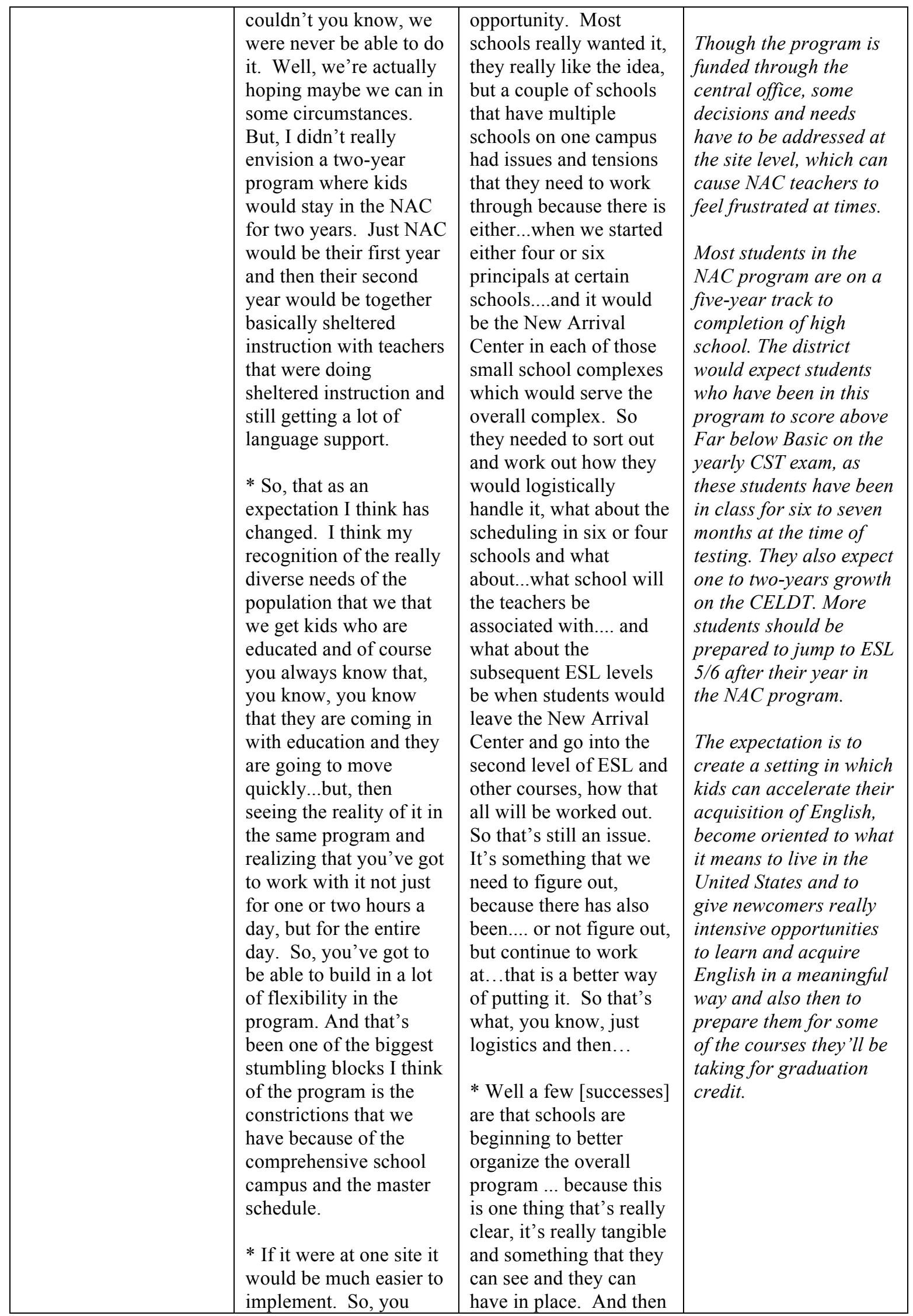




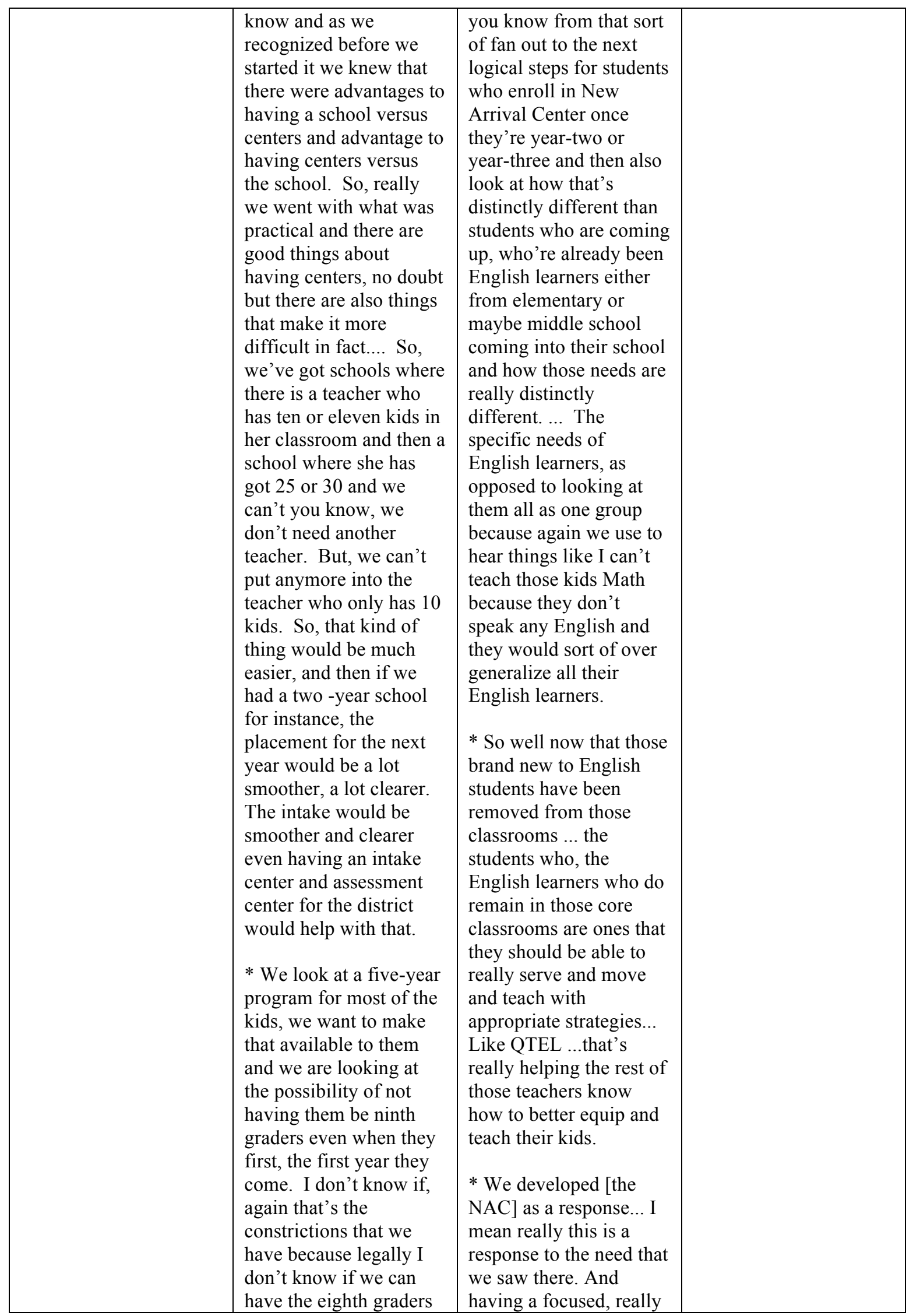




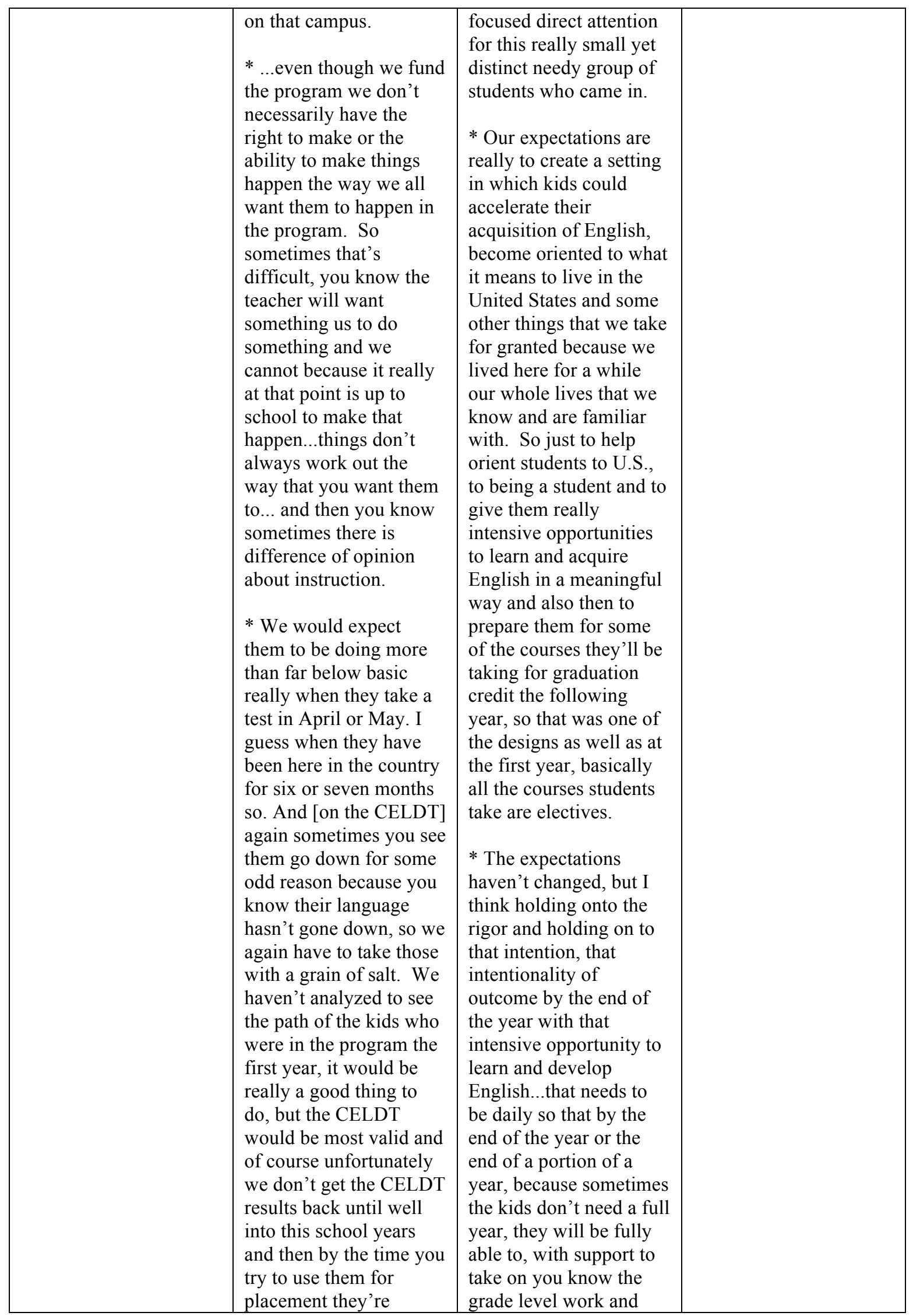




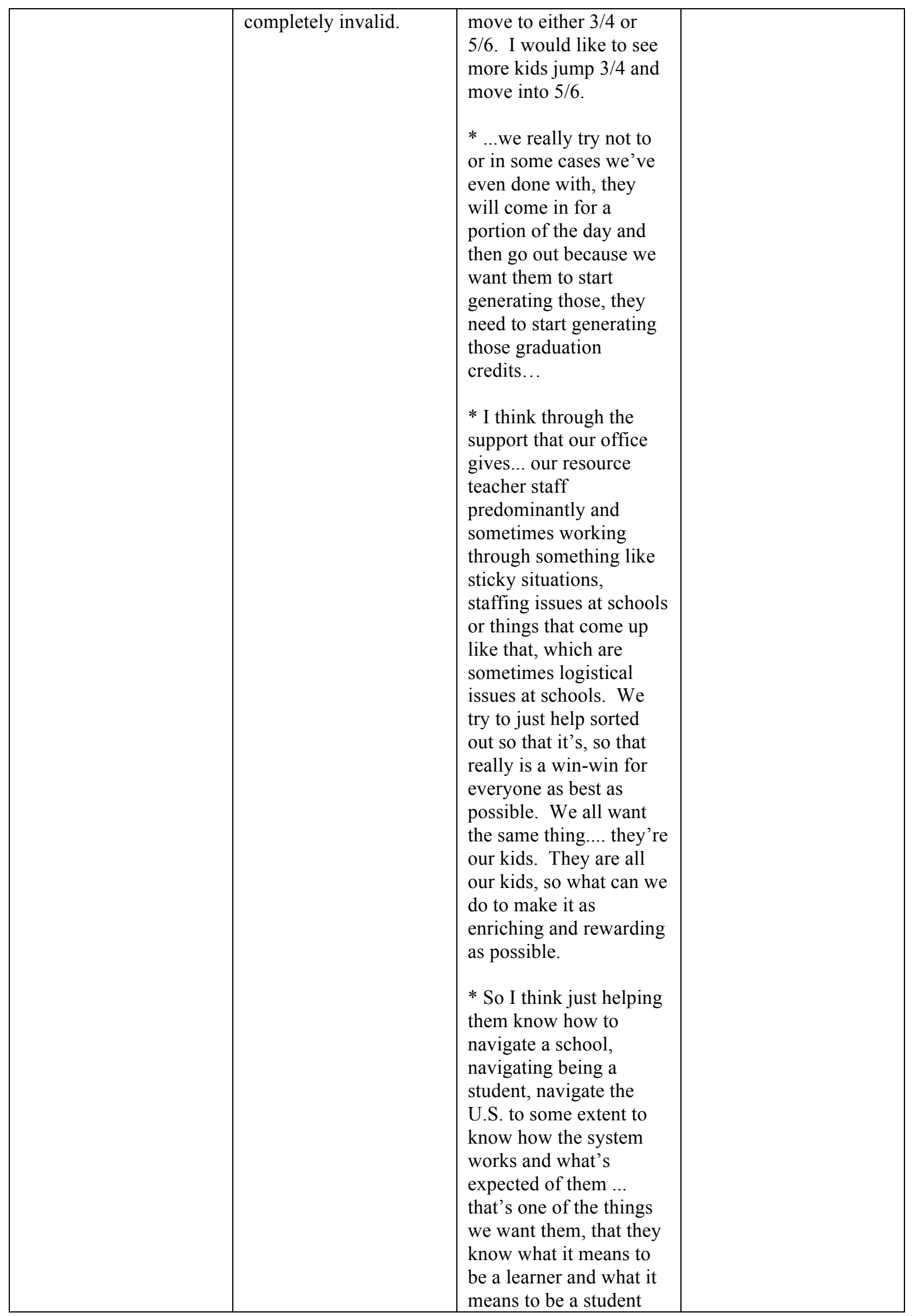




\begin{tabular}{|c|c|c|c|}
\hline & & $\begin{array}{l}\text { and what their } \\
\text { investment needs to be. }\end{array}$ & \\
\hline $\begin{array}{c}\text { Culturally } \\
\text { Responsive } \\
\text { Teaching }\end{array}$ & $\begin{array}{l}\text { * We really encouraged } \\
\text { them to build on the } \\
\text { student's home culture } \\
\text { in the prior experiences } \\
\text { that's what we hope as } \\
\text { part of every lesson. So, } \\
\text { there is a connection } \\
\text { their background } \\
\text { knowledge. Their } \\
\text { primary language, their } \\
\text { kids have it in the } \\
\text { languages where we } \\
\text { can...we [also] have } \\
\text { dictionaries. We do } \\
\text { encourage them to read } \\
\text { in their primary } \\
\text { language again when } \\
\text { there are books available } \\
\text { for them. They aren't in } \\
\text { all the languages and } \\
\text { then we have some } \\
\text { teachers who speak } \\
\text { Spanish and they will } \\
\text { explain some things in } \\
\text { Spanish. We really } \\
\text { discourage the teachers } \\
\text { from using the primary } \\
\text { language very much } \\
\text { because we really want } \\
\text { the kids to learn English, } \\
\text { not all of the kids can } \\
\text { get the directions or an } \\
\text { explanation in their } \\
\text { primary language... } \\
\text { which is not a reason to } \\
\text { deprive others of the } \\
\text { explanation, but it also it } \\
\text { leaves that group of kids } \\
\text { out. } \\
\text { * So the kids help each } \\
\text { other. They use the } \\
\text { primary language a lot } \\
\text { we don't discourage that } \\
\text { except that we really } \\
\text { encourage the use of } \\
\text { English and so there are } \\
\text { times during the day } \\
\text { when we ask the } \\
\text { teachers to make it an } \\
\text { English-only time. You } \\
\text { know and maybe as }\end{array}$ & $\begin{array}{l}\text { * Some schools more } \\
\text { than others [use primary } \\
\text { language] like some of } \\
\text { the schools the majority } \\
\text { of the students are } \\
\text { Spanish speakers.... } \\
\text { Most have a range of } \\
\text { languages in which case } \\
\text { you know it's really } \\
\text { impossible to, for the } \\
\text { teacher at least, to } \\
\text { provide that modeling. } \\
\text { But it is encouraged that } \\
\text { students can use their } \\
\text { language with each } \\
\text { other to help on specific } \\
\text { things. } \\
\text { * If the purpose is using } \\
\text { English and applying } \\
\text { English then you know } \\
\text { [in class] would be the } \\
\text { time....but using their } \\
\text { language and validating } \\
\text { it and respecting it and } \\
\text { demonstrating value and } \\
\text { support for it, that's } \\
\text { something that needs to } \\
\text { be a part of every } \\
\text { classroom...at all the } \\
\text { New Arrival Centers. } \\
\text { * ... there is a possibility } \\
\text { of them going out for a } \\
\text { subject or two...in their } \\
\text { primary } \\
\text { language...that's } \\
\text { something that not all } \\
\text { schools have, you know, } \\
\text { just logistically } \\
\text { establishing a, for } \\
\text { Spanish for example, } \\
\text { Spanish language } \\
\text { science course taught in } \\
\text { Spanish or a history } \\
\text { course taught in } \\
\text { Spanish... we have had } \\
\text { individual ones, but not } \\
\text { really well-organized. }\end{array}$ & $\begin{array}{l}\text { Teachers are } \\
\text { encouraged by } \\
\text { administrators to build } \\
\text { on students' home } \\
\text { cultures as a part of } \\
\text { every lesson. The } \\
\text { students should feel a } \\
\text { connection to their home } \\
\text { culture and language. } \\
\text { Students are encouraged } \\
\text { to read books in their } \\
\text { primary language, and } \\
\text { teachers use primary } \\
\text { language support when } \\
\text { possible (primarily in } \\
\text { Spanish). The teachers } \\
\text { are discouraged from } \\
\text { using primary language } \\
\text { often because the } \\
\text { administrators want to } \\
\text { see students immersed in } \\
\text { as much English as } \\
\text { possible. They also feel } \\
\text { that it would exclude } \\
\text { students from language } \\
\text { backgrounds that do not } \\
\text { have primary language } \\
\text { support. } \\
\text { The kids help each other } \\
\text { with primary language } \\
\text { support, which is not } \\
\text { discouraged, so teachers } \\
\text { are asked to enforce } \\
\text { short periods of English- } \\
\text { only time. They may be } \\
\text { as short as 10-minute } \\
\text { periods. } \\
\text { One administrator, } \\
\text { while acknowledging the } \\
\text { importance of practicing } \\
\text { English, believes that } \\
\text { the students' primary } \\
\text { languages must be } \\
\text { utilized, respected, and } \\
\text { validated within the } \\
\text { NAC program. } \\
\text { If available, some } \\
\text { students have access to } \\
\text { primary language } \\
\text { classes, but they are not }\end{array}$ \\
\hline
\end{tabular}




\begin{tabular}{|c|c|c|c|}
\hline & $\begin{array}{l}\text { short as a } 10 \text {-minute } \\
\text { period but at least } \\
\text { sometime when they } \\
\text { really are going to have } \\
\text { work to get it out in } \\
\text { some level of English } \\
\text { and some teachers you } \\
\text { know will insist on } \\
\text { English more than other } \\
\text { teachers will...As far as I } \\
\text { know all the teachers } \\
\text { speak English to } \\
\text { students nearly virtually } \\
99 \% \text { of the time. }\end{array}$ & & $\begin{array}{l}\text { very common, because } \\
\text { of logistics, in the } \\
\text { district or well } \\
\text { organized at the district } \\
\text { level. }\end{array}$ \\
\hline $\begin{array}{c}\text { Pedagogical } \\
\text { Orientations/Beliefs }\end{array}$ & $\begin{array}{l}\text { * My experience in the } \\
\text { classroom [have helped } \\
\text { me in my position]. } \\
\text { Well, I think being in } \\
\text { Special Ed I gained a } \\
\text { background in } \\
\text { assessment sure I think } \\
\text { that helps. And I gained } \\
\text { a background in } \\
\text { differentiating } \\
\text { instructions so, I think } \\
\text { that helped I've been a } \\
\text { support provider too, as } \\
\text { a support provider and } \\
\text { as a coach I think it } \\
\text { enabled me to help } \\
\text { teachers get started. } \\
\text { And then, again my } \\
\text { background in Special } \\
\text { Ed probably helped me } \\
\text { look at materials to } \\
\text { purchase with a different } \\
\text { eye and then my } \\
\text { background in language } \\
\text { acquisition has helped } \\
\text { me also in purchasing } \\
\text { materials and setting up } \\
\text { the program just } \\
\text { generally. So, yeah, I } \\
\text { am definitely able to use } \\
\text { everything. } \\
\text { * I am kind of } \\
\text { coordinating the } \\
\text { program. How do I } \\
\text { view my role, I view my } \\
\text { role as support basically } \\
\text { I want to know what's } \\
\text { going on in all the } \\
\text { classrooms and in the }\end{array}$ & $\begin{array}{l}\text { * We did a kind of } \\
\text { collaboration [with } \\
\text { schools and principals] } \\
\text { We tried...we are very, } \\
\text { very collaborative and } \\
\text { very open, but } \\
\text { ultimately with the } \\
\text { classrooms and the } \\
\text { assignments, we had } \\
\text { some negotiation that } \\
\text { occurred. You know it's } \\
\text { their school and they're } \\
\text { their students. So, and } \\
\text { we want that, we want } \\
\text { them to feel ownership } \\
\text { for their program as } \\
\text { well. And so they have } \\
\text { a huge say in how it's } \\
\text { all operated...they } \\
\text { actually evaluate the } \\
\text { teachers and we don't, } \\
\text { because they are on the } \\
\text { campus, they're teachers } \\
\text { on the campus. } \\
\text { * We talked about that a } \\
\text { little bit. So sometimes } \\
\text { for ELD and for } \\
\text { something really } \\
\text { specific for a portion of } \\
\text { the day, they might be } \\
\text { regrouped. But we } \\
\text { wanted to keep those } \\
\text { kind of classroom } \\
\text { configurations a little } \\
\text { more mixed. } \\
\text { support and find out } \\
\text { what's needed to help it }\end{array}$ & $\begin{array}{l}\text { One administrator felt } \\
\text { that her experience as a } \\
\text { Special Education } \\
\text { teacher has helped her } \\
\text { in assessing students } \\
\text { and in supporting } \\
\text { teachers in } \\
\text { differentiating } \\
\text { instruction for their } \\
\text { students. In addition, } \\
\text { her background in both } \\
\text { Special Education and } \\
\text { language acquisition } \\
\text { helped her in choosing } \\
\text { materials for the NAC } \\
\text { program. } \\
\text { The administrators } \\
\text { viewed their role as that } \\
\text { of a support to teachers } \\
\text { within the NAC } \\
\text { program, to help } \\
\text { support the program to } \\
\text { grow and flourish. Their } \\
\text { goal is to ensure that } \\
\text { students acquire English } \\
\text { and achieve } \\
\text { academically. The } \\
\text { administrators want to } \\
\text { ensure consistency } \\
\text { within the program and } \\
\text { to collaborate effectively } \\
\text { with site administrators. } \\
\text { One administrator also } \\
\text { felt that she should } \\
\text { support the teachers in } \\
\text { lesson planning, though } \\
\text { with eleven classrooms, } \\
\text { she felt she was unable } \\
\text { to do so as much as she }\end{array}$ \\
\hline
\end{tabular}




\begin{tabular}{|c|c|c|c|}
\hline & $\begin{array}{l}\text { program so that we can } \\
\text { maintain consistency. } \\
\text { So part of my role is to } \\
\text { help ensure that } \\
\text { consistency of program. } \\
\text { Part of my role is to } \\
\text { service the coach to } \\
\text { teachers to help them } \\
\text { with lesson planning to } \\
\text { co-teach, it hasn't } \\
\text { happened a lot with } 11 \\
\text { classroom it happens } \\
\text { less and less but that's -- } \\
\text { I make myself available } \\
\text { to do that and } \\
\text { troubleshooting a link } \\
\text { between the teacher, the } \\
\text { program and the } \\
\text { principal sometimes it's } \\
\text { necessary to have little } \\
\text { bit of intermediary } \\
\text { person in there } \\
\text { advocating for the } \\
\text { programs most of the } \\
\text { teachers do that } \\
\text { themselves but in some } \\
\text { situations its been } \\
\text { necessary to have or } \\
\text { helpful I should say } \\
\text { actually have a district } \\
\text { level person coming in } \\
\text { and sort - and get things } \\
\text { moving or make } \\
\text { something happen that } \\
\text { there wasn't that didn't } \\
\text { happen the way we } \\
\text { wanted it to, so really } \\
\text { it's primarily helping to } \\
\text { maintain the fidelity of } \\
\text { the program } \\
\text { * Well I best support } \\
\text { [teachers] by keeping in } \\
\text { touch with them and we } \\
\text { have had meetings in the } \\
\text { past and those have been } \\
\text { helpful to varying } \\
\text { degrees, but I think just } \\
\text { by keeping in touch by } \\
\text { offering them } \\
\text { opportunities for } \\
\text { professional } \\
\text { development making } \\
\text { sure they take advantage } \\
\text { of opportunities for } \\
\text { professional }\end{array}$ & $\begin{array}{l}\text { progress and to flourish } \\
\text { and to help students to } \\
\text { be successful and to } \\
\text { acquire English and to } \\
\text { achieve academically. } \\
\text { So that's where I work, } \\
\text { but I know that, it's also } \\
\text { in collaboration with the } \\
\text { principals of the school } \\
\text { and the students } \\
\text { themselves. } \\
\text { * These are students } \\
\text { who belong to those } \\
\text { schools. We want them } \\
\text { fully participating and } \\
\text { functioning of the } \\
\text { school, a full part of that } \\
\text { school and yet we're } \\
\text { funding it and we're } \\
\text { providing a lot of the } \\
\text { support. So a challenge } \\
\text { continues I think to be, } \\
\text { to make sure that we are } \\
\text { holding to the integrity } \\
\text { and the quality of the } \\
\text { program, and at the } \\
\text { same time releasing } \\
\text { ownership and } \\
\text { responsibility to schools } \\
\text { to take on that } \\
\text { ownership and } \\
\text { responsibility. Well, } \\
\text { that we recognize fully } \\
\text { that the school are doing } \\
\text { a thousand other things } \\
\text { and a thousand other } \\
\text { programs at the same } \\
\text { time. So I think that's } \\
\text { just a challenge. }\end{array}$ & $\begin{array}{l}\text { would like. } \\
\text { The administrator also } \\
\text { worked with teachers to } \\
\text { maintain the fidelity of } \\
\text { the program, sometimes } \\
\text { serving as an } \\
\text { intermediary between } \\
\text { the principals, the } \\
\text { program, and the } \\
\text { teachers. } \\
\\
\text { While the program } \\
\text { administrators } \\
\text { collaborated with } \\
\text { principals, they } \\
\text { ultimately wanted the } \\
\text { principals to feel } \\
\text { ownership, as the NAC } \\
\text { program was located at } \\
\text { their schools and it } \\
\text { worked with their } \\
\text { students. The principals } \\
\text { were also charged with } \\
\text { evaluating the NAC } \\
\text { teachers, as the teachers } \\
\text { were a part of the } \\
\text { comprehensive site in } \\
\text { which the program was } \\
\text { housed. The students } \\
\text { also needed to be fully } \\
\text { participating in their } \\
\text { schools. Despite the fact } \\
\text { that the central office } \\
\text { was funding and } \\
\text { supporting the program, } \\
\text { they wanted to release } \\
\text { responsibility to the site } \\
\text { while still maintaining } \\
\text { the quality and integrity } \\
\text { of the program. }\end{array}$ \\
\hline
\end{tabular}




\begin{tabular}{|c|c|c|c|}
\hline & $\begin{array}{l}\text { development. } \\
\text { Yesterday, I took one of } \\
\text { newer teacher out } \\
\text { someone classroom and } \\
\text { spent the morning with } \\
\text { her watching what was } \\
\text { going on in the } \\
\text { classroom and talking } \\
\text { about it. }\end{array}$ & & \\
\hline $\begin{array}{l}\text { Academic Self- } \\
\text { Concept }\end{array}$ & $\begin{array}{l}\text { *...one thing... because } \\
\text { their focus is on English } \\
\text { all day long so; yeah I } \\
\text { think one of the biggest } \\
\text { things that we see that's } \\
\text { different is the kids feel } \\
\text { really comfortable in } \\
\text { their school. And I } \\
\text { don't know if you saw } \\
\text { the difference between } \\
\text { the ESL, the schools } \\
\text { without NAC and the } \\
\text { schools with them. It's } \\
\text { a family; it's a home for } \\
\text { those kids because the } \\
\text { teachers just take them } \\
\text { in. } \\
\text { * I think it impacts } \\
\text { immensely because the } \\
\text { teachers -- the kids } \\
\text { know the teachers } \\
\text { really, really care about } \\
\text { them and there are } \\
\text { always encouraging, } \\
\text { they're always telling } \\
\text { them, they can do it, } \\
\text { they're always pushing } \\
\text { them, they're exposing } \\
\text { them to lots of, they take } \\
\text { them on field trips all } \\
\text { the time. I know, } \\
\text { particularly over at } \\
\text { Cambridge, [a NAC } \\
\text { teacher] started a } \\
\text { program for the kids to, } \\
\text { its like a college bound } \\
\text { program, actually I think } \\
\text { it started at Cambridge } \\
\text { not for just NAC kids } \\
\text { but then somehow our } \\
\text { kids ended up being part } \\
\text { of it as time went on. } \\
\text { * ...at one of our } \\
\text { schools, the teachers }\end{array}$ & $\begin{array}{l}\text { * Sometimes what } \\
\text { happens at schools is the } \\
\text { kids want to come back } \\
\text { to the New Arrival } \\
\text { Center, because they } \\
\text { realized once they are } \\
\text { out in the real world that } \\
\text { they were really cared } \\
\text { for and...you know } \\
\text { everything was around } \\
\text { developing their } \\
\text { language, but we really } \\
\text { want to not necessarily } \\
\text { continue to support them } \\
\text { at that level but really } \\
\text { equip them. Equip them } \\
\text { so that they can handle } \\
\text { the challenges in the } \\
\text { academic world... And } \\
\text { the teachers want them } \\
\text { to have that kind of } \\
\text { support and that kind of } \\
\text { success too. So, but } \\
\text { then it's then the } \\
\text { responsibility shifts } \\
\text { more towards the rest of } \\
\text { the school to make sure } \\
\text { that those other pieces } \\
\text { are in place, so it calls to } \\
\text { light those pieces that } \\
\text { may not be as well in } \\
\text { place. } \\
\text { * I think it will really } \\
\text { [impact students } \\
\text { academic self-concept], } \\
\text { I think it has and I hope } \\
\text { they'll continue to be a } \\
\text { strong positive impact } \\
\text { on both those things. } \\
\text { Students are getting an } \\
\text { opportunity to be fully } \\
\text { supported in figuring out } \\
\text { this pretty new world } \\
\text { that they found } \\
\text { themselves in, you can } \\
\text { tal }\end{array}$ & $\begin{array}{l}\text { The administrators felt } \\
\text { the students were very } \\
\text { comfortable in school, } \\
\text { particularly because the } \\
\text { classes were like a } \\
\text { family and they were } \\
\text { able to focus on English } \\
\text { all day long. The } \\
\text { students know that the } \\
\text { teachers care about } \\
\text { them and are } \\
\text { encouraging them to do } \\
\text { well. The teachers } \\
\text { expose the students to } \\
\text { different cultural and } \\
\text { college-going } \\
\text { experiences through } \\
\text { field trips and a college } \\
\text { bound program. } \\
\text { One teacher noticed the } \\
\text { students were feeling } \\
\text { discouraged and so she } \\
\text { started having them } \\
\text { write in a journal twice } \\
\text { a week in their primary } \\
\text { languages. The kids } \\
\text { were able to write } \\
\text { whatever they felt, and } \\
\text { after some time the } \\
\text { teacher noticed a } \\
\text { difference in how the } \\
\text { students were viewing } \\
\text { themselves. They were } \\
\text { far more positive. } \\
\text { Often the kids wanted to } \\
\text { return to the program as } \\
\text { they missed the safe and } \\
\text { caring environment. It } \\
\text { was the job of the NAC } \\
\text { to equip the students } \\
\text { with the skills necessary } \\
\text { to be mainstreamed, and } \\
\text { it becomes the duty of } \\
\text { the rest of the site to }\end{array}$ \\
\hline
\end{tabular}




\begin{tabular}{|c|c|c|c|}
\hline & $\begin{array}{l}\text { were finding that kids } \\
\text { for just really to seemed } \\
\text { kind of discouraged } \\
\text { about themselves and } \\
\text { about life in general so } \\
\text { they started assigning } \\
\text { them to write in a } \\
\text { journal twice a week in } \\
\text { their primary language } \\
\text { and just write about } \\
\text { their day....Whatever } \\
\text { they felt like that day } \\
\text { and the teachers both } \\
\text { said they've seen real } \\
\text { difference in the kid's } \\
\text { self esteem since they } \\
\text { started doing that and } \\
\text { the kids can write } \\
\text { whatever they want if it } \\
\text { something that don't } \\
\text { want the teacher to read } \\
\text { they just fold the page } \\
\text { over. So, the teacher } \\
\text { knows to skip that page } \\
\text { and they will read on } \\
\text { and they make } \\
\text { comments to them and, } \\
\text { yeah it just really... [it } \\
\text { was written in] Spanish } \\
\text { and } 99.9 \% \text { of the kids } \\
\text { do speak Spanish but } \\
\text { they saw the need } \\
\text { ultimately, they saw } \\
\text { need yeah and you know } \\
\text { I think you know with } \\
\text { modification something } \\
\text { like that could work at } \\
\text { other schools. }\end{array}$ & $\begin{array}{l}\text { only imagine. You know } \\
\text { like three weeks ago } \\
\text { I'm, you know, in a } \\
\text { whole other country and } \\
\text { a whole other way of } \\
\text { life and now I'm in this } \\
\text { kind of pristine } \\
\text { institutional looking } \\
\text { building. } \\
\text { * A positive that the } \\
\text { students bring with them } \\
\text { though is they tend to be } \\
\text { overwhelmingly, not } \\
\text { although } \\
\text { overwhelmingly really } \\
\text { highly motivated, much } \\
\text { more motivated than } \\
\text { students who've been } \\
\text { with us for a while. And } \\
\text { that serves them well } \\
\text { and it positions them } \\
\text { well to do well if } \\
\text { properly supported in } \\
\text { the other classes as well, } \\
\text { so. } \\
\text { * Although they do get } \\
\text { that deer in the } \\
\text { headlights look [their } \\
\text { second year] and they } \\
\text { want to come back often } \\
\text { because you know it's } \\
\text { like all so different... }\end{array}$ & $\begin{array}{l}\text { meet these students } \\
\text { needs in a mainstream } \\
\text { environment. Many of } \\
\text { these students entered as } \\
\text { very highly motivated } \\
\text { individuals, which } \\
\text { served them well within } \\
\text { the program, but it is the } \\
\text { programs obligation to } \\
\text { support the students } \\
\text { effectively and maintain } \\
\text { their high motivation. }\end{array}$ \\
\hline $\begin{array}{l}\text { Language } \\
\text { Proficiency } \\
\text { Level }\end{array}$ & $\begin{array}{l}\text { * Our expectation was } \\
\text { that students would be } \\
\text { able to move to an } \\
\text { intermediate level of } \\
\text { ESL for the next school } \\
\text { year. And that they } \\
\text { would be able to be } \\
\text { successful in } \\
\text { mainstream classes that } \\
\text { ideally are sheltered. } \\
\text { Yeah, so they would } \\
\text { have reached that } \\
\text { threshold level of } \\
\text { intermediate } \\
\text { proficiency. We }\end{array}$ & $\begin{array}{l}\text { * I would expect them to } \\
\text { see them in the } \\
\text { beginning as beginners... } \\
\text { You know by, kind of } \\
\text { by definition they are all } \\
\text { entering as beginners. } \\
\text { So I would expect them } \\
\text { [by the end of the year], } \\
\text { to start approaching the } \\
\text { intermediate proficiency } \\
\text { level. I think that for the } \\
\text { most part they would get } \\
\text { through like the } \\
\text { beginning and early } \\
\text { intermediate and then }\end{array}$ & $\begin{array}{l}\text { The administrators } \\
\text { expected for the students } \\
\text { to be able to move to an } \\
\text { intermediate level of } \\
\text { ESL the following year } \\
\text { and they would be able } \\
\text { to function well in } \\
\text { mainstream sheltered } \\
\text { content classes. While } \\
\text { this is not possible for } \\
\text { all students, it was a } \\
\text { goal that had been } \\
\text { increasingly achieved as } \\
\text { the program progressed. } \\
\text { The administrators }\end{array}$ \\
\hline
\end{tabular}




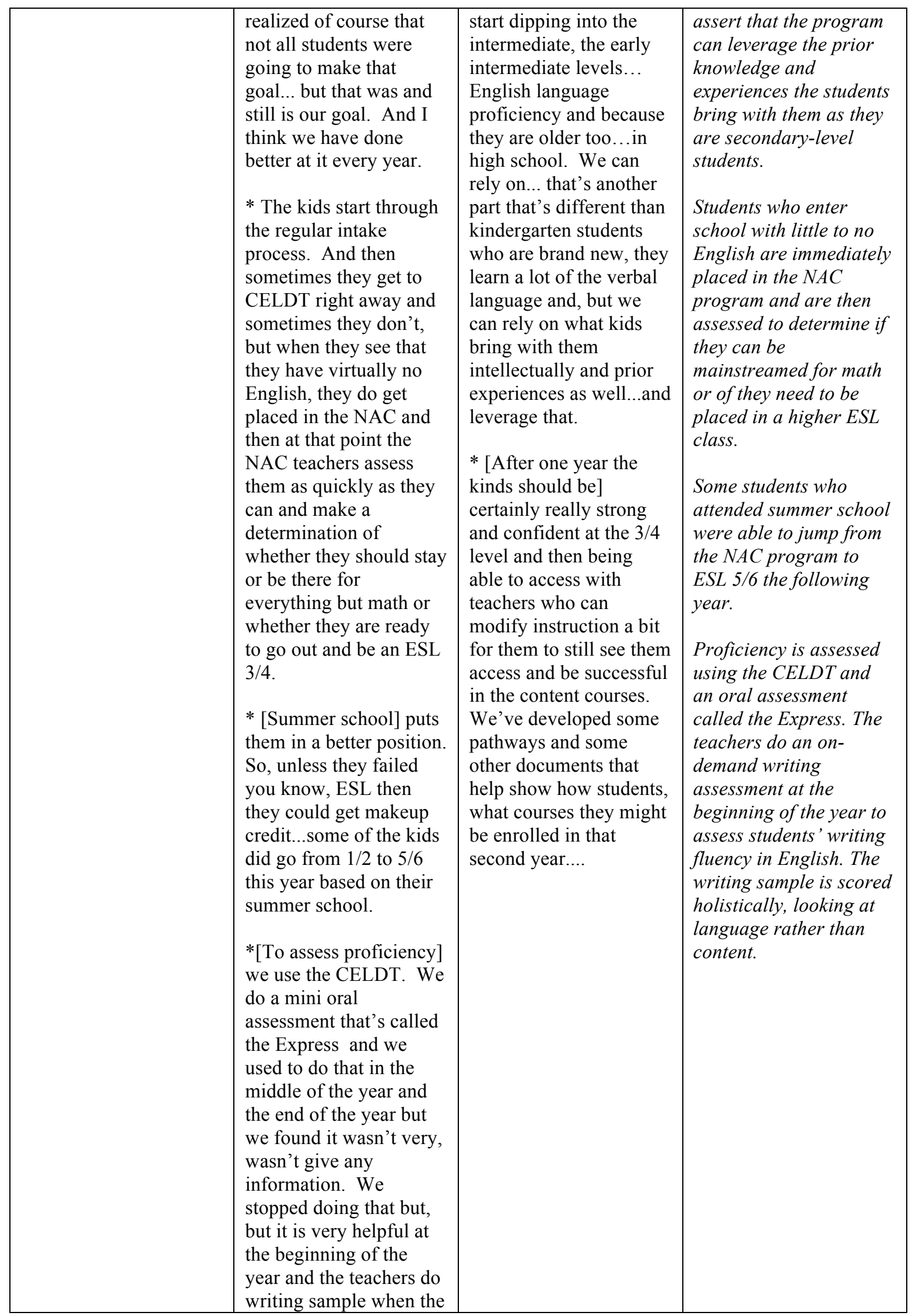




\begin{tabular}{|c|c|c|c|}
\hline & $\begin{array}{l}\text { kids first come in and } \\
\text { sort of a holistic kind of } \\
\text { scoring based on the } \\
\text { language use... not so } \\
\text { much the content. }\end{array}$ & & \\
\hline Academic Skills & $\begin{array}{l}\text { * So, we have one girl at } \\
\text { Cambridge who may be } \\
\text { the valedictorian this } \\
\text { coming year, she was in } \\
\text { the NAC it's first year. } \\
\text { So, as a freshman and } \\
\text { now she is a senior and } \\
\text { we've had a number of } \\
\text { kids jump from } \\
\text { beginning ESL into ESL } \\
\text { 5/6, we've had some go } \\
\text { into mainstream } \\
\text { English, after just their } \\
\text { one year in the NAC } \\
\text { we've had a number of } \\
\text { kids pass the Math } \\
\text { CAHSEE and quite a } \\
\text { few passing with...and } \\
\text { some passing the } \\
\text { English CAHSEE. } \\
\text { * Many of our refugee } \\
\text { students just really do } \\
\text { need the extra year or } \\
\text { two, at least two years in } \\
\text { there and some of them } \\
\text { could probably benefit } \\
\text { from three but we're - I } \\
\text { think there is only one } \\
\text { or two students that } \\
\text { have been in there even } \\
\text { part of their third year } \\
\text { and they were kids who } \\
\text { have never been to } \\
\text { school at all and } \\
\text { definitely refugees } \\
\text { coming from difficult, } \\
\text { difficult situations. }\end{array}$ & $\begin{array}{l}\text { * [Math assessments] } \\
\text { are more of a screening } \\
\text { to determine you know } \\
\text { where they are and what } \\
\text { concepts they do have, } \\
\text { what mathematical } \\
\text { concepts they have and } \\
\text { then the language of } \\
\text { course will all need to } \\
\text { be developed around } \\
\text { that. } \\
\text { * We want to get, for } \\
\text { most kids we want them } \\
\text { to have a good solid } \\
\text { year, so it will go, some } \\
\text { will spill over a little bit } \\
\text { and in a few cases not } \\
\text { many, a handful of } \\
\text { cases, some students } \\
\text { would repeat a second } \\
\text { year or...but they tend } \\
\text { to be students who have } \\
\text { come from refugee } \\
\text { camps...who've never } \\
\text { had any schooling at all } \\
\text { before, who have no } \\
\text { literacy under their belt } \\
\text { and no education at all. } \\
\text { So facing, you know, a } \\
\text { useful curriculum with } \\
\text { one year of education } \\
\text { under your belt is } \\
\text { daunting so they will } \\
\text { benefit from a second } \\
\text { year. }\end{array}$ & $\begin{array}{l}\text { One administrator } \\
\text { reported that a number } \\
\text { of NAC students have } \\
\text { been able to jump to } \\
\text { ESL 5/6, bypassing ESL } \\
\text { 3/4, after the one year } \\
\text { program. A few students } \\
\text { were even able to move } \\
\text { to mainstream English } \\
\text { after one year. Many } \\
\text { NAC students have } \\
\text { passed the high school } \\
\text { exit exam (CAHSEE), } \\
\text { and some have passed } \\
\text { the English CAHSEE. } \\
\text { One NAC student was } \\
\text { possibly going to be the } \\
\text { valedictorian of her high } \\
\text { school class. } \\
\text { Upon entry to the } \\
\text { program, the students' } \\
\text { math skills are screened } \\
\text { utilizing a district- } \\
\text { created math } \\
\text { assessment. The content } \\
\text { language is then } \\
\text { developed around the } \\
\text { students' existing } \\
\text { mathematics skills. } \\
\text { Many refugee students } \\
\text { have needed an extra } \\
\text { year in the program due } \\
\text { to limited formal } \\
\text { schooling. These } \\
\text { students will benefit } \\
\text { from a second year, as } \\
\text { many had never had any } \\
\text { schooling and no } \\
\text { literacy in their primary } \\
\text { languages. }\end{array}$ \\
\hline
\end{tabular}




\section{Appendix Q}

\section{ESL Administrator Interview Analysis}

\begin{tabular}{|c|c|c|c|}
\hline & $\begin{array}{l}\text { Administrator \#1 } \\
\text { ESL Program }\end{array}$ & $\begin{array}{l}\text { Administrator } \# 2 \\
\text { ESL Program }\end{array}$ & Significant Themes \\
\hline Curricula & $\begin{array}{l}\text { * Well, we are like } 10 \\
\text { years out of the last } \\
\text { adoption and it doesn't } \\
\text { look like we're going to } \\
\text { be -- we're still five } \\
\text { years away from a new } \\
\text { adoption... The official } \\
\text { adoption, so we decided } \\
\text { to go ahead and just } \\
\text { fund buying.... new } \\
\text { curriculum for ESL, so. } \\
\text { They've just reinstated } \\
\text { it, but the cycle will still } \\
\text { take [a while]... before } \\
\text { English gets adopted } \\
\text { and again... it will be } \\
\text { another two years. } \\
\text { * [In choosing the } \\
\text { curriculum] we had a } \\
\text { curriculum committee... } \\
\text { and we had about four } \\
\text { different... at the time } \\
\text { there was four different } \\
\text { curriculum options, so } \\
\text { we looked at all four of } \\
\text { them and we had a list } \\
\text { of criteria that we were } \\
\text { looking for and teachers } \\
\text { you know } 15 \text { teachers } \\
\text { went through and looked } \\
\text { at all of them and tried } \\
\text { out some stuff from } \\
\text { them and came back and } \\
\text { their decision was that } \\
\text { they really liked the } \\
\text { Pearson Longman you } \\
\text { know Keys to Learning } \\
\text { and Shining Star, so. } \\
\text { * We have [also] } \\
\text { instituted the WRITE } \\
\text { training... so, we've } \\
\text { incorporated that and } \\
\text { then we've also } \\
\text { incorporated Systematic } \\
\text { ELD which is the -- it's } \\
\text { explicit language }\end{array}$ & $\begin{array}{l}\text { *...as far -- in even } \\
\text { having Office of } \\
\text { Language Acquisition } \\
\text { where you finally had } \\
\text { resource teachers, we } \\
\text { still didn't have.... there } \\
\text { was no curriculum for } \\
\text { ESL. Everyone was just } \\
\text { kind of out there on their } \\
\text { own doing their own } \\
\text { thing and up until } \\
\text { now.... } \\
\text { *... it's been around at } \\
\text { least probably seven or } \\
\text { eight years by now... the } \\
\text { Office of Language } \\
\text { Acquisition has and } \\
\text { when I came on three } \\
\text { years ago, we had a } \\
\text { textbook adoption, so ... } \\
\text { we have these ESL } \\
\text { textbooks, but they're so } \\
\text { new there was no } \\
\text { curriculum written } \\
\text { around it. And it was } \\
\text { when I came in three } \\
\text { years ago that we sat } \\
\text { down and started really } \\
\text { banging out a... a } \\
\text { pacing guide for the } \\
\text { entire school year and } \\
\text { how to work things and } \\
\text { that has evolved like } \\
\text { crazy over the last three } \\
\text { years and now we have } \\
\text { a very strong solid ESL } \\
\text { curriculum for every } \\
\text { single level. We have } \\
\text { assessments for...and we } \\
\text { have baseline } \\
\text { assessments. We have } \\
\text { formal and summative } \\
\text { and full-on language } \\
\text { study and content. } \\
\text { * I think even in just the } \\
\text { three years that I've }\end{array}$ & $\begin{array}{l}\text { The district funded a } \\
\text { new ESL curriculum } \\
\text { three years prior to the } \\
\text { study, as the official } \\
\text { state adoption date was } \\
\text { believed to be too far in } \\
\text { the future. } \\
\text { A curriculum committee, } \\
\text { made up of } 15 \text { ESL } \\
\text { teachers and district } \\
\text { administrators chose } \\
\text { between four } \\
\text { textbook/curricula } \\
\text { options. The teachers } \\
\text { and administrators } \\
\text { chose Pearson } \\
\text { Longman's Keys to } \\
\text { Learning and Shining } \\
\text { Star curricula. } \\
\\
\text { There was no consistent } \\
\text { ESL curriculum prior to } \\
\text { the textbook adoption, } \\
\text { therefore the district } \\
\text { resource teachers were } \\
\text { charged with creating a } \\
\text { pacing guide and } \\
\text { assessments utilizing the } \\
\text { new ESL textbooks. } \\
\text { WRITE curricula and } \\
\text { training and Systematic } \\
\text { ELD training is utilized } \\
\text { for explicit language } \\
\text { development and the } \\
\text { writing component of } \\
\text { the program. } \\
\\
\text { The curriculum and } \\
\text { training has become far } \\
\text { stronger in the last three } \\
\text { years. Teachers are } \\
\text { required to be trained } \\
\text { on the curricula and } \\
\text { explicit language } \\
\text { development piece to } \\
\text { ensure a high quality }\end{array}$ \\
\hline
\end{tabular}




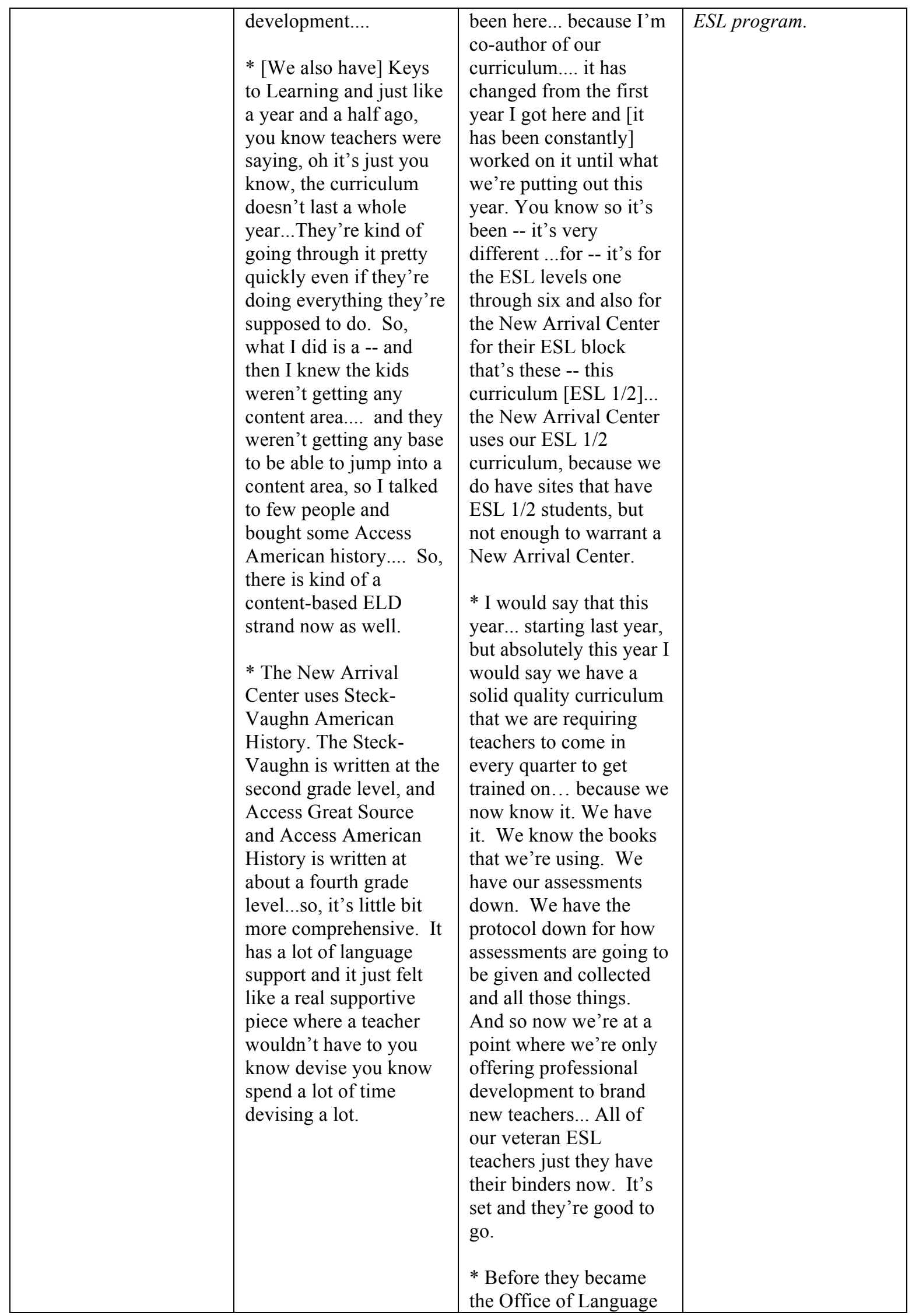




\begin{tabular}{|c|c|c|c|}
\hline & & $\begin{array}{l}\text { Acquisition and I think } \\
\text { that there was so much } \\
\text { to be done and } \\
\text { curriculum just seems } \\
\text { like such this massive } \\
\text { beast that who could } \\
\text { ever tackle that. And so } \\
\text { when I came in there } \\
\text { was the skeleton of a } \\
\text { curriculum and then it } \\
\text { was just refining and } \\
\text { refining. } \\
\text { * I do think that for the } \\
\text { first time, you know } \\
\text { since we've had ESL it } \\
\text { is the strongest it's ever } \\
\text { been. The ESL classes } \\
\text { are very strong, the } \\
\text { teachers have their } \\
\text { curriculum they have, } \\
\text { they all have this binder, } \\
\text { they all... we have had } \\
\text { trainings upon trainings } \\
\text { over each quarter in } \\
\text { over the writing cycle } \\
\text { and the reading cycle } \\
\text { and how it works } \\
\text { together and we've } \\
\text { written language study } \\
\text { guides for every genre } \\
\text { and we have them...to } \\
\text { have this whole cycle } \\
\text { they know exactly what } \\
\text { to do, they have a } \\
\text { pacing guide that's laid } \\
\text { out, so for every quarter } \\
\text { they know exactly } \\
\text { where they should be, } \\
\text { week one, week five, } \\
\text { week nine, it's all laid } \\
\text { out for them. }\end{array}$ & \\
\hline $\begin{array}{l}\text { Language } \\
\text { Instruction }\end{array}$ & $\begin{array}{l}\text { * That's why pacing } \\
\text { guides are so important } \\
\text {...because there was a } \\
\text { few classrooms where } \\
\text { you'd still see the } \\
\text { people who were } \\
\text { working on colors and } \\
\text { clothes like in } \\
\text { February.... You've got } \\
\text { to get through that, } \\
\text { because there is too } \\
\text { much you need to do. } \\
\text { And then you should see }\end{array}$ & $\begin{array}{l}* \text { I mean because our } \\
\text { curriculum is divided } \\
\text { into } 1 / 2,3 / 4 \text {, and } 5 / 6 \\
\text { and while we look } \\
\text { at.... we are a very genre } \\
\text { based, there are so major } \\
\text { differences in that } \\
\text { instruction and so really } \\
\text { working with those } \\
\text { teachers about kind of, } \\
\text { teaching to the high } \\
\text { middle of the class... and } \\
\text { then, scaffolding more }\end{array}$ & $\begin{array}{l}\text { Pacing guides are } \\
\text { distributed to ESL } \\
\text { teachers across the } \\
\text { district to ensure } \\
\text { consistency within the } \\
\text { program. } \\
\text { Shared reading and } \\
\text { language experience are } \\
\text { important components } \\
\text { of the ESL program. } \\
\text { Explicit language }\end{array}$ \\
\hline
\end{tabular}




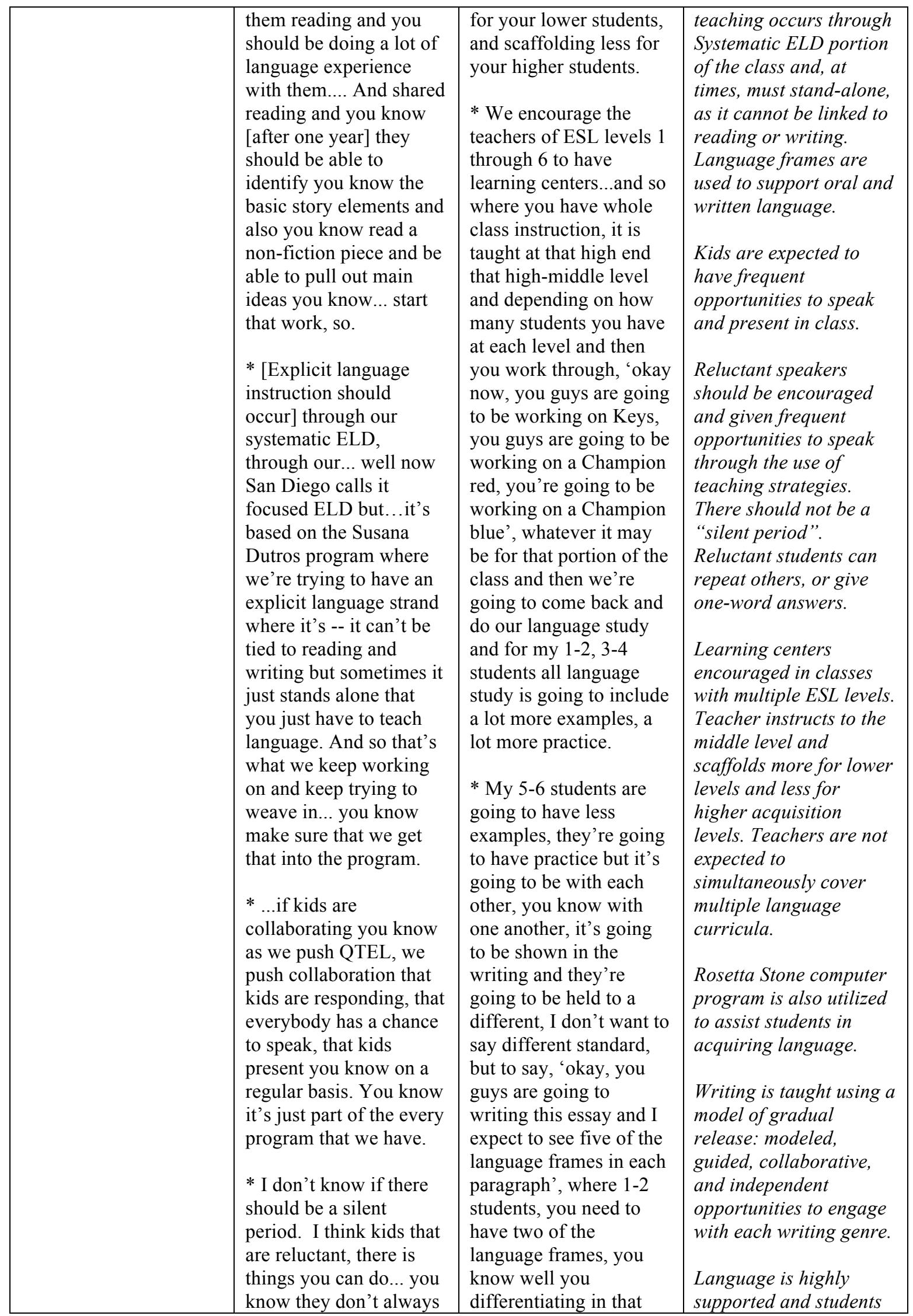




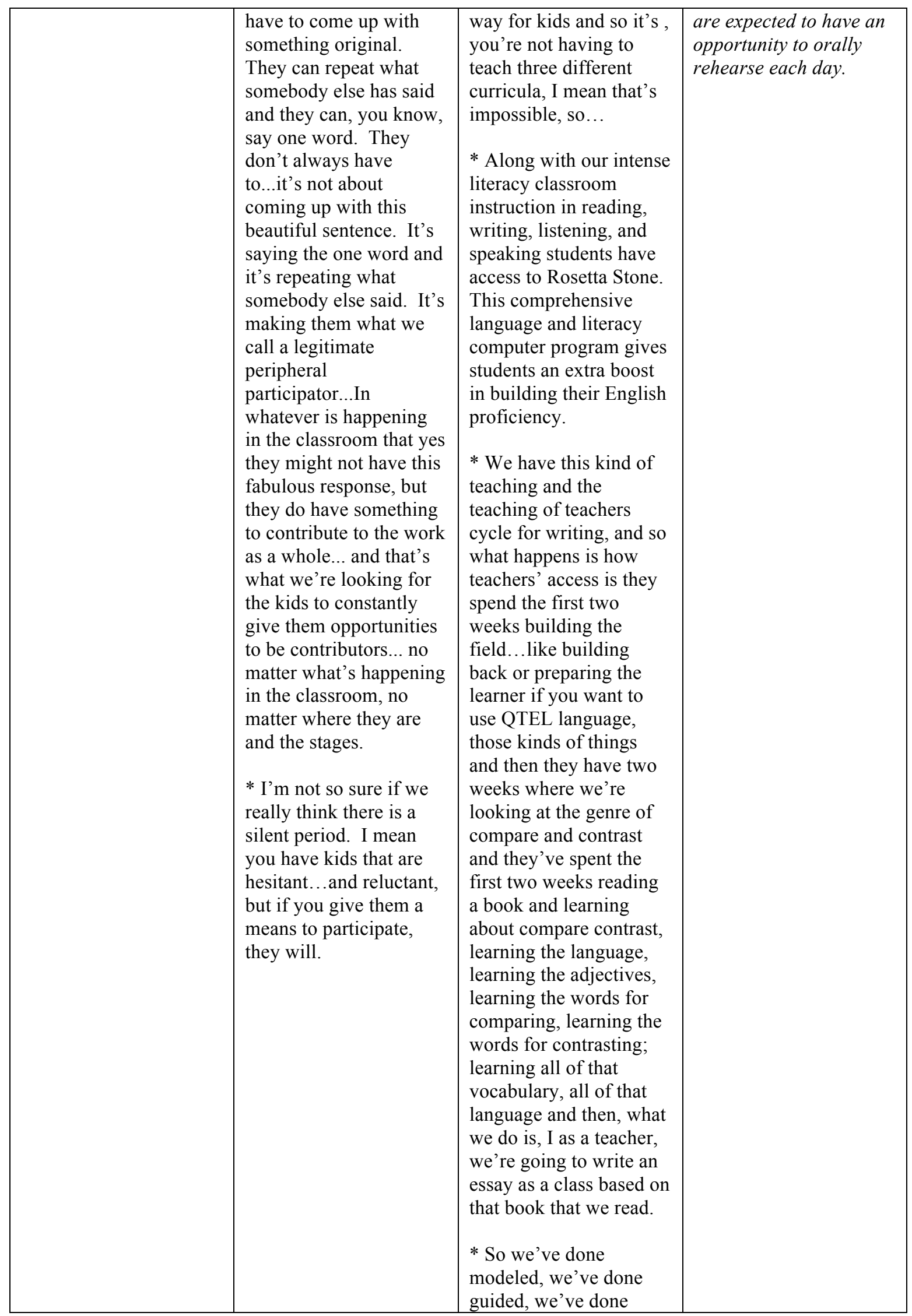




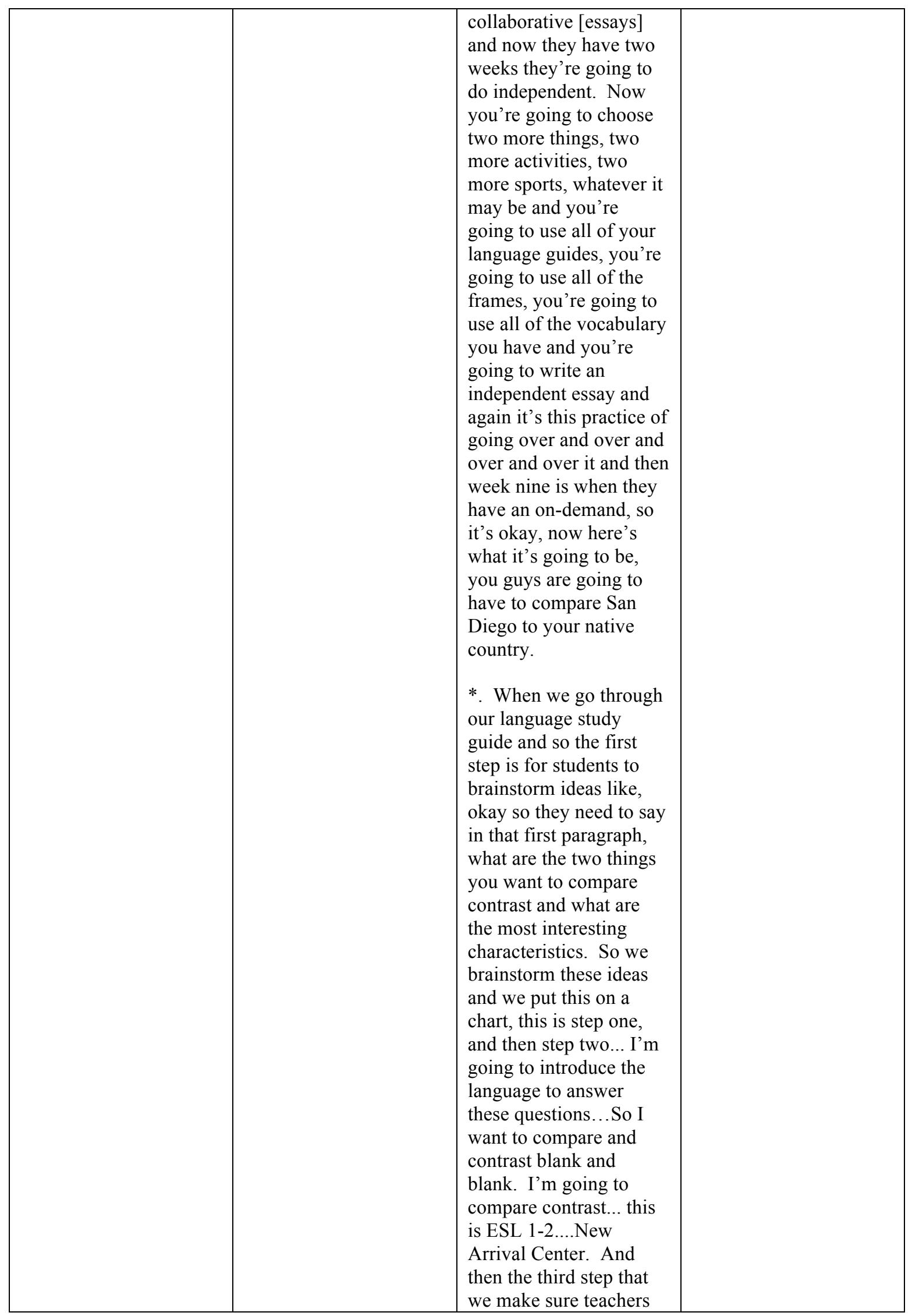




\begin{tabular}{|c|c|c|c|}
\hline & & $\begin{array}{l}\text { do is the oral rehearsal } \\
\text { with linguistic features } \\
\text { and so they don't just } \\
\text { take this and go to } \\
\text { writing, we have the } \\
\text { language, oral language } \\
\text { routines, or you have A } \\
\text { B partners, you have } \\
\text { give one get one, you } \\
\text { have the talking sticks, } \\
\text { you have all those things } \\
\text { where students have to } \\
\text { speak this language } \\
\text { before they ever write it. } \\
\text { And so we have them } \\
\text { practice, and I might say } \\
\text { 'okay, here's the } \\
\text { question so what two } \\
\text { things do you want to } \\
\text { compare and contrast, so } \\
\text { I might say 'Mandy, can } \\
\text { you use one of these } \\
\text { frames' and it's up on a } \\
\text { chart, can you use one } \\
\text { of these frames because } \\
\text { we're doing birds and } \\
\text { bats and can you answer } \\
\text { that question, and you } \\
\text { might say 'oh two things } \\
\text { that I want to compare } \\
\text { contrast are birds and } \\
\text { bats'... Okay next } \\
\text { person now, go to your } \\
\text { partner, your partner } \\
\text { asked the question you } \\
\text { use a different frame } \\
\text { and answer...It's very } \\
\text { highly supported and it's } \\
\text { expected every single } \\
\text { day, that they orally } \\
\text { rehearse. }\end{array}$ & \\
\hline Content Instruction & $\begin{array}{l}\text { * The content area } \\
\text { support has strictly been } \\
\text { through QTEL... that's } \\
\text { why we've just started } \\
\text { making roads into the } \\
\text { content area support, } \\
\text { because we've always } \\
\text { maintained [for those } \\
\text { classes] that nothing is } \\
\text { really going to change } \\
\text { unless we get everybody }\end{array}$ & $\begin{array}{l}\text { * Whereas at Sierra they } \\
\text { don't have as many new } \\
\text { arrivals, they've got like } \\
\text { those intermediate level } \\
\text { kids, so the ELST, she } \\
\text { does a lot of pushing, } \\
\text { there's a lot of co- } \\
\text { planning and co- } \\
\text { teaching in the content } \\
\text { areas and the kids get a } \\
\text { support class, and a lot }\end{array}$ & $\begin{array}{l}\text { QTEL training is the } \\
\text { primary professional } \\
\text { development for content } \\
\text { teachers. It incorporates } \\
\text { strategies for teaching } \\
\text { ELs in mainstream or } \\
\text { sheltered courses. All } \\
\text { teachers at each } \\
\text { secondary site should be } \\
\text { using such strategies to } \\
\text { help students access }\end{array}$ \\
\hline
\end{tabular}




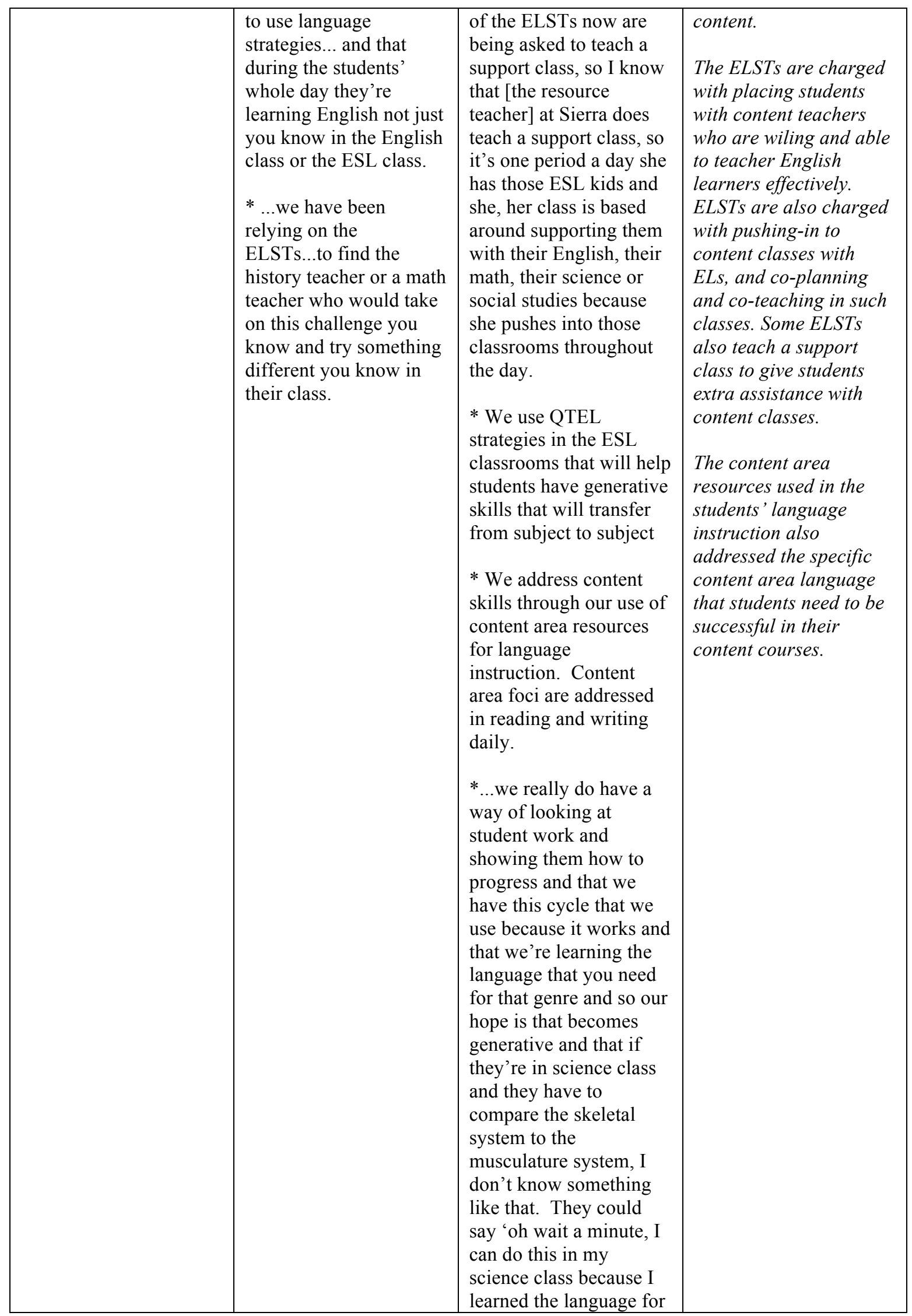




\begin{tabular}{|c|c|c|c|}
\hline & & $\begin{array}{l}\text { this, I know the } \\
\text { language for compare } \\
\text { contrast, no matter what } \\
\text { class I'm in...' }\end{array}$ & \\
\hline $\begin{array}{l}\text { Administrator's } \\
\text { Attitudes/ Program } \\
\text { Expectations }\end{array}$ & $\begin{array}{l}\text { * There have always } \\
\text { been a significant } \\
\text { number of schools that } \\
\text { have never had a } \\
\text { population for ESL. } \\
\text { And so traditionally } \\
\text { those schools have } \\
\text { either you know funded } \\
\text { a lower class size...so } \\
\text { 20, where we would mix } \\
\text { grade levels and a } \\
\text { couple of proficiency } \\
\text { levels, so we would } \\
\text { have sixth, seventh and } \\
\text { eighth grade with } \\
\text { beginners and early } \\
\text { intermediates and that } \\
\text { would usually be } \\
\text { enough students to fund } \\
\text { a full two period course. } \\
\text { But the schools that } \\
\text { can't even do that... that } \\
\text { don't have the numbers } \\
\text { to even do that, you } \\
\text { know we strongly } \\
\text { encourage them to } \\
\text { cluster the kids, so let's } \\
\text { say they have five } \\
\text { beginners or even three } \\
\text { beginners at any grade } \\
\text { level that they find the } \\
\text { teachers who would be } \\
\text { completely on board } \\
\text { with working with these } \\
\text { kids... who would be } \\
\text { completely on board } \\
\text { with doing some... of } \\
\text { using alternative } \\
\text { assessments...and then } \\
\text { we cluster them in those } \\
\text { classes... Placement is } \\
\text { the -- placement is one } \\
\text { of the ongoing issues } \\
\text { that we have you know. } \\
\text { * And every year it } \\
\text { seems like we } \\
\text { continually have to hit } \\
\text { the schools hard and say } \\
\text { okay, how are you } \\
\text { placing them.... What }\end{array}$ & $\begin{array}{l}\text { * Yes, yes [I wanted to } \\
\text { work with English } \\
\text { learners]. That was -- } \\
\text { that's all I had done. I } \\
\text { got my first job ...like } \\
\text { my dad calls and he's } \\
\text { like okay you need to } \\
\text { get a job because I'm } \\
\text { not paying for all of } \\
\text { your college and your } \\
\text { rent and your car...And a } \\
\text { friend had said oh you } \\
\text { should be a teacher's } \\
\text { assistant. I'm like okay. } \\
\text { I mean I had planned to } \\
\text { go pre-law. I mean I } \\
\text { was a pre-law student. I } \\
\text { had you know my whole } \\
\text { direction and I went to } \\
\text { Crawford High School } \\
\text { and interviewed to be a } \\
\text { TA.... So, I taught at } \\
\text { Crawford. Then } \\
\text { because I spoke Spanish } \\
\text { fluently they're like oh } \\
\text { we're going to put you } \\
\text { in ESL. I literally had to } \\
\text { go Google ESL -- I had } \\
\text { never heard of that. } \\
\text { * When I was hired I } \\
\text { was put into the -- they } \\
\text { had a new -- they called } \\
\text { it a Newcomer Center at } \\
\text { that time and I went in } \\
\text { there and it was -- it hit } \\
\text { me like a ton of bricks } \\
\text { that this is my job } \\
\text { like...I'm supposed to do } \\
\text { this. It was not even a } \\
\text { job. It was like a } \\
\text { passion, like oh my god, } \\
\text { never in a million years } \\
\text { did I think I was going } \\
\text { to be a teacher. Never. } \\
\text { And it just hit me like } \\
\text { this is amazing. And I } \\
\text { love this and I happened } \\
\text { to be a fluent Spanish } \\
\text { speaker, which I just } \\
\text { kind of took to be like }\end{array}$ & $\begin{array}{l}\text { Student placement is an } \\
\text { ongoing issue. Some } \\
\text { schools do not have the } \\
\text { numbers to warrant an } \\
\text { ESL class, some are } \\
\text { mixed levels to create } \\
\text { numbers, while other } \\
\text { schools cluster such } \\
\text { students with } \\
\text { mainstream teachers } \\
\text { that are willing and able } \\
\text { to work with such } \\
\text { students. Purposeful } \\
\text { placement is key to } \\
\text { effective instruction for } \\
\text { ESL students. } \\
\\
\text { Professional } \\
\text { development of all } \\
\text { teachers concentrating } \\
\text { on EL strategies should } \\
\text { assist mainstream } \\
\text { teachers in better } \\
\text { educating ESL students. } \\
\text { Support classes are also } \\
\text { used to support students } \\
\text { with their acquisition of } \\
\text { English and their class } \\
\text { work at many sites. The } \\
\text { administrators support } \\
\text { teachers by assisting } \\
\text { them with small group } \\
\text { instruction as needed to } \\
\text { meet the needs of their } \\
\text { ESL students. } \\
\text { The administrators have } \\
\text { a background in } \\
\text { teaching ESL as } \\
\text { classroom teachers. } \\
\text { They have chosen to } \\
\text { specialize in this field } \\
\text { and feel passionate } \\
\text { about ESL students. } \\
\text { They feel pulled thin, as } \\
\text { there is only one } \\
\text { administrator for over } \\
\text { 20 sites. They feel their } \\
\text { major tension is a lack } \\
\text { of time to go out to sites } \\
\text { and work with teachers. } \\
\text { The administrators work }\end{array}$ \\
\hline
\end{tabular}




\begin{tabular}{|c|c|c|c|}
\hline & 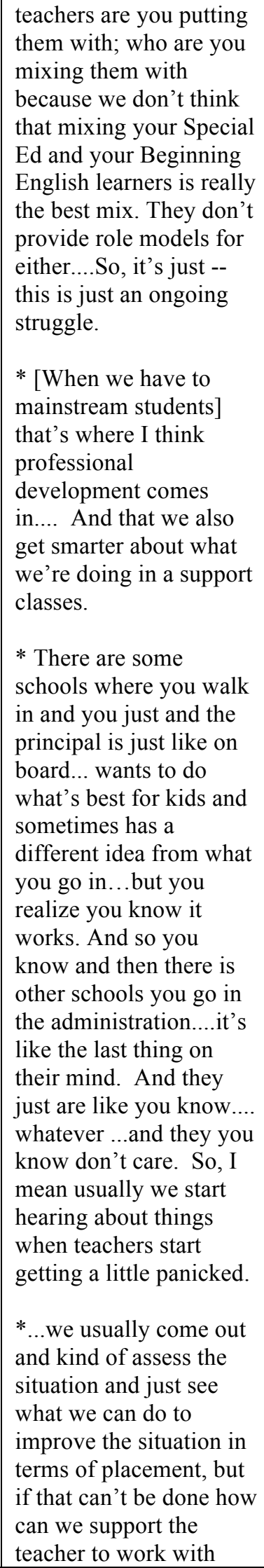 & 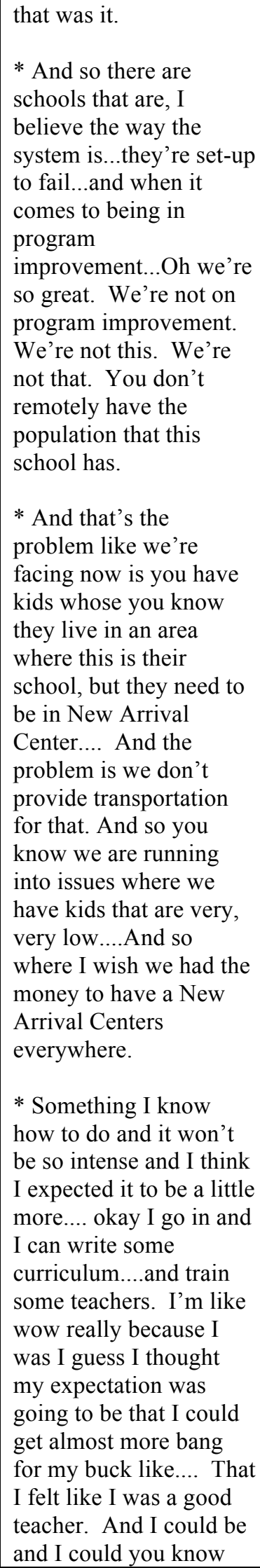 & $\begin{array}{l}\text { increasingly with ELSTS } \\
\text { to assist teachers on } \\
\text { site. } \\
\text { They were hired as } \\
\text { Resource Teachers, but } \\
\text { have had to take on } \\
\text { more central office } \\
\text { responsibilities, which } \\
\text { gives them less time to } \\
\text { co-plan and co-teach } \\
\text { with ESL teachers on } \\
\text { site. There are fewer } \\
\text { resources available as } \\
\text { well due to budget } \\
\text { decreases, but what they } \\
\text { have, they get out to the } \\
\text { sites. They also provide } \\
\text { professional } \\
\text { development and write } \\
\text { curriculum. }\end{array}$ \\
\hline
\end{tabular}




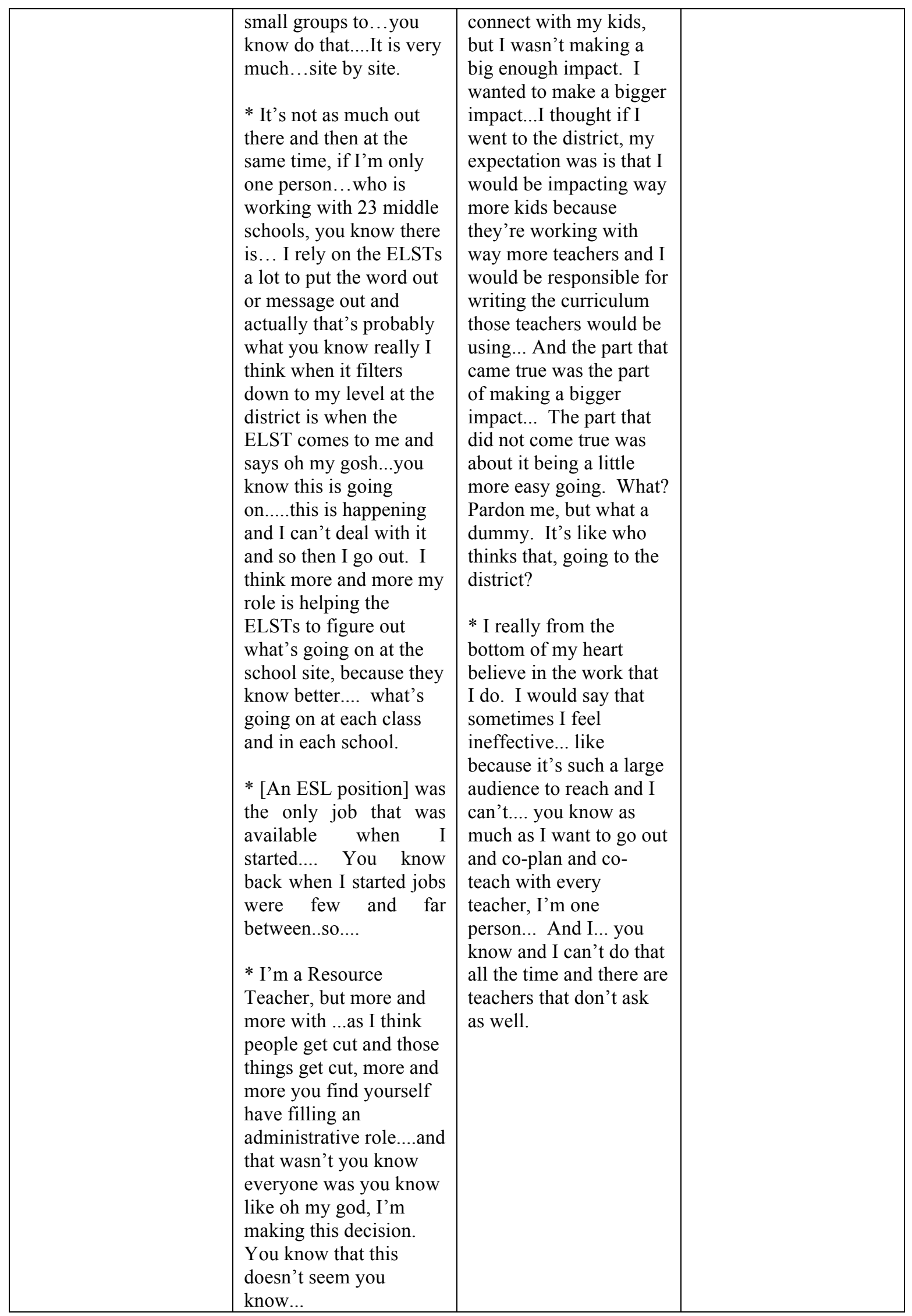




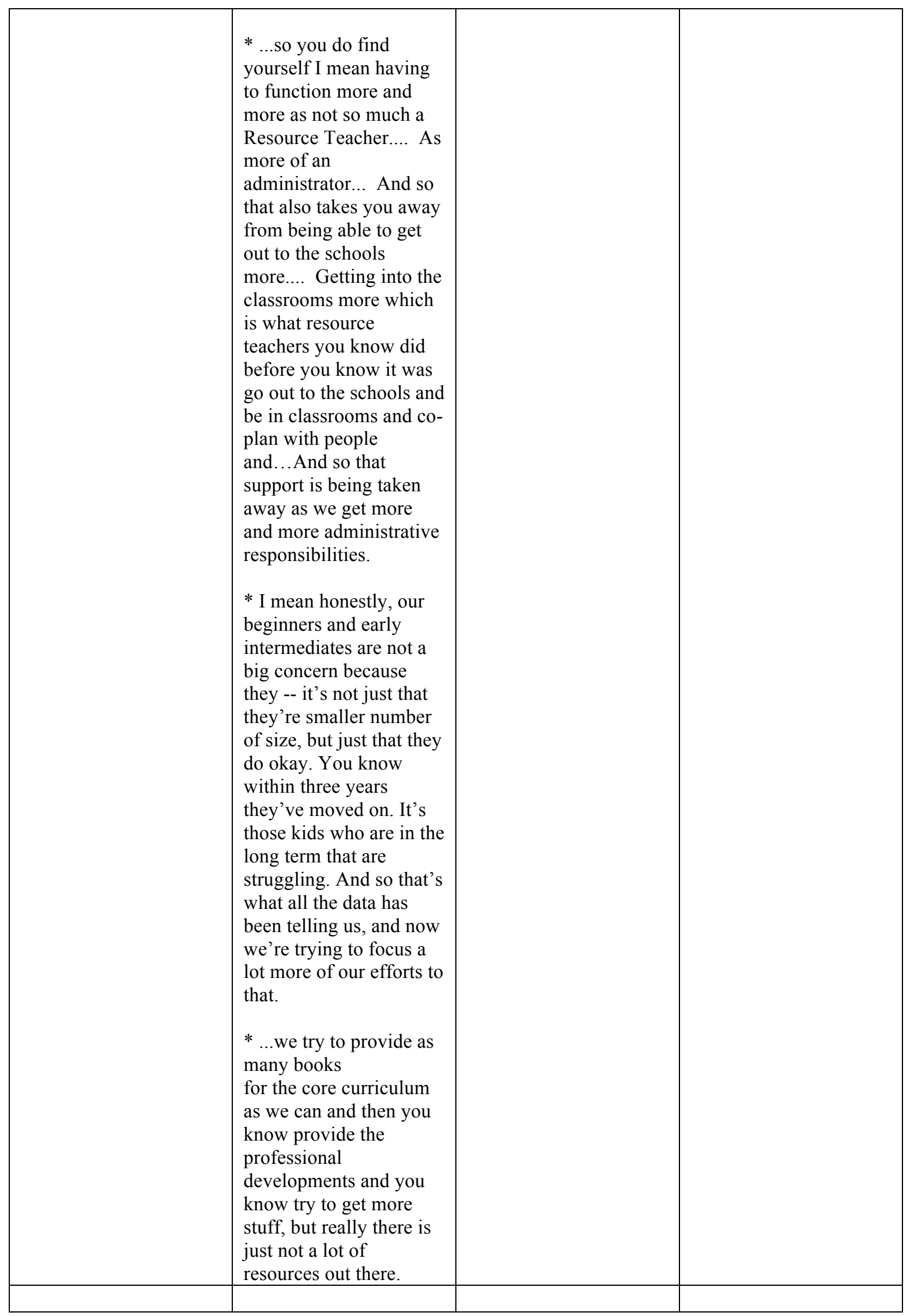




\begin{tabular}{|c|c|c|c|}
\hline $\begin{array}{c}\text { Culturally } \\
\text { Responsive } \\
\text { Teaching }\end{array}$ & $\begin{array}{l}* \text { [To communicate with } \\
\text { parents] we just have a } \\
\text { lot of translation } \\
\text { services...and I mean at } \\
\text { school sites they do } \\
\text { some of them have } \\
\text { specialized people who } \\
\text { come in you know } \\
\text { community liaisons and } \\
\text { you know parent } \\
\text { resource centers that } \\
\text { they use so } \\
\text { communicate you } \\
\text { know... } \\
\text { * Yeah, that's what } \\
\text { should be happening in } \\
\text { those ELAC meetings } \\
\text { [informing parents about } \\
\text { school]. I think one of } \\
\text { the requirements is that } \\
\text { they inform parents } \\
\text { about the structure and } \\
\text { their rights, so. }\end{array}$ & $\begin{array}{l}\text { * For the first time, so } \\
\text { when they're running } \\
\text { around and they don't } \\
\text { know what to do, it's } \\
\text { because they've never } \\
\text { been in an environment } \\
\text { where you sit at a desk } \\
\text { or you listen to a } \\
\text { teacher... they've never } \\
\text { experienced that and } \\
\text { that's a huge wake up } \\
\text { call for teachers. } \\
\text { * You know I'll never I } \\
\text { mean as an ESL teacher } \\
\text { it's something I always } \\
\text { aware of but even I have } \\
\text { caught myself. I'll } \\
\text { never forget I had a girl } \\
\text { from Ethiopia. Oh my } \\
\text { god, so beautiful and so } \\
\text { sweet, never stepped } \\
\text { into a school building } \\
\text { her entire life...she lived } \\
\text { in the bush and was sent } \\
\text { a refugee camp and sent } \\
\text { here and I was having } \\
\text { the kids do an activity } \\
\text { where they had to cut. } \\
\text { And they all had } \\
\text { scissors and I look over } \\
\text { and she is holding her } \\
\text { paper and she is holding } \\
\text { the scissors and she is } \\
\text { trying to cut with two } \\
\text { hands and the paper, and } \\
\text { I realized she has never } \\
\text { seen scissors before. } \\
\text { And I thought oh my } \\
\text { gosh, how can I not } \\
\text { have recognized } \\
\text { that...You know like so } \\
\text { you know if I know that } \\
\text { I can miss that, oh my, I } \\
\text { know that the } 11^{\text {th }} \text { grade } \\
\text { social studies teacher } \\
\text { down the hall that's not } \\
\text { on their mind. }\end{array}$ & $\begin{array}{l}\text { Students come from } \\
\text { many academic } \\
\text { environments, which can } \\
\text { be difficult for many } \\
\text { teachers to understand. } \\
\text { It's important for } \\
\text { teachers to understand } \\
\text { students' cultural and } \\
\text { educational } \\
\text { backgrounds to better } \\
\text { understand the manner } \\
\text { in which students know } \\
\text { how to learn. Many } \\
\text { students are not } \\
\text { educated in a setting } \\
\text { akin to schools in the } \\
\text { U.S. and must be } \\
\text { explicitly taught how } \\
\text { schools function in the } \\
\text { U.S. } \\
\text { Teachers and } \\
\text { administrators utilize } \\
\text { district translation } \\
\text { services to communicate } \\
\text { with parents, and some } \\
\text { sites have parent liaison } \\
\text { and community services } \\
\text { to assist in connecting } \\
\text { parents to schools. The } \\
\text { site English Learner } \\
\text { Advisory Committee is } \\
\text { charged with informing } \\
\text { parents of English } \\
\text { learners about their } \\
\text { rights and happenings at } \\
\text { the site. }\end{array}$ \\
\hline $\begin{array}{c}\text { Pedagogical } \\
\text { Orientations/ } \\
\text { Beliefs }\end{array}$ & $\begin{array}{l}*[\text { My main job is }] \text { trying } \\
\text { to build consistency } \\
\text { across schools for ESL } \\
\text { so that a teacher who's } \\
\text { teaching a level one } \\
\text { class at this school could }\end{array}$ & $\begin{array}{l}\text { * We're so organized } \\
\text { now. I mean we have } \\
\text { dedicated resource } \\
\text { teachers to very specific } \\
\text { areas who go out into } \\
\text { the classrooms and work }\end{array}$ & $\begin{array}{l}\text { A primary role of the } \\
\text { administrators is to } \\
\text { build consistency across } \\
\text { within all ESL programs } \\
\text { around the district. The } \\
\text { consistency can allow }\end{array}$ \\
\hline
\end{tabular}




\begin{tabular}{|c|c|c|c|}
\hline & $\begin{array}{l}\text { potentially collaborate } \\
\text { with the teacher at this } \\
\text { school because we've } \\
\text { got a common } \\
\text { curriculum. } \\
\text { *[I need to] to be able to } \\
\text { provide as many } \\
\text { resources for teachers, } \\
\text { ESL teachers as } \\
\text { possible, because by and } \\
\text { large an ESL teacher at } \\
\text { a school site like is } \\
\text { pretty much working by } \\
\text { themselves. } \\
\text { * ESL teachers kind of } \\
\text { work in isolation, } \\
\text { because what they do } \\
\text { isn't like the regular } \\
\text { English class... And } \\
\text { it's not like any other } \\
\text { class campus, so my } \\
\text { hope was that I could } \\
\text { bring those teachers } \\
\text { together little bit more } \\
\text { so they would work } \\
\text { together. And I think } \\
\text { you know I'm excited } \\
\text { about the new } \\
\text { technology because I } \\
\text { think we'll have a lot of } \\
\text { different venues where } \\
\text { we can just converse } \\
\text { with one another you } \\
\text { know post lessons, get } \\
\text { comments from other } \\
\text { people, you know do all } \\
\text { kinds of things like that, } \\
\text { so... } \\
\text { * I think [being an ESL } \\
\text { teacher] prepared me to } \\
\text { work with teachers and } \\
\text { then to have pretty good } \\
\text { knowledge of what it } \\
\text { would take to what we } \\
\text { or and then I also realize } \\
\text { as like, oh my god, I } \\
\text { wish I would have this }\end{array}$ & 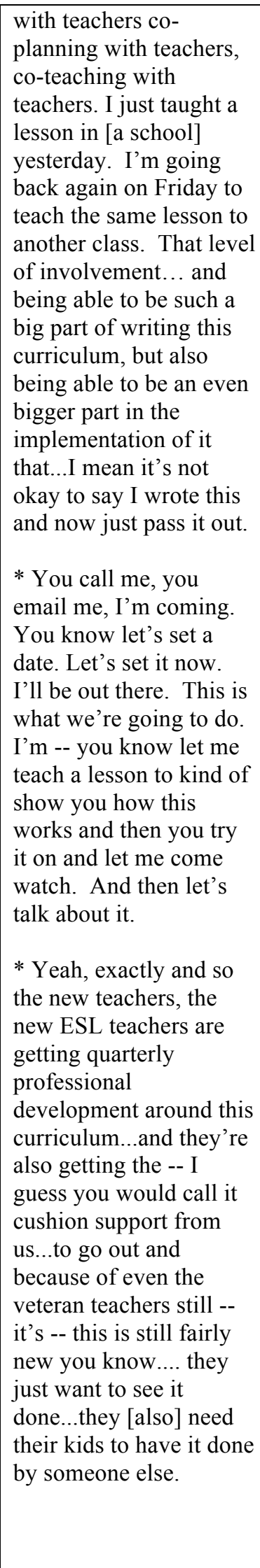 & $\begin{array}{l}\text { for the possibility of } \\
\text { collaboration among } \\
\text { ESL teachers at various } \\
\text { sites. Additionally, the } \\
\text { administrators must } \\
\text { provide resources and } \\
\text { training to the ESL } \\
\text { teachers, in that they are } \\
\text { often the only teacher at } \\
\text { their site servicing the } \\
\text { ESL students. The } \\
\text { program administrators } \\
\text { believe they should co- } \\
\text { plan and co-teach with } \\
\text { the teachers to model } \\
\text { best practices. } \\
\text { The administrators are } \\
\text { excited to utilize } \\
\text { technology to assist the } \\
\text { teachers in } \\
\text { collaborating across the } \\
\text { district. } \\
\text { Both administrators had } \\
\text { experience as ESL } \\
\text { teachers, which gave } \\
\text { them a better } \\
\text { understanding of how } \\
\text { they can best support } \\
\text { such teachers. Along } \\
\text { with resources, they } \\
\text { want to provide the ESL } \\
\text { teachers with time to } \\
\text { collaborate and plan } \\
\text { with one another. } \\
\end{array}$ \\
\hline
\end{tabular}




\begin{tabular}{|c|c|c|c|}
\hline & $\begin{array}{l}\text { because I could have } \\
\text { raised my level of } \\
\text { instruction if I would } \\
\text { have known this before } \\
\text { and so, it does help to } \\
\text { have teaching you know } \\
\text { teaching experience. } \\
\text { * I just see myself as } \\
\text { trying to find the } \\
\text { resources for } \\
\text { teachers...as soon as } \\
\text { possible and then } \\
\text { providing them the time } \\
\text { and opportunity to be } \\
\text { able to you know co- } \\
\text { plan with peers and find } \\
\text { resources. More and } \\
\text { more and more, the } \\
\text { biggest thing teachers } \\
\text { ask for is time...so I } \\
\text { think more and more the } \\
\text { role we should play is } \\
\text { that if that can be funded } \\
\text { then I will do my best to } \\
\text { provide them the } \\
\text { opportunity to plan. }\end{array}$ & & \\
\hline $\begin{array}{l}\text { Academic Self- } \\
\text { Concept }\end{array}$ & $\begin{array}{l}\text { * I've heard reports. } \\
\text {...people saying you } \\
\text { know the student who's } \\
\text { never you know raised } \\
\text { their hand or } \\
\text { volunteered before is } \\
\text { now you know raising } \\
\text { their hand and } \\
\text { volunteering } \\
\text { information and so I'm } \\
\text { hearing more and more } \\
\text { about that...I think } \\
\text { they're more confident. } \\
\text { * I think well I you } \\
\text { know I'm not just } \\
\text { talking about one school } \\
\text { if you look at it as a } \\
\text { whole, I think as a } \\
\text { whole...for the most part } \\
\text { [they are more } \\
\text { confident], there are } \\
\text { going to be pockets } \\
\text { where you know the } \\
\text { instruction wasn't the } \\
\text { strongest, so... who } \\
\text { knows. But maybe their }\end{array}$ & $\begin{array}{l}\text { * One major part of our } \\
\text { curriculum includes } \\
\text { character education. We } \\
\text { discuss the difficulties } \\
\text { and challenges of being } \\
\text { an English learner. } \\
\text { Students have multiple } \\
\text { opportunities to learn } \\
\text { and reflect upon their } \\
\text { experiences. This } \\
\text { allows students to relate } \\
\text { with one another and } \\
\text { build quality } \\
\text { interactions with one } \\
\text { another. } \\
\text { * I believe that students } \\
\text { are more confident } \\
\text { academically and } \\
\text { socially as a result of } \\
\text { their participation in our } \\
\text { program. They are able } \\
\text { to find a niche for } \\
\text { themselves in a very } \\
\text { new and sometimes } \\
\text { overwhelming setting. } \\
\text { Our students are }\end{array}$ & $\begin{array}{l}\text { Teachers report that } \\
\text { students who have never } \\
\text { participated before are } \\
\text { now attempting to } \\
\text { participate in class. } \\
\text { They appear far more } \\
\text { confident as a result of } \\
\text { acquiring language } \\
\text { skills in ESL class. } \\
\text { A part of the curriculum } \\
\text { is character education. } \\
\text { They discuss the } \\
\text { challenges students face } \\
\text { in being English } \\
\text { learners. This gives } \\
\text { students a chance to } \\
\text { reflect upon their } \\
\text { experiences and to } \\
\text { relate to one another } \\
\text { and to build quality } \\
\text { interactions between } \\
\text { students. Students can } \\
\text { find others in their same } \\
\text { position, which is very } \\
\text { comforting in an } \\
\text { overwhelming setting. }\end{array}$ \\
\hline
\end{tabular}




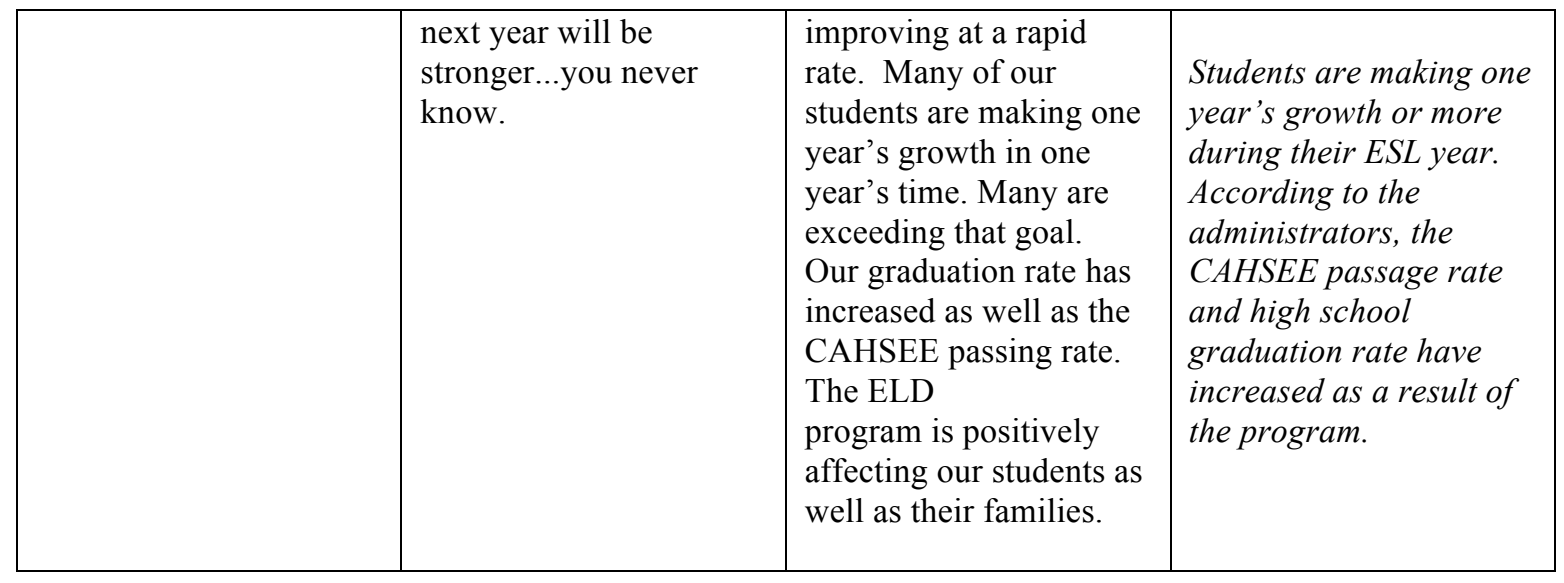




\section{Appendix R}

\section{NAC Focus Group Analysis}

\begin{tabular}{|c|c|c|c|c|}
\hline & $\begin{array}{c}\text { Focus Group \#1 } \\
\text { NAC-Year } 1\end{array}$ & $\begin{array}{c}\text { Focus Group \#2 } \\
\text { NAC-Year } 1\end{array}$ & $\begin{array}{c}\text { Focus Group \#3 } \\
\text { NAC-Year } 2\end{array}$ & Significant Themes \\
\hline $\begin{array}{l}\text { Language } \\
\text { Instruction }\end{array}$ & $\begin{array}{l}\text { * In my house } \\
\text { when I am with my } \\
\text { aunts, uncles and } \\
\text { cousins they want } \\
\text { me to learn English } \\
\text { and they know both } \\
\text { languages. For the } \\
\text { most part they ask } \\
\text { me to speak } \\
\text { English and } \\
\text { because of it I have } \\
\text { been improving in } \\
\text { my classes and I } \\
\text { am asking for less } \\
\text { help. }\end{array}$ & $\begin{array}{l}\text { *My friends who } \\
\text { speak English } \\
\text { sometimes I } \\
\text { understand them } \\
\text { sometimes I don't. } \\
\text { My sister speaks } \\
\text { English, my brother } \\
\text { in law, my dad, my } \\
\text { uncle and they } \\
\text { sometimes speak to } \\
\text { me in English. My } \\
\text { brother in law is } \\
\text { the one that helps } \\
\text { me the most now } \\
\text { that's all he speaks } \\
\text { to me hardly any } \\
\text { Spanish. } \\
\text { * ... my cousins } \\
\text { who live here don't } \\
\text { speak Spanish well } \\
\text { and they help me } \\
\text { understand... when } \\
\text { we go to the store } \\
\text { they will say what } \\
\text { they are talking } \\
\text { about and I answer } \\
\text { them. } \\
\text { * Ms H gave us a } \\
\text { program on the } \\
\text { follow...also games } \\
\text { have helped me a } \\
\text { computer known as } \\
\text { Learning Upgrade } \\
\text { in the computer } \\
\text { like YouTube... I } \\
\text { we can use it at } \\
\text { home it teaches us } \\
\text { words and how to } \\
\text { pronounce them. I } \\
\text { use it at home. } \\
\text { tysics for there that's } \\
\text { for me to }\end{array}$ & $\begin{array}{l}\text { * In school not } \\
\text { much [has helped } \\
\text { me learn English], } \\
\text { but when I am } \\
\text { home listening to } \\
\text { music it helps } \\
\text { because when I like } \\
\text { a song I look up the } \\
\text { lyrics and that helps } \\
\text { me learn new } \\
\text { words by listening } \\
\text { to music. } \\
\text { * Reading and } \\
\text { speaking English } \\
\text { helps me [learn } \\
\text { more English]. }\end{array}$ & $\begin{array}{l}\text { Students commonly } \\
\text { acquire English } \\
\text { through friends and } \\
\text { family members } \\
\text { outside of school. } \\
\text { Many students have } \\
\text { English-speaking } \\
\text { friends and family } \\
\text { members that support } \\
\text { students in learning } \\
\text { English. Students } \\
\text { have to use English } \\
\text { to negotiate their } \\
\text { world outside of } \\
\text { school, such as } \\
\text { speaking to agents } \\
\text { when crossing the } \\
\text { border, which assists } \\
\text { them in learning the } \\
\text { language. } \\
\text { A district educational } \\
\text { computer program, } \\
\text { called Learning } \\
\text { Upgrade, assists } \\
\text { students at home in } \\
\text { practicing language. } \\
\text { Students listen to } \\
\text { songs and translate } \\
\text { the lyrics to learn } \\
\text { more English outside } \\
\text { of school. } \\
\text { Reading in English } \\
\text { also helps students } \\
\text { learn more language } \\
\text { outside of school. }\end{array}$ \\
\hline
\end{tabular}




\begin{tabular}{|c|c|c|c|c|}
\hline & & $\begin{array}{l}\text { bit and I understand } \\
\text { what they say. } \\
\text { * I learn when I go } \\
\text { to Tijuana and I } \\
\text { cross back... the } \\
\text { agents there ask me } \\
\text { and I answer them } \\
\text { in English. They } \\
\text { ask how old I am, } \\
\text { what school I go to, } \\
\text { what grade I am in, } \\
\text { and they ask where } \\
\text { I live and I tell } \\
\text { them... questions } \\
\text { like that and } \\
\text { sometime I get to } \\
\text { chat with them. } \\
\text { * I try to learn } \\
\text { [concepts] } \\
\text { normally, but if } \\
\text { that doesn't work } \\
\text { then I try to come } \\
\text { up with a song to } \\
\text { study the material I } \\
\text { need so I can } \\
\text { memorize it }\end{array}$ & & \\
\hline $\begin{array}{c}\text { Content } \\
\text { Instruction }\end{array}$ & $\begin{array}{l}\text { * My teacher tells } \\
\text { us to learn math } \\
\text { because we will } \\
\text { need it to be } \\
\text { doctors or whatever } \\
\text { we want to be. } \\
\text { * They explain } \\
\text { [difficult concepts] } \\
\text { by writing them on } \\
\text { the board and } \\
\text { showing us how } \\
\text { to...for example do } \\
\text { a division or } \\
\text { multiplication } \\
\text { problem and the } \\
\text { hardest things... } \\
\text { step by step in } \\
\text { order. }\end{array}$ & $\begin{array}{l}\text { * I did [understand] } \\
\text { because when I got } \\
\text { to my science class } \\
\text { I saw the names of } \\
\text { body parts but in } \\
\text { English. } \\
\text { * Math [is easiest] } \\
\text { because what they } \\
\text { are teaching is easy } \\
\text { so far. } \\
\text { * Science [is } \\
\text { easiest] because } \\
\text { when I finished } \\
\text { middle school } \\
\text { that's what we } \\
\text { were learning so I } \\
\text { know the basic } \\
\text { foundation. } \\
\text { * What I } \\
\text { understand easier } \\
\text { than history is } \\
\text { science because } \\
\text { history is pure }\end{array}$ & $\begin{array}{l}\text { * For me [my } \\
\text { easiest class] is } \\
\text { history because its } \\
\text { my favorite subject, } \\
\text { it was my favorite } \\
\text { subject in Mexico } \\
\text { and its my favorite } \\
\text { subject here... the } \\
\text { only thing that } \\
\text { changed is the } \\
\text { language but I still } \\
\text { understand and I } \\
\text { like to focus a lot in } \\
\text { history. } \\
\text { * [The easiest class } \\
\text { is] math because } \\
\text { the teacher writes } \\
\text { everything on the } \\
\text { board and in other } \\
\text { classes they don't } \\
\text {.. it helps to see } \\
\text { what they are } \\
\text { talking about. I } \\
\text { learn more. }\end{array}$ & $\begin{array}{l}\text { The teachers } \\
\text { encourage kids to } \\
\text { learn math by letting } \\
\text { them know they will } \\
\text { need it in the future. } \\
\text { Teachers teach } \\
\text { difficult math } \\
\text { concepts step-by-step } \\
\text { to help them better } \\
\text { understand. They } \\
\text { write it all on the } \\
\text { board, which helps } \\
\text { the kids. } \\
\text { The students find } \\
\text { science easier than } \\
\text { found because history } \\
\text { was purely reading } \\
\text { (in English, whereas } \\
\text { science is more } \\
\text { hands-on and uses } \\
\text { manipulatives. } \\
\text { History was also } \\
\text { difficult as the } \\
\text { students were not }\end{array}$ \\
\hline
\end{tabular}




\begin{tabular}{|c|c|c|c|c|}
\hline & & $\begin{array}{l}\text { reading and } \\
\text { science, well I } \\
\text { understand it. } \\
\text { * For me } \\
\text { mathematics [is } \\
\text { easiest]. In the } \\
\text { beginning I didn't } \\
\text { understand it but } \\
\text { already I see the } \\
\text { work and I can do } \\
\text { it easily in math } \\
\text { and history, except } \\
\text { PE because I don't } \\
\text { understand } \\
\text { teachers. } \\
\text { * Math [is easiest] } \\
\text { because what we } \\
\text { are doing, I already } \\
\text { saw it in } \\
\text { Tijuana...and for } \\
\text { me it's easy } \\
\text { because the } \\
\text { numbers are the } \\
\text { same in Mexico as } \\
\text { they are here. Often } \\
\text { you realize they're } \\
\text { reviewing the } \\
\text { [same concepts] } \\
\text { here... the numbers } \\
\text { are the same but } \\
\text { they are in a } \\
\text { different } \\
\text { language... in } \\
\text { English here and } \\
\text { there in Spanish. } \\
\text { All you have to } \\
\text { know to understand } \\
\text { mathematics are } \\
\text { the numbers in } \\
\text { English. } \\
\text { * PE [is the hardest } \\
\text { class] because the } \\
\text { teacher that my } \\
\text { brother and I have } \\
\text { does not speak } \\
\text { Spanish and he } \\
\text { says to do the } \\
\text { homework but he } \\
\text { speaks purely in } \\
\text { English and usually } \\
\text { we don't } \\
\text { understand him, we } \\
\text { don't do it, and our }\end{array}$ & $\begin{array}{l}\text { * Geometry [is } \\
\text { easiest] because } \\
\text { everything is } \\
\text { written on the } \\
\text { board and we just } \\
\text { copy it. } \\
\text { * The hardest for } \\
\text { me...well, there is } \\
\text { two subjects. There } \\
\text { is biology and } \\
\text { algebra. In biology } \\
\text { there is a teacher } \\
\text { that is so mean to } \\
\text { us because we do } \\
\text { not understand } \\
\text { English and when } \\
\text { we have a questions } \\
\text { he says look at the } \\
\text { instructions, but we } \\
\text { don't understand } \\
\text { the instructions and } \\
\text { we tell her that and } \\
\text { she says well that is } \\
\text { not my problem } \\
\text { you're suppose to } \\
\text { be paying attention. } \\
\text { And Algebra } \\
\text { because the teacher } \\
\text { is Korean and she } \\
\text { has a different } \\
\text { accent so I don't } \\
\text { understand what } \\
\text { she is saying... It's } \\
\text { the way she talks } \\
\text { that I don't } \\
\text { understand. } \\
\text { * I have two hard } \\
\text { classes. In } \\
\text { geometry there are } \\
\text { so many students } \\
\text { that are always } \\
\text { talking and the } \\
\text { teacher is hard to } \\
\text { understand and in } \\
\text { history because I } \\
\text { don't understand } \\
\text { the words they use. } \\
\\
\text { * Science is hard } \\
\text { because most is } \\
\text { taught orally and } \\
\text { then you have to go } \\
\text { in front of class to } \\
\text { explain it. }\end{array}$ & $\begin{array}{l}\text { familiar with U.S. } \\
\text { history. } \\
\\
\text { Math was easiest for } \\
\text { some students, } \\
\text { because they had } \\
\text { already learned the } \\
\text { foundation in their } \\
\text { home countries } \\
\text { (Mexico). Some } \\
\text { reported the math in } \\
\text { Mexico to be higher } \\
\text { than what they } \\
\text { learned so far in the } \\
\text { U.S. They also felt } \\
\text { more comfortable as } \\
\text { they recognized the } \\
\text { numbers for math, } \\
\text { despite the language } \\
\text { being different. } \\
\\
\text { At one site, PE was } \\
\text { hardest for the } \\
\text { students, as the } \\
\text { teacher did not speak } \\
\text { Spanish and they } \\
\text { didn't understand } \\
\text { him. He lowered their } \\
\text { grades since they } \\
\text { could not complete } \\
\text { the work. This was } \\
\text { their only } \\
\text { mainstream class. } \\
\text { Year two students } \\
\text { report they struggle } \\
\text { most with teachers } \\
\text { with whom they } \\
\text { struggle to connect. } \\
\text { One student reported } \\
\text { enjoying history, as it } \\
\text { was his favorite } \\
\text { subject, both in } \\
\text { Mexico and here in } \\
\text { the U.S. Students felt } \\
\text { math was easiest, as } \\
\text { the teachers wrote } \\
\text { the problems on the } \\
\text { board and they could } \\
\text { better understand the } \\
\text { visual. The students } \\
\text { had a difficult time in } \\
\text { classes, such as } \\
\text { science, in which the } \\
\text { subject was taught }\end{array}$ \\
\hline
\end{tabular}




\begin{tabular}{|c|c|c|c|c|}
\hline & & $\begin{array}{l}\text { grade is lowered. } \\
\text { * History is } \\
\text { difficult for me } \\
\text { because I don't } \\
\text { know much about } \\
\text { the history of the } \\
\text { U.S. }\end{array}$ & $\begin{array}{l}\text { * I have a tutor for } \\
\text { history, we do some } \\
\text { work to prepare for } \\
\text { the exam and that } \\
\text { helps me pass it and } \\
\text { in biology I have } \\
\text { tutoring and she } \\
\text { gives us a sample } \\
\text { exam but in } \\
\text { geometry nothing is } \\
\text { easy. } \\
\text { * [Before tests] I } \\
\text { take a look at my } \\
\text { notes, to see if I } \\
\text { remember and in } \\
\text { geometry an oral } \\
\text { review helps even } \\
\text { if its in English... it } \\
\text { works as a } \\
\text { reminder it helps } \\
\text { for my exams. I do } \\
\text { the same in Algebra } \\
\text { I look at my notes } \\
\text { and if I don't know } \\
\text { something I don't } \\
\text { do it because I can't } \\
\text { ask the teacher. }\end{array}$ & $\begin{array}{l}\text { orally and they were } \\
\text { expected to present. }\end{array}$ \\
\hline $\begin{array}{c}\text { Student } \\
\text { Attitude/ } \\
\text { Expectations }\end{array}$ & $\begin{array}{l}\text { * [When I first } \\
\text { came I was] } \\
\text { nervous, I though } \\
\text { no one would speak } \\
\text { Spanish and that I } \\
\text { would be the } \\
\text { oldest. } \\
\text { * The same. I was } \\
\text { afraid because they } \\
\text { are different people } \\
\text { but once you see } \\
\text { the reality of things } \\
\text { you start getting } \\
\text { comfortable with } \\
\text { the people around } \\
\text { you. } \\
\text { *...the same as my } \\
\text { friend [when I first } \\
\text { came] I thought no } \\
\text { one would speak } \\
\text { Spanish, once I got } \\
\text { here I realized that }\end{array}$ & $\begin{array}{l}\text { * I came to study } \\
\text { here because I was } \\
\text { told the diploma } \\
\text { here was valued } \\
\text { more and plus I } \\
\text { didn't get the one } \\
\text { from over there. } \\
\text { * I came to study } \\
\text { here so it can be } \\
\text { easier to get a job. } \\
\text { * [Since my family } \\
\text { moved here before } \\
\text { me] I felt } \\
\text { incomplete, my } \\
\text { family was } \\
\text { incomplete and } \\
\text { apart. } \\
\text { * I felt bad [when } \\
\text { we moved] because } \\
\text { my father's entire } \\
\text { family is here, but }\end{array}$ & $\begin{array}{l}\text { * I struggled [in } \\
\text { Mexico]. I never } \\
\text { had good grades } \\
\text { because I never } \\
\text { listened to the } \\
\text { teachers ... It was } \\
\text { more fun to be with } \\
\text { my friends } \\
\text { * I liked the } \\
\text { teachers [in } \\
\text { Mexico] but they } \\
\text { didn't like me and } \\
\text { we didn't work } \\
\text { well...[my grades] } \\
\text { were good but the } \\
\text { teachers didn't like } \\
\text { me. I was } \\
\text { scandalous. } \\
\text { * I tried my best, I } \\
\text { think [the teachers } \\
\text { in Mexico] were } \\
\text { good. }\end{array}$ & $\begin{array}{l}\text { Students reported } \\
\text { feeling nervous about } \\
\text { starting school in the } \\
\text { U.S. Several believed } \\
\text { no one would spoke } \\
\text { Spanish. After the } \\
\text { students arrived they } \\
\text { reported feeling more } \\
\text { comfortable, } \\
\text { particularly after } \\
\text { realizing that people } \\
\text { did speak Spanish. A } \\
\text { couple also worried } \\
\text { they would be the } \\
\text { oldest in their } \\
\text { classes, but they } \\
\text { realized they were } \\
\text { incorrect after } \\
\text { starting school. In the } \\
\text { beginning, they felt } \\
\text { uncomfortable } \\
\text { participating and } \\
\text { reading aloud in } \\
\text { class, since many felt }\end{array}$ \\
\hline
\end{tabular}




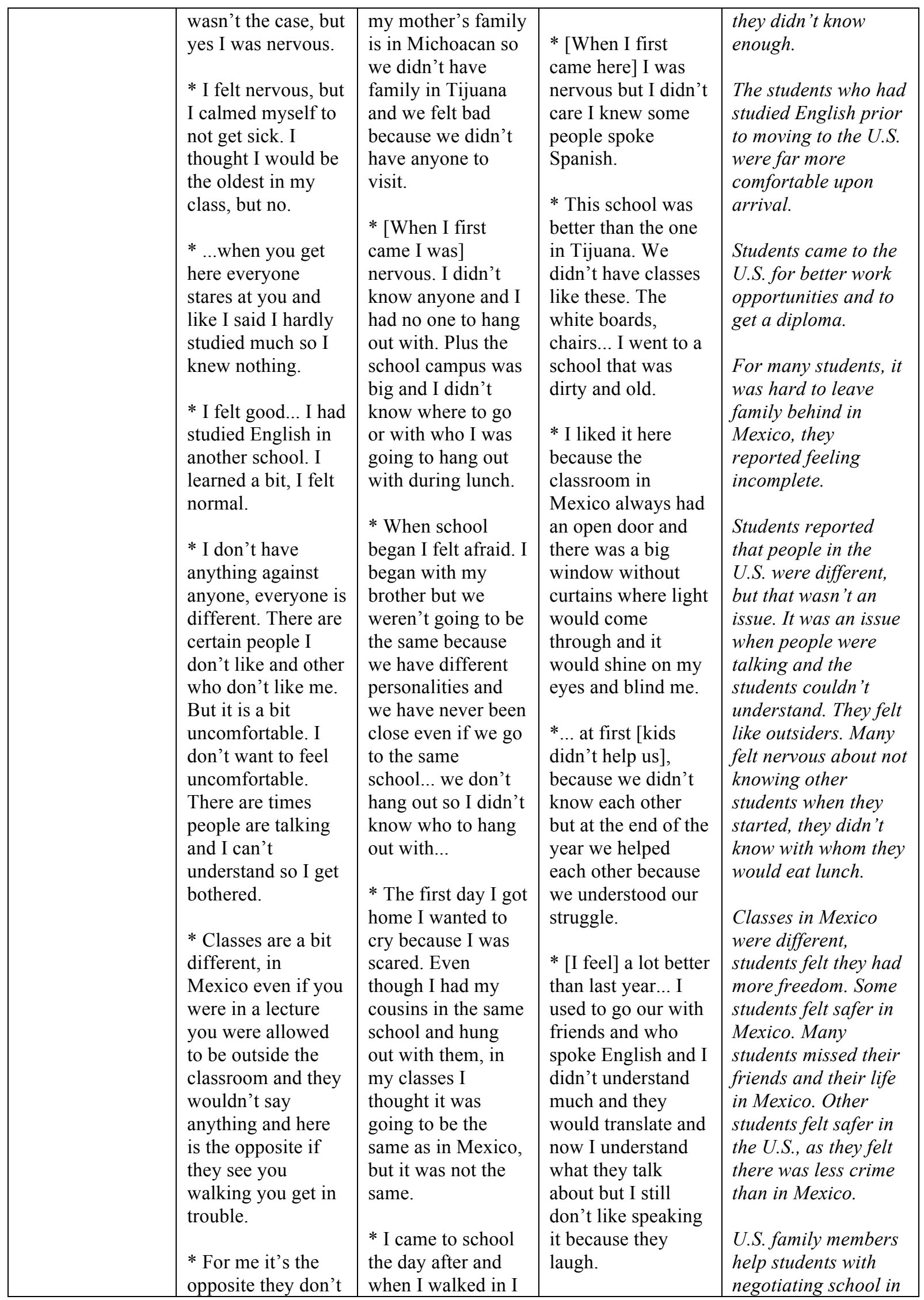




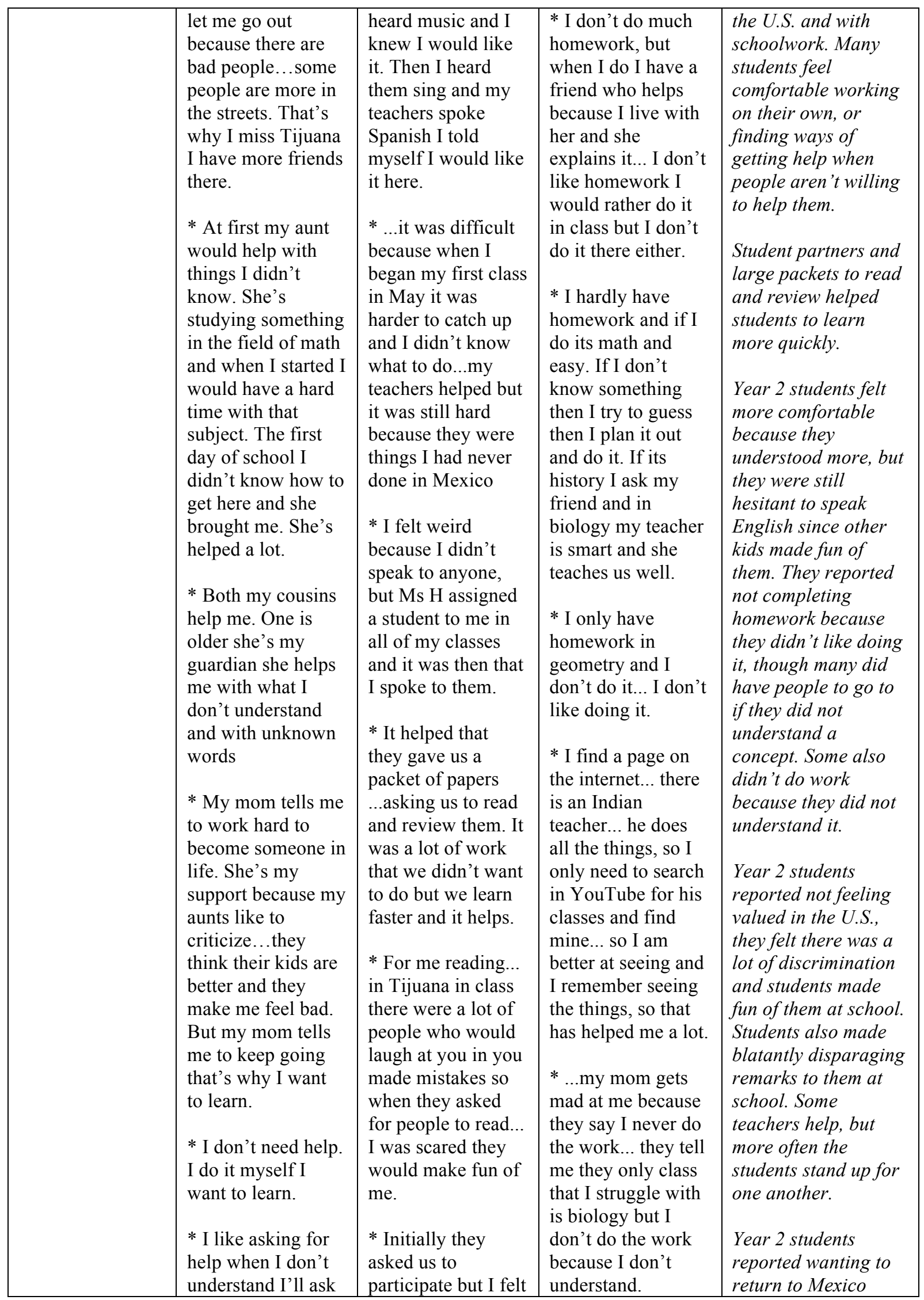




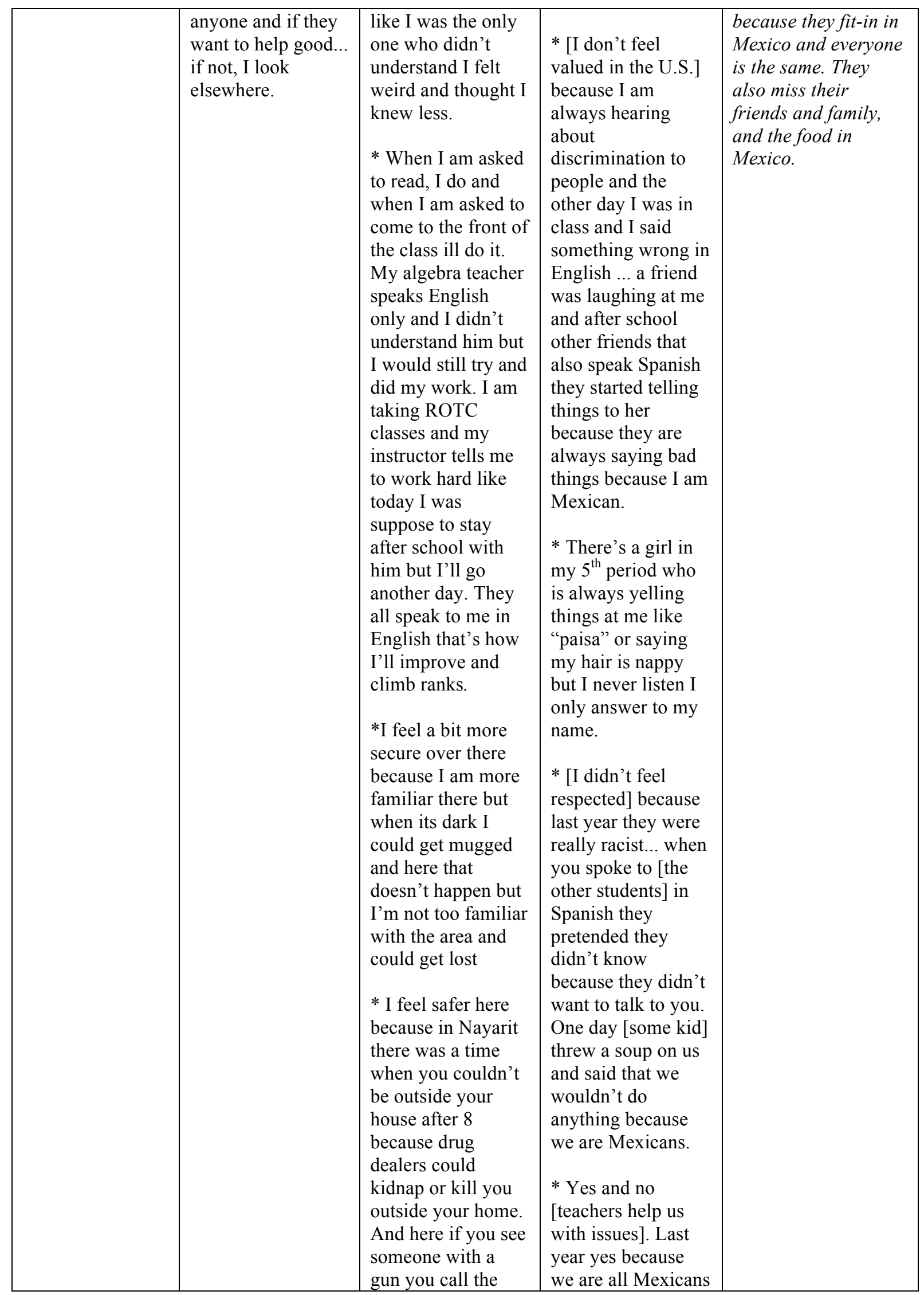




\begin{tabular}{|c|c|c|c|c|}
\hline & & $\begin{array}{l}\text { police and they } \\
\text { wont stop until they } \\
\text { find them. } \\
\text { * I feel the same } \\
\text { there's more } \\
\text { security here but in } \\
\text { Tijuana more } \\
\text { people understand } \\
\text { you. Here if you go } \\
\text { somewhere where } \\
\text { they don't speak } \\
\text { any Spanish you } \\
\text { feel out of place } \\
\text { because you can't } \\
\text { communicate. } \\
\text { * There's a lot of } \\
\text { delinquent, drug } \\
\text { addicts, and here } \\
\text { they set bombs in } \\
\text { schools, hospitals } \\
\text { and in Mexico you } \\
\text { don't hear about } \\
\text { that. Drug addicts } \\
\text { are much younger } \\
\text { here too...but its } \\
\text { better here because } \\
\text { they warn you if } \\
\text { something is } \\
\text { happening in } \\
\text { school. }\end{array}$ & $\begin{array}{l}\text { and we are all } \\
\text { friends so } \\
\text { everybody stood up } \\
\text { for everyone. The } \\
\text { teachers yes, Ms H } \\
\text { or teachers that } \\
\text { knew about that. } \\
\text { * Teachers haven't } \\
\text { done anything but } \\
\text { students laugh } \\
\text { when they hear our } \\
\text { accents and one } \\
\text { friend of mine } \\
\text { spoke up because if } \\
\text { you laugh at one } \\
\text { you are laughing at } \\
\text { all of us. } \\
\text { * [I would rather } \\
\text { live] in Mexico... } \\
\text { all the people are } \\
\text { the same they speak } \\
\text { the same } \\
\text { language... all my } \\
\text { friend are over } \\
\text { there and my } \\
\text { family. I miss the } \\
\text { food everything is } \\
\text { different here I } \\
\text { know I am closer to } \\
\text { the border but still. } \\
\text { * I prefer Mexico } \\
\text { because I have } \\
\text { more friends but I } \\
\text { would stay here... if } \\
\text { I leave it feels like I } \\
\text { have given up. }\end{array}$ & \\
\hline $\begin{array}{c}\text { Cultural } \\
\text { Responsiveness }\end{array}$ & $\begin{array}{l}\text { * Yes, [some } \\
\text { teachers speak to us } \\
\text { in Spanish], but } \\
\text { they want us to } \\
\text { speak English. But } \\
\text { [the students] don't } \\
\text { understand because } \\
\text { they go with Ms } \\
\text { E... and we don't, } \\
\text { we don't } \\
\text { understand. } \\
\text { * [They teach us } \\
\text { about] Veteran's }\end{array}$ & $\begin{array}{l}* \text { [In the beginning] } \\
\text { it helped that the } \\
\text { teachers explained } \\
\text { things in English } \\
\text { and then in } \\
\text { Spanish, whatever } \\
\text { we didn't } \\
\text { understand. } \\
\text { *[They use] videos } \\
\text { to show us how the } \\
\text { culture from here is } \\
\text { different from } \\
\text { Tijuana...The }\end{array}$ & $\begin{array}{l}\text { * In [my first] class } \\
\text { [things were] good } \\
\text { because most were } \\
\text { Mexicans and we } \\
\text { understood each } \\
\text { other. } \\
\text { * [They taught us } \\
\text { about U.S. culture] } \\
\text { in history } \\
\text { class...they played } \\
\text { videos and the } \\
\text { teacher would use } \\
\text { her calendar to }\end{array}$ & $\begin{array}{l}\text { The students } \\
\text { recounted that some } \\
\text { teachers spoke to } \\
\text { them in Spanish, but } \\
\text { they did feel that the } \\
\text { teachers wanted them } \\
\text { to speak English. The } \\
\text { students don't } \\
\text { understand many of } \\
\text { the teachers. } \\
\text { The teachers taught } \\
\text { students about U.S. } \\
\text { holidays using a }\end{array}$ \\
\hline
\end{tabular}




\begin{tabular}{|c|c|c|c|c|}
\hline & $\begin{array}{l}\text { Day ... Fourth of } \\
\text { July....also, just } \\
\text { how [people] } \\
\text { behave. What I } \\
\text { have noticed is that } \\
\text { people are } \\
\text { problematic... } \\
\text { When you want to } \\
\text { do something no } \\
\text { matter how small it } \\
\text { may be they easily } \\
\text { get offended... } \\
\text { because in Mexico } \\
\text { we are used to } \\
\text { joking around. } \\
\\
\text { * Sometimes we } \\
\text { speak Spanish and } \\
\text { they can learn from } \\
\text { us and sometimes } \\
\text { they ask us how to } \\
\text { say certain words } \\
\text { in Spanish... they } \\
\text { ask what is the } \\
\text { difference [between } \\
\text { English and } \\
\text { Spanish } \\
\text { words]...they teach } \\
\text { us English and we } \\
\text { can teach them } \\
\text { Spanish and it can } \\
\text { be better for your } \\
\text { career and all... in } \\
\text { Tijuana it's } \\
\text { important to learn } \\
\text { English and } \\
\text { Spanish. Well, } \\
\text { even here it's } \\
\text { important to learn } \\
\text { Spanish... even } \\
\text { already knowing } \\
\text { English. } \\
\text { * There are some } \\
\text { teachers who } \\
\text { understand you [in } \\
\text { Spanish], } \\
\text { sometimes there's } \\
\text { some we can help. }\end{array}$ & $\begin{array}{l}\text { national anthem } \\
\text { and pledge of } \\
\text { allegiance... they } \\
\text { are songs from the } \\
\text { US we hear every } \\
\text { week and with } \\
\text { books and images. } \\
\text { * I do feel different } \\
\text { being here from } \\
\text { being over there. } \\
\text { Even the people are } \\
\text { different. Over } \\
\text { there they are } \\
\text { always joyful out } \\
\text { on the streets with } \\
\text { very little care and } \\
\text { here they don't } \\
\text { accept people } \\
\text { easily. }\end{array}$ & $\begin{array}{l}\text { show us the } \\
\text { holidays by } \\
\text { assigning pictures } \\
\text { for each holiday. } \\
\text { * The teacher in } \\
\text { American } \\
\text { history...[she taught } \\
\text { us about] what they } \\
\text { did in World War } \\
\text { One in World War } \\
\text { Two and all the } \\
\text { participants of the } \\
\text { United States. } \\
\text { * For holidays they } \\
\text { would play videos } \\
\text { and explain it to us. }\end{array}$ & $\begin{array}{l}\text { calendar and } \\
\text { pictures, in addition } \\
\text { to the manner in } \\
\text { which people act in } \\
\text { the U.S. The students } \\
\text { felt people in the U.S. } \\
\text { were too sensitive, } \\
\text { they were used to } \\
\text { joking with others as } \\
\text { they had done in } \\
\text { Mexico. The people } \\
\text { in the U.S. also } \\
\text { seemed less carefree } \\
\text { and accepting to the } \\
\text { students. The } \\
\text { teachers used videos } \\
\text { and books with } \\
\text { images to } \\
\text { demonstrate U.S. } \\
\text { culture and historical } \\
\text { events. } \\
\text { Some English- } \\
\text { speaking students } \\
\text { asked the NAC } \\
\text { students how to say } \\
\text { certain words in } \\
\text { Spanish and were } \\
\text { curious about the } \\
\text { connections between } \\
\text { English and Spanish. } \\
\text { It helped the NAC } \\
\text { students to feel } \\
\text { valued and it } \\
\text { reinforced the } \\
\text { importance of being } \\
\text { bilingual. Sometimes, } \\
\text { the students are able } \\
\text { help the teachers who } \\
\text { are not fluent } \\
\text { Spanish speakers. }\end{array}$ \\
\hline
\end{tabular}




\begin{tabular}{|c|c|c|c|c|}
\hline $\begin{array}{c}\text { Teachers' } \\
\text { Attitudes/ } \\
\text { Pedagogical } \\
\text { Orientations }\end{array}$ & $\begin{array}{l}\text { * It's easy here. } \\
\text { Teachers are } \\
\text { patient... something } \\
\text { that they are not in } \\
\text { TJ. } \\
\text { * They educate } \\
\text { well, and you learn } \\
\text { fast. } \\
\text { * You feel good } \\
\text { [here], with } \\
\text { teachers and } \\
\text { students. But } \\
\text { teachers are more } \\
\text { patient. } \\
\text { * [Teachers] } \\
\text { welcomed me [to } \\
\text { class] very well and } \\
\text { they were } \\
\text { courteous... They } \\
\text { were courteous } \\
\text { they make you feel } \\
\text { like you're eat } \\
\text { home amongst } \\
\text { family. } \\
\text { * [It helps us when } \\
\text { teachers use] } \\
\text { gestures, pictures, } \\
\text { flashcards, } \\
\text { games...when they } \\
\text { give us chocolate } \\
\text { and rewards. } \\
\text { * [It is difficult to } \\
\text { understand] when } \\
\text { they speak } \\
\text { fast...and the way } \\
\text { they explain } \\
\text { [math]... that's } \\
\text { different. } \\
\text { * They are all easy } \\
\text { because I } \\
\text { understand, my } \\
\text { teachers know how } \\
\text { to explain things } \\
\text { well when we need } \\
\text { to do our work. } \\
\text { * For me all of the } \\
\text { [classes are easy] } \\
\text { because I can } \\
\text { a }\end{array}$ & $\begin{array}{l}\text { * I was [a good } \\
\text { student], but the } \\
\text { last year I had } \\
\text { problems with my } \\
\text { teachers so I } \\
\text { wanted to come to } \\
\text { the US because } \\
\text { they speak well of } \\
\text { this place so I } \\
\text { wanted a } \\
\text { change...My } \\
\text { teacher and I didn't } \\
\text { meet eye to eye, } \\
\text { they would yell at } \\
\text { me because they } \\
\text { said I never did my } \\
\text { homework but I } \\
\text { did. Even if I got } \\
\text { one thing wrong I } \\
\text { would get yelled at. } \\
\text { My teachers were } \\
\text { always rude to } \\
\text { other students and } \\
\text { myself. We always } \\
\text { supported each } \\
\text { other and we even } \\
\text { tried to have a few } \\
\text { teachers fired from } \\
\text { our middle school. } \\
\text { They fought to stay } \\
\text { but they would } \\
\text { even hit some } \\
\text { students. } \\
\text { * I was a good } \\
\text { student but I went } \\
\text { to high school to } \\
\text { take an exam and I } \\
\text { didn't pass it and I } \\
\text { couldn't continue } \\
\text { over there. I } \\
\text { enrolled in a course } \\
\text { online then I came } \\
\text { here. } \\
\text { * I was good [in } \\
\text { Mexico], but still } \\
\text { got yelled at by my } \\
\text { teachers. } \\
\text { * I was here in } \\
\text { middle school for } \\
\text { about } 3 \text { months } \\
\text { they spoke to me } \\
\text { only in English but }\end{array}$ & $\begin{array}{l}\text { * I started with a } \\
\text { teacher who spoke } \\
\text { Spanish even } \\
\text { though she didn't } \\
\text { want us to speak } \\
\text { Spanish but } \\
\text { knowing she } \\
\text { understood was } \\
\text { good. I was happy } \\
\text { they introduced us } \\
\text { one by one she was } \\
\text { a good teacher. } \\
\text { * [When I first } \\
\text { came] the teachers } \\
\text { was really sweet } \\
\text { and helpful } \\
\text { *[It helped me } \\
\text { when] the teacher } \\
\text { would say it in } \\
\text { English and then } \\
\text { repeat it in } \\
\text { Spanish.... and she } \\
\text { would use drawings } \\
\text { and stuff like } \\
\text { that...hand gestures. } \\
\text { * [It was hard } \\
\text { when] they spoke } \\
\text { fast... Also when } \\
\text { they don't speak it } \\
\text { right ... being lazy, } \\
\text { the pronunciation, } \\
\text { or the volume [of } \\
\text { their voices]. } \\
\text { * I suppose [it was } \\
\text { hard] when they } \\
\text { spoke a lot they } \\
\text { would leave me } \\
\text { thinking a lot about } \\
\text { what they meant. } \\
\text { * The teacher } \\
\text { would show us } \\
\text { videos from the } \\
\text { school because the } \\
\text { school would give } \\
\text { information about } \\
\text { how to graduate } \\
\text { and the CAHSEE, } \\
\text { so she would put on } \\
\text { the video and they } \\
\text { were in English she }\end{array}$ & $\begin{array}{l}\text { Many students felt } \\
\text { that the teachers } \\
\text { were more patient in } \\
\text { the United States } \\
\text { than in their home } \\
\text { countries. They felt } \\
\text { the teachers were } \\
\text { welcoming and } \\
\text { courteous and were } \\
\text { supportive of them. } \\
\text { Some students had } \\
\text { struggled in Mexico } \\
\text { and felt the teachers } \\
\text { were more supportive } \\
\text { and encouraging in } \\
\text { the U.S. } \\
\text { While some felt they } \\
\text { explained concepts } \\
\text { well and spoke in an } \\
\text { understandable } \\
\text { manner, others } \\
\text { reported that their } \\
\text { teachers spoke too } \\
\text { fast and explained } \\
\text { concepts in a way } \\
\text { that was new to them. } \\
\text { It was also difficult } \\
\text { for the students if the } \\
\text { teachers did not } \\
\text { pronounce the words } \\
\text { carefully or spoke too } \\
\text { softly. It helped the } \\
\text { students when the } \\
\text { teachers used } \\
\text { pictures, gestures, } \\
\text { and flash cards and } \\
\text { when they rewarded } \\
\text { the students for their } \\
\text { hard work. It also } \\
\text { helped them when } \\
\text { teachers would } \\
\text { repeat English } \\
\text { phrases and make } \\
\text { comparisons between } \\
\text { Spanish and English. } \\
\text { Though many } \\
\text { teachers spoke only } \\
\text { English in class, it } \\
\text { helped the students to } \\
\text { know they could } \\
\text { understand Spanish if } \\
\text { needed. Many } \\
\text { students had also }\end{array}$ \\
\hline
\end{tabular}




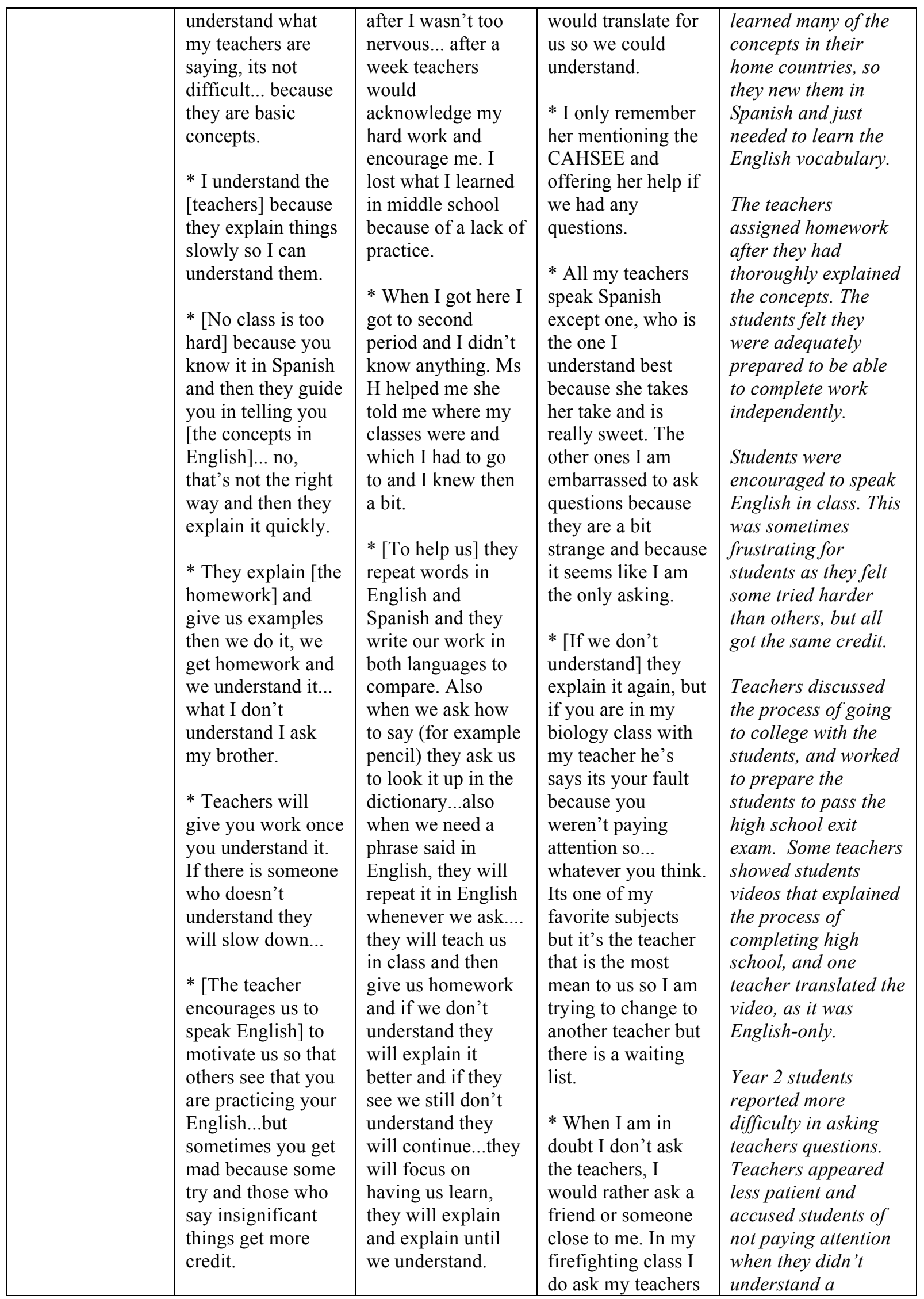




\begin{tabular}{|c|c|c|c|c|}
\hline & & $\begin{array}{l}\text { * [They show us } \\
\text { pictures] for the } \\
\text { weather, a sun for } \\
\text { sunny weather and } \\
\text { also drawings like } \\
\text { for a chair with the } \\
\text { name underneath... } \\
\text { they also showed } \\
\text { us images on the } \\
\text { computer and } \\
\text { internet. } \\
\text { * [Teachers help us } \\
\text { to understand] with } \\
\text { images and they } \\
\text { questions us about } \\
\text { what we don't } \\
\text { understand and } \\
\text { they explain it to us } \\
\text { using the board. } \\
\text { * The teachers have } \\
\text { mentioned the good } \\
\text { [colleges] and how } \\
\text { important it is to } \\
\text { apply to } \\
\text { college...and } \\
\text { we saw a video of } \\
\text { the schools nearby } \\
\text { and what's } \\
\text { necessary to apply. } \\
\text { * They are } \\
\text { preparing us for the } \\
\text { CAHSEE with } \\
\text { certain work for } \\
\text { example with our } \\
\text { autobiography for } \\
\text { the exam. } \\
\text { * When we are in } \\
\text { math class... Ms H } \\
\text { is always yelling at } \\
\text { me because I don't } \\
\text { pay attention and I } \\
\text { get mad because } \\
\text { she doesn't see the } \\
\text { hard work and } \\
\text { effort coming from } \\
\text { my part. } \\
\text { * If I don't } \\
\text { understand } \\
\text { something they go } \\
\text { back to explain it to } \\
\text { me and I still don't } \\
\text { understand they }\end{array}$ & $\begin{array}{l}\text { because I like them } \\
\text { and in my English } \\
\text { class I am scared of } \\
\text { the teacher ...she's } \\
\text { always screaming. } \\
\text { * When I don't } \\
\text { understand in } \\
\text { geometry I ask } \\
\text { questions and he } \\
\text { explains in history } \\
\text { and biology I } \\
\text { understand it all. } \\
\text { * They push you to } \\
\text { speak English and } \\
\text { if you do you're a } \\
\text { good student. } \\
\text { * Its ok to make } \\
\text { mistakes because } \\
\text { everyone makes } \\
\text { mistakes and they } \\
\text { don't get mad if } \\
\text { you make mistakes } \\
\text { * There's only two } \\
\text { teachers [that help] } \\
\text { one in my } 5^{\text {th }} \\
\text { period, but I only } \\
\text { have that class once } \\
\text { a week and she tells } \\
\text { me if I want help I } \\
\text { need to do certain } \\
\text { things and then I } \\
\text { get good feedback. } \\
\text { In my first period } \\
\text { the teacher is really } \\
\text { nice with us she's } \\
\text { always walking } \\
\text { around asking if we } \\
\text { need help she's } \\
\text { always paying } \\
\text { attention to her } \\
\text { students } \\
\text { * My last year } \\
\text { teachers Ms H...the } \\
\text { teacher that I has in } \\
\text { ESL she helped } \\
\text { me... I am not a } \\
\text { student with her } \\
\text { anymore but she } \\
\text { still helps me after } \\
\text { school. }\end{array}$ & $\begin{array}{l}\text { concept. These } \\
\text { students reported } \\
\text { feeling more } \\
\text { comfortable asking } \\
\text { friends and not the } \\
\text { teachers when they } \\
\text { had questions. The } \\
\text { students appear to } \\
\text { feel less connected to } \\
\text { their teachers after } \\
\text { their first year. Some } \\
\text { students returned to } \\
\text { get help from their } \\
\text { NAC teacher after } \\
\text { school. }\end{array}$ \\
\hline
\end{tabular}




\begin{tabular}{|c|c|c|c|c|}
\hline & & $\begin{array}{l}\text { will find a different } \\
\text { way to explain it. } \\
\text { * For us Ms. H, } \\
\text { when we don't } \\
\text { understand, she } \\
\text { reviews and } \\
\text { reviews and } \\
\text { reviews then she } \\
\text { gives us a test to } \\
\text { see how we are } \\
\text { doing. and if we } \\
\text { don't do well she } \\
\text { tells us to pay more } \\
\text { attention and if we } \\
\text { do good she tells us } \\
\text { congratulations. } \\
\text { But yes she lets us } \\
\text { retake tests. } \\
\text { * When my teacher } \\
\text { tells me I am doing } \\
\text { better I feel } \\
\text { motivated and I } \\
\text { feel like working } \\
\text { harder. } \\
\text { * For me, [I feel } \\
\text { good] when I get } \\
\text { good grades on my } \\
\text { tests my teacher } \\
\text { tells me that I have } \\
\text { improved... also } \\
\text { Ms. Sonia, when } \\
\text { we are improving } \\
\text { she gives us } \\
\text { chocolate. }\end{array}$ & & \\
\hline $\begin{array}{l}\text { Academic Self- } \\
\text { Concept }\end{array}$ & $\begin{array}{l}* \text { [In easier classes] } \\
\text { I feel good...I feel } \\
\text { like I know more } \\
\text { things. } \\
\text { *[In harder classes] } \\
\text { I feel belittled, does } \\
\text { that make sense? } \\
\text { So I gain the } \\
\text { courage and I give } \\
\text { it my all. } \\
\text { * Personally, with } \\
\text { things I don't } \\
\text { understand I set my } \\
\text { standards much } \\
\text { higher. }\end{array}$ & $\begin{array}{l}* \text { [In easier classes } \\
\text { I feel] better... } \\
\text { better than } \\
\text { others...better } \\
\text { because it is easier. } \\
\text { * [Harder classes] } \\
\text { lower my self- } \\
\text { esteem. I try a lot } \\
\text { but I stay silent. } \\
\text { They make me feel } \\
\text { a bit incomplete } \\
\text { because I try to get } \\
\text { better but } \\
\text { sometimes I don't } \\
\text { do well but at least } \\
\text { I tried. }\end{array}$ & $\begin{array}{l}\text { * [In easier classes } \\
\text { I feel] good that I } \\
\text { am learning fast. } \\
\text { * When it's an easy } \\
\text { class I only do what } \\
\text { I have to. } \\
\text { * When its easy I } \\
\text { think its stupid for } \\
\text { people not to pass. } \\
\text { * [In harder } \\
\text { classes] it just } \\
\text { makes me feel } \\
\text { angry, because I } \\
\text { know in those }\end{array}$ & $\begin{array}{l}\text { The students reported } \\
\text { that they felt good } \\
\text { and more } \\
\text { knowledgeable in } \\
\text { their easier classes. } \\
\text { They felt like they } \\
\text { were successful since } \\
\text { they were learning } \\
\text { faster. } \\
\text { In their harder } \\
\text { classes, students felt } \\
\text { belittled and anxious. } \\
\text { They would stay } \\
\text { silent in these classes } \\
\text { because they were } \\
\text { nervous and }\end{array}$ \\
\hline
\end{tabular}




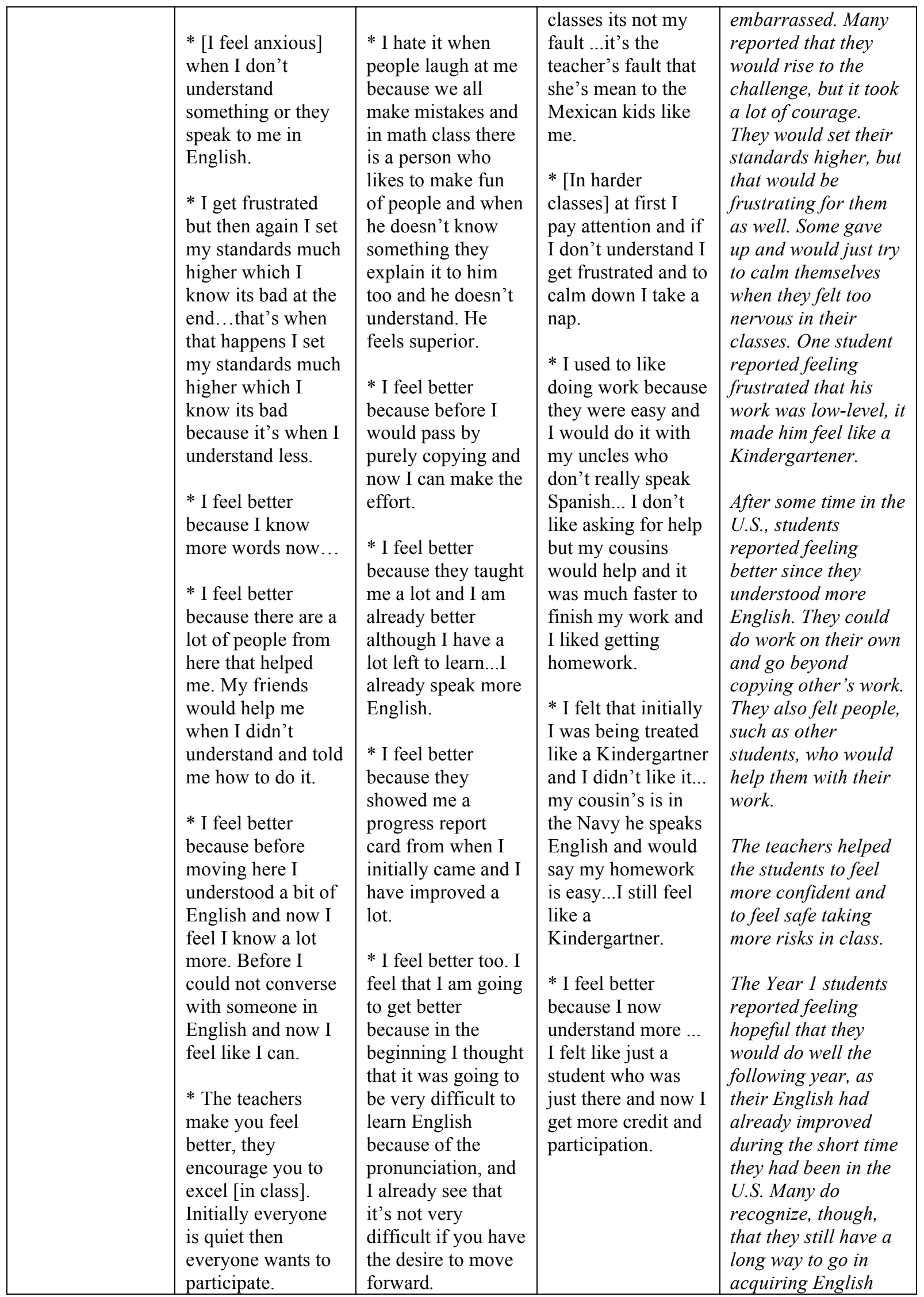




\begin{tabular}{|c|c|c|c|c|}
\hline & & $\begin{array}{l}*[\text { I felt more } \\
\text { confident when] } \\
\text { one time when my } \\
\text { parents and my } \\
\text { teacher met she } \\
\text { told them that I was } \\
\text { more or less } \\
\text { understanding it } \\
\text { and I help my peers } \\
\text { when they don't } \\
\text { understand. } \\
\text { * [Next year] we } \\
\text { are going to learn } \\
\text { more English, we } \\
\text { will learn more } \\
\text { concepts, more } \\
\text { words, how to } \\
\text { write a sentence. } \\
\text { We will not be as } \\
\text { nervous because } \\
\text { we will have a year } \\
\text { of experience.... I } \\
\text { know I still have a } \\
\text { long way to go to } \\
\text { be put in normal } \\
\text { classes but I know I } \\
\text { will learn what I } \\
\text { need to be } \\
\text { prepared. } \\
\text { * I still feel I have } \\
\text { a lot more English } \\
\text { to learn...I feel I } \\
\text { have much to } \\
\text { practice... }\end{array}$ & & $\begin{array}{l}\text { and feeling confident } \\
\text { to be mainstreamed } \\
\text { into higher-level } \\
\text { courses. }\end{array}$ \\
\hline $\begin{array}{c}\text { Language } \\
\text { Proficiency } \\
\text { Level }\end{array}$ & $\begin{array}{l}\text { * [In Mexico] the } \\
\text { only way I studied } \\
\text { English was in } \\
\text { school and the } \\
\text { teachers weren't } \\
\text { very good. They } \\
\text { weren't patient and } \\
\text { they wanted us to } \\
\text { learn everything } \\
\text { fast. } \\
\text { * I studied } \\
\text { [English] for about } \\
\text { nine years, not } \\
\text { consistent, two in } \\
\text { preschool three in } \\
\text { elementary, three in }\end{array}$ & $\begin{array}{l}\text { * My mom sent me } \\
\text { and I regret not } \\
\text { learning much } \\
\text { because I never } \\
\text { thought I would } \\
\text { come study here I } \\
\text { thought I was } \\
\text { staying in Mexico. } \\
\text { Especially because } \\
\text { when I got here and } \\
\text { I knew nothing... I } \\
\text { was not prepared. } \\
\text { * I [studied } \\
\text { English] but the } \\
\text { teacher would let } \\
\text { us do whatever we }\end{array}$ & $\begin{array}{l}\text { * Yes [they moved } \\
\text { me to the NAC } \\
\text { class after two } \\
\text { weeks and things } \\
\text { were better]...the } \\
\text { language, how they } \\
\text { treated me, the } \\
\text { teachers, and I } \\
\text { understood more } \\
\text { and I knew that if I } \\
\text { stayed there I was } \\
\text { going to fail } \\
\text { because I didn't } \\
\text { understand } \\
\text { anything... they } \\
\text { treated me better. }\end{array}$ & $\begin{array}{l}\text { Many students had } \\
\text { studied English in } \\
\text { their home countries, } \\
\text { though most reported } \\
\text { only having learned } \\
\text { basic concepts. Most } \\
\text { felt unprepared when } \\
\text { they entered U.S. } \\
\text { schools. They } \\
\text { reported wishing they } \\
\text { had made a greater } \\
\text { effort to acquire } \\
\text { English before } \\
\text { coming to the U.S. } \\
\\
\text { Some students had } \\
\text { family members who }\end{array}$ \\
\hline
\end{tabular}




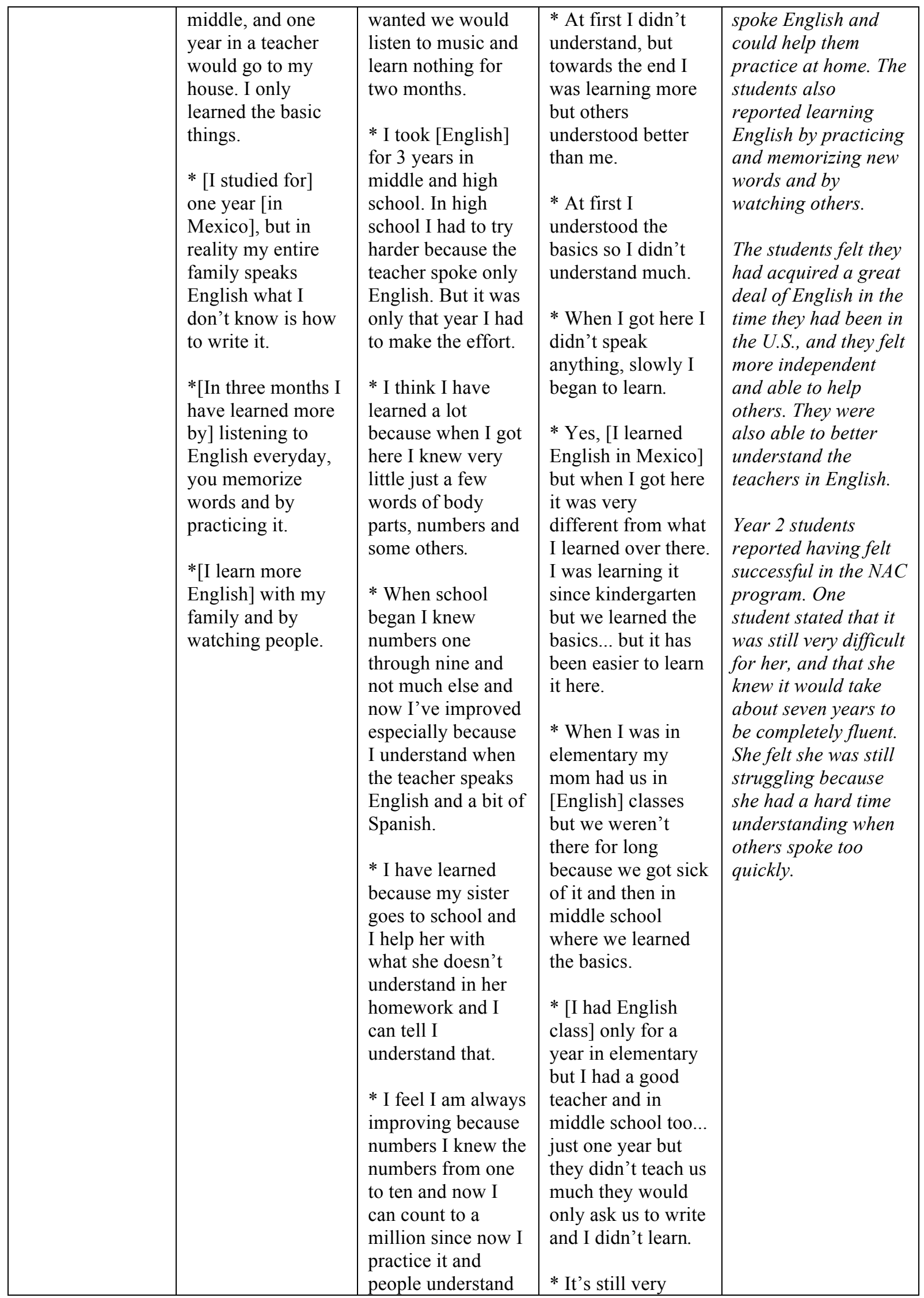




\begin{tabular}{|l|l|l|}
\hline & me. & $\begin{array}{l}\text { difficult because } \\
\text { when we arrived } \\
\text { they told us that for } \\
\text { us to understand } \\
\text { English we need to } \\
\text { pass seven years } \\
\text { and I can not have a } \\
\text { real conversation } \\
\text { step by step we } \\
\text { were studying body } \\
\text { parts they gave } \\
\text { worksheets and } \\
\text { asked us to read } \\
\text { and I learned that. } \\
\text { And they also } \\
\text { taught me English } \\
\text { and now I know } \\
\text { getting it slow } \\
\text { how to write... } \\
\text { compared to when I } \\
\text { speak very fast. } \\
\text { got here I didn't } \\
\text { know anything, } \\
\text { now I listen and } \\
\text { can speak a bit. }\end{array}$ \\
\\
\end{tabular}




\section{Appendix S}

\section{ESL Focus Group Analysis}

\begin{tabular}{|c|c|c|c|c|}
\hline & $\begin{array}{c}\text { Focus Group \#1 } \\
\text { ESL-Year } 1\end{array}$ & $\begin{array}{c}\text { Focus Group \#2 } \\
\text { ESL-Year } 1\end{array}$ & $\begin{array}{c}\text { Focus Group \#3 } \\
\text { ESL-Year } 2\end{array}$ & Significant Themes \\
\hline $\begin{array}{c}\text { Language } \\
\text { Acquisition/ } \\
\text { Practice }\end{array}$ & $\begin{array}{l}\text { * The environment } \\
\text { where you are } \\
\text { helps me [to learn } \\
\text { more English], its } \\
\text { just how Latinos } \\
\text { say things... it } \\
\text { sticks to us or } \\
\text { something. } \\
\text { * Having friends } \\
\text { who only speak } \\
\text { [English] in my } \\
\text { classes and I talk to } \\
\text { them... however I } \\
\text { can speak better as } \\
\text { time goes by we } \\
\text { can have better } \\
\text { conversations. } \\
\text { *[I learned } \\
\text { English] on the } \\
\text { streets. It's very } \\
\text { different here they } \\
\text { teach you grammar } \\
\text { rules because a lot } \\
\text { of Latinos can } \\
\text { speak English but } \\
\text { not to write it. You } \\
\text { can learn it in the } \\
\text { streets but its street } \\
\text { language it's not } \\
\text { formal English its } \\
\text { doesn't understand } \\
\text { because she wants } \\
\text { me to practice. } \\
\text { Even though I feel } \\
\text { read books...she } \\
\text { * Books [help me } \\
\text { learn English]... my } \\
\text { history teacher } \\
\text { pressures me a lot } \\
\text { and she spoke to } \\
\text { my dad and told } \\
\text { him to have me }\end{array}$ & 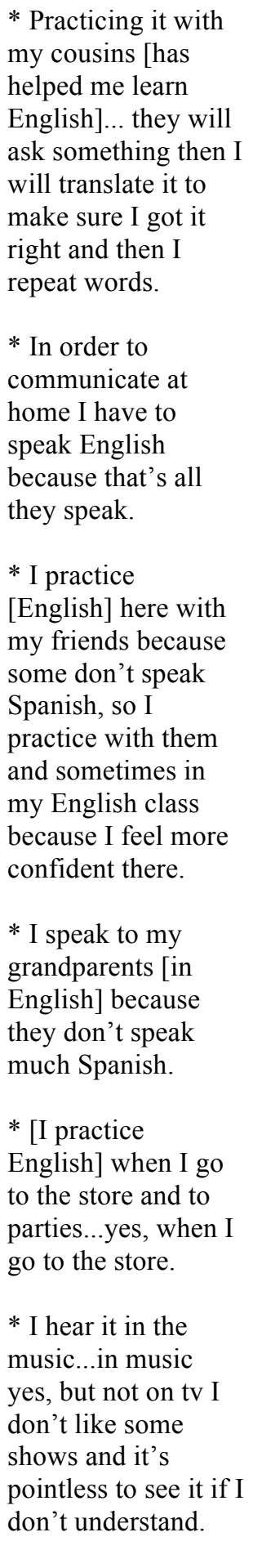 & $\begin{array}{l}\text { * Listening to } \\
\text { teachers and my } \\
\text { friends when they } \\
\text { speak it and read it } \\
\text { [has helped me } \\
\text { learn } \\
\text { English]...reading, } \\
\text { writing, and } \\
\text { listening to it in my } \\
\text { classes. } \\
\text { * Listening to all } \\
\text { my teachers [has } \\
\text { helped me learn } \\
\text { English]. } \\
\text { * Hearing speak } \\
\text { my dad in English } \\
\text { and my brother and } \\
\text { my friends too. } \\
\text { * My friends and } \\
\text { through music [I } \\
\text { have learned } \\
\text { English]. } \\
\text { because I has } \\
\text { * My sister is } \\
\text { helping me learn } \\
\text { more English and } \\
\text { some programs in } \\
\text { the computer... like } \\
\text { Learning Upgrade } \\
\text { and Rosetta } \\
\text { Stone....it did help } \\
\text { here. } \\
\text { * ESL [warned a process. } \\
\text { because we were } \\
\text { beginners and } \\
\text { we're slowly } \\
\text { learning and } \\
\text { evebra because } \\
\text { thing was on } \\
\text { leard and we } \\
\text { best] }\end{array}$ & 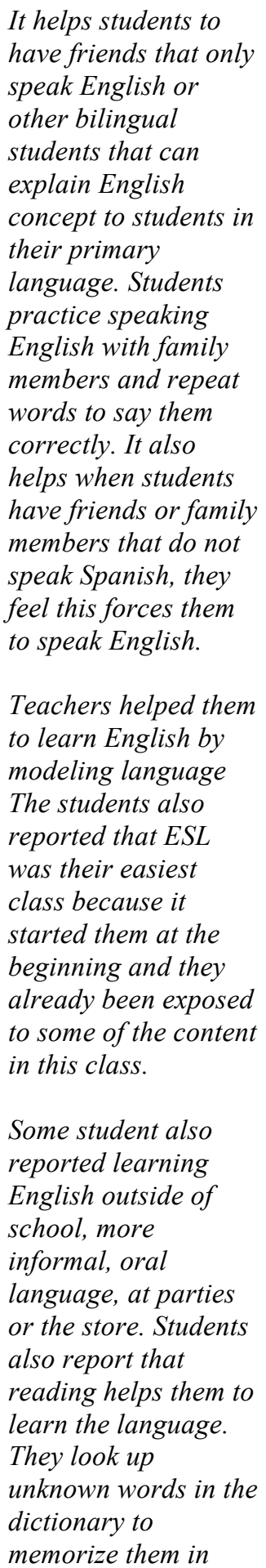 \\
\hline
\end{tabular}




\begin{tabular}{|c|c|c|c|c|}
\hline & $\begin{array}{l}\text { pressured I feel like } \\
\text { its something good. } \\
\text { * Music and TV } \\
\text { [help me]. Music } \\
\text { because when you } \\
\text { listen to it you } \\
\text { come across words } \\
\text { you have heard } \\
\text { before and you } \\
\text { already know the } \\
\text { definition and TV } \\
\text { when you watch it } \\
\text { with subtitles help } \\
\text { too. } \\
\text { * Another problem } \\
\text { is that Spanish is } \\
\text { written how you } \\
\text { hear it and English } \\
\text { is the opposite... } \\
\text { one thing is to } \\
\text { write and speak it. } \\
\text { If we see a movie } \\
\text { and it doesn't have } \\
\text { subtitles we won't } \\
\text { understand it } \\
\text { because the way } \\
\text { you speak it is very } \\
\text { different. Literature } \\
\text { is different; you } \\
\text { don't know it you } \\
\text { look up the } \\
\text { definition. } \\
\text { * Books help me } \\
\text { because if I don't } \\
\text { understand one } \\
\text { word I try figuring } \\
\text { it out with the rest } \\
\text { of the word and I } \\
\text { memorize it for the } \\
\text { future. }\end{array}$ & $\begin{array}{l}\text { * My uncles tell me } \\
\text { to practice it } \\
\text { because that's how I } \\
\text { will learn and my } \\
\text { cousins help with } \\
\text { my homework. I ask } \\
\text { them what it means } \\
\text { and they explain it. }\end{array}$ & $\begin{array}{l}\text { already seen stuff } \\
\text { and because your } \\
\text { barely starting and } \\
\text { its easy. }\end{array}$ & $\begin{array}{l}\text { English. Music and } \\
\text { TV help them at home } \\
\text { to acquire language; } \\
\text { students translate } \\
\text { songs and watch TV } \\
\text { with subtitles to help } \\
\text { them learn English. } \\
\\
\text { One student reported } \\
\text { that computer } \\
\text { programs, such as } \\
\text { Rosetta Stone and } \\
\text { Learning Upgrade, } \\
\text { helped her to learn } \\
\text { English. }\end{array}$ \\
\hline $\begin{array}{c}\text { Content } \\
\text { Instruction/ } \\
\text { Practice }\end{array}$ & $\begin{array}{l}\text { * In math teachers } \\
\text { have been very } \\
\text { helpful they even } \\
\text { translated an entire } \\
\text { exam once and } \\
\text { they are finding } \\
\text { new ways to help. } \\
\text { * In math our } \\
\text { teacher speaks } \\
\text { English but }\end{array}$ & $\begin{array}{l}\text { * The difficult one } \\
\text { is physics because I } \\
\text { don't understand the } \\
\text { teacher...he has a } \\
\text { weird accent he } \\
\text { knows the language } \\
\text { but he speaks it } \\
\text { differently. } \\
\text { * I understand more } \\
\text { in geometry, but not }\end{array}$ & $\begin{array}{l}\text { * Science and } \\
\text { history [were } \\
\text { hardest to } \\
\text { understand] } \\
\text { because its where } \\
\text { they speak the } \\
\text { most. } \\
\text { * History [was } \\
\text { hardest] because } \\
\text { sometimes you had }\end{array}$ & $\begin{array}{l}\text { The students struggle } \\
\text { the most with } \\
\text { language intensive } \\
\text { classes such as } \\
\text { history. They do not } \\
\text { recognize many } \\
\text { words and it is } \\
\text { difficult since it is all } \\
\text { in English. } \\
\text { The students }\end{array}$ \\
\hline
\end{tabular}




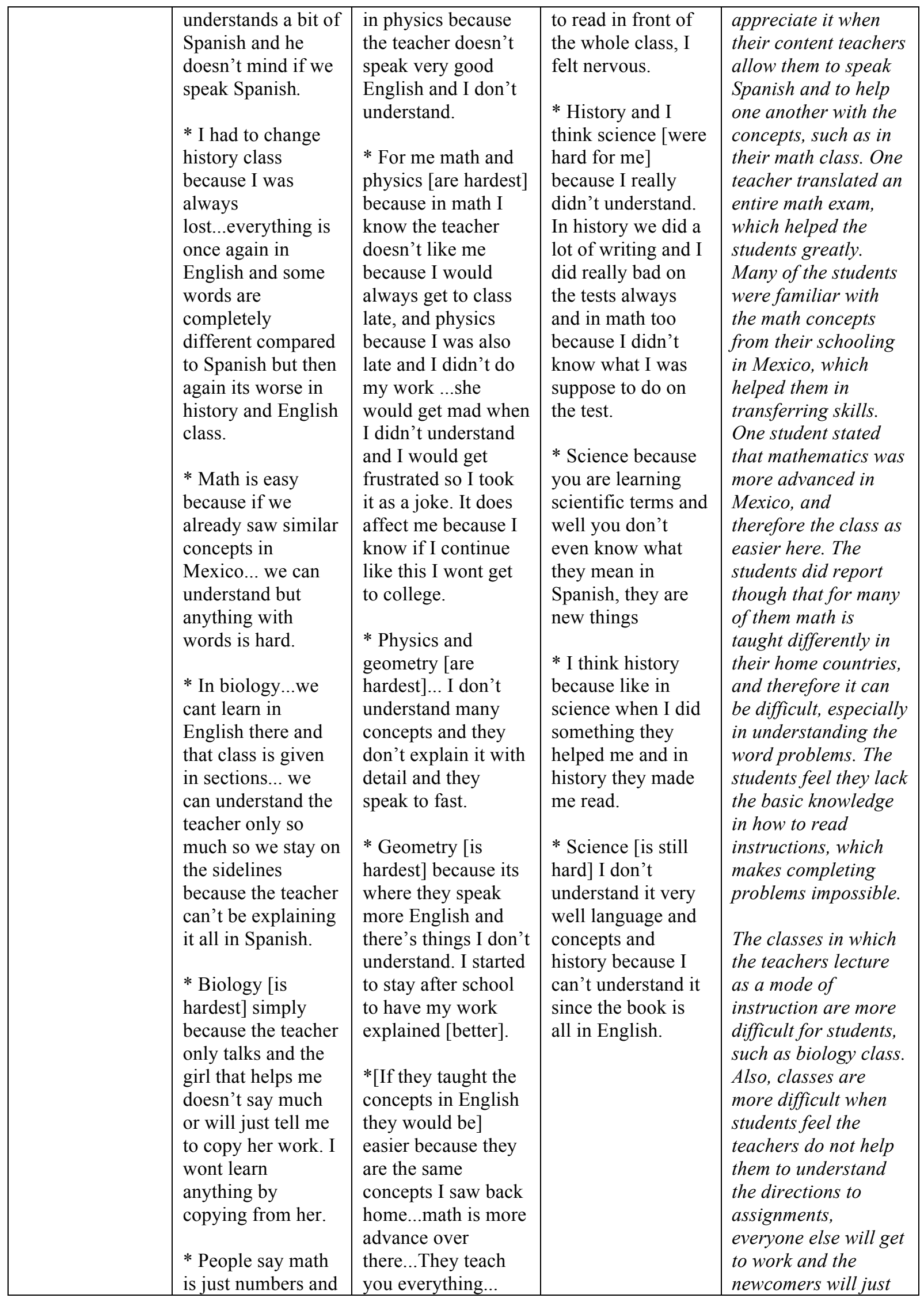




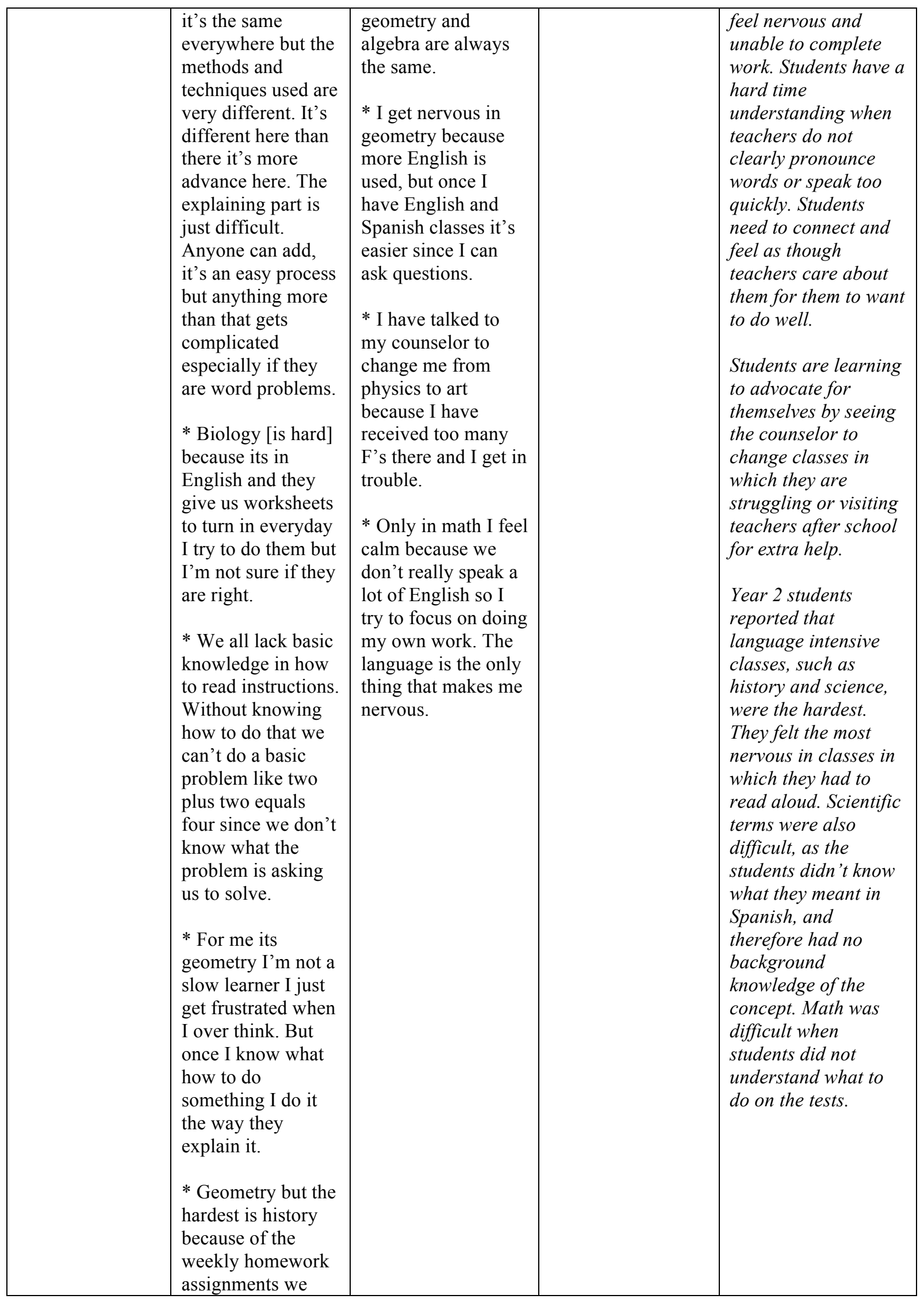




\begin{tabular}{|c|c|c|c|c|}
\hline & $\begin{array}{l}\text { have its like } 20 \\
\text { pages and } \\
\text { everything in } \\
\text { English and since } \\
\text { we just started its } \\
\text { too much work. } \\
\text { * For science class } \\
\text { I feel scared } \\
\text { because I don't } \\
\text { know what they } \\
\text { will ask us to do. } \\
\text { Everyone will get } \\
\text { started on their } \\
\text { work and I won't } \\
\text { know what to do } \\
\text { since the teacher } \\
\text { will not offer to } \\
\text { help. }\end{array}$ & & & \\
\hline $\begin{array}{c}\text { Student } \\
\text { Perceptions/ } \\
\text { Expectations }\end{array}$ & $\begin{array}{l}\text { * [My family } \\
\text { moved to the U.S. } \\
\text { because] they } \\
\text { wanted me to learn } \\
\text { English and have a } \\
\text { better education } \\
\text { here.... they offer } \\
\text { better opportunities } \\
\text { to succeed. } \\
\text { * A better life here } \\
\text { means to come and } \\
\text { suffer. This isn't } \\
\text { life, not knowing } \\
\text { the language; } \\
\text { everyone works too } \\
\text { much, and goes to } \\
\text { school. So for me } \\
\text { this isn't a better } \\
\text { life. } \\
\text { * I was smart I } \\
\text { always got } 10 \text { 's } \\
\text { and here they are } \\
\text { A's but that didn't } \\
\text { help because once I } \\
\text { came here I got } \\
\text { F's. } \\
\text { * I was smart when } \\
\text { I wanted to be } \\
\text { because I got good } \\
\text { grades but then I } \\
\text { lowered them } \\
\text { because it's hard }\end{array}$ & $\begin{array}{l}* \text { [My family moved } \\
\text { to the U.S. for us] to } \\
\text { study and succeed. } \\
\text { * [In school I was] } \\
\text { average not too low } \\
\text { or high...I always } \\
\text { had good grades not } \\
\text { extremely good or } \\
\text { bad. } \\
\text { * [When I first } \\
\text { arrived, I felt] } \\
\text { nervous when they } \\
\text { asked me } \\
\text { something. } \\
\text { * [I felt] nervous but } \\
\text { excited because I } \\
\text { have friends here. } \\
\text { * [I felt] a bit } \\
\text { strange because it } \\
\text { was my first day } \\
\text { here and nervous... } \\
\text { nervous and strange } \\
\text { because I didn't } \\
\text { have friends. } \\
\text { * [When I first } \\
\text { came] I thought no } \\
\text { one spoke Spanish... } \\
\text { * I felt normal } \\
\text { because I had }\end{array}$ & $\begin{array}{l}* \text { [My family } \\
\text { moved to the U.S.] } \\
\text { because my parents } \\
\text { thought it would be } \\
\text { better for me to get } \\
\text { good grades and go } \\
\text { to college. } \\
\text { * [My family } \\
\text { moved us to the } \\
\text { U.S] to have a } \\
\text { better life...to have } \\
\text { a better education } \\
\text { and learn English. } \\
\text { * I did better over } \\
\text { there, I didn't have } \\
\text { so many bad grades } \\
\text { as over here. } \\
\text { * [I was a bit } \\
\text { better] in Mexico I } \\
\text { understood better } \\
\text { because it was in } \\
\text { Spanish. } \\
\text { *[I have] the same } \\
\text { grades here and } \\
\text { there, good ones... } \\
\text { *[On my first day] } \\
\text { I was scared that } \\
\text { no one was going } \\
\text { to speak Spanish... } \\
\text { I felt scared and }\end{array}$ & $\begin{array}{l}\text { Many students moved } \\
\text { to the U.S. because } \\
\text { their families wanted } \\
\text { them to have more } \\
\text { opportunities and } \\
\text { more job and school } \\
\text { prospects. The } \\
\text { students also felt it } \\
\text { was important to be } \\
\text { fully bilingual to have } \\
\text { increased } \\
\text { opportunities. } \\
\\
\text { One student states } \\
\text { that a better life in } \\
\text { the U.S. comes with a } \\
\text { high degree of } \\
\text { suffering, as people } \\
\text { work to much and not } \\
\text { knowing the } \\
\text { language makes the } \\
\text { experience very } \\
\text { negative. Students } \\
\text { are failing courses in } \\
\text { the U.S., though they } \\
\text { did well in their home } \\
\text { countries, which } \\
\text { make it difficult for } \\
\text { them to feel the } \\
\text { opportunities in life } \\
\text { for which they } \\
\text { moved. Students do } \\
\text { not feel as smart as } \\
\text { they cannot } \\
\text { communicate, which }\end{array}$ \\
\hline
\end{tabular}




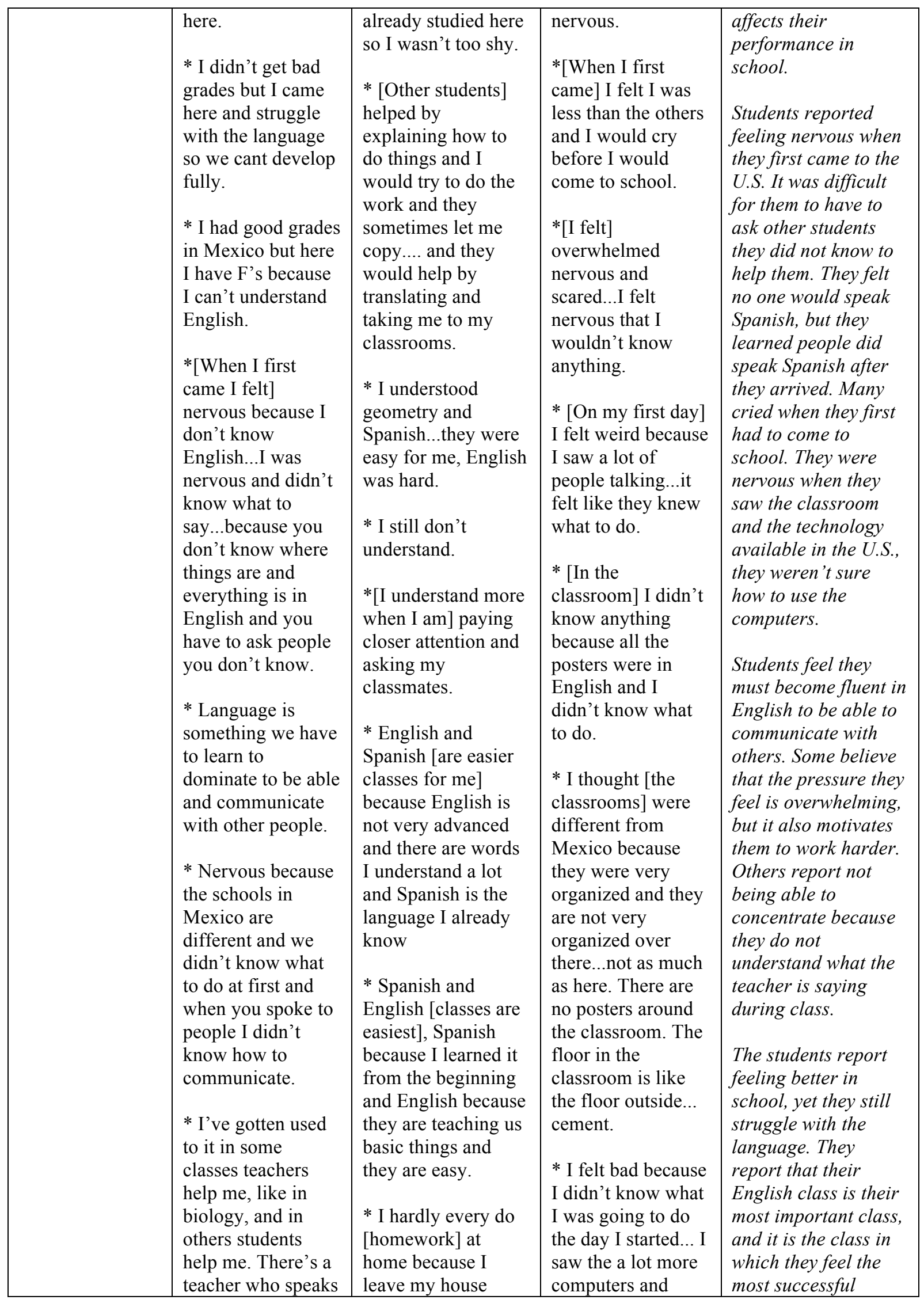




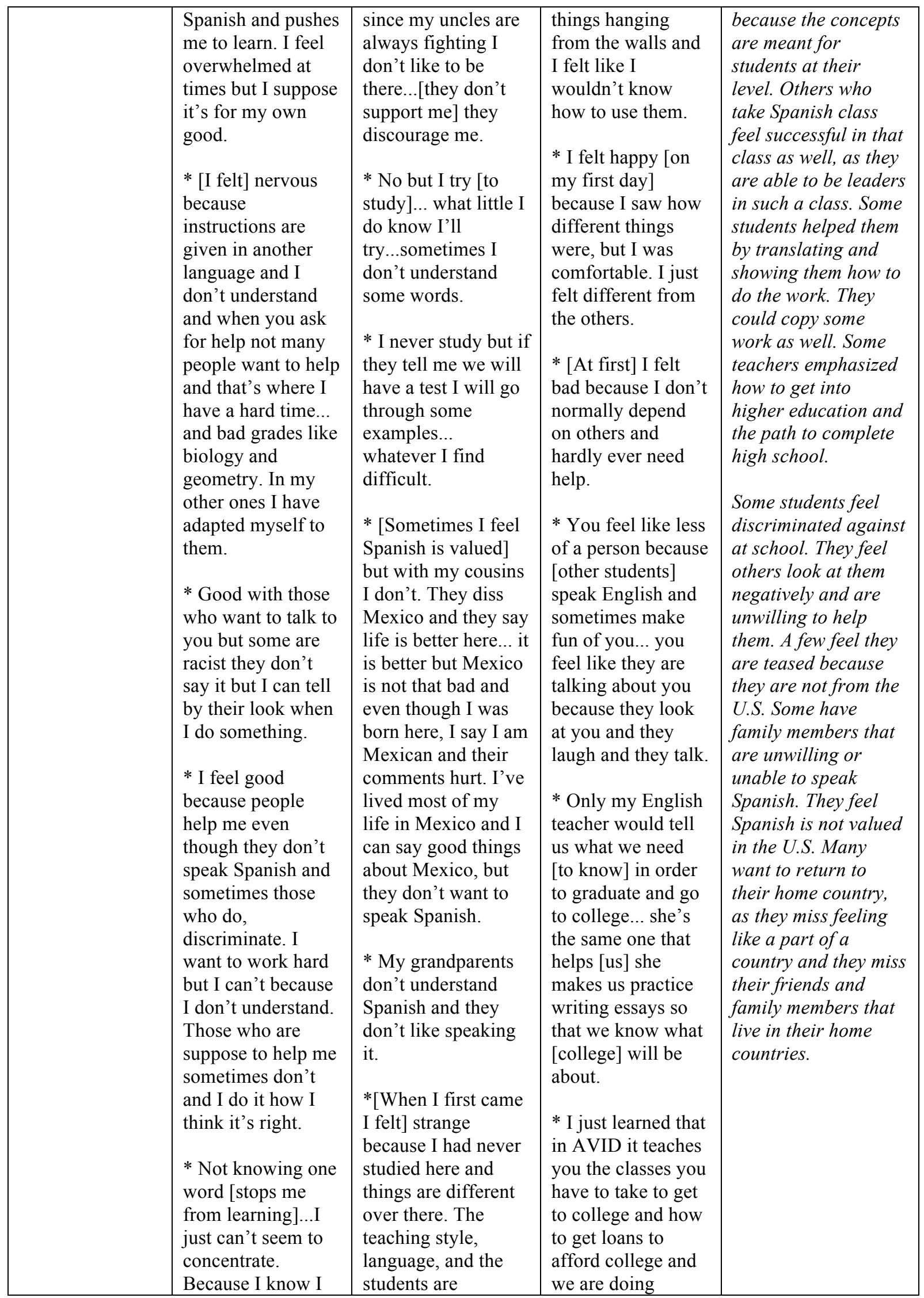




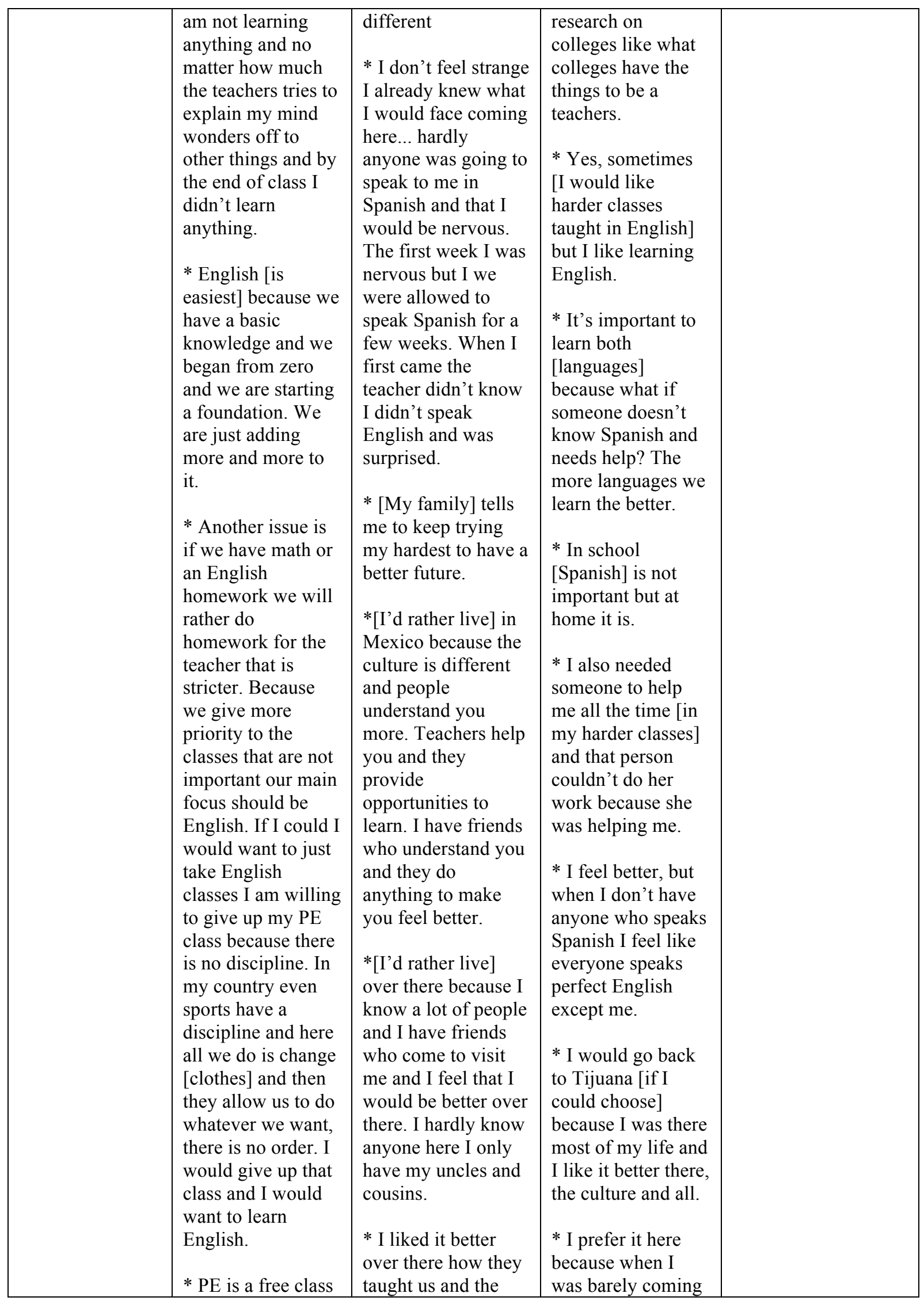




\begin{tabular}{|c|c|c|c|c|}
\hline & $\begin{array}{l}\text { because all we } \\
\text { have to do is } \\
\text { change, play, and } \\
\text { participate. In my } \\
\text { English class I feel } \\
\text { like I am really } \\
\text { taking advantage of } \\
\text { my classes because } \\
\text { I am there for } 2 \\
\text { hours learning new } \\
\text { material, five days } \\
\text { a week. It's a class } \\
\text { I like and I have } \\
\text { problems in my } \\
\text { other ones. }\end{array}$ & $\begin{array}{l}\text { classes that were } \\
\text { assigned to } \\
\text { us...because for } \\
\text { example geometry is } \\
\text { only about angles } \\
\text { and over there they } \\
\text { teach you } \\
\text { everything, its more } \\
\text { advance over there. } \\
\text { * They judge you } \\
\text { here and consider } \\
\text { you strange because } \\
\text { of what you do and } \\
\text { it's not funny. They } \\
\text { say I speak funny } \\
\text { when we play soccer } \\
\text { and in Mexico it's } \\
\text { normal. I know I am } \\
\text { a bit funny but they } \\
\text { shouldn't make fun } \\
\text { of me. }\end{array}$ & $\begin{array}{l}\text { to school here, my } \\
\text { dad said "You are } \\
\text { going to want to } \\
\text { stay there instead } \\
\text { of coming back } \\
\text { here"... I thought } \\
\text { he wasn't right and } \\
\text { now he's trying to } \\
\text { get me to go [back] } \\
\text { and now I don't } \\
\text { want to. I learned } \\
\text { English and } \\
\text { suffered so I want } \\
\text { to stay here. I like } \\
\text { it here. }\end{array}$ & \\
\hline $\begin{array}{c}\text { Culturally } \\
\text { Responsive } \\
\text { Teaching }\end{array}$ & $\begin{array}{l}\text { * In history we } \\
\text { [learn culture]... we } \\
\text { learned about the } \\
\text { conquest, } \\
\text { independence, and } \\
\text { the history. } \\
\text { * I haven't learned } \\
\text { about [U.S. } \\
\text { culture] because I } \\
\text { don't have history, } \\
\text { but as we approach } \\
\text { holidays I notice } \\
\text { how they celebrate } \\
\text { them. } \\
\text { * In school no, but } \\
\text { I am learning about } \\
\text { them on the streets } \\
\text { when there are } \\
\text { certain } \\
\text { celebrations. }\end{array}$ & $\begin{array}{l}* \text { [We learn about } \\
\text { U.S. customs] in } \\
\text { English class when } \\
\text { it's a holiday... for } \\
\text { example } \\
\text { Thanksgiving she } \\
\text { taught us about the } \\
\text { food but I already } \\
\text { knew that. } \\
\text { * I don't think } \\
\text { [teachers do } \\
\text { anything to help us } \\
\text { understand US } \\
\text { costumes and } \\
\text { culture]. } \\
\text { * Here at the school } \\
\text { when I came to } \\
\text { register the } \\
\text { counselor showed } \\
\text { me my classes and } \\
\text { she explained how } \\
\text { things worked and } \\
\text { she said they could } \\
\text { help me in English } \\
\text { and Spanish. }\end{array}$ & $\begin{array}{l}* \text { My friends talked } \\
\text { a lot about } \\
\text { [culture]... and with } \\
\text { some holidays } \\
\text { teachers talked } \\
\text { about that to the } \\
\text { whole class they } \\
\text { tried to teach us. } \\
\text { * TTeachers] also } \\
\text { made us do } \\
\text { research on culture } \\
\text { in the computer. } \\
\text { * Sometimes } \\
\text { [teachers] would } \\
\text { play movies and } \\
\text { make us write } \\
\text { essays about the } \\
\text { customs we learned } \\
\text { about. } \\
\text { *[My culture is not } \\
\text { valued] a lot } \\
\text { because people } \\
\text { don't know the } \\
\text { culture of Mexico } \\
\text { and people will } \\
\text { laugh. } \\
\text { * Yes [people] } \\
\text { motivate me in }\end{array}$ & $\begin{array}{l}\text { The students learn } \\
\text { about U.S. culture in } \\
\text { history class. } \\
\text { Students that are not } \\
\text { enrolled in a history } \\
\text { class do feel they are } \\
\text { explicitly taught } \\
\text { about culture, but } \\
\text { they are taught about } \\
\text { holidays in their ESL } \\
\text { classes. Other } \\
\text { students learn about } \\
\text { the holidays outside } \\
\text { of school, living in } \\
\text { the U.S. One student } \\
\text { stated that students } \\
\text { are not taught about } \\
\text { U.S. culture and } \\
\text { customs. } \\
\text { Year } 2 \text { students } \\
\text { learned about U.S. } \\
\text { customs from friends } \\
\text { and the teachers } \\
\text { taught about } \\
\text { holidays. Teachers } \\
\text { also had them do } \\
\text { research about } \\
\text { culture and customs } \\
\text { on the computer. } \\
\text { Teachers showed } \\
\text { videos and had }\end{array}$ \\
\hline
\end{tabular}




\begin{tabular}{|c|c|c|c|c|}
\hline & & & $\begin{array}{l}\text { learning both } \\
\text { languages because } \\
\text { there's more } \\
\text { opportunities...I } \\
\text { can have more } \\
\text { opportunities here } \\
\text { and in Mexico and } \\
\text { other countries. } \\
\text { * My mom said to } \\
\text { keep our Spanish } \\
\text { because when we } \\
\text { get jobs we can get } \\
\text { paid more and my } \\
\text { dad says when we } \\
\text { get older and go to } \\
\text { courts and we can } \\
\text { translate and the } \\
\text { judge to the } \\
\text { defendants. } \\
\text { * If you know two } \\
\text { languages you are } \\
\text { worth as much as } \\
\text { two people...my } \\
\text { mom tells me there } \\
\text { are better } \\
\text { opportunities in life } \\
\text { with both } \\
\text { languages. } \\
\text { * Teachers say it } \\
\text { all in English only } \\
\text { my English teacher } \\
\text { tells us both } \\
\text { languages are } \\
\text { important. } \\
\text { * Teachers don't } \\
\text { have much to say } \\
\text { [about being } \\
\text { bilingual] they just } \\
\text { give me the class in } \\
\text { English and don't } \\
\text { say anything about } \\
\text { Spanish. }\end{array}$ & $\begin{array}{l}\text { students write about } \\
\text { U.S. culture. } \\
\\
\text { Students don't feel } \\
\text { their culture is } \\
\text { valued, students are } \\
\text { not taught about their } \\
\text { home cultures and do } \\
\text { not see themselves in } \\
\text { the curricula. } \\
\\
\text { Students believe that } \\
\text { it is important to be } \\
\text { fluent in both } \\
\text { languages to have } \\
\text { more opportunities in } \\
\text { life, both in the U.S. } \\
\text { and in students' home } \\
\text { countries. Families } \\
\text { affirm that there are } \\
\text { more job } \\
\text { opportunities for } \\
\text { bilingual people. } \\
\text { Teachers only teach } \\
\text { in English and do not } \\
\text { speak about the } \\
\text { importance of being } \\
\text { bilingual to students. }\end{array}$ \\
\hline $\begin{array}{c}\text { Teacher } \\
\text { Expectations/ } \\
\text { Pedagogical } \\
\text { Orientation }\end{array}$ & $\begin{array}{l}\text { * When I came in } \\
\text { all my teachers } \\
\text { welcomed me } \\
\text { really good. They } \\
\text { even spoke to me } \\
\text { in Spanish even if } \\
\text { it was only a "hi" } \\
\text { and when they }\end{array}$ & $\begin{array}{l}\text { * Almost all the } \\
\text { teachers I have } \\
\text { speak Spanish [so } \\
\text { they welcomed me]. } \\
\text { * [It is difficult in } \\
\text { class when] they } \\
\text { don't speak }\end{array}$ & $\begin{array}{l}* \text { The teachers } \\
\text { were excited [when } \\
\text { I first came] and } \\
\text { they all tried to } \\
\text { speak to me in } \\
\text { Spanish } \\
\text { * Some teachers did }\end{array}$ & $\begin{array}{l}\text { Teachers welcomed } \\
\text { students when they } \\
\text { entered the school. } \\
\text { Teachers attempted } \\
\text { to speak Spanish to } \\
\text { help students feel } \\
\text { more welcome, and } \\
\text { assigned bilingual }\end{array}$ \\
\hline
\end{tabular}




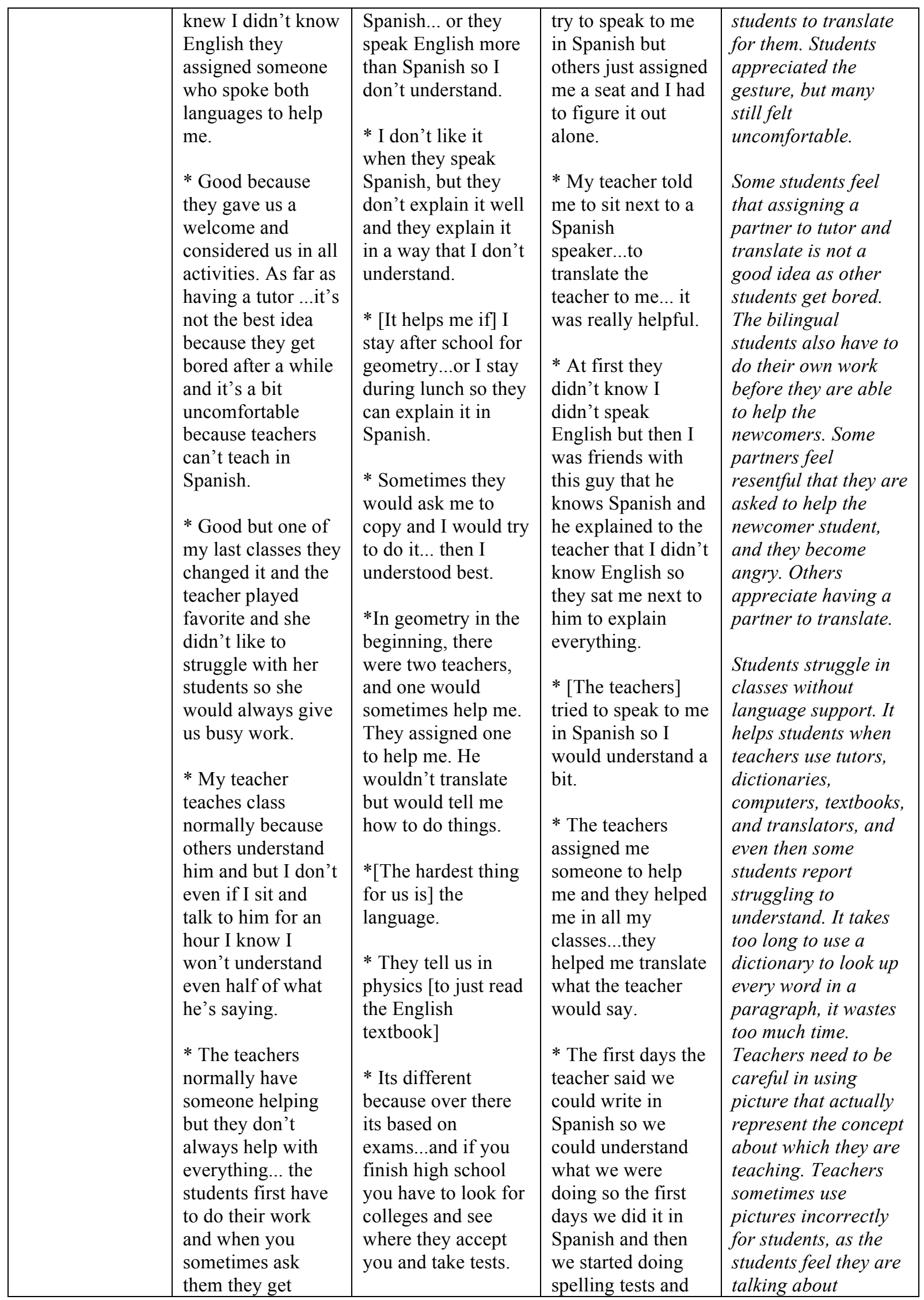




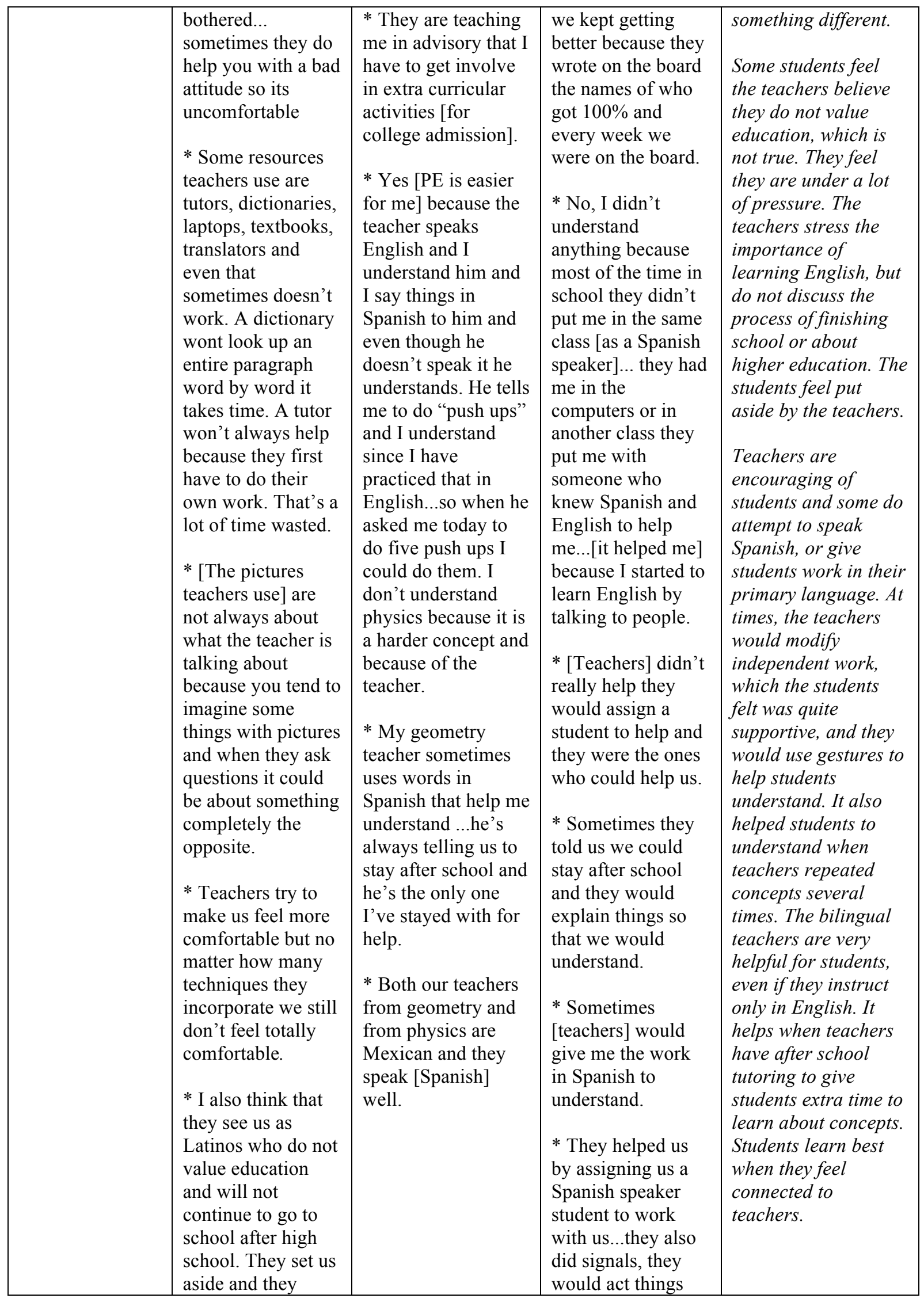




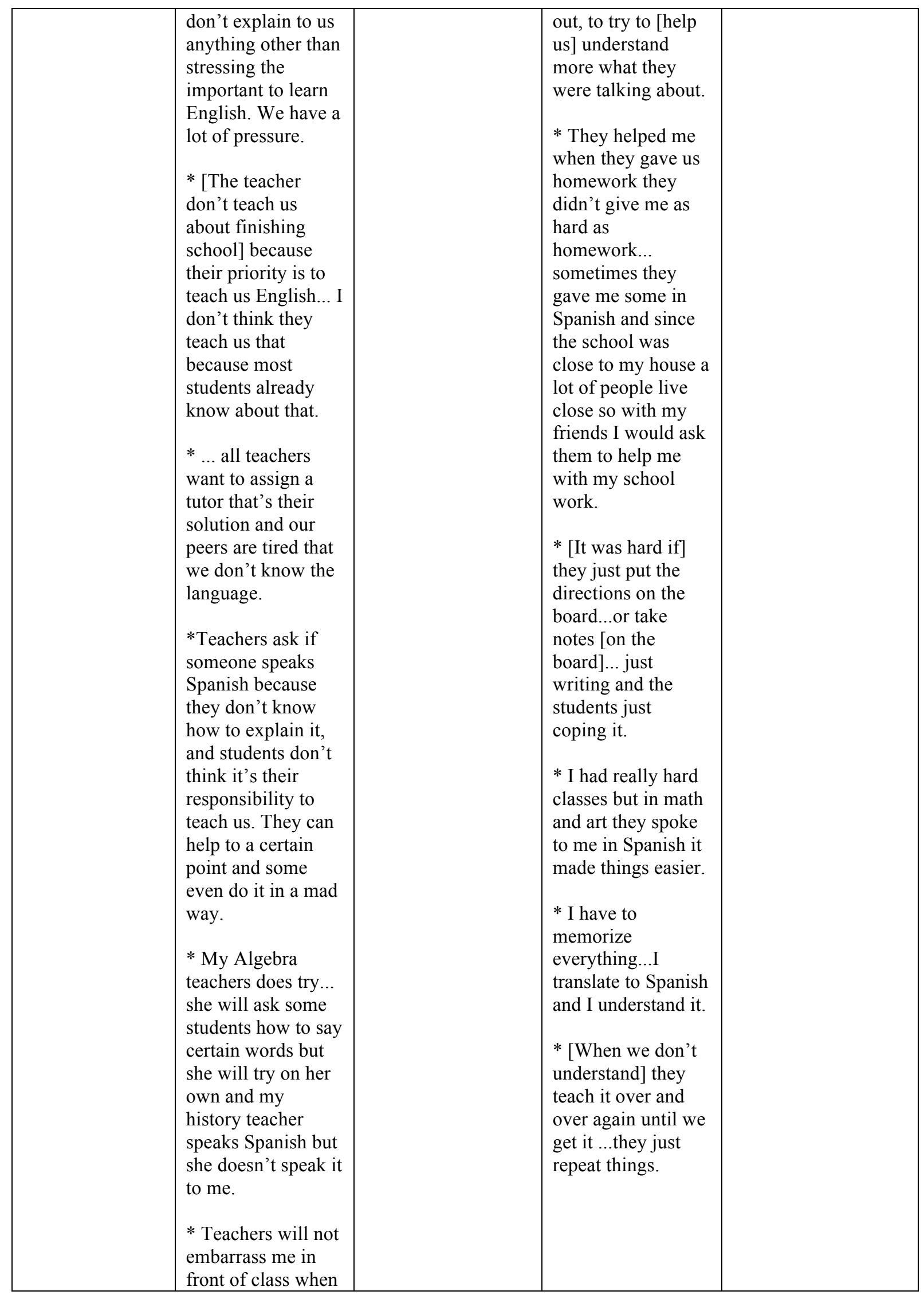




\begin{tabular}{|c|c|c|c|c|}
\hline & $\begin{array}{l}\text { I give an answer } \\
\text { out loud they will } \\
\text { give me good } \\
\text { feedback even } \\
\text { when I clearly } \\
\text { know the answer is } \\
\text { wrong but they do } \\
\text { it so I don't get } \\
\text { discouraged. }\end{array}$ & & & \\
\hline $\begin{array}{c}\text { Academic Self- } \\
\text { Concept }\end{array}$ & $\begin{array}{l}\text { * I feel the same } \\
\text { because I trust } \\
\text { myself and I am } \\
\text { not embarrassed to } \\
\text { talk to people I } \\
\text { don't know... I } \\
\text { socialize well with } \\
\text { other people and } \\
\text { there's people who } \\
\text { like me and want to } \\
\text { help me. } \\
\text { * I don't feel } \\
\text { normal or } \\
\text { comfortable } \\
\text { because I am in a } \\
\text { place I can't } \\
\text { communicate } \\
\text { 100\%. } \\
\text { * We are learning } \\
\text { English with bad } \\
\text { grades but by the } \\
\text { time we fully } \\
\text { understand the } \\
\text { language we will } \\
\text { already have a } \\
\text { record of bad } \\
\text { grades. So we } \\
\text { either learn or do } \\
\text { the work. } \\
\text { * [In easy classes] I } \\
\text { feel good because I } \\
\text { am adjusting } \\
\text { myself to how } \\
\text { things work here, } \\
\text { even though I still } \\
\text { don't know } \\
\text { English. I know } \\
\text { what if I like the } \\
\text { will share it just the }\end{array}$ & $\begin{array}{l}\text { * In my English } \\
\text { class is where I } \\
\text { speak it the most } \\
\text { because I feel more } \\
\text { confident there. } \\
\text { * [The easier classes } \\
\text { make you feel] calm } \\
\text { and confident and } \\
\text { they give you } \\
\text { courage to speak } \\
\text { your mind... I feel } \\
\text { confident as soon as } \\
\text { my first two classes } \\
\text { are over with. } \\
\text { * I don't feel too } \\
\text { nervous anymore } \\
\text { because now we } \\
\text { know what we are } \\
\text { doing for example I } \\
\text { know what to expect } \\
\text { in P.E. We start by } \\
\text { running then do } \\
\text { whatever we want... } \\
\text { then we get a } \\
\text { physical test. } \\
\text { Physics I don't } \\
\text { understand the } \\
\text { language but I still } \\
\text { feel comfortable. } \\
\text { They are all easy for } \\
\text { me its just hard for } \\
\text { me to do my work. } \\
\text { calmer since I } \\
\text { practice the } \\
\text { *[I feel nervous in } \\
\text { harder classes] } \\
\text { because I don't } \\
\text { know how to do the } \\
\text { exercises and I } \\
\text { forget... } \\
\end{array}$ & $\begin{array}{l}\text { *[In easier classes] } \\
\text { I feel better } \\
\text { because I can do it } \\
\text { on my own without } \\
\text { having to ask for } \\
\text { help from other. } \\
\text { *[Harder classes } \\
\text { make me] feel bad } \\
\text { because I can't do } \\
\text { things on my own } \\
\text { and I feel like } \\
\text { crying. } \\
\text { * I felt bad [in hard } \\
\text { classes] and I } \\
\text { would cry out of } \\
\text { frustration that I } \\
\text { couldn't do things. } \\
\text { * [Now] I still get } \\
\text { nervous but I feel a } \\
\text { bit better.. I can do } \\
\text { things on my } \\
\text { own... I still need a } \\
\text { bit of help with } \\
\text { some words but I } \\
\text { don't need } \\
\text { someone by my } \\
\text { side all the time. } \\
\text { * I feel comfortable } \\
\text { [now] because I } \\
\text { understand } \\
\text { English. I feel } \\
\text { better since I know } \\
\text { more words. } \\
\text { felt like I wasn't } \\
\text {.. I felt insecure } \\
\text { because I }\end{array}$ & $\begin{array}{l}\text { Students that feel } \\
\text { confident entering the } \\
\text { school feel able to } \\
\text { maintain confidence. } \\
\text { Most though feel } \\
\text { uncomfortable since } \\
\text { they cannot fully } \\
\text { communicate. In the } \\
\text { beginning, some } \\
\text { worried people were } \\
\text { talking about them } \\
\text { when they couldn't } \\
\text { understand. Students } \\
\text { felt insecure and } \\
\text { embarrassed at } \\
\text { school. } \\
\text { Some students worry } \\
\text { about the poor } \\
\text { grades they receive } \\
\text { as they are acquiring } \\
\text { English. Some don't } \\
\text { know how to remedy } \\
\text { their poor grades. } \\
\text { In the easier classes } \\
\text { the students feel they } \\
\text { have the space to } \\
\text { adjust to life in the } \\
\text { U.S. Students try } \\
\text { harder when they } \\
\text { enjoy the subject and } \\
\text { feel connected and } \\
\text { mafe. The students } \\
\text { want to share what } \\
\text { they have learned } \\
\text { with other students } \\
\text { that need help. In } \\
\text { English class the } \\
\text { students feel the most } \\
\text { comfortable and are } \\
\text { more wiling to } \\
\text { matee and talk } \\
\text { more in }\end{array}$ \\
\hline
\end{tabular}




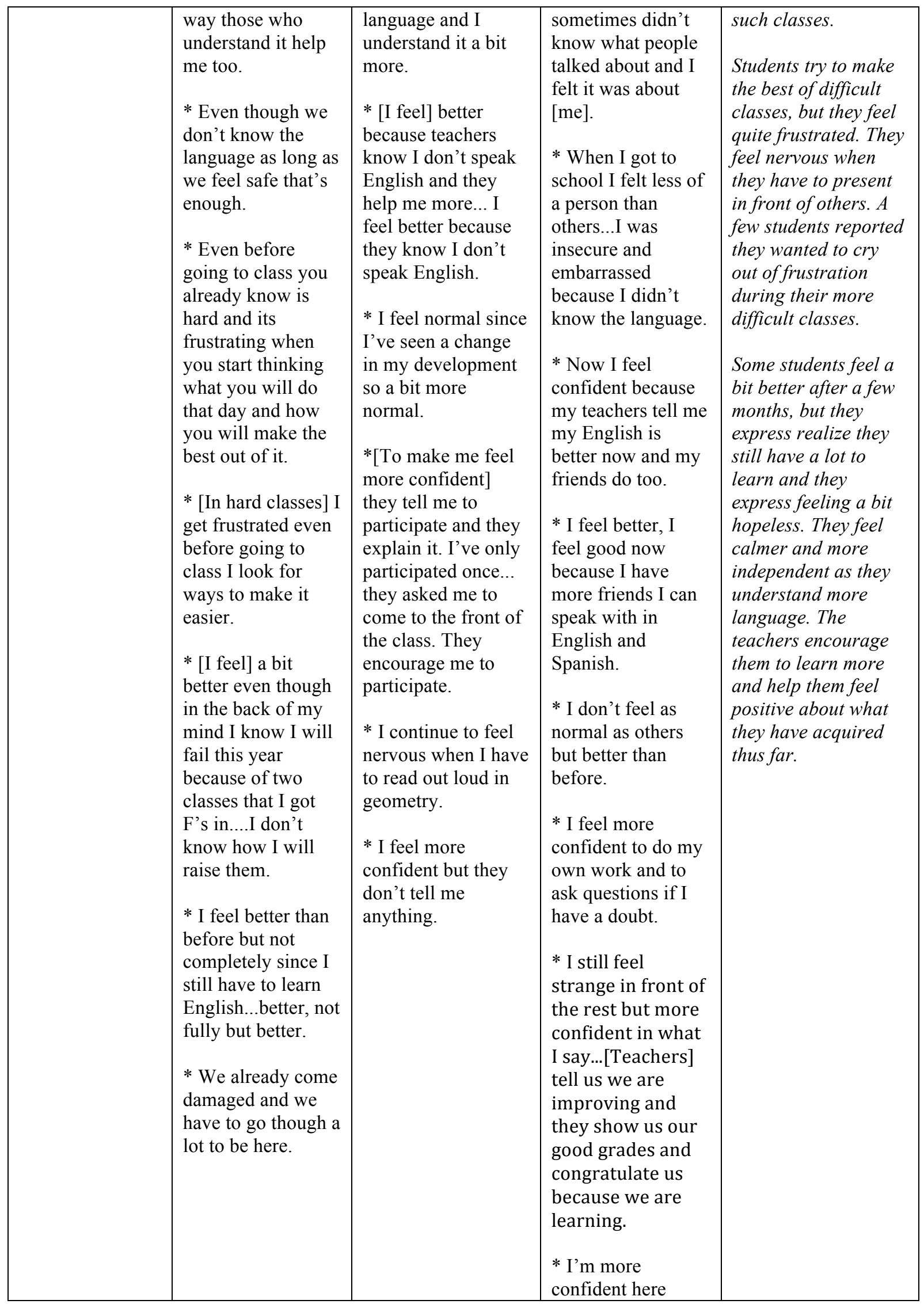




\begin{tabular}{|l|l|l|}
\hline & & [now], because I \\
feel like I have lost \\
a little Spanish and \\
I know my Spanish \\
and English are at \\
the same level... \\
now I prefer it \\
here. \\
* Mrs. M (the \\
counseling \\
secretary) would \\
tell me to come see \\
her in the morning \\
an she would help \\
me...she helped me \\
when I would cry \\
and she would help \\
me learn English. \\
\\
\end{tabular}

